Frank Wolter

\title{
Koordination im internationalen Umweltmanagement
}

Dargestellt an Beispielen aus der Automobilindustrie 


\section{Frank Wolter}

\section{Koordination im internationalen Umweltmanagement}

International tätige Unternehmen sehen sich unterschiedlich restriktiven ökologischen Anforderungen gegenüber. Demgegenüber stehen Forderungen international agierender ökologischer Anspruchsgruppen, möglichst weltweit ein einheitliches ökologisches Vorgehen zu wählen. In diesem Zusammenhang stellt sich die Frage nach einer internationalen Koordination des ökologiegerichteten Unternehmensverhaltens. Die methodisch als konzeptionell-literaturgestützt einzuordnende Arbeit zeigt den ökologiebezogenen Koordinationsbedarf bei internationaler Unternehmenstätigkeit auf und untersucht geeignete Instrumente zur Reduzierung und Deckung dieses Koordinationsbedarfs. Die Arbeit orientiert sich am situativen Kontext der Automobilindustrie und ist mit Erkenntnissen aus Expertengesprächen sowie primär- und sekundärstatistischen Erhebungen unterlegt.

Frank Wolter wurde 1972 in Braunschweig geboren. 1991 trat er sein Studium an der Universität Münster an, welches er 1997 als Diplom-Volkswirt beendete. 1997 nahm der Autor die Tätigkeit am Institut für Marketing der Universität Münster auf. Dort beschäftigte er sich schwerpunktmäßig mit Fragen des Umweltmanagements. Im Juli 2001 erfolgte die Promotion an der Universität Münster. Seit Oktober 2001 ist Frank Wolter Geschäftsführer am Institut für Marketing. 
Koordination im internationalen Umweltmanagement 


\section{SCHRIFTEN ZU MARKETING UND MANAGEMENT}

Herausgegeben von Prof. Dr. Dr. h.c. mult. Heribert Meffert

Band 40

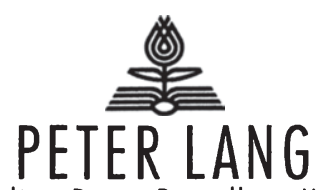

Frankfurt am Main - Berlin. Bern. Bruxelles - New York - Oxford - Wien 


\section{Frank Wolter}

\section{Koordination im internationalen Umweltmanagement \\ Dargestellt an Beispielen aus der Automobilindustrie}

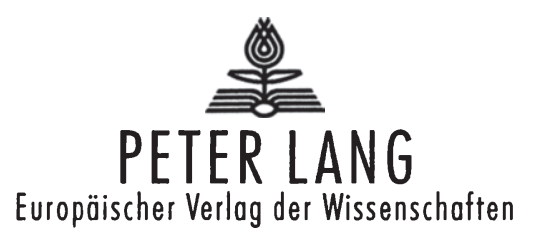


Die Deutsche Bibliothek - CIP-Einheitsaufnahme

Wolter, Frank :

Koordination im internationalen Umweltmanagement :

dargestellt an Beispielen aus der Automobilindustrie / Frank

Wolter. - Frankfurt am Main ; Berlin ; Bern ; Bruxelles ;

New York; Oxford ; Wien : Lang, 2002

(Schriften zu Marketing und Management ; Bd. 40)

Zugl.: Münster (Westfalen), Univ., Diss., 2001

ISBN3-631-39008-4

Open Access: The online version of this publication is published on www.peterlang.com and www.econstor.eu under the international Creative Commons License CC-BY 4.0. Learn more on how you can use and share this work: http://creativecommons. org/licenses/by/4.0.

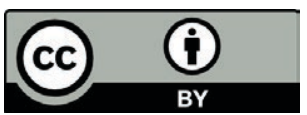

This book is available Open Access thanks to the kind support of ZBW - Leibniz-Informationszentrum Wirtschaft.

Gedruckt auf alterungsbeständigem, säurefreiem Papier.

\author{
D6 \\ ISSN 0176-2729 \\ ISBN3-631-39008-4 \\ ISBN 978-3-631-75063-6 (eBook) \\ (C) Peter Lang GmbH \\ Europäischer Verlag der Wissenschaften \\ Frankfurt am Main 2002 \\ Alle Rechte vorbehalten.
}

Das Werk einschließlich aller seiner Teile ist urheberrechtlich geschützt. Jede Verwertung außerhalb der engen Grenzen des Urheberrechtsgesetzes ist ohne Zustimmung des Verlages unzulässig und strafbar. Das gilt insbesondere für

Vervielfältigungen, Übersetzungen, Mikroverfilmungen und die Einspeicherung und Verarbeitung in elektronischen Systemen.

Printed in Germany 124567

www.peterlang.de 


\section{Meinen Eltern und Angi}


Frank Wolter - 978-3-631-75063-6

Downloaded from PubFactory at 01/11/2019 08:41:13AM

via free access 


\section{Vorwort des Herausgebers}

Trotz fortschreitender Angleichungstendenzen im Umweltrecht unterscheiden sich die ökologiebezogenen Anforderungen an die Fertigung sowie die Produkte in einzelnen Ländern bzw. Märkten derzeit noch sehr stark. Gleichzeitig stehen Unternehmen im internationalen Wettbewerb vor der Notwendigkeit, auch und insbesondere durch konsequente Kostensenkungen ihre Stellung im Wettbewerb zu verteidigen. Eine Verlagerung umweltintensiver Produktionsprozesse in ökologisch weniger restriktive Länder und das Angebot von verschmutzungsintensiven und deshalb kostengüstiger herzustellenden Produkten in diesen Märkten erscheint dabei zumindest auf den ersten Blick ökonomisch vorteilhaft zu sein. Allerdings hat in Anbetracht der weiter zunehmenden internationalen Verflechtung gesellschaftlicher und ökonomischer Systeme auch eine Globalisierung sozio-politischer und ökologischer Anliegen sowie Interessengruppen stattgefunden. Die Proteste der Globalisierungsgegner, die international tätigen Unternehmen u. a. Ökodumping vorwerfen, beweisen dies eindeutig.

Die Beschäftigung von Wissenschaft und Unternehmenspraxis in den Vereinigten Staaten mit dem Thema "Corporate Citizenship“ zeigt die Bedeutung einer länderübergreifenden gesellschaftlichen Legitimität. Diese ist nicht allein vom Unternehmensverhalten in einem Land abhängig, sondern zunehmend auch vom Verhalten an anderen Standorten und in anderen Märkten. Vor diesem Hintergrund gewinnt auch die länderübergreifende Koordination des ökologiegerichteten Unternehmensverhaltens für die internationale Unternehmensführung zunehmend an Bedeutung.

Die wirtschaftswissenschaftliche Forschung hat sich bisher nur in Ansätzen mit dem Thema der internationalen Koordination im Umweltmanagement befasst. Die vorliegende Forschungsarbeit verfolgt die Zielsetzung, einen spezifischen ökologiebezogenen Koordinationsbedarf bei internationaler Unternehmenstätigkeit aufzudecken und geeignete Instrumente zur Reduzierung und Deckung dieses Koordinationsbedarfs zu suchen. Da sich die Ziele und Anforderungen an die internationale, ökologiebezogene Koordination in Bezug auf den Produktund Branchenkontext unterscheiden, orientiert sich der Autor an den spezifischen Rahmenbedingungen der Automobilindustrie. Insbesondere diese Branche steht aufgrund der ökologischen Belastungen, die bei Herstellung, Nutzung und Entsorgung von Automobilen entstehen, im Fokus der Kritik ökologischer Stakeholder. 
Im Rahmen seiner Arbeit ist es dem Verfasser in anschaulicher Weise gelungen, die ökologiebezogenen Herausforderungen in der global tätigen Automobilwirtschaft abzubilden und die Ziele einer internationalen ökologiegerichteten Koordination abzuleiten. Dabei werden die Ziel- und Strategieabhängigkeit der Koordinationskonzepte zutreffend herausgearbeitet und interessante Anwendungsperspektiven für die Gestaltung der Koordination im internationalen Umweltmanagement aufgezeigt. Ein Fokus der Arbeit liegt auf der systematischen Darstellung von Koordinationsinstrumenten zur Reduzierung und Deckung des internationalen, ökologiebezogenen Koordinationsbedarfs. Dazu zeigt der Verfasser das Spektrum möglicher Koordinationsinstrumente im internationalen Umweltmanagement auf und erweitert es. Zur Strukturierung werden diese Maßnahmen nach strukturellen, technokratischen und personenorientierten Koordinationsmaßnahmen systematisiert. Anwendungsbeispiele aus der Automobilindustrie zu den Koordinationsinstrumenten geben dabei einen praxisbezogenen Einblick in den derzeitigen Stand der internationalen, ökologiegerichteten Koordination.

Die Leistung der Arbeit liegt weniger im konzeptionell-theoretischen Bereich als in der Übertragung bewährter Koordinationskonzepte der Organisationsforschung auf die spezifischen Problemstellungen des internationalen Umweltmanagements im Automobilbereich. Die Arbeit zeichnet sich nicht nur durch eine umfangreiche Literaturforschung, sondern auch durch eigenständige empirische Analysen und Befragungen von Experten aus der Automobilindustrie aus. Es ist zu wünschen, dass das Werk auf ein entsprechendes Interesse in einschlägigen Fachkreisen stößt. 


\section{Vorwort des Verfassers}

Es besteht mittlerweile kein Zweifel darüber, dass die Bewältigung der ökologischen Probleme eine der bedeutsamsten Herausforderungen sowohl in Industrie- als auch in Schwellen- und Entwicklungsländern darstellt. Unternehmen stehen dabei in Zeiten der fortschreitenden Globalisierung nicht allein in den Ländern mit anspruchsvollen gesetzlichen Umweltschutzanforderungen in der Verantwortung, einen eigenständigen Beitrag zur Lösung produktions- und produktbezogener Umweltprobleme zu erbringen. Vielmehr wird eine Abkehr von national isolierten Umweltschutzlösungen in ökologischen Vorreiterstaaten hin zu einem international möglichst einheitlichen und proaktiven Umweltmanagement von unterschiedlichen Anspruchsgruppen gefordert. Unternehmen stehen dabei allerdings oft im Spanungsfeld zwischen der Aufrechterhaltung der internationalen Wettbewerbsfähigkeit und der Sicherstellung der gesellschaftlichen Legitimität. In diesem Zusammenhang gewinnt die Frage nach einer internationalen Koordination des ökologiegerichteten Unternehmensverhaltens zunehmend an Bedeutung für die Unternehmensführung. Es ist deshalb um so erstaunlicher, dass bislang umfassende Untersuchungen der Einflussfaktoren auf das internationale ökologische Unternehmensverhalten sowie der koordinationsspezifischen Gestaltungsoptionen in der wirtschaftswissenschaftlichen Literatur weitgehend fehlen.

Vor diesem Hintergrund wird in der vorliegenden Schrift ein spezifischer ökologiebezogener Koordinationsbedarf bei internationaler Unternehmenstätigkeit abgeleitet sowie geeignete Instrumente zur Reduzierung und Deckung dieses Koordinationsbedarfs untersucht. Weiterhin werden Ausgestaltungsempfehlungen für Umweltmanagementsysteme als Konzeptionen zur internationalen ökologiegerichteten Koordination in Bezug auf unterschiedliche strategische Ausrichtungen im internationalen Umweltmanagement abgeleitet. Die Untersuchung orientiert sich dabei am situativen Kontext der Automobilindustrie, da die mittlerweile stark internationalisierte Automobilindustrie trotz großer ökologischer Fortschritte weiterhin aufgrund vielfältiger ökologischer Probleme international in der Kritik ökologischer Anspruchsgruppen steht.

Methodisch ist die Arbeit als konzeptionell-literaturgestützt einzuordnen, wobei die konzeptionellen Überlegungen auf verschiedene Theoriekonzepte aus dem internationalen Management, der Organisationsforschung und nicht zuletzt aus dem Umweltmanagement zurückgreifen. Die theoretischen Ansätze werden mit Erkenntnissen aus im Vorfeld durchgeführten Expertengesprächen, einer sys- 
tematischen Auswertung von Unternehmensberichten, Pressenotizen und ökologiebezogenen Ratingergebnissen sowie primär- und sekundärstatistischen Erhebungen unterlegt. Umfangreiche Recherchen und tiefergehende Einblicke in die Herausforderungen und Abläufe im Umweltmanagement eines international tätigen Automobilherstellers wurden mir dabei durch einen sechswöchigen Aufenthalt in der Umweltschutzabteilung der VolkswAGEN AG ermöglicht. Dort sowie bei FORD Deutschland in Köln führte ich eine Reihe von Expertengesprächen. Weiterhin konnte ich auf die Ergebnisse der Erhebung zum „Europäischen Umweltmanagement-Barometer 1998“, einem europäischen Gemeinschaftsprojekt über die ökologische Verhaltensausrichtung von mehr als 2.500 Unternehmen aus dem verarbeitenden Gewerbe, zurückgreifen, an dem ich beteiligt war.

Die vorliegende Arbeit wurde im Juli 2001 von der Wirtschaftswissenschaftlichen Fakultät der Westfälischen Wilhelms-Universität als Dissertationsschrift angenommen. Die Abfassung der vorliegenden Arbeit war dabei nur mit vielfältiger Unterstützung möglich. Mein besonderer Dank gilt dabei zunächst meinem akademischen Lehrer und Doktorvater, Herrn Professor Dr. Dr. h. c. mult. Heribert Meffert, der bereits in 1997 zu Beginn meiner wissenschaftlichen Arbeit am Institut für Marketing die Themenstellung anregte. In den folgenden Jahren ermöglichte er mir nicht nur die Mitarbeit am Europäischen Umweltmanagement-Barometer und den Aufenthalt bei der VOLKSWAGEN AG, sondern er lieferte auch stets entscheidende Impulse für mein Dissertationsvorhaben. Darüber hinaus förderte er meine fachliche und persönliche Entwicklung in vielfältiger Weise. Herrn Professor Dr. Klaus Backhaus gebührt mein ausdrücklicher Dank für die Übernahme des Zweitgutachtens.

Die fachliche und insbesondere persönliche Hilfestellung meiner Kollegen vom Institut für Marketing möchte ich hier hervorheben. Durch ihre Unterstützung konnte ich größtenteils von anderweitigen Verpflichtungen im Institut während der Abfassung der Arbeit entlastet werden. Nicht zu unterschätzen ist dabei auch der "moralische Beistand" aller Kollegen des Instituts aber auch so mancher Kollegen aus dem Marketing Centrum Münster. Namentlich seien an dieser Stelle insbesondere Herr Dr. Martin Koers und Frau Eva Figge herausgestellt. Herr Dr. Koers stand mir in fachlicher aber auch persönlicher Hinsicht als Coach und Freund zur Seite und gab mir wertvolle Anregungen für die Arbeit. Frau Figge möchte ich für Ihre gründliche Durchsicht des Manuskripts danken. 
Meinen Dank möchte ich weiterhin Frau Dr. Christiane von Finckenstein-Wang gegenüber zum Ausdruck bringen. Ihrer exzellenten Betreuung und ihrem Engagement während meiner Zeit bei VoLKSWAGEN - aber auch in der Zeit danach - habe ich vieles zu verdanken. Erwähnt seien an dieser Stelle auch die stets aufgeschlossenen und hilfsbereiten Mitarbeiter der Abteilung sowie die auskunftsbereiten Experten, die mir ihre Zeit trotz mancher terminlicher Engpässe gerne gewidmet haben. In diesem Zusammenhang möchte ist ebenfalls Herrn Krüger von FORD Deutschland für das umfangreiche Expertengespräch danken.

Nicht zuletzt bin ich meinen Eltern und meiner Großmutter sowie meiner langjährigen Lebensgefährtin Frau Dott. Angela Consolo zutiefst dankbar. Ohne den Rückhalt, das Verständnis und die Liebe dieser Menschen hätte ich die vielfältigen Herausforderungen der Dissertation kaum meistern können.

Frank Wolter 
Frank Wolter - 978-3-631-75063-6

Downloaded from PubFactory at 01/11/2019 08:41:13AM

via free access 


\section{Inhaltsverzeichnis}

Abbildungsverzeichnis................................................................. XVII

Tabellenverzeichnis .....................................................................

Abkürzungsverzeichnis.............................................................. XXIII

A. Wirtschaftswissenschaftliche Zugänge zur Koordination des öko logiegerichteten Verhaltens als Herausforderung an die inter nationale Unternehmensführung

1. Notwendigkeit einer Koordination des ökologiegerichteten Verhaltens bei internationaler Unternehmenstätigkeit ....................................... 1

2. Ökologiegerichtetes Verhalten international tätiger Unternehmen in der wirtschaftswissenschaftlichen Forschung.

2.1 Analyse der Wechselwirkungen zwischen Umweltschutz und internationaler Unternehmenstätigkeit

2.2 Betriebswirtschaftliche Ansätze zur Berücksichtigung des Umweltschutzes bei internationaler Unternehmenstätigkeit

3. Koordination als Aufgabe an der Schnittstelle zwischen internationalem

Management und Umweltmanagement.

4. Ziel und Gang der Untersuchung

B. Ökologiebezogener Koordinationsbedarf bei internationaler Ge schäftstätigkeit in der Automobilindustrie

1. Automobilindustrie als situativer Kontext........................................33

1.1 Internationale Situation der Automobilindustrie............................33

1.2 Ökologische Herausforderungen in der Automobilindustrie .............43 43

2. Interdependenzen als Determinanten des Koordinationsbedarfs ............50

3. Einfluss exogener Kontextfaktoren auf den ökologiebezogenen internationalen Koordinationsbedarf.......................................................6 60

3.1 Umsystemähnlichkeit ...............................................61

3.1.1 Natürliche Gegebenheiten .........................................61

3.1.2 Ökologische Betroffenheit durch lokale öffentliche Anspruchsgruppen....

3.1.3 Ökologische Betroffenheit durch lokale politische Anspruchsgruppen.

3.1.4 Ökologische Betroffenheit durch den Markt 


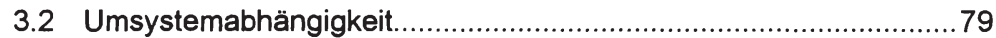

3.2.1 Okologische Betroffenheit durch internationale öffentliche Anspruchsgruppen.

3.2.2 Ökologische Betroffenheit durch internationale institutio nelle und politische Anspruchsgruppen.

3.2.3 Ökologische Betroffenheit durch internationale Marktteilnehmer

3.2.4 OKologische Betroffenheit durch international tätige Wett bewerber

3.3 Ökologische Betroffenheit im Überblick

4. Einfluss endogener Kontextfaktoren auf den internationalen ökologiebezogenen Koordinationsbedarf 93

4.1 Umweltschutz als Unternehmensziel ...........................................93

4.2 Strategische Basisausrichtung im Umweltschutz .........................101

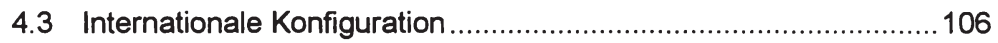

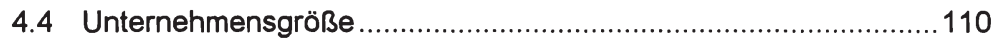

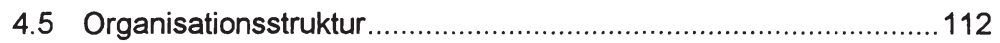

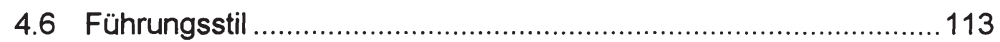

5. Systematisierung der Interdependenzen im situativen Beziehungs rahmen

C. Koordinationskonzeptionen im internationalen Umweltmanagement.121

1. Ziele einer internationalen ökologiegerichteten Koordination . 122

2. Basisstrategische Optionen im internationalen Umweltmanagement als Ausgangspunkt der Koordination.

2.1 Global-proaktive Umweltschutzstrategie .....................................133

2.2 Globale Kostenminimierungsstrategie .......................................142

2.3 Multinationale Umweltschutzstrategie.......................................147

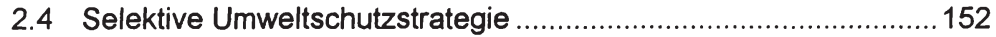

3. Instrumente zur Koordination des internationalen ökologiegerichteten

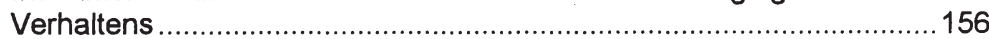

3.1 Systematisierung des Koordinationsinstrumentariums .................. 157

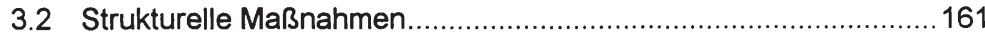

3.2.1 Koordinationsbedarfsreduzierende strukturelle Maß nahmen.....

3.2.2 Koordinationsbedarfsdeckende strukturelle Maß nahmen. 


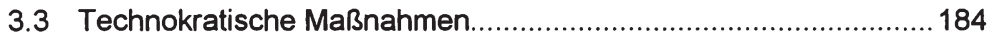

3.3.1 Koordinationsbedarfsreduzierende technokratische Mechanismen.

3.3.2 Koordinationsbedarfsdeckende technokratische Maßnahmen .......................................................... 199

3.4 Personenorientierte Maßnahmen................................................214

3.4.1 Koordinationsbedarfsreduzierende personelle $\mathrm{Ma} \beta$

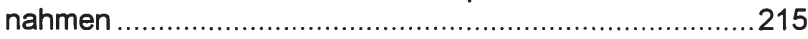

3.4.2 Koordinationsbedarfsdeckende personale Maßnahmen ...221

4. Umweltmanagementsysteme als Koordinationskonzeptionen ...............223

4.1 Aufbau eines Umweltmanagementsystems ................................223

4.2 Ausgestaltungsformen der ökologiegerichteten Koordinations-

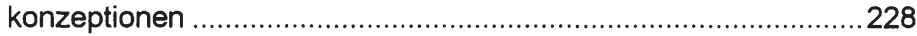

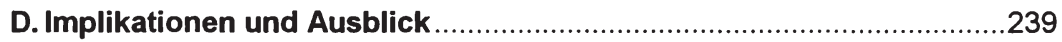

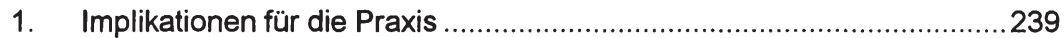

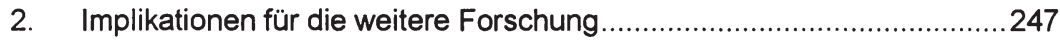


Frank Wolter - 978-3-631-75063-6

Downloaded from PubFactory at 01/11/2019 08:41:13AM

via free access 


\section{Abbildungsverzeichnis}

Abbildung 1: Unterschiedliche Perspektiven zum ökologiebezogenen internationalen Unternehmensverhalten

Abbildung 2: Wechselwirkungen von internationaler Unternehmenstätigkeit und Umweltschutz

Abbildung 3: Integriertes Netzwerk nach NOHRIA und GHOSHAL................. 17

Abbildung 4: Phasen einer ökologischen Unternehmensentwicklung.....

Abbildung 5: Internationales integriertes Produktionsnetzwerk am Bei spiel des VOLKSWAGEN-Konzerns.

Abbildung 6: Ökologische Belastungen durch die Automobilindustrie ......... 44

Abbildung 7: VOC-Reduktion ......................................................... 48

Abbildung 8: Ökologische Handlungsfelder in der Automobilindustrie ......... 49

Abbildung 9: Transformationsprozess von ökologischen Belastungen zu ökologischer Betroffenheit......................................... 55

Abbildung 10: Differenzierung ökologischer Anspruchsgruppen .................. 56

Abbildung 11: Rückkopplungen im internationalen Umweltmanagement ...... 57

Abbildung 12: Ableitung des internationalen ökologiebezogenen Koordinationsbedarfs.

Abbildung 13: Einschätzung der persönlichen gesundheitlichen Beein trächtigung durch ökologische Probleme im internationalen Vergleich

Abbildung 14: Grund der Besorgnis über den Zustand der ökologischen Umwelt im internationalen Vergleich

Abbildung 15: Verzicht auf ein Produkt aus ökologischen Gründen im internationalen Vergleich

Abbildung 16: Entscheidende Kriterien für den Neuwagenkauf in Deutsch land 1999

Abbildung 17: Wichtigkeit des Kraftstoffverbrauchs beim Neuwagenkauf im internationalen Vergleich.

Abbildung 18: Zusätzliche Zahlungsbereitschaft für ein Automobil mit um weltverträglicherem Antriebskonzept

Abbildung 19: Corporate Watch: Greenwash Sweepstakes Award 2000 ...... 82

Abbildung 20: Ökologische Betroffenheit der europäischen Automobil industrie durch unterschiedliche Anspruchsgruppen.

Abbildung 21: Einfluss von Umweltgesetzen auf die Unternehmensleistung. 95

Abbildung 22: Zielbeziehungen zwischen ökologischen und anderen Unternehmenszielen in der europäischen Automobilindustrie 99

Abbildung 23: Internationaler Koordinationsbedarf in Bezug auf strate gische Dimensionen umweltorientierter Basisstrategien 
Abbildung 24: Ökologische Basisausrichtung der deutschen Automobil-

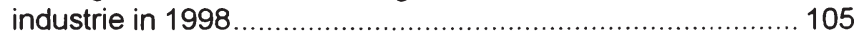

Abbildung 25: Typologisierung von internatonal tätigen Unternehmen ........ 110

Abbildung 26: Ausgewählte Interdependenzen zwischen den Kontext faktoren

Abbildung 27: Profil der Ausprägung unterschiedlicher Kontextfaktoren in der Automobilindustrie zur Ableitung des internationalen ökologiebezogenen Koordinationsbedarfs

Abbildung 28: Ökologische Chancen und Risiken der Automobilhersteller.. 128

Abbildung 29: Umweltpolitik der VoLKSWAGEN AG

Abbildung 30: Strategische Basisorientierungen im internationalen Umweltmanagement

Abbildung 31: Situativer Kontext einer global-proaktiven Umweltschutzstrategie

Abbildung 32: Ausprägungen der global-proaktiven Umweltschutzstrate gie

Abbildung 33: Situativer Kontext für eine globale Kostenminimierungsstrategie 143

Abbildung 34: Ausprägungen einer globalen Kostenminimierungsstrategie 146

Abbildung 35: Situativer Kontext für eine multinationale Umweltschutzstrategie 148

Abbildung 36: Ausprägungen multinationaler Umweltschutzstrategien........ 150

Abbildung 37: Situativer Kontext für eine selektive Umweltschutzstrategie . 153

Abbildung 38: Ausgestaltung der selektiven Umweltschutzstrategie ........... 156

Abbildung 39: Strukturelle Koordinationsmaßnahmen ................................ 162

Abbildung 40: Umweltsteuerkreis der BMW-Gruppe................................. 179

Abbildung 41: Umweltmanagementorganisation der VoLKSWAGEN AG........ 181

Abbildung 42: Verantwortlichkeiten im Umweltschutz.............................. 182

Abbildung 43: Technokratische Koordinationsmaßnahmen ........................ 184

Abbildung 44: Szenarien über mögliche ökologische Orientierungen im europäischen Automobilmarkt......................................... 191

Abbildung 45: Früherkennungsinstrumente des Umwelt-Radar ................... 194

Abbildung 46: Diskussionen und Ereignisse des Jahres 1999 im Themen feld Umwelt und Verkehr - Die Top 20 .............................. 195

Abbildung 47: Standardisierung von ökologiegerichteten Marketingent scheidungen in der deutschen Automobilindustie

Abbildung 48: Aufbau eines Umweltmanagementhandbuches .................... 210

Abbildung 49: Personenorientierte Koordinationsmaßnahmen ................... 215

Abbildung 50: Elemente eines Umweltmanagementsystems (UMS) ........... 226 
Abbildung 51: Internationales Umweltmanagementsystem bei global-proaktiver Basisstrategie

Abbildung 52: Strategiegerechte Ausgestaltung von Koordinationskonzeptionen im internationalen Umweltmanagement.

Abbildung 53: Erweiterung einer Balanced Scorecard um ökologische Zielsetzungen

Abbildung 54: Integrationsmodell zur Verbindung der Outside-in- und Inside-out-Perspektive im Umweltmanagement 
Frank Wolter - 978-3-631-75063-6

Downloaded from PubFactory at 01/11/2019 08:41:13AM

via free access 


\section{Tabellenverzeichnis}

Tabelle 1: Entwicklungslinien in der Automobilindustrie

Tabelle 2: Internationale Abgasvorschriften für Diesel-Pkw im Vergleich.

Tabelle 3: $\quad$ Ökologische Konzeptfahrzeuge unterschiedlicher Hersteller .. 91

Tabelle 4: $\quad$ Überblick über das Koordinationsinstrumentarium im internationalen Umweltmanagement.

Tabelle 5: $\quad$ Typen von Umweltmanagementsystemen und ihre Auspräungsformen 
Frank Wolter - 978-3-631-75063-6

Downloaded from PubFactory at 01/11/2019 08:41:13AM

via free access 


\section{Abkürzungsverzeichnis}

a. $M$

a. a. $\mathrm{O}$.

am Main

AG

am angegebenen Ort

Abb.

Aktiengesellschaft

Anm. d. Verf

Abbildung

Aufl.

Anmerkung des Verfassers

Asw

Auflage

AFTA

Absatzwirtschaft

Asian Free Trade Area

Bd.

Band

BSC

Balanced Scorecard

bspw.

beispielsweise

bzw.

beziehungsweise

ca.

$\mathrm{CH}_{4}$

circa

$\mathrm{CO}$

Methan

$\mathrm{CO}_{2}$

Kohlenmonoxid

Kohlendioxid

d. $h$.

das heißt

DBW

Die Betriebswirtschaft

DIN

Deutsches Institut für Normung

Diss.

Dissertation

DM

Deutsche Mark

EMAS

Environmental Management and Audit Scheme

e. V.

eingetragener Verein

et al.

et alii, et alia, et alteri

etc.

et cetera

EU

Europäische Union

E.B.E.B.

European Business Environmental Barometer

f., ff.

folgende, fortfolgende

FDI

foreign direct investment

GATT

General Agreement on Tariffs and Trade

ggf.

gegebenenfalls

HBM

Harvard Business Manager

HBR

HC

Harvard Business Review

Kohlenwasserstoff

Hrsg.

Herausgeber 
IAA Internationale Automobilausstellung

i. d. R. in der Regel

i. $S$.

im Sinne

insb.

insbesondere

ICC

International Chamber of Commerce

ISO

International Organization for Standardization

JdAV

Jg.

JOAR

JoBR

JoM

JoMR

Jahrbuch der Absatz- und Verbrauchsforschung

Jahrgang

Journal of Advertising Research

Journal of Business Research

Journal of Marketing

Journal of Marketing Research

Kap. Kapitel

Mio. Millionen

Mrd. Milliarden

MS Marketing Science

$\begin{array}{ll}\mathrm{N}_{2} \mathrm{O} & \text { Distickstoffoxid } \\ \text { NAFTA } & \text { North American Free Trade Association } \\ \mathrm{NGO} & \text { Non-governmental Organization } \\ \mathrm{No} . & \text { Number } \\ \mathrm{NO}_{x} & \text { Stickstoffoxid } \\ \mathrm{Nr} . & \text { Nummer }\end{array}$

OECD Organization for Economical Cooperation and Development

o. ä.

o. V.

S.

sog.

Sp.

SUV

Tab.

u. a.

UN

UNEP

Univ.

vgl.

vol.

vs. oder ähnliche ( $n, r, s)$

ohne Verfasser

Seite

so genannte $(n, r, s)$

Spalte

Sporty Utility Vehicle

Tabelle

und andere, unter anderem

United Nations

United Nations Environmental Programme

Universität

vergleiche

Volume

Versus 
WBCSD World Business Council of Sustainable Development

WiSt

WISU

Wirtschaftswissenschaftliches Studium

WTO

Das Wirtschaftsstudium

World Trade Organization

z. B.

ZfB

zum Beispiel

ZfbF

ZFP

\section{Zeitschrift für Betriebswirtschaft}

Zeitschrift für betriebswirtschaftliche Forschung

Zeitschrift für Forschung und Praxis 
Frank Wolter - 978-3-631-75063-6

Downloaded from PubFactory at 01/11/2019 08:41:13AM

via free access 


\section{A. Wirtschaftswissenschaftliche Zugänge zur Koordination des ökologiegerichteten Verhaltens als Herausforderung an die internationale Unternehmensführung}

\section{Notwendigkeit einer Koordination des ökologiegerichteten Ver- haltens bei internationaler Unternehmenstätigkeit}

Das Phänomen der Globalisierung und die zunehmende anthropogene Belastung der natürlichen Umwelt waren in den vergangenen Jahrzehnten prägende gesellschaftliche Themenbereiche, welche insbesondere auch die strategische Unternehmensführung vor neue Aufgaben gestellt haben. ${ }^{1}$ Dabei steht aktuell das Spannungsfeld zwischen internationaler Unternehmenstätigkeit und ökologischer Nachhaltigkeit im Fokus der Betrachtung. ${ }^{2}$

Ein zentrales Element zur Sicherung von Umweltschutz im globalen Wettbewerb ist der internationale ökologische Ordnungsrahmen. In diesem Zusammenhang können die Stockholm Declaration on the Human Environment $(1972)^{3}$ und insbesondere die Rio Declaration on Environment and Development (1992) ${ }^{4}$ als wichtige internationale Konventionen als internationale Leit-

1 Vgl. bspw. Hey, Ch.; Schleicher-Tappeser, R.: Nachhaltigkeit trotz Globalisierung Handlungsspielrăume auf regionaler, nationaler und europăischer Ebene, Berlin u. a. 1998, S. 13.

Vgl. Althammer, W.: Umweltpolitik, internationaler Handel und Wettbewerbsfähigkeit, in: Meffert, H.; Gisholt, O. (Hrsg.): Managementperspektiven und Managementausbildung, Leipzig 1997; Franke, A.: Freier Welthandel und umweltverträgliche Entwicklung, in: IFO Schnelldienst, 21/96, S. 15 - 24; Moltke, K. v.; IISD (Hrsg.): International Environmental Management, Trade Regimes and Sustainability, Winnipeg 1996.

Vgl. zu der Konferenz in Stockholm und den dort aufgetretenen Konflikten zwischen Umweltschutz und Entwicklungszielen Olson, S. S.: International environmental standards handbook, Boca Raton, London, New York, Washington D. C. 1999, S. 8 f.; United Nations (Hrsg.): Report of the United Nations Conference on the Human Environment, Stockholm 1972, http://www.unep.org/Documents/Default.asp?DocumentID=97\&ArticleID= 1501 [11.09.99] sowie Kellersmann, B.: Die gemeinsame, aber differenzierte Verantwortlichkeit von Industriestaaten und Entwicklungsländern for den Schutz der globalen Umwelt, Berlin u. a. 2000, S. 5 f.

Seit der United Nations Conference on Environment and Development von Rio de Janeiro aus dem Jahr 1992 gilt Sustainable Development als international akzeptierte Grundorientierung, die für eine nachhaltige wirtschaftliche, gesellschaftliche und okologische Entwicklung steht. In der Rio Declaration on Environment and Development wurden verschiedene Basisprinzipen entwickelt, die das Leitbild einer nachhaltigen Entwicklung grob skizzieren. Darunter finden sich auch Prinzipien, die einen Bezug zum Umgang mit ókologischen Problemen auf internationaler Ebene beinhalten: die Grundsătze 7, 11 und 12. Vgl. United Nations (Hrsg.): Rio Declaration on Environment and Development, Rio de Janeiro 1992, http://www.unep.org/ Documents/Default.asp? DocumentID=78\&Article/D=1163 [11.09.99]. 
linien im Umweltschutz gelten. ${ }^{5}$ Mit diesen Deklarationen wurde unter dem Leitbild des Sustainable Development ${ }^{6}$ versucht, eine Balance zwischen freiem internationalen Handel, ökonomischem Wachstum sowie ökologischen Zielen bei Beibehaltung der nationalen Souveränität der einzelnen Staaten und gleichzeitiger Förderung internationaler Kooperation zu finden. ${ }^{7}$

„States have, in accordance with the Charter of the United Nations and principles of international law, the sovereign right to exploit their own resources pursuant to their own environmental [and developmental] policies, and the responsibility to ensure that activities within their jurisdiction or control do not cause damage to the environment of other States or of areas beyond the limits of national jurisdiction." ${ }^{8}$

Den Kern dieses ökologischen Ordnungsrahmens bildet eine Vielzahl bi- und multilateraler Verträge zum Schutz regionaler und globaler Umweltgüter. Darunter befinden sich auch einige bedeutende multinationale Umweltschutzabkommen, die nahezu weltweite Gütigkeit erreicht haben. ${ }^{9}$ Das Scheitern der Verhandlungen um das Kyoto-Protokoll in Den Haag zeigt aber deutlich die Schwierigkeiten, Umweltschutzabkommen mit dem Ziel international gültiger und rechtsverbindlicher Vorgaben abzuschließen. ${ }^{10}$

Seit dieser Zeit wird die Ökologiediskussion verstärkt unter dem Begriff der Nachhaltigkeit bzw. Sustainability geführt, dabei ist die Diskussion an Konzepte eines qualitativen Wachstums angelehnt. Vgl. Meffert, H.; Kirchgeorg, M.: Marktorientiertes Umweltmanagement: Konzepte - Strategie - Implementierung mit Praxisfällen, 3. Aufl., Stuttgart 1998, S. $31 \mathrm{ff}$.

Die World Commission on Environment and Development, auch BRUNDTLAND-Kommission genannt, systematisierte in dem Report "Our common future 1987 erstmals das Konzept eines Sustainable Development. Unter Sustainable Development versteht man nach diesem Report: „Development that meets the needs of the present without compromising the ability of future generations to meet their own needs." World Commission on Environment and Development (Hrsg.): Our common future, Oxford 1987. Der BRUNDTLAND-Bericht wurde die Grundlage aller internationalen okologiebezogenen Aktivităten der Vereinten Nationen und regte auf unterschiedlichsten gesellschaftlichen Ebenen einen vertieften Dialog über das Thema Sustainable Development an.

Vgl. Olson, S. S.: International environmental standards handbook, a. a. O., S. 9.

Dieses Zitat gibt das Prinzip 21 der Deklaration von Stockholm bzw. das 2. Prinzip der Deklaration von Rio wieder. Dabei wurde das Prinzip 21 von Stockholm nur um entwicklungspolitische Ziele ergänzt. Die Ergänzung der Deklaration von Rio ist in Klammern beigefügt. Vgl. Olson, S. S.: International environmental standards handbook, a. a. O., S. 34 und 36 .

9 Vgl. dazu bspw. Troge, A.: Schritte zu einer nachhaltigen Weltumweltordnung, in: Fichter, K.; Schneidewind, U. (Hrsg.): Umweltschutz im globalen Wettbewerb - Neue Spielregeln für das grenzenlose Unternehmen, Berlin u. a. 2000, S. 16.

Derartige Verhandlungen werden u. a. vom sog. Trittbrettfahrerproblem erschwert. Die Emissionsverringerung eines Landes ist bei globalen externen Effekten ein internationales offentliches Gut, dessen Nutzen über alle Staaten streut, auch wenn sie sich nicht an einer Emissionsreduzierung beteiligt haben. Vgl. Althammer, W.: Umweltpolitik, internationaler 
Da insgesamt nur für wenige ökologische Probleme multinationale Vertrăge zustande gekommen sind, kann das internationale Umweltrecht nur als „Flickenteppich" ${ }^{11}$ bezeichnet werden. ${ }^{12}$ Demgegenüber geht ein erheblicher Einfluss von nationalen Umweltschutzgesetzen auf die internationale Unternehmenstätigkeit aus. Der sich maßgeblich aufgrund national unterschiedlicher umweltrechtlicher Regelungen ergibt. ${ }^{13}$ Umweltschutz wird aufgrund verschiedener sozio-kultureller, politisch-rechtlicher sowie ökonomischer und ökologischer Gegebenheiten offensichtlich national unterschiedlich diskutiert, definiert und implementiert. Die nationalen Umweltpolitiken beziehen sich dabei primär auf nationale Umweltprobleme bzw. nationale Umweltschutzinteressen. Dies bedeutet nicht, dass nationale Umweltstandards keine internationalen ökologischen Probleme ansprechen, vielmehr stehen diese Bestimmungen oft in keinem Zusammenhang mit Umweltstandards aus anderen Staaten. Zwar zeigen internationale Untersuchungen über den Einsatz von umweltpolitischen Instrumenten eine Angleichung in der Umweltpolitik, es lassen sich aber weiterhin große Unterschiede bezüglich des Umweltschutzniveaus und beim Vollzug der Gesetze feststellen. ${ }^{14}$

Handel und Wettbewerbsfăhigkeit, a. a. O., S. 51 f. sowie Kellersmann, B.: Die gemeinsame, aber differenzierte Verantwortlichkeit von Industriestaaten und Entwicklungsländern for den Schutz der globalen Umwelt, a. a. O., S. 139 - 141.

11 Vgl. zu den wichtigsten Umweltabkommen European Environmental Agency (Hrsg.): Environmental Agreements: Environmental Effectiveness, Copenhagen 1997 sowie Olson, S. S.: International environmental standards handbook, a. a. O., S. 9.

In diesem Zusammenhang nennt der Rat for wirtschaftliche und soziale Fragen der Vereinten Nationen zwei Gründe, warum unkoordinierte nationale Umweltschutzmaßnahmen insbesondere in Bezug auf die internationale Unternehmenstătigkeit kontraproduktiv wirken konnen: "The first reason is the danger that uncoordinated environmental regulations might lead to concentration of polluting industries in areas where environmental regulations are weak or absent. The second reason is that unilateral environmental measures, such as content standards or recycling requirements, can be perceived as non-tariff trade barriers by trade partners, and lead to retaliatory action or trade wars." Economic and Social Council of the United Nations (Hrsg.): Activities of the transnational corporations and management division and its joint units, E/C.10/1993/7, 4 March 1993, S. 23.

Die Sensibilität gegenüber der ökologischen Umwelt ist in verschiedenen Ländern unterschiedlich ausgeprägt. Dies führt auf dem Wege der politischen Willensbildung auch zu unterschiedlichen nationalen Umweltstandards. Diese könnten als eine unterschiedliche Wertschătzung der Okologischen Umwelt in verschiedenen Nationen interpretiert werden.

Vgl. Jăhnike, M.; Weidner, H.: Zum aktuellen Stand der Umweltpolitik im internationalen Vergleich - Tendenzen zu einer globalen Konvergenz?, in: Aus Politik und Zeitgeschichte, 27. Juni 1997, Nr. B27/97, S. $15-24$ sowie Environics International: The Environmental Monitor - Global Public Opinion on the Environment. 1999 International Report, Toronto 1999, S. 56. 
International tätige Unternehmen können bei international divergierenden Umweltschutzbestimmungen ihre Wertschöpfungsaktivitäten in die Länder verlagern, die besonders geringe Umweltschutzansprüche stellen. Dies wird auch als Öko-Dumping bezeichnet. So führen Globalisierungskritiker an, dass sich als Folge der Globalisierung die Machtverhältnisse zwischen den international mobilen und den standortgebundenen Akteuren verschieben und es zu einer Entkopplung von räumlichen, rechtlichen und sozialen Zusammenhängen kommt ${ }^{15}$, was auch als Entgrenzung bezeichnet wird. ${ }^{16}$ Das Überschreiten von politisch-administrativen Steuerungsgrenzen ${ }^{17}$ sowie die Überlastung der Tragfähigkeit ökologischer und sozialer Systeme werden dann der Globalisierung bzw. der zunehmenden internationalen Unternehmenstätigkeit zugeschrieben. ${ }^{18}$

Aufgrund der Verbundenheit der gesellschaftlichen, ökonomischen und ökologischen Systeme ${ }^{19}$ hat analog zur Globalisierung von Unternehmen auch eine Globalisierung sozio-politischer und ökologischer Anliegen und Interessen-

15 Vgl. Hey, Ch.; Schleicher-Tappeser, R.: Nachhaltigkeit trotz Globalisierung - Handlungsspielrăume auf regionaler, nationaler und europäischer Ebene, a. a. O., S. 15 f.

16 DALY und СОВB umschreiben diesen Zusammenhang wie folgt: "transnational companies ... no longer see the national community as their context. They may speak grandly of the 'world community' as their residence, but infact, since no world community exists, they have escaped from community into the gap between communities where individualism has a free reign." Daly, H. E.; Cobb, J. B.: For the Common Good: Redirecting the Economy Toward Community, the Environment and a Sustainable Future, Boston 1989, S. 215, zitiert nach Brodel, D.: Internationales Umweltmanagement: Gestaltungsfelder - Determinanten - Ausprăgungen, Wiesbaden 1996, S. 359 f.

17 Vgl. Steinmann, H.; Scherer, A. G.: Die multinationale Unternehmung als moralischer Aktor - Bemerkungen zu einigen normativen Grundlagenproblemen des interkulturellen Managements, in: Engelhard, J. (Hrsg.): Interkulturelles Management: Theoretische Fundierung und funktionsbereichsspezifische Konzepte, Wiesbaden 1997, S. 24 sowie Pausenberger, E.: Globalisierung der Wirtschaft und die Machteinbußen des Nationalstaats, in: Engelhard, J.; Oechsler, W. A. (Hrsg.): Internationales Management: Auswirkungen globaler Veränderungen auf Wettbewerb, Unternehmensstrategie und Mărkte, Festschrift zum 60. Geburtstag von Klaus Macharzina, Wiesbaden 1999, S. 82 - 87. STEGER identifiziert ahnnliche Charakteristika der Globalisierung. Vgl. Steger, U.: Ist die Deregulierung im Umweltschutz vermeidbar?, in: Akademie der Politischen Bildung (Hrsg.): Umweltschutz zwischen Globalisierung und Deregulierung - Die Grenzen des Rechts in der Risikogesellschaft, Bonn 1998, S. 36.

18 Vgl. Fichter, K.; Schneidewind, U.: Neue Spielregeln für die grenzenlose Okonomie: Eine Einleitung, in: Fichter, K.; Schneidewind, U. (Hrsg.): Umweltschutz im globalen Wettbewerb - Neue Spielregeln für das grenzenlose Unternehmen, Berlin u. a. 2000, S. 1. Globalisierungsskeptiker betonen das Auftreten grenzüberschreitender bzw. globaler Risiken. Vgl. bspw. Martin, H.-P.; Schumann, H.: Die Globalisierungsfalle: Der Angriff auf Demokratie und Wohlstand, Reinbek 1996.

19 Der von MCLUHAN bereits in den 60er Jahren geprägte Begriff des "global village" erscheint in diesem Zusammenhang sowohl aus sozio-kultureller, okonomischer und okologischer Hinsicht treffend. Vgl. Brodel, D.: Internationales Umweltmanagement: Gestaltungsfelder Determinanten-Ausprăgungen, a. a. O., S. 1. 
gruppen stattgefunden. Konflikte werden häufig nicht mehr in dem Land ausgetragen, in dem sie ihren Ursprung haben, sondern in dem Land bzw. den Ländern, in dem die ökologischen Anspruchsgruppen ihre Anliegen am besten durchsetzen können. Mit einer derartigen Konfliktverlagerung sind $z$. T. hohe Gefahren für international tätige Unternehmen verbunden, wie die Auseinandersetzungen zwischen GREENPEACE und SHELL um die Versenkung der Olverladestation BRENT-SPAR und die Chemiekatastrophe im indischen Bhopal beispielhaft zeigen. ${ }^{20}$ Eine weitere Ausdehnung des Freihandels verliert an gesellschaftlicher Akzeptanz, international tätige Großunternehmen geraten dabei zunehmend in die Kritik gesellschaftlicher Anspruchsgruppen, wie die lautstarken und z. T. gewaltsamen Proteste von Globalisierungsgegnern belegen. $^{21}$

Die gleichzeitige Wahrung der gesellschaftlichen Legitimität und der internationalen Wettbewerbsfähigkeit kann als die Herausforderung an die Unternehmensführung im 21. Jahrhundert bezeichnet werden. ${ }^{22}$ Vor diesem Hintergrund kommt der Koordination des ökologiegerichteten Verhaltens international tätiger Unternehmen eine besondere Bedeutung zu.

20 Vgl. Welge, M. K.; Berg, N.: Public Affairs-Management in Multinationalen Unternehmungen, in: Welge, M. K. (Hrsg.): Arbeitspapiere des Lehrstuhls für Unternehmensführung, Nr. 24, Dortmund 1998, S. 1 und 41.

Das Scheitern der Verhandlungen zur weiteren Liberalisierung des Welthandels im Rahmen der WTO-Konferenz im Dezember 1999 in Seattle sowie die Proteste bei der Tagung von Weltbank und Internationalem Währungsfond im September 2000 in Prag können dabei als Beispiele für den wachsenden Einfluss global organisierter Anspruchsgruppen gewertet werden. In Seattle warfen zehntausende Demonstranten der WTO vor, Unternehmen die Möglichkeit zu geben, die Rechte von Arbeitern und Verbrauchern sowie den Umweltschutz zu missachten. Vgl. Pfahl, S.: Internationaler Handel und Umweltschutz - Zielkonflikte und Ansatzpunkte des Interessenausgleichs, Berlin u. a. 2000, S. 93. In Prag haben rund 7.000 Demonstranten ein Ende der angeblich armutsfördernden und okologische Zerstörungen verursachenden Kreditvergabepolitik der Institutionen gefordert. Vgl. Light, J.: Prague: The Aftermath, September 29, 2000, http://www.corpwatch.org/trac Iglobalization/bretton/il9-29.html [07.11.2000].

Globalisierung und Sustainability können nach SEIDEL als die zentralen Schlüsselwörter der umweltorientierten Unternehmensführung im internationalen Kontext gelten. Vgl. Seidel, E.: Instrumente umweltorientierter Unternehmensfuhrung im internationalen Kontext, in: Kramer, M.; Reichel, M. (Hrsg.): Internationales Umweltmanagement und europăische Integration: Deutschland, Polen und Tschechien im Vergleich, Wiesbaden 1998, S. 53. 


\section{2. Ökologiegerichtetes Verhalten international tätiger Unternehmen in der wirtschaftswissenschaftlichen Forschung}

Wie Abbildung 1 verdeutlicht, kann das ökologiegerichtete Verhalten international tätiger Unternehmen aus volkswirtschaftlicher, betriebswirtschaftlicher, ökologiebezogener oder internationaler Perspektive betrachtet werden.

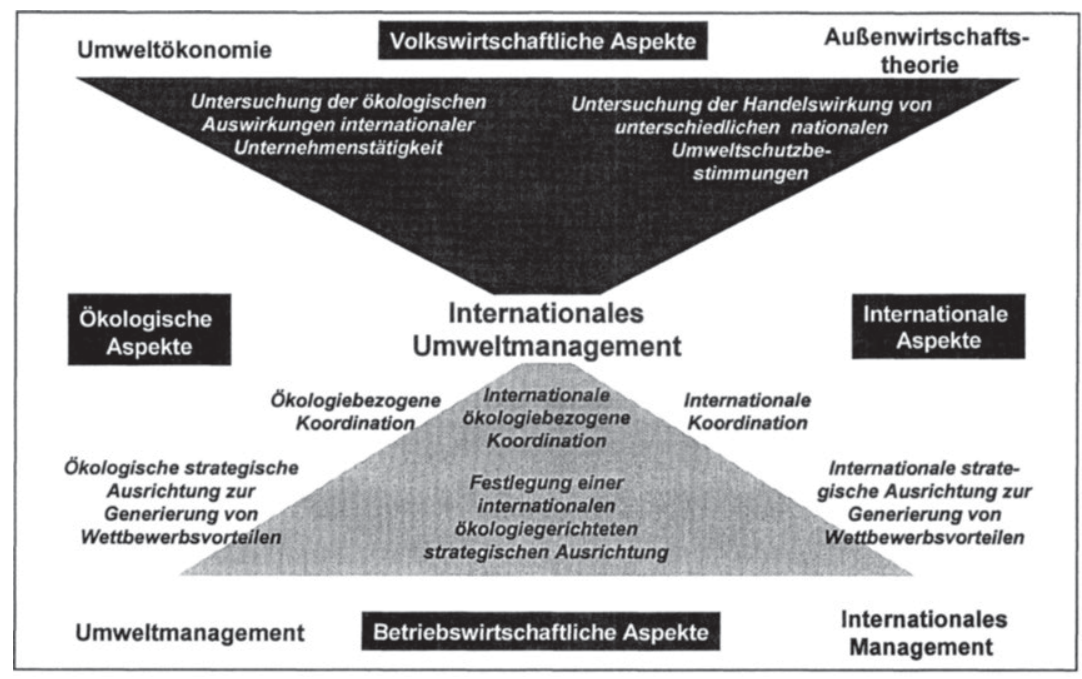

Abbildung 1: Unterschiedliche Perspektiven zum ökologiebezogenen internationalen Unternehmensverhalten

Quelle: Eigene Darstellung

Im Rahmen der vorliegenden betriebswirtschaftlich ausgerichteten Arbeit sollen insbesondere theoretische Ansätze aus dem internationalen Management und dem Umweltmanagement zugrunde gelegt werden. Dennoch bedarf eine Auseinandersetzung mit den betriebswirtschaftlichen Ansätzen zum ökologiegerichteten Verhalten international tätiger Unternehmen und den damit verbundenen Koordinationsentscheidungen einer Darstellung übergeordneter Zusammenhänge. Insbesondere die Analyse der Auswirkungen national differenzierter Umweltpolitiken bei geltendem Handelsrecht auf die internationale Unternehmenstätigkeit ist schon deshalb unerlässlich, da nur bei Kenntnis dieser Rahmenbedingungen fundierte Gestaltungsvorschläge für die Koordination des ökologie- 
gerichteten Verhaltens erarbeitet werden können. ${ }^{23}$ Darüber hinaus gilt es, die ökologischen Effekte zu analysieren, die allein der internationalen Unternehmenstätigkeit zuzuordnenden sind, da international tätige Unternehmen insbesondere deshalb im Mittelpunkt der gesellschaftlichen Kritik stehen. Dabei wurden die Auswirkungen von Umweltschutzmaßnahmen auf das internationale Unternehmensverhalten sowie die ökologischen Auswirkungen der internationalen Unternehmenstätigkeit bisher primär in volkswirtschaftlichen Quellen untersucht.

\subsection{Analyse der Wechselwirkungen zwischen Umweltschutz und internationaler Unternehmenstätigkeit}

Im Rahmen des Heckscher-Ohlin-Ansatzes wurde gezeigt, dass internationaler Freihandel für eine optimale Allokation und eine gesamtgesellschaftliche Wohlfahrtssteigerung sorgt. Handelsbeschränkungen wurden als ökonomisch allokationsverzerrend und wohlfahrtsmindernd abgelehnt. Vor diesem Hintergrund dient das Allgemeine Zoll- und Handelsabkommen (GATT) ${ }^{24}$ dem Ziel, den internationalen Güteraustausch zu liberalisieren. ${ }^{25}$

Das GATT berücksichtigte ursprünglich keine Umweltschutzziele, da es sich losgelöst vom internationalen Umweltrecht entwickelte. Durch Zusatzabkommen sind handelsbeschränkende Maßnahmen aufgrund von national geltenden ökologiebezogenen Produktstandards dann erlaubt, wenn eine Umweltschädigung wissenschaftlich fundiert nachgewiesen wird, es keine andere GATT-konforme Maßnahme zur Problemlösung gibt und sich die Maßnahme nur auf das

23 PORTER führt in diesem Zusammenhang an: „The environment ... affects both a firm 's initial conditions and its managerial choices.." Porter, M. E.: Towards a Dynamic Theory of Strategy, in: Strategic Management Journal, 12. Jg. (1991), S. 115 f.

24 Das GATT wurde 1947 gegründet und stellt seitdem die Welthandelsordnung dar. Die letzte Verhandlungsrunde zum GATT, die sog. Uruguay-Runde in 1994, hatte neben einer Erweiterung des Regelwerks die Gründung der World Trade Organisation (WTO) sowie die Einbettung des GATT in die WTO zum Inhalt. Die WTO hat dabei die Aufgabe, sämtliche in der Uruguay-Runde beschlossenen Verträge zu überwachen. Vgl. Petschow, U. ot al.: Nachhaltigkeit und Globalisierung - Herausforderungen und Handlungsansătze, Berlin u. a. 1998, S. 187.

Als Kernprinzipen des GATT gelten die Meistbegünstigungsklausel, das Prinzip des National Treatment, das Prinzip der Reziprozităt sowie das Verbot mengenmäßiger Beschränkungen. Vgl. bspw. Olson, S.: International Environmental Standards Handbook, a. a. O., S. $21-22$. 
eigene nationale Territorium richtet. $^{26}$ Somit entfalten nationale Umweltstandards $^{27}$ nur in Ausnahmefällen ihre Wirkung auf ausländische Unternehmen.

Die Wechselwirkungen zwischen Umweltschutz und internationaler Unternehmenstätigkeit, die im Folgenden beschrieben werden, sind in Abbildung 2 in einer Übersicht dargestellt.

Die Diskussion um gegenseitige Abhängigkeiten zwischen Umweltschutz und internationalem Unternehmensverhalten ist dabei nicht neu, sondern lässt sich bis in die 70er Jahre zurückverfolgen. Bereits 1971 wurden in der Studie „Industrial Pollution and International Trade " des GATT-Sekretariats die außenwirtschaftlichen Effekte von Umweltschutzmaßnahmen untersucht. Diese Studie fand große Aufmerksamkeit und ihr folgte eine Reihe weiterer Untersuchungen zum Thema „Handel und Umwelt". ${ }^{28}$ Dabei wurden unterschiedliche Einflüsse von national differenzierten produktionsbezogenen sowie produktbezogenen Umweltschutzmaßnahmen auf die internationale Unternehmenstätigkeit festgestellt. Die Auswirkungen von national unterschiedlichen Umweltgesetzen auf die internationale Unternehmenstätigkeit sind im linken Teil der Abbildung 2 dargestellt. Umweltschutzvorschriften können danach einen Einfluss auf die Wettbewerbsfähigkeit eines Unternehmens an einem Standort und damit auch auf Standortentscheidungen ausüben oder zu tarifären bzw. nicht-tarifären Handelshemmnissen führen.

26 Als Ausnahmeregelung von den Basisprinzipien besteht der Artikel $X X$, in dem u. a. der Schutz des menschlichen, tierischen und pflanzlichen Lebens sowie der Erhalt erschøpfbarer Ressourcen als Begründung für handelsbeschränkende Maßnahmen aufgeführt werden. Vgl. Petschow, U. et al.: Nachhaltigkeit und Globalisierung - Herausforderungen und Handlungsansătze, a. a. O., S. 192 - 194.

Da Standards aus theoretischer Sicht durchaus geeignet erscheinen, Umweltbeeintrăchtigungen effizient zu begrenzen, beruhen eine Vielzahl der angesprochenen Maßnahmen auf Umweltstandards. Vgl. Petschow, U. et al.: Nachhaltigkeit und Globalisierung - Herausforderungen und Handlungsansátze, a.a. O., S. 179. Standards sind dokumentierte Vereinbarungen, die technische Spezifizierungen oder andere Kriterien umfassen. Vgl. Olson, S. S.: International Environmental Standards Handbook, a. a. O., S. 136. Zu unterschiedlichen Standards vgl. IISD, UNEP (Hrsg.): Environment and Trade - A Handbook, Winnipeg 2000, S. 10.

28 Kulessa, M. E.: Umweltpolitische Dimensionen des Welthandels, in: Wirtschaft und Umwelt e. V. (Hrsg.): Welthandel und Umweltschutz - Wie handeln wir okologisch?, Münster 1996, S. 41. 


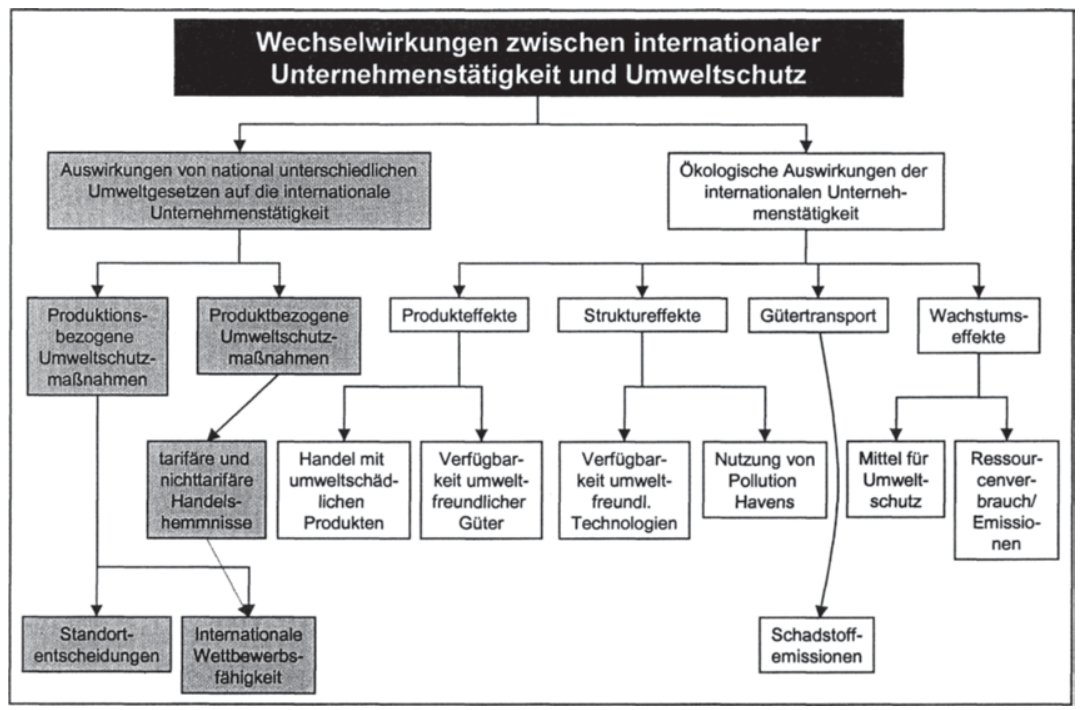

Abbildung 2: Wechselwirkungen von internationaler Unternehmenstätigkeit und Umweltschutz

In Anlehnung an Helm, C.: Sind Freihandel und Umweltschutz vereinbar? Okologischer Reformbedarf des GATTMTO-Regimes, Berlin 1995, S. 22.

STITZEL weist in diesem Zusammenhang auf den „janusköpfigen Charakter" des Umweltschutzes aus wettbewerbsbezogener Sicht hin. ${ }^{29} \mathrm{Er}$ führt an, dass international unterschiedliche Umweltstandards zur Benachteiligung jener Wettbewerber führen, die in ihren Produktionsländern im Vergleich zu ausländischen Konkurrenten einer besonders restriktiven Umweltpolitik unterliegen. Dies gilt in besonderem Maße für Umweltschutzaktivitäten, die nicht direkt mit einem für den Konsumenten "sicht- bzw. erfahrbaren" ökologischen Zusatznutzen der Produkte verbunden sind. ${ }^{30}$ Prozess- bzw. produktionsmethodenbezogene Umweltvorschriften können somit als ein Standortfaktor interpretiert werden. In diesem Zusammenhang wird häufig die Meinung vertreten, dass Unternehmen an Standorten mit niedrigen Umweltstandards Kostenvorteile haben, die es ihnen erlauben, Wettbewerbsvorteile gegenüber Unternehmen aus ökologisch restriktiveren Standorten aufzubauen. Als Folge wird eine Standortverlagerung insbesondere der Unternehmen für wahrscheinlich gehalten, die durch eine anspruchsvolle umweltpolitische Gesetzgebung mit vergleichsweise hohen Kosten belastet werden. ${ }^{31}$

Vgl. Stitzel, M.: Ökologisch orientierte Unternehmensführung - Hemmung der Forderung der Wettbewerbsfähigkeit der Unternehmung, in: Dichtl, E.; Gerke, W.; Kieser A. (Hrsg.): Innovation und Wettbewerbsfähigkeit, Wiesbaden 1987, S. 391.

Vgl. Kirchgeorg, M.: Okologieorientiertes Unternehmensverhalten - Typologien und Erklärungsansatze auf empirischer Grundlage, Gabler 1990, S. $10-11$.

31 In der Literatur spricht man in diesem Zusammenhang von der sog. pollution heavenThese. Verschiedene empirische Untersuchungen aus der Vergangenheit konnten diese 
Produktbezogene Umweltschutzstandards können tarifäre oder nicht-tarifäre Handelsbeschränkungen nach dem GATT begründen. In diesem Fall ist ein Unternehmen gezwungen, die produktbezogenen Anforderungen zu erfüllen, soll der entsprechende Ländermarkt weiterhin bearbeitet werden. Importbeschränkungen, die sich auf die Produktionsmethoden des Importlands beziehen, sind hingegen generell nicht GATT konform. Dennoch versuchen Industriezweige immer wieder, Schutz vor dem Verlust an internationaler Wettbewerbsfähigkeit vom Staat zu fordern. Als Begründung wird in diesem Zusammenhang von Umwelt- bzw. Okodumping ${ }^{32}$ durch die ausländischen Wettbewerber gesprochen. ${ }^{33}$ Wenn Handelshemmnisse einen Markt schützen, können sich ausländische Unternehmen entschließen, die Produktionsmethoden an die Standards des Exportlandes anzupassen bzw. sich für Direktinvestitionen entscheiden, um den Ländermarkt weiterhin bearbeiten zu können.

Während in den 70er Jahren die Auswirkungen von national unterschiedlichen Umweltgesetzen auf die internationale Unternehmenstätigkeit untersucht wurden, nahm zu Beginn der 80er Jahre das Interesse an den Wechselwirkungen zwischen internationaler Unternehmenstätigkeit und Umweltschutz ab. Die Diskussionen sind erst zu Beginn der 90er Jahre vornehmlich von Umweltschutzgruppen wieder aufgegriffen worden. Dabei stehen weniger die Handelseffekte von Umweltschutzmaßnahmen als vielmehr die ökologischen Auswirkungen der internationalen Unternehmenstätigkeit im Fokus der Betrachtung. ${ }^{34}$

These allerdings nicht stützen. Vgl. Pfahl, S.: Internationaler Handel und Umweltschutz Zielkonflikte und Ansatzpunkte des Interessenausgleichs, Berlin u. a. 2000, S. 48 - 55.

Okodumping wird hăufig als Versuch eines Landes verstanden, durch weniger strenge bzw. keine Umweltschutzmaßnahmen einen Wettbewerbsvorteil gegenüber Ländern mit entsprechenden Vorschriften zu erlangen. Vgl. Wied-Nebbing, S.: Wohlfahrtswirkungen des Freihandels bei negativen externen Effekten, in: WiSt, Heft 11, 1997, S. 574. Vgl. zu unterschiedlichen Begriffsauffassungen weiterhin Reuter, G. C.: Nationale Regulierungsunterschiede als komparativer Wettbewerbsvorteil oder Wettbewerbsverzerrung? Eine Studie zur Okodumpingdiskussion als Problem der Welthandelsordnung, Bamberg 1996, S. 113 f. und Sauernheimer, K.; Ködding, G.: Internationale Aspekte der Umweltokonomie, in: Junkernheinrich, M.; Klenner, P.; Wagner, G. R. (Hrsg.): Handbuch zur Umweltökonomie, Berlin 1995, S. 73.

In der Praxis finden sich verschiedene Beispiele für den Versuch, Handelshemmnisse aufgrund von produktionsbezogenen Umweltstandards aufzubauen, wie bspw. den "Thunfischfall" zwischen den USA und Mexiko. Vgl. Kulessa, M. E.: Umweltpolitische Dimensionen des Welthandels, a. a. O., S. 54. Im Zusammenhang mit dem Einsatz von handelsbeschränkenden Maßnahmen zur Umsetzung ókologischer Ziele stellt sich zugleich die Frage nach dem Missbrauchspotenzial durch versteckte Protektion. Vgl. Petschow, U. et al.: Nachhaltigkeit und Globalisierung - Herausforderungen und Handlungsansătze, a. a. O., S. 178.

Vgl. Kulessa, M. E.: Umweltpolitische Dimensionen des Welthandels, a.a.o , S. 41. 
Als ökologische Effekte der internationalen Unternehmenstätigkeit sind neben transportbedingten Schadstoffemissionen Produkt-, Wachstums- und Struktureffekte zu nennen: ${ }^{35}$

- Transportbedingte Schadstoffemissionen und Umweltbelastungen entstehen vornehmlich aufgrund der Nutzung von Verkehrsmitteln für den länderübergreifenden Warentransport. Weiterhin sind aber auch die Umweltwirkungen zu berücksichtigen, die durch die Bereitstellung der Infrastruktur und die Herstellung der Fahrzeuge entstehen. ${ }^{36} \mathrm{Da}$ rund $90 \%$ aller Güter bei interkontinentalem Handel im Schiffstransport bewegt werden, ist der interkontinentale Warentransport Hauptverursacher der Meeresverschmutzung. Intrakontinental dominieren Straßen- und Schienentransporte. Dabei hat die Bedeutung des Straßentransports bis heute stark zugenommen und ist für eine Vielzahl ökologischer Probleme verantwortlich. Der internationale Warentransport ist bspw. für den Verbrauch von ungefähr $1 / 8$ des Weltölaufkommens und den daraus resultierenden Auswirkungen auf die natürliche Umwelt verantwortlich. ${ }^{37}$

- Produkteffekte beschreiben die negativen und positiven Auswirkungen des Austausches von Gütern und Dienstleistungen auf die Umwelt. So führt der grenzüberschreitende Handel mit umweltproblematischen Produkten zu einer internationalen oder sogar weltweiten Verbreitung von Schadstoffen und der Ausbreitung umweltschädigender Konsummuster. Positive Auswirkungen des internationalen Handels auf die natürliche Umwelt sind dann zu erwarten, wenn Unternehmen Umweltschutztechnologien oder vergleichsweise umweltfreundliche Güter in Länder exportieren, die bisher nicht über ein entsprechendes technologisches Wissen bzw. Produktangebot verfügten, oder entsprechende Produktionskapazitäten über Direktinvestitionen vor Ort etablieren. Internationalen Großunternehmen kommt dabei eine wichtige Rolle beim Technologietransfer zwischen technologisch unterschiedlich entwickelten Ländern zu. ${ }^{38}$

Vgl. Althammer, W.: Umweltpolitik, internationaler Handel und Wettbewerbsfahigkeit, a. a. O., S. 52 f. Die Regulierungseffekte, die Althammer noch aufführt werden in diesem Kontext für unwesentlich gehalten. Vgl. auch OECD (Hrsg.): Methodologies for environmental and trade reviews, Paris 1994, OECD/GD(94)103, S. 13.

36 In Europa nahm der grenzüberschreitende Verkehr im Vergleich zum Binnenverkehr überproportional zu, es wird prognostiziert, dass diese Entwicklung weiter anhalten wird. Vgl. Franke, A.: Freier Welthandel und umweltvertrăgliche Entwicklung, a. a. O., S. 16.

Vgl. Altmann, J.: Umweltpolitik, Bern u. a. 1997, S. 98.

"As more nations pursue growth and development objectives through market reform and liberalisation of the foreign direct investment (FDI) regimes, transnational corporations will increasingly become engines of capital formation, human resource development, technology transfer and the exchange of goods and services." United Nations (Hrsg.): Activities of the Transnational Corporations and Management Divisions and its Joint Units, a. a. O., S. 19. Das Wissen über die Besonderheiten des Einsatzes z. B. hoch erklärungsbedürttiger Umweltschutztechnologien kann innerhalb eines Unternehmens transaktionskostengünstiger übertragen werden als über Mărkte. 
- Neben Produkteffekten werden umweltrelevante Einflüsse der internationalen Unternehmenstätigkeit auch in Wachstumseffekten ${ }^{39}$ gesehen. Grenzüberschreitende Unternehmensaktivitäten können dazu beitragen, nationale Wachstumsgren$z^{40}{ }^{40}$ zu überwinden und so zusätzliche Umweltbelastungen herbeiführen. ${ }^{4 \uparrow}$ Auf der anderen Seite wird Wirtschaftswachstum von einigen Autoren als eine notwendige Voraussetzung für nachhaltiges Wirtschaften angesehen, da erst nach Erfüllung der physiologischen Bedürfnisse eine Nachfrage nach sauberer Umwelt artikuliert werden könne. ${ }^{42}$ Die Entkopplung des Umwelt- und Ressourcenverbrauchs vom Wirtschaftswachstum durch Verbreitung eines entsprechenden Knowhows ist dabei Voraussetzung für positive ökologische Wachstumseffekte. ${ }^{43}$

- Von Struktureffekten ${ }^{44}$ des internationalen Handels wird gesprochen, wenn durch differierende nationale Umweltschutzpolitiken ökonomische Transaktionsbeziehungen ausgelöst werden, die allokative und distributive Wirkungen entfalten. Werden umweltbelastende und energieintensive Produktionsprozesse ins umweltpolitisch weniger restriktive Ausland verlagert (Industrieflucht), ändern sich die nationalen Industriestrukturen. Dies kann zu einer Verbesserung der Umweltqualität im restriktiven Land führen. Allerdings ist es auch möglich, dass aufgrund grenzüberschreitender externer Effekte neben einer erhöhten Belastung in jenen Ländern, in die die umweltintensive Produktion verlagert wird, auch eine Umweltverschlechterung im restriktiven Land selbst eintritt. ${ }^{45}$ Weiterhin trägt das ökologisch

39 Wachstumseffekte werden auch Skaleneffekte genannt, vgl. Pfahl, S.: Internationaler Handel und Umweltschutz - Zielkonflikte und Ansatzpunkte des Interessenausgleichs, a. a. O., S. 42.

40 Wachstumsgrenzen können angebotsseitig und nachfrageseitig, z. B. aufgrund einer begrenzten Ressourcenausstattung oder wegen eines bereits ausgeschöpften Nachfragepotenzials eines Landes, erreicht werden.

41 Dieser Betrachtung wird zugrunde gelegt, dass Wachstum stets mit einer Zunahme an Umweltverschmutzung verbunden ist. Zu berücksichtigen ist, dass nach einer allmählichen und dann exponentiell zunehmenden Umweltbelastung bei fortschreitender Industrialisierung, wenigstens bei einigen Industrieländern, eine Entkopplung von Wachstum und Umweltbelastung folgt. Vgl. Altmann, J.: Umweltpolitik, a. a. O., S. 62-64.

42 Dabei ist es von der Art des Wirtschaftswachstums abhängig, welcher okologische Nettoeffekt eintrifft. Vgl. Petschow, U. et al.: Nachhaltigkeit und Globalisierung - Herausforderungen und Handlungsansătze, a. a. O., S. 169. Sollten die Konsummuster der Industrielănder uneingeschränkt auf sich entwickelnde Lănder übertragen werden, bestehen kaum Chancen, die Ziele einer nachhaltigen Entwicklung sicherzustellen. In diesem Zusammenhang sei weiterhin die kontrovers geführte Diskussion um einen ${ }_{n}$ glockenförmigen Verlauf" des Zusammenhangs zwischen Umweltqualität und Wirtschaftswachstum angeführt. Vgl. Grossmann, G. M.; Krüger, A. B.: Economic Growth and the Environment, in: Quarterly Journal of Economics, CX. Jg. (1995), S. 353 - 377; Arrow, K. et al.: Economic Growth, Carrying Capacity, and the Environment, in: Ecological Economics, 15. Jg., (1995), S. 91 95; Jänicke, M. et al.: Umweltentlastung durch industriellen Strukturwandel? Eine explorative Studie uber 32 Industrieländer, Berlin 1992.

Vgl. Pfahl, S.: Internationaler Handel und Umweltschutz - Zielkonflikte und Ansatzpunkte des Interessenausgleichs, a. a. O., S. 42.

44 Die Struktureffekte entsprechen den ${ }_{n} s c a l e$ effects ${ }^{4}$ der Untergliederung der OECD. Vgl. OECD (Hrsg.): Methodologies for environmental and trade reviows, a. a. O., S. 13.

45 Vgl. Meffert, H.; Kirchgeorg, M.: Marktorientiertes Umweltmanagement - Konzeption Strategie - Implementierung mit Praxisfällen, a. a. O., S. 233 ff. 
restriktivere Land möglicherweise die negativen strukturellen Anpassungskosten in Form einer erhöhter Arbeitslosigkeit.

Aufgrund einer weitgehend fehlenden internationalen umweltrechtlichen Koordination durch überstaatliche Institutionen haben international tätige Unternehmen große Freiheitsgrade in Bezug auf ihr ökologiebezogenes Verhalten. Im folgenden Gliederungspunkt sollen deshalb die relevanten betriebswirtschaftlichen Ansätze dargestellt werden, die Aussagen zum ökologiegerichteten Verhalten international tätiger Unternehmen zulassen.

\subsection{Betriebswirtschaftliche Ansätze zur Berücksichtigung des Umweltschutzes bei internationaler Unternehmenstätigkeit}

Die betriebswirtschaftliche Forschung hat die unternehmensführungsbezogenen Herausforderungen der Globalisierung und des Umweltschutzes größtenteils getrennt in den Disziplinen des internationalen Managements bzw. des Umweltmanagements aufgegriffen. Deshalb werden zuerst die im Verlauf dieser Arbeit relevanten Ansätze aus dem Internationalen Management und dem Umweltmanagement getrennt vorgestellt, um darauf aufbauend erste Ansätze eines internationalen Umweltmanagements vorzustellen.

In den 80 er Jahren rückten in Verbindung mit der wachsenden Globalisierung des Wettbewerbs Aspekte des wettbewerbsgerichteten und internationalen Managements in den Mittelpunkt der betriebswirtschaftlichen Diskussion. ${ }^{46}$ Das Erkenntnisobjekt des internationalen Managements ${ }^{47}$ sind grenzüberschreitend tätige Unternehmen. Aufgrund der vielfältigen, sich teilweise überschneidenden Abgrenzungen ${ }^{48}$ von internationalen, multinationalen und transnationalen Unternehmungen und deren Bezug zu der prozessualen Sichtweise der Internationalisierung soll hier der Begriff der international tätigen Unternehmung Verwendung finden. Das international tätige Unternehmen sei durch

46 Vgl. Meffert, H.: Marketingwissenschaft im Wandel - Anmerkungen zur Paradigmendiskussion, in: HHL-Arbeitspapier Nr. 30, Leipzig 1999, S. 5.

Nach MEFFERT und BoLz besteht internationales Marketing-Management ${ }_{n}$ in der Analyse, Planung, Durchfuhrung, Koordination und Kontrolle marktbezogener Unternehmensaktivităten in mehr als einem Land". Meffert, H.; Bolz, J.: Internationales Marketing-Management, 3. Aufl., Stuttgart u. a. 1998, S. 25.

Hinsichtlich der begrifflichen Abgrenzung lässt sich keine einheitliche Auffassung nachweisen. Vgl. zu unterschiedlichen Begriffsabgrenzungen bspw. Plump, D.: Die Koordination des Marketings in multinationalen Unternehmungen: Systemanalyse und Ansătze zur Systemgestaltung, Bamberg 1976, S. 18 - 21; Welge, M.: Management in deutschen multinationalen Unternehmungen: Ergebnisse einer empirischen Untersuchung, Stuttgart 1980, S. 3 ff.; Brodel, D.: Internationales Umweltmanagement: Gestaltungsfelder - Determinanten - Ausprägungen, a. a. O., S. 193- 203. 
substanzielle Beteiligung am Kapital ausländischer Unternehmen mit Einfluss auf sowie Kontrolle über diese Unternehmen, der Produktionstätigkeit in zumindest zwei Ländern und der Orientierung der Entscheidungen des Top-Managements an internationalen Maßstäben gekennzeichnet.

Nach PAUSENBERgER bestimmt dabei insbesondere die Umfeldheterogenität die Spezifika dieses Unternehmenstyps. ${ }^{49}$ Die Konfrontation des internationalen Managements mit länderspezifischen Besonderheiten und tarifären bzw. nichttarifären Maßnahmen bedingt ein höheres Maß an Ungewissheit und Risiko. Dies führt zu einer erhöhten Komplexität ${ }^{50}$ und einem erweiterten Informationsund Koordinationsbedarf. ${ }^{51}$ Nach BACKHAUS kommt deshalb der Koordinationsaufgabe im internationalen Management eine besondere Bedeutung zu. ${ }^{52}$

49 Pausenberger, E.: Pladoyer for eine "Internationale Betriebswirtschaftslehre“, in: Kirch, W.; Picot, A. (Hrsg.): Die Betriebswirtschaftslehre im Spannungsfeld zwischen Generalisierung und Spezialisierung, Wiesbaden 1989, S. 386, zitiert nach Brodel, D.: Internationales Umweltmanagement: Gestaltungsfelder - Determinanten - Ausprägungen, a. a. O., S. 210 f. Die Auffassung von ALBACH, dass die Fragestellungen und Aufgaben der Internationalisierungspraxis keine eigenständigen Gestaltungsempfehlungen bezüglich Gegenstandsbereich und Methodenspektrum bedingen, hat sich nicht durchgesetzt. Vgl. dazu Albach, H.: Betriebswirtschaftslehre als Wissenschaft vom Management, in: ZfB, Ergänzungsheft 1, 1981, S. $13 \mathrm{ff}$.

50 Komplexităt kann als Anzahl möglicher Zustănde, die ein System annehmen kann, definiert werden. Ein System ist dabei eine gegenüber der Umwelt abgegrenzte Gesamtheit von Elementen, die durch Beziehungen miteinander verknüpft sind. Vgl. Malik, F.: Strategie des Managements komplexer Systeme, 5. Aufl., Bern 1996, S. 201.

BRAUCHLIN und WIESMANN führen die Besonderheiten des internationalen Management auf unterschiedliche Differenzen zurück. Dabei bezieht er sich auf Systemdifferenzen, geographische Differenzen und Kulturdifferenzen. Systemdifferenzen beschreiben die in den jeweiligen Ländermärkten unterschiedlichen politischen, rechtlichen, steuerlichen, infrastrukturellen sowie sozialen Systeme, die für das Unternehmen unterschiedliche Freiheitsgrade eröffnen. Geographische Differenzen beziehen sich auf die Entfernung zur übergeordneten Unternehmenseinheit, mogliche Zeit- und Klimadifferenzen. Kulturdifferenzen beschreiben die Wirkung kultureller Unterschiede auf Management- und Verhandlungsstile sowie Entscheidungsprozesse. Vgl. Brauchlin, E.; Wiesmann, D.: Internationales Management, in: Gabler Wirtschaftslexikon, 13. Aufl., Wiesbaden 1993, S. 1676 f.

Auch BEREKOVEN weist darauf hin, dass die für einen Markt getroffenen Entscheidungen im Gesamtzusammenhang mit den Entscheidungen stehen, die für andere Märkte getroffen werden, sich also die Entscheidungen gegenseitig bedingen. Vgl. Berekoven, L.: Internationales Marketing, 2. Aufl., Berlin 1985, S. 21. 
Die Internationalisierungsstrategie hat dabei entscheidenden Einfluss auf den Umfang der internationalen Koordination. ${ }^{53}$ In Anlehnung an PERLMUTER ${ }^{54}$ lassen sich drei unterschiedliche Grundorientierungen im internationalen Management unterscheiden, die sich auch als Entwicklungsprozess der internationalen Geschäftstätigkeit deuten lassen: die ethnozentrische Orientierung, die polyzentrische Orientierung sowie die geozentrische Orientierung. ${ }^{55}$

Die Erfahrungen der letzten Jahre haben gezeigt, dass in vielen Branchen die externen, aber auch die internen Erfolgsvoraussetzungen für eine globale Basisstrategie im Rahmen einer geozentrischen Orientierung nicht oder nicht hinreichend erfüllt waren. ${ }^{56}$ So konnten im Zusammenhang mit der zunehmenden Bildung von gemeinsamen Wirtschaftsblöcken und Freihandelszonen eher regiozentrische Orientierungen beobachtet werden. ${ }^{57}$

Mittlerweile haben sich einige Änderungen im Technologieumfeld ergeben. So ermöglichen Innovationen im Bereich der Fertigungstechnologien die Realisierung von Kostenvorteilen bereits bei kleinen Losgrößen. ${ }^{58}$ Innovationen in der Informations- und Kommunikationstechnologie sowie deren industrie- und länderübergreifende Konvergenz tragen zu weiterer Markttransparenz und steigender Wettbewerbsintensität bei, ermöglichen aber auch eine zunehmende

53 Vgl. Backhaus, K.; Büschken, J.; Voeth, M.: Internationales Marketing, 3. Aufl., Stuttgart 2000, S. $123 \mathrm{f}$.

54 Vgl. Perimutter, H. V.: Three Conceptions of a World Enterprise, in: Revue Economique et Sociale, May 1965 sowie Perlmutter, H. V.: The Tortuous Evolution of the Multinational Corporation, in: Columbia Journal of World Business, Nr. 4, 1969, S. 9 - 18. Die dynamische Perspektive fand durch eine gemeinsame Untersuchung von PERLMUTTER und HEENAN Eingang in die internationale betriebswirtschaftliche Forschung. Vgl. Heenan, D. A.; Perlmutter, H. V.: Multinational Organization Development, Reading (MA) 1979.

$55 \mathrm{Vgl}$. Zu den unterschiedlichen Orientierungen im internationalen Management Perlmutter, H. V.: The Tortuous Evolution of the Multinational Corporation, a. a. O., S. 9-18. Meffert, H.; Bolz, J.: Internationales Marketing-Management, a. a. O., S. 25 - 28 sowie Backhaus, K.; Büschken, J.; Voeth, M.: Internationales Marketing, a. a. O., S. 123 - 126.

56 Als externe Erfolgsvoraussetzungen des globalen Marketing gelten u. a. Betriebsgroßenersparnisse, einheitliche technische Standards sowie das Vorhandensein von sog. cross cultural groups mit übereinstimmenden Verhaltensmustern und homogenen Kundenanforderungen. Vgl. Meffert, H.; Bolz, J.: Internationales Marketing-Management, a. a. O., S. 28.

57 Vgl. Proff, H.; Proff, H. V.: Struktur und Entwicklung der weltweiten Automobilindustrie, in: Proff, H.; Proff, H.V. (Hrsg.): Strategien für die Automobilindustrie: Ansatzpunkte im strategischen Management und in der Industriepolitik, Wiesbaden 1998. S. 96.

58 Vgl. Meffert, H.; Bolz, J.: Internationales Marketing-Management, a. a. O., S. 28. 
Koordination unternehmensinterner Aktivitäten. ${ }^{59}$ Die gestiegene Komplexität und das Erfordernis nach Flexibilität, der Wahrnehmung globaler Kostenvorteile und regionaler Anpassung sowie weltweitem Lernen führten zu dem Bestreben, Vorteile einer differenzierten und einer standardisierten Marktbearbeitung miteinander zu verknüpfen. ${ }^{60}$ Das Ergebnis sind transnationale Unternehmen ${ }^{61}$ mit Netzwerk-Konfigurationen. Dabei werden an unterschiedlichen Produktionsstandorten verschiedene Bauteile oder Produkttypen hergestellt. Je nach strategischer Bedeutung des lokalen Umfeldes und der Ausstattung der lokalen Organisationseinheiten mit Ressourcen und Kernkompetenzen ${ }^{62}$ wird den Netzwerkakteuren eine spezifische Rolle im Netzwerk zugewiesen ${ }^{63}$, sodass diese Unternehmen zur Sicherstellung der Wettbewerbsfähigkeit auf einen gegenseitigen Austausch von Komponenten, Produkten, Ressourcen, Informationen und Personal angewiesen sind. ${ }^{64}$

59 In diesem Zusammenhang gelten die Zunahme von Fusionen und das Auftreten von komplexeren, grenzüberschreitenden Verbundbeziehungen in und zwischen Unternehmen entlang der Wertschöpfungskette als Folge. Vgl. Fichter, K.; Schneidewind, U.: Neue Spielregeln für die grenzenlose Ókonomie: Eine Einleitung, a. a. O., S. 1.

60 MEFFERT weist darauf hin, dass im Spannungsfeld zwischen globalem Wettbewerb und lokalen Bedürfnissen, also im Bereich der Mischstrategien, nach situationsgerechten Kompromissformen zu suchen sei. Vgl. Meffert, H.: Marketing im Spannungsfeld von weltweitem Wettbewerb und nationalen Bedurfnissen, in: ZfB, 1986, Nr. 8, S. $689-712$ sowie Meffert, H.: Voraussetzungen und Implikationen von Globalisierungsstrategien, in: Meffert, H. (Hrsg.): Strategische Unternehmensführung und Marketing, Wiesbaden 1988, S. $266-$ 288; Meffert, H.: Globalisierungsstrategien und ihre Umsetzung im internationalen Wettbewerb, in: DBW, 49. Jg. (1989), Nr. 4, S. 445 - 463.

Vgl. Frese, E.: Grundlagen der Organisation: Konzepte - Prinzipien - Strukturen, 6. Aufl., Wiesbaden 1995, S. 441. Vgl. auch Porter, M. E.: Der Wettbewerb auf globalen Markten: Ein Rahmenkonzept, in: Porter, M. E. (Hrsg.): Globaler Wettbewerb: Strategien der neuen Internationalisierung, Wiesbaden 1989, S. 55 f. BARTLETT und GHOSHAL prägen in diesem Zusammenhang den Begriff des transnationalen Unternehmens. Vgl. Bartlett, C.; Ghoshal, S.: Internationale Unternehmensfuhrung, Frankfurt a. M. 1990.

Vgl. Prahalad, C. K.; Hamel, G.: The Core Competence of the Corporation, in: Bartlett, C.; Ghoshal, S. (Hrsg.): Transnational Management: Text, Cases, and Readings in CrossBorder Management, Third Edition, Boston u. a. 2000, S. 360 - 362.

63 Dies ermơglicht unter Berücksichtigung der lokalen Umfeldbedingungen und Kompetenzen die Wahrnehmung von besonderen Aufgaben im Gefüge des gesamten Netzwerks. BARTLETT und GHOSHAL unterscheiden die Rolle des strategischen Fürers, die mitwirkende sowie die ausführende Rolle und das schwarze Loch. Zu den unterschiedlichen Rollen vgl. Ghoshal, S.; Nohria, N.: Internal Differentiation within Multinational Corporations, in: Strategic Management Journal, 10. Jg. (1989), S. 323 - 337; Bartlett, C.; Ghoshal, S.: Internationale Unternehmensfuhrung, a. a. O., S. $138 \mathrm{ff}$.

In transnationalen Unternehmen werden Ressourcen, Know-how und Aufgaben nach dem Kriterium der wettbewerbsbezogenen Selektion verteilt, sodass die weltweite Wettbewerbsfăhigkeit optimiert wird. Vgl. Backhaus, K.; Büschken, J.; Voeth, M.: Internationales Marketing, a. a. O., S. 50 f. 
Ein derartiges intra-organisationales Netzwerk mit den unterschiedlichen Beziehungen zwischen den einzelnen differenzierten Netzwerkeinheiten ist in Abbildung 3 dargestellt.

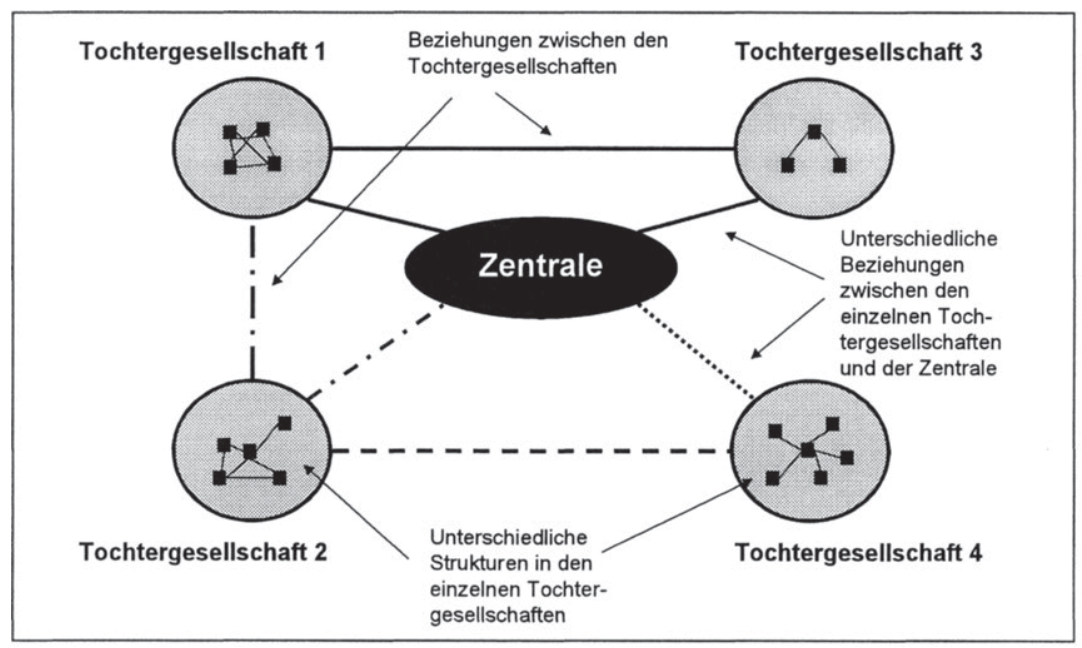

Abbildung 3: $\quad$ Integriertes Netzwerk nach NOHRIA und GHOSHAL

Quelle: Nohria, N.; Ghoshal, S.: The Differentiated Network: Organizing Multinational Corporations for Value Creation, San Francisco 1997, S. 14.

Im internationalen Management lassen sich auch Bezüge zu ökologischen Fragestellungen ausmachen. So wird die natürliche Umwelt explizit im Zusammenhang mit topographischen und klimatischen Gegebenheiten, der Ressourcenausstattung und infrastrukturellen Verhältnissen des Landes erwähnt. ${ }^{65}$ Weiterhin werden den ökologiebezogenen politisch-rechtlichen Rahmenbedingungen sowie dem ökologiebezogenen Konsumentenverhalten in Auslandsmärkten eine hohe Bedeutung beigemessen. Im Gegensatz dazu finden sich nur wenig Ansatzpunkte zu internationalen Umweltschutzstrategien und der internationalen Koordination des ökologiebezogenen Unternehmensverhaltens. ${ }^{66}$ Im Folgenden werden deshalb zunächst die für diese Arbeit relevanten Ansätze aus dem Umweltmanagement dargelegt.

65 Vgl. bspw. Meffert, H.; Bolz, J.: Internationales Marketing-Management, 3. Aufl., Stuttgart u. a. 1998, S. $52 \mathrm{ff}$.

66 In diesem Zusammenhang ist allerdings Hummels zu erwăhnen, der theoriegeleitet die ökonomischen Anreize zur Standardisierung des umweltverträglichen Verhaltens inter- 
Anfang der 90er Jahre erweitert sich die betriebswirtschaftliche Diskussion insbesondere im Marketing vor dem Hintergrund sich verändernder gesellschaftlicher und ökologischer Rahmenbedingungen. ${ }^{67}$ Die Vorstellung, Wirtschaften sei ein funktional autonomer Prozess ${ }^{68}$, wurde unter Rückgriff auf die Systemtheorie abgelöst. Unternehmen gelten danach als offene, zielgerichtete soziotechnische Güter-Umsatz-Systeme. ${ }^{69}$ D. h. ein Unternehmen ist in vielfältiger Weise in eine komplexe Makroumwelt eingebunden, mit der Austauschbeziehungen bestehen. In diesem Zusammenhang ist eine Unternehmung durch okologische Forderungen von Anspruchsgruppen ${ }^{70}$ mittelbar bzw. unmittelbar betroffen. Diese Erkenntnis führt zu der Forderung einer Berücksichtigung der unterschiedlichen Stakeholderinteressen, da ein "Fit" zwischen Unternehmensumwelt und Unternehmen eine Voraussetzung für einen langfristigen Unternehmensfortbestand darstellt. ${ }^{71}$

Die Ursache für eine Berücksichtigung ökologischer Aspekte durch das Management ist häufig nicht direkt auf die Wahrnehmung der Umweltbelastungen als solche zurückzuführen, vielmehr sind Auslöser und Verstärker der Sensibilisierung dazwischengeschaltet, die dafür Sorge tragen, dass sich die ökologischen Probleme im Bewusstsein der Menschen niederschlagen. So wird als ein wesentlicher Bestimmungsfaktor des ökologiegerichteten Unternehmensverhaltens die Betroffenheit durch Ansprüche von Stakeholdern verstanden. ${ }^{72}$

national tătiger Unternehmen untersucht. Vgl. Hummels, H.: Anreize zur Standardisierung umweltverträglichen Verhaltens multinationaler Unternehmen, Frankfurt a. M. u. a. 1998.

Vgl. Meffert, H.: Marketingwissenschaft im Wandel - Anmerkungen zur Paradigmendiskussion, in: Handelshochschule Leipzig (Hrsg.): HHL-Arbeitspapier Nr. 30, S. 5.

„Die Wirtschaft präsentiert sich ... als Prozeß, der allein vom Menschen abhăngig ist und daher in keiner Weise auf die nicht vom Menschen geschaffenen und ihm vorgegebene Natur Rucksicht nehmen muß“. Binswanger, H. C. et al.: Wirtschaft und Umwelt, Stuttgart u. a. 1981, S. 38 .

Vgl. Meffert, H.: Systemtheorie aus betriebswirtschaftlicher Sicht, in: Schenk, K. E. (Hrsg.): Systemanalyse in den Wirtschafts- und Sozialwissenschaften, Berlin 1971, S. 179 sowie Uirich, H.: Die Unternehmung als produktives soziales System: Grundlagen der allgemeinen Unternehmungslehre, Bern 1968, S. 100 ff.; Beer, S.: Kybernetik und Management, Hamburg 1962, S. $21 \mathrm{ff}$.

70 Als Anspruchsgruppe einer Unternehmung zăhlt nach FREEMAN diejenige Personenmehrheit, "who can affect or is affected by the achievements of the firm's objectives." Freeman, R. E.: Strategic Management: A Stakeholder Approach, Marshfield 1984, S. 29.

71 Vgl. zu Ansătzen der Theorie einer sozialen Unternehmensumwelt die Ausführungen von JEHLE. Vgl. Jehle, E.: Unternehmung und gesellschaftliche Umwelt: Grundlagen einer okonomischen Theorie der pluralistischen Unternehmung, Stuttgart 1980.

72 Vgl. Günther, E.; Sturm, A.: Okologieorientierung und okologischer Erfolg, in: Wissenschaftliche Zeitschrift der Technischen Universităt Dresden, 46. Jg. (1997), Nr. 6, S. 78. 
Ökologische Aspekte können dabei bspw. über gesetzliche Regelungen oder direkt über ökologiebezogene Kaufentscheidungen von einer gesellschaftlichen Ebene zu einem unternehmensbezogenen ökologischen Wettbewerbsfeld transformiert werden. Die Phasen der ökologischen Unternehmensentwicklung sind in Abbildung 4 dargestellt. ${ }^{73}$

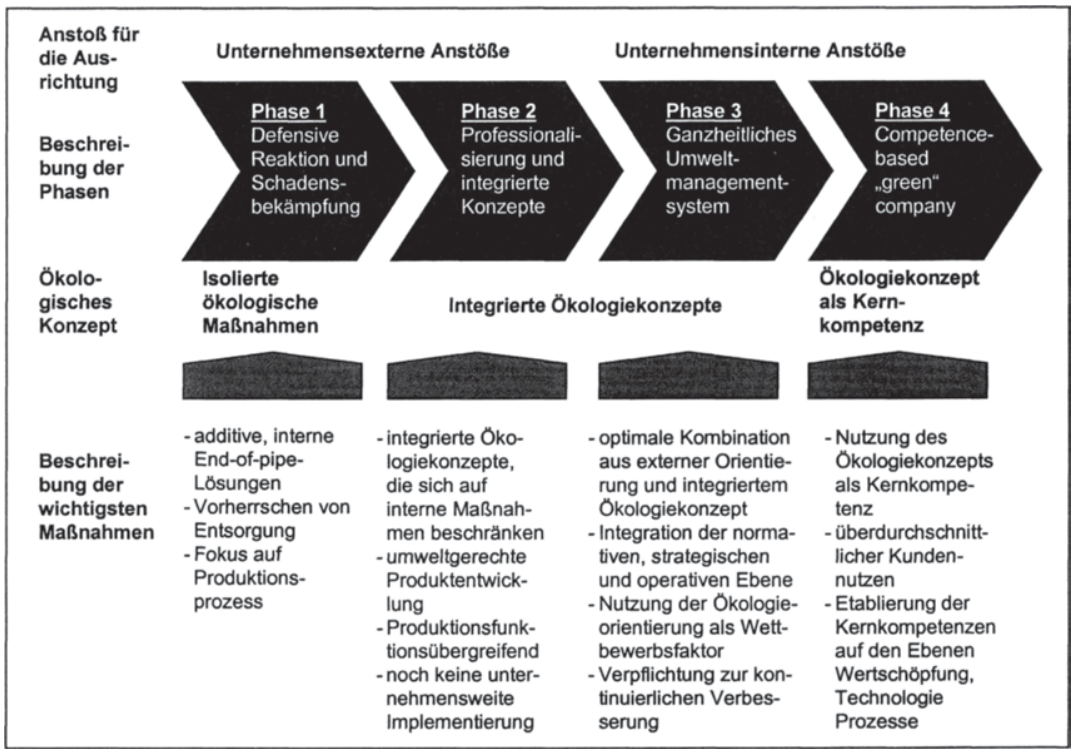

Abbildung 4: $\quad$ Phasen einer ökologischen Unternehmensentwicklung

Quelle: Hipp, Ch.; Reger, G.: Die Dynamik ökologischer Entwicklungsprozesse in Unternehmen - Kernelemente eines ganzheitlichen Umweltmanagementsystems, in: ZfB, Ergänzungsheft 1, 1998, S. 40.

Ende der 70er Jahre führten u. a. Bürgerproteste schrittweise in den USA und Japan, gefolgt von Schweden und den westeuropäischen Staaten, zur Umsetzung einer staatlichen Umweltpolitik. In dieser ersten Phase der ökologischen Unternehmensentwicklung reagierten Unternehmen defensiv auf ökologische Forderungen. Technokratische Ansätze ${ }^{74}$ herrschten vor, bis sie zu Beginn

73 Vgl. hierzu und im Folgenden Hipp, Ch.; Reger, G.: Die Dynamik okologischer Entwicklungsprozesse in Unternehmen - Kernelemente eines ganzheitlichen Umweltmanagementsystems, a. a. O., S. $40-43$.

74 Ziel der defensiven Reaktion war kurzfristige Schadensabwendung durch den Einsatz additiver End-of-pipe-Technologien im fertigungsbezogenen Umweltschutz. Vgl. Tischler, K.: Okologische Betriebswirtschaftslehre, München u. a. 1996, S. 20 f. 
der 80er Jahre vermehrt durch funktionsübergreifende und integrative Ansätze des Umweltschutzes erweitert wurden. Infolge stark zunehmender ökologischer Vorschriften des Gesetzgebers sowie eines höheren Umweltbewusstseins versuchen Unternehmen, den ökologischen Herausforderungen durch höhere Effizienz und Kostenminimierung zu begegnen. Neben fertigungsbezogenen Maßnahmen wurden hier auch produktpolitische Maßnahmen eingeleitet. Ende der 80 er Jahre wurden erste Konzepte einer umweltorientierten Unternehmensführung ${ }^{75}$ entwickelt.

In Verbindung mit einem zunehmenden Wertewandel kamen zu Beginn der 90 er Jahre in den ökologisch fortschrittlichen Industriestaaten zunehmend integrierte, ganzheitliche Ansätze zur Anwendung, die eine Ausweitung des Blickfeldes auf die Öffentlichkeit und gesellschaftliche Anspruchsgruppen sowie ein funktionsübergreifendes Denken in der Unternehmung im Rahmen eines Umweltmanagements forderten. ${ }^{76}$ Eine proaktive Unternehmensstrategie zielt dabei auf die Vorwegnahme von Änderungen der rechtlichen Rahmenbedingungen und der Ansprüche der Stakeholder ab. In diesem Kontext gilt es, ökologische Zielsetzungen mit ökonomischen Unternehmenszielen zu verknüpfen und Umweltschutz nicht als Restriktion, sondern auch als unternehmensbezogene Chance zu verstehen. ${ }^{77}$ Um ein integriertes, übergreifendes Umweltmanagement aufzubauen, bedarf es einer systematischen und integrierten Berücksichtigung ökologiebezogener Problemstellungen im Unternehmen. Ein Umweltmanagementsystem dient in diesem Zusammenhang der Abstimmung von Einzelaktivitäten zur Prozesslenkung mit dem Ziel einer relativen Verbesserung der ökologischen Unternehmensleistung. ${ }^{78}$

75 „Das Umweltmanagement berucksichtigt bei Planung, Durchsetzung und Kontrolle der Unternehmensaktivităten in allen Bereichen Umweltschutzziele zur Vermeidung und Verminderung der Umweltbelastungen und zur langfristigen Sicherung der Unternehmensziele." Meffert, H.; Kirchgeorg, M.: Marktorientiertes Umweltmanagement - Konzeption Strategie - Implementierung mit Praxisfallen, a. a. O., S. 23.

76 Vgl. Meffert, H.: Marketingwissenschaft im Wandel - Anmerkungen zur Paradigmendiskussion, a. a. O., S. 5.

77 Vgl. Dyllick, Th.; Belz, F.; Schneidewind, U.: Okologie und Wettbewerbsfăhigkeit, München, Wien 1997, S. 3-5.

78 Vgl. Epstein, M.; Roy, M.-J.: Managing Corporate Environmental Performance: A Multinational Perspective, in: European Management Journal, 16. Jg. (1998), Nr. 3, S. 292. Durch die systematische Festlegung der Organisationsstruktur, persőnlicher Zustăndigkeiten für Umweltschutzbelange, von okologiebezogenen Maßnahmen, förmlicher Verfahren sowie Abläufen, entstand ein Umweltmanagementsystem (UMS). Vgl. Felix, R.; et al.: Integrierte Managementsysteme: Ansătze zur Integration von Qualităts-, Umwelt- und 
Seit den 90er Jahren lassen sich in einigen Industrieländern große Fortschritte im Umweltschutz verzeichnen. Wissenschaftliche Prognosen deuten aber darauf hin, dass die Effizienzgewinne durch verbesserten betrieblichen Umweltschutz von zusätzlichen Umweltbelastungen aufgrund von Wirtschaftswachstum und Bevölkerungsentwicklung überkompensiert werden und sich die natürlichen Lebensgrundlagen weltweit weiter verschlechtern. ${ }^{79}$ Vor diesem Hintergrund und den ökologischen Forderungen der Gesellschaft erscheint eine Ausrichtung des Unternehmensverhaltens an den Prinzipien des Sustainable Development erforderlich, um der voranschreitenden Umweltzerstörung zu begegnen und langfristig wettbewerbsfähig zu bleiben. ${ }^{80}$ Der Diskussion über Kernkompetenzen folgend kann in diesem Zusammenhang das Ökologiekonzept als eine wesentliche Unternehmenskompetenz begriffen werden. Die Herausbildung ökologiebezogener Kernkompetenzen zur Umsetzung eines intern und extern orientierten OKologiekonzepts gilt dabei als Basis für einen nachhaltigen Wettbewerbsvorteil des Unternehmens. ${ }^{81}$

Während in der internationalen Managementliteratur ökologische Aspekte vernachlässigt wurden, finden sich in der ökologiebezogenen Literatur kaum Bezüge zu den besonderen Herausforderungen bei internationaler Unternehmenstätigkeit. ${ }^{82}$ So wurde in der Vergangenheit primär das ökologiebezogene

Arbeitssicherheitsmanagementsystemen, IWO-Diskussionsbeitrag Nr. 41, St. Gallen 1997, S. 5 - 6 .

79 TROGE verweist dabei auf den Global Environmental Outlook 2000 der UNEP. Vgl. Troge, A.: Schritte zu einer nachhaltigen Weltumweltordnung, in: Fichter, K.; Schneidewind, U. (Hrsg.): Umweltschutz im globalen Wettbewerb - Neue Spielregeln für das grenzenlose Unternehmen, Berlin u. a. 2000, S. 15. Die ökologischen Entwicklungen “..paint a troubling picture of the future, with many critical environmental indicators continuing to decline at their current pace or at increasing speed." World Ressource Institute (Hrsg.): Global Environmental Trends, 1997, http://www.wri.org/wri/ [29.11.00].

80 Nach MEFFERT und KIRCHGEORG werden veränderte umweltpolitische Rahmenbedingungen, ein wachsender okologischer Leidensdruck sowie die wahrgenommene okologische Verantwortung aller Beteiligten die entscheidenden Triebkräfte für eine dauerhafte Entwicklung sein. Dies erfordere u. a. erhebliche Anstrengungen von Seiten der Betriebswirtschaftslehre. Vgl. Meffert, H.; Kirchgeorg, M.: Marktorientiertes Umweltmanagement: Konzeption, Strategie, Implementierung mit Praxisfallen, a. a. O., S. 458 f.

Vgl. Hipp, Ch.; Reger, G.: Die Dynamik okologischer Entwicklungsprozesse in Unternehmen - Kernelemente eines ganzheitlichen Umweltmanagementsystems, a. a. O., S. 43.

Als eine der wenigen Ausnahmen seien hier RUGMANN und VERBEKE erwăhnt, die den Einfluss von nationalen Umweltschutzvorschriften auf die okologiebezogene strategische Ausrichtung von Unternehmen aus ressourcenorientierter Sicht systematisiert haben. Vgl. Rugman, A. M.; Verbeke, A.: Corporate Strategies and Environmental Regulations, in: Strategic Management Journal, 19. Jg. (1998), S. 363 - 375 sowie Rugmann, A. M.; Verbeke, A.: Corporate Strategy and International Environmental Policy, in: Journal of International Business Studies, 29. Jg. (1998), Nr. 4, S. 819-834. 
Unternehmensverhalten in einzelnen Ländern analysiert ${ }^{83}$, während nur vereinzelte empirische Untersuchungen des internationalen ökologiegerichteten Verhaltens vorliegen. ${ }^{84}$

Eine gemeinsame Betrachtung ökologiebezogener und internationaler Herausforderungen bleibt somit in der betriebswirtschaftlichen Literatur bisher weitgehend die Ausnahme. ${ }^{85}$ Lediglich BRODEL zeigt mit seiner Arbeit zum Internationalen Umweltmanagement grundlegende theoretische Ansätze des ökologiegerichteten internationalen Unternehmensverhaltens auf, indem er Erkenntnis-

83

Vgl. Wolter, F.: Umweltmanagement in Europa - eine empirische Untersuchung auf Grundlage des Europäischen Umweltmanagement-Barometers (EBEB), in: Meffert, $H$.; Backhaus, K.; Becker, J. (Hrsg.): Arbeitspapiere der Wissenschaftlichen Gesellschaft für Marketing und Unternehmensführung e. V., Nr. 130, Münster 1999 sowie die dort angegebenen Studien.

Die Arbeiten von GLADWIN und WELLES aus dem Jahr 1976 bieten erste empirische Anhaltspunkte zum ökologiebezogenen Verhalten international tătiger Unternehmen. Die Autoren gelangen zu der Überzeugung, dass: "Environmental protection is generally not viewed as a positive opportunity, but rather as a reluctantly-approached hurdle or operating constraint. ... Geographically, most MNCs have made no attempt to develop a consistent set of environmental policies applicable to their global operations. ... In general, the environmental objectives of MNCs in their overseas operations appear to be a matter of local adoption or accommodation." Vgl. Gladwin, T. N.; Welles, J. G.: Environmental Policy and Multinational Corporate Strategy, in: Studies in International Environmental Economics, New York 1976, S. 183 f. sowie Gladwin, T. N.; Welles, J. G.: Multinational Corporations and Environmental Protection: Patterns of Organizational Adoption, in: International Studies of Management and Organization, 9. Jg. (1976), Spring-Summer, S. $160-184$.

Anfang der 90er Jahre wurde die empirische Forschung zu diesem Thema weitergeführt. Die Vereinten Nationen haben im Report of the Benchmark Corporate Environmental Survey 1993 Daten zur unternehmenspolitischen und strukturellen Verankerung des Umweltschutzes in international tätigen Unternehmen erfasst. Die Ergebnisse legen dabei nahe, dass insbesondere im produzierenden Gewerbe und in der Grundstoffindustrie weltweit gleiche Umweltschutzpolitiken verfolgt und unternehmensbezogen die gleichen strengen Standards angewendet werden. Vgl. United Nations (Hrsg.): Environmental Management in Transnational Corporations: Report on the Benchmark Corporate Environmental Survey, a. a. o. Allerdings sind die Ergebnisse insgesamt kritisch zu hinterfragen. BRODEL spricht in diesem Zusammenhang von der Möglichkeit des sozial erwünschten Antwortverhaltens. Vgl. Brodel, D.: Internationales Umweltmanagement: Gestaltungsfelder - Determinanten - Ausprägungen, a. a. O., S. 419 f.

Schließlich ist die Studie von STRAUBHAAR und WYSs zu erwähnen, in der 1994 das ökologiebezogene Verhalten schweizerischer und deutscher multinationaler Unternehmen untersucht wurde. Zwar zeigte sich, dass an 15 von 22 auslăndischen Standorten einzelne lokale Umweltschutzstandards übererfüllt wurden, allerdings wurden an keinem Standort die strengen Heimatlandvorschriften eingehalten. Vgl. Straubhaar, T.; Wyss M.: Okologisch bedingte Standortarbitrage? Ausländische Direktinvestitionen in Nicht-OECD-Ländern, in: ZfU 1/94, S. $98-121$.

Die stark volkswirtschaftlich geprägte Arbeit von HUMmELS ist in diesem Zusammenhang noch zu erwähnen. Die Erkenntnisse können sowohl zur unternehmensbezogenen Strategiebestimmung als auch für die Formulierung internationaler umweltpolitischer Rahmenbedingungen herangezogen werden. Vgl. Hummels, H.: Anreize zur Standardisierung umweltverträglichen Verhaltens multinationaler Unternehmen, a. a. o. 
objekte und mögliche Zugängen zu einer ökologieorientierten, internationalen Betriebswirtschaftslehre analysiert. Den Kern der Arbeit bildet die Ableitung idealtypischer Ausprägungen internationaler, ökologieorientierter Unternehmensstrategien. ${ }^{86}$

Für den weiteren Verlauf der vorliegenden Arbeit soll an dieser Stelle der Begriff des internationalen Umweltmanagements durch eine Zusammenführung der Definitionen des Umweltmanagements und des internationalen Marketing-Managements eingeführt werden. Danach sind im internationalen Umweltmanagement bei der Analyse, Planung, Durchführung, Koordination und Kontrolle von Unternehmenstätigkeiten bei Geschäftstätigkeit in mehr als einem Land in allen Bereichen Umweltschutzziele zur Vermeidung und Verminderung von Umweltbelastungen und zur langfristigen Sicherung der Unternehmensziele zu berücksichtigen.

Vor dem Hintergrund der Abgrenzung des internationalen Umweltmanagements kann die internationale Koordination des ökologiebezogenen Unternehmensverhaltens als eine zentrale Aufgabe im Rahmen des internationalen Umweltmanagements gelten. Umso mehr ist es überraschend, dass in der betriebswirtschaftlichen Literatur eine Beschäftigung mit diesem Thema die Ausnahme bleibt.

\section{Koordination als Aufgabe an der Schnittstelle zwischen internatio- nalem Management und Umweltmanagement}

Das ökologiegerichtete Verhalten international tätiger Unternehmen steht im Fokus dieser Arbeit. Da die einzelnen Organisationseinheiten eines international tätigen Unternehmens aufgrund einer unternehmensübergreifenden Zielsetzung miteinander verbunden sind, bedarf es der Abstimmung bzw. Steuerung der Entscheidungsträger in den einzelnen Teilbereichen. ${ }^{87}$ Dies kann allgemein als Koordination bezeichnet werden. ${ }^{88}$

86 Vgl. Brodel, D.: Internationales Umweltmanagement: Gestaltungsfelder - Determinanten Ausprăgungen, a. a. 0.

87 Vgl. Frese, E.: Grundlagen der Organisation: Konzept - Prinzipien - Strukturen, a. a. O., S. 13. Ähnlich auch PORTER, der unter Koordination die gemeinsame Nutzung von Informationen, die Zuweisung von Verantwortung sowie die gegenseitige Abstimmung versteht. Vgl. Porter, M. E.: Nationale Wettbewerbsvorteile: Erfolgreich konkurrieren auf dem Weltmarkt, Sonderausgabe, Wien 1993, S. 81.

Vgl. Adam, D.: Investitionscontrolling, 2. Aufl., München, Wien 1997, S. 11 ff. 
Der Koordinationsbegriff gilt als ein zentraler Begriff der Betriebswirtschaftslehre, der in der Literatur sowohl in Bezug auf Begriffsbildung, Problemstellung als auch die Lösungsansätze unter unterschiedlichen Gesichtspunkten beleuchtet wurde. ${ }^{89}$ Dabei beinhaltet der Koordinationsbegriff allgemein "Tatbestände, die aufgrund von Abhängigkeiten der Abstimmung durch Anwendung von Maßnahmen oder Instrumenten bedürfen, wobei diese Abstimmung im Hinblick auf die Optimierung eines definierten Zieles als wünschenswert bzw. notwendig erachtet wird". 90

Gegenseitige Abhängigkeiten können dabei als Interdependenzen ${ }^{91}$ bezeichnet werden. Interdependenzen beziehen sich aus organisationstheoretischer Sicht auf die gegenseitige Abhängigkeit von organisatorischen Einheiten bei ihrer Aufgabenerfüllung. Aus entscheidungstheoretischer Perspektive liegt eine Interdependenz vor, wenn eine Entscheidung einer organisatorischen Einheit bei ihrer Realisation das Entscheidungsfeld einer anderen Einheit zielrelevant verändert. $^{92}$ Sie resultieren aus der Nutzung gemeinsamer, begrenzter Ressourcen, aus einem Ineinandergreifen von Aktivitäten aufgrund von Input-/Outputbeziehungen und damit aus innerbetrieblichen Leistungsverflechtungen sowie aus der gegenseitigen Beeinflussung über Interaktionen mit dem Umsystem des Unternehmens. ${ }^{93}$

89 Vgl. zu unterschiedlichen Koordinationsbegriffen bspw. Hax, H.: Die Koordination von Entscheidungen - Ein Beitrag zur betriebswirtschaftlichen Organisationslehre, Köln u. a. 1965, S. 9; Heinen, E.: Einfuhrung in die Betriebswirtschaftslehre, Wiesbaden 1968, S. 252; Kirch, W.: Die Koordination von Entscheidungen in Organisationen, in: ZfbF, 23. Jg. (1971), Nr. 1, S. 61 f. und Hoffmann, F.: Fuhrungsorganisation, Bd. I: Stand der Forschung und Konzeption, Tübingen 1980, S. 298 - 305, Frese, E.: Grundlagen der Organisation: Konzept - Prinzipien - Strukturen, a. a. O., S. 63-65.

90 Hoffmann, F.: Führungsorganisation, a. a. O., S. 303. Ähnlich auch BACKHAUS, der allgemein das Ziel der Koordination im Erreichen optimaler Zustände für das Gesamtunternehmen sieht. Vgl. Backhaus, K.; Büschken, J.; Voeth, M.: Internationales Marketing, a. a. O., S. 45.

91 Zum Begriff der Interdependenzen vgl. Hoffmann, F.: Fuhrungsorganisation, a. a. O., S. $221-234$ und insbesondere Lassmann, A.: Organisatorische Koordination: Konzepte und Prinzipien zur Einordnung von Teilaufgaben, Wiesbaden 1992, S. 34 - 57sowie die dort angegebene Literatur. Zu empirischen Untersuchungen von Interdependenzen im internationalen Management vgl. Paul, Th.: Globales Management von Wertschopfungsfunktionen, Wiesbaden 1998, S. 93.

92 Vgl. Adam, D.: Planung und Entscheidung, 4. Aufl., Wiesbaden 1997, S. 168 ff.; Lassmann, A.: Organisatorische Koordination: Konzepte und Prinzipien zur Einordnung von Teilaufgaben, a. a. O., S. 46 sowie Frese, E.: Grundlagen der Organisation: Konzept Prinzipien - Strukturen, a. a. O., S. 53.

93 Vgl. Hoffmann, F.: Fuhrungsorganisation, a. a. O., S. 308 sowie Lassmann, A.: Organisatorische Koordination: Konzepte und Prinzipien zur Einordnung von Teilaufgaben, a. a. O., S. $46-47$. 
Interdependenzen können bei internationaler Unternehmenstätigkeit zu Rückkopplungen führen, die bewirken, „dass die Freiheitsgrade bei der Marktbearbeitung und deren Ergebnisse in den einzelnen Ländermärkten nicht unabhängig voneinander sind. Das Agieren auf einem Ländermarkt beeinflusst dann die Art und Weise, wie auf anderen Ländermärkten agiert wird oder agiert werden kann (vice versa)."94 Der Koordinationsbedarf in einem internationalen Unternehmen kann demzufolge als besonders hoch angesehen werden, wenn der Grad der gegenseitigen Abhängigkeit und damit die Komplexität der Beziehungen zwischen den Elementen des Unternehmens aufgrund interner Leistungsverflechtungen und Ressourceninterdependenzen sowie externer Verbundeffekte hoch ist, große räumliche, zeitliche, sachliche und zwischenmenschliche Distanzen ${ }^{95}$ zu überwinden sind und die übergeordnete Zielsetzung selbst unstrukturiert und umfangreich ist oder Zielbeziehungen zwischen Teilzielen bestehen. ${ }^{96}$

Das marktorientierte Umweltmanagement ist aufgrund des funktions- und unternehmensübergreifenden Charakters $^{97}$ ökologischer Problemstellungen durch eine hohe Komplexität und Anpassungsflexibilität und damit durch eine Vielzahl von Interdependenzen geprägt. Der Koordination im Umweltmanagement ist somit ein hoher Stellenwert einzuräumen. Die Koordinationserfordernisse im Umweltbereich sind dabei durch ein Schnittstellenmanagement aufeinander abzustimmen. ${ }^{98}$

94 Backhaus, K.; Büschken, J.; Voeth, M.: Internationales Marketing, a. a. O., S. 46. Der Begriff der Rückkopplung stammt aus der Regelungstheorie und beschäftigt sich mit der Struktur und Regelung von Systemen. Rückkopplungen können als Interdependenzen bezeichnet werden, die auf die Bearbeitung unterschiedlicher Ländermärkte zurückgeführt werden können.

PORTER führt hier an, dass eine große Schwierigkeit darin besteht, die Interessen der Manager der Tochtergesellschaften mit denen der Zentrale in Einklang zu bringen. So würden sich unterschiedliche Tochtergesellschaften häufig eher als Konkurrenten betrachten. Vgl. Porter, M. E.: Nationale Wettbewerbsvorteile: Erfolgreich konkurrieren auf dem Weltmarkt, a. a. O., S. 82. Gegenstand dieser Überlegungen ist die Prämisse, dass neben formalen auch informale Aspekte der Koordination zum Tragen kommen. OUCHI weist bereits 1979 auf diese Probleme hin. Vgl. Ouchi, W. G.: A conceptual Framework for the Design of Organizational Control Mechanisms, in: Management Science, 9. Jg. (1979), Nr. 6, S. 824.

In Anlehnung an Winkler, G.: Koordination in strategischen Netzwerken, Wiesbaden 1999, S. $95 \mathrm{f}$.

97 Vgl. Meffert, H.; Kirchgeorg, M.: Marktorientiertes Umweltmanagement: Konzeption, Strategie, Implementierung mit Praxisfallen, a. a. O., S. 19-22.

98 Vgl. Meffert, H.; Kirchgeorg, M.: Marktorientiertes Umweltmanagement: Konzeption, Strategie, Implementierung mit Praxisfallen, a. a. O., S. 413. 
Die Notwendigkeit, ökologiebezogene Fragestellungen bei der internationalen Unternehmenstätigkeit zu berücksichtigen, und die Tatsache, dass sich nationale ökologiebezogene Anforderungen unterscheiden, führt zu zusätzlichem Koordinationsbedarf. Die Fertigung in unterschiedlichen nationalen Kontexten macht oft eine Anpassung an die dort geltenden ökologiebezogenen Ansprüche notwendig, um die Unternehmenslegitimität wahren zu können. Erfüllen die Produkte eines Unternehmens nicht die Umweltschutzvorschriften eines Exportlandes, kann der Transfer gesetzlich untersagt werden. In Bezug auf den funktionsübergreifenden Charakter ökologischer Problemstellungen und der zunehmenden internationalen Verteilung der Wertschöpfungsaktivitäten kann von einer Vielzahl ökologiebezogener Interdependenzen ausgegangen werden. Vor dem Hintergrund möglicher Rückkopplungen ist zu beachten, dass Entscheidungen über das ökologieorientierte Unternehmensverhalten nicht ländermäßig isoliert, sondern im Gesamtzusammenhang zu treffen sind. ${ }^{99}$

Koordination umfasst in diesem Kontext die notwendige oder wünschenswerte Abstimmung und Ausrichtung dezentraler ökologiebezogener Entscheidungen bei Rückkopplungen zwischen zwei Ländern durch Anwendung von Maßnahmen oder Instrumenten mit dem Ziel, das Verhalten der Organisationsmitglieder auf die Unternehmensziele auszurichten. Dabei ergibt sich die spezifische Zielsetzung der Koordination aus dem Zielsystem der Gesamtunternehmung. Durch den Einsatz von Koordinationsmaßnahmen und -instrumenten entstehen Koordinationskosten, die neben den Anbahnungs-, Vereinbarungs-, Abwicklungs- und Kontrollkosten auch Anpassungskosten umfassen. ${ }^{100}$

Unter Koordinationsmaßnahmen und -instrumenten können generell alle Regelungen verstanden werden, „die Art und Umfang von Interdependenzen zwischen Organisationseinheiten steuem sowie die Abstimmung arbeitsteiliger Prozesse durch Ausrichtung der Entscheidungen dieser autonomen Teilein-

99 Vgl. Bolz, J.: Wettbewerbsorientierte Standardisierung der internationalen Marktbearbeitung: eine empirische Analyse in europäischen Schlusselmärkten, Darmstadt 1992, S. 4.

Der Koordinationskostenbegriff erlangt in der Transaktionskostentheorie einen besonderen Stellenwert. Vgl. dazu Coase, R.: The Nature of the Firm, in: Economica, New Series, 4. Jg. (1937), S. 386 - 405; Picot, A.: Ein neuer Ansatz zur Gestaltung der Leistungstiefe, in: Zeitschrift für betriebswirtschaftliche Forschung, 43. Jg. (1991), Nr. 4, S. 336 - 357; Brockhoff, K.: Management organisatorischer Schnittstellen - unter besonderer Berücksichtigung der Koordination von Marketingbereichen mit der Forschung und Entwicklung, Gottingen 1994, S. 5; Meffert, H.: Marketing: Grundlagen marktorientierter Unternehmensführung: Konzepte - Instrumente - Praxisbeispiele, a. a. O., S. $1022 \mathrm{f}$. 
heiten auf die Untermehmensziele gewährleisten."101 Aufgrund der großen Heterogenität der unterschiedlichen Maßnahmen soll im Rahmen dieser Arbeit in Anlehnung an HofFMANN zwischen Maßnahmen zur Reduzierung und zur Deckung des Koordinationsbedarfs unterschieden werden.

Koordinationserlöse liegen demgegenüber in zusätzlichen Erlösen oder eingesparten Kosten des Gesamtunternehmens, die durch die koordinierte Vorgehensweise realisiert werden können. Da diese Zusatzerlöse bzw. Kosteneinsparungen bei mangelhafter oder fehlender Koordination verloren gehen, werden sie auch als Autonomiekosten bezeichnet. Die Autonomiekosten nehmen mit steigender Koordinationsintensität ab, während die Koordinationskosten zunehmen. Die optimale Koordinationsintensität wird durch das Minimum der Gesamtkostenfunktion bestimmt. ${ }^{102}$

\section{Ziel und Gang der Untersuchung}

Vor dem dargestellten Hintergrund besteht die generelle Zielsetzung der Arbeit in der Aufdeckung eines spezifischen ökologiebezogenen Koordinationsbedarfs bei internationaler Unternehmenstätigkeit sowie der Untersuchung von geeigneten Instrumenten zur Reduzierung und Deckung des Koordinationsbedarfs. In diesem Zusammenhang sollen Ausgestaltungsformen von Konzeptionen zur effektiven und effizienten Gestaltung eines Koordinationssystems für unterschiedliche strategische Orientierungen im internationalen Umweltmanagement abgeleitet werden. Auf diesem Wege können Anhaltspunkte für eine Verbesserung der internationalen Koordination ökologiebezogener Entscheidungen herausgearbeitet werden.

Allgemein kennzeichnet der ökologiegerichtete Koordinationsbedarf bei internationaler Unternehmenstätigkeit die Menge an Abstimmungsmaßnahmen, die zur Ausrichtung des ökologiebezogenen Verhaltens auf die Oberziele der Gesamtunternehmung erforderlich sind. Da die ökologiegerichtete Koordination des internationalen Unternehmensverhaltens je nach Produkt- bzw. Branchen-

101 Hoffmann, F.: Führungsorganisation, a. a. O., S. 316; in Anlehnung an Kieser, A.; Kubicek, H.: Organisation, Berlin, New York 1977, S. 74.

102 Vgl. Benkenstein, M.: F \& E und Marketing: Eine Untersuchung zur Leistungsfăhigkeit von Koordinationskonzeptionen bei Innovationsentscheidungen, a. a. O., S. 18 ff. Dabei lassen sich die Koordinations- und Autonomiekosten bisher nicht zufriedenstellend quantifizieren. Vgl. Albach, H.: Kosten, Transaktionen und externe Effekte im betrieblichen Rechnungswesen, in: Zeitschrift für Betriebswirtschaft, 58. Jg. (1988), Nr. 11, S. $1143-1170$. 
kontext einer situativen Relativierung ${ }^{103}$ bedarf, soll sich die Untersuchung an den spezifischen Rahmenbedingungen der Automobilindustrie ausrichten. Die Automobilindustrie als situativer Kontext der Koordination bietet sich insbesondere aus folgenden Gründen an:

- Die Automobilindustrie umfasst derzeit 16 international tätige Konzerne. ${ }^{104}$ Diese Unternehmen produzieren an verschiedenen Auslandsstandorten und vertreiben ihre Produkte nahezu weltweit.

- In der Automobilindustrie herrscht eine hohe Wettbewerbsintensität bei sinkender Nachfrage in den Triademärkten und hohem Kostendruck. Dabei sehen sich die Hersteller z. T. höchst heterogenen fertigungs- und produktbezogenen Umweltschutzforderungen gegenüber.

- Die Automobilindustrie steht schließlich aufgrund vielfältiger ökologischer Probleme in Bezug auf die Fertigung sowie auf das Produkt wie kaum eine andere Branche in der Kritik ökologischer Anspruchsgruppen, die mittlerweile zumeist ebenfalls international organisiert sind.

Aus der generellen Zielsetzung der Arbeit leiten sich folgende Teilziele als Forschungsschwerpunkte ab, die ebenfalls den Gang der Untersuchung vorgeben:

1. Die Wahl der Automobilindustrie als Untersuchungsbeispiel macht eine Auseinandersetzung mit dieser Branche und eine eingehende Analyse inrer Struktur und Entwicklung notwendig. Im Kontext der vorliegenden Fragestellung werden deshalb in Kapitel B 1 die wesentlichen internationalen Entwicklungen in der Automobilindustrie sowie die aktuellen Herausforderungen der Branche aufgezeigt. Weiterhin ist die Beschreibung der ökologischen Belastungen, die bei Herstellung, Nutzung und Entsorgung der Automobile hervorgerufen werden und als ökologiebezogene Herausforderungen der Automobilindustrie aufgefasst werden können, ein weiterer Teil des Kapitels

103 Der situative Ansatz stellt kontextbezogene, d. h. sich aus dem Umfeld der Unternehmung ergebende Anpassungsnotwendigkeiten in den Vordergrund. Solche Anpassungsnotwendigkeiten ergeben sich entsprechend auch bei der ökologiegerichteten Koordination des internationalen Unternehmensverhaltens. Vgl. zum Kontingenzansatz insbesondere Kast, F.; Rosenzweig, J.: Organization and Management: A Contingency Approach, Tokio 1970; Zeithaml, V. A.; Varadarajan, P.; Zeithaml, C.: The Contingency Approach: It's Foundations and Relevance to Theory Building and Research in Marketing, in: European Journal of Marketing, 22. Jg. (1988), Nr. 8, S. 37 - 64. Eine kritische Würdigung findet sich bei Lehnert, St:: Die Bedeutung von Kontingenzansätzen für das strategische Management, Frankfurt a. M. U. a. 1983, S. 115.

104 Vgl. FOCUS (Hrsg.): Der Markt der Automobile: Daten, Fakten, Trends, 2000, S. 2. 
B 1. Die Erkenntnisse ergeben sich dabei aus einer intensiven Literaturarbeit unter Berücksichtigung automobilspezifischer internationaler und ökologiebezogener Untersuchungen sowie der im Vorfeld durchgeführten Expertenbefragungen.

2. In Kapitel $B 2$ folgt eine Aufarbeitung der theoretischen Grundlagen für das weiterführende Vorgehen. In diesem Zusammenhang werden Interdependenzen als Determinanten des internationalen ökologiegerichteten Koordinationsbedarfs näher gekennzeichnet und exogene und endogene Einflussfaktoren, die auf die Entstehung dieses Koordinationsbedarfs wesentlichen Einfluss nehmen, theoriebezogen abgeleitet. Dabei werden die theoretischen Grundlagen der Koordination bzw. der Koordination im internationalen Kontext unter Bezugnahme auf den Transformationsprozess von ökologischen Belastungen und dem Konstrukt der ökologischen Betroffenheit auf den ökologiespezifischen Kontext der Problemstellung übertragen.

3. In den folgenden Unterkapiteln B 3 und B 4 soll die Notwendigkeit einer internationalen Koordination des ökologiebezogenen Verhaltens in der Automobilindustrie konkretisiert werden. Dazu wird der Einfluss unterschiedlicher situativer Kontextfaktoren auf die Entstehung von ökologiebezogenen Rückkopplungen zwischen Unternehmenseinheiten in unterschiedlichen Ländern untersucht. In diesem Zusammenhang werden Thesen über den Einfluss der unterschiedlichen exogenen und endogenen Kontextfaktoren auf den internationalen ökologiegerichteten Koordinationsbedarf auf Basis von theoriegeleiteten Plausibilitätsüberlegungen aufgestellt. Neben einer generellen Charakterisierung der unterschiedlichen Ländermärkte stehen im Rahmen der vorliegenden Arbeit die Interdependenzen zwischen den Landesgesellschaften und der Muttergesellschaft in der Autombilindustrie im Fokus der Betrachtung. Dabei werden auch die Interaktionsbeziehungen der jeweiligen Landesgesellschaft zu ihrer lokalen, natürlichen und aufgabenbezogenen Umwelt, insbesondere zu den lokalen ökologischen Anspruchsgruppen, berücksichtigt. Weiterhin wird untersucht, inwieweit international oder global agierende Anspruchsgruppen auf die Interdependenzen zwischen unterschiedlichen Landesgesellschaften einwirken können. 
Weiterhin werden unterschiedliche endogene Faktoren bzgl. ihres Einflusses auf die Entstehung eines internationalen ökologiegerichteten Koordinationsbedarfs in der Automobilindustrie untersucht. Zur Berücksichtigung des spezifischen Branchenhintergrunds wird auf im Vorfeld dieser Arbeit durchgeführte explorative Befragungen und eine Auswertung von automobilspezifischen Publikationen sowie verschiedene automobil- und ökologiespezifische Studien zurückgegriffen. In Kapitel B 5 werden die Interdependenzen zwischen einzelnen Faktoren im situativen Kontext dargestellt und der Einfluss ausgewählter Kontextfaktoren auf den ökologiegerichteten internationalen Koordinationsbedarf in einer Übersicht synopsenhaft zusammengefasst. Dabei wird die Ausprägung der einzelnen Kontextfaktoren auf Basis von Expertenurteilen für die Automobilindustrie skizziert.

4. Aufbauend auf den Ausführungen im Kapitel B sollen in Kapitel $C$ zunächst unterschiedliche internationale, ökologiebezogene Koordinationsziele abgeleitet und basisstrategische Optionen im internationalen Umweltmanagement insbesondere in Bezug auf den produkt- und fertigungsbezogenen Umweltschutz erarbeitet werden. Auf Grundlage der Erkenntnisse aus der betriebswirtschaftlichen Zielforschung werden dabei die Koordinationsziele in die unternehmensbezogene Zielhierarchie einbezogen und mögliche Ziele im internationalen Umweltmanagement abgeleitet. Da die strategische Ausrichtung im internationalen Umweltmanagement selbst zur Koordination verschiedener potenzieller internationaler Interdependenzen beiträgt und gleichzeitig als Ausgangspunkt zur Festlegung der konkreten Koordinationsziele dient, folgt eine Darstellung der in der Literatur diskutierten strategischen Basisoptionen im internationalen Umweltmanagement. Aufbauend darauf werden die Anwendungsvoraussetzungen der Strategien, ihre strategischen Ziele sowie insbesondere die jeweilige strategiekonforme Ausrichtung im produkt- und fertigungsbezogenen Umweltschutz abgeleitet.

5. Ein zentrales Ziel der Arbeit stellt die Darstellung von Koordinationsinstrumenten dar, die zur Reduzierung und Deckung des internationalen Koordinationsbedarfs eingesetzt werden können. Zu diesem Zweck soll in Kapitel C 3 das klassische, vor allem aus der Organisationsforschung bekannte Koordiantionsinstrumentarium auf die vorliegende ökologiebezogene Problemstellung übertragen und erweitert werden. Die systematische Darstellung des Einsatzes entsprechender Instrumente in der Unternehmenspraxis erfolgt dabei grundsätzlich anhand von Beispielen aus der Auto- 
mobilindustrie und zeigt, wie Automobilhersteller ihre ökologiebezogenen Tätigkeiten internatonal koordinieren.

6. Ein weiteres Ziel der Arbeit ist es, aufbauend auf den Koordinationszielen der einzelnen Basisstrategien im internationalen Umweltmanagement in Kapitel C 4 Tendenzaussagen über geeignete strategiekonforme Koordinationskonzeptionen abzuleiten. In diesem Zusammenhang wird ein Umweltmanagementsystem als Koordinationskonzeption aufgefasst, welches strategiebezogen unterschiedliche Koordinationsinstrumente umfasst. Mittels Plausibilitätsüberlegungen sowie unter Bezugnahme auf bestehende Koordinationskonzeptionen werden Ausgestaltungsformen für die Automobilindustrie dargestellt.

7. Abschließend werden in Kapitel D die Untersuchungsergebnisse auf andere Branchen übertragen und damit verallgemeinert. In diesem Zusammenhang werden Empfehlungen für die Unternehmenspraxis zur Ausgestaltung von strategiekonformen Umweltmanagementsystemen als unternehmensübergreifende Koordinationskonzeptionen abgegeben. Weiterhin werden offene Forschungsfragen aufgearbeitet, die als Anregung für eine weitere wissenschaftliche Beschäftigung mit dem internationalen Umweltmanagement dienen sollen. In diesem Zusammenhang werden eine Erweiterung der Balanced Scorecard als internationales Koordinationssystem und eine stärkere Berücksichtigung ressourcenorientierter Ansätze im Umweltmanagement diskutiert.

Methodisch kann die Arbeit somit als konzeptionell-literaturgestützt eingeordnet werden, wobei die konzeptionellen Überlegungen auf verschiedene Theoriekonzepte zurückgreifen und mit Erkenntnissen aus Expertengesprächen, einer systematischen Auswertung von Unternehmensberichten, Pressenotizen und ökologiebezogenen Ratingergebnissen sowie primär- und sekundärstatistischen Erhebungen unterlegt werden. ${ }^{105}$ Insbesondere die Ergebnisse der Erhebung

105 In diesem Zusammenhang sind $u$. a. die Ergebnisse aus folgenden, öffentlich nicht zugänglichen Studien zu finden: ${ }_{n}$ The Environmental Monitor - Global Public Opinion on the Environment" von ENVIRONICS INTERNATIONAL LTD. aus Kanada, der "Corporate Responsibility Industry Report Automobile: A Corporate Responsibility Survey of 19 Companies of the Industry" der OEKOM RESEARCH AG aus München sowie der Report "Sustainability in der Automobilindustrie: Das Automobil im năchsten Jahrtausend“ von der SUSTAINABLE ASSET MANAGEMENT GROUP (SAM) aus Zürich.

Der dritte "Environmental Monitor" wurde in 1999 durch eine persönliche bzw. telefonische Befragung von jeweils 1.000 zufällig ausgewählten Erwachsenen in insgesamt 27 Ländern erhoben, die ungefăhr $65 \%$ der Weltbevőlkerung repräsentieren. Der 42 Fragen umschlie- 
zum „Europäischen Umweltmanagement-Barometer", einem europäischen Gemeinschaftsprojekt unter maßgeblicher Beteiligung des Instituts für Marketing, sind dabei hervorzuheben.

Das Europäische Umweltmanagement-Barometer („European Business Environmental Barometer"- kurz E.B.E.B.) ist ein Instrument zur Erhebung und Beschreibung des Standes und der Umsetzung des betrieblichen Umweltmanagements. Es dient einem systematischen und kontinuierlichen Monitoring des ökologiegerichteten Unternehmensverhaltens auf empirisch abgesicherter Basis. Die Daten des Umweltmanagement-Barometers werden anhand einer Umfrage unter Unternehmen des produzierenden Gewerbes und damit auch der Automobilindustrie in regelmäßigen Abstănden von 2 - 3 Jahren in den teilnehmenden Ländern erhoben und ausgewertet. ${ }^{106}$

Bende Fragebogen vergleicht die Einschătzung der Bevölkerung zu ökologiebezogenen Fragestellungen. Vgl. http://www.environics.net/eil/iemnew/.

Als internationale Rating-Agentur liefert die OEKOM RESEARCH AG fundierte und unabhängige Informationen über die ökologische und soziale Performance von Unternehmen. OEKOM arbeitet in einem internationalen Netzwerk von Umwelt- und Sozialorganisationen sowie mit unabhängigen Experten. Vgl. http://www.oekom.de/ag/german/index wir ueber uns.htm.

SAM identifiziert Sustainability-orientierte Unternehmen in Leader- und Pionier-Bereichen. Aufbauend auf klar definierten Methoden nach ökonomischen, okologischen und sozialen Kriterien. Vgl. http://www.sam-group.com/d/about/research.cfm.

Die 15 Kernfragen umfassende Befragung ist standardisiert und wurde 1998 in 11 europäischen Lăndern durchgeführt. Im Rahmen des E.B.E.B. werden Mitglieder der Unternehmensführung in einer schriftlichen Befragung um Auskunft bezüglich unterschiedlicher Aspekte der unternehmensspezifischen Umweltschutzausrichtung gebeten. Der Bezugsrahmen der Untersuchung berücksichtigt endogene und exogene Erklärungsvariablen des umweltorientierten Unternehmensverhaltens und schenkt in diesem Zusammenhang der ökologischen Betroffenheit besondere Bedeutung. Die im Rahmen dieser Arbeit verwendeten Daten unterscheiden allerdings nicht zwischen Automobilherstellern und Zulieferern. Da die Unternehmen bei der schriftlichen Erhebung nur nach ihrer Branchenzugehörigkeit gefragt wurden, kőnnen Automobilhersteller und -zulieferer nicht getrennt ausgewiesen werden. Vgl. zu dieser Umfrage Wolter, F.: Umweltmanagement in Europa - eine empirische Untersuchung auf Grundlage des Europäischen Umweltmanagement-Barometers (EBEB), a. a. O. 


\section{B. Ökologiebezogener Koordinationsbedarf bei internationaler Geschäftstätigkeit in der Automobilindustrie}

\section{Automobilindustrie als situativer Kontext}

\subsection{Internationale Situation der Automobilindustrie}

Das Automobil als Basiskonzept hat sich seit seiner Erfindung vor mehr als 100 Jahren kaum verändert. ${ }^{107}$ Im Gegensatz dazu lassen sich in der Automobilindustrie bis heute verschiedene Transformationen ausmachen, die von einer handwerklichen Fertigung weniger Automobile in einzelnen Ländern bis zur gegenwärtigen nahezu globalen Ausweitung der Industrie reichen. Die dargestellten Transformationen wurden vor allem durch Innovationen ausgelöst, die den Pionierunternehmen jeweils über mehrere Jahre einen Wettbewerbsvorteil verchafft haben. In diesem Zusammenhang war für die Automobilindustrie auch die systematische Erweiterung der Absatzregionen ${ }^{108}$ über Landesgrenzen hinweg schon früh ein strategisch bedeutendes Thema. Der Prozess ist heute noch nicht abgeschlossen und entwickelt sich differenziert nach den wichtigsten Produktionsländern und Herstellern. ${ }^{109}$

Diese Entwicklungen, insbesondere die Internationalisierungsphasen der Branche, sind im Überblick in Tabelle 1 nachgezeichnet.

107 "A hundred years after the first practical vehicle, the automobile is still a four-wheeled, internally powered personal transport apparatus for road use, designed to carry a driver and a few passengers." Altshuler, A. et al.: The Future of the Automobile: The Report of MIT's International Automobile Program, London, Sydney 1984, S. 11.

BECKER nennt dies Arealstrategie; vgl. Becker, J.: Marketing-Konzeptionen: Grundlagen des strategischen Marketing-Managements, München 1990, S. $256 \mathrm{ff}$.

Vgl. Liebehenschel, Th.: Okologieorientierte Produkt- und Dienstleistungspolitik: Rahmenbedingungen und Trends am Beispiel der Automobilindustrie, Marburg 1999, S. 170 sowie Burmann, G.: Marktarealstrategien der internationalen Automobilhersteller, in: Hünerberg, R. et al. (Hrsg.): Internationales Automobilmarketing: Wettbewerbsvorteile durch marktorientierte Unternehmensführung, Wiesbaden 1995, S. 124 ff. 


\begin{tabular}{|c|c|}
\hline $\begin{array}{l}\text { Ende des } 19 . \\
\text { Jahrhunderts }\end{array}$ & $\begin{array}{l}\text { - Handwerkliche Fertigung von Automobilen in Deutschland und Frankreich } \\
\text { - Exporte in das benachbarte Ausland (ethnozentrische Orientierung) }\end{array}$ \\
\hline $\begin{array}{l}\text { Mitte der } 20 \mathrm{er} \\
\text { Jahre }\end{array}$ & $\begin{array}{l}\text { - Innovationen in der Automobilproduktion durch FORD/SLOAN in den USA } \\
\text { - Beginn der Massenfertigung von standardisierten Automobilen } \\
\text { - Export von Fertigteilen und Montage im Ausland } \\
\text { - Aufbau von Tochtergesellschaften/Kauf bestehender Automobilmanufaktu- } \\
\text { ren durch amerikanische Hersteller wegen steigender Handelsschranken } \\
\text { (polyzentrische Orientierung) }\end{array}$ \\
\hline $\begin{array}{l}\text { Ende der } 50 \mathrm{er} \\
\text { Jahre }\end{array}$ & $\begin{array}{l}\text { - Fallende Zollschranken in Europa lassen europäische Hersteller Volumen- } \\
\text { effekte erzielen } \\
\text { - Verbindung von Massenproduktion und Differenzierung durch europäische } \\
\text { Hersteller, die primär in die Überseemärkte exportieren } \\
\text { - Aufbau erster europäischer Tochtergesellschaften im Ausland }\end{array}$ \\
\hline $\begin{array}{l}\text { Ende der 60er } \\
\text { Jahre }\end{array}$ & $\begin{array}{l}\text { - Japanische Hersteller revolutionieren Automobilproduktion } \\
\text { - schlanke Produktion erlaubt Kostensenkungen / Qualitätssteigerungen } \\
\text { - Aufbau von japanischen Tochtergesellschaften in Europa und den USA } \\
\text { aufgrund steigender Handelshemmnisse (geozentrische Orientierung) }\end{array}$ \\
\hline $\begin{array}{l}\text { Mitte der } 80 e r \\
\text { Jahre }\end{array}$ & $\begin{array}{l}\text { - Restrukturierung und Reengineering in der europäischen und amerikani- } \\
\text { schen Automobilindustrie } \\
\text { - Verlagerung der Produktion in Billiglohnländer } \\
\text { - Eintritt neuer Wettbewerber insbesondere aus Südostasien }\end{array}$ \\
\hline $\begin{array}{l}\text { Anfang der } \\
90 \text { er Jahre }\end{array}$ & $\begin{array}{l}\text { - Sättigungstendenzen in den Triademärkten } \\
\text { - wirtschaftliche Erholung in Schwellenländern, hohe Wachstumsraten } \\
\text { - Aufbau / Ausbau der Automobilproduktion in Wachstumsmärkten durch } \\
\text { Hersteller der Triade }\end{array}$ \\
\hline $\begin{array}{l}\text { Mitte der } 90 \mathrm{er} \\
\text { Jahre }\end{array}$ & $\begin{array}{l}\text { - Wirtschaftliche Abkühlung und Asienkrise führen zu Überkapazitäten } \\
\text { - Differenzierungsdruck aufgrund unterschiedlicher nationaler und segment- } \\
\text { spezifischer Kundenwünsche } \\
\text { - Fusionen, Übernahmen, Kapitalbeteiligungen, Kooperationen } \\
\text { - Kostendruck führt zu Plattformstrategie und Gleichteilepolitik - } \\
\text { Realisierung von Economies of Scale bei hoher Produktdifferenzierung } \\
\text { - Aufbau regiozentrischer und transnationaler Unternehmensnetzwerke } \\
\text { (Ansätze transnationaler Orientierungen) }\end{array}$ \\
\hline
\end{tabular}

Tabelle 1: $\quad$ Entwicklungslinien in der Automobilindustrie

Quelle: Eigene Darstellung

Die Entwicklung der Automobilindustrie begann mit der handwerklichen Fertigung weniger Automobile in Deutschland und Frankreich um 1880. Schon vor dem ersten Weltkrieg wurden erste Fahrzeuge in die benachbarten Ländermärkte exportiert (ethnozentrische Orientierung). ${ }^{110}$

Nach dem ersten Weltkrieg revolutionierten HENRY FORD und ALFRED SLOAN mit der Fließbandfertigung die Produktion und begründeten so die standardisierte Massenfertigung. Die amerikanischen Automobilherstellern FORD und GENERAL MOTORS betrieben in dieser Zeit eine Ausweitung ihrer Absatzmärkte nicht über den Export, sondern gründeten Tochtergesellschaften bzw. übernahmen bestehende Unterneh-

110 Vgl. Burmann, G.: Marktarealstrategien der internationalen Automobilhersteller, a. a. O., S. 124. 
men, da die Auslandsmärkte häufig durch Einfuhrzölle geschützt und die Transportkosten relativ hoch waren. Die Tochtergesellschaften waren an die lokalen Anforderungen angepasst und in ihren Entscheidungen autonom (polyzentrische Orientierung). ${ }^{11}$

Als Ende der 50er und zu Beginn der 60er Jahre der Abbau der Zollschranken in Europa begann, konnten die europäischen Hersteller ihre Absatzmärkte innerhalb Europas erweitern und Volumeneffekte nutzen. Es gelang den europäischen Herstellern eine Verbindung von Massenproduktion und Differenzierung. Im Gegensatz zu den amerikanischen Herstellern konnten sie eine wesentlich größere Vielfalt an Automobilen anbieten und wurden den differenzierten lokalen Bedingunskonstellationen, wie den Unterschieden im Durchschnittseinkommen, der Automobilbesteuerung sowie den geographischen Gegebenheiten, besser gerecht. Neben den europäischen Märkten wurden nun auch aussichtsreiche Ländermärkte außerhalb der Triade bearbeitet. Diese Märkte waren oft durch Handelshemmnisse geschützt. ${ }^{112}$ Dabei spielte für europäische Hersteller neben dem Aufbau von Tochtergesellschaften und Joint Ventures ${ }^{113}$ im Gegensatz zu amerikanischen Herstellern insbesondere der Export eine hohe Bedeutung. ${ }^{114}$

Ende der 60er Jahre wurde in der japanischen Automobilbranche unter maßgeblicher Beteiligung des leitenden Produktionsingenieurs OHNO des Herstellers TOYOTA ein Durchbruch in der Organisation der Automobilproduktion erzielt, der zu niedrigeren Kosten und geringeren Entwicklungszeiten beitrug - die sog. schlanke Produktion. ${ }^{115}$ Unterstützt durch die japanischen Handelshäuser als auch durch die Abstimmung der Strategien zwischen Banken, Industrie und Staat (MITI) setzten die japanischen Automobilhersteller eine globale Arealstrategie durch. Kennzeichen dieser Strategie ist ein

111 FORD begründet 1931 seine unabhängige Automobilproduktion in England und Deutschland sowie 1934 in Frankreich. GENERAL MOTORS hingegen kaufte bereits bestehende Produktionsstätten. So erwirbt GENERAL MOTORS 1925 VAUXHALL in England, 1929 ADAM OPEL in Deutschland. In Japan wurden Mitte der 20er Jahre Produktionsstätten errichtet, die aber 1939 aufgrund anderer gesetzlicher Rahmenbedingungen geschlossen wurden. Auch in Brasilien produzieren beide Hersteller seit den 20er Jahren Automobile. Vgl. Altshuler, A. et al.: The Future of the Automobile: The Report of MIT's International Automobile Program, a. a. O., S. 17 f., 30 und 38.

112 So zählte bspw. Brasilien in den 60er Jahren zu einem aussichtsreichen Markt für die Automobilindustrie. Dieser Markt wurde ab 1956 durch Importbeschränkungen und kontinuierlich steigende Local Content-Bestimmungen (nach fünf Jahren sollten $95 \%$ des $\mathrm{Ge}$ wichts eines Pkws aus brasilianischer Fertigung sein) gekennzeichnet. Zugang wurde sieben international tätigen Herstellern damals erst nach Abgabe hoher Investitionszusagen gewăhrt. Vgl. Humphrey, J.; Oeter, A.: Motor-Industry Policies in Emerging Markets, in: Humphrey, J. et al. (Hrsg.): Global Strategies and Local Realities: The Auto Industry in Emerging Markets, London, New York 2000, S. 45.

113 VOLKSWAGEN gilt als einer der wenigen europäischen Hersteller, der bereits sehr früh auch durch Direktinvestitionen Produktionsstandorte außerhalb Europas aufbaute. So gründet VOLKSWAGEN 1953 in Brasilien, 1956 in Südafrika, 1964 in Mexiko und 1980 in Argentinien Tochtergesellschaften. Auch PEUGEOT beginnt die Automobilproduktion in Argentinien im Jahr 1965.

114 Vgl. Altshuler, A. et al.: The Future of the Automobile: The Report of MIT's International Automobile Program, a. a. O., S. $18-29$.

$115 \mathrm{Zu}$ den Hintergründen der schlanken Produktion vgl. Womack, J. P.: Die zweite Revolution in der Automobilindustrie: Konsequenzen aus der weltweiten Studie aus dem Massachusetts Institute of Technology, 6. Aufl., Frankfurt a. M., New York 1992, S. 54 - 78. 
hohes Maß an länderübergreifender Integration (geozentrische Orientierung) mit dem Ziel, eine weltweite Wettbewerbsfähigkeit aufzubauen und Globalisierungsvorteile zu nutzen. ${ }^{116}$ Die anfängliche Exportorientierung der japanischen Hersteller wurde nach 1979 aufgegeben, da die hohen Handelsungleichgewichte mit Nordamerika und Europa zu einem Wiederaufleben protektionistischer Strömungen und zum Aufbau von Handelshemmnissen führten. Anstelle der Exportorientierung waren die japanischen Hersteller nunmehr gezwungen, ihren Anteil am Weltmarkt durch Direktinvestitionen insbesondere in Nordamerika und Europa weiter auszubauen. ${ }^{117}$

Mitte der 80er Jahre beschäftigten sich die amerikanischen und europäischen Herstellern intensiv mit dem Konzept der schlanken Produktion. Anfang der 90er Jahre begannen sie angepasste Ansätze des Konzepts in intensiven Restrukturierungs- und Reengineeringprozessen umzusetzen. ${ }^{118}$ Neben den europäischen, nordamerikanischen und japanischen Herstellern haben auch Hersteller aus Schwellenländern diese Produktionsmethode adaptiert. So treten in den 90er Jahren weitere Anbieter aus asiatischen Ländern als neue Konkurrenten mit preisaggressivem Marktauftritt in den internationalen Automobilmarkt ein. ${ }^{119}$

Anfang der 90er Jahre änderten viele Schwellenländer aufgrund großer ökonomischer Probleme inre Politik der Importsubstitution und öffneten ihre Märkte. ${ }^{120}$ Auch der Zusammenbruch des sog. Ostblocks führte zu einer weiteren Offnung bisher geschützter Märkte. Dabei erscheint es notwendig, aufgrund unterschiedlicher Rahmenbedingungen in diesen Märkten, diese in Gruppen einzuteilen. ${ }^{121}$ Es können Märkte am Rande von Triademärkten (Integrationsländer) ${ }^{122}$, Märkte, die sich im Prozess

116 Vgl. Burmann, G.: Marktarealstrategien der internationalen Automobilhersteller, a. a. O., S. 125.

117 Vgl. Altshuler, A. et al.: The Future of the Automobile: The Report of MIT's International Automobile Program, a. a. O., S. 32.

118 Zur Ausbreitung der schlanken Produktion vgl. Womack, J. P.: Die zweite Revolution in der Automobilindustrie: Konsequenzen aus der weltweiten Studie aus dem Massachusetts Institute of Technology, a. a. O., S. 86 ff. Die Initiative KVP ${ }^{2}$ von VOLKSWAGEN im Jahr 1993 kann bspw. in diesem Zusammenhang gesehen werden. Vgl. Volkswagen (Hrsg.): Geschaftsbericht 1993, Wolfsburg 1994, S. 15 f.

119 Diese Hersteller gingen insbesondere aus finanzstarken asiatischen Unternehmen hervor. Hierbei handelt es sich insbesondere um die bekannten koreanischen Anbieter DAEWOO und HYUNDAl. Vgl. Koers, M.: Steuerung von Markenportfolios: Ein Beitrag zum Mehrmarkencontrolling am Beispiel der Automobilwirtschaft, Frankfurt a. M. 2001, S. 25.

120 Vgl. Humphrey, J.; Oeter, A.: Motor-Industry Policies in Emerging Markets, a. a. O., S. 46 ff. sowie Womack, J. P.: Die zweite Revolution in der Automobilindustrie: Konsequenzen aus der weltweiten Studie aus dem Massachusetts Institute of Technology, a. a. O., S. 278 f.

121 Vgl. Lung, Y.: Is the Rise of Emerging Countries as Automobile Producers an Irreversible Phenomenon?, in: Humphrey, J. et al. (Hrsg.): Global Strategies and Local Realities: The Auto Industry in Emerging Markets, London, New York 2000, S. 19 - 24.

122 Als Integrationsländer an den Rändern der Triadeländer können Länder wie Polen und Tschechien (EU-Beitrittskandidaten) bzw. Mexiko (NAFTA) gelten. Die Automobilindustrie in diesen Ländern orientiert sich an den Anforderungen aus den Triademärkten. Die Qualifizierung der Arbeitskräfte in den Integrationsländern ist dabei zumeist vergleichsweise hoch. So können oft bereits nach kurzer Zeit Qualităts- und Produktivitätsfortschritte durchgesetzt und internationale Standards erreicht werden. Die Leistungen des FORD-Werkes in 
einer regionalen Integration befinden ${ }^{123}$ und Märkte, in denen eine nationale Automobilindustrie im Aufbau begriffen ist, unterschieden werden. ${ }^{124}$ Mittel- bis langfristig werden in diesen Ländern ein ökonomischer Aufschwung und optimistische Wachstumsraten des Pkw-Absatzes prognostiziert. ${ }^{125}$ Darüber hinaus wird eine baldige Sättigung der Automobilnachfrage in den Triademärkten vorhergesagt, es wird prognostiziert, dass sich mittelfristig lediglich ein Wachstum im Neuwagenmarkt von $1-3 \%$ einstellt. $^{126}$

Hermosillo (Mexiko) bzw. der Volkswagen-Werke in Puebla (Mexiko) sowie in Polkowice und Poznan (Polen) und in Bratislava (Slowakische Republik) zeigen dies deutlich.

Länder, die sich im Prozess einer regionalen Integration befinden, umfassen Länder wie Argentinien und Brasilien als Mitglieder des Mercosur oder die Schwellenländer der ASEAN-Region. Die Asian Free Trade Area (AFTA) soll bis 2010 zu einer Freihandelszone ohne Zölle werden. Dabei sollen bis 2002 die Zölle im Automobilsektor auf maximal $5 \%$ sinken. Brunei, Burma, Cambodia, Indonesien, Laos, Malaysia, die Philippienen, Singapur, Thailand und Vietnam gehören zur AFTA. Australien und Neuseeland denken über einen Beitritt zur AFTA nach. Weiterhin schlossen die Länder Argentinien und Brasilien eine Vereinbarung über eine gemeinsame Automobilpolitik im Mercosur. Für Pkw und Nutzfahrzeuge gilt dabei ein gemeinsamer Außenzoll von $35 \%$, die Zölle für Kfz-Teile und -Komponenten sollen bis 2006 schrittweise von 7 bis $11,7 \%$ auf 14 bis $18 \%$ erhöht werden. Während zwischen den Staaten der Freihandelszonen die Handelsschranken abnehmen, bleibt die Abschottung nach außen erhalten. Die regionale Integration erlaubt eine hohe Arbeitsteilung zwischen den einzelnen Produktionsstandorten in der Region. FORD produziert bspw. in Brasilien die an die lokalen Bedürfnisse der Region angepassten Kleinwagen FIESTA und KA, während in Argentinien das Mittelklassemodell ESCORD gefertigt wird. Gleiches gilt für die Arbeitsteilung bezüglich Motoren und anderen Teilen. Vgl. VDA (Hrsg.): Auto 2000: Jahresbericht des Verbands der Automobilindustrie e. V., Frankfurt a. M. 2000, S. $70 \mathrm{f}$.

Einzelne Lănder, die eine eigene nationale Automobilindustrie aufbauen möchten, umfassen Länder wie China oder möglicherweise Russland. Die Schaffung einer nationalen Automobilindustrie bedarf der Unterstützung etablierter Anbieter durch Technologietransfer sowie eines großen Heimatmarktes, der die Produktion absorbieren kann. Internationale Automobilhersteller sind in diesem Zusammenhang aber nur bereit, sich lokal anzusiedeln und Technologien in ein Joint Venture einzubringen, wenn sie dadurch einen privilegierten Marktzugang erhalten. Die Märkte sind deshalb durch Importbeschränkungen geschützt. Local Content-Bestimmungen sollen den Aufbau einer nationalen Zuliefererindustrie unterstützen helfen. So ist der Volkswagen Konzern seit 1984 mit einem Joint Venture, der SHANGHAI-VolKSWAGEN AUTOMOBIL-GeSELLSCHAFT, in China als Hersteller tätig. Bereits 1988 konnten weitere Vertrăge über die Lizenzfertigung mit der FIRST AUTOMOBILE WORKS (FAW) aus Changchun unterzeichnet werden, die 1991 in die Gründung der FAWVOLKSWAGEN AUTOMOTIVE COMPANY unter Beteiligung von Volkswagen und Audi führte. Vgl. Volkswagen (Hrsg.): Geschäftsbericht 1988, Wolfsburg 1989, S. 77 ff. sowie Volkswagen (Hrsg.): Geschäftsbericht 1991, Wolfsburg 1992, S. 48 f.

125 In den Märkten in Südamerika, im ehemaligen Ostblock, der VR China und in Indien wurde ein baldiges Einsetzen einer Massenmotorisierung erwartet, die bspw. im Fall der VR China auch ein politisches Ziel darstellt. Im Jahr 2010 soll sich nach politischer Vorgabe aus 1994 jede chinesische Familie ein Auto leisten können. Der VolKSWAGENKONZERN prognostiziert allein für die VR China einen langfristigen Bedarf von 456 Mio. Fahrzeugen, ebensoviel wie der gesamte Pkw Bestand weltweit im Jahr 1991. Vgl. Lung, Y.: Is the Rise of Emerging Countries as Automobile Producers an Irreversible Phenomenon?, a. a. O., S. 17 sowie UPI (Hrsg.): Folgen einer globalen Motorisierung, UPI-Bericht Nr. 35, Heidelberg 1995, S. 9.

126 Vgl. Humphrey, J. et al.: Introduction, in : Humphrey, J. et al. (Hrsg.): Global Strategies and Local Realities: The Auto Industry in Emerging Markets, London, New York 2000, S. 3. 
Vor diesem Hintergrund begannen die Automobilhersteller verstärkt, neue Märkte in den Wachstumsmärkten der Schwellenländer zu erschließen. Dabei wuchsen die Produktionskapazitäten in den sog. Emerging Markets allerdings schneller als das tatsächliche Absatzpotenzial. Die Prognosen erwiesen sich zusätzlich vor dem Hintergrund der wirtschaftlichen Krise in Asien, Südamerika und Russland ab 1997 nicht immer als zutreffend. ${ }^{127}$ Weltweite Überkapazitäten waren die Folge, die in Zusammenhang mit dem hohen Wettbewerbsdruck zu regelrechten Preiskriegen führten, sodass einige Hersteller nur noch in geschützten Märkten, in Nischen oder aufgrund ihrer finanzwirtschaftlichen Tätigkeiten Profite erzielen konnten. ${ }^{128}$

Zu Beginn des 21. Jahrhunderts ist die Automobilindustrie durch eine starke internationale Ausrichtung und einen hohen wettbewerbsinduzierten Kostendruck gekennzeichnet. Die Nutzbarmachung von Economies of Scale und Scope ist zu einer Überlebensnotwendigkeit geworden. ${ }^{129}$ Auf dem globalen Automobilmarkt besteht ein Spannungsfeld zwischen einer Anpassung der Fahrzeuge an den jeweiligen Käufergeschmack bzw. an lokale Bedürfnisse (segmentspezifische Differenzierung) und einer möglichst umfassenden Vereinheitlichung möglichst vieler Teile des Fahrzeugs, um kostengünstig produzieren zu können (kostenorientierte Standardisierung). ${ }^{130}$ Vor diesem Hintergrund wählen die meisten Automobilhersteller eine gesamtmarktabdeckende segmentspezifische Differenzierung und positionieren sich als international tätige Full-Line-Anbieter. ${ }^{131}$

Kritisch steht das UPI zur These der Sättigung des Automobilmarktes. Vgl. UPI (Hrsg.): Folgen einer globalen Motorisierung, a. a. O., S. 9-14.

Vgl. dazu die Gegenüberstellung der Wachstumsvoraussagen für den Automobilabsatz in verschiedenen Schwellenländern und die tatsächlich eingetretene prozentuale Veränderung in Humphrey, J. et al.: Introduction, a. a. O., S. 2 sowie VDA (Hrsg.): Auto 2000, a. a. O., S. 68 sowie Diez, W.: Plattform-Strategien: Irrweg oder Konigsweg in der Modellpolitik?, Arbeitspapier Nr. 2 des Instituts für Automobilwirtschaft, Gelsenkirchen 1999, S. 4.

Vgl. Koers, M.: Steuerung von Markenportfolios: Ein Beitrag zum Mehrmarkencontrolling am Beispiel der Automobilwirtschaft, a. a. O., S. 25.

Vgl. Lung, Y.: Is the Rise of Emerging Countries as Automobile Producers an Irreversible Phenomenon?, a. a. O., S. 24 f. sowie Dudenhöfer, F.: Outsourcing, Plattorm-Strategien und Badge Engineering: Marktentwicklung bei austauschbaren Produkten, in: WiSt, Heft 3 , 1997, S. 144.

130 Vgl. VDA (Hrsg.): Auto 2000: Jahresbericht des Verbands der Automobilindustrie e. V., a. a. O., S. 200. MACHARZINA beschreibt den Automobilmarkt als blockiert-global, da das ökonomische Vereinheitlichungsstreben der Unternehmen aufgrund des Zwangs zur Anpassung (Kundenwünsche, Rechtsvorschriften, Handelsblockaden, ..) abgeblockt wird. Vgl. Macharzina, K.: Unternehmensfürung: Das internationale Managementwissen; Konzepte - Methoden - Praxis, 3. Aufl., Wiesbaden 1999, S. 695 f.

131 Vgl. Diez, W.: Plattform-Strategien: Irrweg oder Königsweg in der Modellpolitik, a. a. O., S. $3 \mathrm{f}$. Dahinter steht die Überlegung, dass ein breites Produktprogramm auch zielgruppenspezifische Angebote ermöglicht. Vgl. Heise, G.: Internationale Marktsegmentierung im Automobilmarketing, Wiesbaden 1997, S. 40. 
Um den Verdrängungswettbewerb in der Automobilindustrie ${ }^{132}$ in allen Fahrzeugsegmenten bestehen zu können, verfolgen die Automobilhersteller unterschiedliche Strategien. Outsourcing ${ }^{133}$, Gleichteile- ${ }^{134}$ und Plattform-Strategien ${ }^{135}$ sowie Badge Engineering ${ }^{136}$ werden in diesem Zusammenhang von den Herstellern als Möglichkeiten aufgegriffen, trotz differenzierter Angebote möglichst hohe Kostenersparnisse zu erzielen. Neben Kooperationen mit anderen Herstellern beim Bau von Nischenfahrzeugen ${ }^{137}$ ist die Führung unterschiedlicher Marken in einem Markenportfolio eine Erfolg versprechende Möglichkeit, das Angebotsspektrum zu erweitern und gleichzeitig das Absatzvolumen zu

132 Vgl. Liebehenschel, Th.: Okologieorientierte Produkt- und Dienstleistungspolitik: Rahmenbedingungen und Trends am Beispiel der Automobilindustrie, a. a. O., S. 171.

Vgl. hierzu und im Folgenden Diez, W.: Plattform-Strategien: Irnweg oder Königsweg in der Modellpolitik, a. a. O., S. 14 -18 sowie Dudenhöfer, F.: Outsourcing, Plattform-Strategien und Badge Engineering: Marktentwicklung bei austauschbaren Produkten, a. a. O., S. 145 -149 .

Im Rahmen des Outsourcing erfolgt eine Verlagerung von Wertschöpfungsaktivităten auf externe Zulieferer bzw. Kooperationspartner. Die Reduktion der Wertschöpfungstiefe ermöglicht eine Konzentration auf Kernkompetenzen und damit die Realisierung von Kostenvorteilen. Wird die Fahrzeugentwicklung selbst auf Systemlieferanten übertragen, spricht man von Simultaneous Engineering. Vgl. Dyer, J. H.: Specialized Supplier Networks as a Source of Competitive Advantage: Evidence from the Auto Industry, in: Strategic Management Journal, 17. Jg. (1996), S. $271 \mathrm{ff}$.

Bei Anwendung einer Gleichteile-Strategie wird auf Teile-Baukästen und Module zurückgegriffen, die eine technische Differenzierung zwischen Modellen und Varianten aufgrund ihres variablen Einsatzes in einem gewissen Rahmen ermöglichen. Vgl. zur GleichteileStrategie auch Sanchez, R.: Strategic Product Creation: Managing New Interactions of Technology, Markets and Organizations, in: European Management Journal, 14. Jg. (1996), Nr. 2, S. 126.

135 Eine Plattform stellt die Gesamtheit derjenigen Komponenten, Schnittstellen und Funktionen dar, die über eine Produktfamilie hinweg standardisierbar und zeitlich stabil sind. Eine Differenzierung zwischen Modellen erfolgt lediglich durch eine sichtbar andersartige Gestaltung der Karosserie und des Interieurs. Die Verwendung gleicher Plattformen ermöglicht so eine kostengünstige Produktion von Nischenfahrzeugen und Varianten. PlattformStrategien werden heute von vielen Automobilherstellern angewendet.

136 Badge Engineering bedeutet die Vermarktung identischer Automobile unter verschiedenen Marken. Es kann Ergebnis einer Kooperation zwischen zwei Herstellern sein oder aber innerhalb der Marken eines Herstellers betrieben werden. Kritisch ist in diesem Zusammenhang allerdings zu vermerken, dass angefangen mit der Gleichteile- über die Plattform-Strategie bis zum Badge Engineering der Grad der Austauschbarkeit zwischen den Automobilen zunimmt. Eine traditionelle Differenzierung durch technische Eigenschaften eines Automobils wird erschwert.

137 So bauten VOLKSWAGEN und FORD in einem Joint Venture den FORD GALAXY und den VOLKSWAGEN SHARAN (Badge Engineering). Mittlerweile hat VoLKSWAGEN das Joint Venture komplett übernommen und fertigt neben SHARAN und GALAXY zusätzlich den SEAT ALHAMBRA. 
erhöhen. $^{138}$ Mehrmarkenstrategien ${ }^{139}$ werden dabei durch Fusionen oder Übernahmen anderer Marken bzw. dem eigenen Aufbau neuer Marken möglich. $^{140}$

Die in den letzten Jahren erfolgte Bildung von Wirtschaftsräumen erfordert eine weitere Entwicklung der internationalen Führungssystematik. Da mit zunehmender Verflechtung der Unternehmen untereinander die Autonomie einzelner Landesgesellschaften abnimmt und sich Know-how eher in regionalen Kompetenzzentren konzentriert, entwickeln sich eher regionale Strukturen. ${ }^{141}$ Im Automobilmarkt lassen sich demzufolge Regionalisierungstendenzen beobachten. ${ }^{142}$ Regional orientierte Unternehmen verflechten ihre Standorte in den Regionen untereinander, Know-how und Synergien werden in den Regionen konzentriert und erworben. Die Unternehmenszentrale wird in der Führung entlastet, da Entscheidungsbefugnisse tendenziell eher in den Regionen belassen werden. ${ }^{143}$ So hat bspw. FORD seine Absatzmärkte in unterschiedliche Regionen eingeteilt und die Entwicklungskompetenz für Neuwagen entsprechend festgelegt. Während in Europa verstärkt Klein- und Mittelklassewagen entwickelt

138 Viele Hersteller versuchen dabei, ihre internationale Marktabdeckung durch den Kauf von Marken zu erweitern. Dabei spielt neben dem Erwerb einer zusätzlichen Marke auch der Zugang zu einem ausländischen Vertriebsnetz eine wichtige Rolle. Vgl. Dudenhöfer, F.: Outsourcing, Plattorm-Strategien und Badge Engineering: Marktentwicklung bei austauschbaren Produkten, a. a. O., S. 144. Die Fusion von DAIMLER BENZ und CHRYSLER sowie die spätere Kapitalbeteiligung von DAIMLERCHRYSLER an MITSUBISHI wird oft mit diesem Argument begründet.

Eine Mehrmarkenstrategie umfasst eine parallele Führung mehrerer selbstständiger Marken, die auf denselben Produktbereich ausgerichtet sind. Vgl. zur Mehrmarkenstrategie Meffert, H.; Perrey, J.: Mehrmarkenstrategien - Ein Beitrag zum Management von Markenportfolios, in: Meffert, H.; Backhaus, K.; Becker, J. (Hrsg.): Arbeitspapiere der Wissenschaftlichen Gesellschaft für Marketing und Unternehmensführung e. V., Nr. 121, Münster 1998; Meffert, H.: Mehrmarkenstrategien - immer die beste Option?, in: asw, Sondernummer Oktober 1999, S. 82 - 87 und Laforet, S.; Saunders, J.: Managing Brand Portfolios: Why Leaders Do What They Do, in: JoAR, Nr. 1, 1999, S. 51-66 und Koers, M.: Steuerung von Markenportfolios: Ein Beitrag zum Mehrmarkencontrolling am Beispiel der Automobilwirtschaft, a. a. O., S. 34 - 41.

Die fehlende Präsenz eines Herstellers mit Produktionsstätten in den wichtigen bzw. in potenziell wichtigen und durch Handelsschranken geschützten Märkten wird häufig durch Kooperationen bzw. Fusionen mit dort ansässigen Herstellern zu lösen versucht. Vgl. FOCUS (Hrsg.): Der Markt der Automobile: Daten, Fakten, Trends, 1999, S. 1.

Vgl. Mirow, M.: Entwicklung internationaler Fürungsstrukturen, in: Macharzina, K.; Oesterle, M.-J. (Hrsg.): Handbuch Internationales Management: Grundlagen - Instrumente - Perspektiven, Wiesbaden 1997, S. 655 f.

142 Vgl. Liebehenschel, Th.: Okologieorientierte Produkt- und Dienstleistungspolitik: Rahmenbedingungen und Trends am Beispiel der Automobilindustrie, a. a. O., S. 170.

143 Vgl. Proff, H.; Proff, H. V.: Struktur und Entwicklung der weltweiten Automobilindustrie, a. a. O., S. 96. 
und verkauft werden, liegt in den USA die Verantwortung für die Limousinen und die sog. SUVs ${ }^{144}$. So werden unter der Marke FORD in Europa andere Modelle angeboten als in den USA. Die enge Verknüpfung innerhalb einer Region zeigt sich bei FORD bspw. daran, dass FORD Deutschland einen Großteil seiner Produktion innerhalb Europas exportiert. ${ }^{145}$

Eine Konfiguration als regiozentrisches Unternehmen kann dabei als Vorstufe eines transnationalen Unternehmens angesehen werden. Die zunehmende Bearbeitung länder- und regionenübergreifender Segmente durch die Automobilhersteller führt bei fallenden Handelsschranken zwischen den Integrationsräumen $^{146} \mathrm{zu}$ weiteren Veränderungen in den internationalen Organisationsstrukturen der Unternehmen. Eine gleichzeitige Realisierung von Globalisierungs- und Lokalisierungsvorteilen durch eine globale Streuung von Ressourcen und Fähigkeiten sowie der Zuordnung differenzierter und spezialisierter Rollen für die Tochtergesellschaften führt zu der Koordinationsform eines sog. integrierten Netzwerks. In der Automobilindustrie lassen sich Tendenzen ausmachen, die eine derartige Entwicklung andeuten.

Der Übergang vom regiozentrischen zum transnationalen Unternehmen kann bspw. bei VOLKSWAGEN beobachtet werden. Das internationale Produktionsnetzwerk des VoLKSWAGEN-Konzerns ist in Abbildung 5 dargestellt. ${ }^{147}$

\footnotetext{
144 SUV steht für Sporty-Utility-Vehicle.

145 Herr KRÜGER, der Konzernumweltbeauftragte bei FORD, schildert dies im Interview.

146 In der jüngsten Vergangenheit wurden Handelsabkommen zwischen der EU und Südafrika sowie der EU und Mexiko geschlossen. China verhandelt über einen WTO-Beitritt. Zwar werden in allen Fällen Ausnahmeregelungen für die Automobilindustrie geschlossen, aber der Trend zu mehr Freihandel ist erkennbar. Vgl. zu den Details der Abkommen bzw. Verhandlungen VDA (Hrsg.): Auto 2000: Jahresbericht des Verbands der Automobilindustrie e. V., a. a. O., S. $70 \mathrm{ff}$.

Die Lănder mit Produktionsstätten der Marken VOLKSWAGEN, SKODA, SEAT und AUDI in diesen Regionen sind durch die Markensymbole gekennzeichnet. Die entsprechenden Absatzzahlen des Konzerns aus dem Jahr 1999 sind für die einzelnen Regionen angegeben. Die in den Regionen gefertigten Produkte sind angegeben, die Austauschbeziehungen von Produkten, Teilen und Informationen sind durch die Linien grob skizziert.
} 


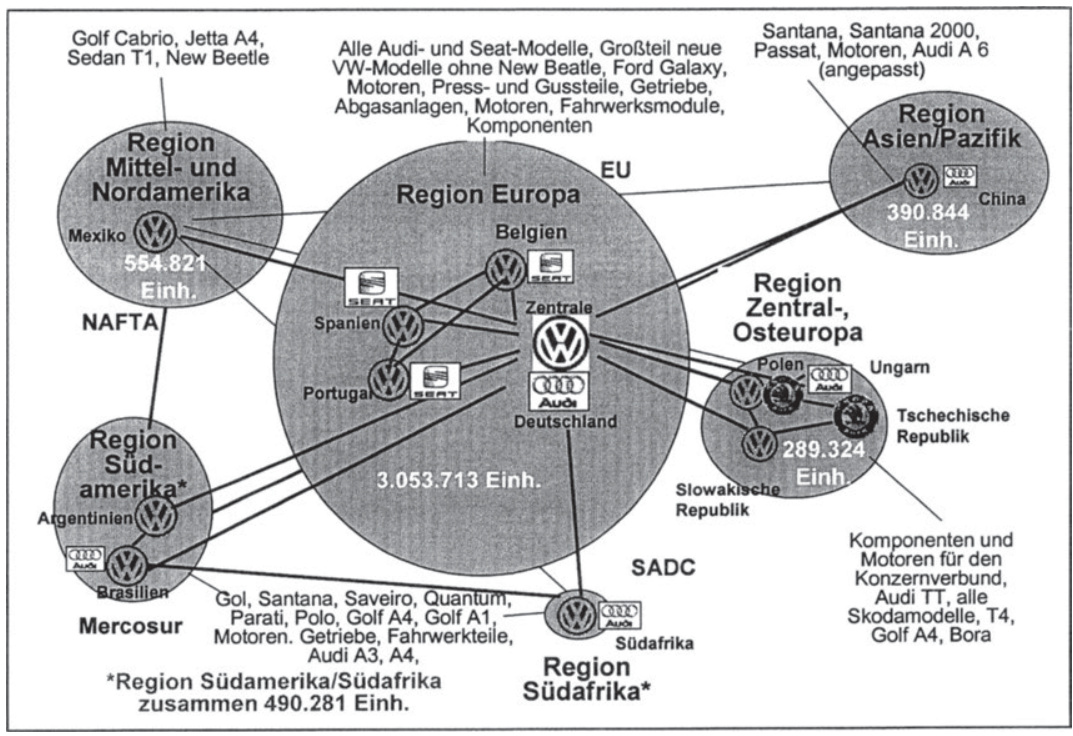

Abbildung 5: Internationales integriertes Produktionsnetzwerk am Beispiel des VOLKSWAGEN-Konzerns

Quelle: Eigene Darstellung

VOLKSWAGEN hat seine Absatzmärkte in unterschiedliche Regionen aufgeteilt. In Südamerika und China liegt die Entwicklungskompetenz für Automobile, die in den jeweiligen Landesmärkten abgesetzt werden sollen. So wurden der GoL, PARATI, QUANTUM, SANTANA und SAVEIRO in Südamerika und der angepasste AUDI A6 sowie der neue SANTANA 2000 im chinesischen Markt entwickelt. Dabei sind die Produktionsstätten in einer Region zumeist stark untereinander verbunden, es bestehen Austauschbeziehungen zwischen den Produktionsstätten einzelner Marken und zwischen verschiedenen Ländern (Motoren, Gussteile etc.).

Im Rahmen der von VolKsWAGEN verfolgten Plattformstrategie werden Fahrzeuge der Marken AUDI, SEAT, SKODA und VOLKSWAGEN auf der gleichen Plattform aufgebaut. Diese Plattformen werden zentral entwickelt und an wenigen Standorten gefertigt. Ihr Einsatz erfolgt dann in unterschiedlichen Modellen der Marken landes- und regionenübergreifend. ${ }^{148}$ Ähnlich wird in Bezug auf verschiedene Gleichteile und Motoren verfahren, die plattformübergreifend eingesetzt werden. Dies erfordert eine straffe und komplexe Koordination und Kon-

148 Vgl. dazu Koers, M.: Steuerung von Markenportfolios: Ein Beitrag zum Mehrmarkencontrolling am Beispiel der Automobilwirtschaft, a. a. O., S. 32. 
trolle sowie einen regen Austausch von Mitarbeitern, Technologie, Kapital, Vorprodukten und Informationen zwischen der Muttergesellschaft und den Tochtergesellschaften sowie zwischen den Tochtergesellschaften der Marken untereinander. ${ }^{149}$

\section{2 Ökologische Herausforderungen in der Automobilindustrie}

Das Automobil ist heute in den Ländern der Triade der bedeutendste Verkehrsträger. Auch eine Vielzahl von Schwellenländern steht kurz vor einer Massenmotorisierung, zumindest steigen die Zulassungszahlen dort rasant an. ${ }^{150}$ Mit dem großen Erfolg des Automobils und seiner Durchdringung vieler Lebensbereiche der modernen Gesellschaft sind aber auch verschiedene ökologisch bedenkliche Entwicklungen verbunden.

Die wichtigsten ökologischen Belastungen, die dem Automobil zugeschrieben werden, sind in Abbildung 6 in sog. Belastungsmatrizen ${ }^{151}$ im Überblick dargestellt. Dabei werden die ökologischen Belastungen im linken Teil der Abbildung auf die unterschiedlichen Lebensphasen Produktion, Nutzung und Verwertung sowie die Bereitstellung der Infrastruktur bezogen. Im rechten Teil der Abbildung sind die ökologischen Belastungen nach ihrem Wirkungsradius differenziert. Dabei wurden die ökologischen Belastungen ebenfalls lebenszyklusübergreifend erfasst.

149 Vgl. Proff, H.; Proff, H. V.: Struktur und Entwicklung der weltweiten Automobilindustrie, a. a. O., S. $95 \mathrm{ff}$.

150 Weltweit existiert heute ein Fahrzeugbestand von ca. 500 Millionen Pkw, in der EU besaßen $1997 \mathrm{im}$ Durchschnitt 442 Personen von 1.000 Einwohnern einen Pkw, in den USA waren es 496 Personen und in Japan 373. Im selben Jahr wurden in der EU 78,7\%, in den USA $85,6 \%$ und in Japan $58 \%$ der Personentransporte mit dem Pkw durchgeführt. Vgl. ECDG for Energy and Transport, Eurostat (Hrsg.): EU Transport in Figures: Statistical Pocket Book 2000, Brüssel 2000, S. 134.

151 Das Instrument der ökologischen Belastungsmatrix soll eine lebenszyklusweite Betrachtung der relevanten Umweltdimensionen darstellen, zur Komplexitätsreduktion und Übersichtlichkeit beitragen sowie als Grundlage für strategische Managemententscheidungen dienen. Vgl. Dyllick, Th.; Belz, F.; Schneidewind, U.: Okologie und Wettbewerbsfăhigkeit, a. a. O., S. 9 ff. 


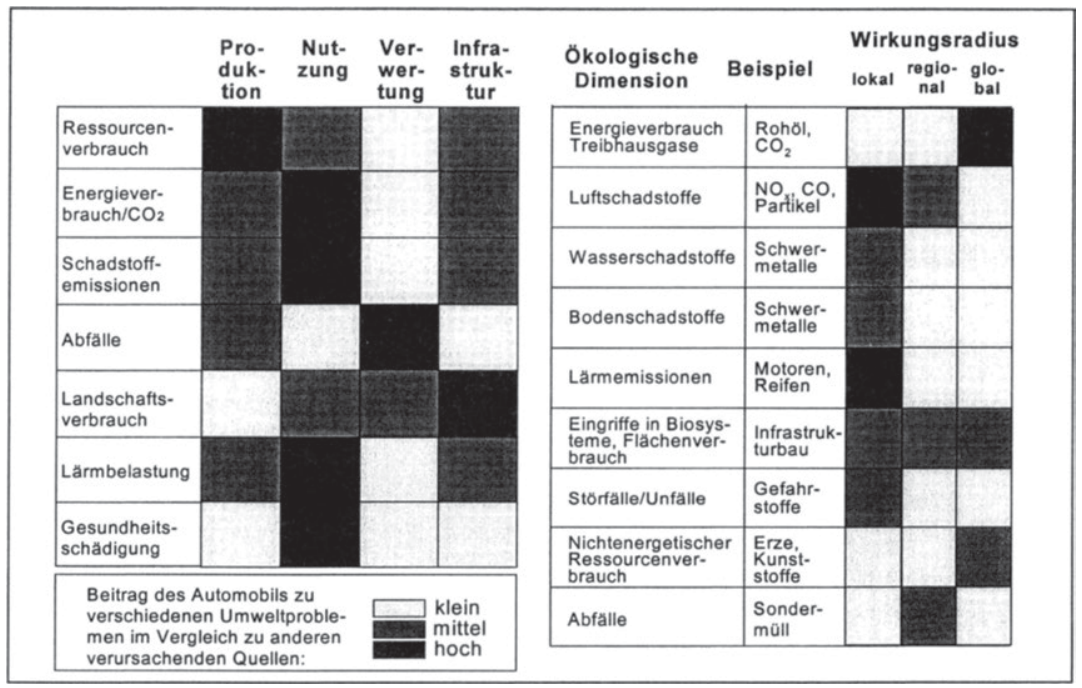

Abbildung 6: Ökologische Belastungen durch die Automobilindustrie

Quelle: Liebehenschel, Th.: Okologieorientierte Produkt- und Dienstleistungspolitik: Rahmenbedingungen und Trends am Beispiel der Automobilindustrie, Marburg 1999, S. 179 sowie Sustainable Asset Management (Hrsg.): Sustainability in der Automobilindustrie: Das Automobil im nächsten Jahrtausend, Zürich 1998, S. 8.

Diese wichtigsten ökologischen Belastungen der Automobilindustrie sind im Folgenden nach den Lebenszyklusphasen geordnet aufgeführt:

\section{- Produktion:}

Für die Herstellung von Automobilen ist zur Gewinnung und Bearbeitung der Rohstoffe und Materialien der Einsatz großer Energiemengen notwendig, dies führt zu entsprechenden $\mathrm{CO}_{2}$-Emissionen, die zur Klimaerwärmung beitragen. $\mathrm{Da}$ ein Auto zu einem großen Teil aus Stahl, aber auch Leichtmetallen wie Aluminium und Titan sowie aus Kunststoffen besteht, werden bei der Herstellung auch andere nicht-regenerative Ressourcen verbraucht. Damit zeichnet die Automobilindustrie auch für den Teil der Tätigkeiten entlang der Wertkette Verantwortung, die nicht direkt von ihr vorgenommen werden. In den Werken der Automobilhersteller werden die Metalle gestanzt und geformt, gegen Korrosion geschützt und lackiert. Dabei gilt insbesondere die Lackierung als ökologisch bedenklich, da hier Lösungsmittelemissionen in die Luft bzw. ins Wasser abgegeben werden können. Bei der Produktion werden weiterhin Hilfs- und Betriebsstoffe wie Schmieröle benötigt, und es fallen Abfälle an, die bei unsachgemäßer Behandlung zu Boden- und Grundwasserkontaminationen beitragen können. ${ }^{152}$ In entscheidendem Ausmaß wer-

152 So weisen die an den Standorten des VOLKSWAGEN-Konzerns durchgeführten Umweltaudits dem Umgang mit wassergefährdenden Stoffen eine hohe ökologische Bedeutung zu, wie Herr MOGg von VOLKSWAGEN bestätigt. Energie- und Schadstoffbilanzen für die 
den die ökologischen Belastungen in dieser Phase von den eingesetzten Produktionsprozessen, Materialien, Umweltschutzmaßnahmen und -techniken mitbestimmt.

\section{- Nutzung:}

Lebensphasenübergreifende Analysen haben ergeben, dass etwa $80 \%$ der Umweltwirkungen des Automobils während der Nutzung des Fahrzeugs anfallen. ${ }^{153}$ Durch die Verbrennung von Benzin und Diesel trägt das Automobil dabei insbesondere zur lokalen Luftverschmutzung bei. ${ }^{154}$ Die Feinstaubemissionen ${ }^{155}$, Stickoxid $\left(\mathrm{NO}_{\mathrm{x}}\right)$, Kohlenmonoxid $(\mathrm{CO})$ sowie Kohlenwasserstoff $(\mathrm{HC})$ gelten als Mitverursacher des Sommersmogs ${ }^{156}$, der Versauerung der Böden, des Waldsterbens, von Schäden an Wassersystemen und Materialschäden an Bauwerken sowie menschlichen Gesundheitsschäden. ${ }^{157}$ Diese ökologischen Probleme treten vornehmlich in Ballungsräumen auf. ${ }^{158}$ Neben den lokalen bzw. regionalen Wirkungen der Luftschadstoffe, die bei der Pkw-Nutzung freigesetzt werden, tragen Kohlen-

Produktion verschiedener Verkehrsmittel finden sich u. a. bei Pfaffenbichler, P.: Energieund Schadstoffbilanz bei der Herstellung und Verteilung verschiedener Verkehrsmittel bis zur Auslieferung an den Kunden, in: Institut für Verkehrsplanung und -Technik der TU Wien (Hrsg.): Beitrăge zu einer ökologisch und sozial vertrăglichen Verkehrsplanung, 1/98, Wien 1998.

Vgl. VDA (Hrsg.): Auto 2000: Jahresbericht des Verbands der Automobilindustrie e. V., a. a. O., S. 134 .

154 Zu den Auswirkungen der Luftschadstoffe vgl. auch Liebehenschel, Th.: Okologieorientierte Produkt- und Dienstleistungspolitik: Rahmenbedingungen und Trends am Beispiel der Automobilindustrie, a. a. O., S. $173 \mathrm{ff}$.

Staubpartikel, die so klein sind, dass sie nicht von der Nase herausgefiltert werden, stehen in Verdacht, chronische Bronchitis, Asthma und andere Atemwegserkrankungen, HerzKreislauferkrankungen und Krebs hervorzurufen. Vgl. Teufel, D., et al.: Externe Gesundheitskosten des Verkehrs in der Bundesrepublik Deutschland - im Auftrag von Greenpeace Deutschland, in: Umwelt- und Prognose-Institut e. V. (Hrsg.), UPI-Bericht Nr. 43, 2. Aufl., Heidelberg 1999, S. 5.

Die Verbindung von Stickoxiden und Kohlenwasserstoffen unter Einwirkung von UV-Strahlung führt zur Entstehung bodennahen Ozons. Im Sommer führen die hohen Ozonwerte dazu, dass immer hăufiger Bürger ihre Aktivităten in der freien Natur unterlassen müssen. Vgl. Liebehenschel, Th.: Okologieorientierte Produkt- und Dienstleistungspolitik - Rahmenbedingungen und Trends am Beispiel der Automobilindustrie, a. a. O., S. 43 sowie Moon, D.: Environmental Aspects of Sustainable Mobility, in: European Commission (Hrsg.): Workingpaper, Version 5, 3 July 2000, S. 6.

Die Atemwege werden durch diese Schadstoffe geschadigt, die Lungenfunktion beeintrăchtigt und das Risiko für Leukämie, Krebs und Allergien steigt. Allein in Deutschland tragen die Feinstaubemissionen zu ca. 25.000 Todesfällen pro Jahr bei. Vgl. dazu Dienst für Gesamtverkehrsfragen des Eidgenössischen Verkehrs- und Energiewirtschaftsdepartments (Hrsg.): Monetarisierung der verkehrsbedingten externen Gesundheitskosten - Synthesebericht, Bern 1996 sowie Teufel, D., et al.: Externe Gesundheitskosten des Verkehrs in der Bundesrepublik Deutschland - im Auftrag von Greenpeace Deutschland, a. a. O., Sowie die Angaben des österreichischen Umweltministeriums, http://www.bmu. gv.at/u verkehr laerm/ [06.11.00].

Dabei führen Smog und Saurer Regen auch zu grenzüberschreitenden Umweltproblemen bei. OECD, ECMT (Hrsg.): Final Report of the Joint OECD/ECMT Project on Urban Travel and Sustainable Development: Principle Conclusions Executive Summery, Paris 1994, S. 4. 
dioxid $\left(\mathrm{CO}_{2}\right)$, Distickstoffoxid $\left(\mathrm{N}_{2} \mathrm{O}\right)$ und Methan $\left(\mathrm{CH}_{4}\right)$ zum globalen Treibhauseffekt bei. In diesem Zusammenhang kommt dem $\mathrm{CO}_{2}$ eine besonders hohe Bedeutung zu, da es sich durch nachgeschaltete Katalysatoren aus dem Abgas nicht herausfiltern lässt. Vor dem Hintergrund des erwarteten Wachstums des Verkehrsaufkommens wird für die Zukunft ein weiterer Anstieg der verkehrsbedingten $\mathrm{CO}_{2}$ Emissionen erwartet. ${ }^{159}$ Weiterhin werden insbesondere nicht-regenerierbare Energiereserven verbraucht, die zukünftigen Generationen nicht mehr zur Verfügung stehen. $^{160}$

- Verwertung und Entsorgung:

Nach der Nutzung werden große Teile des Automobils einem Recycling zugeführt. Durch das Recycling lassen sich viele der eingesetzten Rohstoffe, insbesondere Stahl und Aluminium, zurückgewinnen. ${ }^{161}$ Es kann davon ausgegangen werden, dass zumindest ein Großteil des Metallanteils des Fahrzeugs wiederverwertet wird. ${ }^{162}$ Neben den vergleichsweise geringen Umweltbelastungen, die durch das Recycling hervorgerufen werden, treten ökologische Belastungen aufgrund nicht recycelbarer Abfälle bzw. Sondermüll auf. ${ }^{163}$ Während Abfälle und Sondermüll in den Industrieländern großteils einer geregelten Entsorgung zugeführt werden können, ist dies in Schwellen- und Entwicklungsländern oft nicht der Fall. Boden- und Grundwasserkontaminationen am Standort der Demontage und auf Deponien können die Folge sein.

- Infrastruktur:

Für die Nutzung der Pkw stellt das Vorhandensein einer entsprechenden Infrastruktur in Form von Autobahnen, Straßen, Parkplätzen, Tankstellen etc. eine Grundvoraussetzung dar. ${ }^{164}$ Diese ist allerdings mit einen hohen Flächenver-

159 So wird auf Basis heutiger Trends geschätzt, dass bis 2010 der $\mathrm{CO}_{2}$-Beitrag des Verkehrs im Vergleich zu 1990 in der EU um $40 \%$ höher sein wird. Derzeit beträgt der Anteil des Verkehrs an den $\mathrm{CO}_{2}$-Emissionen in der EU bereits 26\%. Vgl. Moon, D.: Environmental Aspects of Sustainable Mobility, a. a. O., S. $6 \mathrm{f}$.

Es wird angenommen, dass der Autoverkehr im Zeitraum von 1995 bis 2030 rund 60 Milliarden Tonnen Erdöl oder fast die Hälfte der heute registrierten Welterdölreserven verbrauchen wird. Der Beitrag des Autoverkehrs zum globalen Treibhauseffekt lag 1995 bei 4,4 Milliarden Tonnen $\mathrm{CO}_{2}$-Äquivalent und wird aller Voraussicht nach bis zum Jahr 2030 auf über $10 \mathrm{Mrd}$. Tonnen $\mathrm{CO}_{2}$-Äquivalent pro Jahr ansteigen. Vgl. UPI (Hrsg.): Folgen einer globalen Motorisierung, a. a. O., S. 6 - 9.

161 Ein Automobil in der EU bestand im Jahr 1995 im Durchschnitt zu ungefähr $64 \%$ aus Stahl, zu $7 \%$ aus Aluminium und $3 \%$ aus weiteren Metallen. Der Kunststoffanteil eines Automobils beträgt 7,5\%. Vgl. ECDG for Energy and Transport, Eurostat (Hrsg.): EU Transport in Figures: Statistical Pocket Book 2000, a. a. O., S. 112.

Derzeit werden in der EU durchschnittlich ca. $75 \%$ des Fahrzeuggewichts recycelt, dieser Anteil soll in den nächsten Jahren aufgrund der verabschiedeten Altautorichtlinie bis 2005 auf $85 \%$ und bis 2015 auf $95 \%$ steigen.

163 Global prognostiziert das UPI im Zeitraum von 1995 bis zum Jahr 2030 den Anfall von ca. 90 Mrd. Tonnen Abfällen aus Produktion und Entsorgung von Automobilen, darunter allein $1 \mathrm{Mrd}$. Tonne hochgiftiger Shredderabfälle. Vgl. UPI (Hrsg.): Folgen einer globalen Motorisierung, a. a. O., S. 20.

164 Es besteht derzeit ein weltweites Straßennetz von ungefăhr $28.000 .000 \mathrm{~km}$ Länge und ein Autobahnnetz von $208.000 \mathrm{~km}$ Länge. ECDG for Energy and Transport, Eurostat (Hrsg.): EU Transport in Figures: Statistical Pocket Book 2000, a. a. O., S. 134. 
brauch und damit der Versiegelung fruchtbaren Landes verbunden. ${ }^{165}$ Die zunehmende Dichte des Verkehrs führt weiterhin immer häufiger zu einer Übernutzung der vorhandenen Infrastruktur, dies zeigt sich an Staus auf Autobahnen, ruhendem Verkehr in Ballungsräumen ${ }^{166}$ und Parkraumproblemen in Innenstädten sowie einer zunehmenden Lärmbelastung der Bevölkerung. ${ }^{167}$ Dabei hat auch der weitere Ausbau der Infrastruktur in der Vergangenheit immer nur kurzfristig zu einer Entlastung geführt. Ein weiterer Ausbau würde dabei insbesondere im eng besiedelten Europa viele Städte bis zur Unkenntlichkeit verändern, bisher intakte Naturlandschaften zerstören und zu prohibitiv hohen Kosten führen. ${ }^{168}$

Aufgrund der ökologischen Belastungen, die durch die Automobilindustrie verursacht werden, kommt der Lösung der lokalen und globalen ökologischen Probleme als Folgen der Mobilität eine herausragende Bedeutung zu. ${ }^{169}$ Wie Abbildung 6 deutlich zeigt, überwiegen die ökologischen Probleme der Automobilindustrie in der Herstellungs- und Nutzungsphase. Die Suche nach technologischen Innovationen oder neuen Prozessen zur Verminderung der herstellungsbezogenen Umweltbelastungen kann deshalb durchaus als eine zentrale Aufgabe des Umweltmanagements in der Automobilindustrie bezeichnet werden. Neue ökologieverträglichere Herstellverfahren können in diesem Kontext neben einer erhöhten Rechts- und Arbeitssicherheit sowie der Erfüllung externer ökologischer Anforderungen auch Kostenersparnisse bewirken. ${ }^{170}$ In der folgenden Abbildung sind unterschiedliche Maßnahmen zur Reduzierung der VOC-Emissionen im Lackierprozess abgebildet. Der Einsatz

165 Nach Berechnungen des UPI wird in den Industrieländern für Fahren und Parken eine Fläche von ca. $100 \mathrm{~m}^{2}$ pro Automobil verbraucht. Heute beläuft sich der Flächenverbrauch für den Autoverkehr auf ca. $50.000 \mathrm{~km}^{2}$, vor dem Hintergrund der Annahme, dass der Automobilabsatz weiter steigt, kann dieser Flächenverbrauch bis 2030 auf bis zu 200.000 $\mathrm{km}^{2}$ steigen und damit die Fläche versiegeln, die bis zu 80 Mio. Menschen ernähren könnte. Vgl. UPI (Hrsg.): Folgen einer globalen Motorisierung, a. a. O., S. 40 f.

Die volkswirtschaftlichen Kosten der Staus werden für die OECD-Länder auf ca. $2 \%$ des BSP geschätzt. Vgl. OECD, ECMT (Hrsg.): Final Report of the Joint OECD/ECMT Project on Urban Travel and Sustainable Development: Principle Conclusions Executive Summery, Paris 1994, S. 4.

Rund $26 \%$ aller Europäer sind Lärmbelastungen von mehr als $65 \mathrm{db}(\mathrm{A})$ ausgesetzt. Dies gilt aus medizinischer Sicht als inakzeptabel. Vgl. http://www.bmu.gv.at/u verkehr laerm/ [06.11.00] sowie Müller-Wenk, R.: Life-Cycle Impact Assessment of Road Transport Noise, in: IWO-Diskussionsbeitrag Nr. 77, St. Gallen 1999.

168 Vgl. OECD, ECMT (Hrsg.): Final Report of the Joint OECD/ECMT Project on Urban Travel and Sustainable Development: Principle Conclusions Executive Summery, Paris 1994, S. 5.

169 Vgl. Dietz, W.; Brachat, H.: Grundlagen der Automobilwirtschaft, Ottobrunn 1994, S. 31; Liebehenschel, Th.: Okologieorientierte Produkt- und Dienstleistungspolitik - Rahmenbedingungen und Trends am Beispiel der Automobilindustrie, a. a. O., S. 171.

170 Vgl. Volkswagen (Hrsg.): Jahresbericht des fertigungsbezogenen Umweltschutzes 1998/ 1999, Wolfsburg 1999, S. 7 ff. 
von Wasserlacken erfordert dabei neben dem Einsatz anderer Lacke auch die Ausstattung der Lackieranlagen mit einer grundlegend anderen Basistechnologie. Zur Erfüllung des anspruchsvollen Grenzwertes von $35 \mathrm{~g} / \mathrm{m}^{2}$ kann bei Nutzung der Wasserlacktechnologie auf verschiedene zusätzliche Vermeidungsmaßnahmen verzichtet werden. Dies kann zu geringen Umweltschutzkosten führen, als die wietere Nutzung konventioneller Lackieranlagen.

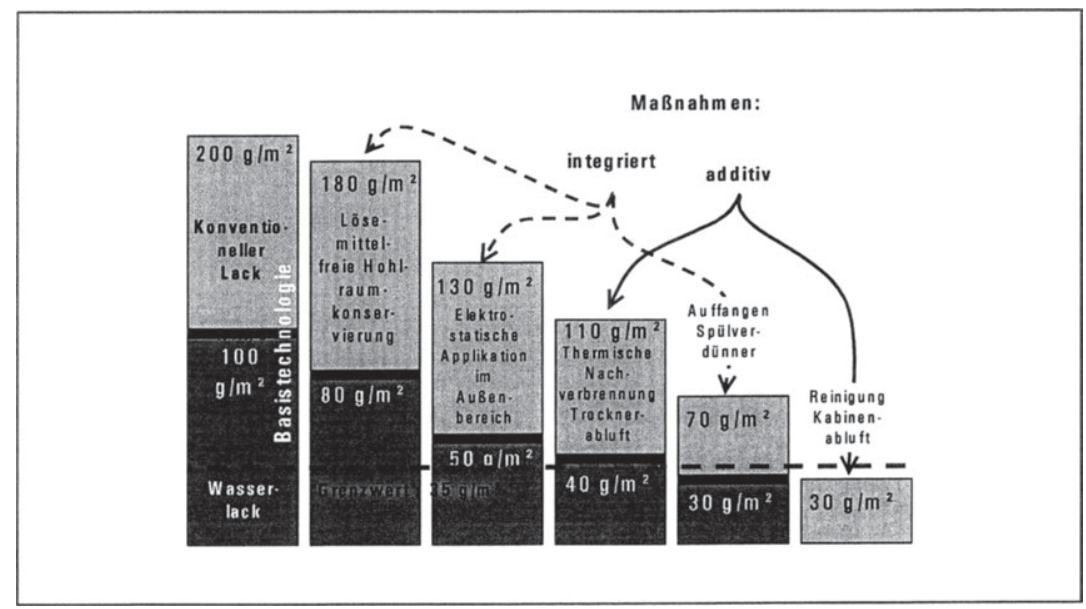

Abbildung 7: $\quad$ VOC-Reduktion

Quelle: VOLKSWAGEN, interne Grafik.

Die Umweltbelastungen in der Gebrauchsphase des Automobils können als besonders hoch angesehen werden. Die Forschungs- und Entwicklungsabteilungen der Automobilindustrie beschäftigen sich maßgeblich mit der Verminderung der Schadstofffreisetzung ${ }^{171}$ und der Senkung des Kraftstoffverbrauchs. ${ }^{172}$ So kann die Senkung des Kraftstoffverbrauchs durch Verbesserungen an den Antriebsaggregaten, dem Leichtbau und anderen Kraftübertragungskonzepten erreicht werden.

171 In der jüngeren Zeit hat die Verminderung der Partikelemissionen von Dieselfahrzeugen und die Diskussion um die Einführung einer entsprechenden Pflicht zur Anbringung von Partikelfiltern zur Entwicklung eines Partikelfilters durch die Firma PEUGEOT geführt. Im Jahr 2000 hat PEUGEOT den Partikelfilter im Modell PEUGEOT 607 HDI 2,2 erstmals eingesetzt, später soll der Filter in allen HDI Autos zum Einsatz kommen. Vgl. oekom research (Hrsg.): Corporate Responsibility Industry Report Automobile: A Corporate Responsibility Survey of 19 Companies of the Industry, München 2000, S. 119.

Diese Priorisierung bestătigt auch eine aktuelle Umfrage. Vgl. Fokus (Hrsg.): Der Markt der Automobile: Daten, Fakten, Trends, 1999, S. 18. 
Zur Reduzierung der $\mathrm{CO}_{2}$-Emissionen werden neben den Maßnahmen zur Kraftstoffiverbrauchsreduzierung auch Möglichkeiten zum Einsatz alternativer Treibstoffe geprüft. Alkohole und Erdgas, Rapsöl und in weiterer Zukunft Wasserstoff gelten hier als mögliche Alternativen. Während Rapsöl, Alkohole, aber auch Erdgas bereits als Treibstoffe in einzelnen Ländermärkten auf Basis bestehender Technik zum Einsatz kommen, fehlt beim Einsatz von Wasserstoff bspw. in einer sog. Brennstoffzelle noch eine marktfähige Lösung. Wasserstoff müsste schließlich auch in ausreichenden Mengen gewonnen und bereitgestellt werden. ${ }^{173}$ Es werden aber auch Maßnahmen zur Verkehrslenkung entwickelt und Car-Sharing-Programme erstellt, um ökologiebezogenen Herausforderungen sowie der Stauproblematik zu begegnen.

Zur Erfüllung der Recyclinganforderungen und der Vermeidung des Anfalls von ökologisch problematischen Abfällen nach der Nutzung wird bereits bei der Konzeption von Automobilen auf Demontagefreundlichkeit und Wiederverwertbarkeit der eingesetzten Materialen geachtet.

In Abbildung 8 sind unterschiedliche ökologiebezogene Handlungsfelder in der Automobilindustrie den Lebenszyklusphasen zugeordnet.

\begin{tabular}{|c|c|c|c|c|}
\hline Herstellung & \multicolumn{3}{|c|}{ Nutzung } & Recycling/Abfall \\
\hline $\begin{array}{l}\text { - Abwasser- } \\
\text { behandlung } \\
\text { - Auffang- } \\
\text { becken } \\
\text { - Abwasser- } \\
\text { behandlung } \\
\text {-... } \\
\text { - Lacklerung } \\
\text { - Wasserlacke } \\
\text { - Pulverlacke } \\
\text { - ... } \\
\text { Emissions- } \\
\text { verminderung } \\
\text { - Kraftstoff- } \\
\text { befüllung } \\
\text { - ... }\end{array}$ & $\begin{array}{l}\text { Antriebe/Kraft- } \\
\text { übertragung } \\
\text { - Ottomotor } \\
\text { - FSI } \\
\text { - Magermotor } \\
\text { - Dleselmotor } \\
\text { - Pumpe-Düse } \\
\text { - Common Rail } \\
\text { - Elektroantrieb } \\
\text { - Hybridantrieb } \\
\text { - (Gas, Benzin, } \\
\text { Elektro) } \\
\text { - Schwungrad- } \\
\text { nutzautomatik } \\
\text { - Brennstoffzellen }\end{array}$ & $\begin{array}{l}\frac{\text { alternative }}{\text { Kraftstoffe }} \\
\text { - Alkohol } \\
\text { (Ethanol) } \\
\text { - Gas } \\
\text { (LPG/NPG) } \\
\text { - Rapsōl } \\
\text { - Wasserstoff } \\
\text { (flüssig, } \\
\text { gasformig) } \\
\text { - (Elektrizităt) }\end{array}$ & $\begin{array}{l}\text { Leichtbau } \\
\text { - Aluminium } \\
\text { - Magnesium } \\
\text { - Titan } \\
\text { - Kunststoff- } \\
\text { verbindungen }\end{array}$ & $\begin{array}{l}\text { Design for } \\
\text { Disassembly } \\
\text { - Einsatz } \\
\text { recyclefăhiger } \\
\text { Materiallen } \\
\text { - Stoffaus- } \\
\text { schlusslisten } \\
\text { - PVC } \\
\text {-... } \\
\text { - Einsatz von } \\
\text { nachwachsen- } \\
\text { den Rohstoffen }\end{array}$ \\
\hline
\end{tabular}

Abbildung 8: $\quad$ Ökologische Handlungsfelder in der Automobilindustrie Quelle: Eigene Darstellung

173 Solange die Herstellung des Wasserstoffs unter Einsatz konventioneller Energien geschieht, lässt sich auch kein positiver okologischer Effekt mit dem Wasserstoffantrieb verbinden, allein der Emissionsort hătte sich verlagert. 
Im folgenden Kapitel werden die Interdependenzen, die bei internationaler Unternehmenstätigkeit eine Koordination des ökologiegerichteten Verhaltens notwendig machen, hergeleitet.

\section{Interdependenzen als Determinanten des Koordinationsbedarfs}

Interdependenzen ergeben sich generell daraus, dass alle Unternehmensteilaufgaben aus der Unternehmensgesamtaufgabe abgeleitet werden und deshalb zusammenhängen. ${ }^{174}$ Zur näheren Kennzeichnung unterschiedlicher Interdependenzen können sie in materielle (physische Produktionsfaktoren, Produkte sowie finanzielle Ressourcen) und informationelle Interdependenzen (Knowhow, Wissen) sowie nach dem Kriterium unterschiedlicher Intensität in gepoolte, sequenzielle und reziproke Interdependenzen unterteilt werden. ${ }^{175}$ Bei gepoolten Interdependenzen ${ }^{176}$ liegt eine indirekte Abhängigkeit zwischen den Tochtergesellschaften vor. Dabei wird bei Ineinandergreifen von Aktivitäten zwischen sequenziellen und reziproken Interdependenzen unterschieden. Sequenzielle Interdependenzen liegen vor, wenn der Output einer Unternehmenseinheit zum Input einer anderen Einheit wird. Von sequenziell-informationellen Interdependenzen kann gesprochen werden, wenn ein Standort über Informationen bspw. zu den ökologischen Anforderungen der Konsumenten vor Ort verfügt und diese einseitig an die Zentrale weitergibt bzw. die Zentrale von diesen Informationen abhängig ist. Reziproke Interdependenzen beschreiben schließlich gegenseitige Leistungsverflechtungen zwischen Tochtergesellschaften aus unterschiedlichen Ländern. Wechselseitig-informationelle Interdependenzen bestehen bei der Notwendigkeit wechselseitigen Informationsaustauschs.

Art und Umfang der Interdependenzen, die bei internationaler Unternehmenstätigkeit in Bezug auf das ökologiegerichtete Verhalten anfallen, hängen von situativen Kontextfaktoren ab. Im Rahmen dieser Arbeit ist demnach zu fragen, welche Faktoren auf die Entstehung eines internationalen ökologiebe-

174 Vgl. Hoffmann, F.: Führungsorganisation, a. a. O., S. 308.

175 Vgl. dazu Thompson, J. D.: Organizations in Action, New York u. a. 1967, S. 54 f. Die unterschiedlichen Interdependenzen führen in der dargestellten Reihenfolge zu zunehmend komplexeren ökologiebezogenen Abhängigkeiten zwischen den Standorten.

Nach FRESE können hier Ressourcen- und Marktinterdependenzen unterschieden werden. Frese, E.: Grundlagen der Organisation: Konzept - Prinzipien - Strukturen, a. a. O., S. 57 f. Gepoolte Interdependenzen entstehen in einem Unternehmen bspw. aufgrund einer Konkurrenz um knappes Kapital, gemeinsame Nutzung von Ressourcen oder Dienstleistungen. 
zogenen Koordinationsbedarfs wesentlichen Einfluss nehmen. In der Literatur werden dabei generell endogene und exogene Faktoren in Bezug auf ihren Einfluss auf den Koordinationsbedarf untersucht. ${ }^{177}$ Als exogene Faktoren werden jene Faktoren verstanden, auf die das Unternehmen nur indirekt Einfluss nehmen kann, wohingegen endogene Faktoren im direkten Einflussbereich des Managements liegen.

Zur Ableitung unterschiedlicher exogener Kontextfaktoren der internationalen Unternehmenstätigkeit ist der Komplexität der Aufgabenumwelt besondere Aufmerksamkeit zu widmen. Komplexität kann dabei auch als Heterogenität der Aufgabenumwelt aufgefasst werden. ${ }^{178}$ Generell lassen sich die internationalen unternehmensbezogenen Umsysteme nach den relationalen Kriterien der Ähnlichkeit ${ }^{179}$ und der Abhängigkeit ${ }^{180}$ differenzieren. $^{181}$

Ähnlichkeit bedeutet, dass Ländermärkte in Bezug auf ökonomische, soziokulturelle, politisch-rechtliche und ökologische Rahmenbedingungen im Vergleich entweder als differente oder kongruente Umsysteme gelten können. ${ }^{182}$ Sowohl differente als auch kongruente Umsysteme werden dabei von interna-

177 Vgl. dazu Hoffmann, F.: Fürungsorganisation, a. a. O., S. 135 sowie die dort angegebene Literatur.

178 HoffMANN beschreibt die Komplexităt im Sinne der Heterogenität als bedeutsamen Umweltaspekt. Vgl. Hoffmann, F.: Fuhrungsorganisation, a. a. O., S. 103.

Die Homogenităt bzw. Differenzierung der externen Umwelt beschreibt eine Determinante des Koordinationsbedarfs. Vgl. Plump, D.: Die Koordination des Marketings in multinationalen Unternehmungen: Systemanalyse und Ansătze zur Systemgestaltung, a. a. O., S. 105 sowie Macharzina, K.: Unternehmensfuhrung: Das internationale Managementwissen; Konzepte - Methoden - Praxis, a. a. O., S. 17 - 23.

Austauschrelationen zu Elementen der Aufgabenumwelt und damit verbundene Machtund Kontrollmöglichkeiten können für Unternehmen zu einer Abhängigkeitssituation führen, die eine Zielerreichung beeinträchtigt oder gar in Frage stellt. HOFFMANN bezieht sich dabei insbesondere auf Ressourcenabhängigkeiten auf der Input- und Outputseite. Das Ausmaß der Abhängigkeit ergibt sich danach aus der Zusammensetzung des relevanten Umweltbereichs und der Stellung der Unternehmung innerhalb dieses Bereichs. Dies bedeutet, dass die Unternehmung von solchen Elementen der Aufgabenumwelt abhängig ist, die gegenüber der Unternehmung eine gewisse Macht besitzen. Vgl. Hoffmann, F.: Führungsorganisation, a. a. O., S. $110 \mathrm{ff}$.

Vgl. hierzu und im Folgenden Brodel, D.: Internationales Umweltmanagement: Gestaltungsfelder - Determinanten - Ausprägungen, a. a. O., S. $366 \mathrm{ff}$.

DÜLFER hat in diesem Zusammenhang ein Ordnungsmuster zur Analyse internationaler Umwelten entworfen. Vgl. Dülfer, E.: Zum Problem der Umweltberücksichtigung im „Internationalen Management", in: Pausenberger, E. (Hrsg.): Internationales Management, Stuttgart 1981, S. $29 \mathrm{ff}$. 
tional tätigen Unternehmen genutzt, um potenzielle Verbundvorteile ${ }^{183} \mathrm{zu}$ erzielen. Um diese potenziellen Wettbewerbsvorteile in tatsächliche Wettbewerbsvorteile zu überführen, bedarf es der internationalen Gestaltung des Wertschöpfungssystems $^{184}$. Die zwischen den geographisch verteilten Unternehmenseinheiten bestehenden Interdependenzen lassen sich dabei gezielt zur Realisierung positiver Verbundeffekte nutzen.

Die gegenseitige externe Abhängigkeit von Ländermärkten ist ebenfalls geeignet, die Wertschöpfung länderübergreifend zu beeinflussen. Dabei können zwischen den Ländermärkten Interdependenzen bestehen oder die einzelnen Ländermärkte sind voneinander entkoppelt. Die strukturelle Entkopplung von Umsystemen wird aus Sicht der internationalen Unternehmenstätigkeit oft als Hindernis bezüglich des Aufbaus von Wettbewerbsvorteilen angesehen. ${ }^{185} \mathrm{Da}$ bei können die zunehmenden Verknüpfungen zwischen Märkten und gesellschaftlichen Systemen sowohl positive als auch negative Verbundeffekte aufgrund von Interdependenzen mit dem externen Umsystem bedingen. Die Umsystemabhängigkeit ergibt sich dabei aufgrund von nicht-institutionellen Verknüpfungen auf gesellschaftlicher Ebene. In diesem Zusammenhang kann auch von ökologiebezogenen Ausstrahlungseffekten ${ }^{186}$ gesprochen werden, die sich sowohl auf die ökologischen Eigenschaften des Produktes, als auch der Produktion bzw. des standortbezogenen Umweltschutzes beziehen können. Dieses Phänomen kann auch als Global-Village-Effekt bezeichnet werden. ${ }^{187}$ Weiterhin

183 Als Verbundvorteile können internationale Erfahrungseffekte, Kostendegressionseffekte, Arbitrageeffekte, Economies-of-Scope-Effekte, Entwickungsstand-Portfolio-Effekte, CrossSubsidizing-Effekte und Risikodiversifikationseffekte unterschieden werden. Unterscheiden sich die Umsysteme zu stark, können Komplexitătsbarrieren ein Unternehmen daran hindern, Verbundvorteile aufzubauen. Vgl. Brodel, D.: Internationales Umweltmanagement: Gestaltungsfelder - Determinanten - Ausprăgungen, a. a. O., S. 371 - 387.

Die Gestaltung des Wertschöpfungssystems kann auch als Konfiguration bezeichnet werden. Konfiguration ist nach PORTER als struktureller Aufbau der internationalen Unternehmensaktivităten zu verstehen. Dies umschließt die extremen Entscheidungen über die Streuung bzw. Konzentration von Unternehmensaktivitatten und damit den Zentralisationsgrad von Entscheidungsstrukturen. Vgl. Porter, M. E.: Der Wettbewerb auf globalen Märkten: Ein Rahmenkonzept, a. a. O., S. $61 \mathrm{ff}$.

185 In diesem Fall stehen internationale Transferbarrieren dem Aufbau von Verbundeffekten entgegen. Vgl. Brodel, D.: Internationales Umweltmanagement: Gestaltungsfelder - Determinanten - Ausprăgungen, a. a. O., S. $387 \mathrm{ff}$.

Vgl. zu Ausstrahlungseffekten in der internationalen Markenpolitik von Automobilherstellern Waltermann, B.: Internationale Markenpolitik und Produktpositionierung: markenpolitische Entscheidungen im europäischen Automobilmarkt, Wien 1989, S. $174 \mathrm{ff}$.

187 Vgl. Brodel, D.: Internationales Umweltmanagement: Gestaltungsfelder - Determinanten Ausprăgungen, a. a. O., S. 383 - 386. 
können institutionelle Verknüpfungen zwischen Ländermärkten ${ }^{188}$, wie dies in der EU und der NAFTA oder bei bi- bzw. multinationalen Abkommen der Fall ist, zu einer Umsystemabhängigkeit führen. ${ }^{189}$ Zumeist verstärken sich dabei die Global-Village-Effekte, je stärker zwei Ländermärkte institutionell verbunden sind.

Die Berücksichtigung ökologischer Aspekte durch das Management findet häufig nicht direkt aufgrund eigener Umweltbelastungen, noch aufgrund des Zustandes der ökologischen Umwelt vor Ort statt, sondern kann durch die ökologische Betroffenheit eines Unternehmens durch Ansprüche von Stakeholdern erklärt werden. ${ }^{190}$ Die ökologische Betroffenheit eines Unternehmens gilt dabei als theoretisches Konstrukt, das als subjektiv wahrgenommene oder objektive Größe operationalisiert werden kann. Die von Entscheidungsträgern im Unternehmen wahrgenommene Intensität ökologischer Ansprüche sowie das bei Nichterfüllung dieser Ansprüche möglicherweise zu erwartende Sanktionspotenzial durch die Anspruchsgruppen kann als subjektive Betroffenheit bezeichnet werden. Als objektive Betroffenheit einer Unternehmung wird die verursachte Beeinträchtigung der Unternehmensziele durch ökologische Anspruchsgruppen und Institutionen verstanden. ${ }^{191}$

Bei internationaler Unternehmenstätigkeit kann davon ausgegangen werden, dass sich die ökologische Betroffenheit in den einzelnen Ländermärkten aufgrund differenzierter Kontextfaktoren voneinander unterscheidet. In Anlehnung an das Modell der ökologischen Transformation ${ }^{192}$ soll die ökologische Be-

188 Institutionelle Verknüpfungen bzw. internationale Governance-Effekte liegen dann vor, wenn zwischen Regierungen der Stamm- und der Gastländer vertragliche Beziehungen bestehen oder internationale Institutionen länderübergreifend verbindliche oder unverbindliche wertschöpfungsrelevante Normen erlassen, die für Stamm- und Gastländer einschlägig sind. Vgl. Brodel, D.: Internationales Umweltmanagement: Gestaltungsfelder - Determinanten - Ausprägungen, a. a. O., S. 368.

PLUMP untersucht in diesem Zusammenhang die Integration der Märkte, die zumeist einem größeren wirtschaftlichen und / oder politischen System angehören. Vgl. Plump, D.: Die Koordination des Marketings in multinationalen Unternehmungen: Systemanalyse und Ansătze zur Systemgestaltung, a. a. O., S. 107.

Dies spiegeln auch empirische Untersuchungen in Bezug auf das ökologische Verhalten wider. Vgl. Wolter, F.: Umweltmanagement in Europa - eine empirische Untersuchung auf Grundlage des Europäischen Umweltmanagement-Barometers (EBEB), a. a. O., S. 25.

Vgl. Kirchgeorg, M.: Okologieorientiertes Unternehmensverhalten, Typologien und Erklärungsansătze auf empirischer Grundlage, a. a. O., S. 59 ff.

Vgl. Belz, F.: Okologische Wettbewerbsfelder in der Lebensmittelbranche, in: Der Markt, 33. Jg. (1994), Nr. 129, S. 51 ff. sowie Dyllick, Th.; Belz, F.; Schneidewind, U.: Okologie und Wettbewerbsfăhigkeit, a. a. O., S. $39 \mathrm{ff}$. 
troffenheit bei internationaler Unternehmenstätigkeit erklärt werden. In diesem Kontext können die Ebenen der lokalen Unternehmensumwelt, der Anspruchsgruppen und der Unternehmung selbst betrachtet werden. Dabei kann das Unternehmen lokal bzw. regional und global durch Forderungen von Anspruchsgruppen ökologisch betroffen sein.

Herkömmlicherweise stehen in diesem Kontext marktbezogene Anspruchsgruppen wie Konsumenten und Wettbewerber im Vordergrund der betriebswirtschaftlichen Betrachtung. Im Vorfeld von marktlichen Veränderungen wirken bei ökologiebezogenen Problemstellungen aber zumeist öffentliche und politische Anspruchsgruppen. ${ }^{193}$ Diesem Modell liegt die Grundannahme zugrunde, dass ökologische Probleme auf stofflich-energetischer Ebene entstehen und zu gesellschaftlichen ökologischen Anforderungen führen. Dabei werden grundsätzlich öffentliche, institutionelle bzw. politische und marktbezogene Anspruchsgruppen ${ }^{194}$ unterschieden. ${ }^{195}$ Diese Anspruchsgruppen können auch als externe Lenkungssysteme der Unternehmung aufgefasst werden. Das Transformationsmodell ist in Abbildung 9 im Überblick dargestellt.

193 Die Diffusion von Ereignissen in einzelnen Umweltebenen als Gegenstand der offentlichen Diskussion wird von Lebenszyklusmodellen gesellschaftlicher Anliegen abgebildet. In diesen Modellen wird die Rolle von offentlichen und politischen Anspruchsgruppen in unterschiedlichen Entwicklungsphasen deutlich. Vgl. Meffert, H.; Kirchgeorg, M.: Marktorientiertes Umweltmanagement: Konzeption, Strategie, Implementierung mit Praxisfallen, 3. Aufl., Stuttgart 1998, S. 97 ff. sowie die dort aufgeführte Literatur.

194 Das WORLD BUSINESS COUNCIL fOR SUSTAINABle DeVELOPMENT nennt in diesem Zusammenhang verschiedene Stakeholder als wichtige Teilnehmer an der okologischen Debatte:

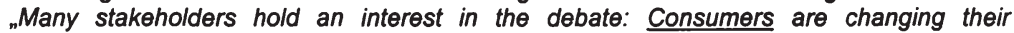
consumption habits based on the information they receive. Consumer Organizations are pushing companies to produce more environmentally and socially sound goods and services. They provide influential information to consumers. Retailers are a critical link in the production/consumption chain as they are the principal interface between producers and consumers. They have the potential to inform consumers and help to create a pull from the marketplace for more eco-efficient and sustainable products. They are increasingly selling services, which may eventually substitute for some products. ... NGOs take an active role in the debate around consumption by informing and influencing consumers and business. They can help pinpoint areas that need to be further examined. Governments must provide a framework of policy measures that encourages innovation among small and big companies alike. Such a framework would avoid unnecessarily prescriptive regulation." http://www.wbcsd.org/sustain1.htm - stakeholder [13.09.2000].

Im Rahmen des Europäischen Umweltmanagement-Barometers wurde durch faktoranalytische Verdichtung der anspruchsgruppenbezogenen Betroffenheitsindikatoren eine ăhnliche Aufteilung abgeleitet. Vgl. Wolter, F.: Umweltmanagement in Europa - eine empirische Untersuchung auf Grundlage des Europäischen Umweltmanagement-Barometers (EBEB), a. a. O., S. 22. 


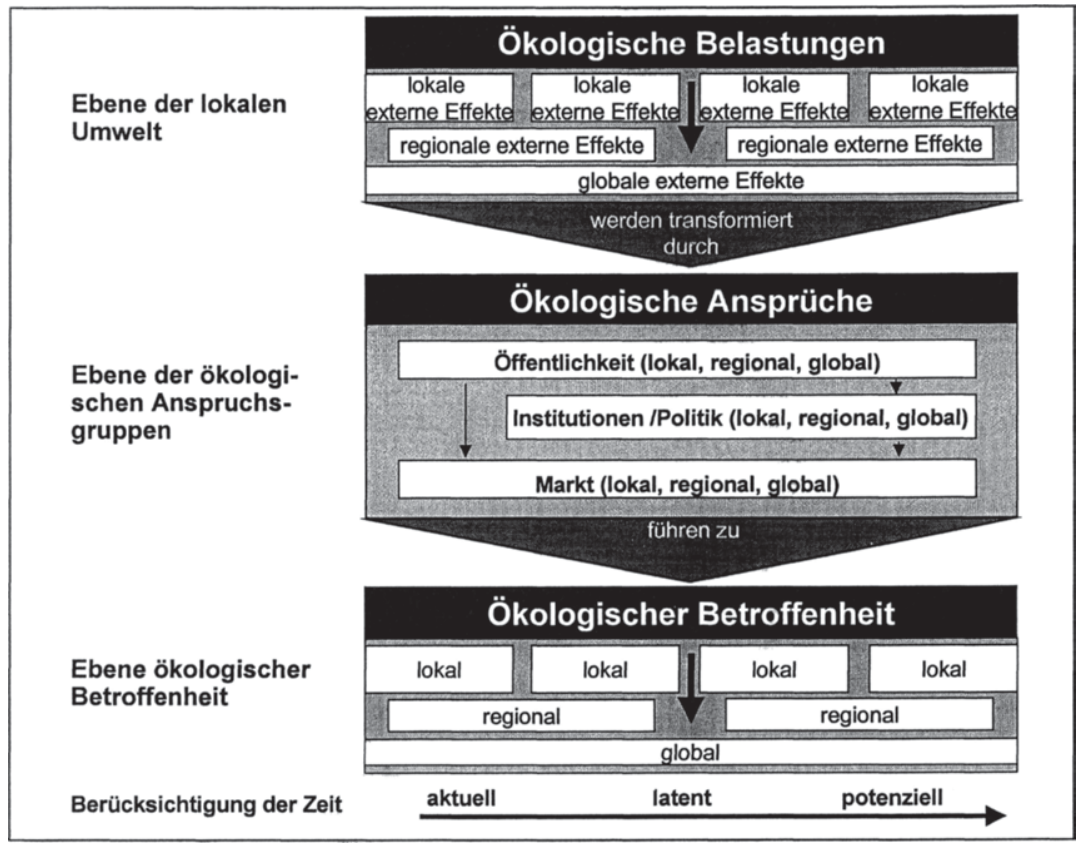

Abbildung 9: Transformationsprozess von ökologischen Belastungen zu ökologischer Betroffenheit

In Anlehnung an Dyllick, Th.; Belz, F.; Schneidewind, U.: Okologie und Wettbewerbsfähigkeit, München, Wien 1997, S. 40.

Die wichtigsten öffentlichen, institutionellen bzw. marktbezogenen Anspruchsgruppen sind in Abbildung 10 in Bezug auf den georgraphischen Ort ihrer Einflussnahme geordnet. Aufgrund der fortschreitenden regionalen Integrationsprozesse sollen lokale und regionale Anspruchsgruppen im Rahmen dieser Arbeit dabei weitgehend gemeinsam abgehandelt werden.

In Anlehnung an den Prozess der ökologischen Transformation sind die lokalen Kontextfaktoren, insbesondere die ökologischen Belastungen auf lokaler Ebene, Ausgangspunkt des Transformationsprozesses. Im Kontext ökologiebezogener Kriterien seien zwei nationale Umfelder dann als ähnlich zu kennzeichnen, wenn die exogenen Kontextfaktoren und damit auch die ökologische Betroffenheit durch Anspruchsgruppen in diesen Ländern nach Art und Ausmaß 
ähnlich ausgeprägt sind. ${ }^{196}$ Wenn sich die lokalen Kontextfaktoren voneinander unterscheiden, sind national bzw. regional differenzierte Reaktionen der Anspruchsgruppen zu erwarten.

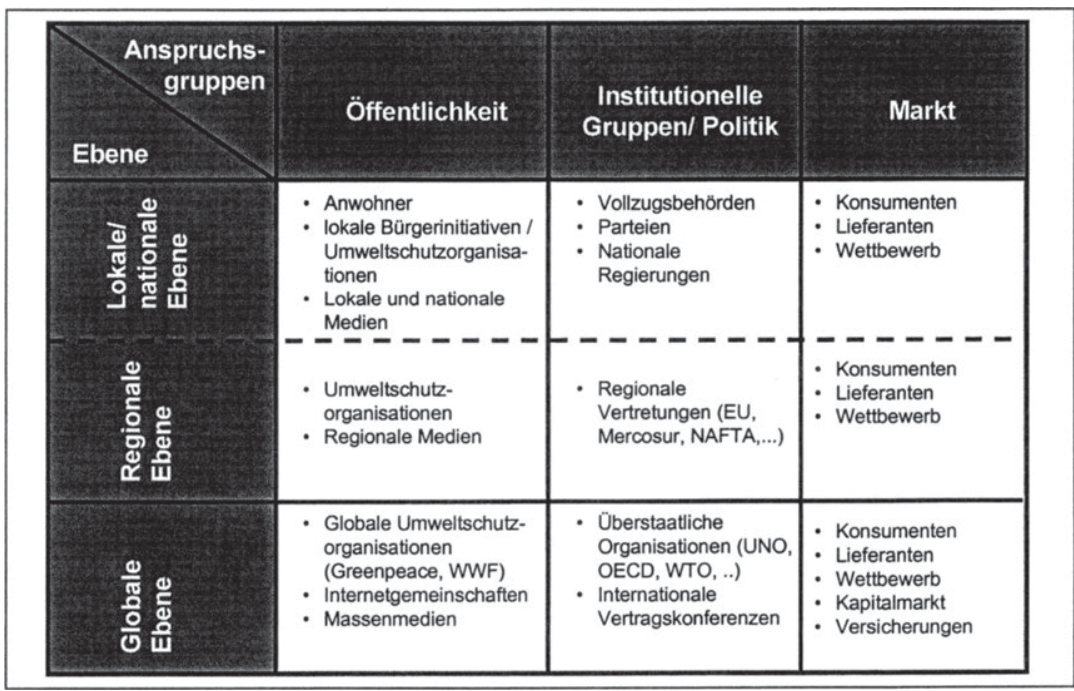

Abbildung 10: $\quad$ Differenzierung ökologischer Anspruchsgruppen

In Anlehnung an Dyllick, Th.; Belz, F.; Schneidewind, U.: Okologie und Wettbewerbsfähigkeit, München, Wien 1997, S. 98.

Eine externe Abhängigkeit bzw. Kopplung der Umsysteme liegt dann vor, wenn das ökologiebezogene Unternehmensverhalten in einem Land aufgrund von externen Rückkopplungen das Verhalten in einem anderen Ländermarkt oder in allen Ländermärkten beeinflusst. Die Kopplung von Umsystemen ergibt sich aufgrund von wirtschaftlichen, kulturellen, politischen oder medialen Vernetzungen und kann insbesondere in Integrationsräumen wie Zollunionen, Freihandelszonen und Wirtschaftsgemeinschaften beobachtet werden. ${ }^{197}$ Eine Abhängigkeit unterschiedlicher Umsysteme bezüglich ökologiebezogener Fragestellungen liegt somit dann vor, wenn ökologiebezogene Anspruchsgruppen einen länderübergreifenden Einfluss auf das Unternehmen ausüben können.

196 In diesem Kontext ist das Sanktionspotenzial der unterschiedlichen Anspruchsgruppen gegenüber der Unternehmung bei der Ableitung der Okologischen Betroffenheit zu berücksichtigen.

197 Vgl. Plump, D.: Die Koordination des Marketings in multinationalen Unternehmungen: Systemanalyse und Ansätze zur Systemgestaltung, a. a. O., S. 134. 
Als endogene Kontextfaktoren der Unternehmung können ökologiespezifische Kontext- bzw. Einflussfaktoren, wie die internationale Konfiguration, der Managementstil oder die Unternehmensgröße differenziert werden. Die Beurteilung des Managements, inwieweit ökologische Ziele komplementär zu ökonomischen Zielen sind, bestimmt dabei grundsätzlich, ob und in welchem Umfang ökologische Ziele überhaupt von einem Unternehmen verfolgt werden. Dadurch werden die Wahl der strategischen ökologischen Ausrichtung vorgegeben, die organisatorische Verankerung des Umweltschutzes beeinflusst und somit das Entstehen eines internationalen ökologiebezogenen Koordinationsbedarfs maßgeblich bestimmt. In Abbildung 11 sind unterschiedliche Arten von Rückkopplungen zusammengefasst, die bei internationaler Geschäftstätigkeit den internationalen ökologiebezogenen Koordinationsbedarf erhöhen können.

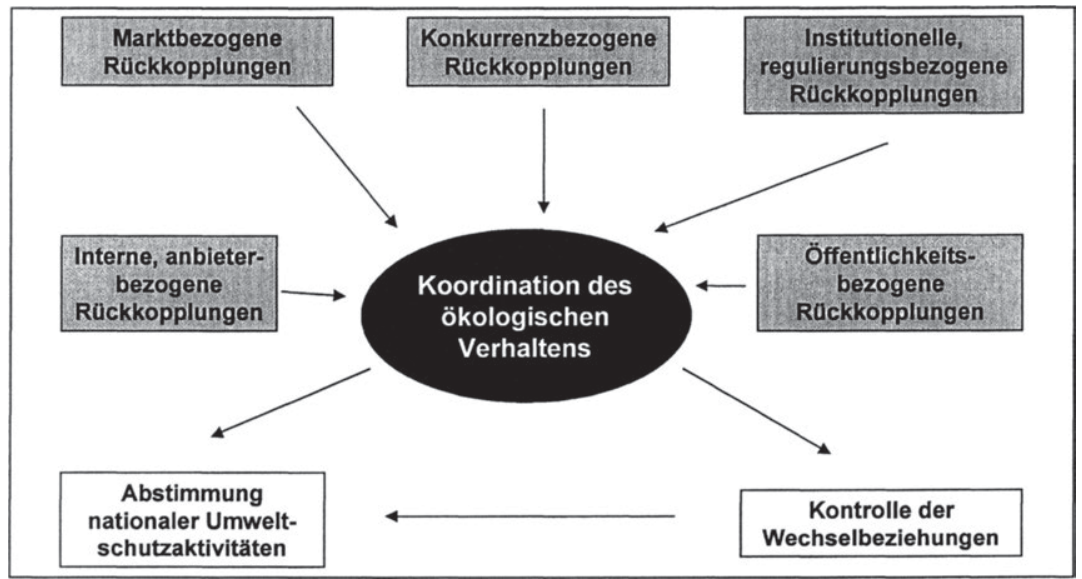

Abbildung 11: Rückkopplungen im internationalen Umweltmanagement In Anlehnung an Backhaus, K.; Büschken, J.; Voeth, M.: Internationales Marketing, 3. Aufl., Stuttgart 2000, S. 68.

Diese Rückkopplungen können sowohl innerhalb der Unternehmung als auch durch Interdependenzen mit dem externen Umsystem verursacht werden.

\section{- Interne Rückkopplungen:}

Gepoolte, sequenzielle und reziproke Interdependenzen, die bei internationaler Unternehmenstätigkeit zwischen den unterschiedlichen nationalen Einheiten auftreten und auf die Freiheitsgrade des lokalen ökologiegerichteten Unternehmensverhalten wirken, seien als interne, anbieterbezogene Rückkopplungen bezeichnet. 
- Rückkopplungen mit dem externen Umsystem:

Interdependenzen mit dem externen Umsystem können weiter differenziert werden:

- Interdependenzen zwischen zwei national unterschiedlichen Unternehmenseinheiten können auch aufgrund der gesellschaftlichen und medialen Verknüpfung von ökologischen Anspruchsgruppen, wie international tätigen Umweltschutzgruppen, zustande kommen. ${ }^{198}$ Umweltschädigendes Verhalten an einem Standort kann dann an einem anderen Standort an die Offentlichkeit gebracht werden. Es entstehen öffentlichkeitsbezogene Rückkopplungen.

- Die Abhängigkeiten, die zwischen unterschiedlichen Ländermärkten aufgrund institutioneller Verknüpfungen entstehen, können ebenfalls zu Rückkopplungen führen. ${ }^{199}$ Werden durch internationale Institutionen wertschöpfungsrelevante verbindliche oder unverbindliche Normen erlassen, die zur extraterritorialen Anwendung von ökologiebezogenen Stammlandvorschriften aufrufen, entstehen Rückkopplungen. Diese Arten von Rückkopplungen seien im Folgenden als institutionelle, regulierungsbezogene Rückkopplungen bezeichnet.

- Unternehmensentscheidungen in einem Ländermarkt können aufgrund von Rückkopplungen über den Markt die Entscheidungen in einem anderen Ländermarkt beeinflussen. Åndert bspw. ein Nachfrager aufgrund der Tätigkeiten eines Unternehmens in einem Auslandsmarkt sein Kaufverhalten im Inland, entstehen Rückkopplungen. Derartige Rückkopplungen können auch aufgrund von Interdependenzen über andere Märkte, wie Rohstoff- oder Kapitalmärkte, entstehen und sollen deshalb übergreifend als marktbezogene Rückkopplungen bezeichnet werden. ${ }^{200}$

198 BRODEL nennt dies nicht-institutionelle Verknüpfungen; vgl. Brodel, D.: Internationales Umweltmanagement: Gestaltungsfelder - Determinanten - Ausprägungen, a. a. O., S. 368.

Institutionelle Verknüpfungen liegen bspw. dann vor, wenn zwischen unterschiedlichen Regierungsstellen von Stamm- und Gastländern vertragliche Beziehungen wie Entwicklungshilfeabkommen, internationale Garantiesysteme für Investitionen etc. bestehen. Vgl. hierzu und im Folgenden Brodel, D.: Internationales Umweltmanagement: Gestaltungsfelder - Determinanten - Ausprägungen, a. a. O., S. 367 f.

BACKHAUS wählt für Rückkopplungen, die aufgrund des grenzüberschreitenden Informations- und Kommunikationsverhaltens der Nachfrager bei Beschaffungsprozessen entstehen, die Bezeichnung "nachfragerbezogene Ruckkopplungen". Vgl. Backhaus, K.; Büschken, J.; Voeth, M.: Internationales Marketing, a. a. O., S. 56 ff. 
- Vor dem Hintergrund der Relativität von Wettbewerbspositionen sind auch die Tätigkeiten von Konkurrenten in einem Auslandsmarkt eine Ursache für die Beeinflussung der Handlungen eines Unternehmens im Inlandsmarkt und umgekehrt. Die Interdependenzen zwischen Märkten, die aufgrund der Maßnahmen des Wettbewerbs entstehen, sollen dabei als konkurrenzbezogene Rückkopplungen bezeichnet.

Vor dem Hintergrund der unterschiedlichen Interdependenzen ist das internationale ökologiegerichtete Verhalten eines Unternehmens im Sinne der unternehmensbezogenen Oberziele dann aufeinander abzustimmen, wenn die Autonomiekosten die Kosten einer Koordination übersteigen. ${ }^{201}$ Autonomiekosten entstehen dabei durch die Bildung quasi autonomer Teilsysteme. Anders ausgedrückt erzeugen die unterschiedlichen Interdependenzen Koordinationsbedarf, wenn ein Unternehmen gleichzeitig auf unterschiedlichen Ländermärkten tätig ist und zwischen den Ländermärkten ein abgestimmtes ökologiebezogenes Verhalten den Gesamtgewinn der Unternehmung verbessert. ${ }^{202}$

Da in den häufigsten Fällen in einer Entscheidungssituation weder die Autonomie- noch die Koordinationskosten genau bestimmt werden können, sind an dieser Stelle Plausibilitätsurteile anzustellen. ${ }^{203}$ Um die Einflüsse einzelner Kontextfaktoren auf die Entstehung des Koordinationsbedarfs deutlich werden zu lassen, erscheint trotz der Vielzahl von Interdependenzen zwischen diesen Kontextfaktoren eine disaggregierte Untersuchung einzelner endogener und exogener Kontextfaktoren zunächst angebracht.

201 Diesem Ansatz liegt die entscheidungsorientierte Koordinationstheorie zugrunde. Diese verzichtet darauf, alle Interdependenzen bei der organisatorischen Abstimmung vollständig abzubilden. Vielmehr wird die optimale Koordinationsintensităt als Minimum der Differenz aus Koordinations- und Autonomiekosten bestimmt. Vgl. Benkenstein, M.: F \& E und Marketing: Eine Untersuchung zur Leistungsfähigkeit von Koordinationskonzeptionen bei Innovationsentscheidungen, a. a. O., S. 18 f. Der Begriff der Autonomiekosten sowie die Abbildung über den Zusammenhang von Autonomiekosten und Kommunikationskosten geht auf EMERY zurück. Vgl. Emery, J.: Organizational Planning and Control: Theory and Technology, London 1969, S. $29 \mathrm{ff}$.

Vgl. Backhaus, K.; Büschken, J.; Voeth, M.: Internationales Marketing, a. a. O., S. 67.

In der Unternehmenspraxis sind derartige Zusammenhänge kaum erfassbar, da eine isolierte Erfassung der beiden Kostenarten im Rahmen der Kostenrechnung nicht vorgesehen ist. Vgl. Plump, D.: Die Koordination des Marketings in multinationalen Unternehmungen: Systemanalyse und Ansătze zur Systemgestaltung, a. a. O., S. 100 f. 
Die im weiteren Verlauf der Arbeit untersuchten endogenen und exogenen Einflussfaktoren sind in Abbildung 12 dargestellt.

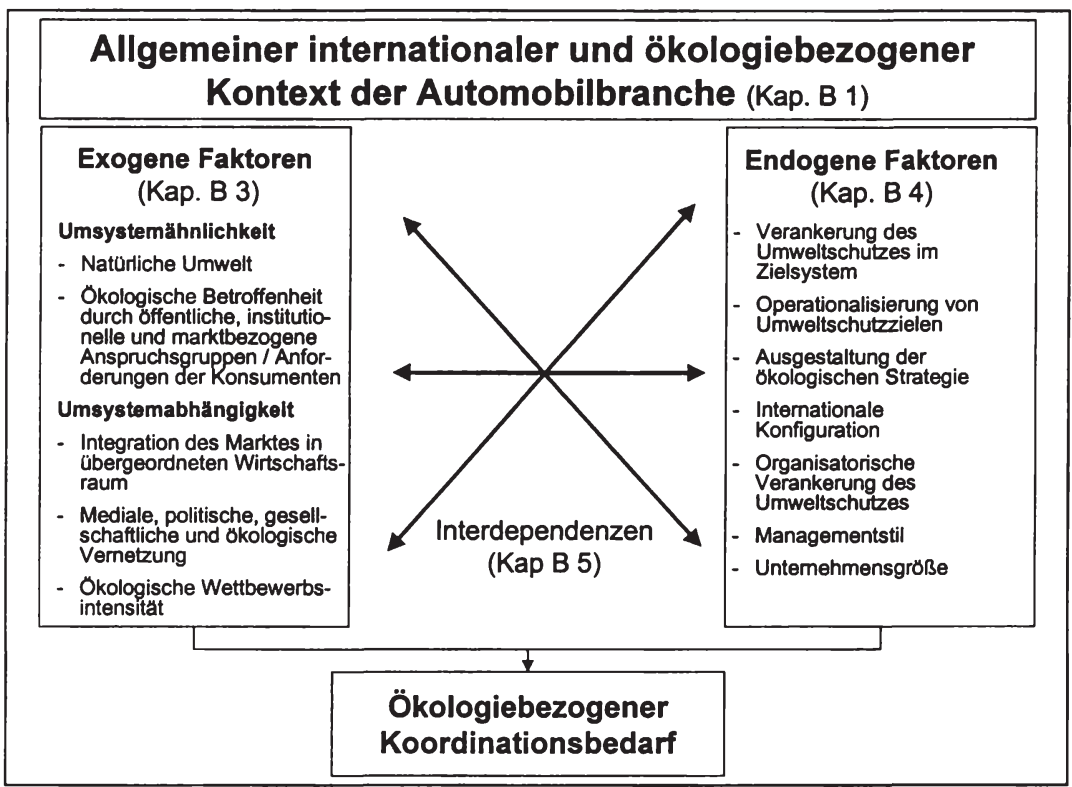

Abbildung 12: Ableitung des internationalen ökologiebezogenen Koordinationsbedarfs

Quelle: Eigene Darstellung

\section{Einfluss exogener Kontextfaktoren auf den ökologiebezogenen internationalen Koordinationsbedarf}

Der Einfluss exogener Kontextfaktoren auf den ökologiebezogenen internationalen Koordinationsbedarf wird in diesem Kapitel analysiert. Zu diesem Zweck wird die ökologische Betroffenheit als maßgeblicher Auslöser ökologiebezogener Unternehmensaktivităten in unterschiedlichen Ländern untersucht, um Aussagen über den Koordinationsbedarf abzuleiten. Aufgrund des Fehlens länderübergreifender Untersuchungen zur Wirkung der ökologischen Betroffenheit auf den Koordinationsbedarf in einem international tätigen Unternehmen werden im Folgenden auf Basis von Plausibilitätsüberlegungen Thesen über diesen Zusammenhang abgeleitet. Dazu wird insbesondere auf die Umsystemähnlichkeit bzw. die Ähnlichkeit der ökologischen Ansprüche sowie die Umsys- 
temabhängigkeit zur Begründung des internationalen ökologiebezogenen Koordinationsbedarfs zurückgegriffen.

\subsection{Umsystemähnlichkeit}

Im Folgenden werden die lokalen Kontextfaktoren, insbesondere die lokalen ökologischen Ansprüche auf ihre Ähnlichkeit untersucht werden. In Bezug auf die vorliegende Fragestellung wird davon ausgegangen, dass der internationale ökologiebezogene Koordinationsbedarf eines Automobilherstellers, der bisher Ländermärkte mit weitgehend ähnlichen ökologiebezogenen Problemen und anspruchsgruppen- bzw. konsumentenbezogenen Anforderungen bearbeitet hat, bei Eintritt in einen Markt mit stark unterschiedlichen ökologischen Rahmenbedingungen zunimmt. Eine standardisierte Bearbeitung dieses Marktes ist dann nicht oder nicht vollständig möglich. Bei international verteilter Wertschöpfung und hohen internen Leistungsverflechtungen steigt der internationale ökologiebezogene Koordinationsbedarf somit bei Abnahme der Homogenität des Umfeldes an.

\subsubsection{Natürliche Gegebenheiten}

Von größter Bedeutung für die Unternehmensführung ist die ökologische Umwelt. $^{204} \mathrm{Da}$ sich die ökologische Situation in den einzelnen Ländermärkten bzw. an den Produktionsstandorten international stark unterscheidet, lassen sich bereits hier Ähnlichkeiten bzw. Unterschiede im Umsystem feststellen. Standortbezogene Besonderheiten in Bezug auf die natürlichen Gegebenheiten $^{205}$ können differenzierte Anpassungsmaßnahmen seitens eines Unternehmens notwendig machen. So können die lokalen ökologiebezogenen Bedingungen bewirken, dass Umweltbelastungen bei Produktion oder Nutzung schädigender wirken als an anderen Orten $^{206}$, oder Produktionstechnologien

204 Vgl. Macharzina, K.: Unternehmensfuhrung: Das internationale Managementwissen; Konzepte - Methoden - Praxis, a. a. O., S. 23.

Der Begriff der natürlichen Gegebenheiten ist dem Schichtenmodell der Umweltdifferenzierung von DÜLFER entnommen, er steht für die gesamte natürliche Umwelt des Unternehmens, wie der Oberflächengestalt, klimatische Einflüsse etc. Vgl. Dülfer, E.: Zum Problem der Umweltberucksichtigung im "Intemationalen Management", a. a. O., S. 29 ff.

Vgl. Epstein, M.; Roy, M.J.: Managing Corporate Environmental Performance: A Multinational Perspective, a. a. O., S. $288 \mathrm{f}$.

In Ballungsräumen ist bspw. eine höhere Belastung der Bevőlkerung durch Lärm und Luftschadstoffe zu erwarten als in dünn besiedelten Răumen. In Städten wie Los Angeles oder Mexiko City bildet sich aufgrund geographischer Besonderheiten leicht Smog, sodass hier die Luftschadstoffe der Automobile wesentlich stärker zu einer gesundheitlichen Schädigung der Bevölkerung beitragen als anderenorts. Vgl. Greenpeace (Hrsg.): Acht Wochen 
aufgrund der geographischen Lage, des Klimas, der Luftdruck- oder Luftfeuchtigkeitsverhältnisse zu qualitativ unterschiedlichen Ergebnissen führen bzw. sich nicht anwenden lassen. Vor diesem Hintergrund erhöht sich die Komplexität der ökologiebezogenen Unternehmensentscheidungen. Die Kosten der Gestaltung und Steuerung international verteilter Wertschöpfungsaktivitäten steigen, je unterschiedlicher die ökologischen Rahmenbedingungen ausfallen. Dabei kann davon ausgegangen werden, dass der Grad der notwendigen Anpassungen von Produktionsanlagen und Produkten um so größer ausfällt, je unterschiedlicher die lokalen őkologischen Rahmenbedingungen sind. ${ }^{207}$

Je differenzierter die lokalen natürlichen (öologischen) Gegebenheiten, desto größer ist der internationale ökologiebezogene Koordinationsbedarf.

Die lokalen ökologischen Kontextfaktoren in einem Ländermarkt gelten weiterhin als Ausgangspunkt für aktuelle und potenzielle branchenbezogene Herausforderungen bzw. ökologische Ansprüche. In diesem Kontext ist die ökologische Situation in unterschiedlichen Ländern zu untersuchen.

Dabei zeigt sich ein heterogenes Bild. Insbesondere Industrie- und Entwicklungs- bzw. Schwellenländer unterscheiden sich dabei deutlich. Allein die Bevölkerung in den Mittelmeerländern Spanien, Griechenland und Italien hält die ökologische Situation im eigenen Land für ähnlich gesundheitsgefährdend wie die Bevölkerung in Schwellenländern. Da sich in den vergangenen Jahrzehnten die Umweltqualität in vielen Industrieländern verbessert hat, erscheint die ökologische Situation insbesondere in Schwellen- und Entwicklungsländern durch hohe ökologische Belastungen geprägt zu sein. Abbildung 13 zeigt die Einschätzung der Bevölkerung in Bezug auf die persönliche gesundheitliche Beeinträchtigung durch ökologische Probleme im internationalen Vergleich.

mit dem Luftmessbus in Mexiko City: Megastadt im Megastau, http://www.greenpeace.de IGP DOK 3P/BROSCHUE/AKTION/CO9IA01.HTM [25.01.2001].

207 Differenzierte lokale Bedingungen erfordern oft neue und andere Lossungen durch den Hersteller, dies kann zu einem erhöhten internationalen Koordinationsbedarf beitragen. Erfahrungen und Technologien aus anderen Standorten können im produktionsbezogenen Umweltschutz vor Ort dann nur begrenzt angewendet werden. BRODEL beschreibt u. a. diesen Zusammenhang als Komplexitătsbarrieren. Vgl. Brodel, D.: Internationales Umweltmanagement: Gestaltungsfelder - Determinanten - Ausprägungen, a. a. O., S. 386 f. 


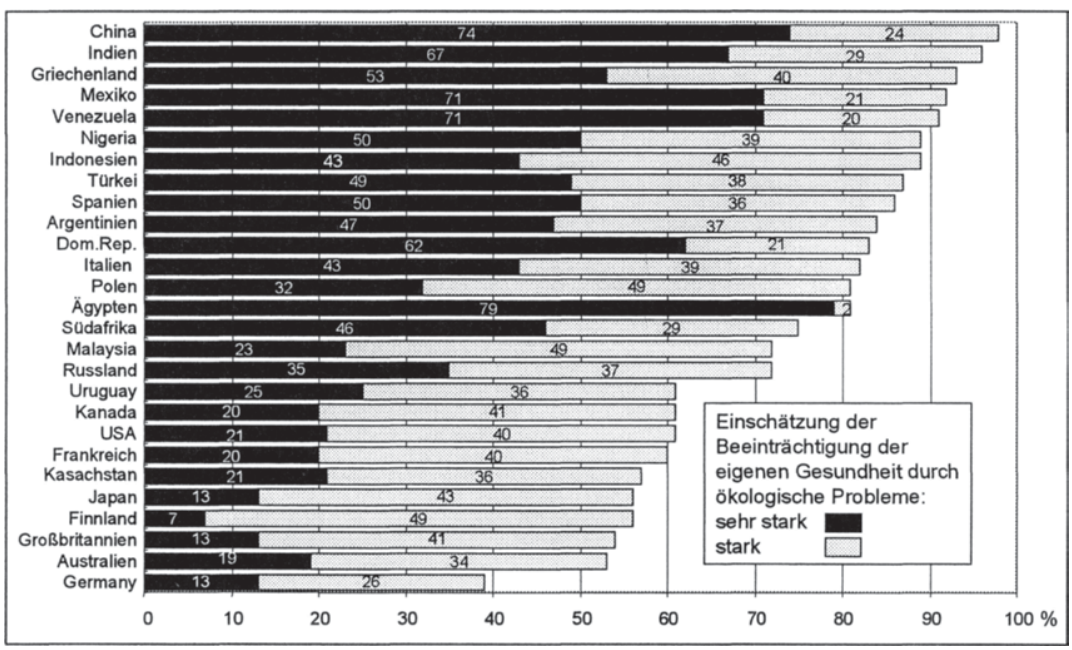

Abbildung 13: $\quad$ Einschätzung der persönlichen gesundheitlichen Beeinträchtigung durch ökologische Probleme im internationalen Vergleich

Quelle: Environics International (Hrsg.): The Environmental Monitor - Global Public Opinion on the Environment: 1999 International Report, a. a. 0., S. 23.

In diesem Kontext zeigen Untersuchungen, dass die durch Pkw verursachte Luftverschmutzung in einigen Ballungsräumen bereits ein gesundheitsgefährdendes Ausmaß angenommen hat. Während die Belastungen durch Autoabgase in den Industrieländern aufgrund von Umweltschutzgesetzen und des technologischen Fortschritts teilweise zurückgegangen sind ${ }^{208}$, kann in Entwicklungs- und Schwellenländern die ökologische Situation oft als prekär beschrieben werden. ${ }^{209}$ Die gesundheitliche Beeinflussung besonders in den Millionen-

208 Dabei haben die aktuell gültigen nationalen Umweltschutzgesetze einen wesentlichen Einfluss auf den Anfall an ökologischen Belastungen in einem Land. So führten strenge gesetzliche Abgasvorschriften insbesondere in den Ländern der Triade bei Luftschadstoffen zu einer spürbaren Entlastung der Umwelt. Nach Angaben des VDA sind so in Deutschland seit Ende der 80er Jahre die Abgasemissionen bis Ende der 90er Jahre drastisch reduziert worden. Vgl. VDA (Hrsg.): Auto 2000: Jahresbericht des Verbands der Automobilindustrie e. V., a. a. O., S. 148.

Obwohl Chinas Automobilindustrie noch sehr jung ist und der private Besitz eines Pkws noch nicht die Regel darstellt, sind die aktuellen Unfallzahlen, Staus und ökologischen Probleme des Individualverkehrs in den Städten Chinas durchaus mit denen von entwickelten Ländern vergleichbar. So zählen die Automobile in der VR China zu den emissionsreichsten der Welt. Sie verursachen trotz ihrer vergleichsweise geringen Anzahl eine Luftverschmutzung, die mit der in Tokio und Los Angeles bei einem 10 mal höheren Fahrzeugbestand vergleichbar ist. Vgl. Madhavan, S.: Mobility at a Price, in: Humphrey, J. et al. (Hrsg.): Global Strategies and Local Realities: The Auto Industry in Emerging Markets, 
städten der Schwellenländer wird in hohem Maße der Luftverschmutzung und insbesondere dem Straßenverkehr zugeschrieben. ${ }^{210}$ Dabei ist davon auszugehen, dass das Ausmaß der ökologischen Probleme an einem Standort ökologiebezogene Forderungen von Anspruchsgruppen nach sich ziehen kann.

In Anbetracht der als differenziert zu kennzeichnenden ökologiebezogenen Rahmenbedingungen können sich auch die lokalen ökologiebezogenen Forderungen von Seiten der Anspruchsgruppen unterscheiden. Inwieweit ökologische Ansprüche lokal artikuliert werden, hängt allerdings nicht allein von der lokalen ökologischen Situation ab, sondern wird auch von anderen Kontextfaktoren, wie dem Wissen um die ökologischen Zusammenhänge, kulturellen ${ }^{211}$ und gefühlsmäßigen Einstellungen zur ökologischen Umwelt, ökonomischen und sozialen Bedingungen, dem Rechtsystem etc. bestimmt. ${ }^{212}$

\subsection{2 Ökologische Betroffenheit durch lokale öffentliche Anspruchs- gruppen}

Steht ein Unternehmen aufgrund ökologisch bedenklicher Verhaltensweisen in der Kritik öffentlicher Anspruchsgruppen ${ }^{213}$, wird von einer öffentlichen Exponiertheit des Unternehmens gesprochen. Die Unternehmung ist nun einer öf-

XINHUA werden in den meisten Städten Chinas die staatlichen Grenzwerte für Luftschadstoffe drastisch überschritten. So werden in Peking die Stickoxid-Emissionen um $100 \%$ und in Taiyuan die Schwefeldioxid-Emissionen um $400 \%$ überschritten.

$\mathrm{Da}$ in Schwellenländern die Großstädte sehr schnell wachsen, wird in Zukunft ein noch größerer Teil der Bevölkerung von den ókologischen Belastungen des Automobils betroffen sein. In Chinas Ballungsräumen ist die Umweltverschmutzung durch Luftschadstoffe so hoch, dass in einem Zeitraum von 1997 bis 2020 allein in den Städten Beijing. Chongqing, Shenyang, Shanghai und Guangzhou ungefăhr 200.000 Menschen an den Folgen der Luftverschmutzung sterben werden. Zur ökologischen Situation in China vgl. Dasgupta, S.; Wang, H.; Wheeler, D.: Surviving Success: Policy Reform and the Future of Industrial Pollution in China, World Bank Policy Research Department Working Paper, 1997, S. 5.

Vgl. zur Landeskulturforschung Harbison, F.; Myers, Ch. A.: Management in the Industrial World. An International Analysis, New York et al. 1959; Keller, E. v.: Management in fremden Kulturen. Ziele, Ergebnisse und methodische Probleme der kulturvergleichenden Managementforschung, Bern, Stuttgart 1982; Hofstede, G.: Organization-Related Value Systems in Forty Countries, Working Paper 78-22, European Institute für Advanced Studies in Management, Brüssel 1978 sowie Schmid, S.: Multikulturalităt in der internationalen Unternehmung: Konzepte - Reflexionen - Implikationen, Wiesbaden 1996, insbesondere S. $229 \mathrm{ff}$.

212 Vgl. Dülfer, E.: Zum Problem der Umweltberucksichtigung im „Internationalen Management", a. a. O., S. $29 \mathrm{ff}$.

213 Als Anspruchsgruppen kommen Einzelpersonen als direkt Betroffene oder Personengruppen aufgrund ihres Wissens (Experten und Wissenschaftler) oder ihrer gesellschaftlichen Position (Politiker, Umweltschutzverbände, Journalisten) in Betracht. Vgl. Achleitner, P. M.: Sozio-politische Strategien multinationaler Unternehmen, in: Schriftenreihe Betriebswirtschaft der Hochschule St. Gallen, Bd. 13, Bern, Stuttgart 1985, S. 95 f. 
fentlichen, zumeist moralisch geführten Diskussion ausgesetzt und verlässt den "Bereich privatrechtlicher Verfasstheit und privatautonomer Entscheidungs- und Gestaltungsfreiheit". Dabei ist die Unternehmung Beeinflussungen sowie Beurteilungskriterien unterworfen, die ansonsten nur für öffentliche Institutionen gelten. $^{214}$

Ökologische Anspruchsgruppen wie Anwohner, Bürgerinitiativen, Umweltschutzgruppen und die lokalen Medien verfügen als Machtmittel über die Möglichkeit, öffentlichen Druck zu mobilisieren. Die Infragestellung der gesellschaftlichen Legitimität unternehmensbezogener Verhaltensweisen soll Politik und Konsumenten bewegen, durch gesetzliche Maßnahmen oder Kaufboykotte eine Änderung des Unternehmensverhaltens zu bewirken. DYLLICK wies bereits 1986 auf die Gefahr für Unternehmen hin, in gesellschaftspolitische Auseinandersetzungen in den Bereichen Gesundheits-, Umwelt- und Konsumentenschutz sowie Beziehungen zur Dritten Welt verwickelt zu werden. Für die Zukunft der Unternehmen können sie eine bedeutend größere Rolle spielen als das Bestehen im täglichen Kampf um Marktanteile. ${ }^{215}$

Inwieweit ökologische Probleme ihren Niederschlag im Bewusstsein der Bevölkerung bereits gefunden haben, zeigt die international hohe Besorgnis über den Zustand der natürlichen Umwelt. ${ }^{216}$ Insbesondere in den Entwicklungs- und Schwellenländern ist die Sensibilisierung gegenüber ökologischen Problemen in den vergangenen Jahren angestiegen. ${ }^{217}$ Dies spricht für eine zunehmende

214 Vgl. Dyllick, Th.: Management der Umweltbeziehungen: Offentliche Exponiertheit von Unternehmen als Herausforderung for Management und Unternehmenspraxis, in: Die Unternehmung, 42. Jg. (1988), Nr. 3, S. 195.

215 Vgl. Dyllick, Th.: Die Beziehungen zwischen Unternehmung und gesellschaftlicher Umwelt, in: Die Betriebswirtschaftslehre, 46. Jg. (1986), Nr. 3, S. 373 f.

Heute wird diese Einschätzung insbesondere von international tătigen Großunternehmen geteilt. Wie dies bspw. in der Rede von PHIL WATTS, Managing Director der Ölgesellschaft Shell, auf der 33. Weltkonferenz der Internationalen Handelskammer in Budapest im April 2000 zum Ausdruck kommt. „We should also be seeking broader debate about a number of challenging dilemmas which affect business but which business alone cannot solve. For example: The direct responsibility of business in relation to human rights. The question of global standards on pay and working conditions as against those that differ in response to national economic and social circumstances. And whether businesses or institutions should impose developed world standards of environmental controls even when developing countries have different priorities." Phil Watts: Globalisation and the Corporation: when preparation meets opportunity, Budapest 2000, http://www.shell.com/library/speech/ [26.08.2000].

216 Vgl. Environics International (Hrsg.): The Environmental Monitor - Global Public Opinion on the Environment: 1999 International Report, a. a. O., S. 17.

217 Vgl. Environics International (Hrsg.): The Environmental Monitor - Global Public Opinion on the Environment: 1999 International Report, a. a. O., S. 16. 
Angleichung des Umweltbewusstseins. Es lassen sich allerdings, wie Abbildung 14 deutlich zeigt, international große Unterschiede in Bezug auf den Grund für die hohe Besorgnis feststellen.

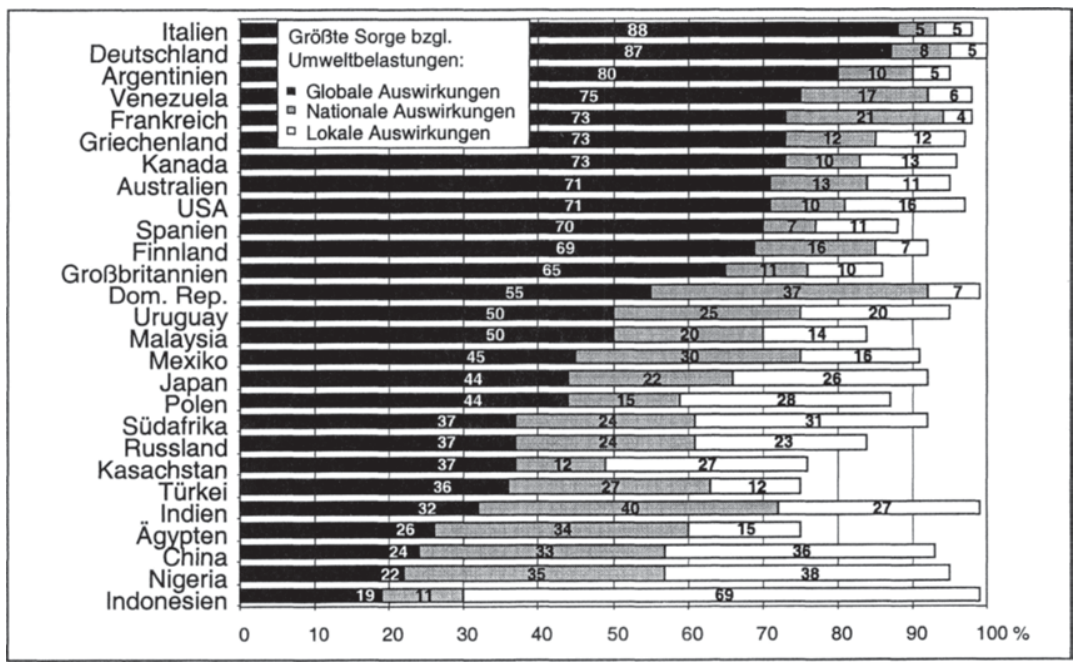

Abbildung 14: Grund der Besorgnis über den Zustand der ökologischen Umwelt im internationalen Vergleich

Quelle: Environics International (Hrsg.): The Environmental Monitor - Global Public Opinion on the Environment. 1999 International Report, a. a. o., S. 19.

Die Bevölkerung in den Industrieländern ist eher über die globalen ökologischen Auswirkungen der Umweltprobleme besorgt, während in den Entwicklungs- und Schwellenländern die Sorge um die lokalen Umweltauswirkungen im Mittelpunkt steht. Diese Unterschiede hängen sicherlich in hohem Maße mit der lokalen ökologischen Situation zusammen. Die Forderung nach Senkung des $\mathrm{CO}_{2}-$ Ausstoßes von Pkw zur Vermeidung einer fortschreitenden Erderwärmung wird somit in ärmeren Ländern kaum eine Rolle spielen. Vielmehr kann erwartet werden, dass der Schwerpunkt der ökologischen Forderungen in diesen Ländern auf der Verringerung von Emissionen durch Produktionsstandorte und Autoabgase liegt. ${ }^{218}$

218 Speziell in Indien, Südafrika, Venezuela, aber auch in Großbritannien und Griechenland wird die Luftbelastung durch Pkw als das am drăngendsten zu lösende ökologische Problem betrachtet. Vgl. Environics International (Hrsg.): The Environmental Monitor Global Public Opinion on the Environment. 1999 International Report, a. a. O., S. 20. 
Je unterschiedlicher die ökologischen Forderungen in einzelnen Ländermärkten ausfallen, desto unterschiedlicher sind die Bedingungen für Unternehmen, die gesellschaftliche Legitimität vor Ort zu wahren. Somit lässt sich vermuten, dass aufgrund unterschiedlicher ökologischer Forderungen interne anbieterbezogene Rückkopplungen entstehen, die den internationalen ökologiegerichteten Koordinationsbedarf erhöhen.

Je unterschiedlicher die gesellschaftlichen, ökologiebezogenen Forderungen international ausgeprägt sind, desto höher ist der intemationale ökologiebezogene Koordinationsbedarf aufgrund interner anbieterbezogener Rückkopplungen.

In diesem Zusammenhang ist einschränkend zu vermerken, dass lokale ökologiebezogene Ansprüche nicht in jedem Fall zu einer ökologischen Betroffenheit eines Unternehmens beitragen. Die Bereitschaft der Öffentlichkeit, ökologische Forderungen auch durchzusetzen und die Politik aufzufordern, entsprechende Gesetze zu erlassen bzw. die eigenen Kaufentscheidungen zu ändern, ist von weiteren lokalen Einflussfaktoren abhängig. So kann die Bevölkerung bspw. die Arbeitsplatzsicherheit bzw. die ökonomische Entwicklung höher bewerten als den Schutz der natürlichen Umwelt. Empirische Untersuchungen zeigen, dass eine Mehrheit der Bevölkerung in den Industrieländern Umweltschutz für wichtiger hält als ökonomisches Wachstum. Diese Einschätzung wird allerdings in weit geringerem $\mathrm{Maße}$ von der Bevölkerung in den ärmeren Ländern geteilt. ${ }^{219}$ Weiterhin können kulturelle Einflussfaktoren und politische Rahmenbedingungen dazu beitragen, dass die Erfolgsaussichten eines persönlichen Engagements für den Umweltschutz als gering eingeschätzt wird und deshalb unterbleibt. ${ }^{220}$ Diese Einflussfaktoren beeinträchtigen den Erfolg einzelner Anspruchsgruppen, ihre ökologischen Forderungen durchzusetzen.

219 Generell bestätigt dies aber die These, dass die Bewertung der ökologischen Umwelt mit dem Einkommensniveau zusammenhängt. Dabei ist anzumerken, dass Umweltschutz im Vergleich zum ökonomischen Wachstum zunehmend höher bewertet wird. Mittlerweile setzt sich diese Einstellung auch in einigen Wachstumsmärkten wie bspw. China durch. Allein in Ländermärkten, die aufgrund ökonomischer Krisensituationen auch mit hohen sozialen Problemen zu kämpfen haben, wie bspw. Mexiko und Russland, wurde dem ökonomischen Wachstum eine höhere Bedeutung beigemessen, als in den Vorjahren. Vgl. Environics International (Hrsg.): The Environmental Monitor - Global Public Opinion on the Environment: 1999 International Report, a. a. O., S. 41.

Die Einschätzungen der Möglichkeit einer persönlichen Einflussnahme auf ökologische Probleme unterscheidet sich international gravierend. Vgl. zur Beurteilung der persönlichen Einflussnahme auf die ókologische Situation Environics International (Hrsg.): The Environmental Monitor - Global Public Opinion on the Environment. 1999 International Report, a. a. O., S. 37. 
Damit sinkt das mögliche Sanktionspotenzial dieser Gruppen und verringert die ökologische Betroffenheit der Unternehmung.

Der internationale ökologiebezogene Koordinationsbedarf aufgrund lokaler Forderungen öffentlicher Anspruchsgruppen fällt umso geringer aus, je geringer das Sanktionspotenzial dieser Gruppen ist.

\subsection{3 Ökologische Betroffenheit durch lokale politische Anspruchs- gruppen}

Ökologische Forderungen, die einen Wiederhall in der breiten Öffentlichkeit gefunden haben, werden zumeist von politischen Institutionen aufgegriffen. Die Politik legt dann verbindliche Regeln für das ökologiebezogene Unternehmensverhalten fest. ${ }^{221}$ Derartige Regelungen können die Handlungsfreiheit und die Kostensituation der Unternehmen am Markt beeinflussen. Derzeit unterscheiden sich die ökologiebezogenen rechtlichen Bestimmungen international noch sehr stark. Dies gilt sowohl für den produkt- als auch den prozessbezogenen Umweltschutz.

An den Standorten der Triade gelten vergleichsweise anspruchsvolle Umweltschutzgesetze, die sich tendenziell untereinander angleichen. ${ }^{222}$ Die Abgasbestimmungen als produktbezogene Umweltvorschriften unterscheiden sich in der Welt derzeit allerdings noch sehr stark, wie die exemplarische Gegenüberstellung unterschiedlicher Abgasvorschriften für Diesel-Pkw in Tabelle 2 zeigt. Dabei sind nicht allein die Höhe der Anforderungen, sondern auch die Art der Messung der Abgasgrenzwerte different. Die europäischen und US-amerikanischen Grenzwerte sind somit nicht direkt miteinander vergleichbar. ${ }^{223}$

221 Vgl. Achleitner, P. M.: Sozio-politische Strategien multinationaler Unternehmen, a. a. O., S. $95 \mathrm{f}$.

222 Trotz gewisser lokaler Unterschiede innerhalb der EU wird im Rahmen dieser Arbeit von einer zunehmenden Angleichung der Umweltschutzgesetzgebung ausgegangen, wie bspw. auch Herr MOGG von VOLKSWAGEN im Interview anmerkt.

Dies geht auf das Interview mit Herrn RössLer von VolKsWAGEN zurück. 


\begin{tabular}{|c|c|c|c|c|c|}
\hline \multicolumn{6}{|c|}{ Europa (ECE 15 + EUDC in $\mathrm{g} / \mathrm{km}$ ) } \\
\hline Diesel & Gültig ab & $\mathrm{CO}$ & $\mathrm{HC}$ & NOx & PM \\
\hline EUROI & 01.07 .92 & 2,88 & 0,2 & 0,78 & 0,14 \\
\hline EURO ॥ & 01.01 .96 & 1,06 & 0,19 & 0,73 & 0,1 \\
\hline EURO III & 01.01 .00 & 0,64 & 0,06 & 0,5 & 0,05 \\
\hline EURO IV & 01.01 .05 & 0,5 & 0,05 & 0,25 & 0,025 \\
\hline \multicolumn{6}{|c|}{ Thalland (ECE A $83-01$ (B) ab 1999 9412/EC in $\mathrm{g} / \mathrm{km}$ ) } \\
\hline \multirow[t]{3}{*}{ Dlesel } & Gültig ab & $\mathrm{CO}$ & $\mathrm{HC}$ & NOx & PM \\
\hline & 01.01 .1997 & 2,72 & & & 0,14 \\
\hline & 01.01 .1999 & 1,0 & & & 0,08 \\
\hline \multicolumn{6}{|c|}{ Australlen (ECE R 83 in $\mathrm{g} / \mathrm{km}$ ) } \\
\hline Diesel & Gültig ab & $\mathrm{CO}$ & HC & NOx & PM \\
\hline (Euro l) & 1995/96 & 2,88 & 0,2 & 0,78 & 0,14 \\
\hline (Euro II) & $2002 / 03$ & 1,06 & 0,19 & 0,73 & 0.1 \\
\hline (Euro IV) & $2006 / 07$ & 0,5 & 0,05 & 0,25 & 0,025 \\
\hline \multicolumn{6}{|c|}{ Argentinien (ECE $15+$ EUDC in $\mathrm{g} / \mathrm{km}$ ) } \\
\hline \multirow[t]{5}{*}{ Diesel } & Gültig ab & $\mathrm{CO}$ & $\mathrm{HC}$ & NOx & PM \\
\hline & 1994 & 24,0 & 2,1 & 2,0 & \\
\hline & 1998 & 12,0 & 1,2 & 1,4 & 0,373 \\
\hline & 1998 & 6,2 & 0,5 & 1,43 & $0,16-0,31$ \\
\hline & 2000 & 2,0 & 0,3 & 0,6 & 0,124 \\
\hline
\end{tabular}

Tabelle 2: Internationale Abgasvorschriften für Diesel-Pkw im Vergleich Quelle: eigene Zusammenstellung auf Basis der Daten von http://www.dieselnet.com/standards.html [04.10.2000].

Das Umweltrecht in vielen Schwellen- und Entwicklungsländern ist erst im Aufbau begriffen, häufig wenig detailliert und in der Anwendbarkeit eingeschränkt. So bestehen in einigen Fällen für die Automobilindustrie keine oder nur unzureichende Regelungen, wie bspw. in Südafrika. ${ }^{224}$

Zur Beschreibung der umweltpolitischen Situation in den Entwicklungs- und Schwellenländern kann auf die in Kapitel B 1 erfolgte Gruppierung zurückgegriffen werden:

- Integrationsländer am Rande der Triademärkte:

Die Umweltpolitik in diesen Ländern orientiert sich zunehmend an den Umweltschutzbestimmungen der jeweiligen Wirtschaftsblöcke (EU, NAFTA). Obwohl eine konsequente Angleichung in den Integrationsländern noch nicht stattgefunden hat ${ }^{225}$, wird diese aber in naher Zukunft zu erwarten sein. ${ }^{226}$

224 Dort werden durch die Umweltgesetzgebung nur wenige Vorschriften erlassen, die primär auf die ortsansässigen Minen zugeschnitten sind. Obwohl durchaus einige Vorschriften auch für die Automobilindustrie einschlägig sind, erläutert Herr MOGg im Interview, dass diese Vorschriften oft nicht weit gestaffelt und wenig detailliert seien.

225 Vgl. hierzu und im Folgenden Lung, Y.: Is the Rise of Emerging Countries as Automobile Producers an Irreversible Phenomenon?, a. a. O., S. 19-24.

226 So hat VOLKSWAGEN bei der Errichtung eines neuen Produktionsstandortes in Polen von Anfang an die strengen europäischen Umweltschutzvorschriften zugrunde gelegt, da durch 
- Schwellenländer im Prozess einer regionalen Integration:

In diesen Ländern wird die Umweltgesetzgebung zuerst untereinander harmonisiert werden. Dabei steht derzeit aber noch der Aufbau eines konsistenten Umweltrechts und dessen Implementierung im Vordergrund. So orientieren sich die Umweltbehörden in Süd- und Mittelamerika bereits informativ an der europäischen und nordamerikanischen Umweltgesetzgebung. ${ }^{227}$ In Südafrika gibt es Berater aus dem nordamerikanischen Raum, die der Regierung beim Aufbau eines Umweltgesetzes helfen. Weiterhin zeigen wissenschaftliche Untersuchungen über das Erfolgspotenzial unterschiedlicher Umweltschutzmaßnahmen in diesen Ländern, dass mit einer weiteren Verschärfung der Umweltgesetze zu rechnen ist. ${ }^{228}$

- Wachstumsmärkte, die einen eigenen Automobilmarkt entwickeln:

Länder, die im Begriff sind, eine eigene Automobilindustrie aufzubauen, weisen derzeit oft wenig anspruchsvolle Umweltschutzbestimmungen auf. Ob und in welchem Zeitraum sich die ökologischen Rahmenbedingungen an die der Industrieländer angleichen, ist mit hoher Unsicherheit behaftet. Aufgrund der sich teilweise stark verschlechternden ökologischen Situation kann aber eine Verschärfung der Umweltgesetze erwartet bzw. bereits beobachtet werden. ${ }^{229}$

Gesetzliche Vorschriften bezüglich der ökologiebezogenen Produkteigenschaften müssen in jedem Land eingehalten werden. So wird bspw. Neufahrzeugen, welche die gesetzlichen Abgasreinigungsvorschriften eines Landes nicht erfüllen, die Zulassung in diesem Markt verweigert. Die Fahrzeuge sind damit in diesem Land unverkäuflich. Je unterschiedlicher die produktbezogenen Umweltschutzbestimmungen sind, desto geringer fallen für einen Hersteller die Möglichkeiten zur Nutzung von Verbundvorteilen, bspw. der Nutzung von Skaleneffekten, aus. ${ }^{230}$ Weiterhin steigt in diesem Fall der Kommunikations- und Koordinationsbedarf zwischen unterschiedlichen Ländermärkten bzw. Produktionsstätten, wenn Leistungen zwischen den Ländermärkten ausgetauscht werden sollen. Ab einem bestimmten Grad der Heterogenität nehmen die Koordinationskosten ein prohibitiv hohes Niveau an, sodass in diesem Fall von einer Komplexitätsbarriere gesprochen werden kann. Dies führt zu regullerungsbe-

den in naher Zukunft zu erwartenden EU-Beitritt mit schärferen Umweltschutzbestimmungen zu rechnen ist. Diese Einschätzung gibt Herr MOGg von VoLKSWAGEN ab.

In Brasilien gibt es bereits eine Reihe von Gesetzen im fertigungsbezogenen Umweltschutz, die zumeist aus den Industrieländern "kopiert" wurden.

Vgl. Farraz, C.; da Motta, R. S.: Automobile Pollution Control in Brazil, Rio De Janeiro, 1999.

229 Vgl. Dasgupta, S.; Wang, H.; Wheeler, D.: Surviving Success: Policy Reform and the Future of Industrial Pollution in China, World Bank Policy Research Department Working Paper, 1997.

In Europa produzierte Pkw sind somit mit einem zumindest teilweise anderen Antriebsstrang (Motor mit Auspuff und Abgasregeltechnik) zu versehen, um die amerikanischen Abgasgrenzwerte einzuhalten. Dies erhöht den internationalen ökologiebezogenen Koordinationsbedarf, wie auch Herr MAYR-RAUCH von VolKSWAGEN bestătigt. 
zogenen Rückkopplungen, die den internationalen Koordinationsbedarf erhöhen.

Je unterschiedlicher die gesetzlichen Vorschriften in Bezug auf den produktbezogenen Umweltschutz sind, desto höher ist der internationale ökologiebezogene Koordinationsbedarf aufgrund regulierungsbezogener Rückkopplungen.

Produktionsbezogene Umweltschutzvorschriften können nach GATT nicht zum Aufbau von Handelshemmnissen herangezogen werden. Die Möglichkeit, die Produktion in die Länder zu verlagern, in denen geringe Umweltschutzvorschriften gelten, könnte somit zu geringeren Produktionskosten führen. Nach der einmaligen Entscheidung für einen Produktionsstandort und der Festlegung, welche Produkte und Fahrzeugteile in welchem Umfang von welchen Standorten produziert werden, besteht nur dann weiterer Koordinationsbedarf, wenn sich die produktionsbezogenen ökologischen Anforderungen und damit die relativen Kosten der einzelnen Standorte verändern. Vor diesem Hintergrund fält der internationale Koordinationsbedarf im produktionsbezogenen Umweltschutz umso höher aus, je dynamischer sich die umweltrechtlichen Rahmenbedingungen verändern. ${ }^{231}$ Dies gilt selbstverständlich auch für den produktbezogenen Umweltschutz, der durch eine hohe Technologiedynamik und einen ökologischen Innovationswettbewerb geprägt ist. ${ }^{232}$ Stehen technische Lösungen im Umweltschutz zur Verfügung, können diese schnell obligatorisch werden.

Je dynamischer sich die umweltrechtlichen Rahmenbedingungen verändern, desto höher ist der internationale ökologiebezogene Koordinationsbedarf.

\subsection{4 Ökologische Betroffenheit durch den Markt}

Wie bereits dargestellt, können sich ökologische Forderungen oder ökologische Knappheiten auch am Markt niederschlagen. Aus Unternehmenssicht können sich marktliche Einflüsse in Form von Kosten niederschlagen, bspw. wenn aufgrund von ökologischen Knappheiten Abfallgebühren, Abwasserabgaben oder Rohstoffpreise steigen. Auch die Berücksichtigung eines erhöhten Haftungsrisikos bei ökologisch belastenden Produktionsprozessen oder Produkten bei der

231 Vgl. dazu auch Hoffmann, F.: Führungsorganisation, a. a. O., S. $107 \mathrm{ff}$. 232

Diese Stellungnahme machte Herr KRŬGER von FORD im Interview. 
Festlegung von Versicherungsprämien oder Kreditbedingungen ${ }^{233}$ sind in diesem Zusammenhang anzusprechen. Schließlich drücken sich ökologiebezogene marktliche Einflüsse auch im Nachfrageverhalten der Konsumenten aus. ${ }^{234}$ All dies kann zu einer marktbezogenen Betroffenheit des Unternehmens auf lokaler, regionaler oder globaler Ebene führen.

Heute sehen sich Unternehmen häufiger als in der Vergangenheit Kunden gegenüber, die beim Kauf die Umweltverträglichkeit von Produkten als Standard fordern und bei Fehlen entscheidender ökologischer Eigenschaften ein Produkt bzw. eine Marke meiden. Dieses Verhalten unterscheidet sich aufgrund nationaler Kontextfaktoren, wie dem Angebot an gleichwertigen ökologischen Produktalternativen, dem Umweltbewusstsein der Konsumenten und kulturellen Besonderheiten, um nur einige Faktoren zu nennen.

Deutlich wird durch die folgende Abbildung, dass in den Industrieländern, insbesondere in den Ländern Australien, Kanada, Deutschland und den USA, wesentlich häufiger Produkte und Marken gemieden werden, als dies in Japan und in Entwicklungs- und Schwellenländern der Fall ist. Die hohe Rate an Erwägern zeigt dabei, dass bei Vorhandensein entsprechender ökologischerer Alternativen Konsumenten eine durchaus höherer Anteil von Konsumenten diese Angebote wahrnehmen würden.

233 Eine Änderung des Umwelthaftungsrechts hat in Brasilien so in $1999 \mathrm{zu}$ einer Zertifizierungswelle nach ISO 14001 geführt. Vgl. Volkswagen (Hrsg.): Umweltbrief, Nr. 11/99, Wolfsburg 1999, S. 10.

234 Vgl. Dyllick, Th.; Belz, F.; Schneldewind, U.: Okologie und Wettbewerbsfähigkeit, a. a. O., S. 25 - 28. 


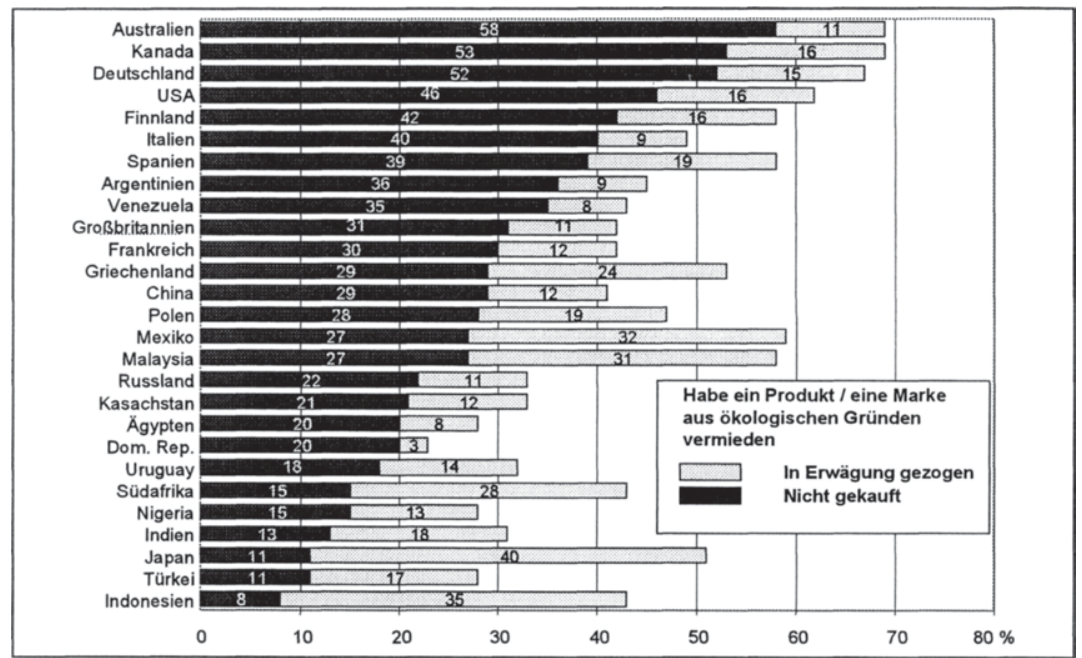

Abbildung 15: Verzicht auf ein Produkt aus ökologischen Gründen im internationalen Vergleich

Quelle: Environics International (Hrsg.): The Environmental Monitor-Global Public Opinion on the Environment: 1999 International Report, a. a. o., S. 39.

Im Hinblick auf das Konsumentenverhalten in der Autombilindustrie ist anzumerken, dass der Umweltverträglichkeit die Eigenschaft eines Hygienefaktors zukommt. ${ }^{235}$ So zeigen empirische Untersuchungen einerseits, dass Konsumenten beim Kauf ihres Neuwagens nicht primär ökologische Eigenschaften in den Vordergrund stellen, sondern vielmehr Nutzendimensionen wie Zuverlässigkeit, Sicherheit, Preis, Motorisierung, Prestige etc. die Kaufentscheidung dominieren. Andererseits ist die hohe Bedeutung des Kraftstoffverbrauchs für das Kaufverhalten zu hervorzuheben, der maßgeblich die Unterhaltskosten eines Pkw bestimmt. In diesem Zusammenhang ist zu erwähnen, dass der Kraftstoffverbrauch aus Verbrauchersicht das maßgebliche Kriterium zur Messung der Umweltfreundlichkeit eines Pkw darstellt. ${ }^{236}$ Folgende Abbildung zeigt dabei entscheidende Kriterien für den Neuwagenkauf in Deutschland im Jahr 1999.

235 Vgl. Liebehenschel, Th.: Ökologieorientierte Produkt- und Dienstleistungspolitik Rahmenbedingungen und Trends am Beispiel der Automobilindustrie, a. a. O., S. 171 sowie Dietz, W.: Das Handbuch für das Automobilmarketing: Strategien, Konzepte, Instrumente, Landsberg/Lech 1996, S. 338.

236 Vgl. Liebehenschel, Th.: Ökologieorientierte Produkt- und Dienstleistungspolitik Rahmenbedingungen und Trends am Beispiel der Automobilindustrie, a. a. O., S. 275. 


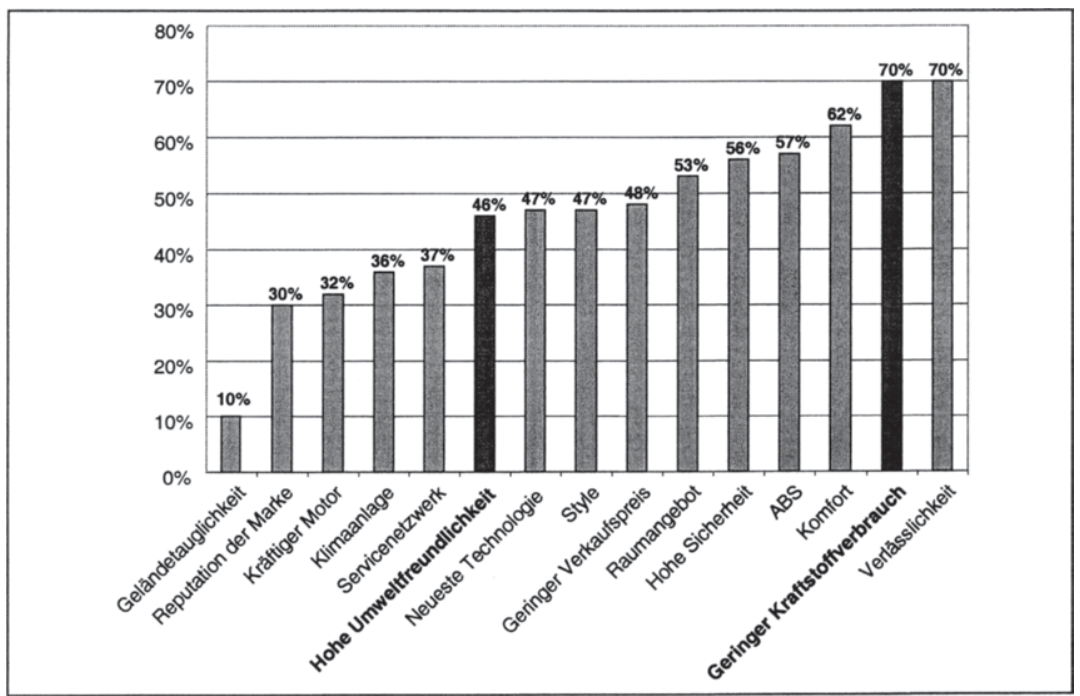

Abbildung 16: Entscheidende Kriterien für den Neuwagenkauf in Deutschland 1999

Quelle: Fokus (Hrsg.): Der Markt der Automobile: Daten, Fakten, Trends, 1999, S. 24.

Aufgrund der hohen Bedeutung des Kraftstoffverbrauchs für die Kosten eines Pkw in der Nutzungsphase erscheint es wenig verwunderlich, dass der Kraftstoffverbrauch in allen Ländermärkten eine moderate bis wichtige Rolle beim Automobilkauf spielt. Dabei hat der Kraftstoffverbrauch für die Kaufentscheidung in Schwellenländern eine etwas höhere Bedeutung als in Industrieländern, wie die folgende Abbildung zeigt. Insbesondere in der VR China, einem wichigem Wachstumsmarkt insbesondere für die Automobilindustrie, kommt dem Kraftstoffverbrauch eine besonders hohe Bedeutung zu. Es kann in diesem Kontext angenommen werden, dass sich diese nationalen Unterschiede in der Beurteilung des Kraftstoffverbrauchs beim Neuwagenkauf wenigstens zu einem großen Teil durch die lokalen Preisunterschiede bzw. dem relativen Kraftstoffpreis im Verhältnis zum Einkommen erklären lassen. 


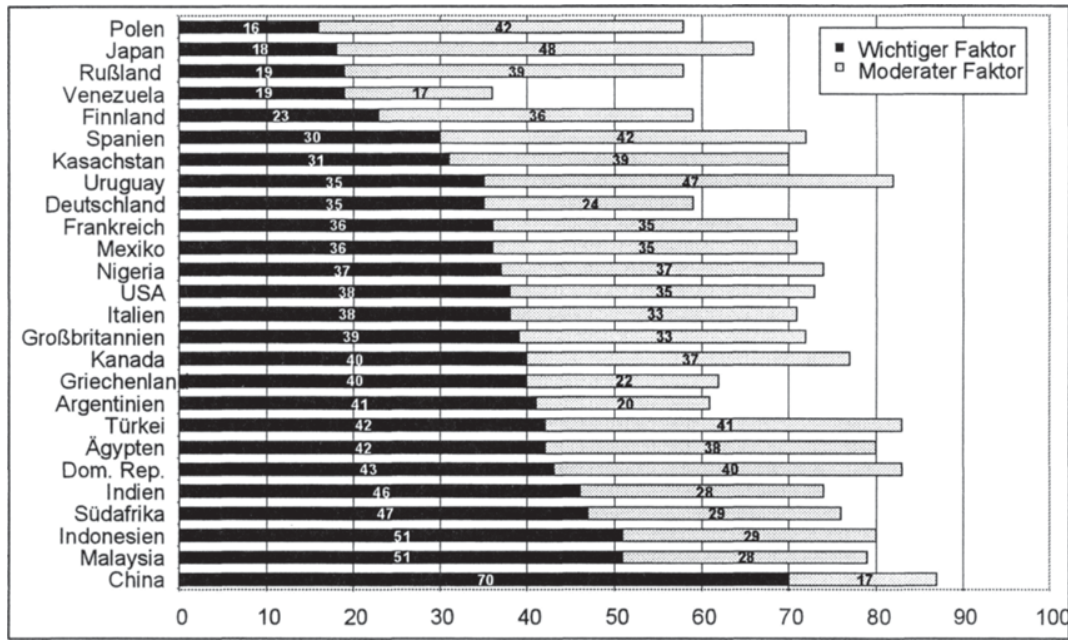

Abbildung 17: Wichtigkeit des Kraftstoffverbrauchs beim Neuwagenkauf im internationalen Vergleich

Quelle: Environics International (Hrsg.): The Environmental Monitor -Global Public Opinion on the Environment: 1999 International Report, a. a. o., S. 51.

In Abbildung 18 ist die Bereitschaft der Konsumenten abgebildet, für einen Pkw mit ökologischem Antriebskonzept einen um 10 \% höheren Preis zu entrichten, als für ein Automobil mit konventionellem Antrieb. In diesem Kontext ist darauf zu verweisen, dass die Eigenschaften des Fahrzeugs durch das ökologische Antriebskonzept nicht nachteilig beeinflusst werden. Diese Prämisse gilt für die heute verfügbaren ökologischen Fahrzeugkonzepte z. T. nur mit großen Einschränkungen. Die bekundete Bereitschaft, für ein ökologieverträgliches Automobil einen Aufpreis zu bezahlen, kann dabei insgesamt als hoch bezeichnet werden; sie liegt durchschnittlich bei ca. $40 \%$ (starke Zustimmung). ${ }^{237}$ In nahezu allen Ländern war die Mehrheit bereit, einen entsprechenden Aufschlag zu zahlen (moderate Zustimmung), allein in Japan liegt die Zustimmung knapp unter 50 \%. Dabei sind es insbesondere die besser verdienenden Konsumenten und diejenigen, die in stark belasteten Ballungsgebieten

237 Vgl. dazu auch die Ergebnisse des Trend Profils zur finanziellen Belastbarkeit der deutschen Autofahrer für umweltfreundliche Autos; dort wurde eine durchschnittliche zusätzliche Zahlungsbereitschaft für verbrauchsärmere Fahrzeuge in Höhe von 2.800 DM ermittelt. Vgl. Axel Springer Verlag (Hrsg.): Auto Bild-Trend Profil: Auto - Umwelt - Zukunft, Hamburg 1995, S. 57. 
wohnen, die eine hohe Bereitschaft aufweisen, für Umweltschutz mehr zu bezahlen. ${ }^{238}$

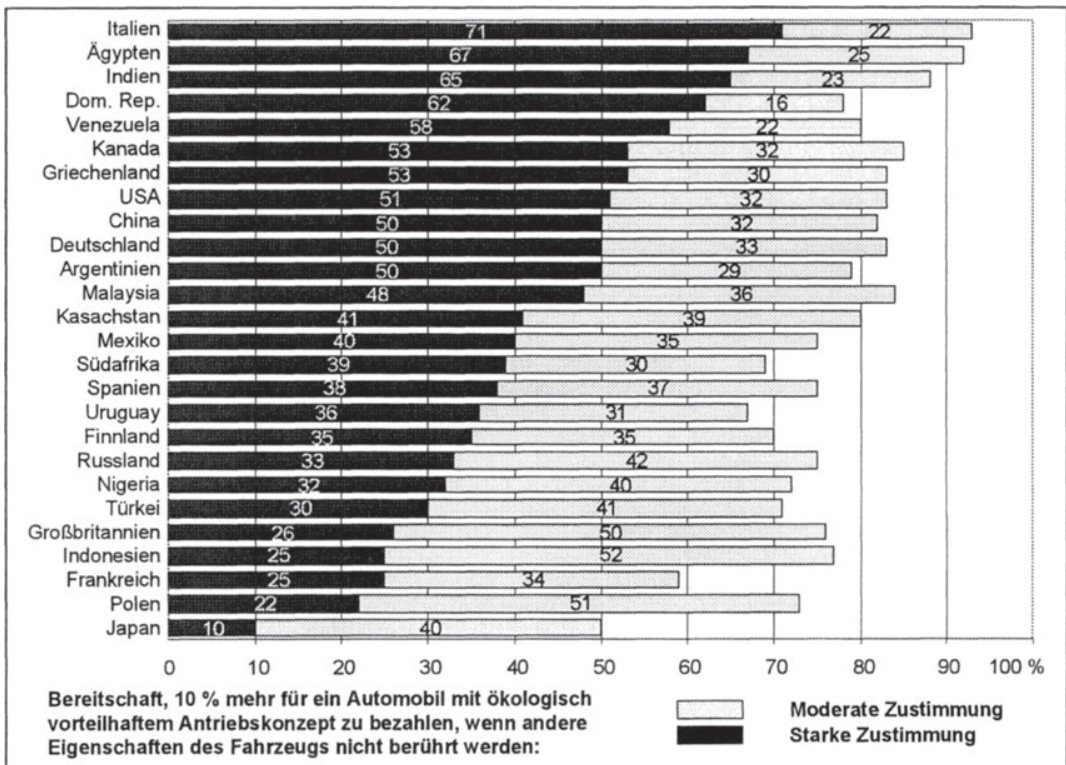

Abbildung 18: Zusätzliche Zahlungsbereitschaft für ein Automobil mit umweltverträglicherem Antriebskonzept

Quelle: Environics International (Hrsg.): The Environmental Monitor - Global Public Opinion on the Environment: 1999 International Report, a. a. o., S. 53.

In diesem Zusammenhang ist allerdings darauf zu verweisen, dass die Bekundung, ein ökologiegerechtes Fahrzeug zu kaufen, häufig in einer realen Kaufsituation nicht umgesetzt wird. ${ }^{239}$ So haben 1995 viele Konsumenten die Automobilindustrie aufgefordert, Fahrzeuge mit geringerer Motorisierung und geringerem Verbrauch zu bauen. Entgegen dieser Forderung hat sich das

238 Environics International (Hrsg.): The Environmental Monitor -Global Public Opinion on the Environment: 1999 International Report, a. a. O., S. 52.

239 In diesem Zusammenhang wird von dem sog. Divergenzproblem zwischen Umweltbewusstsein und -verhalten gesprochen. Vgl. Monhemius, K. Ch.: Divergenzen zwischen Umweltbewusstsein und Kaufverhalten - Ansatze zur Operationalisierung und empirische Ergebnisse, in: Meffert, H. (Hrsg.): Arbeitspapiere des Instituts für Marketing, Nr. 38 , Münster 1990 sowie Monhemius, K. Ch.: Umweltbewußtes Kaufverhalten von Konsumenten - Ein Beitrag zur Operationalisierung, Erklärung und Typologie des Verhaltens in der Kaufsituation, Frankfurt, Bern 1992. 
Kaufverhalten in den vergangenen Jahren aber stark in Richtung einer stärkeren Motorisierung bewegt. ${ }^{240}$

Im Folgenden sollen die generellen Unterschiede im Kaufverhalten zwischen den Automobilmärkten in der Triade und in den Schwellenländern dargestellt und auf das ökologiegerichtete Kaufverhalten bezogen werden.

- In Entwicklungsländern ist das Einkommensniveau entscheidend für die Art der Verkehrsmittelwahl. Der Erwerb eines Pkws ist dort nur einer zahlenmäßig kleinen Oberschicht möglich, die es als Statussymbol betrachtet. In den Wachstumsgesellschaften der Schwellenländer herrschen eher traditionelle Werte vor, die das Konsumentenverhalten beeinflussen. ${ }^{241} \mathrm{Da}$ das Automobil der Mehrzahl der weniger wohlhabenden Nachfrager in diesen Ländern als Familien-, Geschäfts- und Freizeitauto gleichermaßen dient, werden robuste, zuverlässige und preisgünstige Fahrzeuge verstärkt verlangt. ${ }^{242}$ Ökologische Aspekte finden in Verbindung mit dem Kraftstoffverbrauch Berücksichtigung. Vor dem Hintergrund der massiven ökologischen Belastungen insbesondere in den urbanen Ballungsräumen kann bei wohlhabenden Konsumenten ein gewisses Interesse für abgas- und verbrauchsärmere Fahrzeuge erkannt werden.

- In den Triademärkten bestimmen hingegen die spezifischen Konsumentenpräferenzen das Kaufverhalten für Automobile. Kennzeichnend für diese Gesellschaften sind Wertewandelprozesse, die einen relativen Bedeutungsverlust traditioneller und materieller Wertemuster zugunsten von postmateriellen und hedonistischen Grundorientierungen zur Folge haben. ${ }^{243}$ Das Automobil dient demzufolge immer weniger als Prestigeobjekt und Statussymbol, sondern in immer stärkerem $\mathrm{Maße}$ als ein Mittel zum Ausdruck des eigenen Lebensstils. ${ }^{244}$ In diesem Zusammenhang ist auch der Trend zu Cabrios, Roadster und Off-road-Fahrzeugen, den sog. SportyUtility-Vehicles (SUV) ${ }^{245}$, zu sehen ${ }^{246}$, die z. T. aus ökologischer Sicht allein auf-

240 Vgl. Llebehenschel, Th.: Ökologieorientierte Produkt- und Dienstleistungspolitik - Rahmenbedingungen und Trends am Beispiel der Automobilindustrie, a. a. O., S. $276 \mathrm{ff}$.

Diese Werte können mit Schlagwörtern wie Arbeitsethos, Fleiß, Sparsamkeit, dem Willen, ein gutes Mitglied der Gesellschaft zu sein, Besitz schaffen und Bewahren etc. beschrieben werden. Vgl. Schmitt, A.: Die psychologischen Rahmenbedingungen des Automobilmarketing, in: Hünerberg, R. et al. (Hrsg.): Internationales Automobilmarketing: Wettbewerbsvorteile durch marktorientierte Unternehmensführung, Wiesbaden 1995, S. 64.

Dies geht auf das Interview mit Herrn RaKowSKI von VoLKSWAGEN zurück.

Vgl. hierzu und im Folgenden Schmitt, A.: Die psychologischen Rahmenbedingungen des Automobilmarketing, a. a. O., S. $61 \cdot 79$.

Dies führt zu einer zunehmenden Individualisierung der Nachfrage. Die hierarchische Gliederung der Mărkte nach Preis, Hubraumklassen und Leistung wird heute zunehmend durch eine horizontale Differenzierung nach individuellen Nutzungsweisen und stilistischen Ausgestaltungen abgelöst bzw. ergänzt. Vgl. Dlez, W.: Plattform-Strategien: Irrweg oder Königsweg in der Modellpolitik, a. a. O., S. 5 sowie Clef, U.: Mehrmarkenstrategie für die Pole-Position, in: asw, Sondernummer Oktober 1999, S. 72.

Insbesondere in den USA sind Sporty-Utility-Vehicles (SUV), Minivans und Pick-ups (zusammen als Light Trucks bezeichnet) besonders erfolgreich. Von den in den USA 1999 verkauften Automobilen sind $49 \%$ Light Trucks. Vgl. VDA (Hrsg.): Auto 2000: Jahresbericht des Verbands der Automobilindustrie e. V., a. a. O., S. 17 f. Auch in Europa gewinnt 
grund des vergleichsweise hohen Kraftstoffverbrauchs kritisch zu beurteilen sind. ${ }^{247}$

- Im US-amerikanischen Automobilmarkt lässt sich bereits ein Abebben des hedonistischen Konsums beobachten. Rationale Kaufargumente gewinnen insbesondere in der Oberklasse wieder an Gewicht. ${ }^{248}$ Ökologiebezogene Kriterien finden dabei durchaus Eingang in die Kaufentscheidung.

- In den westeuropäischen Gesellschaften können seit den 70er Jahren zwei Entwicklungslinien ausgemacht werden: Anomie und Hedonismus. ${ }^{249}$ Das Automobil dient demzufolge immer weniger als Prestigeobjekt und Statussymbol, sondern in immer stärkerem Maße als ein Mittel zum Ausdruck des eigenen Lebensstils. Insbesondere in Deutschland, zunehmend aber auch in anderen europäischen Ländern, erwarten die Konsumenten eine ökologische Orientierung der Hersteller. ${ }^{250}$

- Der Mangel an Individualisierungsbedürfnissen in Japan und in Südostasien kann schließlich als gravierendster Unterschied zu den westlichen Märkten charakterisiert werden. ${ }^{251}$ Ökologische Kriterien werden beim Automobilkauf hier

diese Kategorie im Zusammenhang mit der fortschreitenden Segmentierung zunehmend an Bedeutung. Vgl. Clef, U.: Mehrmarkenstrategie für die Pole-Position, in: asw, Sondernummer Oktober 1999, S. 73.

246 Das hohe Sicherheitsgefühl, der Raumkomfort und die unterschiedlichen Einsatzmöglichkeiten dieser Fahrzeuge sowie das Bedürf́nis der urbanen Bevölkerung, wieder Năhe zur Natur zu verspüren, haben zum Erfolg dieser Fahrzeugkategorie ebenso beigetragen wie der in den USA vergleichsweise preiswerte Kraftstoff.

247 So bezeichnet der Verkehrsclub Deutschland (VCD) gelăndegängige Jeeps, die aus Gründen des Lifestyle gekauft werden, als Öko-Flop. Vgl. VCD (Hrsg.): Zehn Jahre AutoUmweltliste des VCD: Eine Erfolgsbilanz, Bonn 2000, http://www.vcd.org/themen/ auli10.html [31.08.2000].

248 Weniger reines Prestigedenken als die Orientierung am Preis-Leistungsverhăltnis bzw. am „Wert" des Automobils prăgt das Kaufverhalten. Dabei avancieren Raumkomfort, Solidität, Qualität und Sicherheit zu dominanten Kaufkriterien.

249 Unter Anomie kann der Bedeutungsverlust verbindlicher gesellschaftsübergreifender Wertemuster und Normen verstanden werden, der mit einer wachsenden Skepsis gegenüber staatlichen Institutionen und Einrichtungen einhergeht. Die Ablehnung der blinden Fortschrittsgläubigkeit und zunehmendes ökologisches Bewusstsein lassen sich hierunter subsumieren. Das Anwachsen von Individualismus und Genusswerten kann im Gegensatz dazu als Hedonismus bezeichnet werden. Vgl. Schmitt, A.: Die psychologischen Rahmenbedingungen des Automobilmarketing, a. a. O., S. 64.

250 Schließlich ist aber zu beachten, dass innerhalb Europas durchaus größere Unterschiede bezüglich der Einstellungen zum Automobil festgestellt werden kőnnen. Vgl. dazu bspw. Heise, G.: Internationale Marktsegmentierung im Automobilmarketing, Wiesbaden 1997 und Kleebinder, H. P.: Das öffentliche Meinungsbild zum Themenfeld Mobilităt im europäischen Vergleich, Forschungsbericht Prof. Dr. H. Weinold, St. Gallen 1994.

251 Neben einem hohen Sozialprestige richten sich die Erwartungen der Kunden an das Raumangebot und umfassende Ausstattungspakete. Die Technik muss hingegen allein eine problemlose und zuverlässige Nutzung im Alltag gewährleisten. Vor dem Hintergrund der einschneidenden Wirtschaftskrise in Asien in 1997 gewinnen aber wieder Bescheidenheit und Sparsamkeit an Bedeutung beim Autokauf. So stieg der Absatz von Kraftwagen nicht, wăhrend sich insbesondere Kleinstwagen mit einer Steigerung von $31 \%$ in 
im Vergleich zu den anderen Triademärkten am wenigsten in Kaufentscheidungen einbezogen.

Es lässt sich an dieser Stelle feststellen, dass das ökologiebezogene Kaufverhalten in den Automobilmärkten generell als heterogen einzustufen ist. Dies drücken auch die Marktanteile der unterschiedlichen Fahrzeugklassen in den Triademärkten aus. ${ }^{252}$ Dabei kann davon ausgegangen werden, dass die tatsächliche Zahlungsbereitschaft für ökologische Produktangebote nicht besonders hoch ausfällt. Besonders ökologisch ausgerichtete Fahrzeugkonzepte, die vom Konsumenten einen hohen Mehrpreis fordern, werden sich somit vorerst nur in der Nische durchsetzen können. Sollen die ökologischen Nischen in unterschiedlichen Ländermärkten mit ökologischen Produktalternativen bearbeitet werden, fällt der Koordinationsbedarf aufgrund anbieterbezogener Rückkopplungen umso höher aus, je unterschiedlicher die Konsumentenanforderungen an ein derartiges Auto sind.

Je unterschiedlicher die monetär alimentierten ökologischen Konsumentenanforderungen in den einzelnen Ländermärkten ausfallen, desto höher ist der internationale Koordinationsbedarf aufgrund anbieterbezogener Rückkopplungen.

\subsection{Umsystemabhängigkeit}

Im Zusammenhang mit der Globalisierung lässt sich eine zunehmende ökonomische, gesellschaftliche und technologische Kopplung zwischen einzelnen Ländern beobachten. Dem technischen Fortschritt in der Informations- und Telekommunikationstechnik, der zu einer steigenden Geschwindigkeit und Reichweite sowie einem wachsenden Umfang der international verbreiteten Informationen geführt hat, kommt in diesem Zusammenhang eine wichtige Rolle $\mathrm{zu}^{253}$

1999 wesentlich besser verkauften. Vgl. VDA (Hrsg.): Auto 2000: Jahresbericht des Verbands der Automobilindustrie e. V., a. a. O., S. 18.

Vgl. zu den internationalen Marktanteilen unterschiedlicher Fahrzeugklassen Heise, G.: Internationale Marktsegmentierung im Automobilmarketing, Wiesbaden 1997, S. 65.

Zur Rolle der Medien im Kontext des Umweltschutzes vgl. Pietschmann, M.: Medienstrategien aus der Sicht einer Umweltschutzorganisation, in: Fichter, K.; Schneidewind, U. (Hrsg.): Umweltschutz im globalen Wettbewerb: Neue Spielregeln für das grenzenlose Unternehmen, Berlin et al. 2000, S. 163 - 170 sowie ZerfaB, A.: Machen Medien Mărkte? - Zur Rolle der Medien im ökologischen Wettbewerb, in Fichter, K.; Schneidewind, U. (Hrsg.): Umweltschutz im globalen Wettbewerb: Neue Spielregeln für das grenzenlose Unternehmen, Berlin et al. 2000, S. $171-175$. 


\subsection{1 Ökologische Betroffenheit durch internationale öffentliche Anspruchsgruppen}

Der Einfluss der Medien und des Internet ${ }^{254}$ kann im Zusammenhang mit ökologiebezogenen Fragestellungen wie folgt beschrieben werden:

"In our CNN world, there is no hiding place. The World Wide Web and new media technologies have increased transparency, given stakeholders instant access to information, and are changing business/customer relationships fundamentally. “255

Aufgrund dieser zunehmenden internationalen Vernetzung kann das ökologiebezogene Verhalten eines Unternehmens in einem Ländermarkt somit durch die Medien auch länderübergreifend bekannt werden und Auswirkungen auf den Geschäftserfolg des Gesamtunternehmens ausüben. Somit können insbesondere Massenmedien den Zugang zu einer breiten Öffentlichkeit herstellen und die Akzeptanz und Legitimität von Unternehmen auch über Landesgrenzen hinweg beeinträchtigen, wie folgender Auszug aus dem Internet von CORPWATCH, einer ökologischen Anspruchsgruppe, die u. a. auf irreführende ökologiegerichtete Werbung aufmerksam machen möchte ${ }^{256}$, zeigt.

254 So ist in einem Diskussionsforum von SHELL bsw. folgendes zu lesen: "The problems generated by the irresponsible activities of Your Company in Niger Delta, I mean the crimes committed by Your employees against nature and local population that has no real rights and is not able to defend herself against Your powerful company cannot be accepted by responsible consumers of oil products all over the world. .. You should be aware of the fact that Your practices have luckily been made public by some widespread mass media and that western consumers would not like to make themselves to accomplices buying Shell products any more. Just be aware again that it'll come times when democratic governments will rule in Nigeria. They will punish the heaviest of the crimes against indigenous population and environment which You're still committing without any sense of conscience." http://134.146.1.137/forums/Thread.cfm?CFApp=2\&Thread ID =17\&mc=9\# Message708 [12.08.2000].

255 Mark Moody-Stuart: Putting Principles Into Practice: The Ethical Challenge to Global Business, http://www.shell.com/library/speech/, Sao Paulo 2000 [26.08.2000]. MoodYSTUART ist Vorsitzender des Committee of Managing Directors (CMD) der Royal Dutch/Shell Gruppe und der Shell Transport and Trading Company. Die Rede wurde auf The World Congress of the International Society of Business, Economics and Ethics in Sao Paulo, Brasilien, am 19.07.2000 gehalten.

"CorpWatch gives out bimonthly Greenwash Awards to corporations that put more money, time and energy into slick PR campaigns aimed at promoting their eco-friendly images, than they do to actually protecting the environment. Nominations for these Awards come from you, when you send in a print, electronic or television ad to us, along with the reasons why you think it deserves an Award." Vgl. http://www.corpwatch.org/greenwash. 


\section{Ford Motor Company}

FORDenies global warming, FORDelays solutions, and FORDupes the public

In honor of this Award, Ford Chairman William Clay Ford, Jr. will receive a used bicycle for commuting to work.

This Prize is Awarded for:

Fooling Children into Believing that Ford is Environmentally Friendly (while making the most polluting cars of any major auto maker)

Offering Outrageous Greenwash advertising (attempting to preserve and expand its markets by posing as a friend of the environment)

Raving about its Environmental Programs (while lobbying against air standards and downplaying the threat of global warming)

Daring to Honor Heroes for the Planet (when Ford is a major Corporate Climate Culprit)

\section{HAS FORD DRIVEN YOU CRAZY LATELY?}

If you are offended by a company spending millions on promoting an environmental image while simultaneously doing business that destroys the planet, Ford's latest programs might drive you nuts.

On April 15th Ford sponsored the Heroes for the Planet concert in San Francisco. From where does Ford derive the moral authority to Associate itself with environmental heroes? Nowhere. But it has the financial clout to pull it off.

The Heroes for the Planet march, concert and media extravaganza was the grand kickoff for one of the most expensive and intensive environmental advertising campaigns the world has ever seen. Ford recently announced that all corporate brand advertising will have an environmental theme. It expects to spend as much on this greenwashing as they do to roll out a new line of cars.

To do so Ford has bought the services of one of the best anti-environmental PR firms in the business, Hill and Knowlton. John Stauber, editor of PR Watch, a group which tracks the public relations industry says the company is notorious. "Hill and Knowlton are the propagandists for the worst polluting corporations on the planet, and proud of it."

Ford has also bought nearly forty percent of Time magazine's special Earth Day 2000 edition. This exclusive sponsorship of Time's "How to Save the Earth and the Heroes for the Planet Who Are Making It Happen" reads more like one big greenwash advertisement from Ford than a serious piece of journalism.

Even worse, Ford is the exclusive advertiser in two special issues of TIME FOR KIDS, reaching 2.8 million students in elementary schools in the U.S. alone. Ford's ads surround the special edition announcing the nine winners of the TIME FOR KIDS Environmental Challenge. (Anyone wishing to bet that anti-car, public transport activists were even considered, please contact us at BrooklynBridgeSales.com) 
Ford has the money to make us listen to their rhetorlc. But let's take a look at their record. We'll focus on this Earth Day's theme: Global Warming.

Emissions from cars and light trucks account for about one-quarter of ALL carbon emissions (by far the largest greenhouse gas). And Ford is the world's number two auto maker, so it is a major player. How have they done so far? According to the Union of Concerned Scientists:

- Ford's cars are the worst carbon emitters of any major auto maker.

- Ford's light trucks (SUVs) are the worst carbon emitters of any major auto maker.

- Ford is the second worst polluter overall of any auto maker.*

- Ford's fuel efficiency trend is the worst of any auto maker.

- In the 10 years after global warming first became a public concern, average vehicle emissions for Ford INCREASED by $7.4 \%$, the most of the three major automakers.

- Between 1990 and 1998, emissions from new Ford cars rose from about 21 million tons of $\mathrm{CO}_{2}$ to about 27 million tons, just a hair below GM, the larger company.

Ford still downplays the threat of global warming. On its website, it trots out the tired argument that developing countries must be part of the solution. Nowhere does Ford note that cars and light trucks in the U.S. alone account for more $\mathrm{CO}_{2}$ emissions than all but four countries in the world (the U.S., Russia, China and Japan). Meanwhile Ford is riding the wave of globalizationexpanding its markets in the developing countries that it says should be part of the solution. And of course, it is promoting itself as an environmental hero in these very nations.

Ford tells us it has seen the green light. Not that it's done anything wrong, mind you, but it's going to fix things anyway. It will build fuel-efficient, hybrid and electric cars.

We hope so. But should we believe it, when company officials fail to admit to obvious past and present responsibility? We at the Corporate Watch Greenwash Awards are optimists, but with corporate environmentalism, seeing is believing.

And so far, all we see is greenwash.

*(DaimlerChrysler is ranked slightly worse, due to a higher proportion of SUV's in their fleet. Isuzu, which makes only light trucks and not cars, is not included in this ranking.)

Abbildung 19: Corporate Watch: Greenwash Sweepstakes Award 2000

Quelle: http://www.corpwatch.org/trac/climate/gwiord.html $[07.11$. 2000]

Öffentliche Anspruchsgruppen Sie spielen eine zentrale Rolle für das Entstehen öffentlichkeitsbezogener Rückkopplungen zwischen Ländermärkten. Insbesondere öffentliche ökologische Anspruchsgruppen versuchen diese öffentlichkeitsbezogenen Rückkopplungen zu nutzen. In jüngster Zeit haben sich einige dieser Gruppen auf internationaler Ebene formiert, um ihre Forderungen erfolgreicher durchsetzen zu können. Verschiedene international organisierte Nicht-Regierungsorganisationen (NGOs) ${ }^{257}$, wie bspw. GreenPEACE ${ }^{258}$, sind

257 NGOs können als Organisationen verstanden werden, die als primäres Ziel die Förderung von sozialen und / oder ökologischen Belangen haben und nicht die Sicherung der ökonomischen Macht in Märkten oder der Politik als Hauptaufgabe verfolgen. Vgl. Bendell, J.: Jenseits der Selbstregulierung von Umweltmanagement: Einige Grundgedanken zur wachsenden Bedeutung von Business-NGO-Partnerschaften, in: Fichter, K.; Schneidewind, U. (Hrsg.): Umweltschutz im globalen Wettbewerb: Neue Spielregeln für das grenzenlose Unternehmen, Berlin et al. 2000, S. 153.

GREENPEACE beschreibt sich wie folgt: „Greenpeace is a global environmental campaigning organisation. ... The Greenpeace organisation consists of Greenpeace International (Stichting Greenpeace Council) in Amsterdam and Greenpeace offices around the world. 
dabei zu ernstzunehmenden Kräften und einem Gegengewicht zu international tätigen Unternehmen ${ }^{259}$ geworden. ${ }^{260}$ NGOs greifen in diesem Kontext auf ähnliche Instrumente zur Durchsetzung ihrer Forderungen zurück, wie lokale öffentliche Anspruchsgruppen, sie nutzen aber zusätzlich ihre Präsenz in anderen Ländermärkten ${ }^{261}$ und insbesondere die Medien. ${ }^{262}$

Greenpeace currently has a presence in 40 countries. " http://www.greenpeace.org/report98 lindex.html [13.08.1999].

International tätigen Großunternehmen wird dabei aufgrund ihrer Größe und Macht eine hohe Verantwortung für die ökologische Umwelt zugesprochen. Diese Unternehmen mit inren rund 449.000 weltweiten Beteiligungen bestimmen einen Großteil der industriellen Tätigkeiten entlang der Wertkette, von der Extraktion und Verarbeitung von Ressourcen bis zur Herstellung und dem Verkauf von Produkten und Dienstleistungen. Vor diesem Hintergrund werden international tätige Unternehmen u. a. für insgesamt $50 \%$ der den Treibhauseffekt auslösenden Spurengase und einen Großteil der Ausbeutung natürlicher Ressourcen verantwortlich gemacht. Vgl. hierzu insbesondere Karliner, J.: The Corporate Planet: Ecology and Politics in the Age of Globalization, 1997 zitiert nach: http://www. corpwatch.org/trac/feature/planet/ fact 1.html [07.11.00] sowie Pausenberger, E.: Globalisierung der Wirtschaft und die Machteinbußen des Nationalstaats, a. a. O., S. $80 \mathrm{ff}$. und United Nations (Hrsg.): Activities of the Transnational Corporations and Management Divisions and its Joint Units, a. a. O., S. 19.

260 Ausdruck der hohen Bedeutung der NGOs ist die Tatsache, dass sie gemeinsam weltweit 1,1 Billionen US \$ umsetzen, mehr finanzielle Hilfe leisten als die Weltbank und mehr als 19 Millionen Menschen beschäftigen. Vgl. Bendell, J.: Jenseits der Selbstregulierung von Umweltmanagement: Einige Grundgedanken zur wachsenden Bedeutung von BusinessNGO-Partnerschaften, in: Fichter, K.; Schneidewind, U. (Hrsg.): Umweltschutz im globalen Wettbewerb: Neue Spielregeln für das grenzenlose Unternehmen, Berlin et al. 2000, S. 153.

Auch wenn bspw. VolKsWAGEN selbst noch nicht im Mittelpunkt einer in der Öffentlichkeit breit wirkenden Kampagne durch GREENPEACE gestanden hat, kann dennoch von einer hohen Sensibilität des Automobilherstellers gegenüber derartigen Aktionen ausgegangen werden. Vgl. Volkswagen (Hrsg.): Jahresbericht des produktionsbezogenen Umweltschutzes 1999/2000, Wolfsburg 2000, S. 1.

Neben der Mobilisierung öffentlichen Drucks auf Unternehmen und Regierungen nehmen NGOs auch im Rahmen der Vereinten Nationen an der Gestaltung und Überwachung von multinationalen Umweltschutzabkommen teil. Dabei beschreibt BODE, Executive Director of Greenpeace International, den Einfluss von NGOs in einer Rede vor dem World Economic Forum in Davos wie folgt: "In many countries, we now enjoy relatively clean air, water and food. Much of the progress in developing decent legislative standards has been achieved as a direct result of NGO pressure. The same is true at the international level. Treaties to protect the global commons from nuclear testing, ocean dumping, ozone depletion and overfishing would not have been negotiated as fast, or as comprehensively, without the influence of the international environmental NGOs." Vgl. Bode, Th.: In search of Robin Hood: NGO's foes or partners in the global agenda, Davos 2000, zu finden bei http://www.greenpeace.de/ [12.11.00]. 
Die fortschreitende länderübergreifende Kopplung von Märkten und das Auftreten international tätiger gesellschaftlicher Anspruchsgruppen führt zu sog. Global-Village-Effekten ${ }^{263}$, die den Koordinationsbedarf eines international tätigen Unternehmens erhöhen können.

Der internationale ökologiebezogene Koordinationsbedarf steigt mit zunehmender medialer Verbundenheit zwischen unterschiedlichen Ländermärkten und dem internationalen Auftreten öffentlicher Anspruchsgruppen aufgrund öffentlichkeitsbezogener Rückkopplungen.

\subsection{2 Ökologische Betroffenheit durch internationale institutionelle und politische Anspruchsgruppen}

Neben der Betroffenheit durch lokale umweltrechtliche Bestimmungen, sind Unternehmen in regionalen Integrationsräumen wie der EU oder der NAFTA auch von übernationalen rechtlichen Instanzen betroffen. Weiterhin kann durch internationale Vertragskonferenzen wie dem Montrealer Protokoll oder der Alpenkonvention durchaus eine ökologische Betroffenheit ausgelöst werden.

Im Kontext dieser Arbeit seien insbesondere die 1994 in Kraft getretene Klimakonvention und das seit 1997 in Verhandlung befindliche Kyoto-Protokoll ${ }^{264}$ angesprochen. ${ }^{265}$ Obwohl das Kyoto-Protokoll noch nicht ratifiziert wurde, haben sich einige nationale bzw. regionale Parlamente dem internationalen Klimaschutz verpflichtet und $\mathrm{CO}_{2}$-Minderungsziele festgesetzt. In diesem Zusam-

263 Der Begriff der Global-Village-Effekte spielt auf das Metapher von MCLUHAN von der Welt als globales Dorf an und bringt zum Ausdruck, dass die Bedingungen innerhalb einzelner Staaten und Staatengruppen nicht mehr unabhängig voneinander sind, sondern sich aufgrund der zunehmenden informationellen und sozio-ökonomischen Vernetzung wechselseitig beeinflussen. Vgl. Brodel, D.: Internationales Umweltmanagement: Gestaltungsfelder - Determinanten - Ausprägungen, a. a. O., S. $384 \mathrm{ff}$.

Mit dem Kyoto-Protokoll sollen erstmals verbindliche Emissionsziele für die Zielperiode 2008-2012 für die Industrie- und Transformationsländer festsetzt werden. Die Industriestaaten sollen insgesamt den Ausstoß von sechs klimaschädlichen Gasen bis zur Zielperiode 2008 - 2012 um 5,2 \% gegenüber 1990 verringern, aber in unterschiedlichem Maße: etwa die EU und einige andere europäische Länder um $8 \%$, die USA um $7 \%$, Japan um $6 \%$, Russland um $0 \%$. Einige Industrieländer (Norwegen, Island, Australien) dürfen ihre Emissionen sogar noch steigern. Die Entwicklungsländer werden von der Reduktionsverpflichtung - zumindest in der ersten Zielperiode - ausgenommen. Vgl. Bals, Ch.; Kier, G.; Rück, A.; Treber, M.: Klimaschutz vor Buenos Aires: werden die KyotoSchlupflöcher enger?, Briefing Paper, GERMANWATCH, 1998. Das Protokoll tritt in Kraft, wenn es von mindestens 55 Staaten ratifiziert worden ist und dadurch gleichzeitig mindestens $55 \%$ der 1990 von Industrieländern ausgestoßenen $\mathrm{CO}_{2}$-Emissionen abgedeckt sind. Vgl. http://www.uno.de/presse/1999/unic154.htm.

Vgl. IISD, UNEP: Environment and Trade - A Handbook, Winnipeg 2000, S. 11 - 17. 
menhang hat sich die Automobilindustrie 1995 der deutschen Bundesregierung gegenüber verpflichtet, ihren Teil dazu beizutragen, dass die $\mathrm{CO}_{2}$-Emissionen der Neufahrzeuge durchschnittlich bis zum Jahr 2005 um mindestens $25 \%$ verringert werden können. ${ }^{266}$ Auf Ebene der europäischen Automobilindustrie (ACEA) liegt seit Sommer 1998 eine ähnliche Selbstverpflichtung vor. Die durchschnittlichen $\mathrm{CO}_{2}$-Emissionen der innerhalb Europas verkauften Neufahrzeuge sollen bis 2008 nur noch $140 \mathrm{~g} / \mathrm{km}$ betragen. Dies entspricht einem Durchschnittsverbrauch von $6 \mathrm{~V} / 100 \mathrm{~km}$ für Benziner und 5,3 V/100 km für Dieselfahrzeuge. Die Europäische Kommission hat in 1999 angekündigt, falls die Automobilindustrie in den nächsten Jahren keine Fortschritte verzeichne, gesetzliche Maßnahmen in 2003 zu erlassen, ${ }^{267}$ die dann auch in nationales Recht umzusetzen wären.

Auf internationaler Ebene können schließlich institutionelle Anspruchsgruppen wie die Vereinten Nationen und die OECD enwähnt werden. So verweisen bspw. die Vereinten Nationen darauf, dass international tätigen Großunternehmen im Umweltschutz eine hohe Verantwortung zukomme. ${ }^{268}$ Insbesondere global tätige Automobilhersteller stehen dabei oft im Mittelpunkt der Kritik dieser Anspruchsgruppen. Diese stellen zumeist unverbindliche Leitlinien und Handlungsrichtlinien für Unternehmen auf, die Unternehmen als Leitlinie dienen sollen, den Anforderungen an eine dem Sustainable Development entsprechende Wirtschaftsweise gerecht zu werden. ${ }^{269}$ In diesem Kontext kann

266 Vgl. VDA (Hrsg.): Auto 2000: Jahresbericht des Verbands der Automobilindustrie e. V., a. a. O., S. 138. In den USA gibt es ebenfalls Verbrauchsvorgaben für die Modellflotte einer Automarke. So sind die CAFE (Corporate Average Fuel Economy) Anforderungen für Light Trucks bei $11,5 \mathrm{~V} / 100 \mathrm{~km}$ und für PKW bei $8,6 \mathrm{l} / 100 \mathrm{~km}$. Bei einer $10 \%$ Abweichung von der in Amerika gültigen mpg-Vorgabe werden 5,5 US-\$ Strafe pro Fahrzeug als Strafgebühr erhoben. Vgl. o. V.: Flottenverbrauch von morgen - eine Fata Morgana?, in: ps-report, Nr. 28, 2000.

Vgl. European Commission: Commission Recommendation of 5 Feburary 1999 on the reduction of $\mathrm{CO}_{2}$ emissions from passenger cars, in Official Journal of the European Communities, 13.03.1999.

268 "Transnational corporations were frequently the repositories of scarce technical skills for the preservation and enhancement of the environment, that they conducted activities in sectors that had an impact on the environment and, in that context, efforts needed to be encouraged and mobilized to protect and enhance the environment in all countries." United Nations (Hrsg.): Activities of the Transnational Corporations and Management Divisions and its Joint Units, a. a. O., S. 6.

Eine Auflistung relevanter Verhaltenskodices findet sich u. a. bei: Olson, S. S.: International Environmental Standards Handbook, a. a. O., S. 147 - 160. Anfang der 90er Jahre richteten sich nur ungefähr $10 \%$ der Unternehmen nach derartigen Verhaltenskodices, allerdings wird eine steigende Bedeutung konstatiert. Vgl. United Natlons (Hrsg.): Environmental Management in Transnational Corporations: Report on the Benchmark Corporate Environmental Survey, a. a. O., S. $41 \mathrm{ff}$. 
bspw. die Agenda 21 erwähnt werden, die in Kapitel 30 in Leitlinie 22 von der Privatwirtschaft einschließlich transnationaler Unternehmen fordert, eine auf eine nachhaltige Entwicklung ausgerichtete weltweite Unternehmenspolitik zu erarbeiten. ${ }^{270}$

Dabei sind diese Verhaltenskodices rechtlich nicht bindend, sondern eher als ethisch-normative Regeln zu verstehen. ${ }^{271}$ Ähnliche Verhaltenskodices wurden auch von Institutionen der Wirtschaft, wie der internationalen Handelskammer (ICC) oder dem World Business Council for Sustainable Development (WBCSD) in Eigeninitiative der Wirtschaft aufgestellt. ${ }^{272}$

Aufgrund einer zunehmenden internationalen institutionellen Verknüpfung steigt der intemationale ökologiebezogene Koordinationsbedarf aufgrund institutioneller Rückkopplungen.

270 Weiterhin sollen Tochtergesellschaften in Entwicklungsländern ohne zusätzliche externe Aufwendungen umweltverträgliche Technologien zur Verfügung gestellt werden. Dabei sollen die überseeischen Tochtergesellschaften Verfahren dahingehend abändern, dass sie den ökologischen Gegebenheiten vor Ort Rechnung tragen. Schließlich soll mit den Kommunalbehörden, den nationalen Regierungen und internationalen Organisationen Erfahrungen ausgetauscht werden. Vgl. Bundesministerium für Umwelt, Naturschutz und Reaktorsicherhelt (Hrsg.): Konferenz der Vereinten Nationen für Umwelt und Entwicklung im Juni 1992 in Rio de Janeiro - Dokumente - Agenda 21, Kapitel 30.22, Bonn 1992.

271 Viele der international tätigen Großunternehmen erkennen dabei ihre hohe Verantwortung im Umweltschutz mittlerweile an. In diesem Zusammenhang schließen sie sich bspw. der GLOBAL COMPACT Initiative der Vereinten Nationen freiwillig an. Damit akzeptieren sie als "world business leaders" die Einhaltung von 9 Leitlinien in Bezug auf Menschen- und Arbeitnehmerrechte sowie den Umweltschutz. Vgl. United Nations (Hrsg.): A Compact for the New Century, http://www.unglobalcompact.org/GC/unweb.nst/webprintview/thenine. htm [29.11.2000]. DaimlerChrysler, Fiat, General MOtORS, TOYOTA, VolKSWAGEN und VoLvo sind explizit als Unterstützer dieser Kampagne mit aufgeführt.

272 Die Charta des ICC für eine langfristig tragfähige Entwicklung soll die Unternehmen unterstützen, ihr Engagement für die Umwelt umfassend in die Praxis umzusetzen. Der 3. Grundsatz fordert bezüglich des internationalen ökologiebezogenen Unternehmensverhaltens: "Ausgehend von den gesetzlichen Regelungen die Unternehmenspolitiken, Programme und Umweltschutzmaßnahmen entsprechend dem jeweils neuesten Stand von Wissenschaft und Technik sowie unter Berücksichtigung der Verbraucherbedürfnisse und der Erwartung der Offentlichkeit weiterzuentwickeln und international die gleichen Umweltkriterien anzuwenden. " ICC (Hrsg.): Charta für eine langfristig tragfähige Entwicklung Grundsätze des Umweltmanagement, 1991. "The WBCSD was formed in January 1995 through a merger between the Business Council for Sustainable Development (BCSD) in Geneva and the World Industry Council for the Environment (WICE), an International Chamber of Commerce (ICC) initiative, in Paris." http://www.wbcsd.org/whatis.htm. 


\subsection{3 Ökologische Betroffenheit durch internationale Marktteilnehmer}

Einige Autoren erklären die Finanzmärkte als global am weitesten entwickelte Märkte zu möglichen Triebkräften für eine ökologische Entwicklung. ${ }^{273}$ Verschiedene Investoren haben begonnen, ökologische Aspekte im Investmentgeschäft zu berücksichtigen. ${ }^{274}$ Dem liegt auch die Überlegung zugrunde, dass nachhaltig geführte und operierende Unternehmen ein höheres Wertsteigerungspotenzial aufweisen als vergleichbare, herkömmlich geführte Unternehmen. ${ }^{275}$ Das steigende Interesse von Kapitalanlegern an Unternehmen, die sich ökologisch proaktiv verhalten bzw. Lösungen für aktuelle und zukünftige ökologische Probleme bieten, zeigt sich in der Entwicklung des Volumens für nachhaltige Kapitalanlagen. ${ }^{276}$ Versicherungen und Banken berücksichtigen bei der Bestimmung von Versicherungsbeiträgen und Kreditbedingungen auch ökologische Risiken bei Produktion und Produkten.

Um für den Anleger, aber auch für Banken und Versicherungen die Chancen und Risiken eines Unternehmens aus ökologischen Gesichtspunkten transparent werden zu lassen und diejenigen Unternehmen zu identifizieren, die bezüglich der Umsetzung einer ökologisch nachhaltigen Unternehmensstrategie führend sind, werden von sog. Ratingagenturen ökologische Unternehmensbewertungen durchgeführt. Diese ökologischen Bewertungen beinhalten eine Analyse der ökologischen Betroffenheit durch unterschiedliche ökologische Anspruchs-

273 So werden international tätige Unternehmen, die als Öko-Leader gelten, durch Steuerungsimpulse aus den Finanzmärkten in ihrer ökologischen Ausrichtung gestärkt. Vgl. Flatz, A.: Der Dow Jones Sustainability Index: Eine neue Kraft auf den globalen Finanzmärkten, in: Fichter, K.; Schneidewind, U. (Hrsg.): Umweltschutz im globalen Wettbewerb: Neue Spielregeln für das grenzenlose Unternehmen, Berlin et al. 2000, S. $111 \mathrm{f}$.

Vgl. Schaltegger, S.; Figge, F.: Umweltmanagement und Shareholder Value in den Kriterien des Unternehmenserfolgs, in: Koslowski, P. (Hrsg.): Shareholder Value und die Kriterien des Unternehmenserfolgs. Ethische Ökonomie. Beiträge zur Wirtschaftsethik und Wirtschaftskultur, Heidelberg 1999, S. 217.

Vgl. Flatz, A.: Der Dow Jones Sustainablitity Index: Eine neue Kraft auf den globalen Finanzmärkten, in: Fichter, K.; Schneidewind, U. (Hrsg.): Umweltschutz im globalen Wettbewerb - Neue Spielregeln für das grenzenlose Unternehmen, Berlin u. a. 2000, S. $111 \mathrm{f}$.

Heute sind allein in Deutschland 19 Öko-Fonds zugelassen, die in 1999 zusammen 1,2 Milliarden Mark verwalten. Dies stellt eine Steigerung der Anlagesumme gegenüber 1998 von mehr als $100 \%$ dar. Klaus, I.: Saubere Rendite, in: Die Geldidee, Nr. 18, August 2000, S. 43. Der Anteil nachhaltiger Anlagen in Großbritannien stieg 1999 um $50 \%$ und erreichte einen Anteil von $5 \%$ des gesamten Anlagevolumens. In den USA betrăgt der Anteil bereits $10 \%$ aller Investitionen. Vgl. Flatz, A.: Der Dow Jones Sustainablitity Index: Eine neve Kraft auf den globalen Finanzmärkten, in: Fichter, K.; Schneidewind, U. (Hrsg.): Umweltschutz im globalen Wettbewerb - Neue Spielregeln für das grenzenlose Unternehmen, Berlin u. a. 2000, S. 118 f. 
gruppen als Bewertungsgrundlage. ${ }^{277}$ So kann über den Kapitalmarkt ein Einfluss auf das ökologiebezogene Unternehmensverhalten ausgeübt werden. ${ }^{278}$ Da am Kapitalmarkt das Gesamtunternehmen im Fokus der Betrachtung liegt, können ökologische Risiken in einem Ländermarkt zu marktbezogenen Rückkopplungen führen, die sich bspw. in erhöhten Versicherungsprämien, verschlechterten Kreditbedingungen oder einem niedrigeren Aktienkurs ausdrücken und so den internationalen ökologiebezogenen Koordinationsbedarf erhöhen.

Je stärker Märkte miteinander verbunden sind und ökologiebezogene Kriterien von Marktteilnehmern berücksichtigt werden, desto größer ist der internationale ökologiebezogene Koordinationsbedarf aufgrund marktbezogener Rückkopplungen.

Lehnen Konsumenten in einem Ländermarkt den Kauf von Produkten eines Herstellers ab, weil dieser Hersteller sich in anderen Ländermärkten nicht ökologiegerecht verhält, kann von nachfragebezogenen Rückkopplungen gesprochen werden. ${ }^{279}$ Nachfragebezogene Rückkopplungen stellen dabei einen Spezialfall der marktbezogenen Rückkopplungen dar.

Der ökologiebezogene internationale Koordinationsbedarf fällt aufgrund nachfragerbezogener Rückkopplungen umso größer aus, je stärker unterschiedliche Ländermärkte verbunden sind.

277 Zu den Methoden der Bewertung vgl. Figge, F.: Oko-Rating - Präferenzgerechte Aggregation ökologieorientierter Informationen, Berlin u. a. 2000.

Automobilunternehmen sind zumeist Aktiengesellschaften, deren Aktienkurs durchaus von einer Aufnahme in einen Umweltfond profitieren kann. So wurden bspw. VoLKSWAGEN und BMW in den Dow Jones Sustainability Index aufgenommen. SCHALTEGger und FIGGE prognostizieren für die Zukunft eine weitere Integration von ökologischen und ökonomischen Analyseansătzen in die aufstrebende Disziplin des Sustainable Finance. Vgl. Schaltegger, S.; Figge, F.: Ókologisierung von Finanzmärkten: Neu Steuerungssignale für Unternehmen?, in: Fichter, K.; Schneidewind, U. (Hrsg.): Umweltschutz im globalen Wettbewerb - Neue Spielregeln für das grenzenlose Unternehmen, Berlin u. a. 2000, S. 109.

279 Der FORD FOCUS von FORD in Europa gilt durchaus als okologisch vorteilhaftes Automobil, wie dies bspw. der VCD bestätigt. Vgl. Fokus (Hrsg.): Der Markt der Automobile: Daten, Fakten, Trends, 1999, S. 16. Allerdings wird die FORD MOTOR COMPANY von verschiedenen öffentlichen Anspruchsgruppen aufgrund okologisch zweifelhafter Verhaltensweisen stark kritisiert. So hat die FORD MOTOR COMPANY von der ökologischen Anspruchsgruppe CORPORATE WATCH mehrfach den Greenwash Award für irreführende Werbung mit ökologischen Themen verliehen bekommen. Vgl. http://www.corpwatch.org/greenwash/ford.html. 


\subsection{4 Ökologische Betroffenheit durch international tätige Wettbewerber}

Ökologische Ansprüche können direkt bzw. über (umwelt)politische Maßnahmen indirekt heute oder in Zukunft zu sog. ökologischen Wettbewerbsfeldern führen. Ökologische Wettbewerbsfelder werden als Endpunkt des ökologischen Transformationsprozesses bezeichnet. Die Art und Ausprägung dieser Felder entscheiden darüber, wie sich die Kosten- und Differenzierungsposition von Unternehmen im ökologische Kontext verändert und welche Chancen und Risiken daraus für die Unternehmen entstehen. ${ }^{280} \mathrm{Da}$ die Wettbewerbsposition eines Anbieters von den Maßnahmen der Konkurrenz abhängt, beeinflusst die Internationalisierung von Konkurrenten die eigene Wettbewerbsposition auf dem Heimatmarkt. In diesem Kontext entstehen konkurrenzbezogene Rückkopplungen bereits, wenn ein Konkurrent durch das Betreten eines Auslandsmarktes zusätzliches Wissen oder zusätzliche Größeneffekte erzielen kann. ${ }^{281}$

Die Wettbewerbsintensität in der Automobilindustrie weist ein hohes Niveau auf und bedingt einen zunehmenden Innovationswettbewerb, der eine Differenzierung gegenüber der Konkurrenz erlauben soll. Aufgrund der schnellen Reaktion des Wettbewerbs i. S. von Imitationsstrategien wird eine nachhaltige Verteidigung dieser Vorteile aber erschwert. ${ }^{282}$ Vor dem Hintergrund der ökologischen Betroffenheit der Automobilindustrie beschäftigen sich die Forschungsabteilungen der Automobilhersteller in hohem Maße mit dem Bau verbrauchs- und abgasarmer Fahrzeuge und der Entwicklung innovativer Antriebskonzepte wie der Brennstoffzelle. Neben dem ökologischen Wettbewerb im produktbezogenen Umweltschutz lassen sich auch im prozessbezogenen Umweltschutz Anstrengungen der Hersteller beobachten, eine ökologische Differenzierung anzustreben. In diesem Zusammenhang sei auf den Zertifizierungswettbewerb unter den Herstellern verwiesen. ${ }^{283}$

280 Vgl. Dyllick, Th.; Belz, F.; Schneidewind, U.: Ókologie und Wettbewerbsfähigkeit, a. a. O., S. 57.

281 Vgl. Backhaus, K.; Büschken, J.; Voeth, M.: Internationales Marketing, a. a. O., S. 60 ff.

282 Vgl. Liebehenschel, Th.: Ökologieorientierte Produkt- und Dienstleistungspolitik - Rahmenbedingungen und Trends am Beispiel der Automobilindustrie, a. a. O., S. 289.

Die Ford MOTOR COMPANY weist bspw. darauf hin, als erster Hersteller alle Standorte nach der ISO 14.001 Norm zertifiziert zu haben. Auch andere Hersteller haben sich zum Ziel gesetzt in naher Zukunft alle Standorte zertifizieren zu lassen. 
In diesem Zusammenhang sind es nicht allein die europäischen oder deutschen Hersteller, die sich mit dem Thema Ökologie beschäftigen, sondern die gesamte Branche. Derzeit sind in Deutschland bzw. Europa nahezu alle Hersteller mit der Entwicklung eines Drei-Liter-Autos beschäftigt. ${ }^{284}$ So hat der Bau des $3 \mathrm{~L}$ LUPOS durch den VOLKSWAGEN-Konzern und die neuerliche Ankündigung, bis 2003 ein Ein-Liter-Fahrzeug zu entwickeln ${ }^{285}$, zu einer weiteren Verschärfung des ökologiegetriebenen Wettbewerbs geführt. ${ }^{286}$ Dies zeigt sich insbesondere an den vielfältigen ökologischen Konzeptstudien, welche die Hersteller auf Automobilausstellungen präsentieren.

Der ökologiegerichtete Wettbewerb hat auch dazu beigetragen, dass kleine Nischenanbieter insbesondere im Segment der Elektro- oder Kleinstfahrzeu$\mathrm{ge}^{287}$ sowie neue Wettbewerber wie bspw. die LOREMO AUTOMOTIVE GmbH i. $\mathrm{Gr}^{288}$ oder die HYPERCAR Inc. ${ }^{289}$ versuchen, sich mit eigenen ökologischen Automobilkonzepten im Wettbewerb zu etablieren. Daraus kann geschlossen werden, dass Ökologie in der Automobilindustrie zum Wettbewerbsfaktor avanciert.

284 Dabei kommt der Entwicklung möglichst marktkonformer Fahrzeuge auf Basis der Dieseltechnologie die höchste Bedeutung zu. Vgl. Liebehenschel, Th.: Ókologieorientierte Produkt- und Dienstleistungspolitik - Rahmenbedingungen und Trends am Beispiel der Automobilindustrie, a. a. O., S. 290.

Vgl. o. V.: „Ich werde das Ein-Liter-Auto bauen“, in: Stern.de, 20.09.2000, http://www.stern. de/auto/berichte/ [20.09.2000].

286 Der Hersteller ChRYsLeR hat in 2000 mit dem ESX 3 ebenfalls ein Drei-Liter-Fahrzeug entwickelt. Vgl. o. V.: Chrysler-Forschungsauto ESX 3 leicht und sparsam: Das neueste 3Liter-Auto kommt aus den USA, in: Handelsblatt, Nr. 178, 14.09.2000, S. 53. Aber auch japanische Hersteller wie Toyota und Honda haben Wege gefunden, den Kraftstoffverbrauch weiter zu reduzieren.

287 Vgl. zu Klein- und Kleinstserienanbietern Ostendorf, R. J.: Dynamische Ókologieführerschaft: eine Wettbewerbsstrategie gewinnorientierter Unternehmen - theoretische Darstellung und praktische Überprüfung am Beispiel der Automobilindustrie, Sternenfels 2000.

Diese Firma plant den Bau des LOREMO L22 mit Zweizylinder-Dieselmotor (Pumpe-DüseDirekteinspritzung) mit einem Gewicht von unter $400 \mathrm{~kg}$ und einem Verbrauch von 1,5 Litern zu einem Preis unter 10.000 Euro. Vgl. Stirn, A.: Wunschdenken oder Wunderauto?, in: Spiegel Online-Auto, 06.10.2000, http://www.spiegel.de/auto/showroom/0.1518.97076. 00.html [07.10. 2000].

289 Hinter der HYPERCAR INC. steht AMORY LOVINS. Er hat sich zum Ziel gesetzt, ein Brennstoffzellenauto aus Karbon zu bauen und damit den Automobilmarkt zu revolutionieren. Vgl. Ball, J.: 'Clean' Car Quest Rolls on: Green Guru Lovins's Dream Machine Draws Industry Interest, Few Funds, in: The Wall Street Journal Europe, Thuesday, January 11, 2001, S. 23. 
Die Innovationen, die auf der IAA in Frankfurt (1999) und dem 70. internationalen Autosalon in Genf (2000) ausgestellt wurden, sind in der folgenden Tabelle aufgeführt.

\begin{tabular}{|l|l|}
\hline Hersteller & Ökologische Innovationen und Konzeptfahrzeuge \\
\hline Audi & A2 mit 3-Zylinder-TDI \\
BMW & Wasserstoffbetriebener BWM 750 hI \\
Citroen & Hybrid Konzeptauto Xara \\
Daihatsu & 3L Konzept SIRION 2CD \\
DaimlerChrysler & Brennstoffzellenfahreug Necar 3, Studie Crysler ESX \\
FIAT & Konzeptauto Ecobasic, Multipla Hybrid \\
Ford & Hybrid-Konzept Protegy, THINK-Elektrofahrzeug mit Alurahmen \\
Honda & Hybridfahrzeug Honda Insight, Concept Car FCX mit Brennstoffzelle \\
Mitsubishi & GDi- Technologie im SIGMA \\
Opel & Opel Zafira mit Brennstoffzelle, Astra Eco 4, Konzeptauto G9o \\
Peugeot & HDI Motor (Common Rail DI-Diesel), Rußfiltertechnologie \\
Renault & Hybrid-Geländewagenstudie Koleos \\
SMART & Smart CdI 3L-Auto \\
Toyota & Toyota Prius mit Hybridantrieb, DI-Dieseltechnologie, Yaris eco \\
Volkswagen & Lupo 3L TDI und FSI \\
\hline
\end{tabular}

Tabelle 3: $\quad$ Ökologische Konzeptfahrzeuge unterschiedlicher Hersteller Quelle: Eigene Darstellung

Je höher die Bedeutung von Umweltschutz als Wettbewerbsfaktor ist, desto größer ist der internationale ökologiebezogene Koordinationsbedarf aufgrund konkurrenzbezogener Rückkopplungen.

\section{3 Ökologische Betroffenheit im Überblick}

Die folgende Abbildung zeigt die Betroffenheit der europäischen Automobilindustrie durch einzelne Anspruchsgruppen. Diese Ergebnisse stammen aus der Befragung zum Europäischen Umweltmanagement-Barometer. Die in der folgenden Abbildung dargestellt ökologische Betroffenheit stellt dabei einen europäischen Durchschnittswert dar, der sowohl Mutter- als auch Tochtergesellschaften beeinhaltet. Dennoch lassen sich verschiedene Aussagen auf Basis dieser Daten ableiten. 


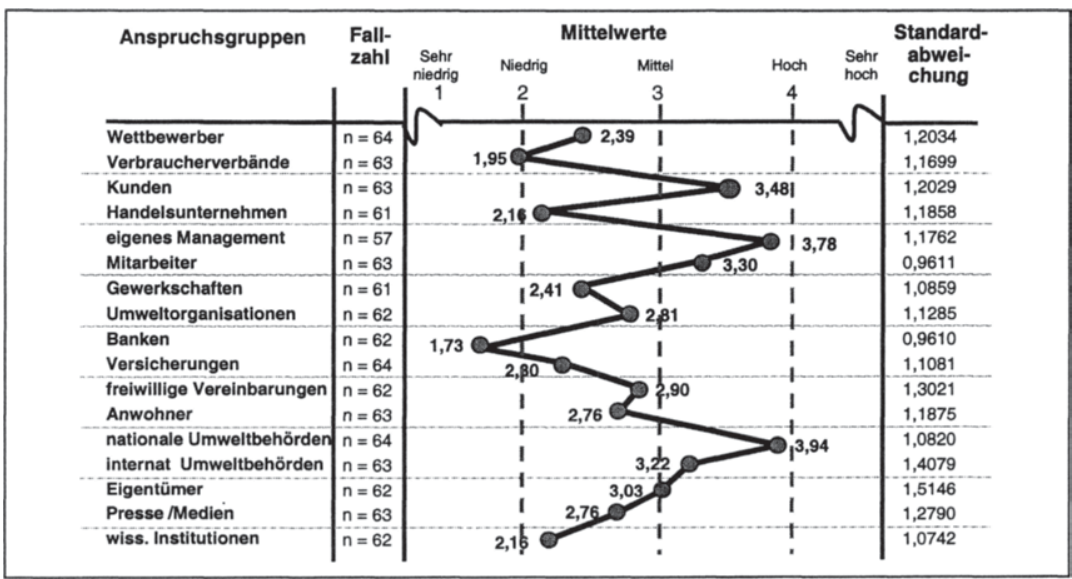

Abbildung 20: Ökologische Betroffenheit der europäischen Automobilindustrie durch unterschiedliche Anspruchsgruppen

Quelle: Eigene Darstellung auf Basis der Daten zum Europäischen Umweltmanagement-Barometer, 1998.

Die ökologische Betroffenheit durch nationale Regulierungsbehörden, Kunden und das eigene Management ist als hoch zu bezeichnen. Kaum eine Rolle spielen hingegen wissenschaftliche Institutionen, Banken und Versicherungen, Verbraucherverbände, der Automobilhandel und Gewerkschaften. Die Betroffenheit durch lokale Umweltschutzforderungen überwiegt die ökologische Betroffenheit durch international tätige Anspruchsgruppen. Dabei ist diese Einschätzung aufgrund der Stichprobenzusammensetzung zu relativieren. Es lässt sich vermuten, dass diese Durchschnittsbetrachtung anders ausgefallen wäre, wenn nur Muttergesellschaften befragt worden wären. ${ }^{290}$ Die vergleichsweise hohe Streuung der Mittelwerte, insbesondere in Bezug auf die Betroffenheit durch Kunden, Wettbewerber, internationale Umweltbehörden, Presse und Medien sowie Eigentümer, zeigt dabei deutlich, dass die ökologische Betroffenheit in unterschiedlichen Ländermärkten differenziert beurteilt wird.

290 Dies bestătigt auch das Interview mit Herrn KRŪGER aus dem Umweltmanagement von FORD Deutschland. 


\section{Einfluss endogener Kontextfaktoren auf den internationalen öko- logiebezogenen Koordinationsbedarf}

In diesem Unterkapitel wird der Einfluss endogener Kontextfaktoren auf den internationalen ökologiebezogenen Koordinationsbedarf untersucht. Dazu werden auf Basis von Plausibilitätsüberlegungen Thesen abgeleitet.

\subsection{Umweltschutz als Unternehmensziel}

Ziele beschreiben einen zukünftigen Zustand, der aus Unternehmenssicht wünschenswert erscheint und gelten als Entscheidungskriterium, Kontrollmaßstab, Anreiz- und Befriedigungsgröße sowie als Koordinationsinstrument in dezentral organisierten Entscheidungseinheiten. ${ }^{291}$ In diesem Kontext kann der langfristigen Gewinnerzielung und der Erhaltung der Wettbewerbsfähigkeit die höchste Priorität im Zielsystem einer Unternehmung eingeräumt werden. ${ }^{292}$ Die Internationalisierung ${ }^{293}$ stellt dabei ebenso ein Instrument zur Erreichung der spezifischen Unternehmensoberziele dar wie der betriebliche Umweltschutz. ${ }^{294}$

Die übergeordneten Ziele einer Unternehmung, also der Unternehmenszweck sowie die Unternehmensidentität, drücken sich in den Unternehmensgrundsätzen bzw. -leitlinien aus, die als konkretisierte Unternehmensphilosophie aufgefasst werden können. ${ }^{295}$ Ökologische Ziele finden im Rahmen der Hand-

291 Vgl. Meffert, H.: Unternehmensziele, in: Schöttle, K. M. (Hrsg.): Jahrbuch des Marketing, Essen 1971, S. 23 f. sowie Strebel, H.: Zielsysteme und Zielforschung, in: Die Betriebswirtschaft, 41. Jg. (1981), Nr. 3, S. 458.

Vgl. zur Rangordnung von Unternehmenszielen und zu Unter-, Ober- und Nebenzielen Heinen, E.: Das Zielsystem der Unternehmung, Wiesbaden 1966; Koch, H.: Aufbau der Unternehmensplanung, in: Gutenberg, E. (Hrsg.): Die Wirtschaftswissenschaften, Bd. 2, Wiesbaden 1977, S. $57 \mathrm{ff}$. Grundlegend zur Auswahl von anzustrebenden Zielen aus einer Anzahl von Zielalternativen vgl. Helnen, E.: Grundlagen betriebswirtschaftlicher Entscheidungen - Das Zielsystem der Unternehmung, 3. Aufl., Wiesbaden 1976, S. 28 ff. Die Ergebnisse der empirischen Zielforschung bestätigen die Bedeutung der langfristigen Gewinnerzielung und der Erhaltung der Wettbewerbsfăhigkeit. Vgl. Meffert, H.; KIrchgeorg, M.: Umweltschutz als Unternehmensziel, in: Specht, G. et al. (Hrsg.): Marketing-Schnittstellen, Stuttgart 1989, S. $179-200$.

Vgl. Backhaus, K.; Büschken, J.; Voeth, M.: Internationales Marketing, a. a. O., S. 54 f.

Vgl. Strebel, H.: Umwelt und Betriebswirtschaftslehre, Berlin 1980, S. 48. Dabei kommt Umweltschutzzielen in Unternehmen durchaus eine hohe Bedeutung zu. So wird die Erweiterung der betriebswirtschaftlichen Zielkonzeption durch ökologische Zielgrößen als Voraussetzung dafür angesehen, um durch eine Vermeidung und Verminderung von Umweltbelastungen ökologiebezogene Chancen wahrzunehmen oder entsprechende Risiken zu mindern. Vgl. Meffert, H.; Kirchgeorg, M.: Ziele und Strategien des betrieblichen Umweltmanagements im Wandel, in: Wagner, G. R. (Hrsg.): Unternehmensführung, Ethik und Umwelt, Wiesbaden 1999, S. 492.

Die Unternehmensphilosophie beinhaltet dabei die allgemeinen Zielvorstellungen und Werte und bildet die Basis für die Bestimmung konkreter Zielsysteme. Diese findet eine 
lungsziele nur Berücksichtigung, wenn sie den übergeordneten Zielen eines Unternehmens dienen bzw. große Nachteile vermeiden helfen. ${ }^{296}$ Dabei kann Umweltschutz mit unterschiedlicher Priorität Eingang in die Unternehmensgrundsätze finden. Je nachdem, ob Umweltschutz

- allein als Kostenfaktor aufgefasst,

- aus gesellschaftlicher Verantwortung heraus betrieben oder

- als Bestandteil des Leistungsprofils und damit als Chance begriffen wird,

ist von einer anderen Wirkung auf den internationalen Koordinationsbedarf auszugehen. Die Beurteilung der Beziehung zwischen ökonomischen und ökologischen Zielen durch das Management ${ }^{297}$ entscheidet dabei in hohem Maß darüber, ob und mit welcher Priorität Umweltschutzziele als Handlungsziele Berücksichtigung finden. ${ }^{298}$ Der internationale ökologiebezogene Koordinationsbedarf entsteht dabei erst durch Berücksichtigung von ökologischen Zielen als Handlungsziele. ${ }^{299}$

Generell werden neutrale, konfliktäre und komplementäre Zlelbeziehungen zwischen ökonomischen und ökologischen Zielen unterschieden. ${ }^{300}$ Während eine neutrale Zielbeziehung zwischen ökonomischen und ökologischen Zielen

Konkretisierung in Form von Unternehmensgrundsätzen als Leitbild für die Ausrichtung des gesamten Unternehmensverhaltens sowie die strategische Planung. Vgl. Meffert, H.: Marketing: Grundlagen marktorientierter Unternehmensführung: Konzepte - Instrumente Praxisbeispiele, a. a. O., S. $73 \mathrm{ff}$.

Vgl. hierzu und im Folgenden Meffert, H.; KIrchgeorg, M.: Marktorientiertes Umweltmanagement: Konzeption, Strategie, Implementierung mit Praxisfällen, a. a. O., S. 181 f.

297 Zum Einfluss des Wertesystems des Managements auf die Handhabung von Entscheidungsproblemen vgl. Macharzina, K.: Unternehmensführung: Das internationale Managementwissen; Konzepte - Methoden - Praxis, a. a. O., S. 93 ff. sowie die dort angegebene Literatur.

So ergab die Studie der Vereinten Nationen zum Umweltmanagement in transnationalen Unternehmen, dass $43 \%$ der Unternehmen eine schriftlich fixierte international gültige Umweltpolitik haben. Die nationalen Ergebnisse unterscheiden sich allerdings stark. Vgl. United Nations (Hrsg.): Environmental Management in Transnational Corporations: Report on the Benchmark Corporate Environmental Survey, a. a. O., S. 13.

Vgl. Meffert, H.: Marketing: Grundlagen marktorientierter Untemehmensführung. Konzepte - Instrumente - Praxisbeispiele, a. a. O., S. 69 ff. sowie die dort angegebene Literatur.

300 Vgl. Meffert, H.; KIrchgeorg, M.: Marktorientiertes Umweltmanagement: Konzeption, Strategie, Implementierung mit Praxisfällen, a. a. O., S. 45 ff. Bei Zielkonflikten konnte kein Konsens zwischen den am Unternehmen beteiligten Interessengruppen, bspw. den Führungskräften aus unterschiedlichen Lăndermärkten, gefunden werden. In diesem Fall sind Konfliktregelungen festzulegen. Vgl. zu unterschiedlichen Möglichkeiten, die Zielintegration im Unternehmen zu erhöhen Barrett, H. J.: Individuelle Ziele und Organisationsziele, in: Wöhler, K.: (Hrsg.) Organisationsanalyse, Stuttgart 1978, S. 68 ff. 
grundsätzlich keinen ökologiebezogenen Koordinationsbedarf begründet $^{301}$, machen länderbezogen unterschiedliche Beurteilungen der Zielbeziehungen eine ökologiebezogene Koordination im internationalen Kontext erforderlich.

In Abbildung 21 sind diesbezüglich unterschiedliche managementbezogene Sichtweisen abgebildet. ${ }^{302}$

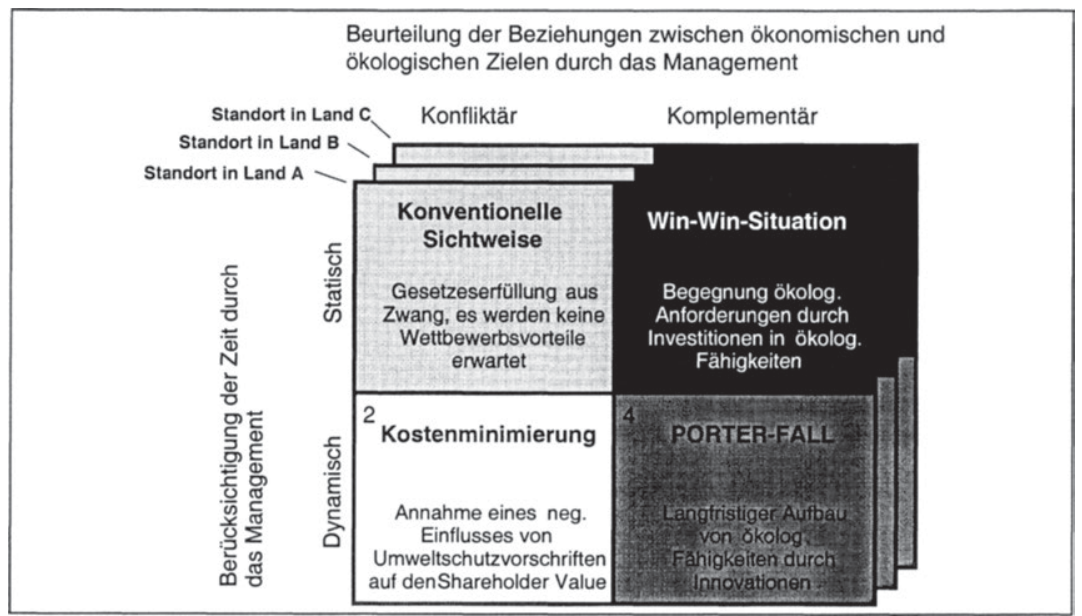

Abbildung 21: Einfluss von Umweltgesetzen auf die Unternehmensleistung In Anlehnung an Rugman, A. M.; Verbeke, A.: Corporate Strategies and Environmental Regulations: An Organizing Framework, in: Strategic Management Journal, Vol. 19 (1998), S. 365.

Die Sichtweisen unterscheiden sich grundlegend sowohl in Bezug auf die Einschätzung der Zielbeziehung von Umweltschutz und Ökologie im internationalen Kontext als auch in Bezug auf die Berücksichtigung der Zeit. Dabei kann das lokale Management an den einzelnen internationalen Standorten zu einer unterschiedlichen Beurteilung der Zielbeziehungen kommen. Wird bspw. am Standort A erwartet, durch eine langfristig ökologische Ausrichtung Wettbewerbsvorteile zu erlangen, in Standort B hingegen bspw. aufgrund anderer Konsumentenpräferenzen angenommen, die Zielbeziehungen zwischen ökonomi-

301 Vgl. Plump, D.: Die Koordination des Marketings in multinationalen Unternehmungen: Systemanalyse und Ansätze zur Systemgestaltung, a. a. O., S. 157.

302 Vgl. hierzu und im Folgenden Rugmann, A. M.; Verbeke, A.: Corporate Strategies and Environmental Regulations: An Organizing Framework, in: Strategic Management Journal, 19. Jg. (1998), S. $364 \mathrm{ff}$. 
schen und ökologischen Zielen seien konfliktär, dann besteht bei Umfeldabhängigkeit ebenfalls eine konfliktäre Beziehung zwischen ökologischen und ökonomischen Zielen für das gesamte Unternehmen und die Unternehmensleitung muss koordinierend tätig werden.

\section{- Feld 1: Die „konventionelle Sichtweise“}

Manager beurteilen den durch den nationalen Gesetzgeber vorgeschriebenen Umweltschutz primär als Kostenfaktor. Die Berücksichtigung ökologischer Belange bei der Produktion geschieht allein aus gesetzlichem Zwang, da eine Nichterfüllung zu hohen Strafen oder dem Genehmigungsentzug führen kann. Auf kurze Sicht wird eine konfliktäre Zielbeziehung zwischen ökonomischen und ökologischen Zielen angenommen. Die Wettbewerbsfähigkeit im internationalen Kontext lässt sich dann durch Umweltschutz kurzfristig nur verschlechtern. Dies gilt besonders bei Konkurrenz aus umweltrechtlich weniger restriktiven Ländern. Ökologische Ziele ergeben sich hier allein aus gesetzlichen Normen, die allerdings kaum als eigenständige ökologische Ziele gelten können.

\section{- Feld 2: „Kostenminimierung“}

In diesem Fall geht das lokale Management ebenfalls von Zielkonflikten zwischen ökonomischen und ökologischen Zielen aus, allerdings wird dabei eine dynamische Sichtweise zugrunde gelegt. Der Anteil von Umweltschutzkosten wird als so hoch angesehen, dass langfristig die Wettbewerbsfähigkeit des Unternehmens als gefährdet gilt. Somit erscheint eine möglichst weitreichende Umgehung von gesetzlichen Umweltvorschriften z. B. durch Verlagerung des Produktionsstandortes in weniger restriktive Länder (sog. pollution heaven) als geeignete Strategie. ${ }^{303}$

- Feld 3: „Win-Win-Situatlon“

In diesem Feld spiegelt sich die Auffassung von Entscheidungsträgern wider, die eine Zielkomplementarltät zwischen ökologischen und ökonomischen Zielen auch kurzfristig vermuten. Dahinter steht auch die Erfahrung, dass Umweltschutz sich durchaus auszahlen kann, wenn flexible umweltpolitische Instrumente eine kosteneffiziente Anpassung an ökolo-

303 WALLEY und WHITEHEAD argumentieren in diesem Zusammenhang, dass der Einfluss erhöhter Umweltschutzkosten auf den Shareholder Value langfristig negativ sei, und durch kostenminimierende Anpassungen des Managements so gering wie möglich zu halten ist. Vgl. Walley, N.; Whitehead, B.: 'It's not easy being green', Harvard Business Review, 72 Jg. (1994), Nr. 3, S. 46 - 52. 
gische Gesetze erlauben. ${ }^{304}$ Kosteneinsparungen ermöglichen eine sog. "Win-Win-Situation". ${ }^{305}$ Ökologische Ziele werden in diesem Fall explizit festgelegt und kontrolliert, soweit interne ökologiegerichtete Anpassungsmaßnahmen auch ökonomisch sinnvoll sind. Eine Verschlechterung der internationalen Wettbewerbsposition stellt sich nicht ein, die Stellung im Wettbewerb kann sich dadurch sogar verbessern.

- Feld 4: „Porter-Fall“

In diesem Fall wird die These vertreten, dass strenge Umweltregulierungen die Innovationstätigkeit der Unternehmen anregen und durch den langfristigen Aufbau von Kernkompetenzen im Umweltbereich Wettbewerbsvorteile geschaffen werden können. So gehen PORTER und VAN DER LINDE davon aus, dass produktionsbezogene Umweltstandards längerfristig zu einer Verbesserung der internationalen Wettbewerbsfähigkeit führen können. ${ }^{306}$ Dies wird, in Anlehnung an das SCHUMPETERSCHE Konzept des dynamischen Wettbewerbs, durch eine proaktive Vorwegnahme von internationalen Entwicklungen und den damit einhergehenden Vorsprüngen in der Forschung und Entwicklung sowie dem Aufbau der entsprechenden Kompetenzen begründet. ${ }^{307}$ Der Einsatz umweltfreundlicher Produktionsmethoden führt zu Technologievorsprüngen und Kostensenkungen, die Herstellung ökologiegerechter Produkte zu Produktimagevorteilen und Umsatzsteigerungen. ${ }^{308}$ Die Erfüllung ökologischer Ziele wird dabei als Bestandteil

304 Vgl. Clarke R. A. et al.: The challenge of going green, in: Harvard Business Review, 72. Jg. (1994), Nr. 4, S. $43-44$.

Vgl. dazu bspw. Gege, M. (Hrsg.): Kosten senken durch Umweltmanagement - 1000 Erfolgsbeispiele aus 100 Unternehmen, München 1997.

PORTER und VAN DER LINDE erlăutern dies wie folgt: ${ }_{n}$ Properly designed environmental standards can trigger innovations that lower the total cost of a product or improve its value. Such innovations allow companies to use a range of inputs more productively - from raw materials to energy to labour - thus offsetting the costs of improving environmental impact and ending the stalemate. Ultimately, this enhanced resource productivity makes companies more competitive, not less.“ Porter, M.; v. Linde, C.: Green and Competitive. Ending the Stalemate, Harvard Business Review, 73. Jg. (1995), Nr. 5, S. 120.

Eine empirische Validierung der PORTER-Hypothese blieb allerdings bis heute aus. Vgl. Albrecht, J.: Environmental costs and competitiveness. A product-specific test of the Porter hypothesis, Working Paper Series, University of Ghent, Faculty of Economics and Business Administration, 1998, S. 17.

${ }_{n}$ After all, the nations that have traditionally been the toughest competitors, like Japan and Germany, have long had energy prices two to three times U. S. levels. Over the long run, this has simply made them use their energy about twice as efficiently. Learning how to do that drove their industrial innovation on a broad front, further widening the competitive gap against a cheap-fuel America." Lovins, A. B.; Lovins L. H.: Climate: Making Sense and 
des Leistungsprofils und damit als Chance der Unternehmung interpretiert. Ökologische Ziele nehmen einen hohen Stellenwert in der Zielhierarchie ein. Möglicherweie findet sogar eine Standortverlagerung von Unternehmensteilen aus weniger restriktiven Ländern in das ökologisch restriktive Land statt.

Je höher die Bedeutung des Umweltschutzes im Zielsystem einer Unternehmung ist, desto größer ist der internationale ökologiebezogene Koordinationsbedarf.

Eine empirische Untersuchung für die europäische Automobilindustrie zeigt, dass nicht von einer generell konfliktären Zielbeziehung zwischen ökologischen und anderen Unternehmenszielen auszugehen ist. Ökologiebezogene Maßnahmen haben demzufolge eine positive Wirkung auf das Unternehmensimage, welches auch eng mit der gesellschaftlichen Legitimität einer Unternehmung zusammenhängt. Die Sicherung der Wettbewerbsfähigkeit und die langfristige Gewinnerzielung werden nach Einschätzung der Unternehmensvertreter positiv durch betrieblichen Umweltschutz beeinflusst. Im Gegensatz dazu werden von ökologiebezogenen Maßnahmen kurzfristig kaum Beiträge zur Kostensenkung, Produktivitäts- oder Umsatzsteigerung enwartet, sodass auch die kurzfristige Gewinnerzielung nicht beeinflusst wird. Die Möglichkeit, durch innovative ökologiegerichtete Produkt- oder Dienstleistungsangebote neue Märkte zu erschlieBen, wird neutral eingeschätzt.

In Abbildung 22 sind die Zielbeziehungen zwischen ökologischen und anderen Unternehmenszielen in der europäischen Automobilindustrie dargestellt.

Making Money, Rocky Mountain Institute (ed.), Colorado 1997, S. 23. Dies lässt sich besonders gut auf die Automobilindustrie übertragen. 


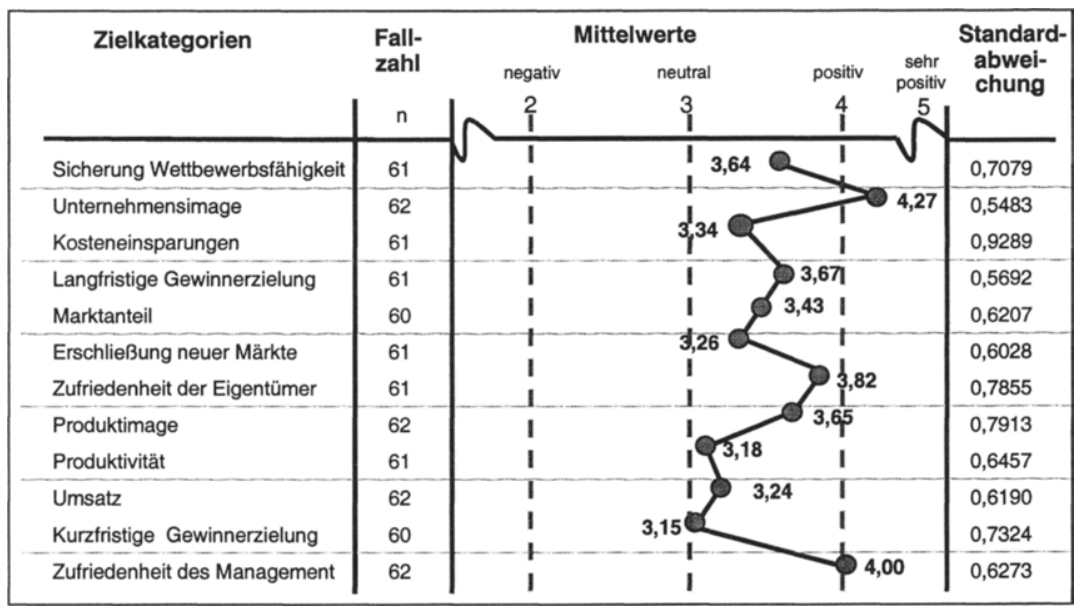

Abbildung 22: Zielbeziehungen zwischen ökologischen und anderen Unternehmenszielen in der europäischen Automobilindustrie

Quelle: Eigene Darstellung auf Basis der Daten des Europäischen Umweltmanagement-Barometers aus 1998

Die Beurteilung der Beziehung zwischen Umweltschutz und einzelnen ökonomischen Zielkategorien fällt heterogen aus, lässt aber den Schluss zu, dass zwischen ökologischen und ökonomischen Zielen kaum größere Zielkonflikte bestehen. Die vergleichsweise geringe Streuung zeigt, dass diese Einschätzung zumindest in Europa mehrheitlich geteilt wird. Die befragten Manager aus der Automobilindustrie beurteilen insbesondere in Bezug auf das Unternehmensimage, die Zufriedenheit von Management und Eigentümern sowie die Sicherung der langfristigen Wettbewerbsfähigkeit und der langfristigen Gewinnerzielung ökologiegerichtete Maßnahmen positiv.

Je konsistenter die Einschätzung der Führungskräfte der einzelnen Landesgesellschaften bezüglich der Zielbeziehungen zwischen ökonomischen und ökologischen Zielen dabei ausfallen, desto geringer ist der internationale ökologiegerichtete Koordinationsbedarf.

Generell gilt, je größer die Konflikte zwischen ökonomischen und ökologischen Zielen und je differenzierter die Beurteilung der Zielbeziehungen durch die nationalen Unternehmenseinheiten ausfällt, desto größer ist der internationale ökologiebezogene Koordinationsbedarf. 
Die auf Ebene der Zentrale fixierten ökologischen Ziele können eine internationale Koordinationsfunktion erfüllen, indem sich alle Unternehmensaktivitäten an den Oberzielen der Unternehmung ausrichten und somit ein aufeinander abgestimmtes Verhalten sichergestellt wird. ${ }^{309}$ Dies bedarf bei unterschiedlicher Beurteilung der Zielbeziehungen einer vorangehenden Lösung bestehender Konflikte zwischen ökonomischen und ökologischen Zielen auf oberster Ebene.

Je größer die Zielkonflikte zwischen ökologischen und ökonomischen Zielen zwischen den Landesgesellschaften ausfallen, desto höher ist der vorangehende Abstimmungs- bzw. Koordinationsbedarf auf der Ebene der Unternehmensführung.

Die auf oberster Ebene der Zielhierarchie festgelegten ökologischen Ziele finden dann Eingang in die Unternehmensgrundsätze. Unternehmensgrundsätze oder die Unternehmensphilosophie stellen dabei eine Ansammlung von allgemeinen Zwecken, Zielen, Potenzialen und Verhaltensweisen dar, die gemeinsam und ohne Ausnahme für alle Unternehmensmitglieder gelten. Sie sollen insbesondere eine Verbindung zwischen Innenwelt und Außenwelt schaffen und eine Grundlage der Zielfestlegung im operativen Geschäft bilden. Dabei sind die Unternehmensgrundsätze lediglich bzgl. des Zielinhaltes, nicht jedoch hinsichtlich des Ausmaßes und des zeitlichen Bezuges bestimmt. ${ }^{310}$ Damit ökologische Ziele eine Koordinationsfunktion erfüllen können, müssen sie operationalisiert werden. Fehlende Operationalität führt zu einer unzureichenden Auseinandersetzung mit Umweltschutzzielen im Unternehmen, lässt Zielkonflikte zwischen ökonomischen und ökologischen Zielen bestehen und erlaubt so national unterschiedliche Interpretationen der Ziele durch das Management. Sollen ökologische Ziele eine internationale Koordinationsleistung erfüllen, sind somit auf oberster Unternehmensebene verbindliche Zielgewichtungen vorzunehmen und operationale ökologische Vorgaben festzulegen.

Je operationaler unternehmensweit geltende Umweltschutzziele definiert werden, desto größer ist die Koordinationsleistung der Ziele und desto geringer der verbleibende internationale ökologiebezogene Koordinationsbedarf.

309 Ohne zielorientierte Gestaltung von Umweltschutzmaßnahmen besteht die Gefahr, dass in einzelnen Funktionsbereichen lediglich im Sinne eines "Muddling Through" in reaktiver Weise auf ökologische Herausforderungen reagiert wird. Vgl. Meffert, H.; KIrchgeorg, M.: Marktorientiertes Umweltmanagement: Konzeption, Strategie, Implementierung mit Praxisfällen, a. a. O., S. 186 f.

310 Vgl. Macharzina, K.: Unternehmensführung: Das internationale Managementwissen; Konzepte - Methoden - Praxis, a. a. O., S. $176 \mathrm{ff}$. sowie die dort angegebene Literatur. 


\subsection{Strategische Basisausrichtung im Umweltschutz}

Je nachdem, ob und in welcher Form Umweltschutzziele bei der Zielplanung Berücksichtigung finden, stellen sich dem Unternehmen verschiedene strategische Optionen zur Erreichung der angestrebten Ziele. ${ }^{311}$ Die strategische Grundhaltung gilt ebenfalls als Determinante des internationalen Koordinationsbedarfs. ${ }^{312}$

Unterschiedliche Ausgestaltungsformen der ökologischen Strategie können im internationalen Kontext zu einem unterschiedlich hohen Koordinationsbedarf beitragen. Dabei können Strategien im Umweltmanagement nach unterschiedlichen Merkmalen klassifiziert werden. In Abbildung 23 sind unterschiedliche strategische Optionen, die den internationalen ökologiegerichteten Koordinationsbedarf beeinflussen, dargestellt.

\begin{tabular}{|c|c|c|c|}
\hline $\begin{array}{l}\text { Klassifikationsmerkmale } \\
\text { umweltorlentlerter }\end{array}$ & \multicolumn{3}{|c|}{$\begin{array}{l}\text { Internationaler Koordinationsbedart bel } \\
\text { unterschledilchen Ausprägungsformen }\end{array}$} \\
\hline Unternehmensstrateglen & niedrig & mittel & hoch \\
\hline Verhaltensbezugsebene & intern & & extern \\
\hline $\begin{array}{l}\text { Anpassungsintensităt } \\
\text { an Umweltschutz- } \\
\text { erfordernisse }\end{array}$ & passiv & & innovativ \\
\hline $\begin{array}{l}\text { Zeitpunkt der Strategie- } \\
\text { entwicklung/Maßnah- } \\
\text { menrealisierung }\end{array}$ & reaktiv & Inittativakth & proaktiv \\
\hline $\begin{array}{l}\text { Art der Strategie- } \\
\text { entwicklung }\end{array}$ & isoliert & & integriert \\
\hline $\begin{array}{l}\text { Durchsetzung von } \\
\text { Strategien }\end{array}$ & individuell & & kooperativ \\
\hline
\end{tabular}

Abbildung 23: Internationaler Koordinationsbedarf in Bezug auf strategische Dimensionen umweltorientierter Basisstrategien

In Anlehnung an Meffert, H.; Kirchgeorg, M.: Marktorientiertes Umweltmanagement: Konzeption, Strategie, Implementierung mit Praxisfällen, 3. Aufl., Stuttgart 1998, S. 202.

311 Das Ziel der Unternehmensstrategie als langfristiger und globaler Verhaltensplan ist dabei die Erschließung und Sicherung von Erfolgspotenzialen. Vgl. hierzu und im Folgenden Meffert, H.; KIrchgeorg, M.: Marktorientiertes Umweltmanagement: Konzeption, Strategie, Implementierung mit Praxisfällen, a. a. O., S. 198 ff.

312 Zu generellen strategischen Grundhaltungen vgl. Miles, R. E.; Snow, C. C.: Organizational Strategy, Structure and Process, New York et al. 1978, S. $29 \mathrm{ff}$. 
Nach MEFFERT und KIRCHGEORG unterscheidet man dabei nach der Verhaltensbezugsebene, der Anpassungsintensität an ökologische Forderungen, dem Zeitpunkt und der Art der Strategieentwicklung sowie der Art der Durchsetzung der Strategien.

Die Bezugsebene des ökologiegerichteten Verhaltens kann sich auf eine eher interne oder stärker externe Ausrichtung beziehen. Während eine innengerichtete Umweltschutzstrategie durchaus zur Realisierung von sog. Win-Win-Situationen aufgrund von Kostensenkungen in der Produktion beitragen kann, gelingt eine Erschließung von Erfolgschancen gegenüber dem Wettbewerb nur durch neue umweltgerechtere Produkte und die Dokumentation der innengerichteten Umweltschutzaktivitäten gegenüber der Öffentlichkeit, also einer externen Strategieausrichtung. Allerdings ist eine auch nach außen gerichtete Umweltschutzstrategie mit einem höheren Koordinationsbedarf verbunden als eine allein innengerichtete Umweltschutzstrategie. In der Automobilindustrie lassen sich zwar nur selten ökologisch dominante Positionierungen erkennen, dennoch richten sich die Hersteller mit Umweltberichten und PR-Kampagnen immer wieder mit ökologischen Themen an die Öffentlichkeit. Wie stark das Thema Umweltschutz in der Öffentlichkeit kommuniziert wird, hängt dabei nach Experteneinschätzung vor allem davon ab, wie dieses Thema in der Unternehmensleitung wahrgenommen wird. Das Interesse einzelner Vorstände hat bspw. dazu geführt, dass VOLVO, FORD und BMW das Thema Umweltschutz regelmäßig kommunizieren. $^{313}$

Je stärker die ökologische Strategie auch auf externe Anspruchsgruppen ausgerichtet ist, desto größer ist der internationale ökologiebezogene Koordinationsbedarf.

Die Anpassungsintensität des Unternehmensverhaltens kann in aktives und passives Verhalten differenziert werden. Dabei bedeutet passives Verhalten, dass eine strategische Ausrichtung im Umweltschutz nicht vorgenommen wird. Bei fehlender strategischer Ausrichtung und damit fehlender ökologischer Zielsetzung findet auch keine gesonderte ökologiebezogene Koordination statt. Das Aktivitätsniveau ist weiter nach einem eher adaptiven oder innovativen ökologischen Verhalten zu unterscheiden. Die Entwicklung und Vermarktung des $3 \mathrm{~L}$ LUPOS durch die VOLKSWAGEN AG kann bspw. als innovatives Verhalten

313 Diese Einschätzung beruht auf dem Expertenurteil von Frau von FINCKENSTEIN-WANG von der VolKSWAGEN AG. Frau VON FINCKENSTEIN-WANG ist für den Bereich Umweltplanung Produktion/Standorte im VoLKSWAGEN-Konzern verantwortlich. 
gedeutet werden. Je innovativer das ökologiebezogene Verhalten eines Unternehmens ausgerichtet ist, desto stärker sind bspw. die Entscheidungen in den Forschungs- und Entwicklungsabteilungen mit den nationalen Marketingabteilungen zu koordinieren.

Je aktiver und innovativer die Verhaltensausrichtung im Umweltmanagement, desto höher ist auch der internationale ökologiebezogene Koordinationsbedarf.

Eng mit der Anpassungsintensität ist auch die zeitliche Ausrichtung der Strategie verbunden, die sich zwischen einer reaktiven und proaktiven Verhaltensausrichtung bewegen kann. Proaktives Verhalten wird weiter in initiativaktives und kontingenzaktives Verhalten unterteilt. Während bei initiativaktivem Verhalten eine Vorreiterrolle im Umweltschutz angestrebt wird, die sich an zukunftsweisenden Leitbildern orientiert, zeichnet sich kontingenzaktives Verhalten durch den Umweltschutz der robusten kleinen Schritte auf Basis von alternativen Szenarien aus. Dabei wird jeweils ein genügend großes Flexibilitätspotenzial eingeplant, um bei sich konkretisierenden Umweltkonstellationen weitere geplante Anpassungen vornehmen zu können. Proaktives Verhalten führt dabei generell zu einem höheren Koordinationsbedarf als reaktives Verhalten. In diesem Zusammenhang ist auf die Risikoneigung der Entscheidungsträger zu verweisen. Mit zunehmender Risikoneigung der Entscheidungsträger sinkt nach BENKENSTEIN der Koordinationsbedarf. ${ }^{314}$ Somit ist kontingenzaktives Verhalten mit einem höheren ökologiebezogenen Koordinationsbedarf verbunden als initiativaktives Verhalten.

Die Vermarktung des Golf ÖKOMATIK durch den VolKSWAGEN-Konzern zu Beginn der 90er Jahre könnte somit als Beispiel für initiativakives Verhalten eines eher risikofreudigen Managements gedeutet werden. Demgegenüber werden bestehende ökologische Fahrzeugkonzepte anderer Hersteller (noch) nicht auf den Markt gebracht. In diesem Zusammenhang können bspw. die Entwicklungsstudien und Prototypen der Hersteller RENAULT (VESTA 2), Volvo (LCP) und GeneRAL MOTORS (TPC) von Anfang der 90er Jahre genannt werden. $^{315}$

Je proaktiver die ökologische Ausrichtung im Umweltmanagement, desto höher ist der internationale ökologiebezogene Koordinationsbedarf.

314 Vgl. Benkenstein, M.: F \& E und Marketing: Eine Untersuchung zur Leistungsfähigkeit von Koordinationskonzeptionen bei Innovationsentscheidungen, a. a. O., S. 89 ff.

315 Vgl. Liebehenschel, Th.: Ökologieorientierte Produkt- und Dienstleistungspolitik Rahmenbedingungen und Trends am Beispiel der Automobilindustrie, a. a. O., S. 196. 
Nach der Art der Strategieentwicklung ist zu unterscheiden, ob die verfolgte Strategie im Sinne eines integrierten Gesamtkonzeptes auf alle Unternehmensfunktionen und -bereiche sowie unter Einbezug unternehmensübergreifender Kooperationen (bspw. mit Zulieferern) bezogen ist oder nur partielle Aspekte des Umweltschutzes im Produkt- oder Produktionsbereich berücksichtigt. Integrierte Unternehmensstrategien stellen dabei gegenüber der Entwicklung von Insellösungen hohe Anforderungen an die Koordination der einzelnen Verhaltensschwerpunkte. So können die zukünftigen noch anspruchsvolleren Abgasvorschriften in den USA nur noch durch gleichzeitige Optimierung von Motorentechnik, Abgasregeltechnik, dem Fahrzeugdesign (Luftwiderstand, Gewicht) und dem Einsatz ökologisch verträglicher Kraftstoffe erreicht werden. $^{316}$

Je integrierter Umweltschutz in einem Unternehmen betrieben werden soll, desto höher ist auch der internationale ökologiebezogene Koordinationsbedarf.

Schließlich stellt sich die Frage nach der Durchsetzung der Strategie. Hier kann eine unternehmensindividuelle oder kooperative Durchsetzung im Branchenverbund angestrebt werden. Im Umweltbereich ermöglicht eine individuelle Durchsetzung eine Differenzierung im Wettbewerb. Handelt es sich um die Umsetzung ökologischer Ziele bzw. die Erfüllung von Forderungen ökologischer Anspruchsgruppen, die kaum zur Profilierung im Wettbewerb geeignet erscheinen, aber mit hohen Kosten verbunden sind, dann eignet sich eher eine kollektive Umsetzung bspw. durch ein Branchenabkommen. Eine kollektive Umsetzung einer ökologischen Strategie ist mit einem höheren Koordinationsbedarf verbunden als die individuelle strategische Ausrichtung, da eine kollektive Ausrichtung eine Abstimmung mit sämtlichen Mitbewerbern bedarf. In diesem Zusammenhang kann die deutsche Selbstverpflichtung der Automobilindustire zur Rücknahme von Altautos als Beispiel dienen. Eine derartige Selbstverpflichtung auf internationaler Ebene hätte zu wesentlich höheren Transaktionskosten geführt.

Der internationale ökologiebezogene Koordinationsbedarf ist bei kooperativer Durchsetzung einer Strategie im Regelfall höher als bei einer individuellen Durchsetzung.

316 Die unterstreichen Herr MAYr-RAUCH und Herrn StOBbE von Volkswagen im Interview. 
Oftmals wird das Problem einer fehlenden Verknüpfung zwischen strategischer und operativer Planung beklagt. ${ }^{317}$ Der Verknüpfung von strategischer und operativer Ebene im Umweltmanagement kommt vor diesem Hintergrund eine zentrale Bedeutung für den Erfolg zu. Bei unzureichender Verbindung der Ebenen kann von einer Implementierungslücke gesprochen werden. ${ }^{318}$ Bei internationaler Geschäftstätigkeit mit den bereits angesprochenen delegationsbedingten Entscheidungsspielräumen des ausländischen Managements kann somit schnell eine Implementierungslücke entstehen. Die Aufdeckung und Beseitigung entsprechender Implementierungslücken führt dann wiederum zu internationalem ökologiebezogenen Koordinationsbedarf.

Je unzureichender die Verknüpfung zwischen strategischer Rahmenplanung und operativer Umsetzung, umso größer ist der internationale ökologiebezogene Koordinationsbedarf.

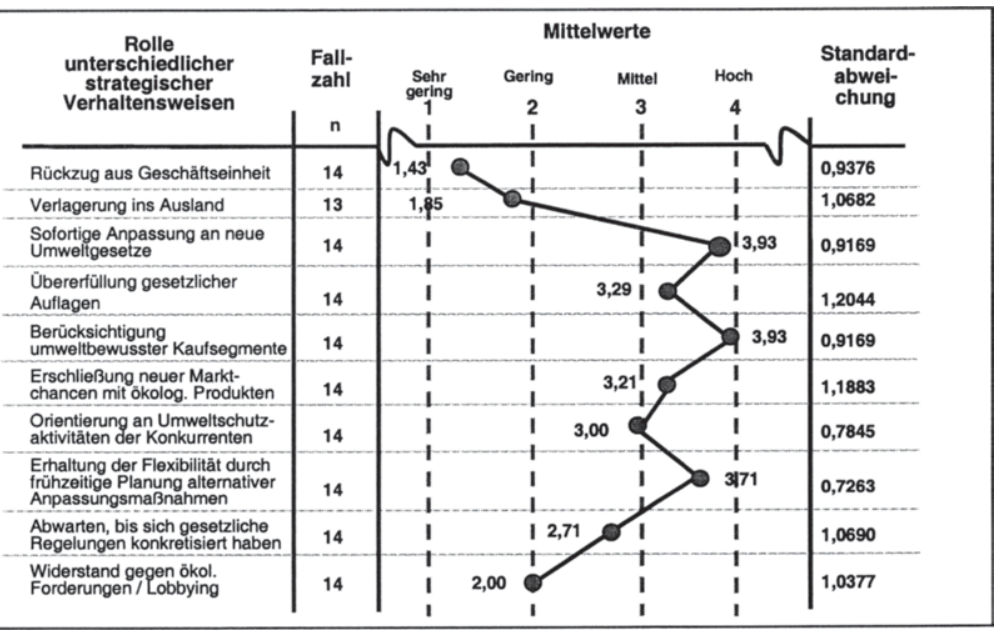

Abbildung 24: Ökologische Basisausrichtung der deutschen Automobilindustrie in 1998

Quelle: Eigene Darstellung auf Basis der Daten des Europäischen Umweltmanagement-Barometers aus 1998.

317 Vgl. Weber, J., Schäffer, U.: Balanced Scorecard - Gedanken zur Einordnung des Konzeptes in das bisherige Controlling-Instrumentarium, WHU- Forschungspapier, Koblenz 1999, S. 18.

Vgl. Hilker, J.: Marketingimplementierung: Grundlagen und Umsetzung am Beispiel ostdeutscher Unternehmen, Wiesbaden 1993; Grimmeisen, M.: Implementierungscontrolling: wirtschaftliche Umsetzung von Change-Programmen, Wiesbaden 1998. 
Im Rahmen des Europäischen Umweltmanagement-Barometers wurden 1998 14 Unternehmen aus der deutschen Automobilindustrie zu der Rolle unterschiedlicher strategischer Verhaltenweisen im Umweltmanagement befragt. In Abbildung 24 ist das entsprechende Mittelwertprofil der Antworten dargestellt. Die deutsche Automobilindustrie richtet demzufolge ihre ökologische Strategie an den Kundenanforderungen aus, wie die starke Berücksichtigung ökologiegerichteter Kundensegmente deutlich zeigt. Die Anpassungsintensität an ökologische Forderungen und der Zeitpunkt der Strategieplanung und Maßnahmenumsetzung lassen auf eine tendenziell proaktive Verhaltensausrichtung schließen. Eine sofortige Anpassung an neue Umweltschutzgesetze wird in der Automobilindustrie für wichtig erachtet. Darüber hinaus wird der frühzeitigen Planung von Umweltschutzmaßnahmen zur Erhöhung der Flexibilität der Anpassungsmaßnahmen eine hohe Bedeutung zugesprochen. Dabei wird auch die Übererfüllung von Umweltschutzgesetzen zumindest teilweise als wichtiger Teil der ökologischen Ausrichtung in der Automobilindustrie aufgefasst. Im Gegensatz dazu spielt eine eher passive Verhaltensausprägung, wie das Abwarten auf die Konkretisierung umweltgesetzlicher Regelungen und Widerstand, bspw. in Form von Lobbying, gegen neue Umweltgesetze nur eine untergeordnete Rolle. In diesem Zusammenhang werden auch Rückzugs- und Standortverlagerungsstrategien aufgrund gestiegener ökologischer Anforderungen als unbedeutende Verhaltensweisen gekennzeichnet.

\subsection{Internationale Konfiguration}

Die internationale Konfiguration als struktureller Aufbau der internationalen Unternehmensaktivitäten - einschließlich der Zahl der Produktionsstandorte und Absatzmärkte sowie deren geographischen Anordnung - erzeugt Rückkopplungen, wenn durch die Konfiguration nationale Einheiten entstehen, die auf gegenseitige Abnahme oder Zulieferung von Ressourcen angewiesen sind. Die Anzahl der Produktionsstandorte und die Leistungsverflechtungen zwischen den Standorten beeinflussen den internationalen Koordinationsbedarf wesentlich. Je mehr internationale Einheiten ein Unternehmen umfasst, desto vielschichtiger müssen die Koordinationsentscheidungen auch in Bezug auf ökologische Fragestellungen gestaltet werden. ${ }^{319}$

319 Vgl. hierzu und im Folgenden Backhaus, K.; Büschken, J.; Voeth, M.: Internationales Marketing, a. a. O., S. 47 Hf. sowie Plump, D.: Die Koordination des Marketings in multinationalen Unternehmungen: Systemanalyse und Ansätze zur Systemgestaltung, a. a. O., S. 165 und MartInez, J. I.; Jarillo, J. C.: Coordination Demands of International Strategies, in: Journal of International Business Studies, 1991, Nr. 3, S. $429-444$. 
Je höher die Anzahl der Unternehmensstandorte und je größer die internen Leistungsverflechtungen zwischen unterschiedlichen nationalen Standorten sind, desto höher ist der internationale ökologiebezogene Koordinationsbedarf.

In diesem Zusammenhang wird auch festgelegt, inwieweit die Fertigung konzentriert bzw. verteilt wird und welchen Zentralisationsgrad ${ }^{320}$ die Entscheidungsstrukturen aufweisen. ${ }^{321}$ Die internationale Konfiguration beruht dabei auf strategischen Entscheidungen im internationalen Management. ${ }^{322}$ Diese Entscheidung hat Auswirkungen auf die anbieterbezogenen Rückkopplungen, denn die Anzahl und die geographische Verteilung der Standorte determinieren den internationalen Koordinationsbedarf. ${ }^{323}$ In der Automobilindustrie können unterschiedliche internationale strategische Ausrichtungen beobachtet werden:

- International ausgerichtete Unternehmen sind schwerpunktmäßig auf den Heimatmarkt konzentrierte und damit zentralisierte Unternehmen, die ihren Unternehmensbestand durch lukrative Auslandsgeschäfte sichern wollen. Das Geschäft wird dabei überwiegend als Exportgeschäft über die internationalen Divisionen abgewickelt. Als Hauptkonkurrent gilt der stärkste inländische Wettbewerber. In der Vergangenheit konnten insbesondere die Automobilhersteller, die sich besonders auf eine Nische spezialisiert haben, wie bspw. BMW, DAIMLER-BENZ und PORSCHE, als internationale Unternehmen gelten. Heute bedienen diese Hersteller mit ihren Automobilen globale Seg-

320 Nach SIMON ist eine Organisation in dem Maße zentralisiert, in dem Entscheidungen auf relativ hohen Ebenen der Hierarchie getroffen werden. Sie ist in dem Maße dezentralisiert, in dem Entscheidungen von der obersten Führungsebene auf untere Ebenen delegiert werden. Vgl. Simon, H. A.; Guetzkow, H. et al.: Centralization vs. Decentralization in Organizing the Controller's Department, New York 1954, S. 1. Im Gegensatz zu SimON spricht ALBACH Von Dezentralisation, wenn der Entscheidungsspielraum entscheidungsgebundener Abteilungen größer als Null ist, von Zentralisation, wenn die weisungsgebundenen Abteilungen keinen eigenen Ermessensspielraum bei ihren Entscheidungen haben. Vgl. Albach, H.: Die Koordination der Planung im Großunternehmen, in: Schneider, E. (Hrsg.): Rationale Wirtschaftspolitik und Planung in der Wirtschaft von heute, Schriften des Vereins für Socialpolitik, Band 45, Berlin 1967, S. 342. Im Folgenden wird der Auffassung von SimON gefolgt. Vgl. hierzu auch Frese, E.: Grundlagen der Organisation, Konzept - Prinzipien - Strukturen, a. a. O., S. 83 ff.

Vgl. Porter, M. E.: Nationale Wettbewerbsvorteile: Erfolgreich konkurrieren auf dem Weltmarkt, a. a. O., S. 77 f.

HARZING hat empirisch drei unterschiedliche Typen von international tătigen Unternehmen nachgewiesen, die den strategischen Basisausrichtungen multinational, global und transnational entsprechen. Vgl. Harzing, A.-W.: An empirical Analysis and Extension of the Bartlett and Ghoshal Typology of Multinational Companies, in: Journal of International Business Studies, 31. Jg. (2000), Nr. 1, S. 111-112. 
mente, die den Pkw als Statussymbol betrachten und weltweit möglichst identische Fahrzeuge verlangen. ${ }^{324} \mathrm{Da}$ die Auslandsmärkte eine immer gröBere Bedeutung für die Hersteller spielen, sollen sie im Folgenden eher als globale Unternehmen bezeichnet werden.

- Multinational ausgerichtete Unternehmen bilden dezentralisierte und voneinander weitgehend unabhängige nationale Einheiten, die aufgrund einer Anpassung an die jeweiligen lokalen Kontextfaktoren durch eine starke Differenzierung Vorteile erzielen sollen. Die Entscheidungskompetenzen sind weitestgehend an die Tochtergesellschaften delegiert. Die Tochtergesellschaften fungieren dabei im Sinne von Profitcentern. ${ }^{325}$ Die weitgehende Dezentralisation und Differenzierung lässt nur wenige interne anbieterbezogene Rückkopplungen im Umweltbereich entstehen und zieht einen vergleichsweise geringen internen internationalen Koordinationsbedarf nach sich. So hat bspw. General Motors durch den Kauf verschiedener Automobilhersteller in der Vergangenheit ein Markenportfolio aufgebaut, wobei die Mehrzahl der Marken multinational ausgerichtet sind. Die Marke OPEL deckt bspw. den deutschen und europäischen Raum ab, während der Konzern Marken wie Chevrolet, Pontiac, Buick oder CADILlac vornehmlich in den Vereinigten Staaten und Nordamerika anbietet. SUZUKI wiederum dient der Marktabdeckung in Japan und Südostasien.

- Global ausgerichtete Unternehmen sind weltmarktorientiert und weisen eine hohe Zentralisation von Entscheidungen auf. Dabei werden ökologische Entscheidungen weitgehend standardisiert. Einzelnen Tochtergesellschaften kommt oft nur der Charakter ausführender Organe zu. Diese Art der Konfiguration soll eine weitestgehende Ausschöpfung von Economiesof-Scale gewähren. Dies erfordert einen höheren internationalen Koordinationsbedarf als im Fall der multinationalen Unternehmung.

- Die Automobilhersteller RENAULT, DAEwOO aber auch BMW sowie die Unternehmen Mercedes-Benz (DaimlerChrysler), Volvo (Ford), Peugeot und Citroen (PSA), LEXUs (TOYOTA) sowie ALPHA ROMEO (FIAT) verfolgen eine

324 Vgl. zu den Beispielen hier und im Folgenden Proff, H.: Internationale Geschäftsfeldstrategien - Entwicklung einer umfassenden Klassifikation und Anwendung auf die Automobilindustrie, in: Proff, H.; Proff, H. V. (Hrsg.): Strategien für die Automobilindustrie: Ansatzpunkte im strategischen Management und in der Industriepolitik, Wiesbaden 1998, S. 120.

325 Vgl. dazu auch Plump, D.: Die Koordination des Marketings in multinationalen Unternehmungen: Systemanalyse und Ansätze zur Systemgestaltung, a. a. O., S. 172. 
globale Ausrichtung. Soweit die Wertschöpfungsaktivitäten auf internationale Standorte verteilt sind und unterschiedliche Ländermärkte beliefert werden sollen, entsteht ein internationaler Koordinationsbedarf.

- Transnational ausgerichtete Unternehmen stellen Mischformen der Extremtypen dar, die sowohl Elemente der multinationalen und der globalen Unternehmen in sich vereinen. Dabei werden die Kompetenzen einer Unternehmung nach dem Kriterium der wettbewerbsbezogenen Selektion weltweit verteilt. So konzentriert ein transnationales Unternehmen manche Ressourcen in der Unternehmenszentrale, andere im Ausland, wieder andere werden unter den verschiedenen lokalen Standorten aufgeteilt. Die daraus resultierende komplexe Konfiguration von verstreuten, aber spezialisierten Vermögenswerten und Kompetenzen führt im Vergleich zu dem höchsten internationalen Koordinationsbedarf. ${ }^{326}$ Der Automobilhersteller VoLKSWAGEN kann bedingt als transnationale Unternehmung aufgefasst werden. ${ }^{327}$

Die internationale strategische Ausrichtung bestimmt maßgeblich den Zentralisations- und Standardisierungsgrad in einem international tätigen Unternehmen und hat auf diesem Weg auch einen entscheidenden Einfluss auf den internationalen ökologiegerichteten Koordinationsbedarf.

Je größer die Entscheidungszentralisation und je größer der Standardisierungsgrad, desto geringer ist der verbleibende internationale ökologiegerichtete Koordinationsbedarf.

Die folgende Abbildung bestätigt diese Aussage. Sie zeigt im Überblick inwieweit für multinational, global und transnational ausgerichtete Unternehmen die Entscheidungskompetenzen dezentralisiert sind, lokale Anpassungen vorgenommen werden und wie groß der Grad der Interdependenzen und Abhängigkeiten ist. ${ }^{328}$

326 Vgl. Bartlett, C.; Ghoshal, S.: Internationale Unternehmensführung, a. a. O., S. 85.

327 Vgl. Kapitel B 2.2.

328 Vgl. Harzing, A.-W.: An empirical Analysis and Extension of the Bartlett and Ghoshal Typology of Multinational Companies, in: Journal of International Business Studies, 31. Jg. (2000), Nr. 1, S. $101-120$. 


\begin{tabular}{|c|c|c|c|}
\hline Strategische Ausrichtung & Multinational & Global & Transnational \\
\hline $\begin{array}{l}\text { Übereinstimmung mit organisatori- } \\
\text { scher Gestaltung und Rolle der } \\
\text { Tochtergesellschaften } \\
\text { - Dezentralisierter Verbund } \\
\text { - Netzwerkstruktur } \\
\text { - Interne Produktionsverflechtungen } \\
\text { - Verlängerter Arm der Zentrale } \\
\text { - Center of excellence }\end{array}$ & $\begin{array}{l}\text { hoch } \\
\text { niedrig } \\
\text { niedrig } \\
\text { niedrig } \\
\text { niedrig }\end{array}$ & $\begin{array}{l}\text { niedrig } \\
\text { niedrig } \\
\text { niedrig } \\
\text { hoch } \\
\text { niedrig }\end{array}$ & $\begin{array}{c}\text { niedrig } \\
\text { hoch } \\
\text { hoch } \\
\text { niedrig/mittel } \\
\text { hoch }\end{array}$ \\
\hline $\begin{array}{l}\text { Lokale Anpassung } \\
\text { - Lokale Produktion } \\
\text { - Lokale F\&E } \\
\text { - Produktmodifikation } \\
\text { - Anpassung des Marketing }\end{array}$ & $\begin{array}{l}\text { hoch } \\
\text { hoch } \\
\text { hoch } \\
\text { hoch }\end{array}$ & $\begin{array}{c}\text { niedrig } \\
\text { niedrig } \\
\text { niedrig } \\
\text { niedrig/mittel }\end{array}$ & $\begin{array}{l}\text { mittel } \\
\text { mittel } \\
\text { hoch } \\
\text { hoch }\end{array}$ \\
\hline $\begin{array}{l}\text { Interdependenzen } \\
\text { - Gesamtumfang an Interdependenzen } \\
\text { - Umfang der Abhängigkeit von der } \\
\text { Zentrale } \\
\text { - Umfang der Abhängigkeit zwischen } \\
\text { den Tochtergesellschaften }\end{array}$ & $\begin{array}{l}\text { niedrig } \\
\text { niedrig } \\
\text { niedrig }\end{array}$ & $\begin{array}{l}\text { hoch } \\
\text { hoch } \\
\text { niedrig }\end{array}$ & $\begin{array}{l}\text { hoch } \\
\text { mittel } \\
\text { hoch }\end{array}$ \\
\hline
\end{tabular}

Abbildung 25: Typologisierung von internatonal tätigen Unternehmen In Anlehnung an Harzing, A.-W.: An empirical Analysis and Extension of the Bartlett and Ghoshal Typology of Multinational Companies, in: Journal of International Business Studies, Vol. 31 (2000), Nr. 1, S. 110.

\subsection{Unternehmensgröße}

Die Messung der Unternehmensgröße kann sich an Indikatoren wie der Mitarbeiteranzahl, der Anzahl der Marktpartner oder am Nettokapital orientieren. ${ }^{329}$ Diese Indikatoren korrelieren dabei in aller Regel miteinander und sind austauschbar. ${ }^{330}$ Dabei gehen von der Unternehmensgröße unterschiedliche Einflüsse auf den internationalen Koordinationsbedarf aus.

$329 \mathrm{Zu}$ unterschiedlichen Größenbegriffen vgl. Hoffmann, F.: Führungsorganisation, a. a. O., S. $150 \mathrm{ff}$.

330 Vgl. Pugh, D. S. et al.: The Context of Organization Structures, in: Administrative Science Quarterly, 14. Jg. (1969), S. 98, zitiert nach Benkenstein, M.: F \& E und Marketing: Eine Untersuchung zur Leistungsfähigkeit von Koordinationskonzeptionen bei Innovationsentscheidungen, a. a. O., S. 69. 
Mit zunehmender Unternehmensgröße nimmt in aller Regel die Entscheidungsdelegation zu, dabei vergrößert sich der Abstand zwischen Führungsspitze und operativer Ebene. ${ }^{331}$ Wenn Tochtergesellschaften in Bezug auf den standortbezogenen Umweltschutz ihre Entscheidungen dezentral treffen können, wächst mit zunehmender Größe auch die Gefahr einer organisatorischen Verselbstständigung und dem Auftreten von Zielkonflikten. Dies führt zu anbieterbezogenen Rückkopplungen und erhöht somit die Kommunikationsund Koordinationsproblematik. Weiterhin haben empirische Untersuchungen gezeigt, dass sich Unternehmen mit zunehmender Größe stärker ökologischen Problemstellungen widmen als kleinere Unternehmen. ${ }^{332}$ Durch Firmenfusionen und strategische Allianzen hat sich die Unternehmensgröße in der Automobilindustrie stark vergößert. International tätige Automobilunternehmen, die nach vollständiger Abdeckung der gesamten Produktpalette und einer weltweiten Präsenz streben, prägen das Bild. ${ }^{333}$ Die international tätigen Autombilhersteller gelten somit als vergleichsweise große Unternehmen.

Mit zunehmender Unternehmensgröße erhöht sich aufgrund anbieterbezogener Rückkopplungen der internationale ökologiebezogene Koordinationsbedarf.

Obwohl mit zunehmender Größe der Koordinationsbedarf tendenziell zunimmt, wird gleichzeitig der Teil der gleichartigen Aufgaben größer. Aufgrund der Homogenität vieler Aufgaben lassen sich Größenvorteile generieren, welche die größeninduzierten Probleme der Koordination verringern. Diese Größenvorteile werden insbesondere von der Automobilindustrie gesucht. ${ }^{334}$ In Bezug auf den Umweltschutz können bspw. durch einen gemeinsamen Rückgriff auf eine zentrale Umweltabteilung Economies-of-Scope realisiert werden. Erfahrungen und Wissen der Mitarbeiter im Umweltschutz lassen sich teilweise auf andere Ländermärkte übertragen, was die Nutzung von internationalen Erfahrungseffekten ermöglicht. Weiterhin können bestimmte Routinen und Tätigkeiten, die in jedem Ländermarkt anfallen, standardisiert werden.

331 Eine zunehmend dezentrale Entscheidungsverteilung hăngt oft eng mit der Heterogenität der internationalen Umsysteme zusammen. Vgl. Hoffmann, F.: Führungsorganisation, a. a. O., S. 158 ff. sowie Benkenstein, M.: F \& E und Marketing: Eine Untersuchung zur Leistungsfähigkeit von Koordinationskonzeptionen bei Innovationsentscheidungen, a. a. O., S. $71 \mathrm{f}$.

So stehen auch mehr Mitarbeiter zur Verfügung, die Umweltschutzaufgaben bearbeiten können. Vgl. Wolter, F.: Umweltmanagement in Europa - eine empirische Untersuchung auf Grundlage des Europäischen Umweltmanagement-Barometers (EBEB), a. a. O., S. 27.

Vgl. FOCUS (Hrsg.): Der Markt der Automobile: Daten, Fakten, Trends, 2000, S. 2.

Vgl. dazu die Ausführungen in Kapitel B 1.1. 


\subsection{Organisationsstruktur}

Die Organisationsstruktur umfasst ein System von Regelungen, welches die Handlungsweisen der Organisationsmitglieder so beeinflussen soll, dass eine effiziente Steuerung des Verhaltens und der Leistungen erreicht wird. ${ }^{335}$ Die grundlegende Strukturdimension stellt dabei die Arbeitsteilung bzw. organisatorische Differenzierung dar. Der Koordinationsbedarf resultiert nun unmittelbar aus der Art und der Intensität zielbezogener Interdependenzen zwischen den arbeitsteiligen Organisationseinheiten und dem Ausmaß der diesen Bereichen eingeräumten Entscheidungsspielräume. Die Art und Intensität der aufgaben- und zielbezogenen Interdependenzen hängen dabei von der gewählten Spezialisierungsart und dem Spezialisierungsgrad ab. Grundlegend werden in Bezug auf die Differenzierung die verrichtungs- bzw. funktionsorientierte und die objektorientierte Spezialisierung unterschieden. ${ }^{336}$ Die Regel stellt dabei eine funktionale Spezialisierung der Organisation auf zweiter Hierarchieebene dar. ${ }^{337}$ Eine Erhöhung des Spezialisierungsgrades führt tendenziell zu einem höheren Koordinationsbedarf. ${ }^{338}$

Die durch Arbeitsteilung und Spezialisierung erfolgte Teilung der Unternehmensgesamtaufgabe ist zur Erfüllung der Unternehmenszielsetzung durch die Schaffung von Schnittstellen aufzuheben. Die Art der geschaffenen Verbindungen beeinflussen dabei ebenfalls den Koordinationsbedarf. In diesem Zusammenhang können vertikale und horizontale Verknüpfungen unterschieden werden. ${ }^{339}$ Während vertikale Koordinationsbeziehungen einseitige Beziehungen in der Linie darstellen und Koordinationsentscheide einer höheren hierarchischen Ebene eine Abstimmung der Entscheidungen auf nachgeordneter Stufe bewirken, liegen bei horizontalen bzw. dezentralen Koordinationsbeziehungen keine Über- oder Unterordnungsverhältnisse vor. Die interdependenten

335 Die durch Regeln geschaffene Ordnung eines sozialen Systems nennt man Organisationsstruktur. Vgl. Schreyögg, G.: Organisation: Grundlagen moderner Organisationsgestaltung, Wiesbaden 1996, S. $11 \mathrm{f}$.

336 Vgl. hierzu und im Folgenden Hoffmann, F.: Führungsorganisation, a. a. O., S. 308 ff. sowie Benkenstein, M.: $F$ \& E und Marketing: Eine Untersuchung zur Leistungsfähigkeit von Koordinationskonzeptionen bei Innovationsentscheidungen, a. a. O., S. 64 ff.

337 Vgl. Schreyögg, G.: Organisation: Grundlagen modemer Organisationsgestaltung, Wiesbaden 1996, S. $142 \mathrm{f}$.

Vgl. Lassmann, A.: Organisatorische Koordination, Konzepte und Prinzipien zur Einordnung von Teilaufgaben, a. a. O., S. 193.

339 Vgl. Steffenhagen, H.: Konflikt und Koordination in Distributionssystemen, Münster 1972 , S. 24 sowie Schreyögg, G.: Organisation: Grundlagen modemer Organisationsgestaltung, Wiesbaden 1996, S. $151 \mathrm{ff}$. 
Entscheidungen müssen hier zwischen den Entscheidungsträgern selbst koordiniert werden, was zu einem höheren Koordinationsaufwand führt. ${ }^{340}$ Generell kann festgehalten werden, dass die Art der Spezialisierung und Verknüpfung einen Einfluss auf den internationalen ökologiegerichteten Koordinationsbedarf hat. Im Umweltmanagement in der Automobilindustrie werden dabei sowohl vertikale als auch horizontale Verknüfungen hergestellt. Stark zentralisierte Unternehmen, wie bspw. TOYOTA, wählen dabei eher vertikale Verknüpfungen, während das Umweltmanagement bspw. im VOLKSWAGENKonzern in höherem Maße dezentralisiert ist. ${ }^{341}$

Vertikale Verknüpfungen zwischen national unterschiedlichen Unternehmensteilen bedingen einen geringeren internationalen ökologiegerichteten Koordinationsbedarf als horizontale Verknüpfungen.

\subsection{Führungsstil}

Das Verhalten des Managements bei der Erfüllung seiner Funktionen, wie Organisation, Planung, Entscheidung, Führung und Kontrolle, prägt einen Management- bzw. Führungsstil. ${ }^{342}$ Durch die Art des Führungsstils werden die Interaktionen innerhalb eines Unternehmens beeinflusst, sodass davon auszugehen ist, dass alternativen Führungsstilen auch ein unterschiedlicher Einfluss auf den internationalen Koordinationsbedarf zukommt. Die möglichen Ausprägungen des Führungsstils werden üblicherweise in einem Kontinuum zwischen autoritär bis partizipativ ${ }^{343}$ angegeben. In diesem Zusammenhang ist die autoritäre Führung ein Ausdruck einer zentralen Koordination, die partizipative bzw. kooperative Führung dagegen eher einer dezentralen Koordination zuzuordnen. ${ }^{344}$ Die Führungsstile unterscheiden sich demnach vor allem darin, in welchem Umfang das Management der ausländischen Tochtergesellschaften in ökologiebezogene Entscheiden einbezogen wird.

340 Vgl. Plump, D.: Die Koordination des Marketings in multinationalen Unternehmungen: Systemanalyse und Ansätze zur Systemgestaltung, a. a. O., S. $164 \mathrm{ff}$.

341 Vgl. dazu auch die Ausführungen in Kapitel C 3.2.1.

342 Vgl. Benkenstein, M.: F \& E und Marketing: Eine Untersuchung zur Leistungsfähigkeit von Koordinationskonzeptionen bei Innovationsentscheidungen, a. a. O., S. 83 ff.

343

Vgl. zu weiterreichenden Unterscheidungen der Führungsstile Tannenbaum, R.; Schmldt, W.: How to Choose a Leadership Pattern, in: Litterer, J. A. (Hrsg.): Leadership and Organization, New York, u. a. S. 122 - 130.

344 Plump, D.: Die Koordination des Marketings in multinationalen Unternehmungen: Systemanalyse und Ansätze zur Systemgestaltung, a. a. O., S. $149 \mathrm{ff}$. 
Da alle Koordinationsaufgaben zentral von einer hierarchisch höhergelagerten Stelle erfüllt werden, hat die autoritäre Führung im Umweltmanagement grundsätzlich einen eher geringeren Koordinationsbedarf der Auslandsniederlassungen zur Folge. Der geringe Bedarf ist darauf zurückzuführen, dass sowohl die Interdependenzen als auch die Entscheidungsspielräume der Auslandstöchter bei dieser Form des Führungsstils äußerst gering sind. Der japanische Automobilkonzern TOYOTA setzt dabei die zentral getroffenen Entscheidungen tendenziell autoritär in den ausländischen Tochtergesellschaften um.

Auf der anderen Seite ist der Koordinationsbedarf in einem eher partizipativ geführten Unternehmen höher, da sich eine Vielzahl von Auslandsmanagern multilateral koordinieren muss und damit die Anzahl der Interdependenzen sowie Entscheidungsspielräume und Zielkonflikte zwischen den Märkten anwachsen kann. Die markenübergreifenden und markenbezogenen Abstimmungsprozesse im internationalen Umweltmanagement der VoLKSWAGEN AG sind Ausdruck eines partizipativen Führungsstils. ${ }^{345}$

Je autoritärer der Führungsstil, desto geringer ist der internationale ökologiebezogene Koordinationsbedarf.

\section{Systematisierung der Interdependenzen im situativen Beziehungs- rahmen}

Die vorangegangenen Überlegungen verdeutlichen, dass die situativen Kontextfaktoren nicht isoliert auf den Koordinationsbedarf im internationalen Umweltmanagement einwirken, sondern zwischen den einzelnen Faktoren wiederum Wechselwirkungen bestehen bzw. diese nicht überschneidungsfrei sind. Somit wird der internationale ökologiebezogene Koordinationsbedarf auch weniger von Einzelfaktoren als vielmehr von Situationsclustern bestimmt. Abbildung 26 verdeutlicht ausgewählte Wechselwirkungen zwischen den Kontextfaktoren, die von empirischen Untersuchungen zum ökologischen Unternehmensverhalten bzw. zum internationalen Management gestützt bzw. plausibel erklärt werden können. ${ }^{346}$

345 Dies basiert auf den Interviews mit Herrn StOBBE und Herrn MINTE von VolksWAGEN.

346 Dabei ist an dieser Stelle darauf hinzuweisen, dass die untersuchten Kontextfaktoren wie auch die dargestellten Interdependenzen zwischen diesen Kontextfaktoren keinen Anspruch auf Vollständigkeit erheben. 


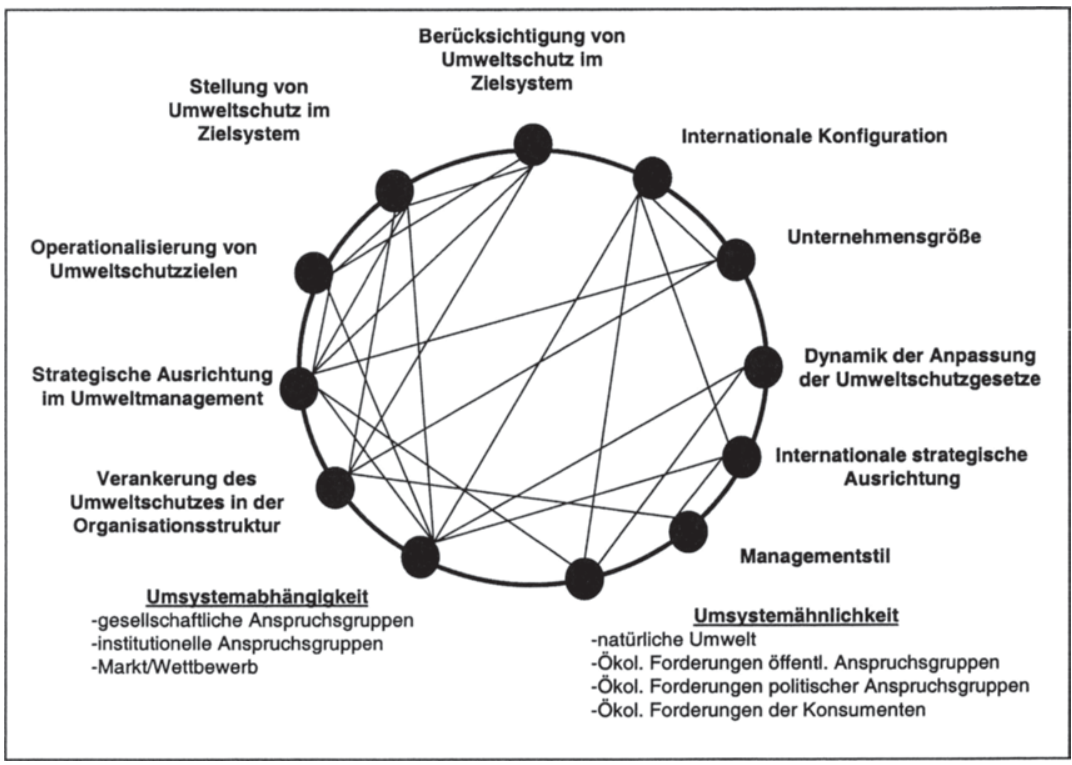

Abbildung 26: Ausgewählte Interdependenzen zwischen den Kontextfaktoren

Quelle: Eigene Darstellung

Bezüglich ihrer Wirkungen auf den Koordinationsbedarf kann zwischen komplementären und substitutiven Beziehungen differenziert werden. Findet bspw. Umweltschutz als Unternehmensziel Berücksichtigung und nimmt der Umweltschutz dabei eine hohe Stellung im Zielsystem ein, wird zumeist eine proaktive strategische Ausrichtung im Umweltmanagement angestrebt. Eine intensive Auseinandersetzung mit Umweltschutzzielen durch das Management kann zur Verabschiedung operativer Zielsetzungen im Umweltschutz führen, die bereits einen Beitrag zur Koordination des ökologiegerichteten Verhaltens der ausländischen Tochtergesellschaften leisten können.

Die internationale Konfiguration, die maßgeblich durch die internationale strategische Ausrichtung beeinflusst wird, bestimmt den Zentralisationsgrad und damit über den Entscheidungsspielraum der ausländischen Tochtergesellschaften. Je nachdem, ob die bearbeiteten Ländermärkte in Bezug auf ökologierelevante Fragestellungen eher ähnlich oder eher different sind, erweist sich eine mehr oder weniger starke Entscheidungszentralisation als günstig. So wählen Unternehmen bspw. bei weitgehend heterogenen Ländermärkten eine multinationale Ausrichtung mit hoher Entscheidungsdezentralisation und Differenzierung 
des Verhaltens. Agieren die Tochtergesellschaften dabei unabhängig voneinander, fält kaum ökologiebezogener Koordinationsbedarf aufgrund anbieterbezogener Rückkopplungen an.

Eine starke Umsystemkopplung kann aber zu externen Rückkopplungen führen, die nach Entscheidungszentralisation und standardisiertem ökologischen Verhalten verlangen. So können sich die einzelnen Kontextfaktoren hinsichtlich ihrer Wirkung gegenseitig verstärken oder abschwächen.

Unabhängig davon, welche einzelnen Kontextfaktoren bzw. welche situativen Cluster die Höhe des Koordinationsbedarfs bestimmen, ist davon auszugehen, dass die Wirkungen der situativen Kontextfaktoren auf den internationalen ökologiebezogenen Koordinationsbedarf indirekter Natur sind. Der Koordinationsbedarf wird direkt von Art und Intensität der internen und externen Interdependenzen sowie von den ökologischen Zlelen des Unternehmens bestimmt.

Auf Basis von unterschiedlichen Expertenurteilen konnte die in Abbildung 27 dargestellte Einschätzung über die Ausprägung der einzelnen endogenen und exogenen Einflussfaktoren in der Automobilindustrie zur Bestimmung des internationalen Koordinationsbedarfs abgeleitet werden. Dabei ist neben dem Durchschnittsprofil auch die Streuung der Einschätzungen angedeutet, welche die Heterogenität der Einschätzungen andeutet. 


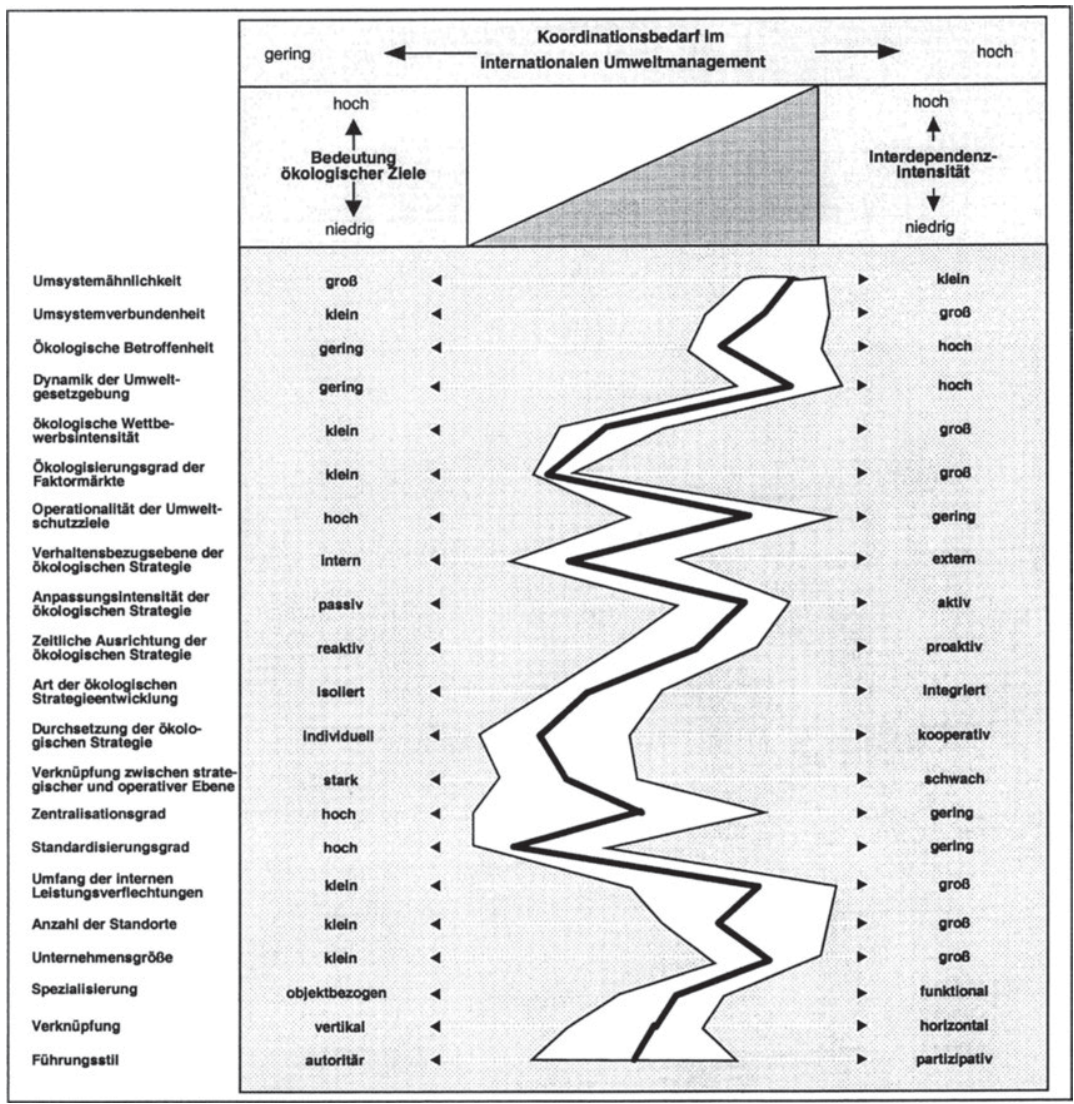

Abbildung 27: Profil der Ausprägung unterschiedlicher Kontextfaktoren in der Automobilindustrie zur Ableitung des internationalen ökologiebezogenen Koordinationsbedarfs

Quelle: Eigene Darstellung

Die Ähnlichkeit der Umsysteme wird als vergleichsweise gering eingestuft, da sich die ökologiebezogenen Kontextfaktoren insbesondere zwischen den Triademärkten und den Schwellenländern z. T. stark unterscheiden. Die Umsystemverbundenheit wird von den Experten hingegen als vergleichsweise hoch bewertet, da zunehmend international organisierte öffentliche und marktliche Umweltschutzgruppen das ökologiegerichtete Unternehmensverhalten an allen Standorten beobachten und beurteilen. Die ökologische Betroffenhelt in der Automobilindustrie wird dabei insbesondere aufgrund der ökologischen Probleme der Produkte als relativ hoch angesehen. Dabei verweisen die Experten 
auf die hohe Dynamik der Umweltgesetzgebung in den Schwellenländern, während sie in den Triademärkten eine weniger schnelle Änderung der automobilbezogenen Umweltschutzgesetze konstatieren. Die ökologische Wettbewerbsintensität im Automobilmarkt wird derzeit hingegen eher als wenig bis mittel stark charakterisiert, da einzig der Kaftstoffverbrauch als ökologisches Kriterium Berücksichtigung in den Kaufentscheidungen der Konsumenten findet. Der ökologiebezogene Wettbewerb in der Automobilindustrie konzentriert sich dabei eher auf die Entwicklung zukunftsfähiger Antriebskonzepte, die allerdings noch nicht marktreif sind.

Die Automobilhersteller weisen oft wenig operationale Umweltschutzziele aus. Somit werden Umweltsachziele im fertigungsbezogenen Umweltschutz zumeist lokal eigenverantwortlich an die national einschlägigen Umweltschutzgesetze angepasst. Dabei ist zwar generell die Umweltpolitik des Unternehmens für alle Standorte verbindlich, allerdings ist die Umweltpolitik nach Meinung der Experten zumeist wenig spezifisch formuliert. Die Verhaltensbezugsebene von Umweltschutzstrategien war bis vor einigen Jahren in der Automobilindustrie überwiegend intern ausgerichtet. Zunehmende Relevanz von Stakeholdern, die Diskussion über den Treibhauseffekt etc. haben aber dazu geführt, dass die Automobilhersteller das Thema Umweltschutz mittlerweile stärker nach außen kommunizieren. Unterstützt wurde dieser Trend nach Expertenschätzung insbesondere durch publikumswirksame Umweltratings.

Dennoch sind die ökologischen Strategien im Durchschnitt weiterhin eher intern ausgerichtet. Die Anpassungsintensität der Umweltschutzstrategien in der Automobilindustrie wird dabei als nur bedingt innovativ eingeschätzt. Produkte als auch Produktionsstätten werden zumeist von den zuständigen Entwicklungs- und Planungsabteilungen eigenständig geplant, wobei die Umweltschutzabteilungen nur in eingeschränktem $\mathrm{Maße}$ in die Planung einbezogen werden. Sofern verbesserter Umweltschutz zu erhöhten Kosten führt, scheitern ökologiebezogene Maßnahmen oft an internen Widerständen. Dies bedingt nach Ansicht der Experten, dass sich die Umweltstandards meist an der bestehenden Gesetzgebung, also adaptiv, orientieren bzw. lediglich unmittelbar bevorstehende Gesetzesvorgaben Berücksichtigung finden. Dies schließt jedoch zeitlich proaktives Handeln und entsprechende Forschungsaktivitäten nicht generell aus. 
Die Art der Strategieentwicklung ist aufgrund der beträchtlichen Größe und der relativ hohen Anzahl der Standorte der meisten Automobilunternehmen tendenziell eher isoliert. Ökologische Problemstellungen werden dabei oft allein in den zuständigen Abteilungen oder an den einzelnen Standorten behandelt. Je mehr allerdings seitens der Öffentlichkeit das Thema Umweltschutz an die Automobilunternehmen herangetragen wird, umso mehr beschäftigen sich auch die Unternehmensleitungen und andere Abteilungen mit ökologischen Problemstellungen. Die Nachhaltigkeits-Diskussion hat nach Einschätzung der Experten dabei maßgeblich dazu beigetragen, dass zunehmend auch Abteilungen wie Regierungsbeziehungen, Personalwesen, Kommunikation etc. in die Diskussion ökologischer Themen einbezogen werden. Da auf internationaler Ebene keine global tätigen Verbände existieren, findet die Durchsetzung der ökologischen Ausrichtung zumeist auf individueller Ebene statt. Allerdings sind in den Regionen der Triademärkte durchaus Verbände der Automobilindustrie präsent, sodass auf regionaler Ebene verschiedene ökologiegerichtete Maßnahmen kollektiv durchgeführt werden. Die Verknüpfung von strategischer und operativer Ebene im Umweltmangement ist in der Automobilindustrie tendenziell stark ausgeprägt, wobei hier strategieabhängige, herstellerspezifische Unterschiede zu beachten sind.

Der Zentralisationsgrad in der Automobilindustrie kann für den produktbezogenen Umweltschutz ähnlich wie der Standardisierungsgrad als vergleichsweise hoch angesehen werden. Aufgrund der Globalisierung nehmen auch die bereits hohen internen Leistungsverflechtungen zwischen national unterschiedlichen Standorten weiter zu. Dabei hängt der Grad der Zentralisation und damit auch der Standardisierungsgrad von der internationalen Ausrichtung der Hersteller ab. In Bezug auf den fertigungsbezogenen Umweltschutz können diese Tendenzaussagen hingegen nicht bestätigt werden. Hier beschreiben die Experten eher dezentrale Steuerungsstrukturen und zumindest für Altanlagen ein eher differenziertes Bild. Demgegenüber werden die neuen Anlagen, auch aufgrund der Wahl zumeist gleicher Systemlieferanten, als ökologisch weitgehend standardisiert charakterisiert. Die Hersteller sind nach Angabe der Experten zumeist eher funktional organisiert, wobei der Umweltschutz auch um eine Vielzahl objektbezogener Strukturen ergänzt wird. Dabei lassen sich bezüglich der internen Verknüpfungen im Umweltschutz und des Führungsstils keine homogenen Aussagen treffen. 
Nachdem in diesem Kapitel am Beispiel der Automobilindustrie aufgezeigt wurde, wie durch den Einfluss endogener und exogener Kontextfaktoren ein internationaler ökologiebezogener Koordinationsbedarf entstehen kann, sollen im folgenden Kapitel unterschiedliche Koordinationsinstrumente auf ihre Eignung zur Reduktion und Deckung des ökologisch bedingten Koordinationsbedarfs analysiert werden. 


\section{Koordinationskonzeptionen im internationalen Umwelt- management}

Unter einer ökologiebezogenen Koordinationskonzeption kann die Gesamtheit der Mechanismen, Maßnahmen und Instrumente verstanden werden, die in einem Unternehmen zur zielgerichteten Koordination des internationalen ökologiegerichteten Verhaltens angewendet wird. ${ }^{347}$

Aufbauend auf der Erkenntnis, dass bei internationaler Geschäftstätigkeit vielfältige ökologiebezogene, wertschöpfungssysteminterne und -externe Rückkopplungen entstehen können, setzt die Ableitung einer Koordinationskonzeption eine Auseinandersetzung mit der Koordination als „wünschenswerte" Abstimmung interdependenter Entscheidungsträger voraus. Da koordinierende Maßnahmen nicht losgelöst von ihren Zielsetzungen betrachtet werden können, ${ }^{348}$ werden im Folgenden zunächst die grundlegenden Ziele der Koordination des ökologiegerichteten Verhaltens sowie basisstrategische Optionen im internationalen Umweltmanagement vor dem Hintergrund der Automobilindustrie dargestellt.

Im Anschluss werden konkrete Instrumente und Maßnahmen gesucht, die Art und Umfang der ökologiebezogenen Rückkopplungen zwischen Organisationsbereichen in unterschiedlichen Ländermärkten steuern und eine Abstimmung des ökologiegerichteten Verhaltens der autonomen nationalen Teileinheiten auf eine gemeinsame Zielsetzung gewährleisten. Hier findet eine Systematisierung des Koordinationsinstrumentariums statt, welches sich im internationalen Umweltmanagement anwenden lässt. Darauf aufbauend gilt es, idealtypische strategiebezogene Kombinationen von Koordinationsinstrumenten zu einer Koordinationskonzeption zusammenzustellen. Zu diesem Zweck wird ein Umweltmanagementsystem als Koordinationskonzeption gewählt. Die aktuelle Auseinandersetzung mit Umweltmanagementsystemen in der Betriebswirt-

347 Vgl. Hoffmann, F.: Führungsorganisation, a. a. O., S. 316.

348 Vgl. Meier, A.: Koordination in der Leistungsorganisation, in: Zeitschrift für betriebswirtschaftliche Forschung, 13. Jg. (1961), S. 538 - 553, hier S. 540; KIrsch, W.: Die Koordination von Entscheidungen in Organisationen, in: Zeitschrift für betriebswirtschaftliche Forschung, 23. Jg. (1971), S. 61 - 82, hier S. 66; Benkenstein, M.: F \& E und Marketing: Eine Untersuchung zur Leistungsfähigkeit von Koordinationskonzeptionen bei Innovationsentscheidungen, a. a. O., S. 122 f.; MacharzIna, K.; Oesterle, M.-J.: Bestimmungsgrößen und Mechanismen der Koordination von Auslandsgesellschaften, in: Macharzina, K.; Oesterle, M.-J. (Hrsg.): Handbuch Internationales Management: Grundlagen - Instrumente - Perspektiven, Wiesbaden 1997, S. 614. 
schaftslehre sowie ihre fortschreitende Verbreitung in der internationalen Unternehmenspraxis, die an der stetig steigenden Zahl an Zertifizierungen nach der EMAS-Verordnung bzw. dem ISO Standard 14.001 ablesbar ist, zeugen von der wachsenden Bedeutung dieser Systeme, die generell der Koordination des ökologiegerichteten Verhaltens dienen.

\section{Ziele einer internationalen ökologiegerichteten Koordination}

Im Rahmen der Umweltschutzkonzeption einer Unternehmung bilden Ziele die Richtgrößen, an denen sich die Basisstrategien und operativen Maßnahmen im internationalen Umweltmanagement ausrichten. Die Koordination durch Ziele kann dabei anhand des Konkretisierungsgrades bzw. nach der zeitlichen Befristung differenziert werden. Unbefristet gültige Ziele sind vielfach in Form von Unternehmensgrundsätzen bzw. -leitlinien in der betrieblichen Umweltpolitik kodifiziert. Das Ziel einer Koordination im internationalen Umweltmanagement ist dabei generell die Ausrichtung der ökologiebezogenen Aktivitäten der Landesgesellschaften auf die Zielsetzung der Gesamtunternehmung. Diese Zielsetzung ist für das weitere Vorgehen allerdings zu allgemein und wenig operational.

Zur Ableitung der Zielsetzungen der Koordination im internationalen Umweltmanagement sollen deshalb die Ziele des internationalen Umweltmanagements als Ausgangspunkt untersucht werden. Die Vorgabe von langfristigen, strategischen Zielen im internationalen Umweltmanagement erfolgt in der strategischen Planung, die sich in der internationalen Umweltmanagementstrategie ausdrückt. Dabei steht neben der zentralisierten und formalisierten Planung im Sinne eines top-down-Planungsprozesses die dezentrale Planung durch einen bottom-up-Planungsprozess. Im Sinne einer Verbindung von topdown- und bottom-up-Planung steht ein am Gegenstromverfahren orientierter Planungsprozess. ${ }^{349}$ Die Betrachtung von Mittel-Zweck-Vermutungen zwischen den Zielen im Zielsystem erlaubt es, aus den Zielen des internationalen Umweltmanagements in einem zweiten Schritt die Ziele der internationalen ökologiegerichteten Koordination abzuleiten. Somit werden im Folgenden die Koordinationsziele im internationalen Umweltmanagement in direkte und indirekte Koordinationsziele unterschieden. Während die indirekten Ziele die Aufgaben inhaltlich konkretisieren und die verschiedenen Ziele des interna-

349 Vgl. Welge, M. K. (Hrsg.); Böttcher, R.; Paul, Th.: Das Management globaler Geschäfte: Grundlagen, Analysen, Handlungsempfehlungen, München, Wien 1998, S. 70. 
tionalen Umweltmanagements zum Gegenstand haben, bestimmen die direkten Ziele der Koordination den Umfang der Koordination und damit das Verhältnis von Autonomie- und Koordinationskosten. ${ }^{350}$

Im Zielsystem einer Unternehmung werden die unterschiedlichen Ziele nach inrem Beitrag zur Ereichung der unternehmensbezogenen Oberziele in eine hierarchische Rangfolge gebracht. Die Ziele im Umweltmanagement und im internationalen Management können dabei als Zwischenziele ${ }^{351}$ zur Erreichung der unternehmensbezogenen Oberziele gewertet werden. ${ }^{352}$ Die Ziele im internationalen Umweltmanagement stellen in diesem Kontext eine Konkretisierung der im Rahmen der ökologischen und internationalen Ausrichtung formulierten Ziele dar. Sie beziehen sich im Gegensatz zu den allgemeinen ökologischen Zielen auf die konkreten Tatbestände der internationalen Tätigkeiten mit Ökologiebezug und definieren wünschenswerte Zustände, die durch zieladäquate Umsetzung im Rahmen des Umweltmanagements und durch eine zielgerechte internationale Koordination anzustreben sind. ${ }^{353}$

Findet durch die Unternehmung eine eigenständige Zielfestlegung in Bezug auf internationale Umweltschutzziele statt, können daraus die inhaltlichen, indirekten Ziele einer internationalen Koordination des ökologiegerichteten Verhaltens abgeleitet werden. Vor diesem Hintergrund kommt der Festlegung der Ziele im internationalen Umweltmanagement durch die Unternehmensführung der Charakter einer conditio sine qua non für die internationale, ökologiegerichtete Koordination zu, da der Einsatz von Koordinationsmaßnahmen in unmittelbarem Zusammenhang mit den Zielen im internationalen Umweltmanagement steht. So erhöhen sich bspw. durch Festlegung anspruchsvollerer Vermeidungsziele, wie bspw. eine internationale Senkung des Kraftstoffverbrauchs der

350 Da die Koordination im internationalen Umweltmanagement nur einen mittelbaren Beitrag zur Erreichung dieser Ziele leisten kann, werden die Koordinationsziele im internationalen Umweltmanagement als indirekte Ziele bezeichnet. Sie stellen den inhaltlichen Ausgangspunkt der Koordination im internationalen Umweltmanagement dar. Vgl. Schweltzer, M.; Friedl, B.: Beitrag zu einer umfassenden Controlling-Konzeption, in: Spremann, K.; Zur, E. (Hrsg.): Controlling, Wiesbaden 1992, S. 143.

351 Vgl. grundlegend zu End- und Zwischenzielen Mahoney, Th. A.; Weitzel, W.: Managerial Models of Organizational Effectiveness, in: Administrative Science Quarterly, 14. Jg. (1969), S. 357 - 365; Frese, E.: Grundlagen der Organisation, Konzept - Prinzipien Strukturen, a. a. O., S. 288.

Vgl. hierzu und im Folgenden auch Kapitel B. 1 und die dort angegebene Literatur.

353 Vgl. Bolz, J.: Wettbewerbsorientierte Standardisierung der internationalen Marktbearbeitung: Eine empirische Analyse in europäischen Schlüsselmärkten, a. a. O., S. 113 sowie die dort angegebene Literatur. 
Fahrzeugflotte in der Automobilindustrie, die Anforderungen an die Informationsbeschaffungs- und -verarbeitungsaktivitäten der Unternehmung. Dies führt zu einer Steigerung des internationalen, ökologiegerichteten Koordinationsbedarfs. $^{354}$

Die mit einem internationalen Umweltmanagement verfolgten Ziele können dabei generell in Effizienz- und Wirkungsziele unterschieden werden. ${ }^{355} \mathrm{Im}$ Folgenden werden die im Rahmen der Automobilindustrie verfolgten Effizienzund Wirkungsziele im internationalen Umweltmanagement abgeleitet, um darauf aufbauend mögliche Koordinationsziele festzulegen.

Da die Wettbewerbsintensität und der Kostendruck in der Automobilbranche sehr hoch sind, müssen auch im Rahmen des internationalen Umweltmanagements Maßnahmen ergriffen werden, die dem Ziel der Kostensenkung dienen. Als Effizienzzlele sind somit Größen-, Erfahrungs- Synergie-, Arbitrage- und Entwicklungsstand-Portfolio-Effekte zu diskutieren. ${ }^{356}$

- Größeneffekte geben die mit einer größeren Ausbringungsmenge verbundenen Effizienzsteigerungen wieder. ${ }^{357}$ Verantwortlich für das Entstehen von Kostendegressionseffekten sind Economies-of-Scale- und Fixkostendegressionseffekte. Die Ausnutzung von Kostendegressionseffekten erfordert i. d. R. eine internationale Produkt- wie auch Prozessstandardisierung. Die Produktstandardisierung drückt sich in der Automobilindustrie durch die internationale Verwendung von gleichen Modulkomponenten wie

354 Vgl. Macharzina, K.: Rahmenbedingungen und Gestaltungsmöglichkeiten bei Umsetzung von globalen Strategieansätzen, in: Schmalenbach-Gesellschaft/Deutsche Gesellschaft für Betriebswirtschaft $\Theta$. V. (Hrsg.): Internationalisierung der Wirtschaft, Stuttgart 1993; Brodel, D.: Internationales Umweltmanagement: Gestaltungsfelder - Determinanten Ausprägungen, a. a. O., S. 441.

Dieses Vorgehen lehnt sich an die Diskussion der mit der internationalen Marktbearbeitung verfolgten Ziele an. Vgl. Buzzel; R. D.: Can You Standardize Multinational Marketing?, in: Harvard Business Review, 46. Jg. (1968), S. 102 - 113, insb. S. 103; Kreutzer, R.: Reif für Global Marketing?, in: Absatzwirtschaft, Sonderausgabe 10 (1985), S. $144-157$, insb. S. 146; Bolz, J.: Wettbewerbsorientierte Standardisierung der internationalen Marktbearbeitung: Eine empirische Analyse in europäischen Schlüsselmärkten, a. a. O., S. 113.

Vgl. hierzu und im Folgenden Brodel, D.: Internationales Umweltmanagement: Gestaltungsfelder - Determinanten - Ausprägungen, a. a. O., S. 371 ff. sowie Bolz, J.: Wettbewerbsorientierte Standardisierung der internationalen Marktbearbeitung: Eine empirische Analyse in europäischen Schlüsselmärkten, a. a. O., S. 114 ff.

357 Vgl. Kogut, B.: International Sequential Advantages and Network Flexibility, in: Bartlett, Ch. A. et al. (Hrsg.): Managing the Global Firm, London, New York 1990, S. $48 \mathrm{ff}$. 
Gleichteilen, Antriebssträngen und Bodengruppen aus. ${ }^{358}$ Dementsprechend erweist es sich tendenziell als vorteilhaft, wenn die Produkte nach Maßgabe der strengsten nationalen produktbezogenen Umweltschutznormen standardisiert hergestellt werden, um insbesondere Economies-of-Scale-Effekte sowie Fixkostendegressionseffekte zu generieren.

- Durch den länderübergreifenden Einsatz von Unternehmens-Know-how im Umweltschutz lassen sich internationale Erfahrungseffekte nutzen. Erfahrungseffekte sind dynamischer Natur und beziehen sich auf die Realisierung von Kostenvorteilen im Zeitablauf, die zu Wettbewerbsvorteilen avancieren können. Je mehr Erfahrung ein Unternehmen bspw. im Leichtbau oder bei der Steigerung der Kraftstoffeffizienz bereits gesammelt hat, desto höher sind die Erfahrungen in diesen Bereichen und desto einfacher lassen sich die Erfahrungen und Routinen auch auf weitere Länder übertragen. Dies führt zu sinkenden Kosten. ${ }^{359}$

- Synergie- oder Economies-of-Scope-Effekte umschreiben das Phänomen, dass die Gesamtkosten zweier gemeinsam durchgeführter Aktivitäten geringer ist als eine getrennte Erstellung. Diese Synergieeffekte können durch eine gemeinsame Nutzung von flexibel einsetzbaren Ressourcen, Anlagen oder Personal generiert werden. So ist im Umweltbereich nicht für jede ausländische Tochtergesellschaft der Aufbau eigener Zentralstellen im Umweltschutz notwendig, wenn bspw. Mitarbeiter aus der Zentrale des Unternehmensverbundes auch Beratungsleistungen für Tochtergesellschaften erbringen können. ${ }^{360}$

- Die Ausnutzung internationaler Faktorunterschiede kann bei internationaler Unternehmenstätigkeit zur Ausschöpfung umweltschutzbezogener Arbitrage-Effekte führen. Sofern Ressourcen in bestimmten Ländern z. B. durch nicht umweltverträgliche Gewinnungsmethoden oder durch nicht nachhaltige

358 Vgl. dazu die Skalensensitivităt ausgewăhlter Prozesse bei der Automobilproduktion bei Doz, Y.: Strategic Management in Multinational Companies, Oxford et al. 1986, S. 69; Meffert, H.; Bolz, J.: Erfolgswirkungen der internationalen Marketingstandardisierung, in: Meffert, H.; Wagner, H.; Backhaus, K. (Hrsg.): Arbeitspapiere der Wissenschaftlichen Gesellschaft für Marketing und Unternehmensführung e. V., Nr. 85, Münster 1994, S. 4 ff.

Vgl. Ghemawat, P.: Dauerhafte Wettbewerbsvorteile aufbauen, in: Simon, H. (Hrsg.): Wettbewerbsvorteile und Wettbewerbsfähigkeit, Stuttgart 1988, S. 32.

Vgl. Porter, M. E.: Wettbewerbsvorteile, Frankfurt a. M. 1986, S. 75; Ghemawat, P.: Dauerhafte Wettbewerbsvorteile aufbauen, in: Simon, H. (Hrsg.): Wettbewerbsvorteile und Wettbewerbsfähigkeit, Stuttgart 1988, S. 33. 
Nutzung international zu Dumpingpreisen angeboten werden, können international tätige Unternehmen diese Ressourcen durch länderübergreifende Logistikprozesse weltweit für alle Produktionsstandorte verfügbar machen. Können Produktionsprozesse in diesen Ländern aufgrund weniger strenger Umweltschutzauflagen kostengünstiger durchgeführt werden, kann die Fertigung in diesen Ländern konzentriert und in andere Länder exportiert werden.

- Bei der Anhebung von Umweltschutzstandards in bestimmten Ländern können international tätige Unternehmen durch eine nationale Rückzugsstrategie und Standortverlagerung ausweichen bzw. rückständige Produkte und Verfahren in jene Länder verlagern, in denen geringere Umweltschutzanforderungen an die Wirtschaft gestellt werden. Durch die Verlagerung von abgeschriebenen und ökologisch veralteten Produktionsanlagen in Schwellenländer können Entwicklungsstand-Portfolio-Effekte realisiert werden.

Die Wirkungsziele im internationalen Umweltmanagement sind anspruchsgruppenbezogen unterschiedlich.

- Gegenüber den lokalen Regulierungsbehörden, den Gesetzgebern und politischen Institutionen ist die Elinhaltung rechtlicher Rahmenbedingungen sowie die lokale Zusammenarbeit mit Behörden und anderen staatlichen Institutionen ein wichtiges Ziel und eine Grundvoraussetzung, um die Betriebsgenehmigung zu erhalten und zu behalten.

- In Bezug auf die nicht institutionellen Anspruchsgruppen sind insbesondere die Anwohner von großer Bedeutung, da sie bei starker Belästigung die Zufahrt des Standorts blockieren können, um so einen Produktionsstillstand herbeizuführen, oder andere Maßnahmen ergreifen, die einen Ausgangspunkt für imageschädigende Medienberichte liefern könnten. ${ }^{361}$ Insbesondere Neuansiedlungen und Erweiterungen sollten deshalb im Konsens mit den lokalen Anspruchsgruppen durchgeführt werden. ${ }^{362}$

361 So hat bspw. VolKSWAgen an seinem Standort in Pacheco (Argentinien) Lärmschutzwände an den Zufahrtsstraßen errichtet, da sich die direkte Nachbarschaft durch den Lärm der Lieferanten-Lkws belästigt fühlte. Diese Information stamt von Herrn MOGG von der VOLKSWAGEN AG.

Vgl. hierzu auch die Maßnahmen des Risikodialogs Meffert, H.; Klrchgeorg, M.: Marktorientiertes Umweltmanagement: Konzeption, Strategie, Implementierung mit Praxisfällen, a. a. O., S. $254 \mathrm{ff}$. 
- Weiterhin sind die Bedenken lokaler Umweltschutzorganisationen und anderer z. T. auch international organisierter öffentlichen Anspruchsgruppen zu berücksichtigen, um die gesellschaftliche Legitimität der Automobilhersteller in allen Märkten wahren zu können. Da nicht-institutionelle öffentliche Rückkopplungen durch Overspill-Effekte auch in anderen Ländern zur öffentlichen Exponiertheit eines Unternehmens beitragen können, sind international an allen Standorten Tätigkeiten zu unterlassen, die zu einem Verlust der gesellschaftlichen Legitimität beitragen könnten. Der Sicherstellung der gesellschaftlichen Legitimität kommt national sowie international der Charakter einer Mindestvoraussetzung zu. ${ }^{363}$ Die Wahrung der gesellschaftlichen Legitimität ist für die Automobilindustrie aufgrund des hohen Beitrags ihrer Produkte zur Umweltverschmutzung dabei besonders wichtig.

- Ein weltweit hohes Umweltimage bei den aktuellen und potenziellen Kunden steigert das Vertrauen der Kunden gegenüber dem Produkt und dem Unternehmen. Ziel im internationalen Umweltmanagement der Automobilindustrie ist es somit, ein möglichst einheitliches Umweltimage der einzelnen Marken aufzubauen, um negative marktbezogene Rückkopplungen zu vermeiden und Dissonanzen beim Produktkauf, der Nutzung und eventuell der Rückgabe nicht aufkommen zu lassen.

- Gegenüber Banken, Eigenkapitalgebern und Versicherungen bestehen ebenfalls verschiedene internationale Ziele im Umweltmanagement. So kann die Minimierung ökologischer Risiken zu geringen Versicherungsprämien und einer höheren Kreditwürdigkeit sowie zu einer besseren Bewertung seitens der Aktionäre beitragen. Weiterhin wird die Wahrnehmung ökologischer Chancen zumeist ebenfalls positiv an der Börse bewertet.

363 "So increasingly, public interest groups are requiring responses from industry sector. This suggests that there is a growing need for companies to acquire a >>social licence<< to operate as well as its more customary regulatory licence." Warhurst, A.: Corporate Citizenship and Corporate Social Investment: Drivers of Tri-Sector Partnerships, in: Journal of Corporate Citizenship, Nr. 1 (2001), Spring 2001, S. 58. 
Die folgende Abbildung zeigt die ökologischen Chancen und Risiken unterschiedlicher Automobilhersteller aus dem Jahr 1998. In diese Untersuchung wurde sowohl die aktuelle internationale Umweltschutzleistung im produkt- als auch im fertigungsbezogenen Umweltschutz zur Abbildung der Sustainability Risiken einbezogen. Die Forschungs- und Entwicklungsaktivitäten der Hersteller fanden bei der Beurteilung der Sustainability Chancen Eingang in die Untersuchung. ${ }^{364}$

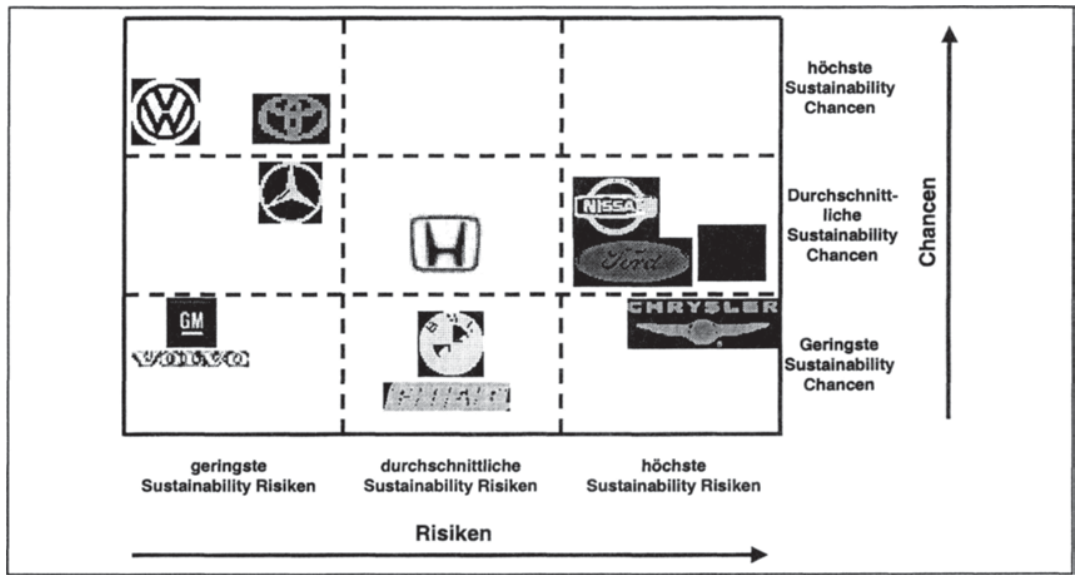

Abbildung 28: Ökologische Chancen und Risiken der Automobilhersteller

Quelle: Sustainable Asset Management (Hrsg.): Sustainability in der Automobilindustrie: Das Automobil im nächsten Jahrtausend, Zürich 1998, S. 28.

Als direkte Koordinationsziele im internationalen Umweltmanagement können in Anlehnung an BARTLETT und GOSHAL die internationale Integration der ökologiebezogenen Aktivitäten sowie die Verknüpfung international verstreuter Kenntnisse, Erfahrungen und Informationen zur Förderung der organisatorischen Lernfähigkeit im Umweltschutz angeführt werden. ${ }^{365}$

364 Vgl. dazu Sustainable Asset Management (Hrsg.): Sustainability in der Automobilindustrie: Das Automobil im nächsten Jahrtausend, Zürich 1998.

Vgl. Bartlett, Ch. A.; Goshal, S.: Managing Innovations in the Transnational Corporation, in: Bartlett, Ch. A.; Doz, Y.; Hedlund, G.: (Hrsg.): Managing the Global Firm, London, New York 1990, S. 212; zitiert nach Bolz, J.: Wettbewerbsorientierte Standardisierung der internationalen Marktbearbeitung: Eine empirische Analyse in europäischen Schlüsselmärkten, a. a. O., S. 123. 
- Die internationale Integration der wichtigsten ökologiebezogenen Unternehmensaktivitäten zielt auf ein den unternehmensübergreifenden ökologischen Grundsätzen, Strategien und Zielen entsprechendes Verhalten der Landesgesellschaften ab. Die Integration kann bspw. durch internationale ökologische Strategien und standardisierte instrumentelle Rahmenkonzepte erreicht werden. Auf diese Weise lassen sich die indirekten Koordinationsziele, wie bspw. die Sicherstellung der gesellschaftlichen Legitimität, erfüllen.

- Durch die Verknüpfung des innerhalb des Unternehmensverbundes verstreuten ökologischen Know-hows und der Förderung der organisatorischen Lernfähigkeit ${ }^{366}$ soll der Aufbau von internationalen ökologischen Erfahrungseffekten gefördert werden. Weiterhin ergibt sich die hohe Bedeutung der internationalen Lernfähigkeit daraus, dass eine Veränderung der umweltrechtlichen Rahmenbedingungen dazu führen kann, dass traditionelle Wettbewerbsvorteile verloren gehen und sich dadurch der Wert vorhandener Ressourcen im Unternehmen verringert. ${ }^{367}$

Die Förderung der organisatorischen Lernfähigkeit durch die Verknüpfung des lokalen ökologischen Wissens und der lokalen Erfahrungen im Umgang mit ökologischen Herausforderungen kann den unternehmensweiten Aufbau von ökologischen Kompetenzen und Fähigkeiten fördern. Gesetzliche Entwicklungen in Vorreiterstaaten, Neuerungen der Wettbewerber und lokales Know-how sowie der unmittelbare Umgang mit Erstnutzern einer ökologiebezogenen technologischen Innovation können durch Direktinvestitionen in Standorte in ökologischen Vorreiterländern erreicht werden. ${ }^{368}$ Die Entwicklung ökologischer Innovationen in der Automobilindustrie erfolgt dabei in den Ländermärkten, in denen entsprechende gesetzliche oder marktbezogenen Rahmenbedingungen herrschen, wie bspw. die Entwicklung des Katalysators und der Brennstoffzelle (USA) und verbrauchsarmer Motoren (Deutschland / Europa) zeigen. national Perspective, a. a. O., S. $291 \mathrm{f}$.

Vgl. Packard, K.; Reinhardt, F.: What Every Executive Needs to Know About Global Warming, in: Harvard Business Review, 78. Jg. (2000), Nr. 4, S. 132.

Vgl. Serapio, M. G. Jr.: Macro-Micro Analyses of Japanese Direct $R$ \& $D$ Investments in the U.S. Automotive and Electronics Industries, in: Management International Review, Nr. 3, 1993, S. 219. 
Durch eine internationale ökologiebezogene Koordination sind Verknüpfungen innerhalb des Unternehmensverbundes herzustellen, um ökologiebezogene Know-how-Vorsprünge einzelner Standorte im Unternehmensverbund nutzen zu können. Auf diese Weise können in einzelnen Ländermärkten im günstigsten Fall bereits vor Umsetzung von ökologiebezogenen gesetzlichen Regelungen marktliche und gesellschaftliche Forderungen von Anspruchsgruppen durch ökologiegerechte Lösungen erfüllt werden. ${ }^{369}$ Ökologische Innovationen können dabei als Grundlage zukünftiger Wettbewerbsvorteile dienen. ${ }^{370}$

Eine Koordination im internationalen Umweltmanagement soll in diesem Kontext zu einer Reduzierung der Autonomiekosten führen. Eine Abstimmung der Intentionen und Verhaltensweisen der Entscheidungsträger im Umweltmanagement setzt somit Informationen voraus. Dabei stehen die Anforderungen an das betriebliche Umweltinformationssystem in direktem Mittel-Zweck-Verhältnis zu den konkreten unternehmensbezogenen Koordinationszielen. ${ }^{371}$ Sämtliche Informationsgewinnungs- und Informationsverarbeitungsaktivitäten zur Verbesserung informationeller und methodischer Grundlagen von Entscheidungen bedingen nämlich den Einsatz von Ressourcen und verursachen somit Abstimmungs- bzw. Koordinationskosten. Die Kosten der Koordination im internationalen Umweltmanagement sind dabei den Autonomiekosten bei nicht koordiniertem Verhalten gegenüberzustellen und möglichst gering zu halten.

369 HART geht in diesem Zusammenhang davon aus, dass in Zukunft ...it appears inevitable that businesses (markets) will be constrained by and depend upon ecosystems (nature). In other words, it is likely that strategy and competitive advantage in the coming years will be rooted in capabilities that facilitate environmentally sustainable economic activity - a natural-resource-based view of the firm." Hart, S. L.: A Natural-Resource-Based View of the Firm, in: Academy of Management Review, 20. Jg. (1995), Nr. 4, S. 991.

Diese Überlegungen lassen sich auf Ansätze aus der Ressourcentheorie zurückführen. Wettbewerbsvorteile gelten als Ergebnis von besonderen Ressourcen und Fähigkeiten der Unternehmung. Fähigkeiten kennzeichnen die „Begabung“ des Managements, vorhandene Ressourcen so zu nutzen bzw. zu integrieren, dass das Unternehmen in die Lage versetzt wird, Aktivitäten durchzuführen, die in einer bestimmten Markt- und Wettbewerbssituation zum Erfolg des Unternehmens am Markt beitragen. Vgl. zur Ressourcentheorie bspw. Meffert, H.: Marketingwissenschaft im Wandel - Anmerkungen zur Paradigmendiskussion, a. a. O., S. 26 und Burmann, Ch.: Strategiewechsel in turbulenten Märkten - Neuere theoretische Ansätze zur Unternehmensflexibilität, in: Meffert, H.; Backhaus, K.; Becker, J. (Hrsg.): Arbeitspapiere der Wissenschaftlichen Gesellschaft für Marketing und Unternehmensführung e. V., Münster 2000, S. 24, sowie die dort angegebene Literatur.

371 Vgl. Plump, D.: Die Koordination des Marketings in multinationalen Unternehmungen: Systemanalyse und Ansätze zur Systemgestaltung, a. a. O., S. $142 \mathrm{ft}$. 
In der folgenden Abbildung ist die international gültige Umweltpolitik für die Marke VOLKSWAGEN abgedruckt. Anhand der unterschiedlichen ökologischen Zielsetzungen kann z. T. auf die Wirkungsziele geschlossen werden, während Effizienzziele nur implizit Erwähnung finden.

\section{Die VolkswaGEN Umweltpolitik}

VOLKSWAGEN hat im Mai 1995 seine bis dahin gültigen Umweltleitlinien zu einer Konzernumweltpolitik weiterentwickelt. Auf dieser Basis haben in den folgenden Monaten die Marken des Konzerns eigene, der jeweiligen Unternehmenskultur entsprechende Umweltpolitiken entwickelt. Den Anfang machte hierbei die Marke VolkswaGeN, deren Leitung im September 1995 eine eigene Umweltpolitik verabschiedete.

\section{Präambel}

VOLKSWAGEN entwickelt, produziert und vertreibt weltweit Automobile zur Sicherstellung individueller Mobilität. Das Unternehmen trägt Verantwortung für die kontinuierliche Verbesserung der Umweltverträglichkeit seiner Produkte und die Verringerung der Beanspruchung der natürlichen Ressourcen unter Berücksichtigung wirtschaftlicher Gesichtspunkte. Es macht daher umwelteffiziente fortschrittliche Technologien weltweit verfügbar und bringt sie über den gesamten Lebenszyklus seiner Produkte zur Anwendung. Es ist an allen Standorten Partner für Gesellschaft und Politik bei der Ausgestaltung einer sozialen und ökologisch nachhaltig positiven Entwicklung.

\section{Grundsätze}

1. Es ist das erklärte Ziel von VOLKSWAGEN, bei all seinen Aktivitäten die Einwirkung auf die Umwelt so gering wie möglich zu halten und mit den eigenen Möglichkeiten an der Lösung der regionalen und globalen Umweltprobleme mitzuwirken.

2. Es ist das Ziel von VolkswageN, hochwertige Automobile anzubieten, die den Ansprüchen seiner Kunden an Umweltverträglichkeit, Wirtschaftlichkeit, Sicherheit, Qualităt und Komfort in gleicher Weise gerecht werden.

3. Zur langfristigen Sicherung des Unternehmens und zur Steigerung seiner Wettbewerbsfähigkeit erforscht und entwickelt VoLKswaGeN ökologisch effiziente Produkte, Prozesse und Konzepte für individuelle Mobilität.

4. Das Umweltmanagement von VolkswaGEN stellt auf der Grundlage der Umweltpolitik sicher, dass gemeinsam mit Zulieferunternehmen, Dienstleistern, Handelspartnern und Verwertungsunternehmen die Umweltverträglichkeit seiner Automobile und Fertigungsstandorte einem kontinuierlichen Verbesserungsprozess unterworfen ist.

5. Der Vorstand von VolkswaGeN überprüft regelmăßig die Einhaltung der Umweltpolitik und -ziele sowie die Funktionsfähigkeit des Umwelt-Management-Systems. Dies schließt die Bewertung der erfassten umweltrelevanten Daten ein.

6. Die offene und klare Information sowie der Dialog mit Kunden, Händlern und der Öffentlichkeit sind für VoLKSWAGEN selbstverständlich. Die Zusammenarbeit mit Politik und Behörden beruht auf einer handlungsorientierten und vertrauensvollen Grundhaltung und bezieht die Notfallvorsorge an den Produktionsstandorten mit ein.

7. Alle Mitarbeiter von VolKSWAGEN werden entsprechend ihren Aufgaben im Umweltschutz informiert, qualifiziert und motiviert. Sie sind zur Umsetzung dieser Grundsătze sowie zur Erfüllung der gesetzlichen und behördlichen Auflagen im Rahmen ihrer jeweiligen Aufgabenstellung verpflichtet.

Abbildung 29: Umweltpolitik der VOLKSWAGEN AG

Quelle: Volkswagen 


\section{Basisstrategische Optionen im internationalen Umweltmanagement als Ausgangspunkt der Koordination}

Vor dem Hintergrund der direkten und indirekten Ziele der internationalen, ökologiegerichteten Koordination in der Automobilindustrie sollen an dieser Stelle unterschiedliche Basisstrategien im internationalen Umweltmanagement als Ausgangspunkt der Koordination diskutiert werden. Die strategische Ausrichtung im internationalen Umweltmanagement ist für die Ausgestaltung der Koordinationskonzeption maßgeblich, da die unterschiedlichen Basisstrategien im internationalen Umweltmanagement in direkter Mittel-Zweck-Beziehung zu den unternehmensbezogenen Oberzielen stehen.

Bei der Untersuchung von Basisstrategien im internationalen Umweltmanagement ist auf die grundlegende Systematisierung von BRODEL zu verweisen, der das Spannungsfeld zwischen kostenorientierter Externalisierung und erfolgszielorientierter Internalisierung in den Mittelpunkt der Betrachtung rückt. Von internationaler ökologischer Internalisierung spricht BRODEL, wenn im Gesamtunternehmensverbund im Vergleich zu einem rein national tätigen Unternehmen ein verbessertes Umweltschutzniveau realisiert wird. Demgegenüber wird als internationale ökologische Externalisierung das "Herunterfahren" der betrieblichen Umweltschutzstandards gegenüber national tätigen Unternehmen bezeichnet. $^{372}$

Je nach den Oberzielen der Unternehmung, den situativen Kontextbedingungen und vorliegenden Externalisierungs- und Internalisierungsvorteilen ${ }^{373}$ können vier Basisstrategien unterschieden werden. Als Typologisierungsdimension der Strategien werden die ökologischen Internalisierungs- und Externalisierungsvorteile als jeweils eigenständige Dimensionen herangezogen, da die jeweiligen Vorteile mit spezifischen Wettbewerbsvorteilen im Zusammenhang stehen. Die Basisstrategien im internationalen Umweltmanagement sind in der folgenden Abbildung dargestellt.

372 Vgl. hierzu und im Folgenden Brodel, D.: Internationales Umweltmanagement: Gestaltungsfelder - Determinanten - Ausprägungen, a. a. O., S. $403 \mathrm{ff}$.

373 Eine detaillierte Darstellung der Externalisierungsvorteile und Internalisierungsvorteile findet sich bei Brodel, D.: Internationales Umweltmanagement: Gestaltungsfelder - Determinanten - Ausprägungen, a. a. O., S. 371 - 389. 


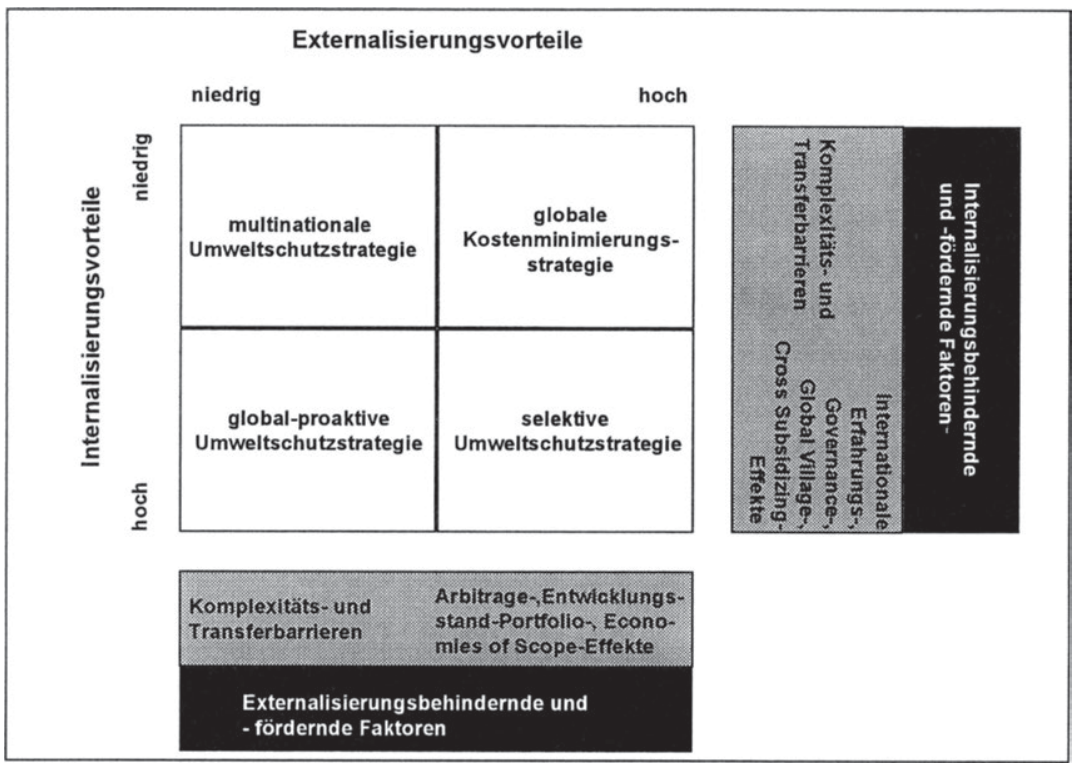

Abbildung 30: Strategische Basisorientierungen im internationalen Umweltmanagement

Quelle: Brodel, D.: Internationales Umweltmanagement: Gestaltungsfelder - Determinanten - Ausprägungen, a. a. 0., S. 408.

In den nachfolgenden Unterkapiteln werden die einzelnen basisstrategischen Umweltschutzorientierungen jeweils charakterisiert. ${ }^{374}$

\subsection{Global-proaktive Umweltschutzstrategie}

Im Rahmen einer global-proaktiven Ausrichtung im internationalen Umweltmanagement erlangen die Effizienzziele der Kordination eine hohe Gewichtung. Insbesondere die Erzielung von Größen-, Synergie- und Erfahrungseffekten erscheint mit einer international ökologisch proaktiven Verhaltensausrichtung gut vereinbar zu sein, während Arbitrage- und EntwicklungsstandportfolioEffekten kaum eine Bedeutung beizumessen ist.

In Bezug auf die Wirkungsziele der Koordination des internationalen ökologiegerichteten Verhaltens ist die Einhaltung lokaler rechtlicher Rahmenbedingungen, die lokale Zusammenarbeit mit den Behörden ebenso wie die Erzielung

374 Vgl. zu den Basisstrategien Brodel, D.: Intemationales Umweltmanagement: Gestaltungsfelder - Determinanten - Ausprägungen, a. a. O., S. 409-412. 
eines Konsenses mit den wichtigsten lokalen Anspruchsgruppen, wie der unmittelbaren Nachbarschaft, lediglich als Basisanforderung zu beurteilen. Das ökologiegerichtete Verhalten des Unternehmens ist darüber hinaus so auszurichten, dass aufgrund der potenziell hohen öffentlichkeitsbezogenen Rückkopplungen die gesellschaftliche Legitimität der Unternehmung insgesamt gewahrt bleibt. Da Umweltschutz in diesem Kontext zumindest einen potenziell wichtigen Wettbewerbsfaktor darstellt, ist durch das Angebot ökologisch vergleichsweise vorteilhafter Produkte und die Wahl einer entsprechenden ökologischen Positionierung im Wettbewerb eine hohe ökologische Reputation aufzubauen. Damit einher geht die Minimierung ökologischer Risiken, um marktbezogene Rückkopplungen über den Finanzsektor möglichst gering zu halten.

Vor diesem Hintergrund kommt der einheitlichen Ausrichtung der ökologiegerichteten Aktivitäten aller Auslandsgesellschaften auf die gemeinsamen ökologischen Ziele als direktem Koordinationsziel eine hohe Bedeutung zu. Die in ökologischen Vorreiterländern gesammelten Erfahrungen und das Wissen bzgl. der Verringerung ökologischer Belastungen durch die Produkte und bei der Fertigung sind durch eine enge interne Verknüpfung über Landesgrenzen hinweg zu transferieren und zur Steigerung der ökologischen Kompetenz des Gesamtunternehmens einzusetzen.

Im Spannungsfeld von Zentralisation und Dezentralisation besteht die Aufgabe des Managements darin, die ökologiegerichteten Entscheidungen, die der Erzielung der Effizienzziele dienen und zu externen Rückkopplungen führen könnten, zu zentralisieren. Es bedarf dabei keiner gesonderten Begründung, dass die Kosten der Koordination möglichst gering zu halten sind.

Bei global-proaktiver Umweltschutzstrategie ist ein international einheitliches Informationssystem zur Früherkennung ökologischer Chancen und Risiken aufzubauen. Die Führungskräfte sind dabei zeitnah über die ökologiebezogenen Entwicklungen in den Ländermärkten und über die ökologische Leistung der Auslandsgesellschaften zu informieren.

Global-proaktive Umweltschutzstrategien sind dann zu erwarten, wenn sich durch eine anspruchsvolle und international weitgehend einheitliche Orientierung im Umweltschutz Vorteile im Wettbewerb erzielen lassen und eine ökologische Externalisierung mit hohen Opportunitätskosten verbunden wäre. ${ }^{375}$ Eine

375 Vgl. hierzu und im Folgenden Brodel, D.: Internationales Umweltmanagement: Gestaltungsfelder-Determinanten - Ausprägungen, a. a. O., S. 410. 
derartige Situation ist insbesondere dann zu erwarten, wenn die endogenen und exogenen Kontextfaktoren zu hohen internen und externen ökologiebezogenen Rückkopplungen bei internationaler Unternehmenstätigkeit führen. Die folgende Abbildung zeigt eine derartige Situation im Überblick.

\begin{tabular}{|l|c|}
\hline \begin{tabular}{|l|l|}
\hline Ausprägung exogener Kontextfaktoren \\
- Umsystemähnlichkeit
\end{tabular} & $\begin{array}{c}\text { hoch } \\
\text { hoch }\end{array}$ \\
\hline Uussystemabhängigkeit & hoch \\
- Bedeutung des Umweltschutzes & $\begin{array}{c}\text { proaktiv } \\
\text { mittel -hoch } \\
\text { hoch }\end{array}$ \\
- Zentrategische Umweltschutzorientierung & hoch \\
\hline Rückkontions- u. Standardisierungsgrad & hoch \\
\hline
\end{tabular}

Abbildung 31: Situativer Kontext einer global-proaktiven Umweltschutzstrategie

Quelle: Eigene Darstellung

Die Wahl einer global-proaktiven Ausrichtung im Umweltmanagement erfolgt idealtypisch bei internationaler Tätigkeit in weitgehend ähnlichen Umsystemen und einer hohen Abhängigkeit der Umsysteme voneinander. Dabei wird Ökologie aktuell bzw. potenziell als relevanter Wettbewerbsfaktor angesehen, der zur Differenzierung gegenüber der Konkurrenz genutzt werden kann. Grundlegend werden dabei ökologische und ökonomische Ziele somit als komplementäre Ziele verstanden.

Eine global-proaktive Umweltschutzstrategie zeichnet sich demnach durch eine unternehmensweit weitgehend einheitliche, ökologisch anspruchsvolle Ausrichtung aus. Dabei orientieren sich die Auslandsgesellschaften an den international strengsten Umweltschutzbestimmungen bzw. hohen unternehmensbezogenen Standards, da in naher Zukunft auch in den Schwellenländern schärfere Anforderungen im fertigungs- und produktbezogenen Umweltschutz erwartet werden. ${ }^{376}$ Die sich abzeichnende Konvergenz in der Wahl umweltpolitischer In-

376 Vgl. Rugmann, A. M.; Verbeke, A.: Corporate Strategies and Environmental Regulations: An Organizing Framework, a. a. O., S. 372. 
strumente, zunehmende internationale ökologiebezogene Rahmenkonventionen sowie gesellschaftsübergreifende Forderungen nach immer anspruchsvolleren Umweltschutzgesetzen und Produkten können in diesem Kontext als Treiber für einen ökologiebezogenen Globalisierungs-Push gelten. Eine proaktive Erfüllung gesetzlicher Anforderungen im produkt- und insbesondere im fertigungsbezogenen Umweltschutz erhöht dabei die Flexibilität gegenüber einer unsicheren Entwicklung der Umweltschutzgesetze und Forderungen der Anspruchsgruppen. ${ }^{377}$ So wurden beim Aufbau des neuen VOLKSWAGENMotorenwerks in Polkowice (Polen) mit Blick auf den zukünftigen Beitritt Polens in die EU bereits heute Umweltschutztechnologien eingesetzt, die sich an deutschen und zukünftigen europäischen Standards orientieren. So können teure Nachrüstungen vermieden werden. ${ }^{378}$

Aufgrund der hohen Kapitalintensität technologischer Umweltschutzentwicklungen bei einer oftmals nur gering ausgeprägten zusätzlichen Zahlungsbereitschaft der Konsumenten für ökologische Produktalternativen ${ }^{379}$ können international tätige Hersteller in nur einem Ländermarkt häufig keine ausreichend große Käuferschaft für ökologische Produktangebote finden, um Erfahrungs-, Größen- und Synergieeffekte realisieren zu können. Ökologische Produktangebote wären dann erheblich teurer als vergleichbare konventionelle Produktalternativen.

Durch den länderübergreifenden Einsatz von Unternehmens-Know-how und Erfahrungen im Umweltschutz lassen sich Kostensenkungseffekte in Forschung und Entwicklung, Fertigung und Vermarktung sowie Demontage und im Recycling nutzen. Dies erfordert i. d. R. eine internationale Produkt-Standardisierung, zumindest aber den umfassenden Einsatz von Gleichteilen und Modulen.

377 In diesem Fall kann von Built-in-Flexibilität gesprochen werden. Vgl. Meffert, H.: Größere Flexibilität als Unternehmenskonzept, in: Schmalenbachs Zeitschrift für betriebswirtschaftliche Forschung, 37. Jg. (1985), Nr. 2, S. 121 - 137.

$378 \mathrm{Vgl}$. Volkswagen (Hrsg.): Jahresbericht des produktionsbezogenen Umweltschutzes 1999/2000, a. a. O., S. 20.

379 Vgl. Llebehenschel, Th.: Ökologieorientierte Produkt- und Dienstleistungspolitik: Rahmenbedingungen und Trends am Beispiel der Automobilindustrie, a. a. O., S. $281 \mathrm{ff}$. 
Die Ausweitung der Gleichteilestrategie, wie derzeit bei VolKSWAGEN, führt somit im Umweltmanagement zu einer Standardisierung der Produktteile auf ökologisch hohem Niveau. Dementsprechend erweist es sich tendenziell als vorteilhaft, wenn die Produkte zumindest nach Maßgabe der strengsten nationalen Umweltschutznormen oder höheren unternehmensbezogenen Standards hergestellt werden.

Kostensenkungspotenziale lassen sich bei internationaler Unternehmenstätigkeit auch über die Erzielung von Größenvorteilen bei Bearbeitung mehrerer Märkte $^{380}$ und Nutzung von Komponenten für unterschiedliche Produkte und Marken sowie durch weltweites Lernen und Risikodiversifikation erreichen. ${ }^{381}$ Die Motorentechnik des 3-L LUPOS von VoLKSWAGEN wird bspw. auch im AUDI $A L_{2}$, dem Drei-Liter-Auto der Konzerntochter AUDI, verwendet. Neben anderen Gleichteilen konnten insbesondere die Erfahrungen im Leichtbau bei der Konstruktion der Karosserie übertragen werden. Die technologische Pionierarbeit in Bezug auf das Antriebsaggregat kann weiterhin auch für andere Fahrzeugmodelle des Konzerns genutzt werden. Die Modulstrategie des VolKSWAGENKonzerns ermöglicht durch den Plattform-übergreifenden Einsatz von Gleichteilen hohe Kostensenkungspotenziale. ${ }^{382}$ Dabei ist selbst bei unterschiedlich hoch ausgeprägter Zahlungsbereitschaft der Nachfrager eine Standardisierung erstrebenswert, wenn sich dadurch Kostensenkungspotenziale erschließen und Entwicklungskosten für nationale Eigenentwicklungen einsparen lassen. ${ }^{383}$

380 TOYOTA bietet bspw. das Modell Prius, ein Hybridfahrzeug mit einem sehr niedrigen Kraftstoffverbrauch, mittlerweile in allen drei Triademärkten an. Nachdem der PRIUS 1997 in Japan eingeführt wurde, ist er leicht modifiziert seit Mitte 2000 auch in Deutschland und in den Vereinigten Staaten erhältlich. Vgl. http://global.toyota.com/ [10.01.2001].

Vgl. Engelhard, J.; Dähn, M.: Theorien der internationalen Unternehmenstätigkeit - Darstellung, Kritik und zukünftige Anforderungen, in: Macharzina, K.; Oesterle, M.-J. (Hrsg.): Handbuch Internationales Management: Grundlagen - Instrumente - Perspektiven, Wiesbaden 1997, S. 25.

382

Vgl. die Ausführungen von dem Vorstandsmitglied PIschetsaieder der VolkswaGen AG auf der "Merrill Lynch Global Autos Conference" in Genf am 27 February 2001 bei http://ir.volkswagen-ir.de/deutsch/.

Whitelock und PIMBlett (1997) argumentieren,: "If these [standardised] products are of the best quality and the lowest price consumers will forego local preferences based on cultural differences and accept a standardised product. To succeed in this new environment companies must produce on a global scale to ensure the scale economies needed to keep quality up and prices down." Whitelock, J.; Pimblett, C.: The Standardisation Debate in International Marketing, in: Journal of Global Marketing, 10. Jg. (1997), Nr. 3, S. 46. Umwelteigenschaften können dabei durchaus als Qualitătseigenschaften aufgefasst werden. 
Im fertigungsbezogenen Umweltschutz gibt es ebenfalls gute Gründe für die Wahl einer global-proaktiven Ausrichtung. In diesem Zusammenhang können ökologische Belastungen und ein hoher Abfallanfall bei der Fertigung als Zeichen für ökologische und ökonomische Verschwendung interpretiert werden. Der Einsatz effizienterer Produktionsverfahren führt dann sowohl zu ökologischen als auch zu ökonomischen Vorteilen. ${ }^{384}$ Ist die Einführung einer Umweltschutztechnik in einem Schwellenland ökonomisch erfolgreich durchgeführt worden, kann dies ökologischen Technologien in diesem Ländermarkt zum Durchbruch verhelfen. ${ }^{385}$ Die Beschleunigung der Diffusion ökologischer Innovationen durch Technologietransfer entspricht einer im Rahmen der Agenda 21 kodifizierten Forderung an Unternehmen aus Industrieländern.

Weiterhin kann die Unternehmung durch Kontakte und Beratungen mit nationalen Behörden und politischen Vertretern auch auf eine Anhebung der Umweltstandards in ökologisch rückständigen Ländern hinarbeiten. Auf diese Weise besteht die Möglichkeit für proaktive Unternehmen, den firmeneigenen Umweltstandard zum Industriestandard zu erheben. In Schwellenländern oder anderen Märkten, die in Bezug auf ökologische Fragestellungen wenig anspruchsvolle Anforderungen stellen, sind dies oft Entwicklungen aus ökologischen Vorreiterstaaten, die durch international tätige Unternehmen eingeführt

384 Hier kann bspw. die Neukonzeption der ${ }^{\circ D u s c h k a b i n e n “ ~ z u r ~ U ̈ b e r p r u ̈ f u n g ~ d e r ~ D i c h t i g k e i t ~}$ von Karosserien im BMW-Werk Rosslyn in Südafrika genannt werden. Wăhrend früher jährlich 30.000 Kubikmeter Wasser direkt in die Kanalisation geleitet wurden, durchläuft das Wasser heute verschiedene Kreisläufe, sodass lediglich 500 Kubikmeter Wasser im Jahr verbraucht werden. Bereits eineinhalb Jahre nach Umbau der Anlage hat sich die Umweltschutzinvestition amortisiert. Vgl. BMW (Hrsg.): BMW Group: Clean Production weltweit, Presseinformation vom 09.12.1999, S. 3 f.

Prozessinnovationen in der mechanischen Bearbeitung der Automobilindustrie tragen bspw. zu einer Senkung des Emulsionsverbrauchs und damit zu einer Senkung von anfallenden Umweltbelastungen durch Kühlschmierstoffe bei. Bei gleicher Qualităt der Werkstücke fallen geringere Kosten an. Die Schmutzpartikel, die sich bei Entfetten der Karosserien (Phosphatierung) zur späteren Lackierung im Abwasser sammeln, können durch Umkehr-Osmose als Filtrationstechnik herausgefiltert werden. Dies führt zu Kostenersparnis und Umweltschutz. Das Verfahren wurde von VolKSWAGEN zum Patent angemeldet. Auch bei der Kraftstoffbefüllung konnte VOLKSWAGEN durch ein innovatives Verfahren, welches ebenfalls zum Patent angemeldet wurde, Investitionskosten von mehreren 100.000 DM vermeiden.

Beispiele aus anderen Branchen finden sich U. a. bei Gege, M. (Hrsg.): Kosten senken durch Umweltmanagement - 1000 Erfolgsbeispiele aus 100 Unternehmen, a. a. 0.; Nehrt, Ch.: Maintainability of First Mover Advantages When Environmental Regulations Differ Between Countries, in: The Academy of Management Review, 23. Jg. (1998), Nr. 1, S. 81 $-83$.

Die ökonomisch erfolgreiche Einführung der Wasserlacktechnologie durch BMW in Südafrika führte dazu, dass auch andere Hersteller neue Lackierereien für Wasserlacktechnologie bauen möchten. Vgl. BMW (Hrsg.): BMW Group: Clean Production weltweit, a. a. O., S. 4. 
werden. So nahm die Entwicklung der Katalysatoren in den USA ihren Ausgangspunkt, während die EU erst mit acht Jahren Verzögerung im Jahr 1991 beschloss, die Abgasgesetzgebung europaweit der US-Norm anzupassen. In Deutschland war es das Unternehmen OPEL, eine Tochter der US-amerikanischen GENERAL MOTORS-Konzerns, das aufgrund der Erfahrungen der Muttergesellschaft nach sehr kurzer Entwicklungszeit Anfang 1985 einen Motor mit geregeltem 3-Wege-Katalysator auf den Markt brachte. Weiterhin war OPEL europaweit der erste Hersteller, der Katalysatoren für die Kompaktklasse anbot und als erster deutscher Hersteller mit der serienmäßigen Ausstattung der Fahrzeuge mit einem Katalysator begann. ${ }^{386}$

In diesem Kontext können das bereits aufgebaute Wissen, die entsprechenden Ressourcen und die Kompetenzen im betrieblichen Umweltschutz als Erfahrungseffekte Markteintrittsbarrieren für lokale Wettbewerber darstellen. ${ }^{387}$ Innovationen können darüber hinaus durch Patente abgesichert werden, die vom Wettbewerber bei fehlenden eigener Innovationsfähigkeit u. U. erworben werden müssen. ${ }^{388} \mathrm{Da}$ derzeit insbesondere in Deutschland und Europa nahezu alle Hersteller mit der Entwicklung eines Drei-Liter-Autos befasst sind, haben diese Hersteller insbesondere gegenüber amerikanischen Herstellern Wettbewerbsvorsprünge in der Dieseltechnologie erlangt. Amerikanische Hersteller hatten festgestellt, dass dies auf Märkten außerhalb Amerikas zu einem zunehmenden Wettbewerbsproblem führen kann und sich im Rahmen der "Partnership for a New Generation of vehicles" (PNGV) zusammengeschlossen, um den Ausbau der Wettbewerbsvorteile deutscher Hersteller nicht zu einer Marktbarriere werden zu lassen. ${ }^{389}$ Auch die in der Vergangenheit aufgebaute ökologische Reputation eines Unternehmens kann dabei zum Aufbau sekundärer Markteintrittsbarrieren für ökologische Folger beitragen. Schließlich demon-

386 Vgl. Meffert, H.: Okologieorientierte Marketing- und Werbestrategie der Marke Opel, Münster, Hamburg 1990, S.15.

Vgl. Rugman, A. M.; Verbeke, A.: Corporate Strategies and Environmental Regulations, a. a. O., S. 369. Vgl. zum Erfolg von Pionierstrategien im internationalen Umweltmanagement Nehrt, Ch.: Maintainability of First Mover Advantages When Environmental Regulations Differ Between Countries, a. a. O., S. $77 \mathrm{ft}$.

$388 \mathrm{Vgl}$. Volkswagen (Hrsg.): Jahresbericht des fertigungsbezogenen Umweltschutzes 1998/1999, Wolfsburg 1999, S. 7 ff.

389 Vgl. Liebehenschel, Th.: Okologieorientierte Produkt- und Dienstleistungspolitik: Rahmenbedingungen und Trends am Beispiel der Automobilindustrie, a. a. O., S. 290. Zu Timingstrategien sowie primären und sekundären Markteintrittsbarrieren im Rahmen ökologischer Basisstrategien siehe grundlegend Meffert, H.; KIrchgeorg, M.: Marktorientiertes Umweltmanagement - Konzeption, Strategie, Implementierung mit Praxisfällen, a. a. O., S. 231 f. 
strieren ökologisch global-proaktiv handelnde Unternehmen ethisch verantwortungsbewusstes Verhalten, welches zur Wahrung der gesellschaftlichen Legitimität und zum Aufbau von Vertrauen führen kann.

Die folgende Abbildung zeigt mögliche Ausprägungen einer global-proaktiven Umweltschutzstrategie. Dabei wird bei der Standardisierung bzw. Differenzierung von Produkten und Fertigungsprozessen ${ }^{390}$ jeweils zwischen einer im Vergleich zu nationalen Wettbewerbern vergleichsweise hohen und niedrigen Umweltqualität der Produkte bzw. Fertigungsprozesse unterschieden. Das ökologische Niveau kann auch als ökologische Internalisierung (international relativ hohes ökologisches Niveau) bzw. Externalisierung (international relativ niedriges ökologisches Niveau) bezeichnet werden.

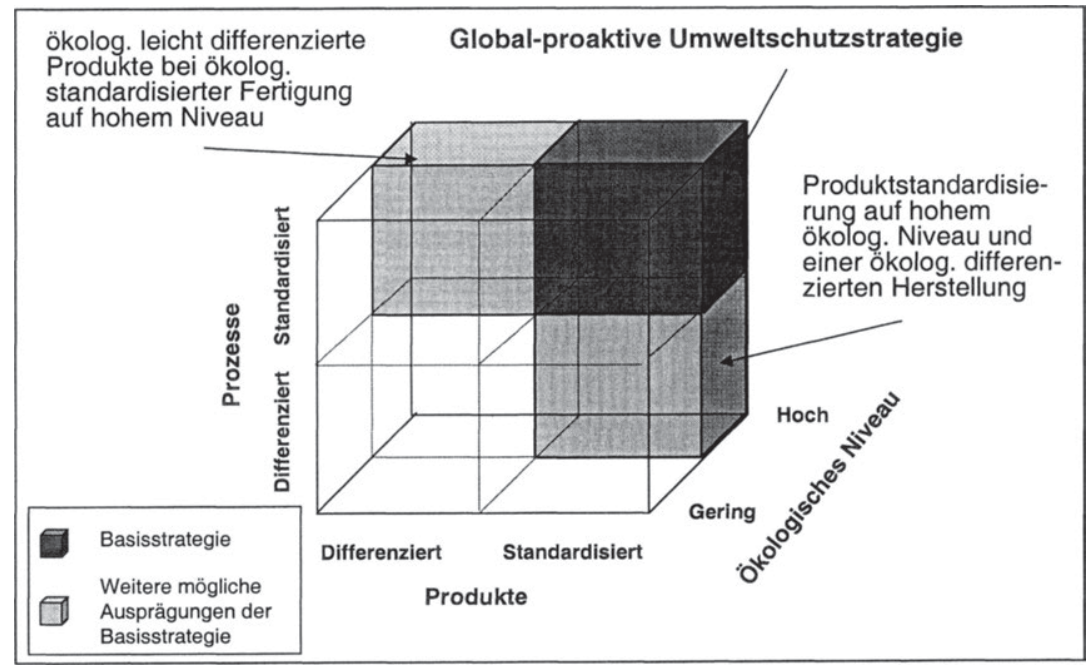

Abbildung 32: Ausprägungen der global-proaktiven Umweltschutzstrategie

Quelle: Eigene Darstellung

390 Die Abbildung zeigt dabei unterschiedliche Intensitätsstufen instrumenteller und prozessualer Standardisierung. Vgl. dazu die Intensitätsstufen instrumenteller und prozessualer Standardisierung in Meffert, H.: Marketing im Spannungsfeld zwischen weltweitem Wettbewerb und nationalen Bedürfnissen: Voraussetzungen und Implikationen von Globalisierungsstrategien, a. a. O., S. 21 ff. sowie Meffert, H.: Perspektiven des Marketing im Spannungsfeld zwischen Standardisierung und Differenzierung, in: Der Markenartikel, Heft 10, 1986, S. 442 - 450. 
Global-proaktive Umweltschutzstrategien zeichnen sich durch eine weitgehende internationale Standardisierung der Wertschöpfungsaktivitäten im Hinblick auf ökologische Fragestellungen aus. Dabei werden Umweltschutzerfordernisse nach Maßgabe international anspruchsvoller Umweltschutzgesetze und Anforderungen berücksichtigt. Die internationale ökologisch proaktive Umweltpolitik bildet dabei die Grundlage für unternehmensweit geltende, weitgehend einheitliche ökologiebezogene Vorgehensweisen und die Festlegung entsprechender Standards.

Eine weitgehende Ausnutzung des Standardisierungspotenzials in Bezug auf die ökologische Produktgestaltung und die Anwendung ökologisch ausgereifter und fortschrittlicher Fertigungstechnologien sind Merkmale einer global-proaktiven Umweltschutzstrategie. Von einer Standardisierung des Produktes wird nur dann abgerückt, wenn sich bspw. Umweltschutzgesetze in zwei Ländern widersprechen oder lokale Gegebenheiten, wie das lokale Kraftstoffangebot, eine vollständige Produktstandardisierung unmöglich machen. Ähnlich verhält es sich mit dem Angebot von Produkten, die auf hohem ökologischen Niveau standardisiert sind, allerdings unter Verwendung differenzierter Fertigungsmethoden hergestellt wurden. Auch in diesem Fall können lokale Rahmenbedingungen die Einhaltung der ökologischen Standards im fertigungsbezogenen Umweltschutz unmöglich machen, wenn bspw. Local-content-Vorschriften in einigen Ländern den Einsatz von lokalen Lacken vorschreiben, die lokalen Zulieferer aber die nach Unternehmensstandard verlangten wasserlöslichen Lacke nicht anbieten können. ${ }^{391}$

In der Automobilindustrie kann eine global-proaktive Ausrichtung im Umweltschutz unter Verwendung international gleicher produkt- und fertigungsbezogener Standards allein aufgrund der großen Unterschiede in den ökologiebezogenen Rahmenbedingunen der Triade- und Schwellenländer derzeit als nicht realisierbar angesehen werden. Aber im Rahmen der Plattform- und Modulstrategien vieler Automobilhersteller ist die Realisierung ökologisch relativ anspruchsvoller Produktstandards notwendig. In diesem Fall werden lediglich einzelne Module, wie bspw. der Motor und die Abgasregeltechnik, an lokale Bedingungen, wie die Kraftstoffqualität, Abgasreinigungsvorschriften und die lokale Zahlungsbereitschaft, angepasst. Im fertigungsbezogenen Umweltschutz kann ebenfalls die Durchsetzung weitgehend vergleichbarer - nicht unbedingt gleicher - Umweltschutzstandards aufgrund der hohen ökologischen Betroffenheit

391 Dies basiert auf den Ausführungen von Herrn Mogg und Herrn Riemann von Volkswagen. 
der Branche von gesellschaftlichen, politisch institutionellen und marktlichen Rückkopplungen beobachtet werden. ${ }^{392}$ Eine derart ausgestaltete globalproaktive Verhaltensausrichtung im internationalen Umweltmanagement üben Automobilhersteller wie BMW, VolkSWAGEN, RENAULT, FIAT und TOYOTA aus. ${ }^{393}$

\subsection{Globale Kostenminimierungsstrategie}

Im Gegensatz zu einer ökologisch-proaktiven Umweltschutzausrichtung werden im Zusammenhang mit der Verfolgung einer Kostenminimierungsstrategie im internationalen Umweltmanagement keine unternehmensübergreifenden Umweltschutzziele verfolgt. Allein einzelnen Effizienzzielen kommt eine hohe Bedeutung im Rahmen dieser strategischen Ausrichtung zu, da insbesondere durch die Realisierung von Größendegressions-, Arbitrage- und Entwicklungsstand-Portfolio-Effekten Kostensenkungspotenziale erreicht werden sollen.

Bei internationaler Kostenminimierungsstrategie wird lediglich die Einhaltung der lokalen rechtlichen Rahmenbedingungen gefordert (Wirkungsziele), wobei im Fall von kostenwirksamen Verschärfungen der lokalen ökologischen Bestimmungen durchaus Standortverlagerungen in Erwägung gezogen werden. Vor dem Hintergrund möglicher ökologischer Störälle und anderer haftungsbezogener Forderungen spielt die Minimierung lokaler ökologischer Risiken eine relevante Rolle.

Da die Kostenminimierungsstrategie im Umweltschutz darauf abzielt, die Marktchancen umweltbelastender Produkte international zu nutzen und fertigungsbezogene Externalisierungsmöglichkeiten bei Realisierung von Kostenersparnissen wahrzunehmen, fehlen unternehmensübergreifende ökologiegerichtete Koordinationsziele weitgehend. ${ }^{394}$ In Bezug auf die Koordination kommt der Zentralisation zur Generierung von Größenvorteilen eine gewisse Bedeutung $\mathrm{zu}$, wobei hier die Unternehmenstätigkeiten tendenziell an den Standorten mit besonders geringen Umweltschutzauflagen konzentriert werden.

392 Herr STOBBe von der Volkswagen AG gab dies im Interview an.

393 Vgl. hierzu bspw. die Ergebnisse der Ratings der Sustainable AsSET MANAGEMENT (SAM) Group aus dem Jahr 1998 sowie OEKOM. Sustainable Asset Management (Hrsg.): Sustainablility in der Automobilindustrie: Das Automobil im nächsten Jahrtausend, a. a. o.; oekom research (Hrsg.): Corporate Responsibility Industry Report Automobile: $A$ Corporate Responsibility Survey of 19 Companies of the Industry, a. a. 0.

394 Vgl. Brodel, D.: Internationales Umweltmanagement: Gestaltungsfelder - Determinanten Ausprägungen, a. a. O., S. 441. 
Als internationales Informationsziel kann die frühzeitige Unterrichtung der Unternehmensführung über geplante Verschärfungen von Umweltgesetzen angesehen werden. Schließlich könnte mit einfach gestalteten Informationssystemen nach schwachen Signalen bezüglich einer zunehmenden Sensibilisierung öffentlicher Anspruchsgruppen gegenüber dem Unternehmen bzw. der Branche gesucht werden.

Die folgende Abbildung zeigt die Ausprägung unterschiedlicher situativer Kontextfaktoren, bei denen eine Kostenminimierungsstrategie idealtypischerweise zur Anwendung kommt, im Überblick.

\begin{tabular}{|l|c|}
\hline \begin{tabular}{|l|l|}
\hline Auspräqung exogener Kontextfaktoren \\
- Umsystemähnlichkeit
\end{tabular} & $\begin{array}{c}\text { gering } \\
\text { gering }\end{array}$ \\
\hline Auspräqunga endogener Kontextfaktoren & \\
- Bedeutung des Umweltschutzes & $\begin{array}{c}\text { gering } \\
\text { reaktiv } \\
\text { Widerstand } \\
\text { hoch } \\
\text { - Strategische Umweltschutzorientierung } \\
\text { - Zentralisations u. Standardisierungsgrad }\end{array}$ \\
\hline Oückentliche Exponiertheit & gering \\
\hline - Interne Rückkopplungen & hoch \\
- Externe Rückkopplungen & gering \\
\hline
\end{tabular}

Abbildung 33: Situativer Kontext für eine globale Kostenminimierungsstrategie

Quelle: Eigene Darstellung

Globale Kostenminimierungsstrategien sind dann zu erwarten, wenn durch fertigungs- und produktbezogenen Umweltschutz keine ökonomischen Vorteile zu erzielen $\operatorname{sind}^{395}$ bzw. einer ökologischen Ausrichtung Wirtschaftlichkeitserwägungen entgegenstehen. Konflikte zwischen einer Kostenorientierung im Wettbewerb und einer proaktiven Umweltschutzstrategie lassen sich bspw. bei FIAT zumindest vermuten. Dort ist zu lesen:

395 Vgl. hierzu und im Folgenden Brodel, D.: Internationales Umweltmanagement: Gestaltungsfelder - Determinanten - Ausprägungen, a. a. O., S. 409 f. 
„As a rule, the environmental targets which Fiat Group Companies set themselves are higher than those envisaged by legislation. The principle of graduality will of course be adopted in cases where improvements with respect to legal provisions require extensive changes in progress. ${ }^{396}$

Im Gegensatz zur Situation bei global-proaktiver Umweltschutzausrichtung versuchen Unternehmen, die eine Kostenminimierungsstrategie wählen, die internationalen Differenzen im Umweltschutz auszunutzen. Dabei lässt sich erwarten, dass die Umsysteme, in denen ein derartiges Unternehmen tätig ist, bzgl. der Umweltschutzanforderungen möglichst unterschiedlich ausgeprägt sind. Weiterhin sollten aufgrund des wenig ökologischen Vorgehens möglichst keine externen Rückkopplungen entstehen und damit eine geringe Umsystemabhängigkeit vorliegen. Eine derartige Situation geht zumeist mit einer geringen Bedeutung des Umweltschutzes und einer hohen Bedeutung des Preises als Wettbewerbsfaktor einher. Da die Konsumenten in diesem Fall nicht gewillt sind, für ökologische Konzepte mehr zu bezahlen oder Einschränkungen bei der Nutzung der Produkte hinzunehmen, gelten ökonomische und ökologische Ziele als konfliktär. Der Misserfolg des Golf ÖKOMATIK des VolKSWAGENKonzerns Mitte der 90er Jahre kann hier als Paradebeispiel für die fehlende Akzeptanz eines ökologischen Fahrzeugkonzeptes gelten.

Eine Kostenminimierungsstrategie im internationalen Umweltmanagement ist eng mit der Volumenstrategie eines Kostenführers verknüpft. Diese wettbewerbsstrategische Ausrichtung steht dabei zumeist einem Umstieg auf integrierte Umweltschutztechnologie entgegen, da dies oft nur mit einem Austausch bestehender kostenoptimierter Prozessabläufe und -technologien möglich ist. $^{397}$ Dabei werden im Rahmen der Kostenminimierungsstrategie alle Möglichkeiten genutzt, um durch Kostensenkungen Wettbewerbsvorteile zu erlangen. In diesem Zusammenhang wird auch versucht, einer Internalisierung von Umweltschutzkosten entgegenzuwirken.

396 Fiat (Hrsg.): Fiat - 1999 Environmental Report, Turin 2000, S. 52.

397 Vgl. Meffert, H.; Kircheorg, M.: Das neue Leitbild Sustainable Development - Der Weg ist das Ziel, in: Harvard Business Manager, 15. Jg. (1993), Nr. 2, S. 34 - 45. 
Bei sich abzeichnenden Verschärfungen ökologiebezogener Gesetze versuchen Unternehmen, mit globaler Kostenminimierungsstrategie durch Lobbying Widerstand zu üben und umweltpolitische Maßnahmen zu blockieren. ${ }^{398}$ So haben verschiedene Automobilhersteller eigens Abteilungen geschaffen, um Einfluss auf die umweltpolitische Arbeit der Regierungen zu nehmen. ${ }^{399}$ Ist eine Anhebung ökologischer Standards auch unter Androhung einer Standortverlagerung und damit einhergehenden lokalen Arbeitsplatzverlusten nicht abzuwenden, können international tätige Unternehmen durch eine nationale Rückzugsstrategie und Standortverlagerung in jene Länder ausweichen, in denen geringere Umweltschutzanforderungen an die Wirtschaft gestellt werden. Es werden dabei die Länder als Produktionsstandorte im Rahmen einer globalen Kostenminimierungsstrategie bevorzugt, in denen geringe Umweltstandards bestehen und möglicherweise sogar staatliche Anreize geschaffen werden, Industrieansiedlungen unabhängig ihrer Umweltbelastungen vorzunehmen.

Als internationaler Externalisierungsvorteil steht insbesondere die Ausnutzung von Arbitrage-Effekten im Mittelpunkt dieser strategischen Ausrichtung. Die Nutzung von Arbitrage-Effekten setzt dabei eine weitgehend integrierte Wertschöpfungskonfiguration voraus. Durch Zentralisierung der Wertschöpfung an den Standorten, die nur geringe Umweltschutzvorschriften aufweisen, können diese Kostenvorteile der Gesamtunternehmung zugute kommen. Die Kostenminimierungsstrategie im internationalen Umweltschutz eignet sich insbesondere für Produkte, für die keine expliziten Umweltschutzrichtlinien einzuhalten sind. Es findet dann eine weitestgehende Standardisierung des ökologieorientierten Verhaltens auf sehr geringem ökologischen Niveau statt. ${ }^{400}$

In der folgenden Abbildung sind Ausprägungen der globalen Kostenminimierungsstrategie dargestellt.

398 Im internationalen Kontext wird gegenüber Unternehmen, die ein ausgeprăgtes Lobbying betreiben, oft der Verdacht geäußert, ihre okonomische Macht zu missbrauchen oder durch Manipulation und Bestechung ihre Ziele zu verfolgen. Vgl. zu den Gestaltungsinstrumenten Lobbying und Bestechung im internationalen Management Welge, M. K.; Berg, N.: Public Affairs-Management in Multinationalen Unternehmungen, a. a. O., S. 35 ff.

Dies bestätigten sowohl Herr Minte von der Volkswagen AG als auch Herr Krüger von FORD im Interview.

400 Vgl. Meffert, H.; KIrcheorg, M.: Das neue Leitbild Sustainable Development - Der Weg ist das Ziel, in: Harvard Business Manager, 15. Jg. (1993), Nr. 2, S. 34 - 45. 


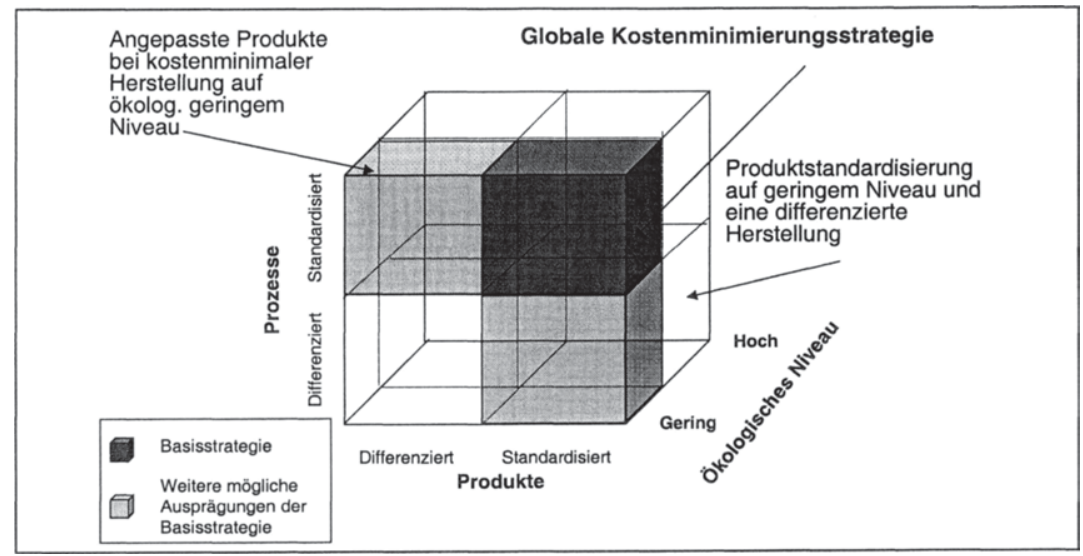

Abbildung 34: Ausprägungen einer globalen Kostenminimierungsstrategie

Quelle: Eigene Darstellung

Einschränkend ist anzumerken, dass bei Existenz unterschiedlicher ökologischer produktbezogener Anforderungen in den Absatzmärkten diese auch durch einen Kostenminimierer im Umweltschutz zu erfüllen sind, wenn die Märkte bearbeitet werden sollen. ${ }^{401}$ Somit kann eine Anpassung der Produkte an die ökologischen Vorgaben bei einer weitgehenden Umgehung anspruchsvoller umweltschutzbezogener Richtlinien in der Fertigung als eine mögliche Ausprägung einer globalen Kostenminimierungsstrategie gelten. Bei Fehlen produktbezogener Vorschriften und unterschiedlichen, aber wenig anspruchsvollen fertigungsbezogenen Umweltgesetzen ist ebenfalls eine Standardisierung der Produkte auf niedrigem Niveau bei Nutzung heterogener Produktionsverfahren eine mögliche Ausgestaltungsalternative der Kostenminimierungsstrategie.

Eine globale Kostenminimierungsstrategie stellt insbesondere für die Automobilindustrie aufgrund der hohen öffentlichen Exponiertheit der Branche eine sehr risikoreiche Strategie dar. ${ }^{402}$ Dennoch ließen sich in der Vergangenheit Anzeichen erkennen, die vermuten lassen, dass eine derartige Strategie auch in der Automobilindustrie verfolgt wurde. So haben einzelne Automobilhersteller

401 Vgl. dazu die Bemerkungen zum GATT in Kapitel A 2.1.

402 Wie schwierig es ist, ein Unternehmen ökologisch proaktiv zu positionieren, welches zuvor über viele Jahre eine Widerstandsstrategie im Umweltschutz verfolgt hat, zeigt sich derzeit bei FORD, dessen ökologiebezogenen Aussagen besonders kritisch von Anspruchsgruppen hinterfragt werden.Vgl. zur Verleihung des Greenwash Awards an FORD das Insert in Kapitel B 3.2.1. 
insbesondere in den 80er Jahren Produktionsstandorte zur Kostensenkung in Schwellenländern angesiedelt. In den Ländern galten und gelten zumeist vergleichsweise geringe Umweltschutzvorschriften. Streben diese Unternehmen dort nicht höhere unternehmenseigene Standards im Umweltschutz an, könnte durchaus eine bewusste ökologische Externalisierung vermutet werden. ${ }^{403}$

\subsection{Multinationale Umweltschutzstrategie}

Bei Verfolgung einer multinationalen Umweltschutzstrategie kommt der Erzielung von Effizienzzielen im Umweltmanagement nur eine untergeordnete Rolle zu. Dies bedeutet allerdings nicht, dass bei einer regionalen Ausrichtung diese nicht wahrgenommen werden. Vielmehr ist die Realisierung von Effizienzeffekten über eine räumlich begrenzte Absatzregion hinaus aufgrund der Differenzen der Kontextfaktoren und der Höhe der Koordinationskosten nicht angestrebt. Aufgrund der Abhängigkeit vom jeweiligen ökologiebezogenen Kontext gewinnen einzelne Wirkungsziele allerdings eine vergleichsweise hohe Bedeutung. So sind an allen Standorten die Erfüllung der lokalen umweltrechtlichen Bestimmungen sowie eine Orientierung an den Forderungen wichtiger lokaler Anspruchsgruppen Ausdruck der multinationalen Orientierung im Umweltschutz. Dies schließt eine enge Beziehung zu den Anwohnern und den lokalen Regulierungsbehörden an den Standorten explizit mit ein. Somit steht die Sicherung der lokalen gesellschaftlichen Legitimität im Vordergrund dieser Strategie.

In diesem Zusammenhang kommt den direkten unternehmensweiten Koordinationszielen nur eine geringe Bedeutung zu. Vielmehr steht die Durchsetzung einer weitreichenden Dezentralisation im Mittelpunkt der Bemühungen multinational ausgerichteter Unternehmungen im Umweltschutz. Die Sicherstellung der lokalen Handlungsflexibilität und die vollständige Anpassung an die lokalen Kundenbedürfnisse sowie die Motivation von Mitarbeitern und Führungskräften für die lokalen ökologischen Ziele haben oberste Priorität.

403 Die nordamerikanischen und europäischen Hersteller versuchten den japanischen Wettbewerbern zu Beginn der 80er Jahre durch die Verlagerung ihrer Produktion in Niedriglohnländer zu begegnen. Vgl. Womack, J. P.: Die zweite Revolution in der Automobilindustrie: Konsequenzen aus der weltweiten Studie aus dem Massachusetts Institute of Technology, a. a. O., S. $86 \mathrm{ff}$. 
Eine multinationale Ausrichtung im internationalen Umweltmanagement zeichnet sich somit durch eine weitgehende Adaption der Auslandsgesellschaften an die lokalen ökologiebezogenen Rahmenbedingungen aus. Diese strategische Ausrichtung erscheint dann sinnvoll, wenn sowohl die ökologischen Externalisierungs- als auch die Internalisierungsvorteile gering ausgeprägt sind. Umweltschutz spielt dabei in den einzelnen Ländermärkten eine differenzierte Rolle, zählt aber nicht zu den bedeutenden Differenzierungsinstrumenten im internationalen Wettbewerb. ${ }^{404}$ Auch sollten bei Entscheidung für eine multinationale Umweltschutzausrichtung ökologiebezogene internationale Rückkopplungen nur in geringem Maß vorliegen. Eine idealtypische Situation, in der es sich anbietet, eine multinationale Umweltschutzstrategie zu wählen, ist in der folgenden Übersicht dargestellt.

\begin{tabular}{|c|c|c|}
\hline & $\begin{array}{l}\text { Auspräqung exogener Kontextfaktoren } \\
\text { - Umsystemähnlichkeit } \\
\text { - Umsystemabhängigkeit }\end{array}$ & $\begin{array}{l}\text { gering } \\
\text { gering }\end{array}$ \\
\hline & $\begin{array}{l}\text { Ausprägung endogener Kontextfaktoren } \\
\text { - Bedeutung des Umweltschutzes } \\
\text { - Strategische Umweltschutzorientierung } \\
\text { - Zentralisations- u. Standardisierungsgrad } \\
\text { - Öffentliche Exponiertheit }\end{array}$ & $\begin{array}{c}\text { differenziert } \\
\text { lokal angepasst } \\
\text { gering } \\
\text { lokal } \\
\text { differenziert }\end{array}$ \\
\hline & $\begin{array}{l}\text { Rückkopplungen } \\
\text { - Interne Rückkopplungen } \\
\text { - Externe Rückkopplungen }\end{array}$ & $\begin{array}{l}\text { gering } \\
\text { gering }\end{array}$ \\
\hline
\end{tabular}

Abbildung 35: Situativer Kontext für eine multinationale Umweltschutzstrategie

Quelle: Eigene Darstellung

Im Rahmen einer multinationalen Umweltschutzstrategie orientieren sich die einzelnen Auslandsgesellschaften lediglich an den jeweiligen Iokalen Umweltschutzanforderungen. So verfolgen HONDA, MITSUBISHI, NISSAN und FORD keine unternehmensweit geltenden Umweltschutzsstandards, sondern schreiben den Tochtergesellschaften lediglich die Einhaltung lokaler Umweltschutzgesetze vor. ${ }^{405}$ Dies kann in einer vergleichsweise geringen ökologischen Relevanz der

404 Vgl. hierzu und im Folgenden Brodel, D.: Internationales Umweltmanagement: Gestaltungsfelder - Determinanten - Ausprägungen, a. a. O., S. 409.

405 Vgl. oekom research (Hrsg.): Corporate Responsibility Industry Report Automobile: A Corporate Responsibility Survey of 19 Companies of the Industry, a. a. 0. 
Wertschöpfung und deshalb weitgehend ausbleibenden externen Rückkopplungen begründet sein. Weiterhin können sich die lokalen Umfelder in Bezug auf die ökologischen Gesetze, abweichende Einstellungen, Motive und Bedürfnisse der Verbraucher sowie insbesondere eine divergierende Zahlungsbereitschaft für Umweltschutz als Gründe ${ }^{406}$ für eine differenzierte Umweltschutzstrategie angeführt werden. Diese Komplexitätsbarrieren ${ }^{407}$ können bewirken, dass ein standardisiertes ökologiebezogenes Vorgehen zu Akzeptanzverlusten bei den Konsumenten vor Ort führen würde. So könnten bspw. Automobilkäufer aufgrund höherer Anschaffungspreise und gleichzeitig fehlenden staatlichen Begünstigungen Aufpreise für nicht vorgeschriebene Abgasreinigungstechnik nicht akzeptieren. Demgegenüber ermöglicht es eine lokale Differenzierung, das Angebot von Umweltqualität nach den Konsumentenpräferenzen bzw. der Zahlungsbereitschaft der Kunden zu richten.

Weiterhin können aufgrund einer Zentralisierung bei Verfolgung einer standardisierten Umweltschutzstrategie Steuerungs- und Kontrollprobleme zwischen der Unternehmenszentrale und einzelnen Tochtergesellschaften bzw. lokalen Repräsentanten auftreten. Solche Reibungsverluste bei der Steuerung und Kontrolle von ausländischen Unternehmensaktivitäten basieren $u$. a. auf zu langen Kommunikationswegen und dem geringeren zentral vorliegenden Wissen über die lokale Situation sowie einem Motivationsverlust der lokalen Manager bei starker Einschränkung ihrer Entscheidungskompetenzen. Dies kann mögliche Kostenvorteile einer Standardisierung überkompensieren bzw. ihre Umsetzung verzögern. ${ }^{408} \mathrm{Im}$ Gegensatz dazu nutzt eine multinationale Ausrichtung im Umweltschutz den Informationsvorteil der lokalen Umweltmanager und erweist sich auch deshalb bei Änderungen von ökologiebezogenen Gesetzen oder anderen lokalen Ansprüchen als flexibel. Es findet eine weitgehende Dezentrali-

406 Vgl. Müller, S.; Kornmeler, M.: Grenzen der Standardisierung im Internationalen Marketing, in: Jahrbuch der Absatz- und Verbrauchsforschung, 1996, Nr. 1, S. 6- 10.

407 Vgl. zum Begriff der Komplexitätsbarrieren Brodel, D.: Internationales Umweltmanagement: Gestaltungsfelder - Determinanten - Ausprägungen, a. a. O., S. 386 f.

408 Reibungsverluste können zwischen der Zentrale und den Tochtergesellschaften bzw. den lokalen Reprăsentanten auftreten. Auf der einen Seite ergeben sich Schwierigkeiten, die Tochtergesellschaften zu managen, weil bspw. [...] "conditions in each locale are too different for long-distance management. "Auf der anderen Seite besteht die Gefahr, dass Manager vor Ort die Standardisierungsstrategie unterlaufen, weil sie ${ }_{n}[. .$.$] feel that they are$ closer to the market and believe that they are better equipped to handle local market conditions." Shoham, A.: Global Marketing Standardization, in: Journal of Global Marketing, 9. Jg. (1995), Nr. 1 / 2, S. 102. Vgl. auch Shoham, A.; Albaum, G.: The Effects of Transfer of Marketing Methods on Export Performance: An Empirical Examination, in: International Business Review, 3. Jg. (1994), Nr. 3, S. 219 - 241. 
sation und damit eine Entlastung der Zentrale bezüglich ökologiegerichteter Entscheidungsaufgaben statt, sodass die Voraussetzungen für eine hohe lokale Motivation der Verantwortlichen im Umweltschutz gegeben ist.

Die Wahl einer wenig integrierten, internationalen Wertschöpfungskonfiguration spricht schließlich ebenfalls für eine multinationale Ausrichtung im Umweltmanagement, da die Kosten einer Abstimmung des Umweltschutzes bei ansonsten weitestgehend fehlender Kommunikationsinfrastruktur zwischen den internationalen Tochtergesellschaften mit vergleichsweise hohen Koordinationskosten verbunden wäre. Globale und transnationale Ausrichtungen der Wertschöpfung können dagegen kaum mit einer multinationalen Umweltschutzstrategie vereinbart werden, da bei stark integrierter Fertigung unternehmensinterne Leistungsverflechtungen die Einhaltung vorgegebener einheitlicher ökologischer Standards notwendig machen.

In der folgenden Abbildung sind die möglichen Ausprägungen einer multinationalen Umweltschutzstrategie skizziert.

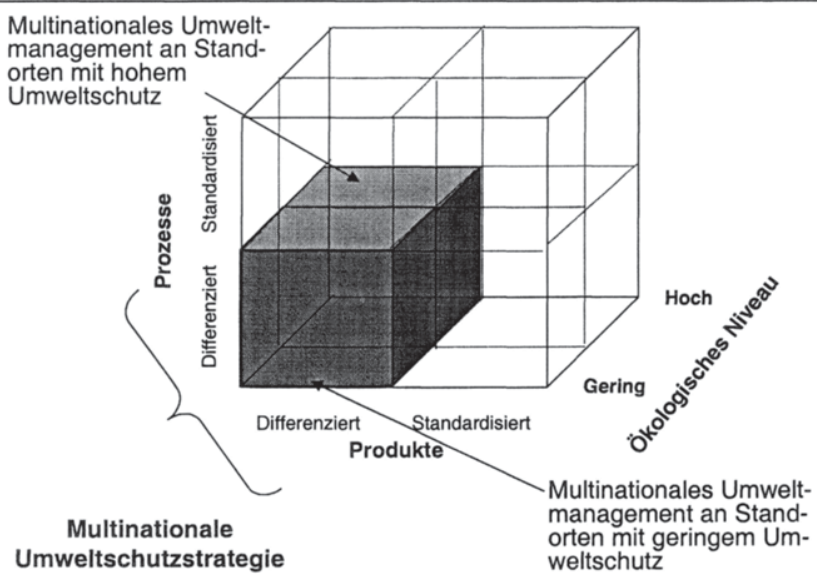

Abbildung 36: Ausprägungen multinationaler Umweltschutzstrategien

Quelle: Eigene Darstellung

Multinationale Umweltschutzstrategien weisen zumeist auf Ebene der Unternehmenszentrale keine eigenständigen Umweltschutzziele auf, sondern setzen eher auf eine Anpassung an lokale Erfordernisse durch eine differenzierte Ausrichtung in Bezug auf ökologische Fragestellungen. Bei multinationaler Um- 
weltschutzausrichtung entscheiden die lokal gültigen produkt- und produktionsbezogenen Umweltschutzvorschriften und die lokalen Anspruchsgruppen der Ländermärkte über die Umweltleistung ${ }^{409}$ der Gesamtunternehmung. Bei hoher Exportabhängigkeit der einzelnen Landesgesellschaften gilt es hingegen, sich dann an die Umweltschutzerfordernisse der Ländermärkte anzupassen, von denen eine hohe Exportabhängigkeit besteht. „Firms based in large home markets like the United States and the European Union (EU) should pay great attention to domestic environmental regulations. In contrast, firms from smaller countries like Canada, most Asian countries and all Latin American countries should focus primarily upon the environmental regulations of their major customers". 410

In der Automobilindustrie finden sich durchaus Ansatzpunkte für multinationale bzw. regionale Ausrichtungen im Umweltschutz. Dabei passen sich die Hersteller zumeist an die unterschiedlichen produkt- und fertigungsbezogenen Anforderungen im Umweltschutz in großen Wirtschaftsräumen an. Insbesondere Automobilkonzerne, die in unterschiedlichen Regionen mit unterschiedlichen Marken präsent sind, wählen eine multinationale Umweltschutzstrategie, da dann kaum externe Rückkopplungen zu enwarten sind. Eine Bestätigung für eine derartige Verhaltensausrichtung könnte bspw. im unterschiedlichen Abschneiden verschiedener Konzernmarken der FORD MOTOR COMPANY in einem Umweltranking gesehen werden. Während bspw. die insbesondere im asiatischen Raum stark präsente Marke MAZDA in einer Untersuchung aus dem Jahr 2000 den letzten Rang (14) belegt, ist die Marke ForD auf dem 8. Platz. ${ }^{411}$ Die europäische Marke Volvo wurde nicht untersucht, allerdings ist der Hersteller für seine ökologisch proaktive Verhaltensausrichtung bekannt. ${ }^{\mathbf{4 1 2}}$

409 Vgl. Zur Messung der Umweltleistung Klelvane, T.: Environmental Performance Evaluation - The Link Between Management Systems and Reality, in: Hitchens, D. M. W. N.; Clausen, J.; Fichter, K.: International Environmental Management Benchmarks: Best Practice Experiences from America, Japan and Europe, Berlin u. a. 1999, S. 103 - 109.

Rugmann, A. M.; Verbeke, A.: Corporate Strategy and International Environmental Policy, in: Journal of International Business Studies, 29. Jg. (1998), Nr. 4, S. 820.

411 Vgl. oekom research (Hrsg.): Corporate Responsibility Industry Report Automobile: $A$ Corporate Responsibility Survey of 19 Companies of the Industry, a. a. O., S. 20.

412 Eine umfassende Darstellung der ökologischen Ausrichtung von Volvo findet sich bspw. bei Plaseckl, B. W. et al.: Environmental Management and Business Strategy: Leadership Skills for the 21st Century, New York et al. 1999, S. $192 \mathrm{ff}$. 


\subsection{Selektive Umweltschutzstrategie}

Eine duale Ausrichtung im internationalen Umweltmanagement zur Erzielung von Vorteilen durch eine ökologische Internalisierung sowie Externalisierung zielt insbesondere auf eine hohe ökonomische Effizienz im internationalen Umweltmanagement $a b$ und verlangt damit nach einer gleichzeitigen Verfolgung von Größen-, Erfahrungs-, Synergie-, Arbitrage- und Entwicklungsstandportfolio-Effekten.

In Bezug auf die Wirkungsziele im fertigungsbezogenen Umweltschutz kann die Einhaltung der lokalen Umweltschutzvorschriften als ausreichend betrachtet werden. Da ein Großteil der Produktion zumeist nicht dort abgesetzt wird, wo sie hergestellt wird, erscheint eine Berücksichtigung weitreichender ökologischer Forderungen der lokalen Anspruchsgrupppen keine große Bedeutung beizumessen zu sein. Zur Sicherstellung der gesellschaftlichen Legitimität in den wichtigsten Absatzmärkten kann auf die ökologische Ausrichtung der Produkte bzw. einzelner Modelle verwiesen werden. Aufgrund des dualen Vorgehens kommt dem Aufbau eines hohen Umweltimages eher eine untergeordnete Bedeutung zu, da dies $u$. U. externe Rückkopplungen erst hervorrufen könnte.

Als direkte Koordinationsziele kommt insbesondere der Förderung der organioatorischen Lernfähigkeit und dem Aufbau ökologischen Wissens bzgl. produktbezogener Belange eine hohe Bedeutung zu. Dies kann durch Aufbau von Forschungs- und Entwicklungsabteilungen in ökologischen Vorreiterländern und eine Verknüpfung dieser mit den Produktionsgesellschaften in den Schwellenländern erreicht werden.

Im Zusammenhang mit den Nebenzielen der Koordination kommt der Realisierung von Zentralisationszielen bei der Produktion von ökologisch standardisierten Produkten eine hohe Bedeutung zu, während im fertigungsbezogenen Umweltschutz Dezentralisationsziele eher im Vordergrund stehen. Aufgrund der Betonung von Effizienzzielen im Rahmen der selektiven Umweltschutzstrategie kommt der Minimierung von Koordinationskosten ein hoher Stellenwert zu.

Die internationalen Informationsziele in Bezug auf den produkt- und fertigungsbezogenen Umweltschutz sind bei selektiver Ausrichtung unterschiedlich. So ist zur Gewährleistung einer proaktiven Ausrichtung im produktbezogenen Umweltschutz ein internationales Frühwarnsystem aufzubauen, welches die Entwicklungen in den wichtigsten Absatzmärkten und den ökologischen Vorreiterländern beobachtet und Prognosen über die zukünftigen Ent- 
wicklungen zulässt. Dagegen sind im fertigungsbezogenen Umweltschutz lediglich die lokalen Entwicklungen zu beobachten.

Im Rahmen einer selektiven Umweltschutzstrategie versuchen Unternehmen demzufolge sowohl durch eine ökologische Internalisierung als auch Externalisierung Vorteile zu generieren, da generell beide Ausrichtungen hohe Vorteile erwarten lassen. In diesem Kontext liegen interne anbieterbezogene Rückkopplungen im produktbezogenen Umweltschutz vor, sodass Vorteile durch eine ökologiegerichtete Standardisierung auf hohem Niveau zumindest für Teile des Produktionsprogramms zu erwarten sind. Weiterhin können durch ein Herunterfahren ökologischer Standards im fertigungsbezogenen Umweltschutz Kostenvorteile generiert werden. In diesem Kontext empfiehlt sich die Anwendung selektiver Umweltschutzausrichtungen lediglich bei geringer Umsystemabhängigkeit und weitgehend ausbleibenden externen Rückkopplungen. Eine derartige Situation ist in der folgenden Abbildung skizziert.

\begin{tabular}{|l|c|}
\hline \begin{tabular}{|l|l|}
\hline Ausprägung exogener Kontextfaktoren \\
- Umsystemähnlichkeit
\end{tabular} & mittel \\
- Umsystemabhängigkeit & gering \\
\hline Ausprägung endogener Kontextfaktoren & \\
- Bedeutung des Umweltschutzes & mittel \\
- Strategische Umweltschutzorientierung & selektiv \\
- Zentralisations- u. Standardisierungsgrad & hoch / mittel \\
- Öffentliche Exponiertheit & gering \\
\hline Rückkopplungen & hoch / mittel \\
- Interne Rückkopplungen & gering \\
\hline
\end{tabular}

Abbildung 37: $\quad$ Situativer Kontext für eine selektive Umweltschutzstrategie Quelle: Eigene Darstellung

Im Rahmen einer selektiven Umweltschutzstrategie sollen durch ein duales Vorgehen sowohl die Chancen hoher Umweltschutzstandards in Bezug auf einzelne Kundengruppen als auch Kostensenkungspotenziale durch die Nutzung produktionsbezogener Externalisierungsmöglichkeiten mit niedrigen Umweltschutzvorschriften wahrgenommen werden. ${ }^{413}$ Umweltschutzziele werden dabei ökonomischen Zielen nachgeordnet. Da ökologiegerichtete Maßnahmen nur

413 Vgl. Brodel, D.: Internationales Umweltmanagement: Gestaltungsfelder - Determinanten Ausprägungen, a. a. O., S. 412. 
dann umgesetzt werden, wenn sie in direkter Beziehung zu ökonomischen Erfolgen stehen oder als gesetzliche Vorschriften als Nebenbedingung beachtet werden müssen, erscheint eine eigenständige Festlegung ökologischer Zielsetzungen auf Ebene der Gesamtunternehmung nicht notwendig.

Das duale Vorgehen kann sich dabei bspw. auf das Angebot ökologisch relativ vorteilhafter Produkte beziehen, während die Produktion unter weitgehender Missachtung ökologischer Faktoren durch eine gezielte Standortwahl zur Externalisierung von Umweltschutzkosten vorgenommen wird. Diese Situation könnte sich dann einstellen, wenn der Umweltvorteil des Produktes zu einem Individualnutzen beim Konsumenten führt, wie dies bspw. bei verbrauchsarmen Pkw der Fall ist. Eine ökologiegerechtere Herstellung des Pkw führt im Gegensatz dazu beim Konsumenten nicht direkt zu einem erfahrbaren Nutzen und unterbleibt aus Kostengesichtspunkten.

Das Unternehmen HONDA belegte aufgrund seiner verbrauchs- und schadstoffarmen Fahrzeuge in den USA in einem Umweltranking, welches sich allein auf die ökologische Bewertung der Produkte bezog, den vordersten Platz.

„By implementing gas-saving technologies and reducing emissions below required levels, Honda produces vehicles with the best environmental performance in today's market. Since 1998, Honda has built most of its Accords and Civics to meet California's LEV stan-dard, which is 57 percent more stringent than EPA's current standard. At the same time, Honda leads other automakers in fuel efficiency with cars that average an industry-best 31.8 miles per gallon. In addition, Honda sells fewer SUVs and light trucks than other automakers, and those it does sell use less fuel and run cleaner than those of competing brands. ${ }^{\text {4114 }}$

Eine Bewertung, die neben dem produktbezogenen auch den fertigungsbezogenen Umweltschutz und die Organisation des Umweltschutzes beurteilte, fiel wesentlich schlechter aus. So belegt die auf Umweltratings spezialisierte Argentur ОЕКОM den Automobilhersteller HONDA im Jahr 2000 lediglich mit einem $C$ bei Noten von A+ bis D-. Damit belegt Honda den 10. Rang von 14 Herstellern. ${ }^{415}$

414 Morey, C. et al.: Pollution Lineup: An Environmental Ranking of Automakers, Union of Concerned Scientists (Hrsg.), March 2000.

415 Vgl. oekom research (Hrsg.): Corporate Responsibility Industry Report Automobile: A Corporate Responsibility Survey of 19 Companies of the Industry, a. a. 0. 
Weiterhin könnte auch die Wahl einer ökologischen Produktpositionierung in einzelnen Ländermärkten oder Käufersegmenten bei gleichzeitigem Anbebot von Produkten in anderen Märkten oder anderen Segmenten, die ökologisch als besonders belastend gelten, als duales Vorgehen gewertet werden. So beteiligt sich FORD in den USA maßgeblich an der Entwicklung der Brennstoffzelle im Rahmen der "California Fuel Cell Partnership" und der Entwicklung umweltfreundlicher Automobile im Rahmen der "Partnership for a New Generation of Vehicles" (PNGV). Gleichzeitig ist der Light Truck Anteil von $54 \%$ der verkauften Pkw bei FORD in den USA vergleichsweise groß. Diese Fahrzeuge gelten als besonders emissionsstark. Die Modelle der Firma ForD zeichneten sich dabei 1998 in Bezug auf den Kraftstoffverbrauch als besonders verbrauchsstark aus. FORD nimmt in einem Öko-Ranking deshalb den drittletzten Platz von 11 Herstellern ein, die „Pollution Performance ${ }^{4}$ wird als "worst" bewertet. ${ }^{416}$

Das selektive Vorgehen erscheint auch dann angebracht, wenn bspw. in den Triademärkten die Umweltverträglichkeit des Fahrzeugs zu den wichtigen Profilierungsdimensionen im Wettbewerb zählt, aufgrund einer mangelnden Sensibilisierung oder Zahlungsbereitschaft dies aber in den Märkten der Schwellenländer nicht der Fall ist.

Das Ziel einer selektiven Umweltschutzstrategie liegt in der kurzfristigen Verbesserung der Wirtschaftlichkeit. In der Automobilindustrie sind selektive Ausrichtungen im Umweltschutz durchaus beobachtbar, wenn nicht gar die Regel.

Die folgende Abbildung zeigt den Ausgestaltungsraum der selektiven Umweltschutzstrategie für das Gesamtunternehmen.

416 Vgl. Ford Motor Company (Hrsg.): Connecting with Society: 1999 Corporate Citizenship Report, Dearborne 2000, S. 62 f. sowie Morey, C. et al.: Pollution Lineup: An Environmental Ranking of Automakers, Union of Concerned Scientists (Hrsg.), March 2000, S. 5 und 9. 


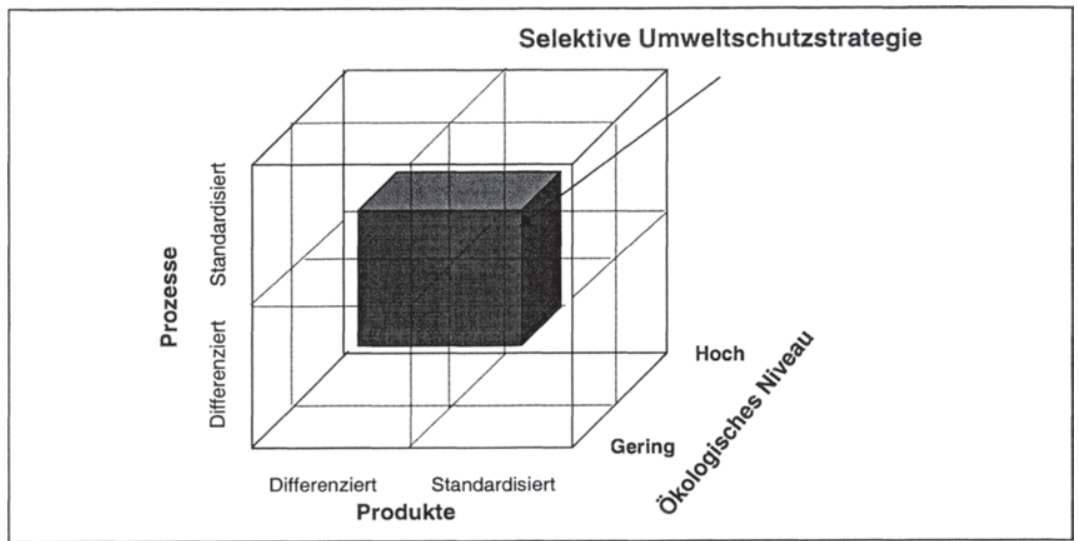

Abbildung 38: Ausgestaltung der selektiven Umweltschutzstrategie

Quelle: Eigene Darstellung

Nachdem die unterschiedlichen strategischen Basisorientierungen im Umweltmanagement dargestellt wurden, findet im folgenden Kapitel eine Systematisierung des im Rahmen des internationalen Umweltmanagement anwendbaren Koordinationsinstrumentariums statt, welches sodann auf die einzelnen Strategien übertragen wird.

\section{Instrumente zur Koordination des internationalen ökologiegerich- teten Verhaltens}

Im Kontext dieser Arbeit werden Koordinationsinstrumente als Regelungen verstanden, die Art und Umfang von internen und externen ökologiebezogenen Rückkopplungen zwischen national unterschiedlichen Organisationseinheiten steuern und eine Abstimmung arbeitsteiliger Prozesse durch Ausrichtung der Entscheidungen autonomer Teileinheiten auf die Koordinationsziele gewährleisten. ${ }^{417}$ Die Instrumente der Koordination im internationalen Umweltmanagement können dabei als alternative bzw. sich ergänzende Möglichkeiten betrachtet werden, die realistisch und praktikabel sind. ${ }^{418}$

417 Vgl. Kleser, A.; Kublcek, H.: Organisation, a. a. O., S. 74, zitiert nach Hoffmann, F.: Führungsorganisation, a. a. O., S. 316.

418 Vgl. Frese, E.: Organisation und Koordination, in: Zeitschrift für Organisation, 41. Jg. (1972), S. 408 sowie Macharzina, K.: Unternehmensführung: Das internationale Managementwissen; Konzepte - Methoden - Praxis, a. a. O., S. 715. 


\subsection{Systematisierung des Koordinationsinstrumentariums}

In der Literatur findet sich eine Reihe unterschiedlicher Klassifikationsschemata zur Typologisierung des Koordinationsinstrumentariums. ${ }^{419}$ Dabei hat sich bis heute keine geschlossene Theorie der Koordination durchgesetzt. ${ }^{420}$ Allerdings hat die Differenzierung des Koordinationsinstrumentariums nach strukturellen, technokratischen und personellen Abstimmungsinstrumenten in der wissenschaftlichen Diskussion breite Akzeptanz gefunden. ${ }^{421}$ Diese grundlegende Systematisierung geht auf KHANDWALLA zurück und basiert auf einem theoretischen Modell zum Zusammenhang von Umweltunsicherheit und Organisationsstruktur. ${ }^{422}$

Die von KHANDWALLA vorgenommene Differenzierung in strukturelle, technokratlsche und personelle Koordinationsinstrumente soll im Rahmen dieser Arbeit grundlegend übernommen werden. Sie erlaubt eine relativ trennscharfe und überschneidungsfreie Zuordnung weiterer, in der Literatur im Rahmen der Organisationslehre, des internationalen Managements sowie des Umweltmanagements diskutierter Koordinationsverfahren. ${ }^{423}$ Dies ermöglicht es, eine relativ umfassende Berücksichtigung alternativer Koordinationsinstrumente im internationalen Umweltmanagement aufzuzeigen. ${ }^{424}$

419 Auflistungen von unterschiedlichen Systematisierungsansătzen des Koordinationsinstrumentariums finden sich bspw. bei Heynen, C.-H.: Die Koordination dezentraler Entscheidungen: Ein verhaltensorientierter Ansatz zur Analyse der Koordination von MarketingEntscheidungen, Münster 1975, S. 48; Benkensteln, M.: F \& E und Marketing: Eine Untersuchung zur Leistungsfähigkeit von Koordinationskonzeptionen bei Innovationsentscheidungen, a. a. O., S. 130 ff. und Wolf, J.: Internationales Personalmanagement: Kontext, Koordination, Erfolg, Wiesbaden 1994, S. 116.

Vgl. Brockhoff, K.; Hauschlldt, J.: Schnittstellen-Management - Koordination ohne Hierarchie, in: Zeitschrift für Organisation, Nr. 6, 1993, S. 396 - 403; Meffert, H.: Marketing: Grundlagen marktorientierter Unternehmensführung: Konzepte - Instrumente - Praxisbeispiele, a. a. O., S. 1013.

Vgl. Macharzina, K.; Oesterle, M.-J.: Bestimmungsgrößen und Mechanismen der Koordination von Auslandsgesellschaften, a. a. O., S. 615.

Vgl. hierzu und im Folgenden Khandwalla, P. N.: Uncertainty and the "Optimal" Design of Organizations, McGill University, Working Paper, Houston 1972 sowie Khandwalla, P. N.: Unsicherheit und die optimale Gestaltung der Organisation, in: Grochla, E. (Hrsg.): Organisationstheorie, 1. Teilband, Stuttgart 1975, S. 140-156.

Vgl. Hoffmann, F.: Führungsorganisation, a. a. O., S. 328.

Vgl. zur kritischen Würdigung des Modells von KHandwalla bspw. Hoffmann, F.: Führungsorganisation, a. a. O., S. 325 - 328; Welge, M.: Management in deutschen multinationalen Unternehmungen: Ergebnisse einer empirischen Untersuchung, a. a. O., S. 305 f.; Frese, E.: Grundlagen der Organisation, Konzept - Prinzipien - Strukturen, a. a. O., S. 401 ff. 
Im Folgenden werden die Koordinationsinstrumente in Anlehnung an HOFFMANN in Anbetracht ihrer unterschiedlichen Wirkungsweise weiter in koordinationsbedarfsreduzierende und -deckende Maßnahmen differenziert. ${ }^{425}$ Dabei können koordinationsbedarfsreduzierende Maßnahmen als solche Eingriffe verstanden werden, welche den Bedarf einer internationalen ökologiegerichteten Koordination durch Verringerung von internen und externen Rückkopplungen veringern. Koordinationsbedarfsdeckende Maßnahmen umfassen hingegen solche Maßnahmen, die Art und Ausmaß verbleibender Rückkopplungen durch Abstimmung arbeitsteiliger Prozesse steuern und die Ausrichtung ökologiegerichteter Entscheidungen relativ autonomer Teileinheiten auf die Ziele im internationalen Umweltmanagement zum Inhalt haben. ${ }^{426}$

Tabelle 4 zeigt die, in den folgenden Unterkapiteln auf die vorliegende Problemstellung übertragenen strukturellen, technokratischen und personellen Koordinationsinstrumente im Überblick. Die Instrumente sind entsprechend inrer Wirkung nach koordinationsbedarfsreduzierenden und -deckenden Maßnahmen weiter systematisiert und im Überblick dargestellt.

\section{Strukturelle Maßnahmen}

Strukturelle koordinationsbedarfsreduzierende Maßnahmen umfassen die Differenzierung bzw. Trennung in ökologiebezogene Inlands- und Auslandsaktivitäten durch Abteilungs-, Sparten- oder Divisionsbildung sowie eine anschlieBende Integration der Tätigkeiten in die internationale Unternehmung. ${ }^{427}$ Eng damit in Zusammenhang steht die Entscheidung über eine Zentralisierung bzw. Dezentralisierung der Entscheidungskompetenzen im internationalen Umweltmanagement. ${ }^{428}$ Die Bildung operationaler Organisationsstrukturen stellt in diesem Kontext quasi das "Rückgrat" einer koordinierenden ökologiebezogenen Aufgabenerfüllung im internationalen Kontext dar. ${ }^{429}$ Verbleibende

425 Vgl. Hoffmann, F.: Führungsorganisation, a. a. O., S. 328; Meffert, H.: Marketing: Grundlagen marktorientierter Untemehmensführung: Konzepte - Instrumente - Praxisbeispiele, a. a. O., S. 1013.

In Anlehnung an Frese, E.: Organisation und Koordination, a. a. O., S. 408, zitiert nach Hoffmann, F.: Führungsorganisation, a. a. O., S. 338.

427 Vgl. Welge, M.: Management in deutschen multinationalen Unternehmungen: Ergebnisse einer empirischen Untersuchung, a. a. O., S. 193.

Vgl. Macharzina, K.; Oesterle, M.-J.: Bestimmungsgrößen und Mechanismen der Koordination von Auslandsgesellschaften, a. a. O., S. 616.

429 Vgl. Brodel, D.: Internationales Umweltmanagement: Gestaltungsfelder - Determinanten Ausprägungen, a. a. O., S. 444. 
Interdependenzen können entweder bewusst vernachlässigt werden oder sie sind durch strukturelle koordinationsbedarfsdeckende Maßnahmen, wie den Einsatz vertikaler bzw. horizontaler uni- und multipersonaler Koordinationsinstrumente sowie durch Einrichtung angepasster Informations- bzw. Kommunikationsstrukturen abzustimmen. ${ }^{430}$ Eine funktions- und länderübergreifende Kommunikation kann in diesem Kontext aufgrund des Querschnittscharakters vieler Umweltschutzprobleme als besonders wichtig angesehen werden.

\begin{tabular}{|c|c|c|c|}
\hline & $\begin{array}{l}\text { Strukturelle } \\
\text { Maßnahmen }\end{array}$ & $\begin{array}{l}\text { Technokratische } \\
\text { Instrumente }\end{array}$ & $\begin{array}{c}\text { Personenorientierte } \\
\text { Maßnahmen }\end{array}$ \\
\hline $\begin{array}{l}\text { Koordinations- } \\
\text { bedarfs- } \\
\text { reduzierende } \\
\text { Maßnahmen }\end{array}$ & $\begin{array}{l}\text { - Differenzierung } \\
\text { - Integration } \\
\text { - Zentralisierung } \\
\text { - Dezentralisierung } \\
\text { - Bewusste Vernach- } \\
\text { lässigung von Inter- } \\
\text { dependenzen }\end{array}$ & $\begin{array}{l}\text { - Ausgestaltung des } \\
\text { Informationssystems } \\
\text { - Informatorische } \\
\text { Vereinheitlichung } \\
\text { - Antizipation } \\
\text { - Ökologische Pro- } \\
\text { gramm-/ Verfahrens- } \\
\text { bereinigung } \\
\text { - Bandbreiten / } \\
\text { Standards } \\
\text { - Verringerung des } \\
\text { Anspruchsniveaus }\end{array}$ & $\begin{array}{l}\text { - Personalmanagement } \\
\text { - Personalaus- } \\
\text { wahl } \\
\text { - Personalent- } \\
\text { wicklung } \\
\text { - Personalbe- } \\
\text { wertung/ } \\
\text { Anreizsystem } \\
\text { - Internationale Dele- } \\
\text { gation von Mitarbei- } \\
\text { tern } \\
\text { - Besuche }\end{array}$ \\
\hline $\begin{array}{l}\text { Koordinations- } \\
\text { bedarfs- } \\
\text { deckende } \\
\text { Maßnahmen }\end{array}$ & $\begin{array}{l}\text { - Uni- und multi- } \\
\text { personale Instru- } \\
\text { mente zur vertikalen } \\
\text { Koordination } \\
\text { - Uni- und multiperso- } \\
\text { nale Instrumente zur } \\
\text { horizontalen / late- } \\
\text { ralen Koordination }\end{array}$ & $\begin{array}{l}\text { - Planung } \\
\text { - Zielvorgaben } \\
\text { - Mittelvorgaben } \\
\text { - Planungssystem } \\
\text { - Interne Märkte / } \\
\text { Verrechnungspreise } \\
\text { - Regeln / Programme } \\
\text { Aktivitätenrei- } \\
\text { henfolgen } \\
\text { - Leitungsprozesse }\end{array}$ & $\begin{array}{l}\text { - Persönlicher } \\
\text { Führungsstil } \\
\text { - Autoritärer } \\
\text { Führungsstil } \\
\text { - Partizipativer } \\
\text { Führungsstil }\end{array}$ \\
\hline
\end{tabular}

Tabelle 4:

Überblick über das Koordinationsinstrumentarium im internationalen Umweltmanagement

Quelle: Eigene Darstellung

\section{Technokratische Instrumente}

Die unmittelbare Funktion der technokratischen koordinationsbedarfsreduzierenden Instrumente ist es, zeit- und kostenintensive einzelfallbezogene Abstimmungsmaßnahmen zu reduzieren, die in der internationalen Unternehmung allein schon aufgrund der räumlichen Distanz zwischen den interdependenten

430 Vgl. Macharzlna, K.: Unternehmensführung: Das internationale Managementwissen; Konzepte - Methoden - Praxis, a. a. O., S. 714. 
Unternehmenseinheiten an gewisse Grenzen stoßen. ${ }^{431}$ Sie dienen der Verringerung externer Abhängigkeiten durch eine ökologische Produkt- bzw. Verfahrensbereinigung zur Reduktion der Umweltunsicherheit. Intern kann die Reduzierung des Anspruchsniveaus bei Festlegung ökologischer Ziele für ausländische Tochtergesellschaften den ökologiebezogenen Koordinationsbedarf im internationalen Umweltmanagement vereinfachen. Weiterhin kann das oberste Management durch Vorgabe von Bandbreiten, innerhalb derer lokale Entscheidungsträger ökologiebezogene Entscheidungen eigenverantwortlich treffen können, entlastet werden. Schließlich kommt der Ausgestaltung des ökologiebezogenen Informationssystems eine hohe koordinationsbedarfsreduzierende Wirkung zu. Neben der internationalen Informationsversorgung der Entscheidungsträger soll durch ein Frühwarnsystem auch eine Antizipation zukünftiger ökologischer Forderungen ermöglicht werden. Technokratische koordinationsbedarfsdeckende Instrumente stellen nicht personengebundene Regelungen zur Steuerung von Abhängigkeitsbeziehungen zwischen organisatorischen Einheiten dar. Allein die Ingangsetzung technokratischer Mechanismen beruht auf bewussten persönlichen Weisungen der entscheidungsberechtigten Führungskräfte. ${ }^{432}$ Sie umfassen die ökologische Planung mit entsprechenden Ziel- und Mittelvorgaben sowie interne Märkte bzw. Lenkpreise. Schließlich zählen Regeln und Programme von operativen Aktivitätenreihenfolgen und Leitungsprozessen zu den deckenden technokratischen Koordinationsmaßnahmen.

\section{Personenorientierte Maßnahmen}

Personenorientierte Maßnahmen zur Koordination des internationalen Umweltmanagements sind nicht-strukturelle Maßnahmen, die nicht oder nur teilweise Gegenstand organisatorischer Regelungen sind. ${ }^{433}$ Während KHANDWALLA zu den personalen Koordinationsmaßnahmen lediglich indirekt wirkende Maßnahmen, wie die Vermittlung einer gemeinsamen Unternehmenskultur, zählt, sollen in Anlehnung an HOFFMANN diese Maßnahmen als koordinationsbedarfsreduzierende personale Maßnahmen aufgefasst werden. Sie umfassen Maßnahmen des internationalen Personalmanagements wie die Personalauswahl

\footnotetext{
431 Vgl. zu den Vorteilen der regelgebundenen Durchführung unterschiedlicher Aktivitätenfolgen vgl. Macharzina, K.; Oesterle, M.-J.: Bestimmungsgrößen und Mechanismen der Koordination von Auslandsgesellschaften, a. a. O., S. $616 \mathrm{f}$.

432 Vgl. Macharzina, K.: Unternehmensführung: Das internationale Managementwissen; Konzepte - Methoden - Praxis, a. a. O., S. 714.

433 Vgl. Hoffmann, F.: Führungsorganisation, a. a. O., S. $336 \mathrm{f.}$
} 
und -beschaffung sowie die Personalentwicklung durch Aus- und Weiterbildung der internationalen Unternehmenseinheiten in ökologiebezogenen Fragestellungen. Neben der Durchführung von Schulungs-, Trainee- und Entwicklungsmaßnahmen zählen auch der internationale Führungskräftetransfer, die Besetzung ausländischer Schlüsselpositionen mit Führungskräften aus der Muttergesellschaft und gegenseitige Besuche der Mitarbeiter zu diesen Instrumenten. In Anlehnung an MEFFERT kann die Unternehmenskultur selbst als koordinationsbedarfsdeckendes Instrument aufgefasst werden. ${ }^{434}$ Die Art des persönlichen Führungsstils zählt schließlich ebenfalls zu den koordinationsbedarfsdeckenden personalen Maßnahmen. Dabei bezieht sich der Führungsstil auf eine persönlichkeitsgebundene und positionsbezogene Autorität zur hierarchischen Ad-hoc-Koordination. ${ }^{435}$

Im Folgenden werden die strukturellen, technokratischen und personenbezogenen Koordinationsinstrumente im internationalen Umweltmanagement eingehend besprochen.

\subsection{Strukturelle Maßnahmen}

Die konsequente Integration von Umweltschutzaktivitäten in sämtliche Entscheidungsprozesse und Arbeitsabläufe (Querschnittscharakter) führt zu erheblichem Abstimmungsbedarf in einer international tätigen Unternehmung. Strukturelle Maßnahmen zur Reduzierung und Deckung des internationalen Koordinationsbedarfs im Umweltmanagement sollen eine optimale Anpassung der Unternehmensstruktur an diese Herausforderungen ermöglichen.

Die folgende Abbildung zeigt die im Rahmen der folgenden Ausführungen zu untersuchenden koordinationsbedarfsreduzierenden und -deckenden strukturellen Maßnahmen im Überblick.

434 Vgl. Meffert, H.: Marketing: Grundlagen marktorientierter Untemehmensführung: Konzepte - Instrumente - Praxisbeispiele, a. a. O., S. 1028.

435 Vgl. Hoffmann, F.: Führungsorganisation, a. a. O., S. $355-358$. 


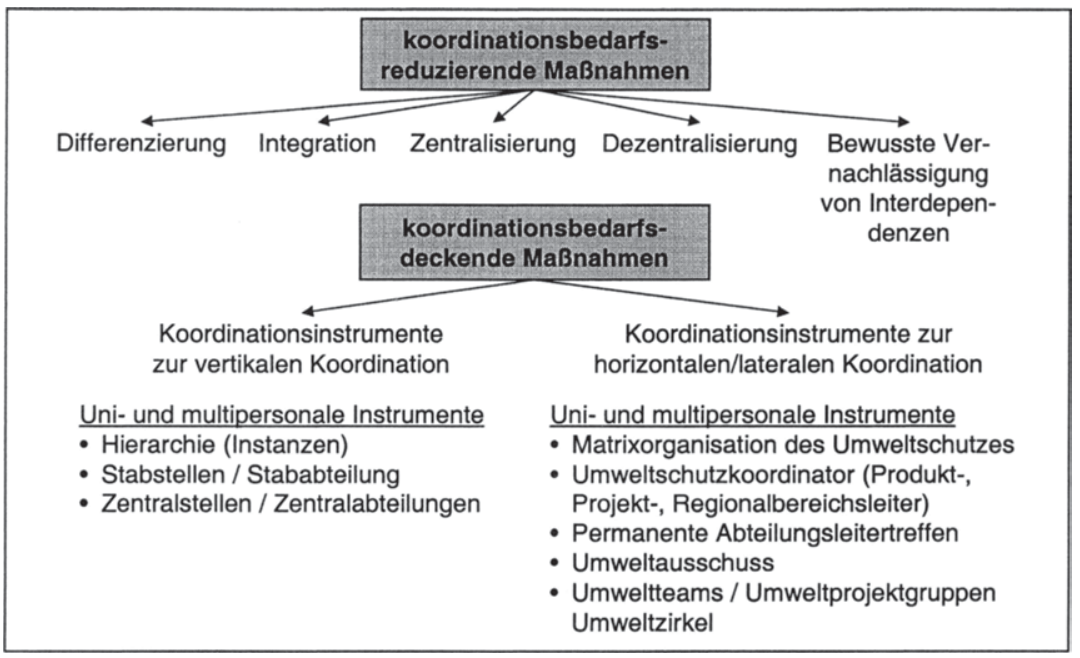

Abbildung 39: Strukturelle Koordinationsmaßnahmen

Quelle: Eigene Darstellung

Am Ende des Unterkapitels wird das Ineinandergreifen der sich z. T. ergänzenden strukturellen Maßnahmen zur Koordiantion im internationalen Umweltmanagement am Beispiel des VoLKSWAGEN-Konzerns aufgezeigt.

\subsubsection{Koordinationsbedarfsreduzierende strukturelle Maßnahmen}

In Abhängigkeit von der Zielsetzung im internationalen Umweltmanagement, den jeweils zu beachtenden gesetzlichen Vorgaben, der bereits vorhandenen Organisationsstruktur und den vorhandenen Mitarbeitern ist über eine geeignete Differenzierung sowie Integration von Umweltschutzaufgaben zu entscheiden. Als Ausdruck der strukturellen Koordination lässt sich in diesem Zusammenhang die organisatorische räumliche Differenzierung ökologiebezogener Aufgaben sowie die Integration des internationalen Umweltschutzes in die formale Organisationsstruktur bezeichnen. ${ }^{436}$ Weiterhin stellt sich die Frage, wo in der Organisation Umweltschutzaufgaben zentralisiert und dezentralisiert werden, d. h. die Zuweisung von Entscheidungskompetenzen kann nach Maßgabe

436 Vgl. hierzu die Ausführungen in Kapitel B 4.5 sowie Welge, M. K.: Management in deutschen multinationalen Unternehmungen: Ergebnisse einer empirischen Untersuchung, a. a. O., S. 193. 
der jeweiligen hierarchischen Verankerung von Befugnissen im Umweltmanagement zentral oder dezentral erfolgen. ${ }^{437}$

\section{Differenzierung}

Die Differenzierung kann sowohl als eine Voraussetzung für das Entstehen von Koordinationsbedarf als auch als ein Koordinationsinstrument gelten, da die Komplexität von Abhängigkeitsbeziehungen innerhalb einer Organisationseinheit durch Differenzierungsmaßnahmen reduziert werden kann. ${ }^{438}$ Wird durch die Bildung organisatorischer Teileinheiten der Abstimmungsbedarf reduziert, kann in Anlehnung an KIESER und KUBICEK auch von prozessorientierter Entkopplung gesprochen werden. ${ }^{439}$ Dies lässt sich auch auf das internationale Umweltmanagement übertragen.

Die Aufgaben im Umweltschutz in einem international tätigen Unternehmen sind vielseitig und umfassen unterschiedlichste Aufgabeninhalte. Art und Anforderungshöhe der Aufgaben im internationalen Umweltschutz unterscheiden sich vor dem Hintergrund der national unterschiedlichen Umweltschutzgesetze und -ansprüche sowie der lokalen Gegebenheiten. Da die Auslandsgesellschaften weiterhin gegenüber Außenstehenden, bspw. den lokalen Regulierungsbehörden, als eigenständige Rechtspersönlichkeiten gelten, geht von einer räumlichen Differenzierung der Umweltschutzaufgaben eine Senkung des ökologiebezogenen Koordinationsbedarfs aus. Der einzelne Mitarbeiter im fertigungsbezogenen Umweltschutz muss sich nicht direkt mit Mitarbeitern, die bspw. für einen Standort in einem anderen Land zuständig sind, koordinieren. Stattdessen bündelt eine höhere Instanz die Abstimmungstätigkeiten in seiner Stelle. ${ }^{440}$ Die Verantwortung für den fertigungsbezogenen Umweltschutz sowie die ökologiebezogenen Angelegenheiten der einzelnen Werke kann durch die Tätigkeit lokaler Umweltschutzabteilungen bzw. Umweltschutzbeauftragter ${ }^{441}$ direkt an den nationalen Standorten übernommen werden. Dieses Vorgehen ist auch in der Automobilindustrie einschlägig.

437 Vgl. hierzu auch Frese, E.: Organisation des Umweltschutzes, in: Frese, E. (Hrsg.): Handwörterbuch der Organisation (HWO), 3. Aufl., Stuttgart 1992, Sp. 2433-2451.

438

Vgl. Hoffmann, F.: Führungsorganisation, a. a. O., S. 328 f.

439

Vgl. Kleser, A.; Kubicek, H.: Organisation, 3. Aufl., Berlin u. a. 1992, S. 102 f.

440 Vgl. Dobry, A.: Die Steuerung ausländischer Tochtergesellschaften: Eine theoretische und empirische Untersuchung ihrer Grundlagen und Instrumente, Gießen 1983, S. 29.

441 Vgl. zu den Aufgaben von Umweltschutzbeauftragten Müller-Chrlst, G.: Umweltmanagement: Umweltschutz und nachhaltige Entwicklung, München 2001, S. $135 \mathrm{ff}$. 


\section{Integration}

Bei einer Integration werden ökologiebezogene Aufgaben, die auf in- und ausländische Unternehmenseinheiten verteilt sind, als Segmente zusammengeschlossen und einer gemeinsamen Leitung unterstellt. Dabei werden zwischen den ausländischen Tochtergesellschaften und der zentralen Unternehmung besondere organisatorisch-personelle Stellenverbindungen etabliert, denen übergreifende Steuerungsaufgaben zugrunde liegen. ${ }^{442}$ Integrierte Strukturen können dazu beitragen, dass Abstimmungsprobleme bspw. im produktbezogenen Umweltschutz bei internen Leistungsverflechtungen inländischer und ausländischer Unternehmensteile durch eine für beide Organisationseinheiten weisungsbefugte höhere Instanz geregelt werden. Vor dem Hintergrund der in der Automobilindustrie an Bedeutung gewinnenden Gleichteile- und Plattformstrategien findet bspw. eine Festlegung von produktbezogenen Umweltschutzverantwortlichen statt, um die internationalen ökologiebezogenen Entscheidungen zu koordinieren.

\section{Zentralisierung}

Die Zuweisung von Entscheidungskompetenzen in der horizontalen Dimension deckt noch nicht den gesamten Alternativenraum der formal-organisatorischen Gestaltung ab. In diesem Zusammenhang kommt der Ausgestaltung des Verhältnisses zwischen Mutter- und Tochtergesellschaften eine wesentliche $\mathrm{Be}$ deutung zu. Der Grad der Entscheidungszentralisation beschreibt dabei die Verteilung von Entscheidungskompetenzen in der Hierarchie. ${ }^{443}$ Je geringer die Zahl der Entscheidungsträger im Umweltmanagement ist und je eingeengter der Entscheidungsbereich der beteiligten Stellen innerhalb der Auslandsgesellschaften ist, desto stärker ist das internationale Umweltmanagement bei der Muttergesellschaft bzw. einer verantwortlichen Unternehmenseinheit zentralisiert. In diesem Kontext gilt die Entscheidungsfreiheit der Auslandsgesellschaf-

442 Vgl. hierzu und im Folgenden Macharzina, K.; Oesterle, M.-J.: Bestimmungsgrößen und Mechanismen der Koordination von Auslandsgesellschaften, a. a. O., S. 615.

443 Vgl. die Ausführungen in Kapitel B 4.3. Da eine zentrale Koordination den höchsten Grad der Koordination und die dezentrale Koordination den geringsten Koordinationsgrad ermöglicht, kann die Koordinationsform als Ausdruck des Koordinationsausmaßes gedeutet werden. Dabei müssen Koordinationsgrad und Form sich allerdings nicht in jedem Fall analog verhalten. Vgl. Plump, D.: Die Koordination des Marketings in multinationalen Unternehmungen: Systemanalyse und Ansătze zur Systemgestaltung, a. a. O., S. $149 \mathrm{ff}$. 
ten auch dann als eingeschränkt, wenn die Muttergesellschaft die ökologiebezogenen Entscheidungen der Tochtergesellschaft genehmigen muss. ${ }^{444}$

Bei einer zentralen Bündelung von Entscheidungskompetenzen ist eine vertikale Konfigurierung der Kommunikationsinfrastruktur notwendig. Die Unternehmenszentrale benötigt zur Entscheidungsvorbereitung Informationen, die häufig nur dezentral in den Auslandsgesellschaften vorliegen und bottom-up zu kommunizieren sind. Im Gegensatz dazu sind die Entscheidungen, Planungen und Programme top-down zu übermitteln. Schließlich sind in regelmäßigen Abständen die Erfolge bei der Umsetzung der zentralen Vorgaben an die Zentrale zu berichten. ${ }^{445}$

Zur Sicherstellung der Zielsetzungen des Gesamtunternehmens kann es notwendig sein, bestimmte ökologiebezogene Entscheidungen zentral zu treffen. So können unterschiedliche Zielsetzungen und Verhaltensweisen der Auslandsgesellschaften zu Rückkopplungen führen, die entweder zu einer Gefährdung der gesamtunternehmensbezogenen Zielsetzungen führen können oder die Wahrnehmung von Chancen durch gemeinsames Handeln verhindern. ${ }^{446}$ Sind bspw. Verbundvorteile durch länderübergreifendes ökologiebezogenes Handeln zu erzielen, kann eine Zentralisierung dieser Entscheidungen im Umweltschutz sinnvoll sein. ${ }^{447}$ So werden die Auslandsstandorte des VOLKSWAGEN-Konzerns zentral in Deutschland geplant. Auf diese Weise können von Beginn an Umweltschutzgesichtspunkte berücksichtigt werden. Dies gilt bspw. für die Ausstattung der Standorte mit einer Abwasserbehandlung. ${ }^{448}$

444 Vgl. Macharzina, K.; Oesterle, M.-J.: Bestimmungsgrößen und Mechanismen der Koordination von Auslandsgesellschaften, a. a. O., S. 616.

Vgl. Brodel, D.: Internationales Umweltmanagement: Gestaltungsfelder - Determinanten Ausprägungen, a. a. O., S. 445 f.

Vgl. Backhaus, K.; Büschken, J.; Voeth, M.: Internationales Marketing, a. a. O., S. $367 \mathrm{ff}$.

447 Eine direkte Unterstellung der Auslandsgesellschaften unter eine konzernübergreifend ausgerichtete Instanz, welche die Koordination des Umweltschutzes international koordiniert, eignet sich dabei nur für Unternehmen mit wenigen Standorten im Ausland. Bei wachsendem Auslandsengagement erscheint hingegen eine eigenståndige organisatorische Berücksichtigung einer internationalen Koordinationsstelle in der Hierarchie sinnvoll, da die obersten Leitungsstellen sonst überlastet werden. In Anlehnung an Welge, M. K.: Management in deutschen multinationalen Unternehmungen: Ergebnisse einer empirischen Untersuchung, a. a. O., S. 193.

Dies führte Herr MOGg von Volkswagen im Interview aus. 
Bei der BMW-Gruppe ist bspw. ein Kernteam um den Konzernbeauftragten für Umweltschutz für alle Genehmigungsverfahren an in- und ausländischen BMWStandorten zuständig. Die Teammitglieder recherchieren die gesetzlichen Anforderungen, bereiten die Prüfung der Standorte auf Altlasten vor, kümmern sich um die sicherheitstechnischen Einrichtungen, das Abfallmanagement oder den Schallschutz innerhalb und außerhalb der geplanten Produktionsstätten. Dabei findet zumeist eine Suche nach standortspezifischen Lösungen statt, die gleichzeitig die Erfüllung der Konzernstandards gewährleisten. Das Team hat dabei auch die Aufgabe, Anregungen zu geben und selbst Vorschläge zur ökologischen Verbesserung zu erarbeiten. ${ }^{449}$

Eine Berichtspflicht und Weisungsgebundenheit der Tochtergesellschaften gegenüber der Unternehmensführung eignet sich allerdings nur insoweit, wie die Stärkung der Entscheidungsautonomie der Zentrale bzw. die Schwächung der Stellung der einzelnen Tochtergesellschaften nicht zu einer Demotivation der Mitarbeiter in den Auslandsgesellschaften und einer geringen Anpassungsflexibilität an Umfeldveränderungen führt. Schließlich kann das in den Auslandsgesellschaften akkumulierte ökologiebezogene Markt- und Entwicklungs-Knowhow von der Zentrale oft nur unzureichend genutzt werden. ${ }^{450}$

Um die Nachteile einer alleinigen Zentralisation auf das Stammhaus zu vermeiden, hat das Lead-Country-Konzept starke Beachtung gefunden. Im Rahmen des Konzeptes wird bezüglich einzelner Leistungen (bspw. ökologische Antriebsaggregate) oder Regionen (Europa, Nordamerika, Japan, Südamerika, etc.) einer Landesgesellschaft die klare Führungsverantwortung über andere nationale Einheiten zugewiesen. Entsprechend liegt die Verantwortung für bestimmte ökologische Aufgabenbereiche typischerweise in dem Land bzw. der Region, in der die Mitarbeiter und Führungskräfte über die höchste Qualifikation, die meisten Ressourcen und die größte Erfahrung verfügen. Weiterhin ist die bewusste Wahl eines Standorts mit besonders hoher ökologiebezogener Wettbewerbsdynamik und besonders stringenten Umweltschutzgesetzen möglich. Aufgabe von derartigen Kompetenzzentren ist der Vertrieb ökologisch besonders fortschrittlicher Fahrzeuge (bspw. 3L-LUPO in Deutschland) oder der

\footnotetext{
449 Vgl. BMW (Hrsg.): Umweltbericht der BMW Group 1999/2000, München 1999, S. 98 f.

450 Vgl. Bartlett, C. A.; Ghoshal, S.: Arbeitsteilung bei der Globalisierung, in: Harvard Manager, 9. Jg. (1987), Nr. 2, S. 49 - 59; Frese, E.; Blies, P.: Konsequenzen der Internationalisierung für Organisation und Management der Muttergesellschaft, in: Macharzina, K.; Oesterle, M.-J. (Hrsg.): Handbuch Internationales Management: Grundlagen - Instrumente - Perspektiven, Wiesbaden 1997, S. 294.
} 
Aufbau besonderer ökologiebezogener Fachkompetenz (bspw. Brennstoffzellenforschung in Kalifornien). Die nationalen Einheiten sind dabei weitgehend gleichberechtigt, sodass der Verlust von Fähigkeiten und lokalem Unternehmertum aufgehoben wird. Der Mangel an klarer Hierarchie bedingt allerdings, dass weiche Koordinationsinstrumente wie Kultur, die Institutionalisierung von Knowhow-Austausch sowie internationales Management-Development stark an Bedeutung gewinnen. ${ }^{451}$

\section{Dezentralisierung}

Liegt ein hoher Grad an Entscheidungsbeteiligung der Auslandsgesellschaften in Bezug auf die ökologiebezogene Entscheidungen vor, dann werden den Auslandsgesellschaften große Entscheidungsspielräume im Umweltmanagement eingeräumt. Im Fall einer Dezentralisierung im internationalen Umweltmanagement werden neben den obersten Führungskräften in der Zentrale weitere Hierarchiestufen am Entscheidungsprozess beteiligt. Bei einer eher gleichberechtigten Rolle der Entscheidungsträger von Zentrale und Auslandsgesellschaften im internationalen Umweltmanagement werden diese in den Prozess der strategischen Planung einbezogen. Im Rahmen einer derartigen Einbeziehung des ausländischen Managements können bspw. die Erfolgsvoraussetzungen unterschiedlicher Strategien im internationalen Umweltmanagement besser überprüft werden, da das Wissen um lokale Konsumentenpräferenzen, gesetzliche Rahmenbedingungen und andere Faktoren berücksichtigt wird. ${ }^{452}$ Eine nationale Bündelung von Umweltschutzaufgaben führt zu relativ geschlossenen Verantwortungsbereichen und einer vergleichsweise hohen Motivation der lokalen Führungskräfte sowie zur Vereinfachung von Abstimmungsproblemen durch räumliche Differenzierung. Bei Entscheidungsdezentralisation, also der Zuweisung von Entscheidungsrechten an die Tochtergesellschaften, und gleichzeitiger Interdependenz der internationalen Wertschöpfungseinheiten ist eine horizontal konfigurierte Kommunikationsinfrastruk-

451 Vgl. Rall, W.: Der Netzwerkansatz als Alternative zum zentralen und hierarchisch gestützten Management der Mutter-Tochter-Beziehungen, in: Macharzina, K.; Oesterle, M.J. (Hrsg.): Handbuch Internationales Management: Grundlagen - Instrumente - Perspektiven, Wiesbaden 1997, S. 672; Mirow, M.: Entwicklung internationaler Führungsstrukturen, in: Macharzina, K.; Oesterle, M.-J. (Hrsg.): Handbuch Internationales Management: Grundlagen - Instrumente - Perspektiven, Wiesbaden 1997, S. 655.

Nach Auffassung von CLEE und DI SCIPIO sollte die strategische Planung und Kontrolle im internationalen Management allerdings zentralisiert werden. Vgl. Clee, G. H.; DI Sciplo, A.: Creating a World Enterprise, in: Douglas, N. D. (Hrsg.): Managing Effectively in the World Marketplace, New York 1983, S. 99, zitiert nach Macharzina, K.; Oesterle, M.-J.: Bestimmungsgrößen und Mechanismen der Koordination von Auslandsgesellschaften, a. a. O., S. 616. 
tur zu schaffen. Dabei findet die Kommunikation unmittelbar zwischen den verknüpften Teilen des Unternehmens statt. ${ }^{453}$

In der Automobilindustrie kann eine weitgehende Dezentralisation von fertigungsbezogenen ökologischen Entscheidungen beobachtet werden. Insbesondere die Hersteller, die keine unternehmensübergreifenden Ziele im fertigungsbezogenen Umweltschutz festlegen, überlassen lokalen Standort- bzw. Werksbeauftragten oder Regionenbeauftragten im Umweltschutz einen hohen Entscheidungsfreiraum. ${ }^{454}$

Eine zunehmende Spezialisierung des ökologiebezogenen Wissens in einzelnen Abteilungen kann allerdings spezifische Denkgewohnheiten, unbewusste Wahrnehmungsfilter und das Entstehen von Abteilungszielen fördern und so zwischen unterschiedlichen Funktionsbereichen Barrieren errichten. Diese Tendenz zur Verselbstständigung kann bei internationaler Verteilung der Wertschöpfung umso größer ausfallen und zu zusätzlichen Konflikten führen. ${ }^{455}$ Gleiches gilt für das Phänomen der Kommunikationsverdünnung, welches bedeutet, dass mit wachsender Größe zunehmend nur noch innerhalb des eigenen, überschaubaren Bereichs Informationen ausgetauscht werden. In Bezug auf den Umweltschutz als Querschnittsaufgabe in einem Unternehmen können diese Einflüsse zu einer Barriere für die erfolgreiche Umsetzung ökologischer Maßnahmen im internationalen Kontext werden. ${ }^{456}$

\section{Bewusste Vernachlässigung von Interdependenzen}

Der ökologiebezogene Koordinationsbedarf kann schließlich auch durch eine bewusste Vernachlässigung erkannter Interdependenzen zwischen organisatorischen Teilbereichen aus unterschiedlichen nationalen Organisationseinheiten reduziert werden. Dieses Verhalten ist immer dann eine sinnvolle Option, wenn die Kosten einer Koordination höher ausfallen als die Autonomiekosten. In

453 Vgl. Brodel, D.: Internationales Umweltmanagement: Gestaltungsfelder - Determinanten Ausprägungen, a. a. O., S. $445 \mathrm{f}$.

454 Vgl. dazu auch die Ausführungen zur Umweltschutzorganisation der VoLKSWAGEN AG am Ende dieses Unterkapitels.

455 Vgl. Schreyögg, G.: Organisation: Grundlagen moderner Organisationsgestaltung, Wiesbaden 1996, S. $173 \mathrm{f}$.

456 Vgl. zur Verselbstständigung einer Umweltschutzabteilung Pfriem, R.: Untemehmenspolitik in sozialökologischen Perspektiven, Marburg 1995, S. 357; Meffert, H.; KIrchgeorg, M.: Marktorientiertes Umweltmanagement: Konzeption - Strategie - Implementierung, a. a. O., S. 404; Müller-Christ, G.: Umweltmanagement: Umweltschutz und nachhaltige Entwicklung, a. a. O., S. 149 f. 
einem derartigen Fall würden auch die internationalen ökologiebezogenen Rückkopplungen kaum zu einer hohen unternehmensbezogenen ökologischen Betroffenheit beitragen. ${ }^{457}$

Die Automobilindustrie in Europa muss aufgrund gesetzlicher Vorschriften (Altautoverordnung) Neuwagen, die nach Januar 2001 verkauft werden, nach der Nutzung zurücknehmen und einem Recycling zuführen. Dabei existieren branchenbezogene Rücknahmeprogramme und Recyclinggarantien derzeit nahezu ausschließlich in Europa. ${ }^{458}$ Die Vorteile einer internationalen Rücknahme- und Recyclinggarantie werden demzufolge von der Mehrzahl der international tätigen Automobilhersteller als geringer bewertet als der Aufbau entsprechender Rücknahme- und Recyclingkapazitäten im nicht-өuropäischen Ausland. In diesem Zusammenhang kann vermutet werden, dass die Automobilhersteller auch kaum externe ökologiegerichtete Rückkopplungen erwarten.

\subsubsection{Koordinationsbedarfsdeckende strukturelle Maßnahmen}

Durch Differenzierung bzw. Integration sowie Zentralisation und Dezentralisation können Strukturen im Umweltmanagement geschaffen werden, die durch Herabsetzen der Komplexität und der Interdependenzen die Notwendigkeit einer Koordination reduzieren. ${ }^{459}$ Im Folgenden sollen koordinationsbedarfsdeckende strukturelle Maßnahmen im internationalen Umweltmanagement diskutiert werden.

Zur Ausrichtung der einzelnen Aufgabenteilgebiete auf die Koordinationsziele im internationalen Umweltmanagement werden horizontale bzw. vertikale Verknüpfungen hergestellt. Vertikale Koordinationsprobleme ergeben sich dabei aus dem vertikalen Beziehungszusammenhang zwischen organisatorischen Teilbereichen, während horizontale Koordinationsnotwendigkeiten auf die Be-

457 Vgl. Hoffmann, F.: Führungsorganisation, a. a. O., S. 335 sowie die dort angegebene Literatur.

BMW bietet als ökologischer Branchenvorreiter eine Rücknahme aller Fahrzeuge in Europa an. Zusătzlich nimmt BMW auch in einigen Gebieten in den USA und Japan Fahrzeuge zurück. Die japanischen Hersteller beteiligen sich in Japan an einer freiwilligen Rücknahmevereinbarung. Generell bieten die Automobilhersteller aber keine international gültigen Rücknahmegarantien an. Vgl. oekom research (Hrsg.): Corporate Responsibility Industry Report Automobile: A Corporate Responsibility Survey of 19 Companies of the Industry, a. a. o.

Gănzlich lässt sich hingegen der ökologiebezogene Koordinationsbedart durch strukturelle koordinationsbedarfsreduzierende Maßnahmen nicht verringern. Vgl. Adam, D. et al.: Koordination betrieblicher Entscheidungen: Die Fallstudie Peter Pollmann, 2. Aufl., Berlin et al. 1998, S. 224. 
rücksichtigung des verrichtungs- oder objektbezogenen Aufgabenzusammenhangs gleicher organisatorischer Ebenen zurückzuführen sind. ${ }^{460}$ Dabei können uni- und multipersonale ${ }^{461}$, dauerhafte oder zeitlich begrenzt tätige Koordinationsorgane zur Deckung des Koordinationsbedarfs beitragen. Die Koordination erfolgt dabei auf dem Wege der persönlichen Kommunikation zwischen Organisationsmitgliedern, wobei der Kommunikationsprozess offiziell sanktioniert bzw. legitimiert ist. ${ }^{462} \mathrm{Zu}$ den vertikalen Abstimmungsinstrumenten zählen grundsätzlich die persönliche Weisung bzw. die Hierarchie. Die Anwendung hierarchischer struktureller Maßnahmen findet ihren Ausdruck in der Struktur des Umweltschutzes in der internationalen Unternehmung.

\section{Hierarchie}

Die Hierarchie $e^{463}$ bzw. die persönliche Weisung durch den direkten Vorgesetzten gilt als reaktionsschneller vertikaler Koordinationsmechanismus, der sich in der Linienorganisation widerspiegelt. Die Linie als Einheit von Leitung und Auftragsempfang fungiert als Dienstweg, über den der Entscheidungs- und Informationsfluss zu erfolgen hat. Somit bedingt die Koordination des internationalen Umweltmanagements vertikale Informations- und Kommunikationswege. Durch eine Einlinienorganisation im Umweltschutz soll dabei gewährleistet werden, dass trotz Arbeitsteilung bei der Durchsetzung einer Umweltschutzkonzeption die Einheitlichkeit gewahrt wird. ${ }^{464}$ Die hierarchische Lösung des Koordinationsbedarfs geht dabei mit einer fallweisen Abstimmung der Interdependenzen einher. Eine Eingliederung des Umweltschutzes in die vorhandene Linienorganisation kann in diesem Kontext durch Aufgabenerweiterung bestehender Stellen und der Schaffung neuer Stellen ${ }^{465}$ in der jeweiligen Linie erfolgen. ${ }^{466}$

$460 \mathrm{Vgl}$. hierzu die Ausführungen in Kapitel B 4.5.

461 Zu unipersonellen und multipersonellen Strukturausrichtungen vgl. Frese, E.: Grundlagen der Organisation, Konzept - Prinzipien - Strukturen, a. a. O., S. $170 \mathrm{ff}$.

Vgl. Brodel, D.: Internationales Umweltmanagement: Gestaltungsfelder - Determinanten Ausprägungen, a. a. O., S. 447.

463 Vgl. zur Abstimmung durch Hierarchie bspw. Schreyögg, G.: Organisation: Grundlagen moderner Organisationsgestaltung, Wiesbaden 1996, S. 154 ff.; Hoffmann, F.: Führungsorganisation, a. a. O., S. $339 \mathrm{ff}$.

464 Vgl. Meffert, H.; Kirchgeorg, M.: Marktorientiertes Umweltmanagement: Konzeption Strategie - Implementierung, a. a. O., S. 400.

465 Zur Eignung funktional-additiver und integrativer Ansătze zur Einbindung des Umweltschutzes in die Organisation vgl. Müller-Chrlst, G.: Umweltmanagement: Umweltschutz und nachhaltige Entwicklung, a. a. O., S. $133 \mathrm{ft}$. 
Die Funktionsfähigkeit des Koordinationsinstruments Hierarchie ist allerdings entscheidend von der Ausgestaltung des Kommunikationssystems abhängig. Bei funktionaler Organisation bedeutet dies eine straffe Einbindung sämtlicher Kommunikationsbeziehungen in vertikale Dienstwege. Dabei ist der hierarchische Kommunikationsfluss vielfältigen Verzerrungen unterworfen, die häufig Rückfrageprozesse notwendig machen, damit die vorgesetzten Instanzen die zur Entscheidung erforderlichen Informationen bekommen. ${ }^{467} \mathrm{Im}$ internationalen Kontext sind hier neben sprachlichen und kulturellen Barrieren oft auch große Entfernungen sowie Zeitzonen zu überwinden, sodass der Koordinations- und Informationsaufwand der leitenden Instanzen weiter erhöht werden.

Das Koordinationssystem stößt dort an seine Grenzen, wo aufgrund des Anwachsens der Aufgabenbereiche die ökologiebezogene Sachkenntnis der übergeordneten Stellen abnimmt und wegen des Umfangs der Aufgabe Leistungsgrenzen erreicht werden. Dabei kann eine ökologische Spezialisierung der Mitarbeiter bei Aufgabenerweiterung klassischer Stellen um Umweltschutzaufgaben aufgrund einer Überbelastung mit anderen Routineaufgaben behindert werden. Insbesondere in der Automobilindustrie hat sich vor dem Hintergrund der komplizierten technologischen und rechtlichen Rahmenbedingungen eine ökologiebezogene Spezialisierung durchgesetzt. Alle international tätigen Automobilhersteller verfügen zumindest in der Muttergesellschaft über eine eigenständige Umweltschutzabteilung. Durch die strikte Einhaltung des Dienstweges gilt eine Linienorganisation im Umweltmanagement weiterhin als unflexibel und kann bei Störfällen gar problematisch werden. Schließlich trägt die Koordination durch persönliche Weisungen in der reinen Linienorganisation nicht der Querschnittsfunktion und dem Dialogerfordernis des Umweltschutzes Rechnung und

466 Die Implementierung ökologischer Unternehmenskonzepte mittels eines top-down Managementprozesses wird dabei oft mit dem viel zitierten Ausspruch "Umweltmanagement ist Chefsache" verbunden. Zu unterschiedlichen Interpretationsmöglichkeiten dieses Ausspruches vgl. Müller-Chrlst, G.: Umweltmanagement: Umweltschutz und nachhaltige Entwicklung, a. a. O., S. 134 f.

In der Unternehmenspraxis findet in diesem Zusammenhang häufig eine Verordnung des Umweltschutzes mittels einer sog. „Bombenwurfstrategie“ ohne Beteiligung der Betroffenen im In- und Ausland statt. Auf diese Weise wird versucht, schnell erste Handlungsänderungen der Mitarbeiter herbeizuführen. Allerdings gilt die Bombenwurfstrategie zur Einführung eines integrierten Umweltmanagements nur als bedingt geөignet. Vgl. zur Managementstrategie des Bombenwurfs Meffert, H.; Kirchgeorg, M.: Marktorientiertes Umweltmanagement: Konzeption - Strategie - Implementierung, a. a. O., S. 396 f.

467 Vgl. Schreyögg, G.: Organisation: Grundlagen moderner Organisationsgestaltung, Wiesbaden 1996, S. $161 \mathrm{ff}$. 
gilt als motivationshemmend. ${ }^{468}$ Insgesamt kann eine reine Linienorganisation im internationalen Umweltschutz und somit auch in der Automobilindustrie als ineffizient und störanfällig gelten.

\section{Stabstellen / Stababteilungen}

Die Einrichtung von Leitungshilfsstellen in Form von Stabstellen oder Stababteilungen dient der Erhöhung der Informationsgewinnungs- und -verarbeitungskapazität der Hierarchie. ${ }^{469}$ Dabei treten bei wachsender Größe verrichtungsund objektdifferenzierter Organisationsstrukturen diejenigen Aufgabenmerkmale in den Mittelpunkt organisatorischer Gestaltung, die in der Primärstruktur nicht berücksichtigt wurden. Es werden Zentralabteilungen oder -bereiche institutionalisiert, die spezifische Koordinationsaufgaben erfüllen. Damit wird eine Realisierung von Spezialisierungsvorteilen, die Vermeidung von Doppelarbeiten, die Gewährleistung der Einheitlichkeit in der Wahrnehmung von Verrichtungsaufgaben und eine Entlastung der Unternehmensführung angestrebt.

Eine Stab-Linien-Organisation im Umweltschutz versucht, die Nachteile der Einlinienorganisation durch die zusätzliche Schaffung von Stabstellen zu kompensieren. In den Stabstellen können Spezialwissen und Erfahrung im Umweltschutz gebündelt werden. Als sog. Fachpromotoren nehmen die Mitarbeiter insbesondere Aufgaben der Entscheidungsvorbereitung, Kontrolle und der Beratung wahr. In diesem Kontext bedarf es einer Einordnung des Stabs in die Unternehmenshierarchie, um die Einbindung des Umweltschutzes in allen Phasen des Entscheidungsprozesses international zu ermöglichen. Vor diesem Hintergrund eignet sich insbesondere eine Anbindung an die Geschäftsleitung.

Dabei sind je nach Unternehmensgröße auch mehrstufige Stabsmodelle denkbar. Insbesondere im internationalen Umweltmanagement von Automobilherstellern bietet sich bei divisionaler Organisation ein zweistufiges Stabsmodell an. Das reine Stabsmodell wird verlassen, wenn ein zentraler Umweltschutzstab mit Zugehörigkeit zur Unternehmensführung die Weisungsbefugnis über die in- und ausländischen Stabstellen erhält. Als Fachpromotoren fehlt den Mitarbeitern der Stabstellen allerdings der formale Einfluss zur Entscheidungsdurchsetzung. Die faktische Durchsetzung von Umweltschutzbelangen ist somit

468 Vgl. Meffert, H.; Kirchgeorg, M.: Marktorientiertes Umweltmanagement: Konzeption Strategie - Implementierung, a. a. O., S. 400.

469 Vgl. zur organisatorischen Teilung des Entscheidungsprozesses in Entscheidungsvorbereitung durch Stäbe und die eigentliche Entscheidung Schreyögg, G.: Organisation: Grundlagen moderner Organisationsgestaltung, Wiesbaden 1996, S. $145 \mathrm{ff}$. 
von der Einstellung des Linienvorgesetzten und der Persönlichkeit des Stabstelleninhabers sowie ihrem fachlichen und zwischenmenschlichen Zusammenspiel abhängig. ${ }^{470}$ Bei fehlender Unterstützung durch den Linienvorgesetzten sind die Mitarbeiter der Geschäftsleitung der Auslandsniederlassungen durch Fachautorität und Überzeugungskraft von der Richtigkeit der ökologiegerichteten Maßnahmen durch die Inhaber der Stabstellen zu überzeugen. Während den Empfehlungen der Stabstellen dann erheblicher Nachdruck verliehen wird, wenn umweltrechtliche bzw. strafrechtliche Konsequenzen drohen ${ }^{471}$, sind "freiwillige" Maßnahmen im Umweltschutz schwieriger zu kommunizieren. Dies führt zu einem hohen Koordinationsbedarf insbesondere bei international unterschiedlichen Einstellungen zum Umweltschutz. ${ }^{472}$

Oft erweist sich die Zusammenarbeit von Stab und Linie zusätzlich als konfliktreich. Fehlende praktische Erfahrungen der Stäbe werden durch die Linienmanager beklagt. Die Vorschläge der Stäbe werden dabei häufig als Bedrohung für die Linienmanager empfunden, da die Stäbe durch ihre Fachkompetenz und die Möglichkeit der Informationsselektion entscheidenden Einfluss auf die Entscheidungen der Linie nehmen können. Der international steigenden Bedeutung des Umweltschutzes trägt eine Stabslösung wenig Rechnung, auch fördert diese Lösung Konflikte zwischen Stab und Linie. ${ }^{473}$ Die Ergänzung der Linie durch Stäbe für Aufgaben des internationalen Umweltschutzes als einfache und schnelle Lösung erweist sich somit oft als nur bedingt geeignet.

470 Vgl. Müller-Christ, G.: Umweltmanagement: Umweltschutz und nachhaltige Entwicklung, a. a. O., S. $145 \mathrm{f}$.

471 Vgl. Frese, E.: Organisation des Umweltschutzes, a. a. O., Sp. 2446; Müller-Christ, G.: Umweltmanagement: Umweltschutz und nachhaltige Entwicklung, a. a. O., S. 146.

472 Vgl. Meffert, H.; KIrchgeorg, M.: Marktorientiertes Umweltmanagement: Konzeption Strategie - Implementierung, a. a. O., S. $402 \mathrm{ff}$.

473 Vgl. Schreyögg, G.: Organisation: Grundlagen moderner Organisationsgestaltung, Wiesbaden 1996, S. 151. 


\section{Zentralstellen / Zentralabteilungen}

Eine Weiterentwicklung der Stab-Linien-Organisation im Umweltschutz stellt die Bildung von Zentralbereichen bzw. zentralen Dienststellen dar. ${ }^{474}$ Die funktionale Eingliederung des Umweltschutzes in die Organisation ermöglicht eine weitere Professionalisierung sowie Spezialisierung. ${ }^{475}$

Dabei kann der Grad der formalen Weisungsbefugnis des zentralen Umweltbereichs unterschiedlich ausgestaltet sein. Zumeist werden bei Einrichtung von Zentralbereichen Informations-, Repräsentations- und Innovationsaufgaben im Umweltschutz als Serviceleistungen für die operativen Bereiche erbracht. Dabei sind formale Weisungsbefugnisse gegenüber anderen Fachstellen nicht nötig. Mit wachsender Bedeutung des Umweltschutzes können dem Zentralbereich aber auch Weisungsbefugnisse gegenüber in- und ausländischen Teilbereichen zugewiesen werden. In diesem Fall steht dem Vorteil der direkten Durchsetzungsmacht in Umweltschutzangelegenheiten die Auflösung der Einheit der Aufgabenerteilung gegenüber. ${ }^{476}$ Konflikte sind aufgrund der Mehrfachunterstellungen der Mitarbeiter in den einzelnen Funktionen vorprogrammiert.

Aufgrund der verschiedenen Interdependenzen bei einer funktionalen Eingliederung des Umweltschutzes aufgrund von Mehrfachunterstellungen gewinnen horizontale und laterale Abstimmungsmechanismen im Umweltschutz zunehmend an Bedeutung. Diese Verknüpfungen sind ihrem Wesen nach eine Form der institutionalisierten Selbstabstimmung, $d . h$. es findet eine direkte Abstimmung der Aktivitäten zwischen gleichberechtigten Aufgabenträgern statt. ${ }^{477}$ In der Automobilindustrie haben sich Zentralabteilungen für den Umweltschutz mittlerweile durchgesetzt.

474 Vgl. Müller-Christ, G.: Umweltmanagement: Umweltschutz und nachhaltige Entwicklung, a. a. O., S. 146.

475 Vgl. Meffert, H.; Kirchgeorg, M.: Marktorientiertes Umweltmanagement: Konzeption Strategie - Implementierung, a. a. O., S. 404.

476 Vgl. Müller-Christ, G.: Umweltmanagement: Umweltschutz und nachhaltige Entwicklung, a. a. O., S. 146.

477 Vgl. Frese, E.: Grundlagen der Organisation, Konzept - Prinzipien - Strukturen, a. a. O., S. $125 \mathrm{f}$. 


\section{Matrixorganisation des Umweltschutzes}

Mit steigender Unternehmensgröße, mit zunehmender Spezialisierung des Wissens und der fortschreitenden Internationalisierung des Geschäfts erweist sich eine einfache funktionale Organisationsstruktur vielfach nicht als ausreichend. ${ }^{478} \mathrm{Da}$ Umweltschutz eine Querschnittsaufgabe darstellt, könnte eine mehrdimensionale Koordinationsstruktur wie die Matrixorganisation dieser Aufgabe am besten gerecht werden. Bei einer Matrixorganisation des Umweltschutzes würde, wie in der funktionalen Organisation, eine Spezialisierung im Umweltbereich erfolgen, wobei diese Funktion in direktem Kommunikationskontakt mit anderen Funktionsbereichen stünde; somit fände eine horizontale Verknüpfung von Entscheidungsträgern statt. Diese Doppel- bzw. Mehrfachunterstellung der ausführenden Personen würde allerdings zu einem hohen Abstimmungsbedarf führen.

In der Automobilindustrie sind eigenständige Matrixorganisationen im Umweltschutz nicht zu beobachten. Die durch eine Matrix hergestellte Gleichrangigkeit von Umweltschutzaufgaben und den Aufgaben anderer Funktionsbereiche erscheint vielfach utopisch. Dies gilt insbesondere für global tätige Automobilhersteller mit unterschiedlichen Produktsparten. Weiterhin konkurrieren gleichzeitig weitere Querschnittsdimensionen wie das Qualitäts- oder Personalmanagement bzw. das Controlling ebenfalls um eine Berücksichtigung in der Organisation. ${ }^{479}$ Bestehende Matrixorganisationen in Großunternehmen wären durch eine zusätzliche Einbindung der Umweltschutzorganisation schließlich überfordert. ${ }^{480}$

Empirische Ergebnisse zeigen, dass die Einrichtung additiver Fachstellen im Umweltschutz für komplexe Innovationsaufgaben häufig weniger geeignet ist als produktnahe Funktionsstellen. ${ }^{481}$ Eine integrierte Einbindung des Umweltschutzes, bei der in letzter Konsequenz alle Stellen bei ihrer Aufgabenerfüllung für den Umweltschutz zuständig und verantwortlich sind, verspricht, Umwelt-

478 Vgl. hierzu und im Folgenden Schreyögg, G.: Organisation: Grundlagen moderner Organisationsgestaltung, Wiesbaden 1996, S. $173 \mathrm{ff}$.

479 Vgl. Müller-Christ, G.: Umweltmanagement: Umweltschutz und nachhaltige Entwicklung, a. a. O., S. 148.

480 Vgl. Meffert, H.; Klrchgeorg, M.: Marktorientiertes Umweltmanagement: Konzeption Strategie - Implementierung, a. a. O., S. 404 f.

481 Vgl. Dyckhoff, H.; Jacobs, R.: Organisation des Umweltschutzes in Industriebetrieben, in: ZfB, 64. Jg. (1994), Nr. 6, S. 717 - 735, hier S. 731. 
schutzaufgaben effektiver lösen zu können. ${ }^{482}$ Abstimmungsprozesse zwischen Entscheidungsträgern aus unterschiedlichen Funktionsbereichen finden dabei auf horizontaler Ebene statt.

Da eine individuelle Selbstkoordination jedes einzelnen Mitarbeiters nicht zweckmäßig erscheint, erfolgt die Abstimmung oft durch offizielle Gruppenentscheidungen in teamorientierten Strukturen. ${ }^{483}$ Die Bildung der Gruppen kann durch die Vorgabe von Kommunikationskanälen, die Ausstattung der Gruppe mit spezifischen Entscheidungskompetenzen und die Vorgabe von Abstimmungsanlässen durch die Unternehmensleitung unterstützt werden. ${ }^{484}$ Zur Selbstabstimmung eignen sich strukturelle Maßnahmen wie bspw. Abteilungskonferenzen, Komitees, Ausschüsse, Konferenzen und Besprechungen. Erste Ansätze zur Integration des Umweltschutzes in das Betriebsgeschehen stellen dabei Umweltausschüsse und Projektgruppen dar. ${ }^{485}$

\section{Umweltschutzkoordinator}

Die Rolle eines Koordinators ${ }^{486}$ zur gleichzeitigen Abstimmung vertikaler und horizontaler Koordinationsnotwendigkeiten zwischen international interdependenten Abteilungen wird zur Handhabung nicht routinemäßiger Probleme eingesetzt, wenn die Unabhängigkeit der organisatorischen Teilbereiche eine zentrale Rolle spielt. Im internationalen Umweltmanagement sind die ausländischen Tochtergesellschaften rechtlich für die Einhaltung der national gültigen Umweltschutzvorschriften verantwortlich, sodass hier von einer gewissen Unabhängigkeit von der Muttergesellschaft bezüglich des Umweltschutzes auszugehen ist. Ein internationaler Umweltschutzkoordinator könnte dann bspw. für die Koordination des Umweltschutzes eines Landes oder einer Region mit dem Umweltschutz der Muttergesellschaft sorgen. Ein Regionenbeauf-

482 Vgl. Müller-Christ, G.: Umweltmanagement: Umweltschutz und nachhaltige Entwicklung, a. a. O., S. 152.

483 Vgl. Wermeyer, F.: Marketing und Produktion - Schnittstellenmanagement aus unternehmensstrategischer Sicht, Wiesbaden 1994, S. 220; Meffert, H.: Marketing: Grundlagen marktorientierter Unternehmensführung: Konzepte - Instrumente - Praxisbeispiele, a. a. O., S. 1026.

Vgl. Kieser, A.; Kubicek, H.: Organisation, 3. Aufl., Berlin u. a. 1992, S. 107.

Vgl. Müller-Christ, G.: Umweltmanagement: Umweltschutz und nachhaltige Entwicklung, a. a. O., S. 153.

In diesem Zusammenhang wird auch der Begriff der "liason role" verwendet, der auf LAWRENCE und LORSCH zurückgeht. Vgl. dazu Lawrence, P. R.; Lorsch, J. W.: Now Management Job: The Integrator, Harvard Business Review, 45. Jg. (1967), Nr. 6, S. 142 151. 
tragter im Umweltschutz, der bspw. sowohl für den fertigungsbezogenen Umweltschutz in der Muttergesellschaft als auch für den Umweltschutz in einer bestimmten Region verantwortlich ist, kann hier als Beispiel dienen. ${ }^{487}$ So gehört der Koordinator des Umweltschutzes für die Region Südamerika/ Südafrika der Marke VolKswAGEN der Abteilung Produktionsstrategie des Konzerns an und ist gleichzeitig für den Umweltschutz der Tochtergesellschaften VW OF SOUTH AFRICA, VW ARGENTINIA und VW DO BRASIL verantwortlich.

\section{Permanente Abteilungsleiterkonferenzen}

Die Einrichtung von permanenten Abteilungsleiterkonferenzen dient der regelmäßigen Abstimmung von Interdependenzen zwischen Abteilungen. In diesem Zusammenhang werden regelmäßig unspezifische Aufgaben zwischen national unterschiedlichen Abteilungen abgestimmt. ${ }^{488}$ Bei internationalen Leistungsverflechtungen zwischen zwei Abteilungen können in regelmäßig stattfindenden Abteilungsleiterkonferenzen u. a. auch Fragestellungen des Umweltschutzes besprochen werden. Bei internationaler Tätigkeit können sich auch die nationalen bzw. regionalen Leiter der Umweltschutzabteilungen regelmäßig treffen, um über die neuesten Entwicklungen und Erfahrungen im Umweltschutz zu beraten.

Dieses Koordinationsinstrument kann allerdings häufig aufgrund der großen Entfernungen sowie damit verbundenen Anreisezeiten und -kosten und der Zeitverschiebung nur begrenzt zur internationalen Koordination im Umweltschutz eingesetzt werden. Die moderne Kommunikationstechnologie ermöglicht zwar den Einsatz von Telefon- oder Videokonferenzen sowie die Nutzung des Internet, allerdings erscheint eine regelmäßige Koordination auch aufgrund der Zeitverschiebung und der sehr hohen Kosten kaum durch permanente Abteilungskonferenzen möglich. Demzufolge finden sich auch in der Automobilindustrie keine Beispiele für permanente Abteilungskonferenzen zur Koordination im internationalen Umweltmanagement.

487 Aufgrund der simultanen Anordnung zweier sich überlagernder Kompetenz- und Weisungssysteme findet so die systematische Institutionalisierung von Kompetenzüberschneidungen in der Organisation statt. Dies kann eine funktionsübergreifende Integration des Umweltschutzes fördern. Vgl. Hoffmann, F.: Führungsorganisation, a. a. O., S. $341 \mathrm{f}$. sowie Schreyögg, G.: Organisation: Grundlagen moderner Organisationsgestaltung, Wiesbaden 1996, S. 172 f.

488 Hier wird auch von der FAYOLSCHEN Brūcke gesprochen. Vgl. Schreyögg, G.: Organisation: Grundlagen moderner Organisationsgestaltung, Wiesbaden 1996, S. 171 f. 


\section{Umweltausschuss}

Zur Lösung spezifischer lateraler Abstimmungsprobleme mit klar umrissener Aufgabe werden Ausschüsse oder Komitees gebildet. Die Aufgabe der internationalen Koordination von Umweltschutzfunktionen wurde Mitte der 70er Jahre der Studie von GLADWIN und WELLES zufolge vornehmlich von Umweltkomitees wahrgenommen. Diese häufig stammlandorientiert ausgerichteten Gremien hatten die Aufgabe, die Umweltpolitik zu formulieren, Verantwortlichkeiten festzulegen sowie die Programme und Erfahrungen der operativen und unterstützenden Einheiten zu integrieren. ${ }^{489}$ Auch Sitzungen, Besprechungen und Konferenzen zählen zu dieser Koordinationsform ${ }^{490}$, die insbesondere im Umweltmanagement als betriebliche Querschnittsfunktion besonders gut Anwendung finden kann. ${ }^{491}$ Der direkte Informationsaustausch zwischen Mitarbeitern oder Führungskräften aus unterschiedlichen Bereichen erlaubt eine situationsadäquate Verbindung von fachlichen und instanziellen Entscheidungsträgern und führt zu einer Substitution der hierarchischen Koordination durch eine demokratische Meinungsbildung.

Der Umweltausschuss ist zumeist relativ hoch in der Unternehmenshierarchie angesiedelt und hat deshalb nicht die Aufgabe, die Umweltschutzprobleme auf der Ausführungsebene zu lösen. Als wesentliche Aufgabe kommt dem Umweltausschuss die Koordination umweltpolitischer Maßnahmen zu. ${ }^{492}$ Zur Koordination internationaler Herausforderungen im Umweltmanagement eignen sich Umweltausschüsse als variable Instrumente der funktionsübergreifenden Zusammenarbeit, wenn die Mitarbeiter aus national unterschiedlichen Standorten nur zeitlich begrenzt von ihrer normalen Funktion für die Mitarbeit im Umweltausschuss freigestellt werden müssen. Hier kann die Erarbeitung von international gültigen Umweltstandards im Rahmen einer international und funktionsübergreifend besetzten Arbeitsgruppen als Beispiel dienen. Die Koordi-

489 Vgl. Gladwin, T. N.; Welles, J. G.: Environmental Policy and Multinational Corporate Strategy, a. a. O., S. 185, zitiert nach Brodel, D.: Internationales Umweltmanagement: Gestaltungsfelder - Determinanten - Ausprägungen, a. a. O., S. 451.

490 Vgl. Schreyögg, G.: Organisation: Grundlagen modemer Organisationsgestaltung, Wiesbaden 1996, S. 171.

491 Ein Vorteil der multipersonalen, integrierten Koordination liegt in der aktiven Einbindung einer größeren Zahl funktionaler Teilbereiche in die Umweltschutzfunktion. Vgl. Brodel, D.: Internationales Umweltmanagement: Gestaltungsfelder - Determinanten - Ausprägungen, a. a. O., S. 451.

492 Vgl. Müller-Christ, G.: Umweltmanagement: Umweltschutz und nachhaltige Entwicklung, a. a. O., S. $156 \mathrm{f}$. 
nation mittels Umweltausschüssen ist dabei auch in der Automobilindustrie verbreitet. ${ }^{493}$

Als Beispiel kann hier der Umweltsteuerkreis der BMW-GRUPPE angeführt werden, dessen Zusammensetzung die folgende Abbildung zeigt.

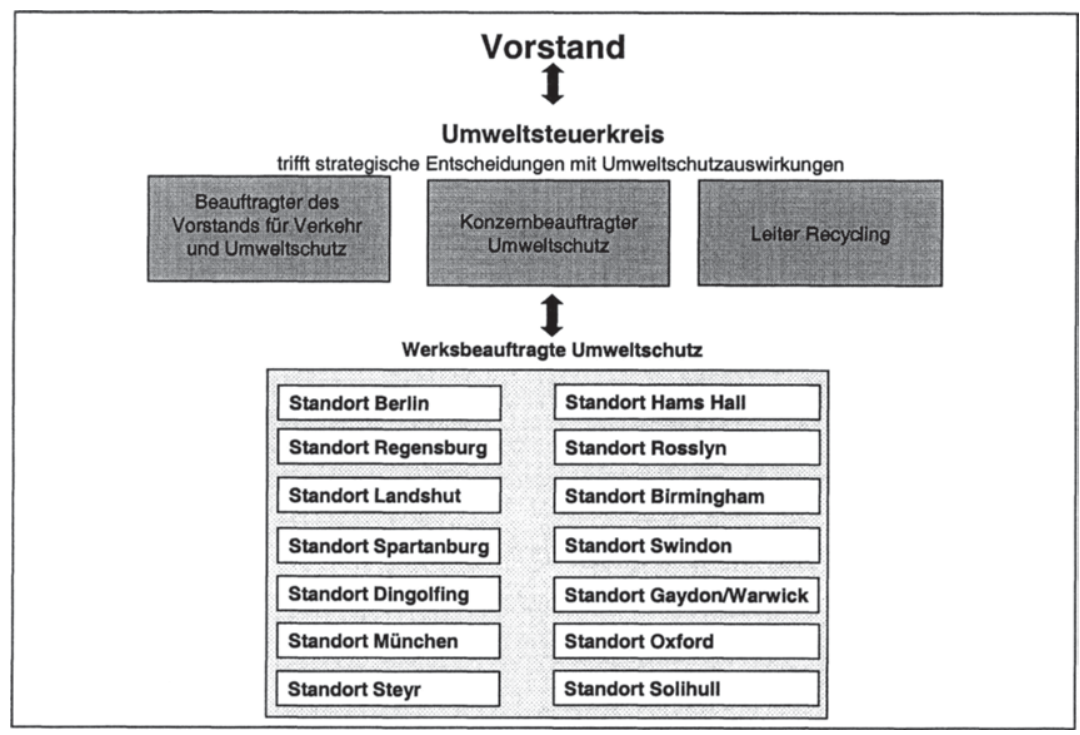

Abbildung 40: Umweltsteuerkreis der BMW-Gruppe

Quelle: BMW (Hrsg.): Umweltbericht der BMW Group 1999/2000, München 1999, S. 97.

Der Steuerkreis fungiert als Schaltzentrale für das Umweltmanagementsystem. Die Mitglieder berichten direkt an den Vorstand und bereiten wesentliche Entscheidungen vor. Der Umweltsteuerkreis ist paritätisch mit Vertretern der Bereiche Umweltschutz, Verkehr und Umwelt sowie Recycling besetzt und trifft sich im Schnitt alle zwei Monate. Je nach abzustimmendem Thema zieht der Umweltsteuerkreis weitere Experten hinzu, etwa aus dem Bereich Forschung \& Entwicklung. Das Gremium setzt nach den Leitlinien des Vorstandes die Prämissen für die unternehmerische Umweltpolitik und vertritt sie nach innen und außen.

493 Vgl. Volkswagen (Hrsg.): Jahresbericht des produktionsbezogenen Umweltschutzes 1999/2000, a. a. O., S. 20. 


\section{Umweltteams}

Bei neuartigen, aber relativ regelmäßig auftretenden Koordinationsaufgaben im internationalen Umweltschutz können interfunktionale Teams bzw. Koordinationsgruppen dauerhaft eingesetzt werden. ${ }^{494}$ In Anlehnung an Qualitätszirkel können diese Teams auch Umweltzirkel genannt werden. Ziel von Umweltzirkeln ist es bspw., die ökologische Qualität der Produktion und der Produkte kontinuierlich zu verbessern. ${ }^{495}$ Dabei werden zumeist selbstausgewählte Probleme unter Anleitung eines geschulten Moderators von Mitarbeitern einer hierarchischen Ebene eigenverantwortlich zu lösen versucht. Die Aufgaben werden dabei in der Arbeitszeit quasi als jeweils zeitlich begrenzte Sonderaufgabe neben dem Tagesgeschäft erledigt. Teams sind auf spezielle Aufgabenbereiche ausgerichtet und gelten als motivations- und kreativitätsfördernd sowie flexibel und auch in dynamischen und instabilen Situationen einsetzbar. ${ }^{496}$

Internationale Teams setzen sich aus nominierten Mitgliedern aus verschiedenen Abteilungen und / oder Ländern zusammen und werden für die Koordination größerer Projekte eingesetzt. Die Organisation einer Umweltleistungsshow oder einer Messe, wo die internationalen Umweltschutztätigkeiten einem interessierten Fachpublikum bzw. der Öffentlichkeit präsentiert werden sollen, könnte hier als Beispiel dienen. Weiterhin könnten derartige Teams auch den ökologiebezogenen Know-how-Transfer unterstützen. ${ }^{497}$ Mitarbeiter aus Ländern, in denen moderne Umweltschutztechnik, ökologiebezogene Produktionsund Planungsverfahren eingesetzt werden, könnten bspw. im Rahmen von Schulungen, Informationsveranstaltungen o. Ä. Mitarbeitern aus anderen Ländern mit gleichen Aufgabengebieten dieses Wissen und ihre Erfahrung übermitteln. Das Audit-Team der VolKswAGEN AG stellt ein derartiges Team dar. ${ }^{498}$

494 Vgl. Hoffmann, F.: Führungsorganisation, a. a. O., S. 344; Schreyögg, G.: Organisation: Grundlagen moderner Organisationsgestaltung, Wiesbaden 1996, S. 173; Remer, A.: Organisationslehre, 4. Aufl., Bayreuth 1996, S. 223 ff.

Vgl. Matzel, M.; Sekul, S.: Integration des Umweltschutzes in die Profit-Center-Organisation, in: Umweltwirtschaftsforum, 3. Jg. (1995), Nr. 3, S. 7.

496 Vgl. Matzel, M.: Die Organisation des betrieblichen Umweltschutzes, Berlin 1994, S. 217 ff.; Müller-Christ, G.: Umweltmanagement: Umweltschutz und nachhaltige Entwicklung, a. a. O., S. 158 f.

497 Vgl. zum Know-how Transfer Brauweiler, J. et al.: Umweltmanagement im Transformationsprozess: Ansatzpunkte für einen internationalen Know-how-Transfer - dargestellt am Beispiel polnischer und tschechischer Unternehmen, in: Umweltwirtschaftsforum, 8. Jg. (2000), Nr. 1, S. $16-25$.

498 Dies entspricht auch den Aussagen von Herrn MogG, dem Leiter des Audit Teams bei VOLKSWAGEN. 
In der Realität werden zumeist unterschiedliche strukturelle Instrumente zur Koordination im internationalen Umweltmanagement ergänzend eingesetzt. Dies zeigt zusammenfassend auch die folgende Darstellung der Umweltschutzorganisation der VOLKSWAGEN AG.

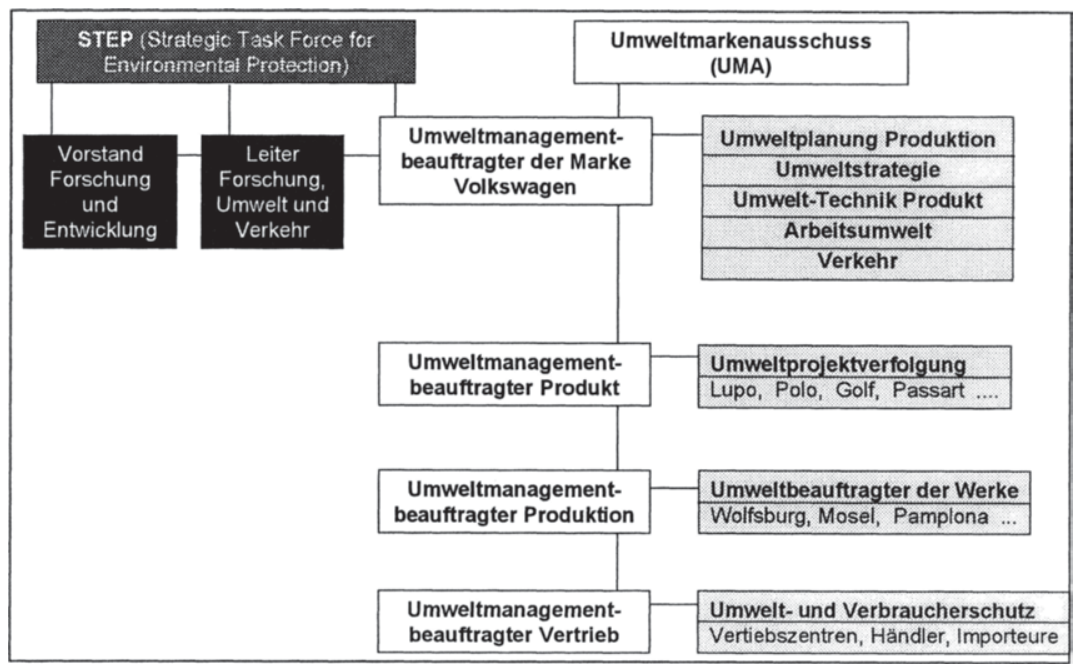

Abbildung 41: Umweltmanagementorganisation der VOLKSWAGEN AG

Quelle: Volkswagen (Hrsg.): Umweltbericht 1999/2000, Wolfsburg 1999, S. 12.

Die Umweltschutzabteilung der VOLKSWAGEN AG ist als Zentralabteilung zu kennzeichnen und als Hauptabteilung dem Bereich Forschung und Entwicklung zugeordnet. So sind der Vorstand, der Markenvorstand und die Führungskräfte gemäß Gesetz verpflichtet, für die Belange des Umweltschutzes Strukturen und Berichtswege aufzubauen, umzusetzen und einschließlich Delegationsmaßnahmen laufend zu überprüfen. Der Leiter Forschung, Umwelt und Verkehr organisiert, realisiert und überwacht den Aufbau und die Umsetzung der Aufgaben, Strukturen und Berichtswege für die Belange des fertigungsbezogenen Umweltschutzes im Auftrag des Vorstandes. Dabei wird der Leiter Forschung, Umwelt und Verkehr durch den Leiter Umwelt, Verkehr und Arbeitsschutz vertreten.

Die Abteilung ist weiter nach Umweltplanung, Produktion, Umweltstrategie, Umwelt-Technik Produkt, Arbeitsumwelt und Verkehr funktionsorientiert spezialisiert. Diese Unterabteilungen nehmen zentrale Umweltschutzaufgaben auch 
für die internationalen Standorte der Marke Volkswagen wahr. Die Koordination der Aktivitäten der Unterabteilungen ist Aufgabe des Umweltmanagementbeauftragten der Marke VOLKSWAGEN, der gleichzeitig Leiter der Hauptabteilung Forschung, Umwelt und Verkehr ist.

Auf lokaler Ebene sind die Werkleiter bzw. Betreiber für den standortbezogenen Umweltschutz und zur Umsetzung der Berichtspflicht verantwortlich. Diese delegieren ihre Umweltschutzaufgaben an die lokalen Umweltschutzbeauftragten, die auch für die Information und Kommunikation mit den lokalen Behörden zuständig sind. Die Umweltschutzbeauftragten sind gegenüber dem Leiter Umwelt, Verkehr und Arbeitsschutz berichtspflichtig. Auch den lokalen Führungskräften obliegt die Pflicht, alle erforderlichen Maßnahmen zur Erfüllung der Umweltschutzziele in ihren Geschäftsbereichen zu ergreifen. Dazu können die Pflichten an Sachkundige für Umweltschutz übertragen werden, die wiederum den Führungskräften gegenüber weisungsgebunden und berichtspflichtig sind. $^{499}$

\begin{tabular}{|l|l|}
\hline Vorstand & $\begin{array}{l}\text { Organisationspflicht, Aufsichtspflicht, Personelle / } \\
\text { materielle Ausstattung, Mittelbereitstellung }\end{array}$ \\
\hline $\begin{array}{l}\text { Leiter des Betriebes } \\
\text { Werkleiter }\end{array}$ & $\begin{array}{l}\text { Bestellung von Betriebsbeauftragten } \\
\text { Freigabe von Personal, Räumen, Einrichtungen, } \\
\text { Geräten für den Umweltschutz, Aufsichtspflicht }\end{array}$ \\
\hline Umweltschutzbeauftragte & $\begin{array}{l}\text { Kontrollieren, Informieren, Berichten } \\
\text { Erfülen der Aufgaben nach §21b WHG, } \\
\text { \$54 BImSchG, §55 KrW-IAbf und ORL 507 } \\
\text { Stellungnahme zu Investitionsentscheidungen }\end{array}$ \\
\hline Führungskräfte & $\begin{array}{l}\text { Einhalten der Gesetze und Veranlassen der } \\
\text { erforderlichen Maßnahmen } \\
\text { Ubertragung von Verantwortlichkeiten }\end{array}$ \\
\hline Sachkundige für & $\begin{array}{l}\text { Verhalten und Arbeiten entsprechend den } \\
\text { Vorgaben der Pflichtenübertragung }\end{array}$ \\
\hline Umweltschutz & Umweltgerechtes Verhalten und Arbeiten \\
\hline
\end{tabular}

Abbildung 42: Verantwortlichkeiten im Umweltschutz

Quelle: Volkswagen, interne Aufstellung

Zur Koordination der Umweltschutzbeauftragten der Werke und der Personen, die für die Umweltprojektverfolgung bei der Entwicklung der unterschiedlichen Fahrzeugmodelle sowie den Umweltschutz in den Vertriebszentren, bei den

$499 \mathrm{Vgl}$. Volkswagen (Hrsg.): Sachkundige für Umweltschutz: Handbuch, Stand 6/99, Organisations-Richtlinie des Vorstandes Nr. 507. 
Händlern und Importeuren zuständig sind, finden auf horizontaler Ebene regelmäßige Treffen zum Erfahrungsaustausch statt. ${ }^{500}$ Die Umweltschutzbeauftragten der Werke stimmen bspw. ca. alle vier Monate in diesen Treffen werksübergreifende Themen des fertigungsbezogenen Umweltschutzes ab. Der Leiter der Organisationseinheit Umwelt, Verkehr und Arbeitsschutz hat dabei gegenüber den Umweltschutzbeauftragten der deutschen Werke die Aufgabe der vertikalen Koordination. Die Umweltschutzbeauftragten der europäischen Standorte sind ebenfalls in den Erfahrungsaustausch eingebunden.

Die ökologiebezogene Koordination der ausländischen Gesellschaften findet aufgrund der fehlenden Weisungsbefugnis der Hauptabteilung gegenüber den ausländischen Tochtergesellschaften maßgeblich durch persönliche Abstimmungsprozesse in zweimal jährlich tagenden Arbeitskreisen statt. Dazu wurden der Umweltmarkenausschuss für die Marke VoLKsWAGEN (UMA) und die Strategic Task Force for Environmental Protection (STEP) für die Koordination zwischen den Marken etabliert. STEP ist als konzernübergreifender Arbeitskreis für die Abstimmung der Umweltschutzstrategien und Maßnahmen der einzelnen Gesellschaften der VolkswAGEN-Gruppe verantwortlich. Dazu gibt es jeweils eine Arbeitsgruppe für Produkte und Verkehr und eine für Produktion und Standorte. STEP erarbeitet weiterhin für die jeweiligen Managementvertreter der Marken und Gesellschaften Vorschläge für Anpassungen der konzernweiten Umweltpolitik bzw. für konzernweite Umweltziele. Der UMA bereitet Entscheidungen der verantwortlichen Managementgremien in Bezug auf die Umweltpolitik, das Umweltmanagement, die Erstellung des Umweltberichtes etc. vor. Weiterhin werden im UMA prozessübergreifende Umweltthemen beraten und für die Marke VolKsWAGEN abgestimmt. Zusätzlich findet im Abstand einiger Jahre eine Konzernumweltkonferenz statt, auf der Leitlinien und Visionen für den Umweltschutz des Konzerns beraten werden. ${ }^{501}$

500 Vgl. auch Kostka, C.; Kostka, S.: Umweltorientierte Personalentwicklung, in: Winter, G. (Hrsg.): Das Umweltbewusste Unternehmen: Die Zukunft beginnt heute, 6. Aufl., München 1998 , S. $437 \mathrm{ff}$.

501 Vgl. hierzu die Angaben im Umweltbericht, Volkswagen (Hrsg.): Umweltbericht 1999/ 2000, Wolfsburg 1999, S. $12 \mathrm{f}$. 


\subsection{Technokratische Maßnahmen}

Technokratische Koordinationsinstrumente stellen nicht strukturelle und nicht personenorientierte Regelungen zur Steuerung von Abhängigkeitsbeziehungen zwischen organisatorischen Teilbereichen dar. Allein die Ingangsetzung technokratischer Mechanismen beruht auf bewussten persönlichen Weisungen der entscheidungsberechtigten Führungskräfte.

Folgende Abbildung zeigt unterscheidliche koordinationsbedarfsreduzierende und -deckende technokratische Maßnahmen im Überblick.

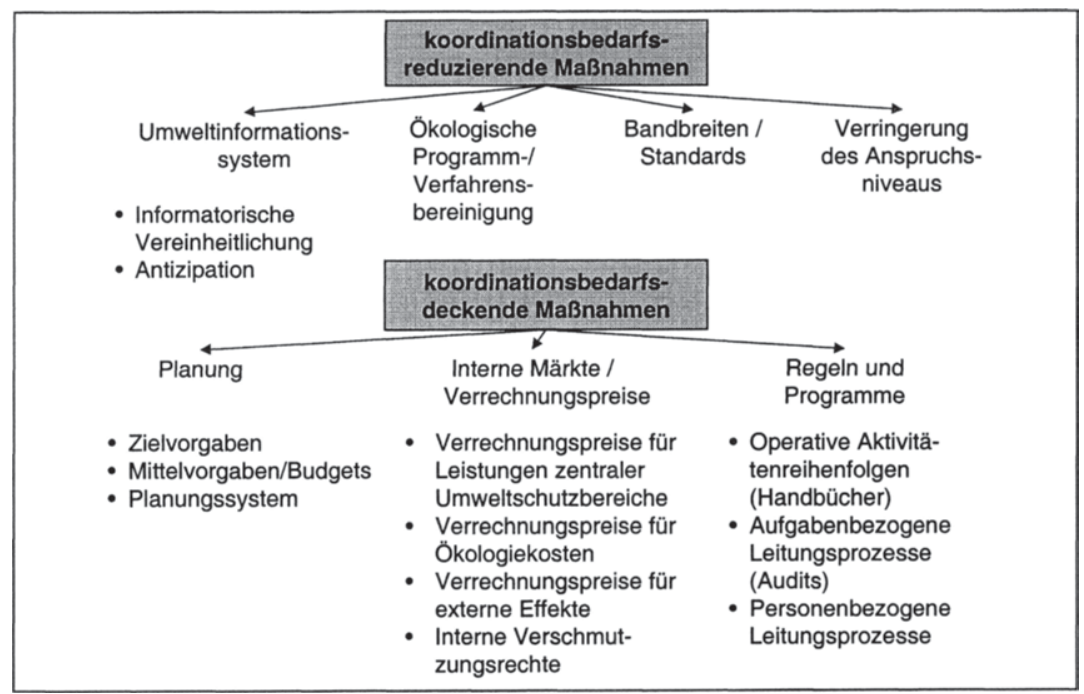

Abbildung 43: Technokratische Koordinationsmaßnahmen

Quelle: Eigene Darstellung

\subsubsection{Koordinationsbedarfsreduzierende technokratische Mechanismen}

Koordinationsbedarfsreduzierende technokratische Mechanismen werden angewendet, um die Umweltunsicherheit zu verringern und in- und externe Rückkopplungen zu vereinfachen bzw. von vornherein zu unterbinden.

\section{Umweltinformationssystem}

Die Sicherstellung der Informationsversorgung und Bereitstellung entscheidungsrelevanter Informationen wird häufig durch die Einführung eines betrieblichen Informationssystems geregelt und durch Methoden der elektronischen 
Datenverarbeitung umgesetzt. ${ }^{502}$ Dabei wurde schon früh auf die verhaltenssteuernde Wirkung des Einsatzes von Informationstechnologie und auf die Möglichkeiten der Erhöhung der Kapazität des Managements für Planung und Steuerung hingewiesen. ${ }^{503} \mathrm{Im}$ Umweltbereich werden sog. betriebliche Umweltinformationssysteme (BUIS) eingesetzt, durch deren adäquate Ausgestaltung auch eine koordinationsbedarfsreduzierende Wirkung im internationalen Umweltmanagement erreicht werden kann. ${ }^{504}$ Der organisationsweiten internationalen Informationstransparenz, also der Kommunikation aktueller Entwicklungen der internen und externen Situation, kommt dabei eine entscheidende Bedeutung zu, da nur so die Verantwortung für die unternehmensweite Umsetzung der Zielsetzung und Strategie im internationalen Umweltmanagement an sämtliche organisatorische Teileinheiten delegiert wird. ${ }^{505}$

Die Schaffung einer entsprechenden internationalen Informationsinfrastruktur (z. B. Anwendungs- und Datenbanksysteme) ist Grundvoraussetzung einer effektiven Koordination. Dies macht allerdings die Berücksichtigung einheitlicher Informations- und Kommunikationstechnologien mit kompatibler Hard- und Soft-

502 Vgl. Arndt, H.-K.; Günther, O.: Betriebliche Umweltinformationssysteme als Knowledge Managementsysteme im Umweltmanagement, in: Umweltwirtschaftsforum, 9. Jg. (2001), Nr. 1, S. 64. Der Aurbau eines betrieblichen Umweltinformationssystems liegt im Aufgabenbereich des Umwelt- oder Öko-Controlling.

503 So stellt WHISLER bereits 1970 fest, dass: ${ }_{n}$ Modern information technology is .. a technology of control." Vgl. Whisler, T. L.: The Impact of Computers on Organizations, Washington, London 1970, S. 15; Dlebold, J.: Bad Decisions in Computer Use, in: Harvard Business Review, 47. Jg. (1969), S. 14 ff., zitiert nach Welge, M.: Management in deutschen multinationalen Untermehmungen: Ergebnisse einer empirischen Untersuchung, a. a. O., S. 326.

Neben der Verringerung des Koordinationsbedarfs wird der Informationstechnologie auch eine Verbesserung der Koordinationsinstrumente Planung und Selbstabstimmung zugesprochen. Vgl. dazu grundlegend Kublcek, H.: Informationstechnologie und organisatorische Regelungen, Berlin 1975, S. 242, zitiert nach Hoffmann, F.: Führungsorganisation, a. a. O., S. 336.

Der Begriff des betrieblichen Umweltinformationssystems wird dabei in der Literatur nicht einheitlich gebraucht. In Anlehnung an MüLLER-CHRIST seinen betriebliche Umweltinformationssysteme hier als Instrumente verstanden, die der Abbildung und Bewertung der vom Unternehmen ausgehenden ökologischen Wirkungen dienen. Sie beinhalten aufeinander abgestimmte, in die Entscheidungsstruktur des Unternehmens eingebettete Verfahren und Instrumente zur Ermittlung, Darstellung und Bewertung betrieblicher Umweltwirkungen. Vgl. Müller-Christ, G.: Umweltmanagement: Umweltschutz und nachhaltige Entwicklung, a. a. O., S. 339.

505 Vgl. Welge, M. K. (Hrsg.); Böttcher, R.; Paul, Th.: Das Management globaler Geschäfte: Grundlagen, Analysen, Handlungsempfehlungen, a. a. O., S. 77. 
ware im In- und Ausland notwendig. ${ }^{506}$ Die zeit- und sachgerechte Verfügung über Informationen in einem internationalen Informationssystem, welches die Landesgesellschaften und die Unternehmenszentrale miteinander verbindet, bedarf der Standardisierung interner und externer Informationsprozesse. ${ }^{507} \mathrm{Da}$ bei ist das Informationssystem unternehmensindividuell und situationsadäquat auszugestalten. ${ }^{508}$

Das konzernweite Umwelt-Know-how der BMW-Gruppe ist bspw. in der Datenbank ZEUS gebündelt. Dort werden alle bei BMW eingesetzten chemischen Stoffe und Zubereitungen sowie Informationen über Werkstoffe, Produktionsprozesse und Recycling der Produktionsabfälle verwaltet. ZEUS ermöglicht ein lückenloses Gefahrstoffmanagement. Die dort gespeicherten EG-Sicherheitsdatenblätter und US-MSDS (Material Safety Data Sheets) können weltweit von allen BMW Mitarbeitern per Knopfdruck abgerufen werden. Die Prüfung der gefahrgutrechtlichen Aspekte sowie eine Klassifizierung der Stoffe werden von einem zentralen Team durchgeführt. Der Vorteil einer zentralen Datenerfassung ist, dass die verantwortlichen Mitarbeiter in den Werken entlastet und mögliche Fehlerquellen verringert werden. ${ }^{509}$

Generell ist betriebsinterner und -externer Informationsbedarf durch ein Umweltinformationssystem zu decken. Intern dient es als Grundlage der betriebsspezifischen Planung, Steuerung und Kontrolle und entfaltet so eine koordinationsbedarfsreduzierende Wirkung. Extern trägt es zur Förderung des Dialogs und des Interessensabgleichs mit den ökologischen Anspruchsgruppen bei und ermöglicht vertrauensbildende Maßnahmen bspw. durch eine zielgruppen-

506 Vgl. Schönert, O.: Integrationspotentiale von Informations- und Kommunikationstechnologien für internationale Unternehmungen, in: Kutschker, M. (Hrsg.): Integration in der internationalen Unternehmung, Wiesbaden 1998, S. 275 ff.

Vgl. Meffert, H.; Bolz, J.: Globalisierung des Marketing bei internationaler Unternehmenstătigkeit, in: Meffert, H.; Wagner, H.; Backhaus, K.: Arbeitspapiere der Wissenschaftlichen Gesellschaft für Marketing und Unternehmensführung e. V., Nr. 64, Münster 1991, S. 25.

508 Werden bspw. Art und Umfang der benötigten Informationen bei hoher Zentralisierung maßgeblich durch die Unternehmenszentrale bestimmt, ist ein top-down-orientiertes Vorgehen umzusetzen, bei dem die Tochtergesellschaften als verlängerter Arm der Zentrale vor Ort den angeforderten Informationsbedarf erheben. Bei hoher Dezentralisierung wird das Informationssystem auf die Bedürfnisse der internationalen Einzelunternehmen zugeschnitten, nur was auch im Interesse der Tochtergesellschaften im Ausland erhoben wurde, kann überhaupt als Information an die Zentrale weitergegeben werden (bottom-up). Vgl. Hünerberg, R.: Intemationales Marketing, Landsberg/Lech 1994, S. 343 ff.; Relchmann, Th.: Trends und Entwicklungen im Euro-Controlling, in: Reichmann, Th. (Hrsg.): DV-gestütztes Unternehmens-Controlling, München 1993, S. 1 - 15, S. 8.

509 Vgl. BMW (Hrsg.): Umweltbericht der BMW Group 1999/2000, München 1999, S. 100 f. 
gerechte Aufbereitung der Umweltinformationen. ${ }^{510}$ Auf diese Weise können u. U. negative Rückkopplungen mit dem externen Umsystem vermieden werden. Um diese Aufgaben erfüllen zu können, ist die Bereitstellung interner und externer Daten notwendig, die durch Methoden der Primär- und Sekundärforschung erhoben werden können. ${ }^{511}$

Ausgangspunkt und zentraler Baustein des Informationssystems ist dabei der Aufbau einer einheitlichen Firmensprache zur Erleichterung der unternehmensinternen Kommunikation. So ist bei einer zentralen Bereitstellung relevanter Umweltschutzvorschriften bspw. für die Fahrzeugentwicklung in Form einer Zusammenstellung aller aktuellen Gesetze und Richtlinien aus den relevanten Märkten, in denen das Produkt vermarktet werden soll, darauf zu achten, dass die Archivierung in einer für den Adressaten verständlichen Sprache stattfindet. Weiterhin sind für den unternehmensinternen Sprachgebrauch einheitliche Definitionen und Abgrenzungen der relevanten ökologiebezogenen Begriffe festzulegen. ${ }^{512}$

Betriebsbezogene Informationen, wie die Umweltleistung unterschiedlicher Standorte, Umweltbilanzdaten für Komponenten, Teile und ganze Produkte sowie Informationen für die Kostenrechnung, sind den Entscheidungsträgern in verschiedenen Abteilungen bereitzustellen. ${ }^{513} \mathrm{Im}$ Finanzbereich ist in Deutschland die Erfassung der Betriebs- und Investitionskosten für Aufgaben des Umweltschutzes durch das Umweltstatistikgesetz vorgeschrieben. Darüber hinaus ist eine unternehmensweit angewendete Umweltkostenrechnung ein wichtiges Informationsinstrument zur ökonomischen Erfolgskontrolle im Umweltmanagement. ${ }^{514}$

510 Vgl. Müller-Christ, G.: Umweltmanagement: Umweltschutz und nachhaltige Entwicklung, a. a. O., S. $340 \mathrm{ff}$.

511 So können die Daten entweder für einen bestimmten Informationszweck erhoben werden oder eine nachtrăgliche Bereitstellung von Daten erfolgt, die ursprūnglich für andere Zwecke oder ohne konkrete Zielsetzung, z. B. aus gesetzlicher Verpflichtung, erhoben wurden. Vgl. Hünerberg, R.: Internationales Marketing, a. a. O., S. 345.

512 Vgl. Meffert, H.; Bolz, J.: Globalisierung des Marketing bei internationaler Unternehmenstătigkeit, a. a. O., S. 25.

513 Vgl. Auerbach, H.; Melssner, H. G.: Controlling im internationalen Marketing-Management, in: Hermanns, A.; Wissmeier, U. K. (Hrsg.): Internationales Marketing Management: Grundlagen, Strategien, Instrumente, Kontrolle und Organisation, München 1995, S. 288.

514 Vgl. zum betriebsinternen Umweltinformationsbedarf Müller-Chrlst, G.: Umweltmanagement: Umweltschutz und nachhaltige Entwicklung, a. a. O., S. $341-345$. 
Die Erhebung umfeldbezogener externer Daten soll im Rahmen eines zielgerichteten Informationsmanagements der Früherkennung von Mustern zukünftiger ökologiebezogener Entwicklungen in den Auslandsmärkten und somit der Senkung der Unsicherheit und der Erleichterung der Entscheidungsfindung dienen. Ein Wettbewerbsvorteil wird folglich darin gesehen, die verfügbaren Informationspotenziale möglichst aller Niederlassungen zu verknüpfen. In Bezug auf die Entwicklung ökologiebezogener Konsumentenpräferenzen oder des Konkurrenzverhaltens haben die Informationen zwar ihre informatorische Basis auf lokaler Ebene, aber erst ihre Zusammenfügung auf eine übergeordnete Ebene erlaubt eine umfassende Beurteilung. ${ }^{515}$ So können globale Erfolgspotenziale und Risiken frühzeitig identifiziert werden.

\section{Informatorische Vereinheitlichung}

In diesem Kontext kommt der informatorischen Vereinheitlichung eine besondere Bedeutung zu. Standardisierung bedeutet die Bereitstellung gleichartiger bzw. vergleichbarer Informationen für alle Auslandsmärkte, die Anwendung analoger Verfahren der Informationserhebung und die Gewährleistung eines über alle Ländermärkte einheitlichen internen Informationsniveaus. ${ }^{516}$

Der Erstellung von Sachbilanzen kommt bspw. im produktbezogenen Umweltschutz eine hohe Bedeutung zu, da daraus nicht nur wichtige Implikationen für den Umweltschutz über den gesamten Lebenszyklus ableitbar sind, sondern auch unterschiedliche Umweltschutzziele besser in eine Prioritätenreihenfolge gebracht werden können. ${ }^{517}$ In diesem Zusammenhang ist bspw. der Bilanzraum in allen Ländermärkten gleich abzugrenzen. Bei unterschiedlicher Fertigungstiefe sind Informationen über die ökologischen Leistungen der Zulieferer in die Bewertung einzubeziehen.

Ein primäres Problem im internationalen Umweltmanagement stellt dabei die mangelnde Verfügbarkeit und Vergleichbarkeit der ökologiebezogenen Informationen aus den einzelnen Landesgesellschaften dar. Soll bspw. das internationale Marktpotenzial eines Fahrzeugs mit ökologisch vorteilhaftem Antriebskonzept, wie bspw. der 3-L LUPO mit Pumpe-Düse-Dieselmotor, abgeschätzt

515 Vgl. Welge, M. K. (Hrsg.); Böttcher, R.; Paul, Th.: Das Management globaler Geschäfte: Grundlagen, Analysen, Handlungsempfehlungen, a. a. O., S. 69.

516 Vgl. Hünerberg, R.: Internationales Marketing, a. a. O., S. 347.

517 Vgl. dazu bspw. die Sachbilanz des LUPO 3L TDI von VoLKSWAGEN, vgl. Volkswagen (Hrsg.): Umweltbericht 1999/2000, Wolfsburg 1999, S. 15. 
werden, sind neben anderen Informationen ${ }^{518}$ die ökologischen Einstellungen der Automobilkäufer sowie die Zahlungsbereitschaft der potenziellen Kunden international möglichst mit einem abgestimmten Erhebungsinstrumentarium zu ermitteln, um vergleichbare Daten für die Absatzprognose erstellen zu können. Aus Effizienzgründen wird auf die Durchführung von Primärforschung in allen Ländermärkten oft verzichtet. Bei Rückgriff auf Sekundärforschung sind allerdings, insbesondere in Schwellen- und Entwicklungsländern, oft wenige oder keine sekundärstatistischen Materialien in Bezug auf ökologiegerichtete Fragestellungen zu finden. Weiterhin ist die Vergleichbarkeit der Erhebungssachverhalte genau zu prüfen. ${ }^{519}$

Ähnliche Problemstellungen treten auch bei der Erfassung unternehmensinterner Informationen auf. Sollen bspw. die Lösemittelemissionen der Lackierereien im internationalen Vergleich erfasst werden, bedeutet dies, dass an allen Standorten mit gleicher Mess- und Regeltechnik die Emissionen von Lösemitteln mit der gleichen Meßmethode in gleichen Maßen (Ermittlung der Konzentration oder des Massestroms) zu ermitteln sind. Dies scheitert oft allein daran, dass in den Schwellenländern die entsprechende Mess- und Regeltechnik nicht vorhanden ist, komplett andere Verfahren angewendet werden oder das Fachpersonal zur Messung fehlt.

\section{Antizipation}

Ziel einer frühzeitigen Information im Umweltmanagement ist die Antizipation von gesetzlichen Maßnahmen, ökologiebezogenen Kundenanforderungen oder sonstigen Risiken, wie bspw. die Ressourcenverknappung. Je früher eine Antizipation der Entwicklung durch ein Unternehmen möglich ist, desto größer ist der weitere Handlungsspielraum der Unternehmung. Dies kann durch den Einsatz von entscheidungsvorbereitenden und entscheidungsunterstützenden Methoden wie Prognose- und Analysetechniken erreicht werden. ${ }^{520}$

$518 \mathrm{Zu}$ denken wäre in diesem Zusammenhang bspw. an Informationen über die gültigen nationalen Abgasgrenzwerte, die Verfügbarkeit der benőtigten Dieselqualitäten an den nationalen Tankstellen, nationale steuerliche Anreizinstrumente zur Unterstützung von ökologisch vorteilhaften Fahrzeugen durch den Staat, vorliegende ökologische Angebote der Wettbewerber etc.

Vgl. zu Ansätzen und Verfahren der internationalen Marktforschung bspw. Meffert, H.; Bolz, J.: Internationales Marketing-Management, a. a. O., S. 81 - 96.

Vgl. Kleser, A.; Kubicek, H.: Organisationsstruktur und individuelles Verhalten als Einflussfaktoren der Gestaltung von Management-Informationssystemen, in: Zeitschrift für Betriebswirtschaftslehre, 44. Jg. (1974), S. 460 ff.; Benkensteln, M.: F \& E und Marketing: Eine Untersuchung zur Leistungsfăhigkeit von Koordinationskonzeptionen bei Innovations- 
Der Einsatz von Prognosetechniken soll Voraussagen über die Wahrscheinlichkeit zukünttiger Ereignisse geben. Nur wenn relative Sicherheit über die Entwicklung der umweltrechtlichen und konsumentenbezogenen ökologischen Anforderungen an das Unternehmen in den einzelnen Ländermärkten besteht, können in der Forschung Entscheidungen darüber getroffen werden, mit welchen Motor- und Abgasreinigungstechniken die zukünftigen Automobile in welchen Ländermärkten ausgestattet werden sollen oder mit welchen Umweltschutz- und Fertigungstechnologien in Zukunft an welchen Standorten gearbeitet werden soll. Die Innovationsentscheidung ist dabei auch von maktbezogenen Entwicklungen, wie der Absatzmarktentwicklung für ökologische Fahrzeugkonzepte wie den PRIUS oder den 3-LITER LUPO und dem technologischen Fortschritt abhängig. ${ }^{521}$ Während langfristige Prognosen der Umfeldentwicklung als Grundlage der Ziel- und Strategiekoordination gelten und hoch aggregierte Prognoseinformationen bereitstellen, dienen mittelfristige Prognosen der Maßnahmenplanung in den nächsten 1- 5 Jahren.

Früherkennungssysteme bestehen aus einer Zusammenstellung von Instrumenten, die eine systematische Vorschau in die Zukunft ermöglichen sollen, um ggf. „schwache Signale“ einer Veränderung entdecken zu können. ${ }^{522}$ Früherkennungssysteme senken den internationalen Koordinationsbedarf, da die ökologiebezogenen Entwicklungen in den verschiedenen nationalen Umfeldern antizipatives Verhalten ermöglichen, um Risiken, aber auch Chancen wahrzunehmen.

Eine Aufgabe eines unternehmensbezogenen Umweltinformationssystems ist die Früherkennung von Umwelttrends im strategischen Umweltmanagement. Als Mittel eignen sich dazu bspw. Delphi-Befragungen und die Szenariotechnik. Im Rahmen von Delphi-Prognosen werden mittels mehrstufiger Befragungen Vorhersagen von Experten erfasst. Aufgrund der im Zeitablauf mehr-

entscheidungen, a. a. O., S. 136. Eine weitere Unterscheidung von Analyse- und Entscheidungstechniken soll im Folgenden nicht vorgenommen werden, da der Übergang zwischen den Instrumentegruppen fließend ist.

Vgl. dazu auch Benkenstein, M.: F \& E und Marketing: Eine Untersuchung zur Leistungsfähigkeit von Koordinationskonzeptionen bei Innovationsentscheidungen, a. a. O., S. 138 f.

Vgl. Ansoff, L.: Managing Surprise and Discontinuity - Strategic Response to Weak Signals, in: ZfbF, 28. Jg. (1976), Nr. 3, S. 129 - 152, Böhler, H.: Früherkennungssysteme, in: Wittmann, W. et al. (Hrsg.): Handwörterbuch der Betriebswirtschaft, 5. Aufl., Stuttgart 1993, Sp. 1256 - 1270. Vgl. zu Früherkennungssystemen im Umweltmanagement Meffert, H.; Kirchgeorg, M.: Marktorientiertes Umweltmanagement: Konzeption, Strategie, Implementierung mit Praxisfällen, a. a. O., S. 145 f.; Müller-Christ, G.: Umweltmanagement: Umweltschutz und nachhaltige Entwicklung, a. a. O., S. $31 \mathrm{ff}$. 
fach wiederholt durchgeführten Befragungen soll eine laufende Anpassung der Prognosen und eine genauere Voraussicht ermöglicht werden. Die DelphiMethode dient dabei häufig auch der Unterstützung der Szenariotechnik. Bei der Szenariotechnik handelt es sich um eine heuristische, subjektiv geprägte Prognose, wobei unterschiedliche Umweltentwicklungen - zumeist eine optimistische, eine pessimistische und eine wahrscheinliche - beschrieben werden. Auf diese Weise lässt sich in unterschiedlichen Szenarien die Gesamtentwicklung von Ländermärkten sowie das Verhalten der Konkurrenz und der ökologischen Anspruchsgruppen untersuchen. ${ }^{523}$

Die Szenariotechnik ist dabei sowohl im Rahmen internationaler als auch ökologischer Fragestellungen besonders gut geeignet ${ }^{524}$ und findet in der Automobilindustrie intensive Anwendung. Beispielhaft seien in der folgenden Abbildung drei Szenarien über mögliche Entwicklungen im europäischen Automobilmarkt aufgezeigt. ${ }^{525}$

\section{Ökologische Orientierung}
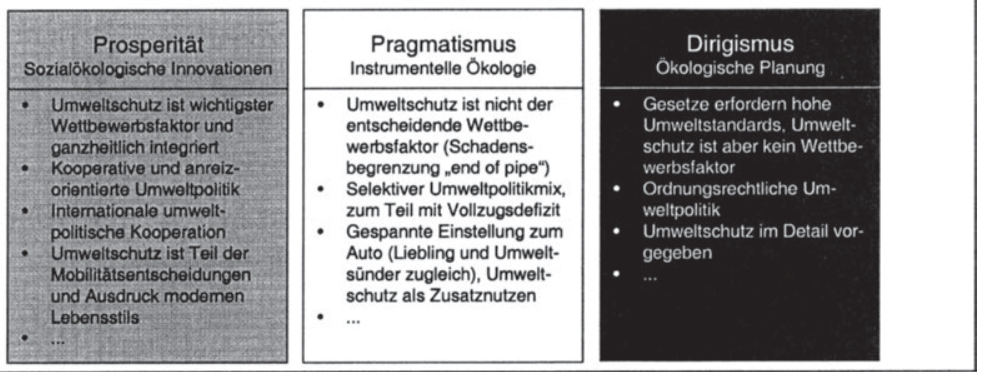

Abbildung 44:

Szenarien über mögliche ökologische Orientierungen im europäischen Automobilmarkt

Quelle: Volkswagen (Hrsg.): Umweltbericht 1999/2000, Wolfsburg 1999, S. 9.

523 Vgl. die Koordinationswirkung unterschiedlicher Prognosetechniken bei Benkenstein, M.: F \& E und Marketing: Eine Untersuchung zur Leistungsfähigkeit von Koordinationskonzeptionen bei Innovationsentscheidungen, a. a. 0 .

Vgl. Hünerberg, R.: Internationales Marketing, a. a. O., S. 430 sowie Meffert, H.; Kirchgeorg, M.: Marktorientiertes Umweltmanagement: Konzeption, Strategie, Implementierung mit Praxisfällen, a. a. O., S. $145 \mathrm{ff}$.

Vgl. dazu ergănzend auch die Angaben in der Golf-Fallstudie in Meffert, H.: Marketing: Grundlagen marktorientierter Unternehmensführung: Konzepte - Instrumente - Praxisbeispiele, a. a. O., S. 1342. 
Diese Szenarien entstanden durch die Zusammenarbeit verschiedener Automobilhersteller und Forschungsinstitute im internationalen Projekt „European Research on Mobility Scenarios (Euromos)". Dort wurden Mobilitätsszenarien für fünf europäische Ballungsräume entwickelt. Die drei in der Abbildung gezeigten Szenarien stellen eine Verknüpfung dieser Automobilmarktszenarien für Europa mit den Szenarien des World Business Council for Sustainable Development (WBCSD) dar. Im Rahmen des Globalen Szenario-Projekts des WBCSD haben Unternehmen aus mehr als 30 Wirtschaftszweigen, darunter auch Automobilhersteller wie VolKswaGEN, drei Szenarien zu denkbaren Reaktionen der Menschheit auf die Herausforderungen, wie Ressourcenverknappung und Umweltzerstörung, aufgezeigt. ${ }^{526}$

Wird bspw. eine Verschärfung des Umweltrechts absehbar, können beim Bau neuer Werke bereits heute Umweltschutztechniken installiert werden, die eine zukünftige Standarderfüllung gewähren. Werden die Umweltschutzgesetze später tatsächlich angepasst, bedarf es keiner weiteren Koordination. Gleiches gilt für die Forschung und Entwicklung von produktbezogenen Innovationen. So kann die Antizipation sinkender Rohstoffreserven, steigender klimapolitischer Restriktionen sowie verschärfter Abgasgrenzwerte durch die Entwicklung zukunftsfähiger Antriebskonzepte, bspw. die Brennstoffzelle, helfen, Abhängigkeiten der Automobilindustrie zu verringern und so den Koordinationsbedarf zu senken.

Ein weiteres, eher operativ anzuwendendes Frühwarninstrument stellt die Untersuchung der Nachrichten, Medienberichte und Pressemitteilungen über umweltrelevante Entwicklungen dar. Ziel ist es, die Entwicklungen herauszusortieren, die für das Unternehmen unter der öffentlichen Meinungsdynamik zu negativen oder positiven Wirkungen führen kann. Die Beobachtung der kumulierten Medienberichte zu einem Umweltthema über die Zeit führt dabei zu einer Diffusionskurve, die Aufschluss über den Lebenszyklus eines öffentlichen Anliegens und damit über eine potenzielle ökologische Betroffenheit der Unternehmung gibt. ${ }^{527}$

526 Vgl. Volkswagen (Hrsg.): Umweltbericht 1999/2000, a. a. O., S. 8 f.

527 Vgl. Dyllick, Th.: OKkologisch bewusste Unternehmensführung: Der Beitrag der Managementlehre, Schriftenreihe der Schweizer Vereinigung für okologisch bewusste Unternehmensführung, Nr. 1, 1989, St. Gallen 1989, S. 241 ff; Steger, U.; WInter, M.: Strategische Früherkennung zur Antizipation ökologisch motivierter Marktveränderungen, in: DBW, 59. Jg. (1996), S. 613 ff.; Meffert, H.; Kirchgeorg, M.: Marktorientiertes Umweltmanagement: Konzeption, Strategie, Implementierung mit Praxisfällen, a. a. O., S. $97 \mathrm{ff.}$ 
Obwohl ökologische Probleme international oft nicht gleichzeitig von der Öffentlichkeit aufgegriffen werden, kann es dennoch von großem Nutzen sein, alle Entscheidungsträger im Umweltmanagement international gleichzeitig über die aktuellen ökologischen Anforderungen in einem Ländermarkt zu informieren, da aufgrund der medialen Vernetzung ein solches Thema durchaus schnell in andere Ländermärkte diffundieren kann.

Um eine ziellose Informationsüberflutung zu verhindern, ist den lokalen Entscheidungsträgern im Umweltmanagement eine Informationsauswahl zur Verfügung zu stellen. Hier bietet es sich an, die Auswahl durch die Betroffenen selbst erfolgen zu lassen. Erforderlich ist dann die Bereitstellung eines adäquaten Mediums, um die entsprechenden Informationen auszutauschen. Während sich in der Praxis sog. interne Newsletter oder Innovationsreporte durchgesetzt haben, wird in Zukunft die Informationsversorgung durch den Einsatz des Intranets eine wesentlich genauere Informationsauswahl durch die Entscheidungsträger erlauben und wesentlich schneller und kostengünstiger sein. ${ }^{\mathbf{5 2 8}}$

Ein wesentlicher Bestandteil des Umweltmanagements bei VOLKSWAGEN ist ein Frühwarnsystem, das Umweltradar, welches im Rahmen des Umwelt-Controlling von der Umweltstrategieabteilung eingesetzt wird. Aufgabe des Umweltradars ist es, Umwelttrends möglichst frühzeitig zu erkennen. Dazu werden die internationalen Märkte, öffentliche Diskussionen, Wettbewerber und Politik sowie technologische Entwicklungen kontinuierlich beobachtet und internationale Experten aus unterschiedlichen Fachbereichen in einer regelmäßig wiederkehrenden Befragung zu zukunftsbezogenen Fragestellungen aus dem Themenfeld Gesellschaft, Automobil und Umwelt im Rahmen einer Delphistudie befragt. Eine weitere Teilaufgabe des Umweltradars ist eine Medienpräsenzanalyse. Dabei werden die Umweltthemen aus dem Verkehrsbereich aus ca. 50 deutschen Tageszeitungen, Wochenmagazinen, Umwelt-, Forschungs- und Automobilpublikationen sowie verschiedenen Internetnachrichtendiensten kontinuierlich ausgewertet. Die zwanzig Themen, über die in den deutschen Medien im Jahr 1999 am intensivsten berichtet wurde, sind in Abbildung 46 aufgelistet.

Die einzelnen Themenbereiche werden über das gesamte Jahr beobachtet. Folgende Abbildung zeigt den Einsatz von unterschiedlichen Früh-

528 Vgl. Welge, M. K. (Hrsg.); Böttcher, R.; Paul, Th.: Das Management globaler Geschäfte: Grundlagen, Analysen, Handlungsempfehlungen, a. a. O., S. 78. 
aufklärungsinstrumenten im stilisierten Lebenszyklus eines öffentlichen ökologischen Anliegens im VoLKSWAGEN-Konzern.

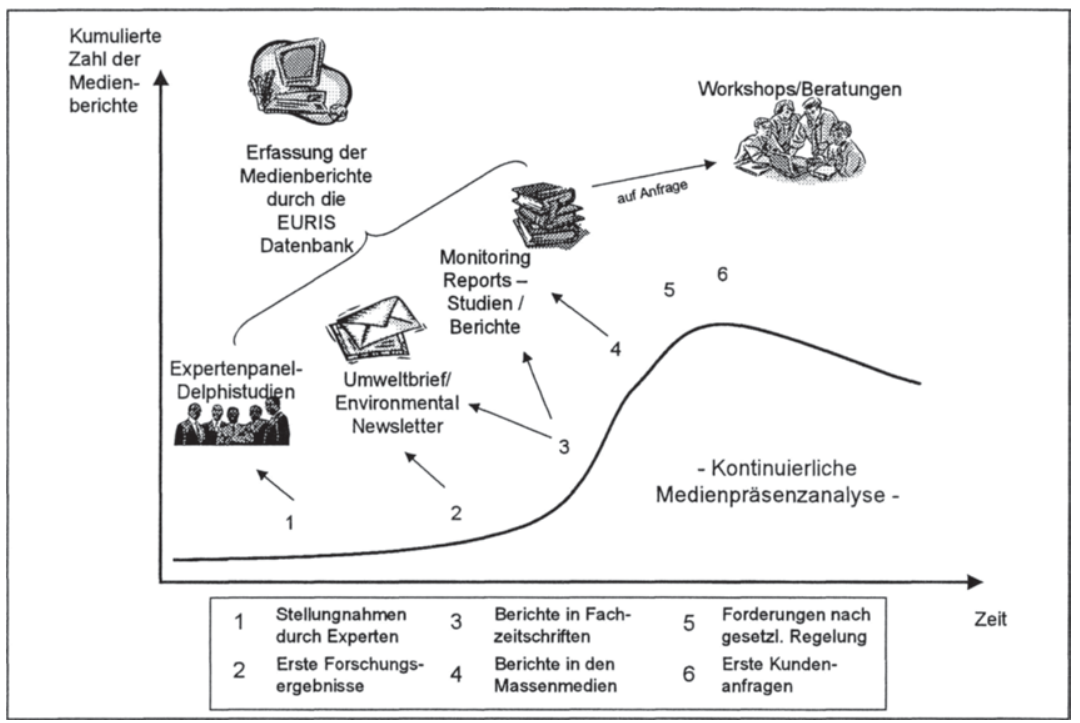

Abbildung 45: Früherkennungsinstrumente des Umwelt-Radar

Quelle: Volkswagen

Bei plötzlichen „Ausschlägen“ der Medienpräsenz werden detaillierte Informationen zusammengestellt und in dem monatlich erscheinenden Umweltbrief an die deutschen Mitarbeiter weitergegeben. Die ausländischen Mitarbeiter werden in einem englischsprachigen "Environmental Newsletter" alle zwei Monate mit Umweltinformationen versorgt. Neben Informationen über die ökologiebezogenen externen Entwicklungen werden auch ökologische Maßnahmen sowie ökologiebezogene Erfolge der Muttergesellschaft bzw. der deutschen Standorte im Umweltbrief dargestellt. Im Environmental Newsletter haben zusätzlich die ausländischen Standorte die Möglichkeit, über ihre Umweltaktivitäten zu berichten. Strategische Themen wie Recycling, neue Antriebstechnologien etc. werden weitergehend in Studien und Berichten analysiert. In diesem Zusammenhang werden auch die Umweltkommunikation der Wettbewerber sowie deren Produktpräsentationen auf Fahrzeugmessen genau beobachtet und ausgewertet. Insbesondere die öffentlichen Meinungsbildungsprozesse zu ökologischen Themen werden in sog. "Monitoring Reports" dargestellt und in Form von Chancen- und Risikoanalysen aus Perspektive des VOLKSWAGEN-Konzerns 
bewertet. Schließlich bildet die Umweltdatenbank „EURIS“ im international zur Verfügung stehenden Intranet den letzten Baustein des Umweltradars. In der Umweltdatenbank sind die Informationen des Umweltradars abgelegt, weiterhin finden sich dort Vorträge, wissenschaftliche Artikel sowie alle bundesdeutschen, umweltrechtlich relevanten Vorschriften. ${ }^{529}$

\begin{tabular}{|c|c|c|c|c|}
\hline Platz & Issue & $\begin{array}{c}\text { Meldungen } \\
\text { insgesamt }\end{array}$ & $\begin{array}{c}\text { Top-Ten- } \\
\text { Platzierungen }\end{array}$ & $\begin{array}{c}\text { Platzierung } \\
1998\end{array}$ \\
\hline $\begin{array}{c}1 \\
2 \\
3 \\
4 \\
5 \\
6 \\
7 \\
8 \\
9 \\
10 \\
11 \\
12 \\
13 \\
14 \\
15 \\
16 \\
17 \\
18 \\
19 \\
20\end{array}$ & $\begin{array}{l}\text { Steuerreform, ökologische } \\
\text { Klima } \\
\text { Transrapid } \\
\text { Verkehrssicherheit } \\
\text { Mineralölsteuer } \\
\text { Altauto-Verordnung } \\
\text { Drei-Liter-Auto } \\
\text { Diesel } \\
\text { Emissionen } \\
\text { Telematik } \\
\text { Kraftstoffe } \\
\text { ÖPNV } \\
\text { Güterverkehr } \\
\text { Kraftstoffverbrauch } \\
\text { Antrieb, Brennstoffzelle } \\
\text { Verkehrswegeplanung/-bau } \\
\text { Tempolimit } \\
\text { Fahrzeugsicherheit } \\
\text { Handel, Auto } \\
\text { Sommersmog/Ozon }\end{array}$ & $\begin{array}{l}288 \\
239 \\
163 \\
148 \\
125 \\
119 \\
98 \\
95 \\
91 \\
86 \\
86 \\
80 \\
78 \\
74 \\
71 \\
65 \\
63 \\
63 \\
62 \\
61\end{array}$ & $\begin{array}{l}10 \\
11 \\
8 \\
9 \\
8 \\
4 \\
5 \\
6 \\
4 \\
5 \\
6 \\
4 \\
5 \\
3 \\
2 \\
1 \\
2 \\
4 \\
2 \\
2\end{array}$ & $\begin{array}{c}1 \\
2 \\
\text { neu in Auswertung } \\
7 \\
3 \\
19 \\
6 \\
\text { nicht unter Top } 20 \\
4 \\
13 \\
16 \\
14 \\
9 \\
12 \\
18 \\
20 \\
10 \\
\text { neu in Auswertung } \\
\text { nicht unter Top } 20 \\
17\end{array}$ \\
\hline
\end{tabular}

Abbildung 46: Diskussionen und Ereignisse des Jahres 1999 im Themenfeld Umwelt und Verkehr - Die Top 20

Quelle: Volkswagen, imug (Hrsg): Jahrbuch Umwelt und Verkehr 1999: Diskussionen und Ereignisse des Jahres 1999 im Themenfeld Umwelt und Verkehr, interne Studie, Wolfsburg 2000, S. 6.

\section{Ökologische Programm- / Verfahrensbereiniqung}

Die ökologische Programm- und Verfahrensbereinigung zählen zu den Maßnahmen, die zu einer Verringerung der Koordinationsparameter beitragen. ${ }^{530}$ Die Verringerung der Koordinationsparameter soll dabei durch die Herstellung von Produkten bzw. Anwendung von Verfahren geschehen, die weitestgehend ökologisch ein einheitliches Niveau erreichen. Bei internen Leistungs-

529 Vgl. Volkswagen (Hrsg.): Umweltbericht 1999/2000, a. a. O., S. 14.

530 HOFFMANN spricht in diesem Zusammenhang von der Rationalisierung der OrganisationsUmweltbeziehung durch Programmbereinigung. Vgl. Hoffmann, F.: Führungsorganisation, a. a. O., S. 336. MEFFERT nennt hier die Verringerung der Koordinationsparameter und bezieht sich insbesondere auf Interdependenzen zwischen Produktion und Marketing. Vgl. Meffert, H.: Marketing: Grundlagen marktorientierter Unternehmensführung: Konzepte Instrumente - Praxisbeispiele, a. a. O., S. 1021. 
verflechtungen werden Pkw-Teile wie Plattformen, Bleche, Motoren und die Innenausstattung teilweise an unterschiedlichen nationalen Standorten gefertigt. Sind einzelne Produkte bzw. Produktteile aufgrund ihrer ökologischen Eigenschaften in einzelnen Ländermärkten nicht zugelassen, führt dies zu internen anbieterbezogenen Rückkopplungen. Können die Produktteile durch weniger ökologisch schädigende Teile ersetzt werden, lassen sich die Rückkopplungen reduzieren, dies kann als ökologische Produktbereinigung bezeichnet werden. Ähnliches gilt für die Eliminierung von Herstellungsverfahren oder ganzen Standorten, die als besonders verschmutzungsintensiv gelten. ${ }^{531}$ Die Verwendung unterschiedlicher Verfahren, bspw. bei der Lackierung, erhöht die Komplexität bei einer gemeinsamen Beschaffung von Lacken und reduziert so die Möglichkeit, durch Bündelung der Einkaufsmengen Kostensenkungen zu erzielen. Die Verwendung ökologisch unterschiedlicher Verfahren bzw. große standortbezogene Unterschiede im Umweltschutz (keine Kläranlage, keine Filteranlagen, ..) könnten auch von ökologischen Anspruchsgruppen aufgegriffen werden. Die Anhebung des Umweltschutzniveaus an den Standorten durch eine ökologische Verfahrensbereinigung kann dann helfen, bestehende Rückkopplungen zu reduzieren bzw. potenzielle Rückkopplungen gar nicht erst entstehen zu lassen. So trägt eine Senkung der Varietät der ökologischen Produktqualität sowie der ökologischen Eigenschaften der Produktionsverfahren zu einer Verringerung von Rückkopplungen bei.

\section{Bandbreiten / Standards}

Eine weitere Maßnahme zur Reduktion des internationalen ökologiebezogenen Koordinationsbedarfs liegt in der Festlegung von Bandbreiten oder Standards im Umweltmanagement, sodass eine Koordination nur erfolgt, wenn - im Sinne des Management by Exception - die zwischen den internationalen Organisationseinheiten ausgetauschten Teile, Vorprodukte, Fahrzeuge oder Informationen von einer vorgegebenen Toleranz abweichen. Erst wenn bspw. ein bestimmter Prozentsatz der in einem Land hergestellten Aggregate von einem vorgegebenen Toleranzwert in Bezug auf bestimmte Abgasbestandteile ab-

531 Insbesondere alte Standorte, bei deren Installation Umweltschutz keine oder nur eine unbedeutende Rolle gespielt hat, gelten in diesem Zusammenhang als besonders verschmutzungsintensiv. Vgl. Epstein, M.; Roy, M.-J.: Managing Corporate Environmental Performance: A Multinational Perspective, a. a. O., S. $287 \mathrm{f}$. 
weicht, werden weiterreichende Koordinationsmaßnahmen in die Wege geleitet, um die angestrebte ökologische Produktqualität wieder zu erreichen. ${ }^{532}$

Im Rahmen der marketingbezogenen Entscheidungen in der deutschen Automobilindustrie zeigt sich ein vergleichsweise hoher Standardisierungsgrad insbesondere der produktbezogenen Entscheidungen, während Preise, Konditionen und die ökologiegerichtete Werbung weniger stark einheitlich durchgeführt werden. Die vergleichsweise hohe Standardabweichung ist dabei allerdings nur bedingt auf den geringen Stichprobenumfang zurückzuführen, sondern ist auch Ausdruck durchaus unterschiedlicher Verhaltensweisen in der Automobilbranche. So wenden 5 (45,5\%) von 11 deutschen Unternehmen aus der Automobilindustrie global weitgehend einheitliche Umweltstandards im fertigungsbezogenen Umweltschutz an, während 6 (54,5\%) lediglich die lokal verlangten Umweltschutzstandards erfüllen. In Bezug auf den produktbezogenen Umweltschutz wählen $8(72,7 \%)$ von 11 Unternehmen überwiegend einheitliche Produktstandards, während nur $3(27,3 \%)$ Unternehmen sich international nach lokalen bzw. regionalen Anforderungen richten.

In der folgenden Abbildung ist die internationale Standardisierung von Marketingentscheidungen in der deutschen Automobilindustrie im Durchschnitt abgebildet. $^{533}$

532 Vgl. Kieser, A.; Kublcek, H.: Organisation, 3. Aufl., Berlin u. a. 1992, S. 103, Frese, E.: Management by Exception, in: Handwörterbuch der Organisation, 1. Aufl., Stuttgart 1969, Sp. 956 - 959; Meffert, H.: Marketing: Grundlagen marktorientierter Unternehmensführung: Konzepte - Instrumente - Praxisbeispiele, a. a. O., S. 1022.

533 Da diese Frage allein in der deutschen Befragung zum Europăischen UmweltmanagementBarometer aufgenommen wurde, fällt die Stichprobe vergleichsweise gering aus. 


\begin{tabular}{|c|c|c|c|c|c|}
\hline \multirow{2}{*}{$\begin{array}{l}\text { Marketingentscheidungen } \\
\text { im internationalen } \\
\text { Umweltmanagement }\end{array}$} & \multicolumn{2}{|c|}{$\begin{array}{c}\text { stark } \\
\text { Fall- differenziert }\end{array}$} & Mittelwerte & $\begin{array}{c}\text { hoch } \\
\text { standardisiert }\end{array}$ & \multirow{2}{*}{$\begin{array}{l}\text { Standard- } \\
\text { abwei- } \\
\text { chung- }\end{array}$} \\
\hline & $\underset{n}{z a h l}$ & 1 & 3 & $4 \quad 5$ & \\
\hline Markenname & 12 & 1 & $\mathbf{I}$ & $19^{\mathrm{V}} 1$ & 1,2673 \\
\hline Produktspezifikation & 12 & I & I & i & 1,2792 \\
\hline Produktpositionierung & 11 & $\begin{array}{l}1 \\
1\end{array}$ & $\begin{array}{l}T \\
1\end{array}$ & $\begin{array}{l}1 \\
1\end{array}$ & 1,2210 \\
\hline Produktdesign & 12 & $\begin{array}{l}1 \\
1\end{array}$ & $\begin{array}{l}1 \\
1\end{array}$ & $\begin{array}{l}1 \\
1\end{array}$ & 1,1645 \\
\hline Preis & 11 & 3,18 & & $\begin{array}{l}1 \\
1\end{array}$ & 1,0787 \\
\hline Konditionen & 11 & 3,18 & ic & 1 & 1,0787 \\
\hline Werbebotschaft & 11 & 3,45 & & 1 & 1,3685 \\
\hline Public Relations & 12 & i & $4,0 \mathrm{C}$ & 1 & 1,2060 \\
\hline Logistik & 11 & $\begin{array}{l}1 \\
1 \\
\end{array}$ & $4,00 \mathrm{~g}$ & $\begin{array}{l}1 \\
1\end{array}$ & 1,2649 \\
\hline Absatzkanalstruktur & 11 & $\begin{array}{l}1 \\
1\end{array}$ & 3,910 & $\begin{array}{l}1 \\
1 \\
\end{array}$ & 1,2210 \\
\hline & & 1 & 1 & 1 & \\
\hline
\end{tabular}

Abbildung 47: $\quad$ Standardisierung von ökologiegerichteten Marketingentscheidungen in der deutschen Automobilindustie

Quelle: Eigene Darstellung auf Basis der Daten zum Europäischen Umweltmanagemement-Barometer 1998.

Im fertigungsbezogenen Umweltschutz könnte auf weitere Koordinationsmaßnahmen dann verzichtet werden, wenn die Landesgesellschaft sicherstellt, dass die geltenden Gesetze des jeweiligen Landes bzw. darüber hinausgehende firmeneigene Mindeststandards eingehalten werden.

\section{Verringerung des Anspruchsniveaus}

Schließlich kann der Koordinationsbedarf durch Verringerung des Anspruchsniveaus wesentlich reduziert werden. Werden bspw. international nicht exakt die gleichen ökologischen Standards im fertigungsbezogenen Umweltschutz vorgeschrieben, sondern aufgrund unterschiedlicher Rahmenbedingungen lediglich vergleichbare Standards ${ }^{534}$ akzeptiert, oder längere Anpassungszeiträume für die Auslandsgesellschaften zugelassen, sinkt der internationale ökologiebezogene Koordinationsbedarf. ${ }^{535}$ Der Volkswagen-Konzern hat sich auch nicht die Erfüllung gleicher fertigungsbezogener Umweltschutzstandards zum

\footnotetext{
534 Dies könnte bspw. bedeuten, dass in einem Schwellenland nicht die Einhaltung der gleichen Standards wie bspw. in Deutschland verlangt wird, sondern eine Anhebung der lokalen Standards über das lokal gesetzlich geforderte Niveau. Hier könnte dann die wirtschaftliche Vertretbarkeit berücksichtigt werden.

535 Vgl. Meffert, H.: Marketing: Grundlagen marktorientierter Unternehmensführung: Konzepte - Instrumente - Praxisbeispiele, a. a. O., S. 1022.
} 
Ziel gesetzt, sondern lediglich die Erfüllung vergleichbarer Standards. Vergleichbare Standards haben zum Ziel, die gesellschaftliche Legitimität aufrechtzuerhalten, ohne zu prohibitiv hohen Koordinationskosten zu führen. ${ }^{536}$

\subsubsection{Koordinationsbedarfsdeckende technokratische Maßnahmen}

Als koordinationsbedarfsdeckende technokratische Maßnahmen können Instrumente der Planung sowie die Verabschiedung genereller Regelungen und Programme verstanden werden. Die Notwendigkeit einer ex ante Koordination durch Planung bzw. Programme und Regeln ergibt sich aus dem internationalen ökologiebezogenen Koordinationsbedürfnis, welches allein durch eine ex post Koordination oft nicht bewältigt werden kann, ohne obere Hierarchiestufen zu überlasten.

\section{Planung}

Planung im internationalen Umweltmanagement bedeutet systematisches, rationales und vorausschauendes Handeln zur Festlegung von Handlungsspielräumen organisatorischer Bereiche sowie zur Strukturierung künftiger Entscheidungssituationen durch Formulierung anzustrebender Ziele und der zur Zielereichung notwendigen Maßnahmen. ${ }^{537}$ Bei der Koordination über Pläne erhalten die ausführenden Stellen oder Mitarbeiter in den ausländischen Standorten in regelmäßigen Abständen Vorgaben, die ihre ökologiegerichteten Tätigkeiten koordinieren. Die Vorgaben werden dabei nach festgelegten Verfahren im Rahmen eines institutionalisierten Planungsprozesses in einem unternehmensübergreifenden Planungssystem erarbeitet. ${ }^{538}$

Eine Koordination durch Planung nutzt bestimmte Ziel- und Mittelvorgaben zur Koordination und bezieht sich zumeist auf eine bestimmte Periode. Dabei ist sie auf eine möglichst präzise Prognose der zukünftigen Umfeldentwicklungen angewiesen. ${ }^{539}$ Die Informationen aus dem Umweltinformationssystem werden im Rahmen des Planungssystems verarbeitet, die geplanten Handlungsziele

536 Dies sagte Herr STOBBE, der Leiter der Hauptabteilung Umwelt, Verkehr und Arbeitsschutz bei VOLKSWAGEN im Interview.

537 Vgl. Koslol, E.: Zur Problematik der Planung in der Unternehmung, in: Zeitschrift für Betriebswirtschaft, 37. Jg. (1967), S. 79, zitiert nach Hoffmann, F.: Führungsorganisation, a. a. O., S. 346.

538 Vgl. Kleser, A.; Kublcek, H.: Organisation, 3. Aufl., Berlin u. a. 1992, S. 114.

539 Vgl. Meffert, H.: Marketing: Grundlagen marktorientierter Untemehmensführung: Konzepte - Instrumente - Praxisbeispiele, a. a. O., S. 1027. 
als Aufgabeninhalte präzisiert und als Ziel- oder Mittelvorgaben zur Abstimmung interdependenter Einzelentscheidungen und -maßnahmen genutzt. ${ }^{540}$

\section{Zielvorgaben}

Eine Koordination durch operative Ziele kann generell durch Zielvorgabe oder Zielvereinbarung erfolgen. Diese Fragestellung ist eng an die Frage des Führungsstils ${ }^{541}$ geknüpft und wird später unter den koordinationsbedarfsdeckenden personalen Maßnahmen behandelt. ${ }^{542}$

Die Vorgabe von ökologiebezogenen Zielen stellt im Rahmen der technokratischen Koordinationsinstrumente das restriktionsfreiste Mittel zur Abstimmung internationaler Interdependenzen dar, da Ziele indirekt koordinieren. Dabei kennzeichnen Ziele Handlungsspielräume weniger eng und räumen den ausländischen Unternehmenseinheiten die Möglichkeit einer selbstständigen Bewertung sowie die Wahl der Mittel zur Erreichung der angestrebten Zustände ein. ${ }^{543}$

Die internationale Umweltschutzstrategie bildet dabei den Rahmen für die Umweltprogrammentwicklung durch die operative Planung sowie die Vorgabe konkreter internationaler Formalziele. Internationale ökologiebezogene Formalziele standardisieren dabei die Inhalte der Umweltpolitik und können in Form von periodischen Vorgaben oder Umweltstandards im fertigungsbezogenen und produktbezogenen Umweltschutz zum Einsatz kommen. ${ }^{544}$ Im fertigungsbezogenen Umweltschutz kann dies $u$. a. durch die Festlegung von international maximal zulässigen Emissionswerten erreicht werden. Als Beispiel kann hier die Begrenzung von Lösemittelemissionen von Karossenlackieranlagen im fertigungsbezogenen Umweltschutz auf maximal $35 \mathrm{~g} / \mathrm{m}^{2}$ für Neuanlagen und $45 \mathrm{~g} / \mathrm{m}^{2}$ für Altanlagen bis Ende $2003 \mathrm{im}$ VOLKSWAGENKonzern genannt werden. Im produktbezogenen Umweltschutz stellt bspw. die Senkung des Kraftstoffverbrauchs um 25 \% bis zum Jahr 2005 gegenüber 1990

540 Vgl. Hoffmann, F.: Führungsorganisation, a. a. O., S. 346.

541 Vgl. zum Führungsstil die Ausführungen in Kapitel B 4.6.

542 Dabei ist diese Frage gleichzeitig auf der Grenze zwischen technokratischen und personenorientierter Koordination einzuordnen. In Anlehnung an HOFFMANN wird der Führungsstil als koordinationsbedarfsdeckende personelle Maßnahme verstanden, vgl. Hoffmann, F.: Führungsorganisation, a. a. O., S. $355 \mathrm{ff}$.

543 Vgl. hierzu und im Folgenden Hoffmann, F.: Führungsorganisation, a. a. O., S. 347 f.

544 Vgl. Macharzina, K.; Oesterle, M.-J.: Bestimmungsgrößen und Mechanismen der Koordination von Auslandsgesellschaften, a. a. O., S. 617. 
für alle in Neufahrzeuge der Unternehmung in Europa eine konkrete Zielsetzung der VolkswAGEN AG dar. Weitere ökologiebezogene Zielvorgaben beziehen sich auf den Einsatz ökologieverträglicher Roh-, Hilfs- und Betriebsstoffe bspw. in Form von Quotenregelungen oder Stoffausschlusslisten.

\section{Mittelvorgaben / Budgets}

Budgets beschreiben eine vollständige Darstellung der geplanten und in Geldeinheiten umgeformten Gesamttätigkeit einer organisatorischen Einheit in einer bestimmten Planperiode. Budgetierung bezeichnet die Technik der Budgetaufstellung und die praktische Anwendung des Budgets als Mittel der Planung, Koordination und Kontrolle. ${ }^{545}$ Durch eine Budgetvorgabe werden knappe Ressourcen nach ihren Zielbeiträgen auf unterschiedliche organisatorische Teilbereiche verteilt. Der Budgetierungsprozess kann dabei auch als Verhandlungsprozess zwischen verschiedenen Organisationseinheiten interpretiert werden, in dem jeder Teilnehmer einen individuell zufriedenstellenden Anteil an der Mittelallokation zu erhalten sucht. ${ }^{546}$

Budgets spielen dabei im Planungsprozess von international tätigen Unternehmen generell eine große Rolle. In zahlreichen Unternehmenseinheiten werden Budgets auf Ebene der Niederlassungen erstellt und dem Management der Zentrale zur Genehmigung vorgelegt. Dort werden die Budgets aggregiert und als Grundlage der Strategieformulierung eingesetzt. Weiterhin werden Budgets auch als Vorgaben für die Leistungsbeurteilung von Niederlassungen verwendet. ${ }^{547}$ Bei der Anwendung von Ökologie-Budgets im internationalen Umweltmanagement werden Ökologiekosten als Steuerungsgröße eingesetzt. ${ }^{548}$ Die Festlegung der Budgets für den Umweltschutzbereich der Tochtergesellschaften kann dabei generell im Bereich des lokalen Managements liegen, welches nur das Gesamtbudget inklusive der Umweltschutzkosten mit der Zentrale aushandelt. Da Umweltschutzaufgaben in einzelnen ausländischen Niederlassungen

545 Vgl. Helser, H. C.: Budgetierung, Berlin 1964, S. 15 und 48, zitiert nach Heynen, C.-H.: Die Koordination dezentraler Entscheidungen: Ein verhaltenstheoretischer Ansatz zur Analyse der Koordination von Marketing-Entscheidungen, a. a. O., S. 97 f.

Vgl. Heynen, C.-H.: Die Koordination dezentraler Entscheidungen: Ein verhaltenstheoretischer Ansatz zur Analyse der Koordination von Marketing-Entscheidungen, a. a. O., S. $97 \mathrm{ff}$.

547 Vgl. Welge, M. K. (Hrsg.); Böttcher, R.; Paul, Th.: Das Management globaler Geschäfte: Grundlagen, Analysen, Handlungsempfehlungen, a. a. O., S. 74 f.

548 Vgl. Günther, E.: Okologieorientierte Bereichssteuerung, in: Umweltwirtschaftsforum, 3. J. (1995), Heft 3, S. 32. 
häufig als unproduktiv und nachrangig aufgefasst werden, kann in diesem Fall der "Kampf um die knappen Mittel" für die lokale Umweltschutzabteilung besonders hart ausfallen, da möglicherweise große interne Widerstände zu überwinden sind. Eine koordinierende Wirkung der Budgets im Umweltbereich kann dann isoliert kaum erwartet werden.

Wird die Budgethöhe für den fertigungsbezogenen Umweltschutz der Gesellschaften zentral festgelegt, treten Konflikte zwischen einzelnen funktionalen Organisationseinheiten auf lokaler Ebene möglicherweise weniger stark auf als bei isolierter Budgetplanung in den Tochtergesellschaften. Die Budgets im Umweltschutz der einzelnen Tochtergesellschaften können dabei einzeln für jeden Auslandsstandort oder gemeinsam für mehrere Standorte festgelegt werden. Die zentral festgelegten Budgetgrenzen determinieren dabei den Entscheidungsspielraum im Umweltschutz. ${ }^{549}$ Bei zentraler Budgetplanung für den fertigungsbezogenen Umweltschutz für mehrere Standorte durch zentrale Entscheidungen ist die Budgetverteilung zwischen den lokalen Umweltschutzaufgaben zu koordinieren, sodass hier zentral Prioritäten für die unterschiedlichen Aufgaben im Umweltschutz festzulegen sind. Dabei könnte bspw. für einzelne homogene Länder bzw. Regionen eine Instanz zur Budgetverteilung eingesetzt werden, die unter Maßgabe der zentralen Ziele eine regionale Aufteilung des Budgets vornimmt. Hier eignet sich bspw. eine Führungskraft im Umweltmanagement aus einem Lead-Country der Region. Diese Budgetsteuerung wäre dabei weniger zentralisiert als die direkte Budgetplanung durch die Zentrale.

Bei der Marke FORD in Europa wird bspw. vom Gesamtbudget für die Produktion der Standorte ein gewisser Prozentsatz an die zentrale Umweltschutzabteilung abgeführt. So werden für die rund 40 Standorte in Europa Mittel in Höhe von rund 30 Mio. US $\$$ für den fertigungsbezogenen Umweltschutz zur Verfügung gestellt. Das Budget ist nicht spezifisch aufgabengebunden, sondern wird durch die zentrale europäische Umweltmanagementabteilung nach vorheriger Prüfung für Umweltschutzaufgaben der europäischen Werke vergeben.

549 Wird die Budgethöhe für den Umweltschutz zentral vorgegeben, können Umweltschutzinvestitionen und laufende Umweltschutzkosten der einzelnen Standorte nur in Höhe des ex post zentral geplanten Budgets durchgeführt werden. Bei plötzlichen Änderungen der Umweltdaten, wie Störälllen oder bei überraschender Einführung eines neuen Gesetzes, sind die im Voraus zumeist für den Jahreszeitraum festgelegten Mittel umzuverteilen. Vgl. Heynen, C.-H.: Die Koordination dezentraler Entscheidungen: Ein verhattenstheoretischer Ansatz zur Analyse der Koordination von Marketing-Entscheidungen, a.a. O., S. 99; Hoffmann, F.: Führungsorganisation, a. a. O., S. 349. 
Die Umweltschutzinvestitionen der Standorte werden somit nicht direkt von den Werken bezahlt, sondern aus dem gemeinsamen Budget beglichen. Dabei können Zielkonflikte zwischen den europäischen Tochtergesellschaften bspw. beim Standortwettbewerb um die Produktion eines neuen Fahrzeugmodells vermieden werden, da die Fertigungskosten nicht bzw. weniger stark aufgrund unterschiedlich hoher Umweltschutzkosten verzerrt werden. ${ }^{550}$

\section{Planungssystem}

Ein integriertes Planungs- und Kontrollsystem im internationalen Umweltmanagement stellt eine geordnete und aufeinander abgestimmte Gesamtheit von verschiedenen Teilplanungen sowie entsprechend in die Arbeitsabläufe eingebauten Kontrollen dar. ${ }^{551}$ Eine Koordination des ökologiebezogenen Verhaltens im internationalen Umweltmanagement erfolgt dabei durch die Abstimmung der Planungs- und Kontrollvorgänge zwischen allen beteiligten Stellen, insbesondere zwischen der Zentrale und den Niederlassungen, aber auch zwischen den Niederlassungen. ${ }^{552}$ Der systematische Aufbau eines Planungssystems gründet auf einer Differenzierung des Gesamtplanungsprozesses in die Aspekte der generellen ökologischen Zielplanung, der umweltstrategischen Planung und der operativen Planung. Planungssysteme umfassen dabei nicht ausschließlich Teilpläne und Kontrollen, sondern sämtliche Planungsverfahren, Elemente und Bestandteile, die in die Planungs- und Kontrolltätigkeit einbezogen werden. ${ }^{553} \mathrm{Da}$ ein Planungssystem verschiedene bereits beschriebene Koordinationsinstrumente umfasst und in der Gestaltung grundsätzlich sehr variabel ist ${ }^{554}$, soll es an dieser Stelle nicht tiefergehend beschrieben werden.

550 Herr KRŬGER von FORD führte dies im Interview aus.

551 Vgl. zu Planungssystemen Hahn, D.: Planungs- und Kontrollrechnung - PuK: Integrierte ergebnis- und liquiditätsorientierte Planungs- und Kontrollrechnung als Führungsinstrument in Industrieunternehmen mit Massen- und Serienfertigung, Wiesbaden 1974, S. 53 ff.; Hahn, D.: PuK, Controllingkonzepte, 5. Aufl., Wiesbaden 1996, S. 56 ff.; Macharzina, K.: Unternehmensführung: Das internationale Managementwissen; Konzepte - Methoden Praxis, a. a. O., S. $322 \mathrm{ff}$.

Vgl. Hünerberg, R.: Intemationales Marketing, a. a. O., S. 434.

553 Vgl. Töpfer, A.: Planungs- und Kontrollsysteme industrieller Unternehmungen: Eine theoretische, technologische und empirische Analyse, Berlin 1976, S. 91; Hoffmann, F.: Führungsorganisation, a. a. O., S. $349 \mathrm{ff}$.

Vgl. Hoffmann, F.: Führungsorganisation, a. a. O., S. 350. 


\section{Interne Märkte / Verrechnungspreise}

Eine Möglichkeit der nicht strukturellen Koordination stellt generell die Koordination über Märkte dar, wobei der Leistungsaustausch bspw. über Marktpreise oder interne Verrechnungspreise erfolgen kann. ${ }^{555} \mathrm{Im}$ Sinne des Profit-CenterGedankens ${ }^{556}$ kann auch im Ökologiebereich eine verursachungsgerechte Leistungsbeurteilung und Koordination der Verantwortlichen angestrebt werden. Diese Verfahren eignen sich dabei insbesondere für den Fall, dass Umweltschutz eine sehr hohe Bedeutung im Unternehmen spielt. ${ }^{557}$ Gegen ein derartiges Vorgehen können allerdings ökologiebezogene Mess- und Bewertungsprobleme angeführt werden. Dabei müssen die Bewertungsansätze bspw. der starken Dynamik und Unsicherheit sowie der mangelnden Quantifizierbarkeit von Umweltinformationen und additiven und synergetischen Effekten von Umweltwirkungen Rechnung tragen. ${ }^{558}$ Eine nachvollziehbare Berechnung von Kosten und Nutzen ${ }^{559}$ des Umweltschutzes erscheint vor diesem Hintergrund kaum realisierbar. Die praktische Umsetzung derartiger Koordinationsformen lässt sich in der Unternehmenspraxis und damit auch in der Automobilindustrie deshalb nur in Ansätzen finden. ${ }^{560}$

555 Vgl. Bühner, R.: Betriebswirtschaftliche Organisationslehre, 9. Aufl., München 1999, S.194 ff.; Meffert, H.: Marketing: Grundlagen marktorientierter Unternehmensführung: Konzepte - Instrumente - Praxisbeispiele, a. a. O., S. 1028; Ossadnlk, W.: Koordination internationaler Konzerne über Verrechnungspreise, in: Betriebswirtschaftliche Forschung und Praxis, 48. Jg. (1996), Heft 1, S. $123-135$.

556 Vgl. zu Profit-Center und Verrechnungspreisen bspw. Frese, E.: Grundlagen der Organisation, Konzept - Prinzipien - Strukturen, a. a. O., S. 380 ff. sowie 461 ff. sowie Kreuter, A.: Verrechnungspreise in Profit-Center-Organisationen, München 1997.

557 Vgl. Matzel, M.; Sekul, S.: Integration des Umweltschutzes in die Profit-Center-Organisation, in: Umweltwirtschaftsforum, 3. Jg. (1995), Nr. 3, S. 10.

$558 \mathrm{Vgl}$. zu ökologischen Bewertungsproblemen und unterschiedlichen Bewertungsmethoden Bundesumweltministerlum, Umweltbundesamt (Hrsg.): Handbuch Umweltcontrolling, 2. Aufl., München 2001, S. 217 - 226.

559 Vgl. zur Kosten-Nutzen-Analyse generell bspw. Kalser, W.: Kosten-Nutzen-Analyse Grundlagen, Theorie, Modell, 4. Aufl., Stuttgart 1995. Zum Einsatz der Kosten-NutzenAnalyse im Umweltmanagement vgl. bspw. Orth, U.: Umweltcontrolling mit erweiterter Kosten-Nutzen-Analyse - dargestellt am Beispiel des Wassereinsatzes zur Pflanzenerzeugung, in: Zeitschrift für angewandte Umweltforschung, 9. Jg. (1996), Heft 2, S. 233 247.

560 Vgl. Müller-Christ, G.: Umweltmanagement: Umweltschutz und nachhaltige Entwicklung, a. a. O., S. 161. 


\section{Verrechnungspreise für Leistungen zentraler Umweltschutzbereiche}

Eine vergleichsweise einfache Lösung stellt der interne bzw. externe Verkauf von Umweltschutzleistungen dar. Besteht für ökologische Leistungen ein Markt, können die Leistungen sowohl extern als auch intern zu Marktpreisen verkauft werden. So könnte bspw. eine zentrale Umweltstrategieabteilung ökologiebezogene Beratungsleistungen externen Partnern sowie den Landesgesellschaften des Unternehmensverbunds anbieten. Der größte Anreiz, die Kosten zur Erstellung der ökologischen Leistung zu minimieren und selbst Ergebnisbeiträge zu generieren, besteht, wenn die Leistung auch auf externen Märkten angeboten wird. Bei fehlenden externen Märkten oder wettbewerbsstrategischen Gründen, die gegen ein externes Leistungsangebot sprechen, sind interne Verrechnungspreise auszuhandeln. ${ }^{561}$

Eine dezentrale Steuerung von zentralen Umweltschutzaufgaben durch Aufträge der unterschiedlichen Auslandsgesellschaften stellt dabei sicher, dass die zentralen Umweltbereiche Kostenbewusstsein zeigen und Aufgaben wahrnehmen, die im Unternehmensverbund nachgefragt werden. ${ }^{562}$ Die interne Marktkoordination des internationalen Umweltmanagements eignet sich zur Bündelung ökologischen Wissens, dem zentralen Aufbau von Erfahrungseffekten und der Realisierung von Economies-of-Scope-Effekten. Isoliert betrachtet geht von dieser Steuerung allerdings kein zentraler Koordinationsimpuls für das ökologiegerichtete Verhalten der ausländischen Gesellschaften aus, vielmehr wird das Verhalten der ökologischen Zentralbereiche koordiniert.

Im VoLKSWAGEN-Konzern ist u. a. die Forschung ein eigenständiger zentraler Bereich und beschäftigt sich großteils mit ökologischen Fragestellungen. In regelmäßigen Abständen werden bestimmte Ergebnisse vorgestellt, die dann von der Entwicklung aufgenommen werden können. Damit die Forschung nicht den Bezug zur Fahrzeugentwicklung verliert, ist sie bei VOLKSWAGEN als Businessunit organisiert und muss interne Aufträge generieren. Die Entwicklung ist dabei Auftraggeber der Forschung. ${ }^{563}$

561 Vgl. Günther, E.: Okologieorientierte Bereichssteuerung, in: Umweltwirtschaftsforum, 3. J. (1995), Heft 3, S. 33.

562 Vgl. zu Dienstleistungsabteilungen und Zentralbereichen Schreyögg, G.: Organisation: Grundlagen moderner Organisationsgestaltung, Wiesbaden 1996, S. 148.

Diese Aussage folgt den Angaben von Herrn MAYR-RAUCH im Interview. 


\section{Verrechnungspreise für Ökologiekosten}

Der Verbrauch von Energie, Wasser und anderen Rohstoffen, die Entsorgung des bei der Produktion anfallenden Abfalls, die Abwasserableitung und der Einsatz von end-of-pipe Umweltschutztechnologien führen zu Kosten. Werden diese Kosten mittels der Prozesskostenrechnung in Verbindung mit Input- und Output-Prozessbilanzen erfasst und aufgeschlüsselt ${ }^{564}$, können sie den verursachenden Bereichen zugeordnet werden. Dies schärtt das Kostenbewusstsein der Abteilungen bzw. Produktionsbereiche und kann zu ökologiebezogenen Anpassungsmaßnahmen beitragen. Im internationalen Kontext fallen aufgrund unterschiedlicher nationaler Umweltschutzabgaben und Ressourcenpreise die Kosten allerdings unterscheidlich aus. ${ }^{565}$

Sollen diese Unterschiede in der Anreizstruktur international angeglichen werden, kann die Unternehmensführung einheitliche Verrechnungspreise für Ökologiekosten festlegen. Dieses Vorgehen kann dann zur Koordination des ökologiegerichteten Verhaltens der ausländischen Tochtergesellschaften eingesetzt werden. Standorten in Schwellenländern könnte durch langsame Erhöhung der Verrechnungspreise ein Anreiz gegeben werden, zusätzliche ökologische Anpassungsmaßnahmen zu unternehmen. So ließe sich durch eine dezentrale Steuerung mittels Verrechnungspreisen das internationale Umweltschutzniveau anheben. Da den lokalen Entscheidungsträgern die Wahl der Mittel zur Senkung der internen Ökologiekosten freigestellt ist, können sich die lokalen Maßnahmen in Anbetracht der unterschiedlichen lokalen Gegebenheiten durchaus voneinander unterscheiden.

\section{Verrechnungspreise für externe Effekte}

Ein noch weitreichenderer Vorschlag zur ökologischen Steuerung ist die Festlegung von Verrechnungspreisen für die externen Effekte der unterschiedlichen nationalen Einheiten. Dabei könnten bspw. auf Basis einer Ökobilanz des Gesamtunternehmens die Menge an Emissionen, Rohstoffen, der Energieverbrauch etc. und damit der Umfang der externen Effekte festgestellt werden. Anschließend werden die Landesgesellschaften mit den von innen verursachten Effekten in Form von Kosten konfrontiert. Dabei sind an dieser Stelle die Erfassungs- und Bewertungsprobleme anzuführen, die ein derartiges Vorgehen

564 Vgl. Bundesumweltministerium, Umweltbundesamt (Hrsg.): Handbuch Umweltcontrolling, 2. Aufl., München 2001, S. 221.

565 In Anlehnung an Günther, E.: OKologieorientierte Bereichssteuerung, in: Umweltwirtschaftsforum, 3. Jg. (1995), Nr. 3, S. 32. 
erschweren. Genau genommen unterscheidet sich international die Höhe der Kosten externer Effekte lokaler Schadstoffe aufgrund unterschiedlicher Knappheiten. Auch hier könnten Verrechnungspreise von der Zentrale vorgegeben werden, um für die Standorte den Anreiz zu erhöhen, ökologische Anpassungsmaßnahmen zu unternehmen. Dabei wäre eine langsame Erhöhung der Verrechnungspreise bis zum Einsatz gewünschter Anpassungsmaßnahmen eine mögliche Option.

Eine Möglichkeit zur Koordination des internationalen ökologiegerichteten Verhaltens wäre der Ausweis der externen Kosten, um die Ökologieorientierung der Ländergesellschaften beurteilen zu können und bspw. ökologische Maßnahmen für die lokalen Standorte zentral festzulegen, die einen besonders hohen Beitrag zu den externen Effekten leisten. Ließen sich die Kosten auf die Nachfrager überwälzen, könnten Rücklagen gebildet werden, um daraus entsprechende ökologische Anpassungsmaßnahmen zu finanzieren. ${ }^{566}$

\section{Interne Verschmutzungsrechte}

Der unternehmensinterne Emissionshandel kann schließlich ebenfalls zur dezentralen Koordination des internationalen ökologischen Verhaltens herangezogen werden. Dabei wird in Bezug auf einen Schadstoff oder einen Einsatzstoff eine maximal zulässige Obergrenze für den Unternehmensverbund festgelegt. Danach werden die Rechte zur Emission eines Stoffes entweder von der Zentrale, bspw. in Form einer Auktion, intern versteigert oder es werden nach dem "Grandfathering-Prinzip“ den bisherigen Emittenten bzw. Nutzern Verschmutzungsrechte kostenlos zugeteilt. Um strategische Ziele zu erreichen, kann die Zahl der Verschmutzungsrechte im Zeitablauf verringert oder abgewertet werden. ${ }^{567} \mathrm{Da}$ die aktuelle Emissionsmenge höher als die Anzahl der Lizenzen ist, bestehen folgende Anpassungsmöglichkeiten:

566 In Anlehnung an Günther, E.: Ókologieorientierte Bereichssteuerung, in: Umweltwirtschaftsforum, 3. Jg. (1995), Heft 3, S. 32 f.

Vgl. Gray, R. H.: Accounting and Environmentalism: An Exploration of the Challenge of Gently Accounting for Accountability, Transparency and Sustainability, in: Accounting Organizations and Society, 17. Jg. (1992), Nr. 5, S. $399-425$, zitiert nach Günther, E.: Ókologieorientierte Bereichssteuerung, in: Umweltwirtschaftsforum, 3. Jg. (1995), Nr. 3, S. 33. 
- Die Geschäftseinheit schränkt ihre Aktivitäten ein, was zumeist eine Produktionsdrosselung bedeutet und wirtschaftlich kaum vertretbar erscheint.

- Die Einheit trägt durch Umweltschutzinvestitionen zur Steigerung der ökologischen Effizienz bei. Werden dadurch die Emissionen soweit gesenkt, dass Emissionsrechte frei werden, können diese auf dem internen Markt angeboten und verkauft werden. Findet sich ein Käufer, finanziert dieser zumindest teilweise die Umweltschutzinvestition.

- Schließlich kann eine am Markt erfolgreiche Landesgesellschaft ihre Produktion ausweiten und ihr Emissionsniveau erhöhen, wenn sie entsprechende Rechte von anderen Einheiten erwirbt.

Ein derartiges Handelssystem bedarf eines umfassenden Informations- und Kommunikationssystems, da jede Geschäftseinheit in der Lage sein muss, die Entwicklung auf dem internen Emissionsrechtemarkt zu verfolgen. Schließlich sind ein Broker zur Abwicklung und Registrierung der Geschäfte sowie eine Controllinginstanz notwendig. ${ }^{568}$ Eine Anwendung von internen Verschmutzungsrechten kann als ergänzende Lösung im Umweltschutz bspw. für die Koordination des $\mathrm{CO}_{2}$-Ausstoßes angewendet werden, der Verkauf von Verschmutzungsrechten für eine Vielzahl von Schadstoffen erscheint aber wenig praktikabel. Derzeit findet ein Verkauf von internen Verschmutzungen allerdings lediglich in der Ölindustrie statt.

\section{Regeln und Programme}

Die Koordination durch Regeln und Programme ergänzt die Aufgabengestaltung nach den Merkmalen der Zentralisation und Dezentralisation, da diese allein oft nicht genügen, um die angestrebten Koordinationsziele zu erreichen. Die Anwendung von internationalen Verfahrensrichtlinien oder Entscheidungsregeln kann dort zum Einsatz kommen, wo eine gewisse Gleichartigkeit der anfallenden Aufgaben und eine relativ stabile, antizipierbare Situation in unterschiedlichen Auslandsgesellschaften vorliegt. ${ }^{569}$ Anwendungsbereiche von Regeln und Programmen sind sowohl operative Aktivitätsfolgen als auch aufgaben- und personenbezogene Leistungsprozesse.

$568 \mathrm{Vgl.} \mathrm{zu}$ einer möglichen Ausgestaltungsform eines internen Emissionshandels Knobel, P.: Der Emissionsrechtehandel bei BP Amoco: Ein praktischer Ansatz, in: Umweltwirtschaftsforum, 8. Jg. (2000), Nr. 1, S. 41 - 45.

569 Vgl. Brodel, D.: Internationales Umweltmanagement: Gestaltungsfelder - Determinanten Ausprägungen, a. a. O., S. 459. 
Die zumeist schriftlich fixierten Regeln und Programme können als geronnene, in der Vergangenheit getroffene Entscheidungen interpretiert werden. ${ }^{570}$ Sie stellen wesentliche Elemente des organisationalen Wissens dar, welches in Verfahrensrichtlinien beziehungsweise Handbüchern zusammengefasst und damit gespeichert wird. ${ }^{571}$ Das Ausmaß, in dem vorab festgelegte und offiziell legitimierte Regeln die Leistungserstellungs- und -verwertungsabläufe steuern, wird als Programmierungs- oder Standardisierungsgrad bezeichnet. Ein zentrales Problem im Zusammenhang mit der Programmierung von Leistungsprozessen ist die Gefahr, dass in Situationen, die eigentlich innovative Lösungen erfordern, Programme angewendet werden. In einem solchen Fall können Chancen aufgrund geänderter Rahmenbedingungen leicht übersehen und der Aufbau neuen Wissens verhindert werden. ${ }^{572}$

\section{Operative Aktivitätsreihenfolgen}

Operative Aktivitätsreihenfolgen umschließen physische Transformationsprozesse wie bspw. Art und Reihenfolge der Arbeitsschritte bei der Lackierung von Automobilen $^{573}$ sowie administrative Prozesse, wie die Handhabung von Informationen bzw. Informationsträgern. Die Festlegung, wie die unterschiedlichen Stellen miteinander verknüpft sind und welche Stellen welche ökologiebezogenen Aufgaben realisieren müssen, ist in der Ablauforganisation festgelegt. In detaillierten Arbeits- und Verfahrensanweisungen wird der Umweltschutz programmiert und in Umweltmanagementhandbüchern und den mitgeltenden Unterlagen ${ }^{574}$ dokumentiert.

570 Vgl. Macharzina, K.: Unternehmensführung: Das internationale Managementwissen; Konzepte - Methoden - Praxis, a. a. O., S. 714.

571

Vgl. March, J. G.; Olsen, J. P.: The Uncertainty of the Past: Organizational Learning under Ambiguity, in: European Journal of Political Research, 3. Jg. (1975), S. 157; Brodel, D.: Internationales Umweltmanagement: Gestaltungsfelder - Determinanten - Ausprägungen, a. a. O., S. 457; Meffert, H.: Marketing: Grundlagen marktorientierter Unternehmensführung: Konzepte - Instrumente - Praxisbeispiele, a. a. O., S. 1026.

Vgl. Brodel, D.: Internationales Umweltmanagement: Gestaltungsfelder - Determinanten Ausprägungen, a. a. O., S. 461.

In diesem Zusammenhang können auch ökologiebezogene Bauvorschriften genannt werden, wie die Installation doppelwandiger Ölauffangbecken bei FoRD. Darauf wies bspw. Herr KRÜGER im Interview hin.

574 Das Umweltmanagementhandbuch erfüllt zum einen die Funktion der Dokumentation der organisatorischen Verfahrensanweisungen, die detailliert alle Zustăndigkeiten und umweltrelevanten Arbeitsabläufe regelt. Weiterhin dient es als verlässliches Nachschlagewerk für alle Informationen, die eine umweltgerechte Gestaltung der Arbeitsabläufe gewăhrleistet. Vgl. Müller-Christ, G.: Umweltmanagement: Umweltschutz und nachhaltige Entwicklung, a. a. O., S. $166 \mathrm{f}$. 
So werden bei BMW bspw. aufbauend auf Erkenntnissen von Demontageanalysen international geltende Leitsätze zur recyclingoptimierten Konstruktion von Bauteilen erarbeitet. Diese Vorgaben zur Umweltverträglichkeit und zur recyclingoptimierten Gestaltung von Bauteilen gliedern sich in die Hauptbereiche Verbindungstechnik, Materialauswahl und recyclingoptimierte Gestaltung und werden in einem Handbuch festgehalten, welches den Konstrukteur bei der Umsetzung der Recyclinganforderungen unterstützen soll. ${ }^{575}$

Das Umweltschutzhandbuch ist dabei in unterschiedliche Ebenen gegliedert, wie Abbildung 48 zeigt. Auf der allgemeinen Handbuchebene erfolgen zumeist eine Zusammenfassung aller umweltrelevanten Tätigkeiten, eine Darstellung der Umweltpolitik sowie die Beschreibung der Aufbauorganisation. Auf der Verfahrensanweisungsebene wird die Ablauforganisation beschrieben. Auf der Ebene der Arbeitsanweisungen werden schließlich die Einzeltätigkeiten arbeitsplatz- bzw. sachgebietsbezogen dargelegt. ${ }^{576}$

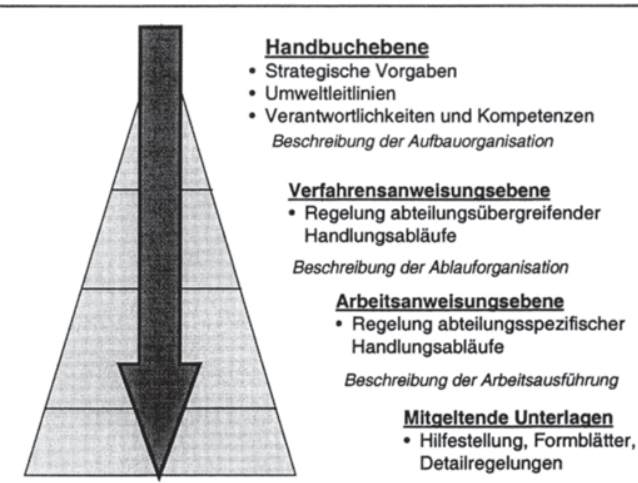

Umsetzung durch Dokumentation

Abbildung 48: $\quad$ Aufbau eines Umweltmanagementhandbuches

Quelle: Wruk, H.-P.: Standardisierte Grundlagen für die Umsetzung von Umweltmanagementsystemen, in: Winter, G. (Hrsg.): Das umweltbewusste Unternehmen: Die Zukunft beginnt heute, 6 . Aufl., München 1998, S. 841.

575 Vgl. BMW (Hrsg.): Umweltbericht der BMW Group 1999/2000, München 1999, S. 110.

576 Vgl. Fellx, R. et al.: Integrierte Managementsysteme: Ansätze zur Integration von Qualitäts-, Umwelt- und Arbeitssicherheitsmanagementsystemen, a. a. O., S. 41; MüllerChrist, G.: Umweltmanagement: Umweltschutz und nachhaltige Entwicklung, a. a. O., S. 163. Eine Darstellung des Umweltschutzhandbuches der BMW AG findet sich bei Heller, M.: Das Umweltmanagementsystem der BMW AG, in: Goralcyk, D.; Heller, M. (Hrsg.): Strategisches Umweltmanagement in der Industrie, Wien 1995, S. $57-81$, hier S. 67. 
Werden die Umwelthandbücher in ihrer Struktur und in Bezug auf ihre Inhalte im In- und Ausland eingeführt, können die Verfahrensanweisungen eine hohe Koordinationswirkung auch im Ausland entfalten. Während die Handbuchebene zumeist nur geringfügig an lokale Gegebenheiten anzupassen ist, steigt der Anpassungsbedarf auf der Verfahrens- und Arbeitsanweisungsebene zumeist stark an. Es empfiehlt sich somit zunächst eine internationale Übernahme der generellen Struktur bei lokaler Anpassung der konkreten Inhalte der Verfahrens- und Arbeitsanweisungen. Die tatsächliche Aufnahme und Umsetzung der ökologischen Zusatzanforderungen durch die lokalen Mitarbeiter sind dabei von der Überzeugungskraft der Unternehmensführung, der ökologischen Einstellungen der Mitarbeiter sowie dem Partizipationsgrad bei der Formulierung der Arbeitsanweisungen abhängig. ${ }^{577}$

In der Automobilindustrie werden Umweltmanagementhandbücher zur Koordination eingesetzt. Dabei findet bspw. bei Volkswagen eine weitgehende Übertragung der zentral geregelten Inhalte des Umweltmanagementhandbuches auf die Auslandsgesellschaften statt, während lokal Anpassungen an die jeweils geltenden rechtlichen und technologiebezogenen Gegebenheiten vorgenommen werden. ${ }^{578}$

\section{Aufgabenbezogene Leitungsprozesse}

Aufgabenbezogene Leitungsprozesse sind wiederholt und regelmäßig angewandte Leitungskonzepte und -strategien wie Planungs-, Budgetierungs- und Kontrollprozesse. Länderspezifisch divergierende Entscheidungsprozesse und Budgetierungsmethoden leisten dabei einer Prozessvarietät Vorschub, welche eine Koordination von Instrumentestrategien zwischen Zentrale und Landesgesellschaft zeitlich ausdehnen, erheblich erschweren oder gänzlich unterbinden. $^{579}$

Einheitliche Budgetierungsprozesse oder eine zentrale Mittelvorgabe könnten diese Koordinationsprobleme lösen helfen. Die Standardisierung von Kontrollprozessen zielt auf eine Vereinheitlichung von Kontrollmethoden und -abläufen ab und macht eine Definition einheitlicher Bewertungsstandards notwendig.

577 Vgl. Müller-Christ, G.: Umweltmanagement: Umweltschutz und nachhaltige Entwicklung, a. a. O., S. $164 \mathrm{f}$.

578 Dies entspricht auch den Aussagen von Herrn MOGg von VolksWAGEN im Interview.

579 Vgl. Landwehr, R.: Standardisierung der internationalen Werbeplanung, Frankfurt, Bern 1988, S. 49, 
Regelmäßig durchgeführte Umweltaudits ${ }^{580}$ im fertigungsbezogenen Umweltschutz zur Risikoanalyse und Umsetzungskontrolle von Umweltschutzmaßnahmen können hier als Beispiel für einen derartigen Kontrollprozess dienen. Die unternehmensübergreifende Vereinheitlichung der ökologiebezogenen Begriffe, Kontrollinstrumente, Kennzahlensysteme und Kontrollmethoden gewährleistet dann eine internationale Vergleichbarkeit von Kontrolldaten. ${ }^{581}$

Im Umweltschutz haben Audits eine hohe Bedeutung erlangt. In den 70er Jahren haben Industrieunternehmen insbesondere in den USA mit der freiwilligen Durchführung von internen Umweltaudits begonnen, um sicherzustellen, dass auch alle relevanten Umweltschutzgesetzte eingehalten werden und das Risiko von Störfällen möglichst klein ausfällt. Bereits 1972 hat bspw. GENERAL МотоRS ein Umwelt-Audit-Programm eingeführt, um sicherzustellen, dass Umweltprobleme in den einzelnen Niederlassungen des Konzerns richtig erfasst und an die Geschäftsleitung korrekt weitergegeben werden. ${ }^{582}$ In den $80 e r$ Jahren wurden in den USA die Regelungen im Zusammenhang mit dem Umgang von Altlasten verschärft. ${ }^{583}$ Umweltaudits wurden von einer immer größeren Zahl von Unternehmen zur Einhaltung der Rechtskonformität (Compliance Audits) ${ }^{584}$ und Gefahrenabwehr durchgeführt und seit 1986 von der amerikanischen Umweltschutzbehörde empfohlen und unterstützt. ${ }^{585}$ In dieser Zeit wurde das Umweltaudit auch von amerikanischen Tochtergesellschaften in Europa angewendet. Ende der $80 e r$ Jahre wurde das Umwelt-Audit von europäischen Unternehmen übernommen und weiterentwickelt. Einem eher ganzheitlichen

580 Umweltaudits oder Öko-Audits sind periodisch und systematisch durchgeführte Bestandsaufnahmen und Prüfungen des Umweltschutzes im Unternehmen im Hinblick auf verschiedene Vorgaben. Vgl. Dyllick, Th.: Basiswissen Umweltmanagementsysteme, St. Gallen 1999, S. 4.

581 Vgl. Kreutzer, R.: Global Marketing - Konzeption eines länderübergreifenden Marketing, Wiesbaden 1989, S. 103; Meffert, H.; Bolz, J.: Globalisierung des Marketing bei internationaler Unternehmenstätigkeit, a. a. O., S. 29.

Vgl. General Motors (Hrsg.): The General Motors 1994 Environmental Report, Detroit 1994, S. 25.

Der Standorteigentümer war verpflichtet, für eine Sanierung aufzukommen, auch wenn er selbst nicht für die Kontamination verantwortlich war. Dies führte bei Erwerb von Grundstücken bzw. Standorten zu der Notwendigkeit, vorab eine Risikoanalyse durchzuführen. Zusătzlich waren die Unternehmen verpflichtet, die Daten über ihre gesamten Emissionen der Öffentlichkeit zugänglich zu machen. Vgl. Bartsch, T.: Erfahrungen mit dem Umwelt / Öko-Audits in den USA, in: Zeitschrift für Umweltrecht, Heft 1/95, S. 14-19.

584 Vgl. zum Begriff des Compliance Audits Dyllick, Th.: Basiswissen Umweltmanagementsysteme, a. a. O., S. 4.

585 Vgl. Schulz, E.; Schulz, W.: Okologiemanagement: So nutzen Sie den Umweltschutz im Betrieb, München 1994, S. 306. 
Verständnis von Umweltmanagement folgend, wurden neben Compliance Audits auch System- und Performance-Audits ${ }^{586}$ durchgeführt. Dabei haben europäische Unternehmen im Gegensatz zu amerikanischen Unternehmen bewusst eine Öffnung nach außen vollzogen und das Audit damit zu einem Instrument ihrer externen Kommunikation gemacht.

Das Umweltaudit dient als Instrument der systematischen, dokumentierten, periodischen und objektiven Beurteilung, wie gut Umweltschutzorganisation, -management und -einrichtungen funktionieren. Dabei sollen die Audits den betrieblichen Umweltschutz durch Erleichterung der Kontrolle von Umweltschutzmaßnahmen durch die Unternehmensführung sowie die Feststellung der Erfüllung unternehmensbezogener umweltpolitischer Vorgaben sowie der Einhaltung behördlicher Auflagen unterstützen. ${ }^{587}$ Umweltaudits finden in der Automobilindustrie seit Jahren auch auf internationaler Ebene Anwendung. ${ }^{588}$

\section{Personenbezogene Leitungsprozesse}

Personenbezogene Leitungsprozesse umfassen Vorgehensweisen und Verfahren, die bei der Auswahl, Einstellung, Aus- und Weiterbildung, Beurteilung und Sanktionierung von Mitarbeitern in Form von standardisierten Verfahren zum Einsatz gelangen. ${ }^{589} \mathrm{Zu}$ diesen technokratischen Maßnahmen zählen das Abhalten festgelegter Schulungen im Umweltschutz für die Mitarbeiter unterschiedlicher Aufgabengebiete sowie festgelegte Beurteilungskriterien, nach denen die ökologische Leistung von Mitarbeitern beurteilt werden kann. Da an dieser Stelle eine hohe Überschneidung zu den personenorientierten Maßnahmen vorliegt, sollen diese Maßnahmen unter dem Gliederungspunkt koordinationsbedarfsreduzierende personelle Maßnahmen genauer behandelt werden. ${ }^{590}$

586 Systemaudits haben eine Überprüfung der Funktionsfähigkeit eines Umweltmanagementsystems, also der errichteten Strukturen und vorgegebenen Abläufe, zum Ziel. Performance Audits bzw. Ziel-Audits dienen der Überprüfung von Umweltleistungen anhand selbstgesetzter Ziele. Vgl. Dyllick, Th.: Basiswissen Umweltmanagementsysteme, a. a. O., S. 4.

Vgl. Internationale Handelkammer (ICC) (Hrsg.): Umweltschutz Audits, Positionspapier des ICC-Executive Boards, Köln 1989, S. 9, zitiert nach Müller-Christ, G.: Umweltmanagement: Umweltschutz und nachhaltige Entwicklung, a. a. O., S. 43.

Herr Mogg, der Leiter des Audit-Teams der Volkswagen AG bestătigt dies im Interview.

Vgl. Hoffmann, F.: Führungsorganisation, a. a. O., S. $354 \mathrm{f}$.

590 Vgl. zur umweltorientierten Personalplanung Müller-Christ, G.: Umweltmanagement: Umweltschutz und nachhaltige Entwicklung, a. a. O., S. $229 \mathrm{ff}$. 


\subsection{Personenorientierte Maßnahmen}

In international tätigen Unternehmen besteht das Problem, dass Menschen aus verschiedenen Kulturbereichen und mit unterschiedlichen Wertvorstellungen mittelbar oder unmittelbar zusammenarbeiten. Hieraus resultieren Abstimmungsprobleme, welche ein homogenes Agieren erschweren. Vor diesem Hintergrund gewinnt die Etablierung einer starken, homogenen Unternehmenskultur einen hohen Stellenwert zur Koordination des internationalen ökologiegerichteten Unternehmensverhaltens. ${ }^{591}$ Personenorientierte Maßnahmen dienen dabei der unmittelbaren „psychologischen Beeinflussung" bspw. mittels Schulungen und direkter persönlicher Kommunikation sowie der indirekten Beeinflussung durch personalpolitische Maßnahmen. Neben diesen koordinationsbedarfsreduzierenden Maßnahmen zählen die Unternehmenskultur sowie die persönlichkeitsgebundene, positionsbezogene Autorität, verstanden als Führungsstil, zu den koordinationsbedarfsdeckenden Instrumenten. Während die koordinationsbedarfsreduzierenden Maßnahmen u. a. der Etablierung einer starken, homogenen Unternehmenskultur ${ }^{52}$ dienen, trägt eine starke, unternehmensweit geltende Organisationskultur selbst zur Deckung des Koordinationsbedarfs bei. Aus diesem Grund wird die Organisationskultur ergänzend unter den koordinationsbedarfsdeckenden Maßnahmen diskutiert. Die folgende Abbildung zeigt die unterschiedlichen personenorientierten koordinationsbedarfsreduzierenden und -deckenden Maßnahmen im Überblick.

591 Vgl. Gladwin, T. N.; Walter, I.: Multinationals under Fire. Lessons in the Management of Conflict, New York et al. 1980, S. 569 f. Insbesondere MARTINEZ und JARILLO weisen auf die steigende Bedeutung "weicher" Koordinationsinstrumente hin. Vgl. Martinez, J. I.; Jarillo, J. C.: The Evolution of Research on Coordination Mechansims in Multinational Corporations, in: Journal of International Business Studies, 1989, Nr. 3, S. 489 - 514.

Unter Unternehmenskultur wird nach SCHEIN die Gesamtheit des gewachsenen Meinungs-, Norm- und Wertgefüges verstanden, welches das Verhalten sowohl der Führungskräfte als auch der Mitarbeiter prägt. Vgl. Schein, E. H.: Organizational Culture and Leadership - A Dynamic View, San Francisco et al. 1985, S. 9. Nach DüLFER ist eine weitere begriffliche Differenzierung der Unternehmens- und der Organisationskultur möglich, auf die im Rahmen dieser Arbeit aber verzichtet wird. Vgl. Dülfer, E.: Organisationskultur: Phănomen, Philosophie, Technologie: Eine Einführung in die Diskussion, in: Dülfer, E. (Hrsg.): Organisationskultur: Phănomen, Philosophie, Technologie, 2. Aufl., Stuttgart 1991, S. 2 ff. 


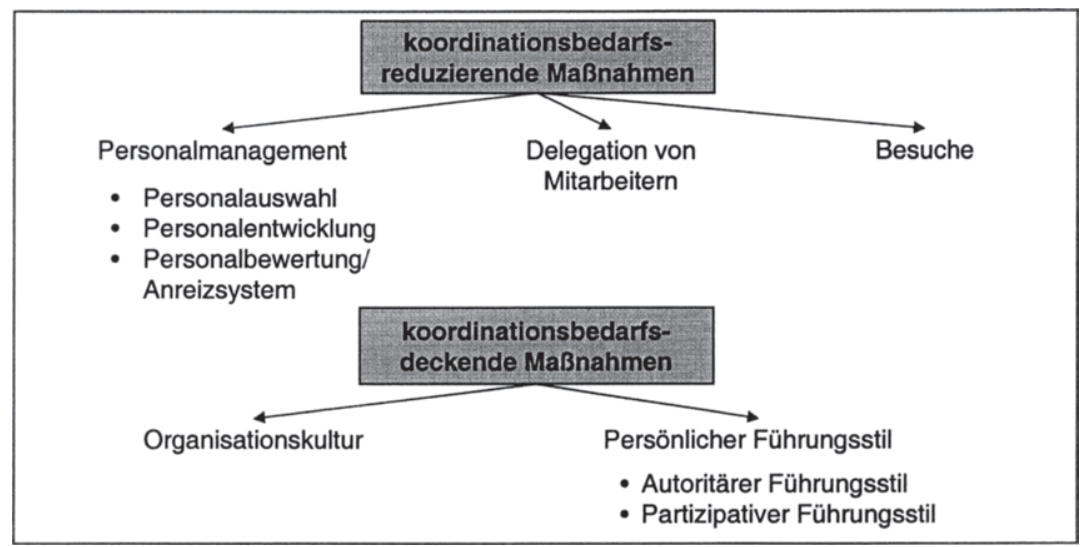

Abbildung 49: Personenorientierte Koordinationsmaßnahmen Quelle: Eigene Darstellung

\subsubsection{Koordinationsbedarfsreduzierende personelle Maßnahmen}

Personale Maßnahmen zur Reduktion des internationalen ökologiebezogenen Koordinationsbedarfs gründen auf der Bemühung, eine grundsätzliche Identifikation der Organisationsmitglieder mit den Unternehmenszielen im internationalen Umweltmanagement herzustellen und eine gemeinsame Unternehmenskultur zu schaffen. Entscheidend für die Koordinationswirkung ist dabei, dass möglichst viele Organisationsmitglieder im In- und Ausland über dieselben Überzeugungen verfügen und diese stark ausgeprägt sind. ${ }^{593} \mathrm{Im}$ Geschäftsbericht der AUDI AG von 1999 ist in diesem Zusammenhang zu lesen: ${ }_{n}$ Aktiver Umweltschutz ist nicht nur eine organisatorische Aufgabe, sondern immer auch eine Frage der Grundeinstellung. Zu unserer Philosophie des Umweltmanagements gehört deshalb auch die Motivation und Sensibilisierung der Belegschaft. ${ }^{\text {(594 }}$

593 Vgl. Hoffmann, F.: Führungsorganisation, a. a. O., S. 336 f. sowie Dobry, A.: Die Steuerung ausländischer Tochtergesellschaften: Eine theoretische und empirische Untersuchung ihrer Grundlagen und Instrumente, a. a. O., S. 147; Frese, E.; Blles, P.: Konsequenzen der Internationalisierung für Organisation und Management der Muttergesellschaft, a. a. O., S. 297.

Vgl. Audl (Hrsg.): Geschäftsbericht 1999, Ingolstadt 1999, S. 27. 


\section{Personalmanagement}

Da eine gemeinsame Unternehmenskultur mit ökologischen Grundsätzen von möglichst vielen Mitarbeitern international getragen werden sollte, gewinnt das ökologiegerichtete Personalmanagement sowohl zur Implementierung einer ökologiegerichteten Kultur als auch zur direkten Koordination an Gewicht. ${ }^{595}$

\section{Personalauswahl}

Hier ist die Berücksichtigung ökologischer Gesichtspunkte bei international ausgestalteten Personalauswahlverfahren zu erwähnen. Da Umweltschutz als Querschnittsaufgabe gilt, sind Stellenbeschreibungen um Anforderungen zu erweitern, die sich aus der Bewältigung der Umweltschutzaufgaben ergeben. In diesem Zusammenhang sind ökologiebezogene Anforderungen sowie die Fähigkeit des interdisziplinären Denkens und des Erkennens komplexer Systemzusammenhänge zu erwähnen. Damit in ausländischen Tochtergesellschaften im Fall einer Anhebung der gesetzlichen oder unternehmensinternen Anforderungen an den betrieblichen Umweltschutz auch auf ausreichend qualifiziertes Personal zurückgegriffen werden kann, könnte eine entsprechende Vorgabe zentral für die Personalbeschaffung vorgeschrieben werden.

\section{Personalentwicklung}

Um Qualifikationsdefizite im Umweltmanagement der Mitarbeiter insbesondere in ökologischen Folgerstaaten auszugleichen, sind Aus- und Weiterbildungsangebote durchzuführen ${ }^{596}$, die durch Mitarbeiter aus der Zentrale bzw. ökologischen Lead-Countries vorbereitet und auch durchgeführt werden können. So zählt die Durchführung von Schulungen für Vertreter des Umweltschutzes der Auslandsstandorte für die zentrale Umweltschutzabteilung der VoLKSWAGEN AG zu den Schwerpunkten der koordinierenden und steuernden Tätigkeiten im Jahr 1999. ${ }^{597}$ Dabei erhöht eine aktive Einbeziehung der lokalen Mitarbeiter in öko-

595 Vgl. hierzu und im Folgenden Meffert, H.; KIrchgeorg, M.: Marktorientiertes Umweltmanagement: Konzeption, Strategie, Implementierung mit Praxisfällen, a.a.o S. 433 ff.

Vgl. Stocker, S.: Mitarbeiterorientierung als Basis eines umweltbewussten Unternehmens, in: Winter, G.: Das Umweltbewusste Unternehmen: Die Zukunft beginnt heute, 6. Aufl., München 1998, S. 108 f.

597 Vgl. Volkswagen (Hrsg.): Jahresbericht des produktionsbezogenen Umweltschutzes 1999/ 2000 , a. a. O., S. 18. 
logiegerichtete Personalentwicklungsmaßnahmen den Erfolg der ökologischen Ausrichtung. ${ }^{598}$

In einige Ländern zeigen Schulungen zur ökologiegerechten Beeinflussung des Mitarbeiterverhaltens nicht den gewünschten Erfolg. Eine Verbindung des Umweltschutzes mit den Themenkreisen Gesundheitsschutz und Arbeitssicherheit können hier u. U. aufgrund der persönlichen und direkten Betroffenheit der Arbeiter zu einem höheren Engagement beitragen. Auch die Verbindung ökologischer Themen mit kulturspezifischen Verhaltensweisen kann helfen, der lokalen Belegschaft ökologische Werte nahezubringen. So wurde die südafrikanische Belegschaft am BMW-Standort Rosslyn während gemeinsamer "DrumSessions" für Umwelt-, Arbeits- und Gesundheitsschutz sensibilisiert. Dazu wurden zwischen den Trommelwirbeln Botschaften verpackt, die von der Belegschaft verstanden wurden. ${ }^{599}$

Globale Management-Trainingsprogramme, in denen den internationalen Führungskräften die Bedeutung ökologischer Aspekte bei der Unternehmensführung, Haftungsregelungen sowie unterschiedliche Instrumente zum Umgang mit den ökologischen Herausforderungen aufgezeigt werden, tragen zu einer einheitlichen Einstellung zum betrieblichen Umweltschutz bei. Ein noch höherer Beitrag zur Implementierung von ökologischen Werten und Normen auch in Schwellenländern kann durch internationale Job Rotation erreicht werden. Werden bspw. führende Positionen im Umweltmanagement in ausländischen Tochtergesellschaften nur bei vorheriger Tätigkeit im Umweltschutz eines ökologischen Vorreiterlandes besetzt, kann neben der Förderung einer gemeinsamen ökologischen Ausrichtung auch ein hoher ökologischer Know-howTransfer erwartet werden. ${ }^{600}$ Insbesondere in Schwellenländern, in denen noch ein geringes Wissen um ökologische Zusammenhänge bei generell hoher Sensibilisierung für den Umweltschutz vorliegt, werden deshalb in der Automobilindustrie über den betrieblichen Bereich hinausgehende Beratungen über

598 Vgl. Domsch, M. et al.: Umweltorientierte Personalentwicklung, in: Winter, J. (Hrsg.): Umweltmanagement, Stuttgart 1997, S. 97 - 124.

599 Vgl. BMW (Hrsg.): BMW Group: Clean Production weltweit, a. a. O., S. 1 f.

600 Vgl. Meffert, H.; Bolz, J.: Globalisierung des Marketing bei internationaler Unternehmenstätigkeit, a. a. O., S. 36. 
Umweltschutzmaßnahmen im privaten Bereich angeboten, um die Mitarbeitermotivation für ökologische Belange zu fördern. ${ }^{601}$

\section{Personalbewertung und Anreizsysteme}

Wichtig erscheint es auch, Anreizsysteme international an ökologischen Kriterien auszurichten. $\mathrm{Zu}$ denken ist hier bspw. an eine Prämierung von Verbesserungsvorschlägen oder an eine Beteiligung der Mitarbeiter am ökonomischen Erfolg ökologischer Verbesserungsmaßnahmen. ${ }^{602}$ Die Ergebnisse der empirischen Erhebung zum Umweltmanagement-Barometer in 1998 ergaben, dass in der deutschen Automobilindustrie knapp $30 \%$ der Mitarbeiter zumindest teilweise in Bezug auf ihre ökologiebezogene Leistung beurteilt werden. Noch geringer ist der Anteil der Unternehmen in der Automobilindustrie, welche die Entlohnung ihrer Mitarbeiter auch an ökologischen Kriterien ausrichten; hier beläuft sich die Anzahl auf weniger als $15 \% .{ }^{603}$ Neben Anreizen sind an dieser Stelle auch Sanktionen zu erwähen. So kann bspw. das schuldhafte Versäumen der auferlegten Pflichten eines Sachkundigen für Umweltschutz bei der VOLKSWAGEN AG zu ordnungs-, zivil- oder strafrechtlichen Konsequenzen führen. $^{604}$

\section{Delegation von Mitarbeitern}

Eine mittelfristige Entsendung von Mitarbeitern ${ }^{605}$ des Umweltschutzes von der Zentrale in ausländische Tochtergesellschaften dient als personalpolitische Maßnahme, um eine Förderung und Verankerung einer einheitlichen Unternehmensphilosophie zu erreichen und zu einer Verbesserung der ökologiebezo-

601 Vgl. dazu generell Müller-Christ, G.: Umweltmanagement: Umweltschutz und nachhaltige Entwicklung, a. a. O., S. 234 ff.; Kostka, C.; Kostka, S.: Umweltorientierte Personalentwicklung, a. a. O., S. 421 ff. sowie BMW (Hrsg.): BMW Group: Clean Production weltweit, a. a. 0 .

602 Vgl. zu möglichen Anreizsystemen im Umweltmanagement Müller-Chrlst, G.: Umweltmanagement: Umweltschutz und nachhaltige Entwicklung, a. a. O., S. 241 ff. sowie grundlegend zur Motivation der Mitarbeiter zu ökologischem Verhalten vgl. Stocker, S.: Mitarbeiterorientierung als Basis eines umweltbewussten Unternehmens, a. a. O., S. $111 \mathrm{ff}$.

Diese Auswertung bezieht sich auf 14 deutsche Unternehmen aus der Automobilindustrie.

604 Vgl. Volkswagen (Hrsg.): Sachkundige für Umweltschutz: Handbuch, a. a. O., Anlage 2, Punkt 6.

605 Die Entsendung in Form einer Delegation ist ein mittelfristiger, ungefăhr 3 bis 4 Jahre andauernder Einsatz eines Stammhausangestellten in einer Tochtergesellschaft im Ausland. Vgl. Oechsler, W. A.: Verfahren zur Auswahl, Vorbereitung und Entsendung von Stammhausdelegierten ins Ausland, in: Macharzina, K.; Oesterle, M.-J. (Hrsg.): Handbuch Internationales Management: Grundlagen - Instrumente - Perspektiven, Wiesbaden 1997, S. $771 \mathrm{f}$. 
genen Steuerung ausländischer Tochtergesellschaften beizutragen. ${ }^{606}$ Bei Neugründungen ausländischer Tochtergesellschaften, aber auch bei der Implementierung von Umweltmanagementsystemen oder neuer Umweltschutztechnologien im Ausland findet ein Transfer von Führungskräften oft aus dem Motiv der Hilfeleistung statt. ${ }^{607}$

Rückt die Bedeutung der Tochtergesellschaft für die Ertragslage oder das Image des Gesamtunternehmens in den Vordergrund, gewinnt das Kontrollmotiv gegenüber der Hilfestellung an Gewicht. ${ }^{608}$ Neben einer kulturprägenden Rolle soll dann durch die Besetzung der geistigen und disziplinarischen Spitze der Hierarchie der Tochtergesellschaft mit Führungskräften aus der Muttergesellschaft eine weitestgehend störungsfreie Kommunikationsbeziehung zwischen der Zentrale und den ausländischen Unternehmensteilen aufgrund der herkunftsbezogenen Prägung der Führungskräfte herbeigeführt werden. Ziel ist es, ein loyales Verhalten des gesamten Subsystems der Tochtergesellschaft in ökologiebezogenen Fragestellungen zu erreichen. ${ }^{609}$ Der Entsendung von Führungskräften kommt damit eine hohe Bedeutung zur Koordination und Steuerung ausländischer Tochtergesellschaften im internationalen Umweltmanagement zu. ${ }^{610}$

606 Die generellen Ziele der Entsendung von Führungskräften wurden empirisch nachgewiesen. Vgl. Pausenberger, E.; Noelle, G. F.: Entsendung von Führungskräften in ausländische Niederlassungen, in: Schmalenbachs Zeitschrift für betriebswirtschaftliche Forschung, 29. Jg. (1977), Nr. 12, S. $346-366$.

607 Vgl. Welge, M. K.: Die effiziente Gestaltung der Mutter-Tochter-Beziehungen in deutschen multinationalen Unternehmungen, in: Pausenberger, E. (Hrsg.): Internationales Management, Stuttgart 1981, S. 45 - 77 sowie Dobry, A.: Die Steuerung ausländischer Tochtergesellschaften: Eine theoretische und empirische Untersuchung ihrer Grundlagen und Instrumente, a. a. O., S. 156.

608 Vgl. Welge, M. K.: Die effiziente Gestaltung der Mutter-Tochter-Beziehungen in deutschen multinationalen Untemehmungen, a. a. O., S. 49 sowie Dobry, A.: Die Steuerung ausländischer Tochtergesellschaften: Eine theoretische und empirische Untersuchung ihrer Grundlagen und Instrumente, a. a. O., S. 159.

Dabei sind Verständnisprobleme nicht endgültig gelöst, sondern in die Auslandsgesellschaft hineinverlagert. Es wird dabei allerdings unterstellt, dass die Vertrauensperson im Ausland die Steuerungsimpulse anhand der vor Ort gewonnenen Kenntnisse zieladäquat umsetzen kann. Vgl. Dobry, A.: Die Steverung ausländischer Tochtergesellschaften: Eine theoretische und empirische Untersuchung ihrer Grundlagen und Instrumente, a. a. O., S. 148.

$610 \mathrm{Zu}$ Auswahlverfahren für geeignete Mitarbeiter und Vorbereitungsverfahren vgl. Oechsler, W. A.: Verfahren zur Auswahl, Vorbereitung und Entsendung von Stammhausdelegierten ins Ausland, a. a. O., S. 771. 
In der Implementierungsphase einer gemeinsamen internationalen Umweltmanagementpolitik oder zur kulturellen Verankerung des Umweltschutzes in der Unternehmenskultur ist es eine wichtige Aufgabe derartiger Transfers, einen Beitrag zur organisationalen Sozialisation zu leisten. Führungskräfte sind als Träger der Umweltpolitik, der Unternehmensphilosophie, der Führungsgrundsätze und des administrativen Know-hows zu verstehen und tragen durch ihre Handlungen zur Erzielung einer homogenen, umweltorientierten Unternehmenskultur bei. ${ }^{611}$ Durch den informellen Austausch von Wissen und Informationen sollen unternehmensbezogene ökologische Werte und Überzeugungen, Rollen und spezifische Fähigkeiten vermittelt werden. ${ }^{612}$ Die entsandten Mitarbeiter gelten dabei als „Agenten der Sozialisation“ der Auslandsniederlassungen bzw. als Katalysatoren des organisatorischen Wandels im Sinne der internationalen Vereinheitlichung der Unternehmenskultur. ${ }^{613}$ In der Automobilindustrie finden sich dabei durchaus Beispiele für die Delegation von Führungskräften im Umweltmanagement. So ist der Umweltmanagementbeauftragte der BMW AG am Standort in Südafrika eine deutsche Führungskraft. ${ }^{614}$

\section{Besuchsverkehr}

Als ein weiteres Instrument der personenorientierten Koordination im internationalen Umweltmanagement ist der internationale Besuchsverkehr anzusehen. ${ }^{615}$ Er dient häufig rein informativen Zwecken, dem persönlichen Kennenlernen, dem Meinungsaustausch, der Schulung oder dem Training etc. Da der Besuchsverkehr mit einer unmittelbaren persönlichen Kontaktaufnahme verbunden ist, findet immer auch eine persönliche, soziale Einflussnahme statt. Daneben wird die Diffusion des im Unternehmensverbund vorhandenen Wissens und des technologischen bzw. managementbezogenen Know-hows verbessert. So können bspw. im Umweltschutz sachkundige Mitarbeiter beim Besuch ausländi-

611 Vgl. Scholz, C.: Die richtige Kulturstrategie schafft Synergien, in: Personalwirtschaft, 20. Jg. (1993), S. 31 - 34 sowie Wolf, J.: Internationales Personalmanagement: Kontext - Koordination - Erfolg, Wiesbaden 1994, S.132 ff.

612 Vgl. Kirsch, W.: Einführung in die Theorie der Entscheidungsprozesse: Entscheidungen in Organisationen, Bd. 3, Wiesbaden 1977, S.. 176, zitiert nach Macharzina, K.; Oesterle, M.-J.: Bestimmungsgrößen und Mechanismen der Koordination von Auslandsgesellschaften, a. a. O., S. 617.

613 Vgl. Macharzina, K.; Oesterle, M.-J.: Bestimmungsgrößen und Mechanismen der Koordination von Auslandsgesellschaften, a. a. O., S. 617 sowie Brodel, D.: Internationales Umweltmanagement: Gestaltungsfelder - Determinanten - Ausprägungen, a. a. O., S. 469.

614 Vgl. BMW (Hrsg.): BMW Group: Clean Production weltweit, a. a. 0.

615 Vgl. Wolf, J.: Internationales Personalmanagement: Kontext - Koordination - Erfolg, Wiesbaden 1994, S. $135 \mathrm{ff}$. 
scher Standorte vor Ort die Umsetzung der Umweltpolitik durch Schulungsmaßnahmen und die Bereitstellung von Arbeitsmaterialien fördern. Die Mitarbeiter des Audit-Teams der VolKswaGeN AG statten zu diesem Zweck den ausländischen Tochtergesellschaften regelmäßig Besuch ab. ${ }^{616}$ Bei Besuchen von Mitarbeitern oder Führungskräften aus den Auslandsgesellschaften in der Unternehmenszentrale bzw. der Gesellschaft in einem ökologischen LeadCountry, können Möglichkeiten des technischen Umweltschutzes vorgestellt werden. Hier finden häufig keine hierarchischen Anweisungen oder Pläne und Programme Anwendung, welche die Auslandsgesellschaften zwingen, die Technologien im eigenen Land einzusetzen. Vielmehr wird durch derartige Besuche auf die Überzeugungskraft der Argumente gesetzt. ${ }^{617}$

Da der internationale Besuchsverkehr aber oft auch in direktem Zusammenhang mit der strukturellen (persönliche Weisungen oder Selbstabstimmung) oder der technokratischen Koordination (personelle Unterstützung bei der Einführung neuer Verfahren) steht, überlagern sich häufig sachrational-formale und persönlich-informelle Koordinationseffekte. ${ }^{618}$

\subsubsection{Koordinationsbedarfsdeckende personale Maßnahmen}

Zu den wichtigsten koordinationsbedarfsdeckenden Instrumenten zählt neben einer einheitlichen Organisationskultur auch der Führungsstil.

\section{Organisationskultur}

Bei der Koordination durch die Unternehmenskultur und -identität erfolgt die Abstimmung ohne explizite organisatorische Regelungen. Gemeinsame ökologiebezogene Werte und Normen sowie die Identifikation mit der ökologischen Ausrichtung einer als eigenständige „Persönlichkeit" wahrgenommenen Organisation führen zu einer informellen Abstimmung von Interdependenzen. ${ }^{619}$ Die Unternehmenskultur basiert dabei nach FRESE auf einer zumindest

616 Diese Aussage bestätigt Herr Mogg im Interview.

617 Vgl. Brodel, D.: Internationales Umweltmanagement: Gestaltungsfelder - Determinanten Ausprägungen, a. a. O., S. $470 \mathrm{f}$.

618 Vgl. Wolf, J.: Internationales Personalmanagement: Kontext - Koordination - Erfolg, Wiesbaden 1994, S. 137 sowie Brodel, D.: Internationales Umweltmanagement: Gestaltungsfelder - Determinanten - Ausprägungen, a. a. O., S. 470.

619 Vgl. Meffert, H.: Marketing: Grundlagen marktorientierter Unternehmensführung: Konzepte - Instrumente - Praxisbeispiele, a. a. O., S. 1028, Meffert, H.; Kirchgeorg, M.: Marktorientiertes Umweltmanagement: Konzeption - Strategie - Implementierung, a. a. O., S. $420 \mathrm{ff}$. 
teilweisen Internalisierung der Unternehmensziele und der diesen zugrunde liegenden Überzeugungen und Werte, die den latenten Widerspruch zwischen ökologischen Unternehmens- und Individualzielen partiell aufhebt und eine implizite Verhaltenssteuerung bewirkt. ${ }^{620}$ In einer Zeit, in der die Grenzen der Nationalstaaten zunehmend an Bedeutung verlieren und gleichzeitig Konflikte zwischen Weltkulturkreisen deutlicher zutage treten, gewinnt eine starke internationale Unternehmenskultur als strategischer Wettbewerbsfaktor an Bedeutung. ${ }^{621}$

\section{Persönlicher Führungsstil}

Als weitere koordinationsbedarfsdeckende Maßnahme kann der Führungsstil im internationalen Umweltmanagement angesehen werden. Der persönliche Führungsstil von Entscheidungsträgern bei der Koordination zwischen national unterschiedlichen Abteilungen bzw. Personen kann dabei autoritär bzw. partizipativ ausgestaltet sein.

\section{Autoritärer Führungsstil}

Im Rahmen einer autoritären Fixierung der ökologischen Ziele durch die Führungsspitze des Gesamtunternehmens stellen diese Ziele für die nicht zu dieser Führungsspitze gehörenden Entscheidungsträger unbeeinflussbare Normen dar. Diese Form der Zielvorgaben trägt wenig zur Motivation der lokalen Entscheidungsträger bei und wird eine Identifikation der Mitarbeiter an den ausländischen Standorten mit den ökologischen Zielen erschweren. Zur Überwachung der Erfüllung der Zielvorgaben in den ausländischen Gesellschaften ist die Einrichtung eines Kontrollsystems maßgeblich. ${ }^{622}$

\section{Partizipativer Führungsstil}

Bei der partizipativen Form der Zielvorgaben misst jeder Entscheidungsträger die von ihm veranlassten Maßnahmen an den selbst mitgetroffenen Zielen und trägt so bereits durch Selbstkontrolle zur Koordination bei. Die Beteiligung bei der Zielausarbeitung trägt dabei auch zur Verminderung von ökonomischen und ökologischen Zielkonflikten an dieser Stelle bei. Eine hohe Identifikation mit den

620 Vgl. Frese, E.: Grundlagen der Organisation, Konzept - Prinzipien - Strukturen, a. a. O., S. $148 \mathrm{ff}$.

621 Vgl. Frese, E.; Blles, P.: Konsequenzen der Internationalisierung für Organisation und Management der Muttergesellschaft, a. a. O., S. 298.

622 Vgl. Benkenstein, M.: F \& E und Marketing: Eine Untersuchung zur Leistungsfähigkeit von Koordinationskonzeptionen bei Innovationsentscheidungen, a. a. O., S. $181 \mathrm{ff}$. 
vereinbarten Zielen bewirkt dabei zumeist eine hohe Koordinationswirkung im internationalen Umweltmanagement. Gegenstand derartiger Zielvereinbarungen können bspw. Materialnutzungsgrade, Energieeffizienzen oder Produktivitätskennziffern sein. ${ }^{623}$

\section{Umweltmanagementsysteme als Koordinationskonzeptionen}

Nachdem die Bandbreite des Koordinationsinstrumentariums vorgestellt wurde, sollen im folgenden Kapitel unterschiedliche Koordinationsinstrumente und -maßnahmen miteinander zu einer Koordinationskonzeption kombiniert werden. Die Ausgestaltung einer solchen Koordinationskonzeption hängt von der basisstrategischen Ausrichtungen im internationalen Umweltmanagement ab. In diesem Zusammenhang beziehen sich die folgenden Ausführungen auf die Ausgestaltung eines Umweltmanagementsystems als systematische Koordinationskonzeption zur Umsetzung der ökologischen Unternehmensziele.

\subsection{Aufbau eines Umweltmanagementsystems}

Ein Managementsystem umfasst organisatorische Maßnahmen zur Beherrschung von Managementprozessen ${ }^{624}$ und zur Sicherstellung der festgelegten Unternehmensziele. Die organisatorischen Maßnahmen beziehen sich dabei sowohl auf aufbauorganisatorische Festlegungen wie Hierarchie, Verantwortlichkeiten und Zuständigkeiten als auch auf ablauforganisatorische Regelungen, bspw. bezüglich der Arbeitsverfahren, des Berichtswesens sowie der Informations- und Entscheidungswege. Managementsysteme bilden somit ein Bindeglied zwischen der strategischen Ausrichtung einer Unternehmung und deren Umsetzung in die Praxis. ${ }^{625}$

623 Vgl. Matzel, M.; Sekul, S.: Integration des Umweltschutzes in die Profit-Center-Organisation, in: Umweltwirtschaftsforum, 3. Jg. (1995), Nr. 3, S. 7, Vgl. Benkenstein, M.: F \& E und Marketing: Eine Untersuchung zur Leistungsfähigkeit von Koordinationskonzeptionen bei Innovationsentscheidungen, a. a. O., S. $179 \mathrm{ff}$.

Die Hauptaufgaben des Managements bestehen im Identifizieren, Organisieren, Führen und Handhaben aller der Wertschöpfung dienenden Prozesse in einer Organisation. Das Management einer Organisation beinhaltet dabei die Festlegung der Unternehmenspolitik, Ziele und Verantwortlichkeiten sowie ihre kontinuierliche Verbesserung durch Planung, Lenkung, Ergebnisbeurteilung und Anpassung. Vgl. dazu Welge, M.: Management in deutschen multinationalen Unternehmungen: Ergebnisse einer empirischen Untersuchung, a. a. O., S. $126 \mathrm{ff}$. und die dort angegebene Literatur.

Vgl. Welge, M.: Management in deutschen multinationalen Untemehmungen: Ergebnisse einer empirischen Untersuchung, a. a. O., S. 129 ff.; Petrlck, K.: Das Konzept der Zusammenführung von Qualitätsmanagement und Umweltmanagement, in: Petrick, K.; Eggert, R. 
Die Diskussion um Umweltmanagementsysteme hat in den 70er Jahren mit der Verschärfung der Haftungsbedingungen bei industrieller Umweltverschmutzung in den USA begonnen. ${ }^{626}$ Ende der 80er und zu Beginn der 90er Jahre wurden die Aufgaben des Umweltmanagements erweitert und ganzheitliche Konzeptionen entwickelt. ${ }^{627}$ Hier setzte sich die Erkenntnis durch, dass es eines systematischen Vorgehens im Umweltmanagement bedarf, um ökologische Unternehmensziele umzusetzen. Ein Umweltmanagementsystem (UMS) stellt dabei einen derartigen systematischen Ansatz dar. Es dient als Teilsystem eines unternehmensübergreifenden Managementsystems der Unterstützung der Führungskräfte ${ }^{628}$ bei der Durchsetzung der Umweltziele durch Bereitstellung einer Organisationsstruktur, Planungstätigkeiten, der Festlegung von Verantwortlichkeiten, der Vorgabe von Methoden, Verfahren, Prozessen und Ressourcen zur Entwicklung, Implementierung, Erfüllung, Bewertung und Aufrechterhaltung der Umweltpolitik. ${ }^{629}$ Damit kann ein Umweltmanagementsystem als Voraussetzung zur Umsetzung eines zielgerichteten, systematischen Umweltmanagements angesehen werden. ${ }^{630}$

(Hrsg.): Umwelt- und Qualitätsmanagementsysteme: Eine gemeinsame Herausforderung, München, Wien 1995, S. 5 f.

Zur Entstehung von Umweltmanagementsystemen bzw. des Umwelt-Audits vgl. Baumast, A.: Die Entstehungsgeschichte des Umwelt-Audit, in: Doktoranden-Netzwerk Öko-Audit e. V. (Hrsg.): Umweltmanagementsysteme zwischen Anspruch und Wirklichkeit: Eine interdisziplinäre Auseinandersetzung mit der EG-Öko-Audit-Verordnung und der DIN EN ISO 14001, Berlin et al. 1998, S. 33 - 56 sowie Gleckman, H.; Krut, R.: Neither International nor Standard: The Limits of ISO 14001 as an Instrument, in Greener Management International, Issue $14 \mathrm{~S} .113-114$.

627 Vgl. dazu auch die Ausführungen in Kapitel A 2.2.

$628{ }_{n}$ As they deal with environmental issues, managers need a rigorous and systematic method to integrate these aspects into the organization's decision-making process. EMS will provide the organization with appropriate data and tools to design, implement, and improve its environmental programs and performance." Epstein, M.; Roy, M.-J.: Managing Corporate Environmental Performance: A Multinational Perspective, a. a. O., S. 292.

629 Diese Formulierung entspricht der Definition des Umweltmanagementsystem-Begriffs in der ISO 14.001. Vgl. Deutsches Institut für Normung (Hrsg.): Umweltmanagementsysteme - Spezifikation mit Anleitung zur Anwendung: Zweisprachige Fassung DIN EN ISO 14.001, 1996, Kap. 3.5. Ähnlich ist in der EMAS-Verordnung zu lesen, dass ein Umweltmanagementsystem als "der Teil des gesamten übergreifenden Managementsystems" definiert wird und ${ }_{n}$ die Organisationsstruktur, Zuständigkeiten, Verhaltensweisen, förmliche Verfahren, Abläufe und Mittel für die Festlegung und Durchführung der Umweltpolitik einschließt".

Vgl. Felix, R. et al.: Integrierte Managementsysteme: Ansắtze zur Integration von Qualitäts-, Umwelt- und Arbeitssicherheitsmanagementsystemen, a. a. O., S. 5 - 6 sowie Thimme, P. M.: Der Wettbewerb zwischen EG-Öko-Audit-Verordnung und DIN ISO 14.001, in: Doktoranden-Netzwerk Öko-Audit e. V. (Hrsg.): Umweltmanagementsysteme zwischen Anspruch und Wirklichkeit - Eine interdisziplinäre Auseinandersetzung mit der EG-Öko-Audit-Verordnung und der DIN ISO 14.001, Berlin u. a. 1998, S. 265. 
Anfang der 90er Jahre entwickelte die British Standards Institution (BSI) den weltweit ersten Standard für ein eigenständiges Umweltmanagementsystem (BS 7750). ${ }^{631} 1993$ erfolgte die Verabschiedung der europäischen EMASVerordnung durch die EU, und in 1996 folgte die Publikation der ähnlich ausgestalteten, weltweit gültigen ISO-Norm 14.001 über Umweltmanagementsysteme. Die Umweltmanagementsystem-Normen geben einen organisatorischen Rahmen für die Einführung, Umsetzung und Überprüfung des Umweltmanagements vor. Jedes Unternehmen kann zwar auch weiterhin ein unternehmensindividuelles UMS entwickeln und dieses installieren. Soll das Umweltmanagementsystem allerdings gegenüber Dritten nach einem gültigen Standard zertifiziert werden, muss sich das Unternehmen für eine Zertifizierung nach der europäischen EMAS-Verordnung oder nach der international gültigen Norm ISO 14.001 entscheiden. ${ }^{632}$

Die Einrichtung von Umweltmanagementsystemen wird international tätigen Unternehmen im Rahmen der OECD-Richtlinien empfohlen:

"Sound environmental management is an important part of sustainable development, and is increasingly being seen as both a business responsibility and a business opportunity. Multinational enterprises have a role to play in both respects. Managers of these enterprises should therefore give appropriate attention to environmental issues within their business strategies. Improving environmental performance requires a commitment to a systematic approach and to continual improvement of the system. An environmental management system provides the internal framework necessary to control an enterprise's environmental impacts and to integrate environmental considerations into business operations. Having such a system in place should help to assure stockholders, employees and the community that the enterprise is actively working to protect the environment from the impacts of its activities".633

631 Vgl. Britlsh Standards Institution (Hrsg.): Specification for Environmental Management Systems. British Standard BS 7750: 1992, London 1992.

Grundlegende gemeinsame Anforderungen von EMAS und ISO 14.001 sind die organisatorische Verankerung des Umweltschutzes im Unternehmen durch ein funktionsfăhiges Umweltmanagementsystem, die Selbstverpflichtung zur Einhaltung aller relevanten Geset$z e$ und Verordnungen als Mindestanforderung, eine regelmäßige Überprüfung des Umweltmanagements sowie die Verpflichtung zur kontinuierlichen Verbesserung der betrieblichen Umweltleistung bzw. des Umweltmanagementsystems. Vgl. zu Unterschieden und Gemeinsamkeiten bspw. Felix, R. et al.: Integrierte Managementsysteme: Ansắtze zur Integration von Qualităts-, Umwelt- und Arbeitssicherheitsmanagementsystemen, a. a. O., S. 17 - 19; Dyllick, Th.: Die EU-Verordnung zum Umweltmanagement und zur Umweltbetriebsprüfung (EMAS-Verordnung) im Vergleich mit der geplanten ISO-Norm 14.001: Eine Beurteilung aus Sicht der Managementlehre, in: Zeitschrift für Umweltpolitik und Umweltrecht, 1995, Nr. 3; Meffert, H.; KIrchgeorg, M.: Marktorientiertes Umweltmanagement - Konzeption, Strategie, Implementierung mit Praxisfällen, a. a. O., S. 418 - 420.

OECD (Hrsg.): OECD Guidelines for Multinational Enterprises: Review 2000: Commentaries, Paris 2000, S. 9. 
Umweltmanagementsysteme dienen dabei der Festlegung und Umsetzung von Umweltzielen, der Umweltpolitik und Umweltprogrammen eines Unternehmens und umfassen dabei planerische, führungsmäßige, organisatorische und personelle Maßnahmen. ${ }^{634}$ Damit bedienen sich Umweltmanagementsysteme generell der im Rahmen dieser Arbeit besprochenen strukturellen, technokratischen und personellen Maßnahmen zur Koordination des ökologiegerichteten Verhaltens der Organisationsmitglieder. In diesem Zusammenhang können Umweltmanagementsysteme als Koordinationskonzeptionen beschrieben werden. Dabei stellt ein Umweltmanagementsystem unabhängig von der verfolgten Strategie einen systematischen Ansatz zur Umsetzung der ökologischen Unternehmensziele dar. ${ }^{635}$ Die folgende Abbildung zeigt die Elemente eines Umweltmanagementsystems.

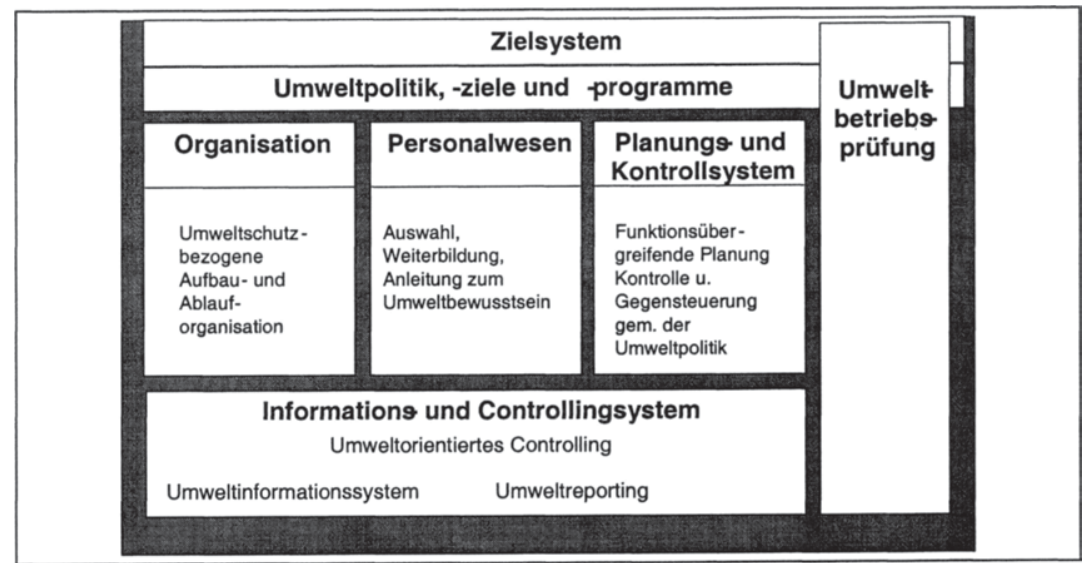

Abbildung 50: Elemente eines Umweltmanagementsystems (UMS)

Quelle: Wagner, G. R.: Betriebswirtschaftliche Umweltökonomie, Stuttgart 1997, S. 208.

In der Automobilindustrie konnte in den vergangenen Jahren ein regelrechter Zertifizierungswettlauf der Hersteller beobachtet werden. So weist das Management der Marke FORD explizit darauf hin, dass FORD als erster Hersteller

634 Vgl. Dyllick, Th.: Basiswissen Umweltmanagementsysteme, a. a. O., S. 3 f.

635 EPSTEIN und RoY weisen darauf hin, dass: ${ }_{n}$ The appropriate EMS framework should enable firms to reap the potential benefits associated with a proactive environmental strategy and allow for both a global integrative or local adaptive environmental strategy." Epstein, M.; Roy, M.-J.: Managing Corporate Environmental Performance: A Multinational Perspective, a. a. O., S. 292. 
alle in- und ausländischen Standorte nach ISO 14.001 zertifiziert hat. ${ }^{636}$ VOLKSWAGEN möchte bis zum Jahr 2003 ebenfalls alle Standorte einer Zertifizierung unterzogen haben.

In Anbetracht unterschiedlicher Ziele und strategischer Orientierungen können Umweltmanagementsysteme unterschiedlichen Zwecken dienen. ${ }^{637}$ Mit DYLLICK und HAMSCHMIDT können die in der folgenden Tabelle aufgeführten grundlegenden Typen von Umweltmanagementsystemen unterschieden werden. ${ }^{638}$

\begin{tabular}{|c|c|c|c|c|c|}
\hline Typ & Infrastruktur & Audlitierung & Controlling & Marketing & PR \\
\hline Zweck & $\begin{array}{l}\text { Organisations- } \\
\text { effizienz }\end{array}$ & Absicherung & Kosteneffizienz & Differenzierung & Profilierung \\
\hline Zlele & $\begin{array}{c}\text { Systematisierung } \\
\text { Motivation }\end{array}$ & $\begin{array}{l}\text { Rechtssicherheit } \\
\text { Risikovorsorge }\end{array}$ & $\begin{array}{l}\text { Prozessbeherr- } \\
\text { schung } \\
\text { Kostensenkung }\end{array}$ & $\begin{array}{c}\text { Ökologische } \\
\text { Differenzierung }\end{array}$ & $\begin{array}{l}\text { Imagebildung } \\
\text { Einflussnahme }\end{array}$ \\
\hline $\begin{array}{l}\text { Anspruchs- } \\
\text { gruppe }\end{array}$ & Management & $\begin{array}{l}\text { Management } \\
\text { Behörden } \\
\text { Kunden } \\
\text { Banken } \\
\text { Versicherungen }\end{array}$ & Management & $\begin{array}{l}\text { Management } \\
\text { Kunden } \\
\text { Konkurrenz }\end{array}$ & $\begin{array}{l}\text { Management } \\
\text { Öffentlichkeit }\end{array}$ \\
\hline $\begin{array}{l}\text { Maßnah- } \\
\text { men }\end{array}$ & $\begin{array}{c}\text { Planung } \\
\text { Organisation } \\
\text { Schulung } \\
\text { Motivation } \\
\text { Umsetzung } \\
\text { Kontrolle } \\
\text { Weiterentwick- } \\
\text { lung }\end{array}$ & $\begin{array}{l}\text { Notfallvorsorge } \\
\text { Schulung } \\
\text { Motivation } \\
\text { Ablauflenkung } \\
\text { Dokumentation } \\
\text { Überwachung } \\
\text { Auditierung }\end{array}$ & $\begin{array}{l}\text { Stoffflussanalyse } \\
\text { Ökobilanzen } \\
\text { Kennzahlen } \\
\text { Reporting } \\
\text { Integration in das } \\
\text { Rechnungswesen }\end{array}$ & $\begin{array}{c}\text { Marktforschung } \\
\text { Konkurrenzanalyse } \\
\text { Produktanalyse } \\
\text { Werbung } \\
\text { Verkaufsförderung }\end{array}$ & $\begin{array}{c}\text { Kommunikation } \\
\text { Interaktion mit } \\
\text { Anspruchs- } \\
\text { gruppen } \\
\text { Offentlichkeits- } \\
\text { arbeit }\end{array}$ \\
\hline
\end{tabular}

Tabelle 5:

Typen von Umweltmanagementsystemen und ihre Ausprägungsformen

Quelle: In Anlehnung an Hamschmidt, J.; Dyllick, Th.: Nutzen Managementsysteme? Vom Umwelt- zum Sustainability-Managementsystem, IWÖ-Diskussionsbeitrag Nr. 82, St. Gallen 2000, S. 126.

636 Diese Angaben hat Herr KRÜGER von FORD im Interview bestätigt.

637 "In addition to improving environmental performance, instituting an environmental management system can provide economic benefits to companies through reduced operating and insurance costs, improved energy and resource conservation, reduced compliance and liability charges, improved access to capital, improved customer satisfaction, and improved community and public relations." OECD (Hrsg.): OECD Guidelines for Multinational Enterprises: Review 2000: Commentaries, Paris 2000, S. 9.

638 Vgl. Hamschimdt, J.; Dyllick, Th.: Nutzen Managementsysteme? Vom Umwelt- zum Sustainability-Managementsystem, IWÖ-Diskussionsbeitrag Nr. 82, St. Gallen 2000, S. 124 ff. sowie Dyllick, Th.; Hamschmidt, J.: Wirksamkeit und Leistung von Umweltmanagementsystemen: Eine Untersuchung von ISO 14.001 zertifizierten Unternehmen in der Schweitz, Zürich 2000. 
Während der Tpy „Infrastruktur" dem Schaffen von Systematik und Transparenz im Umweltmanagement sowie der Stärkung der Mitarbeitermotivation dient und in seiner grundlegenden Form lediglich die Systemanforderungen der ISONorm 14.001 verwirklicht, weisen die übrigen Typen spezifische strategische Ziele auf, die in unterschiedlichem Umfang dem Schaffen von Rechtssicherheit und der Risikovorsorge, dem Nachweis effektiver Prozessbeherrschung, als Instrumente effizienten Ressourcen- und Kostenmanagements, der ErschlieBung ökologischer Differenzierungs- und Wettbewerbspotenziale, der Imagebildung in der Öffentlichkeit oder der Einflussnahme auf Politik und Öffentlichkeit dienen. Da diese Typen von Umweltmanagementsystemen einen idealtypischen Charakter aufweisen, werden sie in der Unternehmenspraxis und damit auch in der Automobilindustrie zumeist kombiniert eingesetzt. ${ }^{639}$

\subsection{Ausgestaltungsformen der ökologiegerichteten Koordinations- konzeptionen}

Anhand der im Kapitel C 2 dargestellten Strategien im internationalen Umweltmanagement und den in Kapitel $\mathrm{C} 3$ untersuchten Koordinationsinstrumenten sollen nun strategiekonforme Koordinationsmuster für die Automobilindustrie abgeleitet werden. Diese schlagen sich in unterschiedlichen Ausgestaltungsformen von Umweltmanagementsystemen nieder. Da sich internationale Umweltmanagementsysteme zur Koordination des ökologiegerichteten Unternehmensverhaltens in der Automobilindustrie derzeit noch in der Umsetzungsphase befinden, ist an dieser Stelle eine konkrete Überprüfung der strategiekonformen Ausgestaltung der Koordinationskonzeptionen nur im Ansatz möglich. Die folgenden Überlegungen stellen deshalb Empfehlungen in Form von Tendenzaussagen bezüglich der Ausgestaltung jeweils geeigneter Umweltmanagementsysteme dar.

Während für eine global-proaktive, multinationale und selektive Verhaltensausrichtung in der Automobilindustrie ein internationales Umweltmanagementsystem zur Koordination erforderlich ist, erscheint der Aufbau eines unternehmensweiten Umweltmanagementsystems bei Verfolgung einer Kostenminimierungsstrategie im internationalen Umweltmanagement vor dem Hintergrund weitgehend fehlender internationaler Zielsetzungen im Umweltschutz nicht zwingend. Da sich Kostenminimierungsstrategien in der Automobil-

639 Vgl. Hamschmidt, J.; Dyllick, Th.: Nutzen Managementsysteme? Vom Umwelt- zum Sustainability-Managementsystem, a. a. O., S. 124. 
industrie heute nicht beobachten lassen, kann eine weitergehende Beschreibung an dieser Stelle unterbleiben.

\section{Koordinationskonzeption bei global-proaktiver Verhaltensausrichtung}

Zur Umsetzung der Ziele bei global-proaktiver Verhaltensausrichtung im internationalen Umweltmanagement der Automobilindustrie ist das Umweltmanagementsystem im Unternehmensverbund auf oberster Ebene zu installieren. Auf diese Weise wird gewährleistet, dass durch die Unternehmensführung eine unternehmensweit und international verbindliche Umweltschutzpolitik verabschiedet und die Grundlage für eine zielgerichtete Koordination im Umweltmanagement gelegt wird. Weiterhin wird an dieser Stelle die Verantwortung für den Umweltschutz in der internationalen Geschäftsleitung und in den Führungsetagen der Landesgesellschaften festgelegt. Das Umweltmanagementsystem auf Ebene der Unternehmenszentrale (bspw. auf Konzernebene) ist dann mit den Umweltmanagementsystemen auf lokaler Ebene zu verknüpfen. Dabei gewährleistet eine Verknüpfung des unternehmensübergreifenden Umweltmanagementsystems mit der lokalen Ebene eine Anpassung an spezifische, vor Ort geltende Rechtsprechung, ökologische Ansprüche und sonstige Kontextfaktoren. Hier sind allerdings nur Änderungen durchzuführen, die eine Einheitlichkeit der ökologischen Ausrichtung nicht durchbrechen. So kann bspw. bei VolksWAGEN durchaus ein Interesse des lokalen Managements an einer engeren Anbindung, mehr Erfahrungsaustausch und einer Verknüpfung der Umweltmanagementsysteme festgestellt werden. ${ }^{640}$

Aufgrund der bei global-proaktiver Umweltschutzausrichtung zumeist vorliegenden reziproken Leistungsverflechtungen zwischen den Auslandsgesellschaften untereinander und der externen Rückkopplungen erscheint die Bildung integrierter Strukturen im Umweltmanagment zur Überwindung möglicher Gegensätze zwischen inländischen und ausländischen organisatorischen Einheiten im produkt- wie fertigungsbezogenen Umweltschutz eine notwendige strukturelle Koordinationsmaßnahme. Um einer Überforderung der Führungskräfte auf oberster Ebene vorzubeugen und eine Professionalisierung im Umweltschutz zu ermöglichen, empfiehlt sich in diesem Kontext die Bildung eines Zentralbereichs für Umweltschutz, wie dies bspw. im VolKSWAGENKonzern der Fall ist. ${ }^{641}$

\footnotetext{
640 Die Ausführungen gehen auf ein Interview mit Herrn MOGG zurück.

641 Vgl. dazu das Beispiel am Ende von Kapitel C 3.2.
} 
In der Zentralabteilung findet eine Bündelung ökologischer Fach- und Entscheidungskompetenzen statt. Eine Aufgabe dieser Umweltschutzabteilung ist dabei die Harmonisierung des Auftritts nach außen, bspw. gegenüber überstaatlichen Institutionen wie der EU, internationalen Industrieverbänden wie dem WBCSD, oder international organisierten Umweltschutzorganisationen. Hier werden Imagebildungsziele und eine Profilierung gegenüber gesellschaftlichen Anspruchsgruppen zu erreichen versucht. Dies erfordert eine aktive Kommunikation mit den Anspruchsgruppen und bedarf der Bereitstellung adäquater Informationen für diese Gruppen. So sind die zentralen Umweltschutzabteilungen in der Automobilindustrie zumeist auch für die externe Umweltkommunikation mit ökologischen Anspruchsgruppen, Regierungsvertretern etc. verantwortlich. $^{642}$

Eine weitere Aufgabe der Zentralabteilung liegt in der internationalen Abstimmung der Gestaltung der internen Wertschöpfungsprozesse. ${ }^{643}$ In diesem Zusammenhang kommt der Begleitung und Unterstützung der Unternehmensführung bei der Aufstellung von operativen Umweltschutzzielen und Standards zur Konkretisierung der internationalen Umweltschutzpolitik und zur Erfüllung der strategischen Ziele ein hohes Gewicht zu. In Bezug auf die Einhaltung von unternehmensweit geltenden ökologischen Mindeststandards im produkt- und fertigungsbezogenen Umweltschutz ist die Ausstattung dieser Zentralabteilung mit formaler Weisungsbefugnis gegenüber bestimmten Stellen in den Auslandsgesellschaften anzuraten. Dies ist allerdings erst in Ansätzen im VOLKSWAGEN-Konzern umgesetzt worden. So hat der Leiter Umwelt, Verkehr und Arbeitsschutz des VOLKSWAGEN-Konzerns gegenüber den Umweltschutzbeauftragten in Deutschland ein fachliches Weisungsrecht und koordiniert den europäischen Erfahrungsaustausch. ${ }^{644}$

Um international einheitliche Umweltstandards und -vorgaben durchzusetzen, wäre ein ökologiegerichtetes Lead-Country-Konzept eine denkbare Möglichkeit. Dabei orientiert sich der gesamte Unternehmensverbund an den ökologischen Vorgaben einer Gesellschaft, die in einem ökologischen Vorreiterland angesiedelt ist. Dort werden das ökologiebezogene Know-how und Wissen

642 Herr KRŨGER bestätigt dies iim Interview.

643 Vgl. Brodel, D.: Internationales Umweltmanagement: Gestaltungsfelder - Determinanten Ausprägungen, a. a. O., S. 448.

644 Vgl. Volkswagen (Hrsg.): Sachkundige für Umweltschutz: Handbuch, a. a. o. Organisationsrichtlinie des Vorstandes Nr. 507. 
aufgebaut und international bereitgestellt. Die zentrale Umweltschutzabteilung ist in diesem Land angesiedelt und international für die Durchsetzung der Umweltschutzziele sowie die Koordination des ökologiegerichteten Verhaltens der Auslandsgesellschaften zuständig. Diese Form der hierarchischen Koordination kann in der Automobilindustrie beobachtet werden. Hersteller wie Fiat und Audi sowie Renault und PSA Peugeot Citroen geben bspw. an, die Umweltstandards des Heimatlandes weltweit vorzuschreiben. ${ }^{645}$

Eine alleinige hierarchische Koordination über Anweisung kann allerdings im Bereich des Umweltschutzes zu Problemen führen. Da sich nationale gesetzliche Vorgaben im Umweltschutz wechselseitig ausschließen können und andere lokale Gegebenheiten gegen eine vollkommene Übernahme der ökologischen Anweisungen aus dem Lead-Country in der Automobilindustrie sprechen, müsste für eine optimale Planung in der Zentralabteilung für Umweltschutz das Wissen über die lokalen Umweltgesetze und andere Umfeldfaktoren vorliegen. Dies kann insbesondere im Fall der größeren Automobilhersteller mit Tätigkeit in einer Vielzahl von Ländermärkten als wenig wahrscheinlich angesehen werden. Allein das operative Problem, die relevanten Informationen aus den Auslandsmärkten zu erhalten und die Vielzahl der Detailangaben zentral verarbeiten zu können, spricht gegen diese Lösung. Schließlich ist die negative Wirkung auf die Motivation der lokalen Führungskräfte ein wichtiger Grund, die einer allein hierarchischen Lösung entgegensteht. ${ }^{646}$

In Anbetracht der aufgezeigten Nachteile erscheint eine partizipative Form der Entscheidungsfindung in internationalen Kollegien oder Umweltausschüssen eher geeignet zu sein, den gewünschten organisatorischen Wandel durchzusetzen. So können auf hoher Ebene angesetzte Umweltausschüsse unter Einbeziehung der international betroffenen Fachabteilungen wie der Forschung \& Entwicklung, Beschaffung, Produktion etc. bei der Formulierung der operativen Ziele und Umweltstandards mitwirken. Diese Abstimmung könnte durch die zentrale Umweltschutzabteilung moderiert und geleitet werden. Die Tätigkeit in heterogenen Umfeldern macht eine Anwendung absolut gleicher Umweltschutzstandards häufig nicht oder nicht sofort möglich. In diesem Fall sind Mindeststandards, Bandbreiten und Übergangszeiträume einvernehmlich festzulegen.

645 Vgl. oekom research (Hrsg.): Corporate Responsibility Industry Report Automobile: A Corporate Responsibility Survey of 19 Companies of the Industry, a. a. 0.

646 Vgl. Brodel, D.: Internationales Umweltmanagement: Gestaltungsfelder - Determinanten Ausprägungen, a. a. O., S. 449. 
Diese Form der partizipativen Zielvereinbarung unter internationaler Beteiligung lässt bessere Resultate erwarten als eine hierarchische Form der Koordination. Dies gilt insbesondere auch deshalb, weil die Vertreter aus den einzelnen Standorten ihr Wissen über die lokalen ökologiebezogenen Rahmenbedingungen sowie ihr Know-how mit in die Entscheidungsfindung einbringen können. Es kann erwartet werden, dass die Motivation der Führungskräfte zur Umsetzung der gemeinsam aufgestellten ökologischen Ziele höher ist als bei hierarchischer Anweisung durch die Zentrale. Die Hersteller VOLKSWAGEN und BMW verfügen bspw. über eine derartige partizipative Form der Koordination. ${ }^{647}$

Die von den Umweltausschüssen erarbeiteten Vorgaben sollten schließlich von oberster Stelle ratifiziert und verkündet werden. ${ }^{648}$ Dies signalisiert die Bedeutung des Umweltschutzes für das Gesamtunternehmen und erleichtert die Durchsetzung auf allen Ebenen der Organisation. Diese Führung im Gegenstromverfahren ermöglicht eine Konkretisierung der Umweltpolitik auf der Grundlage von Zielvereinbarungen. Eventuelle Abweichungen von diesen Zielvereinbarungen können nach dem Prinzip der progressiven Planung (bottomup) eine entsprechende Anpassung der strategischen Rahmenplanung bewirken. Auf diese Weise lassen sich vor dem Hintergrund bestehender Informationsasymmetrien die Detailkenntnisse aus den Auslandsgesellschaften mit den Zielen des Unternehmensverbundes verbinden. ${ }^{649}$

Eine global-proaktive Verhaltensausrichtung im internationalen Umweltmanagement macht überdies eine Standardisierung von Planungs-, Informationsund Kontrollprozessen im Rahmen eines unternehmensübergreifenden ÖkoControlling erforderlich. ${ }^{650}$ Im Rahmen der Einrichtung einer zentralen Umwelt-

647 Vgl. dazu die Ausführungen in Kapitel C 3.2.

648 So argumentiert BARTLETT in seinen Ausführungen zur Steuerung multinationaler Konzerne: "It soon becomes evident that primary responsibility of top management is not to manage such activities directly, but to create and manage an organizational context that will provide the means for the appropriate resolution of decision and coordination of activities. "Bartlett, Ch. A.: Building and Managing the Transnational: The New Organizational Challenge, in: Porter, M. E. (Hrsg.): Competition in Global Industries, Boston 1986, S. 386.

649 Vgl. zur Führung im Gegenstromverfahren Hamprecht, M.: Controlling von Konzernplanungssystemen: theoretische Ableitung und betriebliche Realität führungsstrukturabhängiger Ausprägungsmuster, Wiesbaden 1996, S. 129 f.; Brockhoff, K.; Hauschildt, J.: Schnittstellen-Management - Koordination ohne Hierarchie, in: Zeitschrift für Organisation, Heft 6, 1993, S. $396-403$.

650 Vgl. zur Bewertung des Umweltmanagementsystems Clausen, J.: Monitoring von Umweltleistung und Umweltmanagementsystem - Eine Panelbefragung von Unternehmen: 1. Jahr 1997/1998, in: Diskussionspapier des IÖW 43, St. Gallen 1998. 
schutzabteilung kann das Öko-Controlling verankert werden. Weiterhin können erfahrene Mitarbeiter aus dem Zentralbereich Umweltschutz den Auslandsgesellschaften bei der Umsetzung der internationalen Umweltpolitik mit Dienstleistungen zur Seite stehen. Auf diese Weise können bspw. die Umweltaudits an den ausländischen Standorten durch ein zentrales Audit-Team durchgeführt werden. Ebenso können ökologiegerichtete Schulungen und Beratungsleistungen von der Zentralabteilung erbracht werden. Das Audit-Team im VoLKsWAGEN-Konzern kann hier als Beispiel dienen. Die Möglichkeit der Erzielung von Synergieeffekten wird so mit einer weiteren Professionalisierung im Umweltschutz verbunden.

Das prozessgerichtete Vorgehen im Umweltmanagement ist in Umweltmanagementhandbüchern zu kodifizieren. Nach der Festlegung von konkreten Maßnahmen im produkt- und fertigungsbezogenen Umweltschutz sind Routinetätigkeiten durch detailierte Verfahrensanweisungen zu programmieren. Dies gewährleistet das Herunterbrechen ökologischer Ziele durch Festlegung von operativen Aktivitätenreihenfolgen bis auf die unteren Ebenen der Organisation. Je ähnlicher die einzelnen Standorte mit Produktions- und Umweltschutztechnologie ausgestattet sind, desto eher lassen sich die beschriebenen Aktivitätenreihenfolgen auf diese Standorte übertragen.

Ergänzend kann eine zentrale Steuerung im fertigungsbezogenen Umweltschutz über die Vergabe von Umweltschutzbudgets durchgeführt werden. Dies ermöglicht eine vergleichsweise zeitnahe Anpassung der internationalen Umweltschutzstandards. Die Steuerung der Umweltschutzbudgets durch die zentrale Umweltschutzabteilung ähnlich der Praxis von FoRD wäre hier denkbar. Die Anwendung marktorientierter Anreizsysteme im Umweltschutz bedarf auch aufgrund bisher weitgehend fehlender Erfahrungen mit diesen Instrumenten und möglicherweise vorhandenen Widerständen eher einer längerfristigen Vorbereitung.

Zur Aktivierung der Führungskräfte sowie der Mitarbeiter der Auslandsgesellschaften für den Umweltschutz empfiehlt sich eine Ergänzung um „weiche“ kulturprägende Koordinationsinstrumente. In der Implementierungsphase erscheint dazu die Entsendung von erfahrenen Mitarbeitern der Zentralabteilung Umweltschutz in die Auslandsniederlassungen eine Option darzustellen. Zur Steigerung der lokalen Akzeptanz des internationalen Umweltschutzprogramms und der Motivation könnten aber u. U. Führungskräfte mit lokaler Nationalität einen höheren Beitrag leisten. Somit ist die Durchführung von Weiterbildungs- 
und Schulungsmaßnahmen durch die zentrale Umweltschutzabteilung sowie ein kontinuierlicher Dialog mit den Führungskräften der Auslandsgesellschaften über Chancen und Risiken im Umweltschutz sowie die ökologischen Ziele, Strategien und Maßnahmen und ihre Umsetzung ein wichtiger ergänzender Schritt zur Koordination, der bspw. im VoLKSWAGEN-Konzern durchgeführt wird. $^{651}$

Der Aufbau eines idealtypischen Umweltmanagementsystems für die unternehmensübergreifende Koordination des internationalen ökologiegerichteten Unternehmensverhaltens ist in der folgenden Abbildung dargestellt. Dieses Umweltmanagementsystem stellt in groben Zügen das Umweltmanagementsystem der VOLKSWAGEN AG dar.

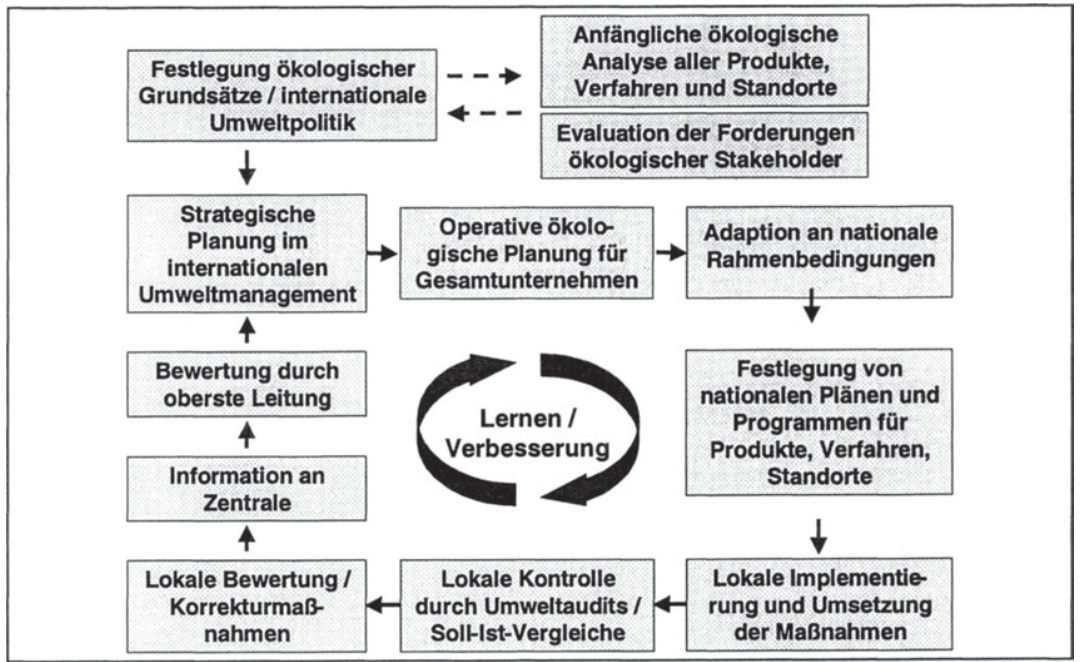

Abbildung 51: Internationales Umweltmanagementsystem bei global-proaktiver Basisstrategie

Quelle: Eigene Darstellung

Die zentral von der Unternehmensführung verabschiedete Umweltpolitik ist Ausgangspunkt für die Festlegung der internationalen Umweltschutzstrategie und der strategischen Umweltschutzziele. Diese zentralen Vorgaben werden in den international besetzten Umweltausschüssen (STEP, UMA) weiter operationalisiert. Dabei werden unterschiedliche lokale Rahmenbedingungen der Län-

651 Vgl. dazu die Ausführungen im Kapitel C 3.2. 
dermärkte bzw. Standorte im fertigungsbezogenen Umweltschutz durch die Vorgabe von Anpassungszeiträumen berücksichtigt. Schließlich werden die internationalen Umweltschutzformalziele in Form von Zielvorgaben oder Standards fixiert, die als Rahmen für die Festlegung der nationalen Umweltschutzpolitiken dienen. Auf lokaler Ebene werden darauf aufbauend Pläne und Programme für Produkte, Verfahren und den standortbezogenen Umweltschutz abgeleitet. Nach lokaler Implementierung und Umsetzung der Maßnahmen in den Auslandsgesellschaften wird in regelmäßigen Abständen die Wirksamkeit der ergriffenen Maßnahmen durch lokale Audits im Sinne von Soll-Ist-Vergleichen überprüft. Diese Audits werden großteils durch das Audit-Team begleitet. Die Audit-Ergebnisse können dann als Grundlage für die Ausgestaltung von lokalen Anpassungsmaßnahmen dienen. Darüber hinaus sind die lokalen Audit-Ergebnisse an die Zentrale weiterzuleiten. Im Sinne von Best Practice Studien ${ }^{652}$ können dabei auch die positiven Erfahrungen auf andere Landesgesellschaften übertragen werden. Weiterhin werden die Ergebnisse auf Ebene der Zentralabteilung für Umweltschutz als Grundlage für die externe Kommunikation, bspw. in Umweltberichten, genutzt und zur regelmäßigen Anpassung der unternehmensweiten Umweltschutzpolitik herangezogen.

Eine global-proaktive Umweltschutzstrategie wird den externen Ansprüchen an international tätige Automobilhersteller letzlich am ehesten gerecht. Hier werden die im Rahmen der Agenda 21 und anderen Verhaltenskodices geforderten und von der Automobilindustrie oft zugesagten Verhaltensweisen umgesetzt. Automobilhersteller, die eine global-proaktive Umweltschutzstrategie verfolgen, sichern ihre gesellschaftliche Legitimität und können darüber hinaus als ökologische Vorreiter auch von positiven Imageeffekten profitieren, wenn sie sich auch entsprechend extern ausrichten. Somit ist es Aufgabe eines unternehmensübergreifenden Umweltmanagementsystems, die organisatorische Effizienz sicherzustellen und eine ökologische Imagebildung und gesellschaftliche Profilierung zu ermöglichen.

652 Vgl. zum ökologischen Benchmarking Schneidewind, U.; Dyllick, Th.: Ökologisches Benchmarking, in: DBW, 57. Jg. (1997), Nr. 4, S. $569-572$. 


\section{Koordinationskonzeption bei multinationaler Verhaltensausrichtung}

Zur Umsetzung einer multinationalen Umweltschutzstrategie in der Automobilindustrie ist die Etablierung weitreichender unternehmensübergreifender Formalziele im Umweltschutz wenig geeignet. Vielmehr gilt es, auf lokaler bzw. regionaler Ebene Umweltmanagementsysteme an die spezifischen Rahmenbedingungen anzupassen.

In Anbetracht der zunehmenden regionalen Homogenisierung wird die multinationale Ausrichtung in der Automobilindustrie zunehmend von einer eher regional geprägten Ausrichtung im Umweltschutz abgelöst. Da innerhalb der Regionen eine hohe Umsystemähnlichkeit und eine starke Umsystemverbundenheit vorliegen, zwischen den unterschiedlichen Regionen aber nur eine geringe Ähnlichkeit und Abhängigkeit zu vermuten ist, wird den Regionen von multinational ausgerichteten Automobilherstellern, wie bspw. GENERAL MOTORS und FORD, eine hohe Autonomie bei der Umsetzung ökologischer Maßnahmen zuerkannt. Dabei erscheint es allerdings angebracht, auf Ebene der Region ein länderübergreifendes Umweltmanagementsystem zu etablieren. Demgemäß spielt eine ökologiebezogene Standardisierung von Produkten und Fertigungsprozessen zwischen den Regionen in der Automobilindustrie eine geringe Rolle, während innerhalb der Region ein hoher Standardisierungsbedarf ausgemacht werden kann.

Ähnlich verhält es sich auch in Bezug auf die Standardisierung von Managementprozessen. Die Informationssysteme in den einzelnen Regionen sind dabei nach Maßgabe der regionalen Informationsbedürfnisse der Führungskräfte sowie der Anspruchsgruppen standardisiert auszugestalten. In diesem Fall sind Unterstützungs- und Serviceleistungen nicht durch eine global zuständige Zentralabteilung im Umweltschutz bereitzustellen, sondern von regionenbezogenen Zentralabteilungen zu erbringen. Die Marke FORD ist auf diese Weise regionenspezifisch organisiert. Allein die Schaffung einer Plattform zum überregionalen Austausch von Best Practice Fällen im Umweltschutz fällt dabei in den Aufgabenbereich einer zentralen Umweltschutzstelle auf Konzernebene. ${ }^{653}$ Da der Etablierung einer unternehmensweit einheitlichen Unternehmenskultur im Rahmen einer multinationalen Umweltschutzstrategie in der Automobilindustrie kein besonderer Stellenwert beizumessen ist, werden regionenüberschreitende Besuchsaktivitäten der Führungskräfte sowie Schulungen im Umweltschutz weitgehend ausbleiben.

653 Die Angaben gehen auf Herrn KRÜGER von FORD zurück. 


\section{Koordinationskonzeption bei selektiver Verhaltensausrichtung}

Zur Erreichung der Ziele einer selektiven Ausrichtung im internationlen Umweltschutz der Automobilindustrie erscheint die Etablierung unternehmensweiter Umweltschutzziele lediglich für bestimmte Produkte bzw. Produktlinien angebracht. So ist im produktbezogenen Umweltschutz durch eine länderübergreifende Standardisierung und Zentralisierung die Realisierung von Größeneffekten anzustreben. Die Erfahrungen in der Entwicklung ökologisch relativ vorteilhafter Produkte können dabei durch Zentralisierung der Forschungs- und Entwicklungstätigkeiten in ökologiebezogenen Lead-Countries gewonnen werden. Hier sind ökologiegerichtete Erfahrungseffekte zu generieren, die bei Bedarf auf andere Ländermärkte zu übertragen sind. So betreiben Automobilhersteller wie FORD, DaIMLERChrysler und VolKSWAGEN in Kalifornien gemeinsam Forschungsanstrengungen zur Entwicklung von Brennstoffzellen im Rahmen der Fuel-Cell-Partnership.

Durch die Wahl von Standorten in Ländern mit vergleichsweise geringen Umweltschutzvorschriften können Umweltschutzkosten bei der Fertigung umgangen werden. Durch Export der kostengünstig produzierten Automobile in Ländermärkte mit höheren fertigungsbezogenen Umweltschutzvorgaben werden diese Kostenvorteile international genutzt. Erfüllen bestimmte Produkte die im Zeitablauf gestiegenen ökologischen Anforderungen in den Ländermärkten der Triade nicht mehr, könnten sie bspw. weiterhin in Schwellenländern abgesetzt werden. Dieses Vorgehen bedarf integrierter Strukturen und einer Entscheidungszentralisation auf hoher Ebene. Das Mittel der hierarchischen Weisung ist zur Durchsetzung selektiver Orientierungen im produktbezogenen Umweltschutz als kostengünstiges und effektives Instument zu wählen. In der Automobilindustrie kann eine starke Zentralisierung produktpolitischer, ökologiegerichteter Entscheidungen bei gleichzeitig hoher Dezentralisierung der Entscheidungen im fertigungsbezogenen Umweltschutz beobachtet werden. ${ }^{654}$ Zur Erreichung der Informationsziele ist eine Zentralisierung der Informationsverarbeitung und Entscheidungsfindung im produktbezogenen Umweltschutz notwendig, während für den fertigungsbezogenen Umweltschutz lediglich dezentrale Informationssysteme aufzubauen sind. So liegen in der Zentralabteilung für Umweltschutz in der VolKSWAGEN AG keine Informationen über die umweltrechtlichen Anforderungen in den unterschiedlichen Ländermärkten vor, diese werden nur dezentral in den Auslandsmärkten vorgehalten.

654 Vgl. dazu die Experteneinschătzung am Ende des B-Kapitels. 
Die Gefahr einer selektiven Ausrichtung im Umweltschutz zeigt sich in der Wirkung auf das ökologiebezogene Unternehmensimage. So ist es bspw. der FORD MOTOR COMPANY trotz hoher kommunikationspolitischer Aufwendungen und umfangreicher Forschungs- und Entwicklungstätigkeiten in Bezug auf die Entwicklung der Brennstoffzelle noch nicht gelungen, ihr wenig ökologisches Image abzulegen. Dies liegt mitunter auch daran, dass der Anteil der verkauften verbrauchsstarken und emissionsreichen Automobile (SUV) in den USA besonders groß ist. ${ }^{655}$

Zur Verringerung externer Rückkopplungen könnte die Wahl unterschiedlicher Marken für ökologisch stark differenzierte Produkte bzW. eine umfangreiche Verlagerung von ökologisch bedenklichen Wertschöpfungsaktivitäten auf Zulieferer als koordinationsbedarfsreduzierende Maßnahme angewendet werden. Dennoch wird ein duales Vorgehen im Umweltschutz auch schwer gegenüber der eigenen Belegschaft zu vermitteln sein.

655 Vgl. dazu auch den Auszug aus der Homepage von CorpWatch im B-Kapitel. 


\section{Implikationen und Ausblick}

Trotz der spezifischen Bedingungskonstellation der Untersuchung und der vor dem Hintergrund des situativen Kontextes der Automobilindustrie gewonnenen Aussagen lassen sich die Ergebnisse der Arbeit auch auf andere Branchen übertragen, sodass sie als weitgehend verallgemeinerungsfähig gekennzeichnet werden können. Im Folgenden gilt es, für die einzelnen Basisstrategien im internationalen Umweltmanagement allgemein gültige, idealtypische Koordinationskonzeptionen abzuleiten. In diesem Kontext sind die Ergebnisse der Untersuchung sowohl für die Unternehmenspraxis als auch für die weitere Forschung auf der Schnittstelle zwischen internationalem Management und Umweltmanagement relevant.

\section{Implikationen für die Praxis}

Als zentrale Hinweise zur Gestaltung der Koordination des ökologiegerichteten Unternehmensverhaltens bei internationaler Geschäftstätigkeit empfiehlt sich ein Gestaltungsprozess, an dessen Ausgangspunkt die Bestimmung des Koordinationsbedarfs vor dem Hintergrund des spezifischen situativen Kontextes steht. Dabei ist neben dem direkten Einfluss der lokalen umweltrechtlichen Bestimmungen und der ökologischen Zahlungsbereitschaft der Konsumenten in den einzelnen Ländermärkten auch der Einfluss international organisierter Anspruchsgruppen zu berücksichtigen. Dazu zählen neben institutionellen und marktlichen Gruppen auch und insbesondere international tätige Umweltschutzorganisationen und Finanzdienstleister. Eine Sensibilisierung der Unternehmensführung gegenüber den unterschiedlichen Stakeholderinteressen kann dabei als Grundvoraussetzung für die Zukunftsfähigkeit der Unternehmung gewertet werden. Die zunehmende Beschäftigung amerikanischer Unternehmen und Wissenschaftler mit dem Thema "Corporate Citizenship" bestätigt diesen Trend.

Neben einer umfassenden Berücksichtigung exogener Faktoren hat die Untersuchung ergeben, dass auch endogene Kontextfaktoren einen erheblichen Einfluss auf das Entstehen eines ökologiegerichteten Koordinationsbedarfs haben. Eine fehlende unternehmensweite Auseinandersetzung mit ökologischen Zielen und Strategien sowie international unterschiedlichen Einstellungen dem betrieblichen Umweltschutz gegenüber kann zu einem weitgehend unko- 
ordinierten, ökologischen Verhalten der einzelnen Auslandsgesellschaften und möglicherweise zu prohibitiv hohen Autonomiekosten führen.

Die Darstellung der allgemeinen Koordinationsziele kann als Anregung zur unternehmensbezogenen Festlegung der Koordinationsziele gelten und soll die unternehmensinterne Diskussion über potenzielle Zielkonflikte zwischen ökonomischen und ökologischen Zielen anregen. Die Darstellung der basisstrategischen Optionen im internationalen Umweltmanagement und die grobe Beschreibung des situativen Kontextes, in denen diese Strategien idealtypischerweise zum Einsatz kommen, kann als Hilfestellung bei der Wahl einer strategischen Basisorientierung dienen.

Die ausführliche Diskussion der unterschiedlichen Koordinationsinstrumente sowie die praktischen Umsetzungsbeispiele können in Verbindung mit der Beschreibung der Koordinationsziele der unterschiedlichen Basisstrategien als Leitfaden zur Festlegung einer unternehmensindividuellen Koordinationskonzeption genutzt werden. In der folgenden Abbildung sind die Koordinationsziele unterschiedlicher Basisstrategien im internationalen Umweltmanagement sowie zu empfehlende Ausgestaltungsformen strategiekonformer Koordinationskonzeptionen bzw. Umweltmanagementsysteme in einer Übersicht zusammengefasst.

Eine global-proaktive Ausrichtung im internationalen Umweltmanagement zielt auf die Umsetzung von Größen-, Synergie- und Erfahrungseffekten, die durch eine weitgehende Standardisierung der ökologiebezogenen produkt- und fertigungsbezogenen Tätigkeiten erreicht werden sollen. Dabei soll aufgrund der hohen Ökologieorientierung eine Differenzierung gegenüber dem Wettbewerb und eine ökologische Profilierung gegenüber den wichtigsten Stakeholdern erreicht werden. Ziel der Koordination ist es demzufolge, ein international einheitliches ökologiegerichtetes Verhalten der Gesellschaften im In- und Ausland sicherzustellen. Weiterhin ist durch eine geeignete Standortwahl in ökologischen Vorreiterländern und entsprechenden Forschungs- und Entwicklungsvorhaben ökologiespezifisches Wissen aufzubauen, welches durch eine enge internationale Verknüpfung an allen Standorten nutzbar zu machen ist. Dies bedingt den Aufbau eines einheitlichen betrieblichen Umweltinformationssystems zur Antizipation ökologischer Anforderungen und zur zentralen Bereitstellung von Informationen zur externen Kommunikation mit den Stakeholdern. 


\begin{tabular}{|c|c|c|c|c|c|}
\hline 흘 & $\begin{array}{l}\text { Strategische Ausrichtung } \\
\text { im internationalen } \\
\text { Umweltmanagement }\end{array}$ & $\begin{array}{l}\text { Global- } \\
\text { proakiv }\end{array}$ & $\begin{array}{l}\text { Kostenmini- } \\
\text { mierung }\end{array}$ & $\begin{array}{c}\text { Multi- } \\
\text { national }\end{array}$ & Selektiv \\
\hline ত্র & Effizienzziele & $\begin{array}{l}\text { : Grōßeneffekte } \\
\text { : Synergieeffekte } \\
\text { Erftahrungseffekte }\end{array}$ & $\begin{array}{l}\text { : Größeneffekte } \\
\text { Arbitrage-Effekte } \\
\text { Entwicklungsstand- } \\
\text { Portfolio-Effekte }\end{array}$ & $\begin{array}{l}\text { - Lokale/regionale } \\
\text { Effizizienzziele }\end{array}$ & $\begin{array}{l}\text { : Größeneffekte } \\
\text { Synergieeffekte } \\
\text { : Errahrungseffekte } \\
\text { A Arbitrage Effekte } \\
\text { - Entwicklungsstand- } \\
\text { Porttolio-Effekte }\end{array}$ \\
\hline 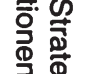 & Wirkungsziele & $\begin{array}{l}\text { : Differenzierung } \\
\text { Profilierung }\end{array}$ & $\begin{array}{l}\text { : Lokale Absicherung } \\
\text { Kosteneffizienz }\end{array}$ & $\begin{array}{l}\text { - Lokal/regional } \\
\text { - differenziert } \\
\text { - Absicherung }\end{array}$ & $\begin{array}{l}\text { Produktdifferenzierung } \\
\text { : Produktprofilicung } \\
\text { Prokale Absicherung }\end{array}$ \\
\hline 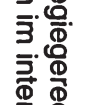 & Koordinationsziele & $\begin{array}{l}\text { - Einheitliches ōkologie- } \\
\text { - Gerichtetes Verhalten } \\
\text { Wissensuafbau und } \\
\text { Verknüpfung }\end{array}$ & $\begin{array}{l}\text { - Kostenminimale } \\
\text { Produktion }\end{array}$ & $\begin{array}{l}\text { - Lokale/regionale } \\
\text { Handlungsflexibilität }\end{array}$ & $\begin{array}{l}\text { - Wissensaufbau im } \\
\text { produktbezogenenen } \\
\text { - Kosteltschutz } \\
\text { Kostentinimale } \\
\text { Produktion }\end{array}$ \\
\hline 胥 올 & Informationsziele & $\begin{array}{l}\text { - Einheitliches Informations- } \\
\text { system zur Antizipation I } \\
\text { extermen Kommunikation }\end{array}$ & $\begin{array}{l}\text { - Lokale } \\
\text { Frühwarnung }\end{array}$ & $\begin{array}{l}\text { - Lokal/regional } \\
\text { angepasst }\end{array}$ & $\begin{array}{l}\text { - Einheitliches Informations- } \\
\text { system für productutbezoge- } \\
\text { nen Umweltschutz }\end{array}$ \\
\hline 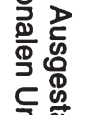 & $\begin{array}{l}\text { Umweltmanage- } \\
\text { mentsystem }\end{array}$ & $\begin{array}{l}\text { - Unternehmens- } \\
\text { übergreiffend integriert }\end{array}$ & - Lokal differenziert & $\begin{array}{l}\text { - Lokalregional } \\
\text { differenziert }\end{array}$ & $\begin{array}{l}\text { - Einheitlich integriert für } \\
\text { den productbbezogenen } \\
\text { Umweltschutzz } \\
\text { - Lokal differenziert für } \\
\text { den fertigungsbezoge- } \\
\text { nen Umweltschutz }\end{array}$ \\
\hline 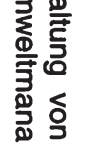 & $\begin{array}{l}\text { Strukturelle } \\
\text { Koordinationsmaßnahmen }\end{array}$ & $\begin{array}{l}\text { - Entscheidungszen- } \\
\text { tralisation } \\
\text { :Zuntralabteilung } \\
\text { : Umweltausschusse } \\
\text { - Umweltprojektgruppen }\end{array}$ & $\begin{array}{l}\text { - Entscheidungs- } \\
\text { dezentralisation }\end{array}$ & 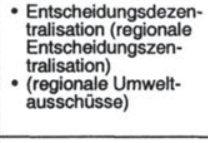 & 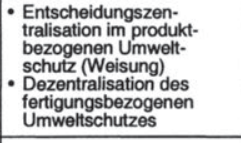 \\
\hline 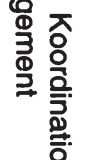 & $\begin{array}{l}\text { Technokratische } \\
\text { Koordinationsmaßnahmen }\end{array}$ & 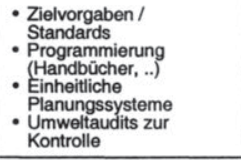 & $\begin{array}{l}\text { - Steuerung über } \\
\text { Marktpreise }\end{array}$ & $\begin{array}{l}\text { - Lokalregional } \\
\text { differenziert }\end{array}$ & $\begin{array}{l}\text { :Zielvorgaben } \\
\text { :Standards } \\
\text { Untassende } \\
\text { Programmierung } \\
\text { (Handbücher, ..) }\end{array}$ \\
\hline 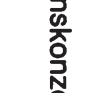 & $\begin{array}{l}\text { Personelle } \\
\text { Koordinationsmaßnahmen }\end{array}$ & $\begin{array}{l}\text { - Internationales Job } \\
\text { Aotation } \\
\text { : Besuche } \\
\text { Unternehmenskultur }\end{array}$ & & $\begin{array}{l}\text { - Lokalregional } \\
\text { differenziert }\end{array}$ & \\
\hline
\end{tabular}


Zur Umsetzung einer global-proaktiven Umweltschutzorientierung ist ein unternehmensübergreifendes Umweltmanagementsystem aufzubauen. Dies bedingt eine Entscheidungszentralisation auf hoher Ebene. Eine Abstimmung des internationalen ökologiegerichteten Verhaltens allein durch hierarchische Weisungen beeinträchtigt allerdings in aller Regel die lokale Akzeptanz ökologischer Ziele und Maßnahmen und führt zu Reaktanzen, welche die Koordinationseffizienz einschränken können. Die Motivation der lokalen Führungskräfte für den Umweltschutz ist entscheidend für die lokale Umsetzung des ökologischen Unternehmensverhaltens. Eine partizipative Erarbeitung der ökologischen Grundsätze und der strategischen Ziele im Umweltschutz durch einen paritätisch mit Führungskräften aus den Auslandsgesellschaften besetzten Umweltausschuss bzw. der Einsatz von Umweltprojektgruppen führt dann zwar zu einem höheren Koordinationsbedarf bei der Zielfestlegung, senkt aber im Folgenden Durchsetzungsbarrieren und hält die Motivation der lokalen Führungskräfte aufrecht.

Der Aufbau eines Zentralbereichs für Umweltschutz in einem ökologischen Vorreiterland bietet dabei die Möglichkeit der Professionalisierung des Umweltschutzes, der Aneignung von Wissen und der Sammlung von Erfahrungen mit restriktiven Umweltschutzvorgaben. Das Wissen und die Erfahrungen können kodifiziert und zentral gespeichert werden, um als Best Practice Beispiele der gesamten Organisation zu dienen. Weiterhin kann die zentrale Umweltschutzabteilung als Dienstleistungsabteilung die Aufgabe eines internationalen Agenten des organisatorischen Wandels übernehmen und Schulungsieistungen etc. durchführen.

Mittels Festlegung operationaler Zielvorgaben, bspw. in Form von internationalen Mindeststandards im Umweltschutz, kann eine international einheitliche Ausrichtung der Auslandsstandorte erreicht werden. Zur Festlegung standardisierter Aktivitätenreihenfolgen und der Festlegung vorgegebener Berichtswege eignen sich strukturell und inhaltlich ähnlich aufgebaute Umweltmanagementhandbücher. Eine weitgehende Standardisierung der Kommunikations- und Informationssysteme stellt eine grundlegende Voraussetzung dar, um Informationen sowohl für die interne Steuerung und Entscheidungsvorbereitung als auch für die externe Kommunikation bereitstellen zu können. Ein erster Schritt dazu ist in der Festlegung international einheitlicher Definitionen für Begriffe wie Abfall, Abwasser, Emissionen etc. zu sehen. Aufbauend darauf sind die Meßmethoden und die Meßzyklen festzulegen, um die Voraussetzungen für ein funktionierendes, internationales Öko-Controlling zu ermöglichen. Dazu ist 
es notwendig, sich international auf einheitliche Kennzahlen festzulegen, die im Folgenden Grundlage der Steuerung und Kontrolle sind. Mittels regelmäßig durchgeführter Umweltaudits ist die Zielerreichung an allen Auslandsstandorten schließlich einer Überprüfung zu unterziehen.

Eine besondere Bedeutung sollte "weichen" Koordinationsmaßnahmen zukommen, um Widerstände gegen ökologische Ziele abzubauen. Die Ausprägung einer einheitlichen Unternehmenskultur in Bezug auf den Umweltschutz kann dabei durch Schulungen und Besuche, aber auch durch ein internationales Job Rotation für Führungskräfte gefördert werden. So bietet es sich an, die Anreizsysteme der Führungskräfte zumindest z. T. an die Erfüllung der ökologischen Ziele zu koppeln. Zur Förderung des ökologischen Verhaltens der Belegschaft sind insbesondere die kulturellen Eigenheiten des Gastlandes und der Stand des Wissens um die ökologischen Zusammenhänge besonders zu berücksichtigen.

Im Rahmen der KostenminImierungsstrategie sollen Größen-, Arbitrageund Entwicklungsstand-Portfolio-Effekte erzielt werden. Dabei ist lediglich sicherzustellen, dass am jeweiligen Produktionsstandort die gesetzlich relevanten Umweltschutzbestimmungen eingehalten werden. Da Kostenführer mit hohem Standardisierungsgrad der Produkte bei Minimierung des Einsatzes von Roh- und Betriebsstoffen auch Ansätze einer ökologischen Effizienz erkennen lassen $^{656}$, ist mittels lokaler Umweltmanagementsysteme auch die Verbesserung der ökologischen Kosteneffizienz durch Aufspüren und Ausnützen von Kostensenkungspotenzialen zu verfolgen. ${ }^{657}$ Das Ziel der Koordination bei Kostenminimierungsstrategie ist es, die kostenminimale Herstellung der Produkte sicherzustellen. Dazu ist es erforderlich über bevorstehende Anpassungen im Umweltschutz durch ein Frühwarnsystem informiert zu werden, um ggf. eine Verlagerung der Produktion an einen anderen Standort frühzeitig veranlassen zu können.

Bei Verfolgung dieser Strategie ist der Aufbau einer unternehmensübergreifenden Koordinationskonzeption im Rahmen eines internationalen Umweltmanagements nicht efforderlich. Dies gilt insbesondere auch deshalb, weil eine aktive Steuerung der Wahrnehmung von Externalisierungschancen aufgrund des

656 Vgl. Meffert, H.; KIrcheorg, M.: Das neue Leitbild Sustainable Development - Der Weg ist das Ziel, in: Harvard Business Manager, 15. Jg. (1993), Nr. 2, S. 34 - 45.

Vgl. Hamschmidt, J.; Dyllick, Th.: Nutzen Managementsysteme? Vom Umwelt- zum Sustainability-Managementsystem, a. a. O., S. 127. 
vorhandenen Marktversagens bei externen Effekten überflüssig erscheint. Die Einführung von Verrechnungspreisen für Ökologiekosten könnte dazu beitragen, dass Standortentscheidungen aufgrund der lokal höheren Umweltschutzkosten, bspw. aufgrund von höheren Abwasser- und Abfallgebühren oder der Vorschrift, Vermeidungstechnologien zu installieren, quasi automatisch in ökologisch weniger restriktive Länder gelenkt werden. ${ }^{658}$ Vor diesem Hintergrund erscheint die Installation einfacher Umweltmanagementsysteme allein auf lokaler Ebene sinnvoll. Diese dienen dann primär der Überwachung der lokalen Gesetzeserfüllung bzw. zur Risikovorsorge. Dies bedingt eine weitgehende Entscheidungsdezentralisation im Umweltschutz.

Da im Rahmen der Kostenminimierungsstrategie gezielt eine internationale Externalisierung zur Ausnutzung ökonomischer Vorteile angestrebt wird, können entsprechende Unternehmen der Kritik verschiedenster Anspruchsgruppen und dabei insbesondere dem Vorwurf des Ökodumping ausgesetzt sein. Eine „Verschleierung" ökologisch kritischer Produktionen wäre in diesem Fall eine Möglichkeit zur Verringerung der externen Rückkopplungen. Hersteller können ökologisch bedenkliche Fertigungsprozesse auf die Zulieferindustrie auslagern oder durch einen international uneinheitlichen Auftritt ihr ökologisch fragwürdiges Verhalten zu verbergen versuchen. Eine derartige Verhaltensausrichtung widerspricht allerdings allgemein akzeptierten ethisch-moralischen Vorstellungen, wie sie z.B. in internationalen Verhaltenskodices festgehalten werden. ${ }^{659}$ Somit gefährdet ein derartiges Unternehmensverhalten langfristig die gesellschaftliche Legitimität und führt bei späterem Einlenken zu hohen Umstellungskosten.

Bei Wahl einer multinationalen (regionalen) Ausrichtung im internationalen Umweltmanagement sind die Ziele im Umweltschutz auf lokaler Ebene (bzw. regionenbezogen) an die spezifischen Rahmenbedingungen anzupassen. Durch Differenzierung sollen die lokalen (regionalen) ökologischen Anforderungen besser erfüllt werden. Das Ziel der Koordination ist dabei in einer weitreichenden Erhaltung der Handlungsflexibilität der lokalen (regionalen) Führungskräfte in Bezug auf ökologiegerichtete Entscheidungen zu sehen. Die Informationssysteme sind an den einzelnen Standorten nach Maßgabe der lokalen (regionalen) Informationsbedürfnisse der Führungskräfte sowie der Anspruchsgruppen auszugestalten.

658 Vgl. Brodel, D.: Internationales Umweltmanagement: Gestaltungsfelder - Determinanten Ausprägungen, a. a. O., S. 441.

659

Vgl. dazu die Ausführungen in Kapitel B 3.2.2. 
Das Koordinationssystem ist bei multinationaler Umweltschutzausrichtung auf lokaler (regionaler) Ebene zu etablieren, da operationale (regionen-)übergreifende Zielsetzungen im Umweltmanagement weitgehend fehlen. Dies bedingt eine Dezentralisation der Entscheidungen im Umweltschutz und eignet sich bei stark heterogenen lokalen (bzw. interregionalen) Kontextfaktoren, geringen internen (interregionalen) Leistungsverflechtungen und einer geringen Umsystemverbundenheit (zwischen den Regionen). Demgemäß spielt eine ökologiebezogene Standardisierung von Produkten und Fertigungsprozessen (überregional) eine ebenso geringe Rolle wie die Standardisierung von Managementprozessen. Der Einsatz technokratischer Koordinationsinstrumente erfolgt ebenso wie der Einsatz personeller Koordinationsmaßnahmen lokal (regional) differenziert.

Durch eine externe Auditierung des Umweltmanagements der lokalen Auslandsgesellschaften kann nach außen das Vorhandensein ökologischer Ziele und Programme sowie Rechtssicherheit dokumentiert werden. Sollten im Rahmen einer multinationalen Umweltschutzausrichtung externe Rückkopplungen auftreten, könnte sich ein Unternehmen allerdings der Kritik international organisierter Anspruchsgruppen ausgesetzt sehen. Dies erscheint besonders dann wahrscheinlich, wenn sich das unternehmensbezogene Umweltschutzniveau in unterschiedlichen Ländern (Regionen) stark voneinander unterscheidet. Bei Verfolgung einer Mehrmarkenstrategie werden diese externen Rückkopplungen allerdings weniger wahrscheinlich auftreten. Zur Verringerung dieser Rückkopplungen könnte das ökologische Niveau der einzelnen Unternehmen und ihrer Produkte aneinander angeglichen werden. Dazu können lokale (regionale) Führungskräfte gemeinsam in einem Umweltausschuss Mindestvorgaben im Umweltschutz erarbeiten. Die Umsetzung der Maßnahmen und insbesondere die Wahl der Mittel kann dann den einzelnen Standorten überlassen bleiben. Ein global zentrales Controlling erscheint in diesem Zusammenhang ebenfalls nicht notwendig, sodass allein auf lokaler (regionaler) Ebene entsprechende Maßnahmen zu ergreifen sind.

Im Rahmen einer international selektiven Umweltschutzausrichtung gilt es, sowohl Kosten- als auch Differenzierungsziele im Umweltschutz zu erreichen. Eine Profilierung mit ökologischen Produktangeboten und Differenzierung vom Wettbewerb erfolgt dabei in den Ländermärkten, in denen die Konsumenten der Ökologie einen hohen Stellenwert bei ihrer Kaufentscheidung beimessen. In Ländermärkten, in denen Umweltschutz nicht zur Profilierung eingesetzt werden kann, wird inm auch eine geringere Bedeutung zuerkannt. 
Da der Umweltschutz in der Fertigung für den Konsumenten oft nur eine untergeordnete Rolle spielt, ist hier eine kostenminimale Anpassung - unter bewusster Ausnutzung der Möglichkeit einer ökologischen Externalisierung Ziel der selektiven Ausrichtung im Umweltschutz. Ziel der Koordination bei dieser strategischen Orientierung ist es, eine kostenminimale Herstellung der Produkte sicherzustellen und gleichzeitig den Wissens- und Kompetenzaufbau im produktbezogenen Umweltschutz zu ermöglichen und dieses Wissen international verfügbar zu machen. Dies impliziert den Aufbau eines internationalen Informationssystems im produktbezogenen Umweltschutz.

Die Koordinationskonzeption bei Verfolgung einer selektiven Umweltschutzstrategie im Umweltschutz ist für den produktbezogenen und den fertigungsbezogenen Umweltschutz unterschiedlich auszugestalten. Während im produktbezogenen Umweltschutz ein weitgehend einheitliches Umweltmanagementsystem auf zentraler Ebene einzurichten ist, können im fertigungsbezogenen Umweltschutz Umweltmanagementsysteme auf lokaler Ebene etabliert werden. In Bezug auf die strukturellen Koordinationsmaßnahmen ist im produktbezogenen Umweltschutz eine Entscheidungszentralisation anzuraten, im fertigungsbezogenen Umweltschutz können die ökologiebezogenen Entscheidungen dagegen dezentral getroffen werden. Als technokratische Koordinationsinstrumente im produktbezogenen Umweltschutz eignen sich Zielvorgaben, Standards sowie eine weitreichende Programmierung von Prozessen. Bei selektiver Ausrichtung im Umweltschutz erscheint der Aufbau einer einheitlichen und starken ökologischen Unternehmenskultur kaum möglich, so dass personalen Koordinationsmaßnahmen eine äußerst geringe Bedeutung beizumesen ist.

Eine selektive Umweltschutzstrategie zeichnet sich aus ökologischer Sicht durch ein geringes $M a ß$ an Konsistenz aus und könnte sich insbesondere vor dem Hintergrund der Tätigkeiten internationaler ökologischer Anspruchsgruppen langfristig als störanfällig erweisen. Der Aufbau eines ökologischen Images in einigen Ländermärkten oder in Bezug auf einzelne Produkte könnte sich dann nicht nur als „sunk cost", sondern auch als kontraproduktiv erweisen, da umweltschutzbezogene Glaubwürdigkeitsverluste möglicherweise auch auf andere Beurteilungsdimensionen abfärben. ${ }^{660}$

660 Vgl. Brodel, D.: Internationales Umweltmanagement: Gestaltungsfelder - Determinanten Ausprägungen, a. a. O., S. 412. 
Abschließend ist anzumerken, dass allein eine global-proaktive Umweltschutzausrichtung langfristig einen "Fit“ zwischen gesellschaftlichen, institutionellen und marktlichen Ansprüchen einerseits und der Unternehmung andererseits gewährleistet und dem Leitbild eines Sustainable Development gerecht wird.

\section{Implikationen für die weitere Forschung}

Die Ergebnisse der Arbeit liefern nicht allein Hinweise für die Unternehmenspraxis, sondern auch Anregungen für die weitere Forschung im Bereich des internationalen Umweltmanagements. Eine Bestätigung der getroffenen Tendenzaussagen und Thesen sowie die Beantwortung weiterführender Fragestellungen müssen zukünftigen Forschungsvorhaben vorbehalten bleiben. In Anbetracht der Aktualität der Herausforderungen im internationalen Umweltmanagement zeichnen sich verschiedene Ansatzpunkte für vertiefende Forschungsarbeiten ab.

1. Die Ergebnisse der vorliegenden Untersuchung sind im Rahmen von empirischen Studien auch in anderen Branchen zu validieren.

- So ist der Einfluss des situativen Kontextes auf das Entstehen eines internationalen ökologiebezogenen Koordinationsbedarfs empirisch zu validieren. Vor dem Hintergrund der Vielzahl der möglichen endogenen und exogenen Einflussfaktoren könnte eine faktorenanalytische Verdichtung dieser detailanalytisch erfassten Kontextfaktoren zu intervenierenden Variablen einen sinnvollen ersten Schritt darstellen. In diesem Kontext sind die unterschiedlichen Einflüsse sowie die Wechselwirkungen zwischen verschiedenen Einflussfaktoren darzustellen und in ihrer Wirkung auf den internationalen Koordinationsbedarf kausalanalytisch zu bewerten.

- Weiterhin sind die Ziele im internationalen Umweltmanagement sowie die daraus folgenden Koordinationsziele empirisch zu überprüfen. In diesem Zusammenhang sind auch die Annahmen zur Hierarchie der Ziele im Zielsystem zu validieren. Neben der Erfassung weiter zu differenzierender ökonomischer, psychographischer und ökologischer Ziele sind in diesem Kontext auch interne ökologiegerichtete Entwicklungs- und Lernziele aufzunehmen. Aus der Gewichtung der einzelnen Ziele lassen sich Rückschlüsse auf die basisstrategische Ausrichtung im internationalen Umweltmanagement ziehen. 
- In einem weiteren Schritt ist die Ausgestaltung der Koordinationskonzeption zu messen. Dazu ist die Anwendung der unterschiedlichen koordinationsbedarfsreduzierenden und -deckenden Maßnahmen zu erheben. Auch sind die gewählten Koordinationskonzeptionen einer Effizienzbewertung zu unterziehen, wobei die Effizienzkriterien zuvor strategiekonform zu gewichten sind.

- Abschließend gilt es, optimale, strategiekonforme Koordinationskonzeptionen abzuleiten. Dabei ist insbesondere zu überprüfen, inwieweit die strukturellen, technokratischen und personellen Maßnahmen zur effizienten Koordination beitragen und welche Einflussfaktoren darüber hinaus die Effizienz der Koordinationskonzeptionen beeinflussen.

Aufgrund unterschiedlicher ökologiebezogener situativer Bedingungsrahmen verschiedener Branchen erscheint es wenig ratsam, die Validierung der Untersuchungsergebnisse zur Koordination des internationalen ökologiegerichteten Unternehmensverhaltens im Rahmen einer einzigen empirischen Studie vorzunehmen. Vielmehr sollte sich die empirische Überprüfung auf jeweils eine Branche beschränken.

2. Ein weiterer Forschungsbereich resultiert aus der Notwendigkeit, die von BRODEL theoriegeleitet aufgestellten Basisstrategien im internationalen Umweltmanagement empirisch zu validieren. Dazu sind die unterschiedlichen basisstrategischen Ausrichtungen im internationalen Umweltmanagement durch geeignete Messindikatoren zu operationalisieren. Die Messindikatoren können unterschiedliche ökologiegerichtete Verhaltensweisen bezeichnen, die anhand einer faktoranalytischen Verdichtung zu internationalen ökologiegerichteten Grundhaltungen zusammengefasst werden können. Dabei kann grundlegend auf das Vorgehen von KIRCHGEORG zur Analyse der strategischen Ausrichtung des ökologieorientierten Unternehmensverhaltens zurückgegriffen werden. ${ }^{661}$

3. Vor dem Hintergrund der Koordination des ökologiegerichteten Verhaltens im Rahmen einer global-proaktiven Verhaltensausrichtung ergibt sich ein weiterer Forschungsbereich aus der Notwendigkeit, ein internationales ÖkoControlling aufzubauen. Im Mittelpunkt steht dabei die Frage, inwieweit das klassische Controlling mit dem Öko-Controlling zu verbinden ist, um ein opti-

661 Vgl. Kirchgeorg, M.: OKologieorientiertes Unternehmensverhalten: Typologien und Erklärungsansätze auf empirischer Grundlage, a. a. o. 
males Zusammenspiel von Koordinations- und Steuerungsinstrumenten zur internationalen Abstimmung international tätiger Unternehmen zu erreichen.

- Aufbauend auf einer Analyse der Controllingobjekte und der Bestimmung des Controllingbedarfs stellt dabei die strukturelle Ausgestaltung eines den Erfordernissen des internationalen Umweltmanagements Rechnung tragenden Controllingsystems ein Ziel des Forschungsfeldes dar, welches eng an die Erkenntnisse der vorliegenden Arbeit anknüpft. Dabei ist insbesondere der Frage nachzugehen, welchen Beitrag die Balanced Scorecard in diesem Kontext leisten kann.

- Eine Balanced Scorecard könnte sich auch deshalb zur internationalen Koordination besonders eignen, weil die im Rahmen der vorliegenden Untersuchung abgeleiteten Koordinationsziele unterschiedlichen Perspektiven der Scorecard zuzuordnen sind. In diesem Zusammenhang ist zu diskutieren, ob ökologische Ziele in die vorhandenen Perspektiven einer Scorecard zu integrieren sind bzw. in eigenen Perspektiven Eingang in die Scorecard finden. Die folgende Abbildung skizziert beispielhaft, in welchen Perspektiven unterschiedliche Anspruchsgruppen Berücksichtigung finden könnten.

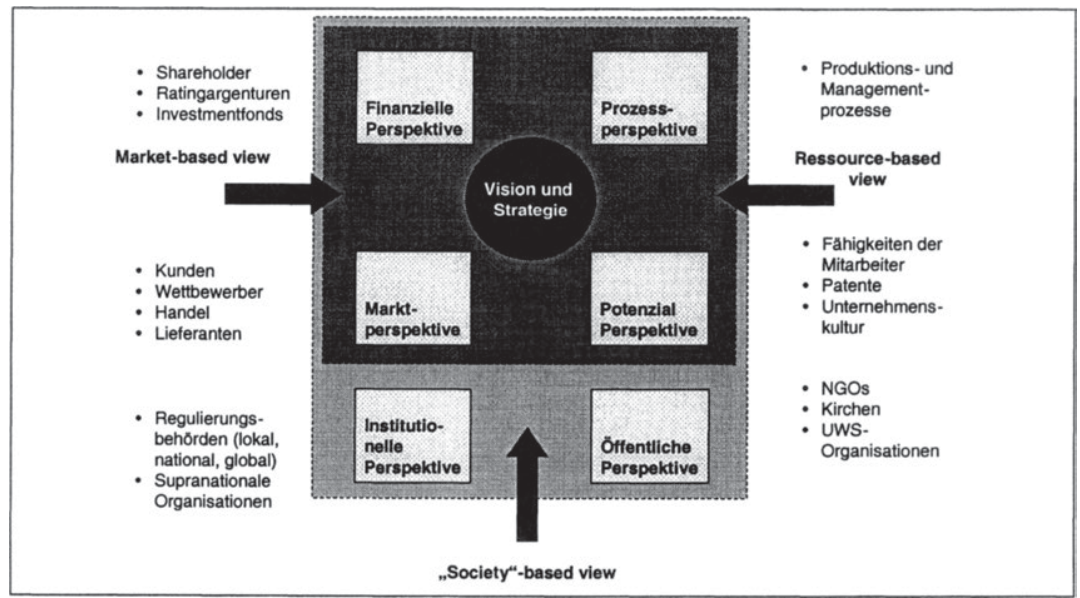

Abbildung 53: Erweiterung einer Balanced Scorecard um ökologische Zielsetzungen

Quelle: Eigene Darstellung 
Dabei wurden sowohl eine Integration der Stakeholderinteressen in die klassischen Perspektiven vorgenommen als auch zwei neue Perspektiven eingeführt, die quasi als Basisperspektiven die Aufrechterhaltung der gesellschaftlichen Legitimität in allen Märkten sicherstellen soll.

- Die größte Herausforderung eines derartigen Forschungsvorhabens liegt letztlich in der Verknüpfung der einzelnen Scorecard-Perspektiven zu einem übergreifenden Geschäftsmodell sowie in der Ableitung geeigneter Kennzahlen zur Steuerung.

4. Die betriebswirtschaftliche Forschung rekrutiert zur Erklärung des ökologiegerichteten Unternehmensverhaltens zumeist auf aktuelle exogene Kontextfaktoren, wie die Betroffenheit durch ökologische Anspruchsgruppen. Dieses Vorgehen kann den proaktiven Aufbau von Kompetenzen und Fähigkeiten zur Verminderung ökologischer Belastungen nur begrenzt erklären, da die diesen Belastungen zugrunde liegenden ökologischen Probleme möglicherweise erst in weiter Zukunft durch öffentliche, politische und marktliche Anspruchsgruppen aufgegriffen und in sog. ökologische Wettbewerbsfelder transformiert werden. ${ }^{662}$

- Vor dem Hintergrund dieser Problemstellung ist im Rahmen eines weiteren Forschungsvorhabens der Erkenntnisbeitrag der Ressourcentheorie zur Erklärung des internationalen ökologiegerichteten Verhaltens zu evaluieren. Die Ansätze aus der Ressourcentheorie versuchen dabei, die Entstehung von überdurchschnittlichen Renditen aus Wettbewerbsvorteilen zu erklären. Dabei gelten Wettbewerbsvorteile als Ergebnis von besonderen Ressourcen und Fähigkeiten ${ }^{663}$ der Unternehmung. Vor dem

Ökologische Wettbewerbsfelder bezeichnen die ökologischen Probleme einer Branche, deren Lösung die Erlangung von Wettbewerbsvorteilen ermöglicht bzw. deren Nichterfüllung mit beträchtlichen Wettbewerbsnachteilen einhergeht. Vgl. hierzu die Ausführungen zum Modell der ökologischen Transformation in Kapitel B 2 sowie Dyllick, Th.; Belz, F.; Schneldewind, U.: Ókologie und Wettbewerbsfähigkeit, a. a. O., S. 5 f.

Fähigkeiten kennzeichnen die "Begabung" des Managements, vorhandene Ressourcen so zu nutzen bzw. zu integrieren, dass das Unternehmen in die Lage versetzt wird, Aktivitäten durchzuführen, die in einer bestimmten Markt- und Wettbewerbssituation zum Erfolg des Unternehmens am Markt beitragen (inside-out Perspektive). In einem anderen Kontext und/oder zu einem späteren Zeitpunkt können diese Aktivitäten jedoch erfolgsirrelevant sein und sogar zu einer Belastung für das Unternehmen werden. Vgl. Meffert, H.: Marketingwissenschaft im Wandel - Anmerkungen zur Paradigmendiskussion, a. a. O., S. 26 und Burmann, Ch.: Strategiewechel in turbulenten Märkten - Nevere theoretische Ansätze zur Untemehmensflexibilität, a. a. O., S. 24. 
Hintergrund der ökologischen Herausforderung des Treibhauseffektes erklären PACKARD und REINHARDT den Zusammenhang bspw. wie folgt:

"Although it may be decades before people in Munich or Minneapolis have to worry about tropical diseases, regulations designed to crub climate change are already being proposed. So in a business sense, at least, global warming is here, posing a threat to business as usual. For instance, it is likely that governments will impose additional taxes on fossil fuel consumption and require that cars and appliances use less energy. Such regulatory programs will change asset values. .. They will also increase the value of goods and services as diverse as renewable-energy technology, process-control equipment, and telecommunications services - such as teleconferences - that substitute for transportation.“664

Der proaktive Aufbau von Kompetenzen und Fähigkeiten ist somit insbesondere vor der Umsetzung von gesetzlichen Regelungen bzw. marktlichen und gesellschaftlichen Forderungen von Anspruchsgruppen im gesamten Unternehmen zu fördern, wenn Wettbewerbsvorteile auch in Zukunft gesichert werden sollen. ${ }^{665}$ Ein erfolgreicher Wettbewerb um die "markets of the future" setzt dabei nach HAMEL und PRAHALAD frühzeitige Investitionen in Ressourcen und Fähigkeiten eines Unternehmens voraus. $^{666}$

- Da sich mit dem resourced-based und market-based-view die Erkenntnis durchgesetzt hat, dass Wettbewerbsvorteile sowohl auf internen als auch externen Faktoren beruhen, erscheint die gleichzeitige Berücksichtigung der inside-out- und outside-in-Perspektive (sog. dynamic capability Ansatz) notwendig. ${ }^{667}$ Das Ziel eines diesbezüglichen Forschungsvorhabens ist in der Entwicklung eines Integrationsmodells für diese beiden

664

Vgl. Packard, K.; Relnhardt, F.: What Every Executive Neөds to Know About Global Warming, in: Harvard Business Review, 78. Jg. (2000), Nr. 4, S. 132.

665

HART hat in vor diesem Hintergrund festgestellt, dass in Zukunft „.. it appears inevitable that businesses (markets) will be constrained by and depend upon ecosystems (nature). In other words, it is likely that strategy and competitive advantage in the coming years will be rooted in capabilities that facilitate environmentally sustainable economic activity - a natural-resource-based view of the firm. "Hart, S. L.: A Natural-Resource-Based View of the Firm, a. a. O., S. 991.

Vgl. Hamel. G.; Prahalad, C. K.: Corporate Imagination and Expeditionary Marketing, in: Harvard Business Review, 69. Jg. (1991), Nr. 4, S. $81 \mathrm{f}$.

Vgl. Meffert, H.: Marketingwissenschaft im Wandel - Anmerkungen zur Paradigmendiskussion, a. a. O., S. 29 und Hart, S. L.: A Natural-Resource-Based View of the Firm, a. a. O., S. 986; Burmann, Ch.: Strategiewechel in turbulenten Märkten - Nevere theoretische Ansătze zur Unternehmensflexibilităt, a. a. O.; Teece, D. J.; Pisano, G.; Shuen, A.: Dynamic Capabilities and Strategic Management, inStrategic Management Journal, 18. Jg (1997), Nr. 7, S. $509-533$. 
Ansätze im Umweltmanagement und ihrer empirischen Überprüfung zu sehen.

Die folgende Abbildung skizziert einen möglichen Ansatz für ein derartiges Modell. Dabei wurde das Modell zur ökologischen Transformation, welches die Entstehung ökologischer Wettbewerbsfelder aufgrund exogener Einflussfaktoren erklärt, mit einem Modell zur Erläuterung der Entstehung von Wettbewerbsvorteilen aufgrund endogener Faktoren verbunden. Sollen latente oder potenzielle ökologiebezogene Wettbewerbsfelder in Zukunft erfolgreich bearbeitet werden, gilt es, bereits heute in der Unternehmung entsprechende Ressourcen aufzubauen und ökologiegerichtete Routinen und Fähigkeiten zu entwickeln.

Ebene der ökologischen Umwelt

Ebene der ökologischen Anspruchsgruppen

Ebene ökologischer Wettbewerbsfelder

Wettbewerbsvorteils-Ebene

FähigkeitenEbene

RoutinenEbene

RessourcenEbene

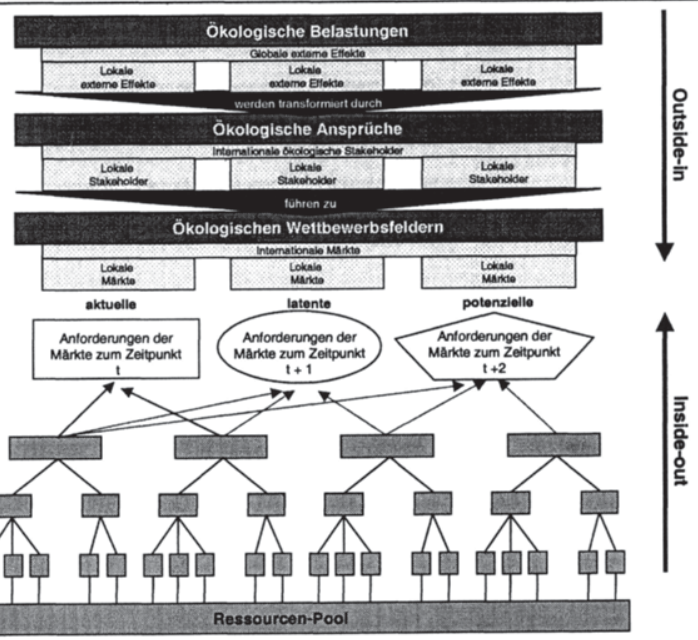

Abbildung 54: Integrationsmodell zur Verbindung der Outside-in- und Inside-out-Perspektive im Umweltmanagement

Quelle: Eigene Darstellung

Insgesamt zeichnet sich ab, dass die internationale Koordination des ökologiegerichteten Verhaltens auch weiterhin an Bedeutung gewinnen wird. Insbesondere die in der Aufgabenumwelt zu beobachtenden Entwicklungen, wie die sich fortsetzende Angleichung der umweltrechtlichen Rahmenbedingungen in den Schwellenländern und der Bedeutungszuwachs international organisierter ökologischer Anspruchsgruppen geben zu der Erwartung Anlass, dass die gesellschaftliche Legitimität von international tätigen Unternehmen nur durch eine 
ökologisch global-proaktive Verhaltensausrichtung aufrechtzuerhalten ist. Vor dem Hintergrund des ökologischen Problemdrucks muss sich die Unternehmenspraxis darauf einstellen, dass auch in Schwellenländern in naher Zukunft ökologisch bedenkliche Produkte und Produktionsverfahren nicht mehr akzeptiert werden. Indessen ist die Wissenschaft aufgerufen, durch eine verstärkte Integration ökologiebezogener Fragestellungen in die betriebswirtschaftliche Forschung Ansätze aufzuzeigen, die eine wirtschaftlich, sozial und ökologisch nachhaltige Entwicklung fördern. 
Frank Wolter - 978-3-631-75063-6

Downloaded from PubFactory at 01/11/2019 08:41:13AM

via free access 


\section{Literaturverzeichnis}

Achleitner, P. M.: Sozio-politische Strategien multinationaler Unternehmen, in: Schriftenreihe Betriebswirtschaft der Hochschule St. Gallen, Bd. 13, Bern, Stuttgart 1985.

Adam, D. et al.: Koordination betrieblicher Entscheidungen: Die Fallstudie Peter Pollmann, 2. Aufl., Berlin u. a. 1998.

Adam, D.: Investitionscontrolling, 2. Aufl., München, Wien 1997.

Adam, D.: Planung und Entscheidung, 4. Aufl., Wiesbaden 1997.

Akademie der Politischen Bildung (Hrsg.): Umweltschutz zwischen Globalisierung und Deregulierung - Die Grenzen des Rechts in der Risikogesellschaft, Bonn 1998.

Albach, H.: Kosten, Transaktionen und externe Effekte im betrieblichen Rechnungswesen, in: Zeitschrift für Betriebswirtschaft, 58. Jg. (1988), Nr. 11 , S. $1143-1170$.

Albach, H.: Betriebswirtschaftslehre als Wissenschaft vom Management, in: ZfB, Ergänzungsheft 1, 1981.

Albach, H.: Die Koordination der Planung im Großunternehmen, in: Schneider, E. (Hrsg.): Rationale Wirtschaftspolitik und Planung in der Wirtschaft von heute, Schriften des Vereins für Socialpolitik, Band 45, Berlin 1967, S. $332-438$.

Albrecht, J.: Environmental costs and competitiveness. A product-specific test of the Porter hypothesis, Working Paper Series, University of Ghent, Faculty of Economics and Business Administration, 1998.

Altmann, J.: Umweltpolitik, Bern u. a. 1997.

Altshuler, A. et al.: The Future of the Automobile: The Report of MIT's International Automobile Program, London, Sydney 1984.

Ansoff, L.: Managing Surprise and Discontinuity - Strategic Response to Weak Signals, in: ZfbF, 28. Jg. (1976), Nr. 3, S. $129-152$.

Arndt, H.-K.; Günther, O.: Betriebliche Umweltinformationssysteme als Knowledge Managementsysteme im Umweltmanagement, in: Umweltwirtschaftsforum, 9. Jg. (2001), Heft 1.

Arrow, K. et al. Economic Growth, Carrying Capacity, and the Environment, in: Ecological Economics, 15. Jg. (1995), S. $91-95$.

Audi AG (Hrsg.): Geschäftsbericht 1999, Ingolstadt 1999. 
Auerbach, H.; Meissner, H. G.: Controlling im internationalen Marketing-Management, in: Hermanns, A.; Wissmeier, U. K. (Hrsg.): Internationales Marketing Management: Grundlagen, Strategien, Instrumente, Kontrolle und Organisation, München 1995, S. 281 - 308.

Axel Springer Verlag (Hrsg.): Auto Bild-Trend Profil: Auto - Umwelt - Zukunft, Hamburg 1995.

Backhaus, K., Büschken, J.; Voeth, M.: Internationales Marketing, 3. Aufl., Stuttgart 2000.

Ball, J.: 'Clean' Car Quest Rolls on: Green Guru Lovins's Dream Machine Draws Industry Interest, Few Funds, in: The Wall Street Journal Europe, Thuesday, January 11, 2001, S. 23.

Bals, Ch.; Kier, G.; Rück, A.; Treber, M.: Klimaschutz vor Buenos Aires: werden die Kyoto-Schlupflöcher enger?, Briefing Paper, GERMANWATCH, 1998.

Barrett, H. J.: Individuelle Ziele und Organisationsziele, in: Wöhler, K. (Hrsg.): Organisationsanalyse, Stuttgart 1978.

Bartlett, Ch. A.; Doz, Y.; Hedlund, G. (Hrsg.): Managing the Global Firm, London, New York 1990.

Bartlett, Ch. A.; Ghoshal, S. (Hrsg.): Transnational Management: Text, Cases, and Readings in Cross-Border Management, Third Edition, Boston u. a. 2000.

Bartlett, Ch. A.; Ghoshal, S.: Internationale Unternehmensführung, Frankfurt a. M. 1990.

Bartlett, Ch. A.; Goshal, S.: Managing Innovations in the Transnational Corporation, in: Bartlett, Ch. A.; Doz, Y.; Hedlund, G. (Hrsg.): Managing the Global Firm, London, New York 1990, S. 215 - 255.

Bartlett, Ch. A.; Ghoshal, S.: Arbeitsteilung bei der Globalisierung, in: Harvard Manager, 9. Jg. (1987), Heft 2, S. $49-59$.

Bartlett, Ch. A. et al. (Hrsg.): Managing the Global Firm, London, New York 1990.

Bartsch, T.: Erfahrungen mit dem Umwelt / Öko-Audits in den USA, in: Zeitschrift für Umweltrecht, Heft 1/95, S. $14-19$.

Baumast, A.: Environmental Management in Europe: Results of the European Business Environmental Barometer (E.B.E.B) 1997/1998, in: IWOe Discussion Paper No. 79, St. Gallen 2000. 
Baumast, A.: Die Entstehungsgeschichte des Umwelt-Audit, in: DoktorandenNetzwerk Öko-Audit e. V. (Hrsg.): Umweltmanagementsysteme zwischen Anspruch und Wirklichkeit: Eine interdisziplinäre Auseinandersetzung mit der EG-Öko-Audit-Verordnung und der DIN EN ISO 14001, Berlin u. a. 1998, S. $33-56$.

Becker, J.: Marketing-Konzeptionen: Grundlagen des strategischen MarketingManagements, München 1990.

Beer, S.: Kybernetik und Management, Hamburg 1962.

Belz, F.: Ökologische Wettbewerbsfelder in der Lebensmittelbranche, in: Der Markt, 33. Jg. (1994), Nr. 129, S. $51-61$.

Bendell, J.: Jenseits der Selbstregulierung von Umweltmanagement: Einige Grundgedanken zur wachsenden Bedeutung von Business-NGOPartnerschaften, in: Fichter, K.; Schneidewind, U. (Hrsg.): Umweltschutz im globalen Wettbewerb: Neue Spielregeln für das grenzenlose Unternehmen, Berlin u. a. 2000, S. 153 - 162.

Benkensteln, M.: F \& E und Marketing: Eine Untersuchung zur Leistungsfähigkeit von Koordinationskonzeptionen bei Innovationsentscheidungen, Wiesbaden 1987.

Berekoven, L.: Internationales Marketing, 2. Aufl., Berlin 1985.

Binswanger, H. C. et al.: Wirtschaft und Umwelt, Stuttgart u. a. 1981.

BMW (Hrsg.): BMW Group: Clean Production weltweit, Presseinformation vom 09.12.1999.

BMW (Hrsg.): Umweltbericht der BMW Group 1999/2000, München 1999.

Bode, Th.: In search of Robin Hood: NGO's foes or partners in the global agenda, Davos 2000.

Böhler, H.: Früherkennungssysteme, in: Wittmann, W. et al. (Hrsg.): Handwörterbuch der Betriebswirtschaft, 5. Aufl., Stuttgart 1993, Sp. 1256 $-1270$.

Bolz, J.: Wettbewerbsorientierte Standardisierung der internationalen Marktbearbeitung: Eine empirische Analyse in europäischen Schlüsselmärkten, Darmstadt 1992.

Brauchlin, E.; Wiesmann, D.: Internationales Management, in: Gabler Wirtschaftslexikon, 13. Auflage, Wiesbaden 1993.

Brauweiler, J. et al.: Umweltmanagement im Transformationsprozess: Ansatzpunkte für einen internationalen Know-how-Transfer - dargestellt am Beispiel polnischer und tschechischer Untemehmen, in: Umweltwirtschaftsforum, 8. Jg. (2000), Nr. 1, S. 16-25. 
British Standards Institution (Hrsg.): Specification for Environmental Management Systems. British Standard BS 7750: 1992, London 1992.

Brockhoff, K.: Management organisatorischer Schnittstellen - unter besonderer Berücksichtigung der Koordination von Marketingbereichen mit der Forschung und Entwicklung, Göttingen 1994.

Brockhoff, K.; Hauschildt, J.: Schnittstellen-Management - Koordination ohne Hierarchie, in: Zeitschrift für Organisation, Nr. 6, 1993, S. $396-$ 403.

Brodel, D.: Internationales Umweltmanagement: Gestaltungsfelder - Determinanten - Ausprägungen, Wiesbaden 1996.

Bühner, R.: Betriebswirtschaftliche Organisationslehre, 9. Aufl., München 1999.

Bundesministerium für Umwelt, Naturschutz und Reaktorsicherheit (Hrsg.): Konferenz der Vereinten Nationen für Umwelt und Entwicklung im Juni 1992 in Rio de Janeiro - Dokumente -Agenda 21, Kapitel 30.22, Bonn 1992.

Bundesumweltministerium, Umweltbundesamt (Hrsg.): Handbuch Umweltcontrolling, 2. Aufl., München 2001.

Burmann, Ch.: Strategiewechsel in turbulenten Märkten - Neuere theoretische Ansätze zur Unternehmensflexibilität, in: Meffert, H.; Backhaus, K.; Becker, J. (Hrsg.): Arbeitspapiere der Wissenschaftlichen Gesellschaft für Marketing und Unternehmensführung $\Theta$. V., Münster 2000.

Burmann, G.: Marktarealstrategien der internationalen Automobilhersteller, in: Hünerberg R. et al. (Hrsg.): Internationales Automobilmarketing: Wettbewerbsvorteile durch marktorientierte Unternehmensführung, Wiesbaden 1995, S. 121 - 142.

Buzzel, R. D.: Can You Standardize Multinational Marketing?, in Harvard Business Review, 46. Jg. (1968), S. 102 - 113.

Clausen, J.: Monitoring von Umweltleistung und Umweltmanagementsystem Eine Panelbefragung von Unternehmen: 1. Jahr 1997/1998, in: Diskussionspapier des IÖW 43, St. Gallen 1998.

Clee, G. H.; Di Scipio, A.: Creating a World Enterprise, in: Douglas, N. D. (Hrsg.): Managing Effectively in the World Marketplace, New York 1983.

Clef, U.: Mehrmarkenstrategie für die Pole-Position, in: asw, Sondernummer Oktober 1999, S. $72-80$. 
Coase, R.: The Nature of the Firm, in: Economica, New Series, 4. Jg. (1937), S 386 - 405.

Daly, H. E.; Cobb, J. B.: For the Common Good: Redirecting the Economy Toward Community, the Environment and a Sustainable Future, Boston 1989.

Dasgupta, S.; Wang, H.; Wheeler, D.: Surviving Success: Policy Reform and the Future of Industrial Pollution in China, World Bank Policy Research Department Working Paper, 1997.

Deutsches Institut für Normung (Hrsg.): Umweltmanagementsysteme - Spezifikation mit Anleitung zur Anwendung: Zweisprachige Fassung DIN EN ISO 14.001, 1996, Kap. 3.5.

Dichtl, E.; Gerke, W.; Kieser A. (Hrsg.): Innovation und Wettbewerbsfähigkeit, Wiesbaden 1987.

Diebold, J.: Bad Decisions in Computer Use, in: Harvard Business Review, 47. Jg. (1969).

Dienst für Gesamtverkehrsfragen des Eidgenössischen Verkehrs- und Energiewirtschaftsdepartments (Hrsg.): Monetarisierung der verkehrsbedingten externen Gesundheitskosten - Synthesebericht, Bern 1996.

Dietz, W.; Brachat, H.: Grundlagen der Automobilwirtschaft, Ottobrunn 1994.

Diez, W.: Plattform-Strategien: Irrweg oder Königsweg in der Modellpolitik, Arbeitspapier Nr. 2 des Instituts für Automobilwirtschaft, Gelsenkirchen 1999.

Dietz, W.: Das Handbuch für das Automobilmarketing: Strategien, Konzepte, Instrumente, Landsberg/Lech 1996.

Dobry, A.: Die Steuerung ausländischer Tochtergesellschaften: Eine theoretische und empirische Untersuchung ihrer Grundlagen und Instrumente, Gießen-Wieseck 1983.

Doktoranden-Netzwerk Öko-Audit e. V. (Hrsg.): Umweltmanagementsysteme zwischen Anspruch und Wirklichkeit: Eine interdisziplinäre Auseinandersetzung mit der EG-Öko-Audit-Verordnung und der DIN EN ISO 14001, Berlin u. a. 1998.

Domsch, M. et al.: Umweltorientierte Personalentwicklung, in: Winter, J. (Hrsg.): Umweltmanagement, Stuttgart 1997, S. 97 - 124.

Douglas, N. D. (Hrsg.): Managing Effectively in the World Marketplace, New York 1983.

Doz, Y.: Strategic Management in Multinational Companies, Oxford u. a. 1986. 
Dudenhöfer, F.: Outsourcing, Plattform-Strategien und Badge Engineering: Marktentwicklung bei austauschbaren Produkten, in: WiSt, Heft 3, 1997, S. $145-149$.

Dülfer, E. (Hrsg.): Organisationskultur: Phänomen, Philosophie, Technologie, 2. Aufl., Stuttgart 1991.

Dülfer, E.: Organisationskultur: Phänomen, Philosophie, Technologie: Eine Einführung in die Diskussion, in: Dülfer, E. (Hrsg.): Organisationskultur: Phänomen, Philosophie, Technologie, 2. Aufl., Stuttgart 1991.

Dülfer, E.: Zum Problem der Umweltberücksichtigung im „Internationalen Management", in: Pausenberger, E. (Hrsg.): Internationales Management, Stuttgart 1981, S. 1-44.

Dyckhoff, H.; Jacobs, R.: Organisation des Umweltschutzes in Industriebetrieben, in: ZfB, 64. Jg. (1994), Heft 6, S. $717-735$.

Dyer, J.H.: Specialized Supplier Networks as a Source of Competitive Advantage: Evidence from the Auto Industry, in: Strategic Management Journal, 17. Jg. (1996), S. 271 - 291.

Dyllick, Th.; Belz, F.; Schneidewind, U.: Ökologie und Wettbewerbsfähigkeit, München, Wien 1997.

Dyllick, Th.: Basiswissen Umweltmanagementsysteme, St. Gallen 1999.

Dyllick, Th.: Die EU-Verordnung zum Umweltmanagement und zur Umweltbetriebsprüfung (EMAS-Verordnung) im Vergleich mit der geplanten ISO-Norm 14.001: Eine Beurteilung aus Sicht der Managementlehre, in: Zeitschrift für Umweltpolitik und Umweltrecht, 1995, Nr. 3, S. $299-339$.

Dyllick, Th.: Ökologisch bewusste Unternehmensführung: Der Beitrag der Managementlehre, Schriftenreihe der Schweizer Vereinigung für ökologisch bewusste Unternehmensführung, Nr. 1, 1989, St. Gallen 1989.

Dyllick, Th.: Management der Umweltbeziehungen: Öffentliche Exponiertheit von Unternehmen als Herausforderung für Management und Unternehmenspraxis, in: Die Unternehmung, 42. Jg. (1988), Nr. 3, S. $190-205$.

Dyllick, Th.: Die Beziehungen zwischen Unternehmung und gesellschaftlicher Umwelt, in: DBW, 46. Jg. (1986), Nr. 3.

Dyllick, Th.; Hamschmidt, J.: Wirksamkeit und Leistung von Umweltmanagementsystemen: Eine Untersuchung von ISO 14.001 zertifizierten Unternehmen in der Schweitz, Zürich 2000. 
Dyllick, Th.; Belz, F.; Schneldewind, U.: Ökologie und Wettbewerbsfähigkeit, München, Wien 1997.

ECDG for Energy and Transport, Eurostat (Hrsg.): EU Transport in Figures: Statistical Pocket Book 2000, Brüssel 2000.

Economic and Social Council of the United Nations (Hrsg.): Activities of the transnational corporations and management division and its joint units, E/C.10/1993/7, 4 March 1993.

Emery, J.: Organizational Planning and Control: Theory and Technology, London 1969.

Engelhard, J. (Hrsg.): Interkulturelles Management: Theoretische Fundierung und funktionsbereichsspezifische Konzepte, Wiesbaden 1997.

Engelhard, J.; Dähn, M.: Theorien der internationalen Unternehmenstätigkeit Darstellung, Kritik und zukünftige Anforderungen, in: Macharzina, K.; Oesterle, M.-J. (Hrs g.): Handbuch Internationales Management: Grundlagen - Instrumente - Perspektiven, Wiesbaden 1997, S. 23 44.

Environics International (Hrsg.): The Environmental Monitor -Global Public Opinion on the Environment. 1999 International Report, Toronto 1999.

Epstein, M.; Roy, M.-J.: Managing Corporate Environmental Performance: $A$ Multinational Perspective, in: European Management Journal, Vol. 16 (1998), Nr. 3., S. $284-296$.

European Commission: Commission Recommendation of 5 Feburary 1999 on the reduction of $\mathrm{CO}_{2}$ emissions from passenger cars, in: Official Journal of the European Communities, 13.03.1999.

European Environmental Agency (Hrsg.): Environmental Agreements: Environmental Effectiveness, Copenhagen 1997.

Farraz, C.; da Motta, R. S.: Automobile Pollution Controll in Brazil, Rio De Janeiro, 1999.

Felix, R.; et al.: Integrierte Managementsysteme: Ansätze zur Integration von Qualitäts-, Umwelt- und Arbeitssicherheitsmanagementsystemen, IWÖ-Diskussionsbeitrag Nr. 41, St. Gallen 1997.

Flat (Hrsg.): Fiat - 1999 Environmental Report, Turin 2000.

Fichter, K.; Schneidewind, U. (Hrsg.): Umweltschutz im globalen Wettbewerb: Neue Spielregeln für das grenzenlose Unternehmen, Berlin u. a. 2000. 
Fichter, K.; Schneidewind, U.: Neue Spielregeln für die grenzenlose Ökonomie: Eine Einleitung, in: Fichter, K.; Schneidewind, U. (Hrsg.): Umweltschutz im globalen Wettbewerb - Neue Spielregeln für das grenzenlose Unternehmen, Berlin u. a. 2000, S. 1 - 11.

Figge, F.: Öko-Rating - Präferenzgerechte Aggregation ökologieorientierter Informationen, Berlin u. a. 2000.

Flatz, A.: Der Dow Jones Sustainability Index: Eine neue Kraft auf den globalen Finanzmärkten, in Fichter, K.; Schneidewind, U. (Hrsg.): Umweltschutz im globalen Wettbewerb: Neue Spielregeln für das grenzenlose Unternehmen, Berlin u. a. 2000, S. 111 - 120.

FOCUS (Hrsg.): Der Markt der Automobile: Daten, Fakten, Trends, 1999.

FOCUS (Hrsg.): Der Markt der Automobile: Daten, Fakten, Trends, 2000.

Ford Motor Company (Hrsg.): Connecting with Society: 1999 Corporate Citizenship Report, Dearborne 2000.

Franke, A.: Freier Welthandel und umweltverträgliche Entwicklung, in: IFO Schnelldienst, 21/96, S. $15-24$.

Freeman, R. E.: Strategic Management: A Stakeholder Approach, Marshfield 1984.

Frese, E.: Grundlagen der Organisation: Konzept - Prinzipien - Strukturen. 6. Aufl., Wiesbaden 1995.

Frese, E. (Hrsg.): Handwörterbuch der Organisation (HWO), 3. Aufl., Stuttgart 1992.

Frese, E.: Organisation des Umweltschutzes, in: Frese, E. (Hrsg.): Handwörterbuch der Organisation (HWO), 3. Aufl., Stuttgart 1992, Sp. 2433 2451.

Frese, E.: Organisation und Koordination, in: Zeitschrift für Organisation, 41. Jg. (1972), S. $404-411$.

Frese, E.: Management by Exception, in: Handwörterbuch der Organisation, 1. Aufl., Stuttgart 1969, Sp. 956 - 959.

Frese, E.; Blies, P.: Konsequenzen der Internationalisierung für Organisation und Management der Muttergesellschaft, in: Macharzina, K.; Oesterle, M.-J. (Hrsg.): Handbuch Internationales Management: Grundlagen - Instrumente - Perspektiven, Wiesbaden 1997, S. 287 - 308.

Gege, M. (Hrsg.): Kosten senken durch Umweltmanagement - 1000 Erfolgsbeispiele aus 100 Unternehmen, München 1997. 
General Motors (Hrsg.): The General Motors 1994 Environmental Report, Detroit 1994.

Ghemawat, P.: Dauerhafte Wettbewerbsvorteile aufbauen, in: Simon, $H$. (Hrsg.): Wettbewerbsvorteile und Wettbewerbsfähigkeit, Stuttgart 1988 , S. 18 - 29.

Ghoshal, S.; Nohria, N.: Internal Differentiation within Multinational Corporations, in: Strategic Management Journal, 10. Jg. (1989), S. $323-337$.

Gladwin, T. N.; Walter, I.: Multinationals under Fire. Lessons in the Management of Conflict, New York u. a. 1980.

Gladwin, T. N.; Welles, J. G.: Environmental Policy and Multinational Corporate Strategy, in: Walter, I. (Hrsg.): Studies in International Environmental Economics, New York u. a. 1976, S. 177 - 225.

Gladwin, T. N.; Welles, J. G.: Multinational Corporations and Environmental Protection: Patterns of Organizational Adoption, in: International Studies of Management and Organization, Vol. 9, 1976, SpringSummer, S. $160-184$.

Gleckman, H.; Krut, R.: Neither International nor Standard: The Limits of ISO 14001 as an Instrument, in: Greener Management International, 1996, Issue 14, S. $111-124$.

Goralcyk, D.; Heller, M. (Hrsg.): Strategisches Umweltmanagement in der Industrie, Wien 1995.

Gray, R. H.: Accounting and Environmentalism: An Exploration of the Challenge of Gently Accounting for Accountability, Transparency and Sustainability, in: Accounting Organizations and Society, Jg. 17 (1992), Nr. 5, S. $399-425$.

Greenpeace (Hrsg.): Acht Wochen mit dem Luftmessbus in Mexiko City: Megastadt im Megastau, http://www.greenpeace.de/GP DOK 3P/ BROSCHUE/AKTION/C09IA01.HTM [25.01.2001].

Grimmeisen, M.: Implementierungscontrolling: wirtschaftliche Umsetzung von Change-Programmen, Wiesbaden 1998.

Grochla, E. (Hrsg.): Organisationstheorie, 1. Teilband, Stuttgart 1975.

Grossmann, G. M.; Krüger, A. B.: Economic Growth and the Environment, in: Quarterly Journal of Economics, Vol. CX, 1995.

Günther, E.: Ökologieorientierte Bereichssteuerung, in: Umweltwirtschaftsforum, 3. Jg. (1995), Heft 3, S. $30-34$. 
Günther, E.; Sturm, A.: Ökologieorientierung und ökologischer Erfolg, in: Wissenschaftliche Zeitschrift der Technischen Universität Dresden, 46. Jg. (1997), Heft 6.

Gutenberg, E. (Hrsg.): Die Wirtschaftswissenschaften, Bd. 2, Wiesbaden 1977.

Hahn, D.: PuK, Controllingkonzepte, 5. Aufl., Wiesbaden 1996.

Hahn, D.: Planungs- und Kontrollrechnung - PuK: Integrierte ergebnis- und liquiditätsorientierte Planungs- und Kontrollrechnung als Führungsinstrument in Industrieunternehmen mit Massen- und Serienfertigung, Wiesbaden 1974.

Hamschmidt, J.; Dyllick, Th.: Nutzen Managementsysteme? Vom Umweltzum Sustainability-Managementsystem, IWÖ-Diskussionsbeitrag Nr. 82, St. Gallen 2000.

Harbison, F.; Myers, Ch. A.: Management in the Industrial World. An International Analysis, New York u. a. 1959.

Hart, S. L.: A Natural-Resource-Based View of the Firm, in: Academy of Management Review, 20. Jg. (1995), Nr. 4, S. 986 - 1014.

Harzing, A.-W.: An empirical Analysis and Extension of the Bartlett and Ghoshal Typology of Multinational Companies, in: Journal of International Business Studies, Vol. 31 (2000), Nr. 1, S. $101-120$.

Hax, H.: Die Koordination von Entscheidungen - Ein Beitrag zur betriebswirtschaftlichen Organisationslehre, Köln u. a. 1965.

Heenan, D. A.; Perlmutter, H. V.: Multinational Organization Development, Reading (MA) 1979.

Heinen, E.: Grundlagen betriebswirtschaftlicher Entscheidungen - Das Zielsystem der Unternehmung, 3. Aufl., Wiesbaden 1976.

Heinen, E.: Einführung in die Betriebswirtschaftslehre, Wiesbaden 1968.

Heinen, E.: Das Zielsystem der Unternehmung: Grundlagen betriebswirtschaftlicher Entscheidungen, Wiesbaden 1966.

Heise, G.: Internationale Marktsegmentierung im Automobilmarketing, Wiesbaden 1997.

Heiser, H. C.: Budgetierung, Berlin 1964.

Heller, M.: Das Umweltmanagementsystem der BMW AG, in: Goralcyk, D.; Heller, M. (Hrsg.): Strategisches Umweltmanagement in der Industrie, Wien 1995, S. $57-81$.

Helm, C.: Sind Freihandel und Umweltschutz vereinbar? Ökologischer Reformbedarf des GATTWTO-Regimes, Berlin 1995. 
Hermanns, A.; Wissmeier, U. K. (Hrsg.): Internationales Marketing Management: Grundlagen, Strategien, Instrumente, Kontrolle und Organisation, München 1995.

Hey, Ch.; Schleicher-Tappeser, R.: Nachhaltigkeit trotz Globalisierung Handlungsspielräume auf regionaler, nationaler und europäischer Ebene, Berlin u. a. 1998.

Heynen, C.-H.: Die Koordination dezentraler Entscheidungen: Ein verhaltensorientierter Ansatz zur Analyse der Koordination von MarketingEntscheidungen, Münster 1975.

Hilker, J.: Marketingimplementierung: Grundlagen und Umsetzung am Beispiel ostdeutscher Unternehmen, Wiesbaden 1993.

Hipp, Ch.; Reger, G.: Die Dynamik ökologischer Entwicklungsprozesse in Unternehmen - Kernelemente eines ganzheitlichen Umweltmanagementsystems, in: ZfB, Ergänzungsheft 1, 1998, S. 40 - 43.

Hitchens, D. M. W. N.; Clausen, J.; Fichter, K. (Hrsg): Intemational Environmental Management Benchmarks: Best Practice Experinces from America, Japan and Europe, Berlin u. a. 1999.

Hoffmann, F.: Führungsorganisation, Bd. I: Stand der Forschung und Konzeption, Tübingen 1980.

Hofstede, G.: Organization-Related Value Systems in Forty Countries, Working Paper 78-22, European Institute für Advanced Studies in Management, Brüssel 1978.

Hummels, H.: Anreize zur Standardisierung umweltverträglichen Verhaltens multinationaler Unternehmen, Frankfurt a. M. u. a. 1998.

Humphrey, J. et al. (Hrsg.): Global Strategies and Local Realities: The Auto Industry in Emerging Markets, London, New York 2000.

Humphrey, J. et al.: Introduction, in: Humphrey, J. et al. (Hrsg.): Global Strategies and Local Realities: The Auto Industry in Emerging Markets, London, New York 2000, S. 1 - 15.

Humphrey, J.; Oeter, A.: Motor-Industry Policies in Emerging Markets, in: Humphrey, J. et al. (Hrsg.): Global Strategies and Local Realities: The Auto Industry in Emerging Markets, London, New York 2000, S. $42-71$.

Hünerberg R. et al. (Hrsg.): Internationales Automobilmarketing: Wettbewerbsvorteile durch marktorientierte Unternehmensführung, Wiesbaden 1995.

Hünerberg, R.: Internationales Marketing, Landsberg/Lech 1994. 
ICC (Hrsg.): Charta für eine langfristig tragfähige Entwicklung - Grundsätze des Umweltmanagement, 1991.

IISD, UNEP (Hrsg.): Environment and Trade - A Handbook, Winnipeg 2000.

Internationale Handelkammer (ICC) (Hrsg.): Umweltschutz Audits, Positionspapier des ICC-Executive Boards, Köln 1989.

Jähnike, M.; Weidner, H.: Zum aktuellen Stand der Umweltpolitik im internationalen Vergleich - Tendenzen zu einer globalen Konvergenz?, in: Aus Politik und Zeitgeschichte, 27. Juni 1997, Nr. B27/97, S. 15 24.

Jänicke, M. et al.: Umweltentlastung durch industriellen Strukturwandel? Eine explorative Studie über 32 Industrieländer, Berlin 1992.

Jehle, E.: Unternehmung und gesellschaftliche Umwelt: Grundlagen einer ökonomischen Theorie der pluralistischen Unternehmung, Stuttgart 1980.

Junkernheinrich, M.; Klenner, P.; Wagner, G. R. (Hrsg.): Handbuch zur Umweltökonomie, Berlin 1995.

Karliner, J.: The Corporate Planet: Ecology and Politics in the Age of Globalization, 1997 zitiert nach: http://www.corpwatch.org/trac/ feature/planet/ fact 1.html [07.11.00].

Kast, F., Rosenzweig, J.: Organization and Management: $A$ Contingency Approach, Tokio 1970.

Keller, E. v.: Management in fremden Kulturen. Ziele, Ergebnisse und methodische Probleme der kulturvergleichenden Managementforschung, Bern, Stuttgart 1982.

Kellersmann, B.: Die gemeinsame, aber differenzierte Verantwortlichkeit von Industriestaaten und Entwicklungsländern für den Schutz der globalen Umwelt, Berlin u. a. 2000.

Khandwalla, P. N.: Uncertainty and the "Optimal" Design of Organizations, McGill University, Working Paper, Houston 1972.

Khandwalla, P. N.: Unsicherheit und die optimale Gestaltung der Organisation, in: Grochla, E. (Hrsg.): Organisationstheorie, 1. Teilband, Stuttgart 1975 , S. $140-156$.

Kieser, A.; Kubicek, H.: Organisationsstruktur und individuelles Verhalten als Einflussfaktoren der Gestaltung von Management-Informationssystemen, in: Zeitschrift für Betriebswirtschaftslehre, 44. Jg. (1974), S $449-474$.

Kieser, A.; Kubicek, H.: Organisation, Berlin, New York 1977. 
Kirch, W.: Die Koordination von Entscheidungen in Organisationen, in: ZfbF, 23. Jg. (1971), Nr 1, S. $61-82$.

Kirch, W.; Picot, A. (Hrsg.): Die Betriebswirtschaftslehre im Spannungsfeld zwischen Generalisierung und Spezialisierung, Wiesbaden 1989.

Kirchgeorg, M.: Ökologieorientiertes Unternehmensverhalten - Typologien und Erklärungsansätze auf empirischer Grundlage, Wiesbaden 1990.

Kirsch, W.: Einführung in die Theorie der Entscheidungsprozesse: Entscheidungen in Organisationen, Bd. 3, Wiesbaden 1977.

Kirsch, W.: Die Koordination von Entscheidungen in Organisationen, in: ZfbF, 23. Jg. (1971), S. $61-82$.

Klaus, I.: Saubere Rendite, in: Die Geldidee, Nr. 18, August 2000, S. 14 -17.

Kleebinder, H. P.: Das öffentliche Meinungsbild zum Themenfeld Mobilität im europäischen Vergleich, Forschungsbericht Prof. Dr. H. Weinold, St. Gallen 1994.

Kleivane, T.: Environmental Performance Evaluation - The Link Between Management Systems and Reality, in: Hitchens, D. M. W. N.; Clausen, J.; Fichter, K. (Hrsg.): International Environmental Management Benchmarks: Best Practice Experinces from America, Japan and Europe, Berlin u. a. 1999, S. $103-109$.

Knobel, P.: Der Emissionsrechtehandel bei BP Amoco: Ein praktischer Ansatz, in: Umweltwirtschaftsforum, 8. Jg. (2000), Heft 1, S. $41-45$.

Koch, H.: Aufbau der Unternehmensplanung, in: Gutenberg, E. (Hrsg.): Die Wirtschaftswissenschaften, Bd. 2, Wiesbaden 1977.

Koers, M.: Steuerung von Markenportfolios: Ein Beitrag zum Mehrmarkencontrolling am Beispiel der Automobilwirtschaft, Frankfurt a. M. 2001.

Kogut, B.: International Sequential Advantages and Network Flexibility, in: Bartlett, Ch. A. et al. (Hrsg.): Managing the Global Firm, London, New York 1990, S. $47-68$.

Kosiol, E.: Zur Problematik der Planung in der Unternehmung, in: Zeitschrift für Betriebswirtschaft, 37. Jg. (1967), S.

Koslowski, P. (Hrsg.): Shareholder Value und die Kriterien des Unternehmenserfolgs. Ethische Ökonomie. Beiträge zur Wirtschaftsethik und Wirtschaftskultur, Heidelberg 1999.

Kostka, C.; Kostka, S.: Umweltorientierte Personalentwicklung, in: Winter, G. (Hrsg.): Das Umweltbewusste Unternehmen: Die Zukunft beginnt heute, 6. Aufl., München 1998, S. 913 - 936. 
Kramer, M.; Reichel, M. (Hrsg.): Internationales Umweltmanagement und europäische Integration: Deutschland, Polen und Tschechien im Vergleich, Wiesbaden 1998.

Kreuter, A.: Verrechnungspreise in Profit-Center-Organisationen, München 1997.

Kreutzer, R.: Global Marketing - Konzeption eines länderübergreifenden Marketing, Wiesbaden 1989.

Kreutzer, R.: Reif für Global Marketing?, in: Absatzwirtschaft, Sonderausgabe 10 (1985), S. $144-157$.

Kubicek, H.: Informationstechnologie und organisatorische Regelungen, Berlin 1975.

Kulessa, M. E.: Umweltpolitische Dimensionen des Welthandels, in: Wirtschaft und Umwelt e. V. (Hrsg.): Welthandel und Umweltschutz - Wie handeln wir ökologisch?, Münster 1996, S. 41-60.

Kutschker, M. (Hrsg.): Integration in der internationalen Unternehmung, Wiesbaden 1998.

Laforet, S., Saunders, J.: Managing Brand Portfolios: Why Leaders Do What They Do, in: JoAR, Nr. 1, 1999, S. 51-66.

Landwehr, R.: Standardisierung der internationalen Werbeplanung, Frankfurt, Bern 1988.

Lassmann, A.: Organisatorische Koordination, Konzepte und Prinzipien zur Einordnung von Teilaufgaben, Wiesbaden 1992.

Lawrence, P. R.; Lorsch, J. W.: New Management Job: The Integrator, Harvard Business Review, 45. Jg. (1967), Nr. 6, 142 - 151.

Leavitt, H. J.: Applied Organizational Change in Industry: Structural, Technological and Humanistic Approaches, in: March, J. G. (Hrsg.): Handbook of Organizations, Chicago 1965, S. $1144-1170$.

Lehnert, St:: Die Bedeutung von Kontingenzansätzen für das strategische Management, Frankfurt a. M. u. a. 1983.

Liebehenschel, Th.: Ökologieorientierte Produkt- und Dienstleistungspolitik: Rahmenbedingungen und Trends am Beispiel der Automobilindustrie, Marburg 1999.

Light, J.: Prague: The Aftermath, September 29, 2000, http://www.corpwatch. org/trac/ globalization/bretton/il9-29.html [07.11.2000].

Lovins, A. B.; Lovins L. H.: Climate: Making Sense and Making Money, Rocky Mountain Institute (ed.), Colorado 1997. 
Lung, Y.: Is the Rise of Emerging Countries as Automobile Producers an Irreversible Phenomenon?, in: Humphrey, J. et al. (Hrsg.): Global Strategies and Local Realities: The Auto Industry in Emerging Markets, London, New York 2000, S. $16-41$.

Macharzina, K.: Unternehmensführung: Das internationale Managementwissen; Konzepte - Methoden - Praxis, 3. Aufl., Wiesbaden 1999.

Macharzina, K.: Rahmenbedingungen und Gestaltungsmöglichkeiten bei Umsetzung von globalen Strategieansätzen, in: Schmalenbach-Gesellschaft/Deutsche Gesellschaft für Betriebswirtschaft e. V. (Hrsg.): Internationalisierung der Wirtschaft, Stuttgart 1993, S. 29 - 55.

Macharzina, K.; Oesterle, M.-J. (Hrsg.): Handbuch Internationales Management: Grundlagen - Instrumente - Perspektiven, Wiesbaden 1997.

Macharzina, K.; Oesterle, M.-J.: Bestimmungsgrößen und Mechanismen der Koordination von Auslandsgesellschaften, in: Macharzina, K.; Oesterle, M.-J. (Hrsg.): Handbuch Internationales Management: Grundlagen - Instrumente - Perspektiven, Wiesbaden 1997, S. 609 -640 .

Madhavan, S.: Mobility at a Price, in: Humphrey, J. et al. (Hrsg.): Global Strategies and Local Realities: The Auto Industry in Emerging Markets, London, New York 2000, S. 95 - 121.

Mahoney, Th. A.; Weitzel, W.: Managerial Models of Organizational Effectiveness, in: Administrative Science Quarterly, 14. Jg. (1969), S. 357- 365.

Malik, F.: Strategie des Managements komplexer Systeme, 5. Aufl., Bern 1996.

March, J. G. (Hrsg.): Handbook of Organizations, Chicago 1965.

March, J. G.; Olsen, J. P.: The Uncertainty of the Past: Organizational Learning under Ambiguity, in: European Journal of Political Research, 3. Jg. (1975), S. $147-171$.

Mark Moody-Stuart: Putting Principles Into Practice: The Ethical Challenge to Global Business, Sao Paulo 2000, http://www.shell.com/library/ speech, [26.08.2000].

Martin, H.-P.; Schumann, H.: Die Globalisierungsfalle. Der Angriff auf Demokratie und Wohlstand, Reinbek 1996.

Martinez, J. I.; Jarillo, J. C.: Coordination Demands of International Strategies, in: Journal of International Business Studies, 1991, Nr. 3, S. 429 444. 
Martinez, J. I.; Jarillo, J. C.: The Evolution of Research on Coordination Mechansims in Multinational Corporations, in: Journal of International Business Studies, 1989, Nr. 3, S. 489 - 514.

Matzel, M.: Die Organisation des betrieblichen Umweltschutzes, Berlin 1994.

Matzel, M.; Sekul, S.: Integration des Umweltschutzes in die Profit-CenterOrganisation, in: Umweltwirtschaftsforum, 3. Jg. (1995), Nr. 3, S. 6 -11 .

Meffert, H.: Marketing: Grundlagen marktorientierter Unternehmensführung: Konzepte - Instrumente - Praxisbeispiele, 9. Aufl., Wiesbaden 2000.

Meffert, H.: Marketingwissenschaft im Wandel - Anmerkungen zur Paradigmendiskusion, in: Handelshochschule Leipzig (Hrsg.): HHLArbeitspapier Nr. 30, Leipzig 1999.

Meffert, H.: Mehrmarkenstrategien - immer die beste Option?, in: asw, Sondernummer Oktober 1999, S. $82-87$.

Meffert, H.: Ökologieorientierte Marketing- und Werbestrategie der Marke Opel, Münster, Hamburg 1990.

Meffert, H.: Globalisierungsstrategien und ihre Umsetzung im internationalen Wettbewerb, in: DBW, 49. Jg. (1989), Nr. 4, S. 445 - 463.

Meffert, H.: Voraussetzungen und Implikationen von Globalisierungsstrategien, in: Meffert, H. (Hrsg.): Strategische Unternehmensführung und Marketing, Wiesbaden 1988, S. $266-288$.

Meffert, H. (Hrsg.): Strategische Unternehmensführung und Marketing, Wiesbaden 1988.

Meffert, H.: Perspektiven des Marketing im Spannungsfeld zwischen Standardisierung und Differenzierung, in: Der Markenartikel, Heft 10, 1986, S. 442 - 450.

Meffert, H.: Marketing im Spannungsfeld zwischen weltweitem Wettbewerb und nationalen Bedürfnissen: Voraussetzungen und Implikationen von Globalisierungsstrategien, in: Meffert, $H_{\text {.; }}$ Wagner, $H$. (Hrsg.): Arbeitspapiere der Wissenschaftlichen Gesellschaft für Marketing und Unternehmensführung e. V., Nr. 27, Münster 1986.

Meffert, H.: Marketing im Spannungsfeld von weltweitem Wettbewerb und nationalen Bedürfnissen, in: ZfB, 1986, Nr. 8, S. 689 - 712.

Meffert, H.: Größere Flexibilität als Unternehmenskonzept, in: ZfbF, 37. Jg. (1985), Heft 2, S. $121-137$. 
Meffert, H.: Systemtheorie aus betriebswirtschafticher Sicht, in: Schenk, K. E. (Hrsg.): Systemanalyse in den Wirtschafts- und Sozialwissenschaften, Berlin 1971, S. 174 - 206.

Meffert, H.: Unternehmensziele, in: Schöttle, K. M. (Hrsg.): Jahrbuch des Marketing, Essen 1971, S. $22-34$.

Meffert, H.; Bolz J.: Internationales Marketing-Management, 3. Aufl., Stuttgart u. a. 1998.

Meffert, H.; Bolz, J.: Erfolgswirkungen der internationalen Marketingstandardisierung, in: Meffert, H.; Wagner, H.; Backhaus, K. (Hrsg.): Arbeitspapiere der Wissenschaftlichen Gesellschaft für Marketing und Unternehmensführung e. V., Nr. 85, Münster 1994.

Meffert, H.; Bolz, J.: Globalisierung des Marketing bei internationaler Unternehmenstätigkeit, in: Meffert, H.; Wagner, H.; Backhaus, K.: Arbeitspapiere der Wissenschaftlichen Gesellschaft für Marketing und Unternehmensführung e. V., Nr. 64, Münster 1991.

Meffert, H.; Kirchgeorg, M.: Ziele und Strategien des betrieblichen Umweltmanagements im Wandel, in: Wagner, G. R. (Hrsg.): Unternehmensführung, Ethik und Umwelt, Wiesbaden 1999, S. 491 - 508.

Meffert, H.; Kirchgeorg, M.: Marktorientiertes Umweltmanagement: Konzepte - Strategie - Implementierung mit Praxisfällen, 3. Aufl., Stuttgart 1998.

Meffert, H.; Kirchgeorg, M.: Umweltschutz als Unternehmensziel, in: Specht, G. et al. (Hrsg.): Marketing-Schnittstellen, Stuttgart 1989, S. 179 200.

Meffert, H.; Perrey, J.: Mehrmarkenstrategien - Ein Beitrag zum Management von Markenportfolios, in: Meffert, H.; Backhaus, K.; Becker, J. (Hrsg.): Arbeitspapiere der Wissenschaftlichen Gesellschaft für Marketing und Unternehmensführung e. V., Nr. 121, Münster 1998.

Meier, A.: Koordination in der Leistungsorganisation, in: Zeitschrift für betriebswirtschaftliche Forschung, 13. Jg. (1961), S. 538 - 553.

Miles, R. E.; Snow, C. C.: Organizational Strategy, Structure and Process, New York et al. 1978.

Mirow, M.: Entwicklung internationaler Führungsstrukturen, in: Macharzina, K.; Oesterle, M.-J. (Hrsg.): Handbuch Internationales Management: Grundlagen - Instrumente - Perspektiven, Wiesbaden 1997, S. 641 -662 .

Monhemius, K. Ch.: Umweltbewusstes Kaufverhalten von Konsumenten - Ein Beitrag zur Operationalisierung, Erklärung und Typologie des Verhaltens in der Kaufsituation, Frankfurt, Bern 1992. 
Monhemius, K. Ch.: Divergenzen zwischen Umweltbewusstsein und Kaufverhalten - Ansätze zur Operationalisierung und empirische Ergebnisse, in: Meffert, H. (Hrsg.): Arbeitspapiere des Instituts für Marketing, Nr. 38, Münster 1990.

Moon, D.: Environmental Aspects of Sustainable Mobility, in: European Commission (Hrsg.): Workingpaper, Version 5, 3 July 2000.

Moltke, K. v.; IISD (Hrsg.): International Environmental Management, Trade Regimes and Sustainability, Winnipeg 1996.

Morey, C. et al.: Pollution Lineup: An Environmental Ranking of Automakers, Union of Concerned Scientists (Hrsg.), March 2000.

Müller, S.; Kornmeier, M.: Grenzen der Standardisierung im Internationalen Marketing, in: GfK (Hrsg.): Jahrbuch der Absatz- und Verbrauchsforschung, 1996, Nr. 1,.S. $4-29$.

Müller-Christ, G.: Umweltmanagement: Umweltschutz und nachhaltige Entwicklung, München 2001.

Müller-Wenk, R.: Life-Cycle Impact Assessment of Road Transport Noise, in: IWÖ - Diskussionsbeitrag Nr. 77, St. Gallen 1999.

Nehrt, Ch.: Maintainability of First Mover Advantages When Environmental Regulations Differ Between Countries, in: The Academy of Management Review, Jg. 23 (1998), Nr. 1, S. $77-97$.

Nohria, N.; Ghoshal, S.: The Differentiated Network: Organizing Multinational Corporations for Value Creation, San Francisco 1997.

o. V.: Chrysler-Forschungsauto ESX 3 leicht und sparsam: Das neueste 3-LiterAuto kommt aus den USA, in: Handelsblatt, Nr. 178, 14.09.2000.

o. V.:„ICh werde das Ein-Liter-Auto bauen", in: Stern.de, 20.09.2000, http://www.stern. de/auto/berichte/ [20.09.2000].

o. V.: Flottenverbrauch von morgen - eine Fata Morgana?, in: ps-report, Nr. 28, 2000.

o. V.: http://134.146.1.137/forums/Thread.cfm?CFApp=2\&Thread ID=17\& mc= 9\# Message708 [12.08.2000].

o. V.: http://global. toyota.com/ [10.01.2001].

o. V.: http://www.bmu.gv.at/u verkehr laerm/ [06.11.00].

o. V.: http://www.corpwatch.org/greenwash/ford.html. [07.11.2000].

o. V.: http://www.corpwatch.org/trac/climate/gwford.html [07.11.2000].

o. V.: http://www.corpwatch.org/trac/greenwash/ford.html [07.11.2000]. 
O. V.: http://www.dieselnet.com/standards.html [04.10.2000].

O. V.: http://www.greenpeace.org/report98 /index.html [13.08.1999].

O. V.: http://www.uno.de/presse/1999/unic154.htm [06.05.2001].

o. V.: http://ir.volkswagen-ir.de/deutsch/. [05.05.2001].

o. V.: http://www.wbcsd.org/whatis.htm. [28.03.2000].

OECD, ECMT (Hrsg.): Final Report of the Joint OECD/ECMT Project on Urban Travel and Sustainable Development: Principle Conclusions Executive Summery, Paris 1994.

OECD (Hrsg.): OECD Guidelines for Multinational Enterprises: Review 2000: Commentaries, Paris 2000.

OECD (Hrsg.): Methodologies for environmental and trade reviews, Paris 1994, OECD/GD(94) 103.

Oechsler, W. A.: Verfahren zur Auswahl, Vorbereitung und Entsendung von Stammhausdelegierten ins Ausland, in: Macharzina, K.; Oesterle, M.-J. (Hrsg.): Handbuch Internationales Management: Grundlagen - Instrumente - Perspektiven, Wiesbaden 1997, S. 769 - 784.

oekom research (Hrsg.): Corporate Responsibility Industry Report Automobile: A Corporate Responsibility Survey of 19 Companies of the Industry, München 2000.

Olson, S. S.: International Environmental Standards Handbook, Boca Raton, London, New York, Washington D. C. 1999.

Orth, U.: Umweltcontrolling mit erweiterter Kosten-Nutzen-Analyse - dargestellt am Beispiel des Wassereinsatzes zur Pflanzenerzeugung, in: Zeitschrift für angewandte Umweltforschung, 9. Jg. (1996), Heft 2, S. $233-247$

Ossadnik, W.: Koordination internationaler Konzerne über Verrechnungspreise, in: Betriebswirtschaftliche Forschung und Praxis, 48. Jg. (1996), Heft 1, S. $123-135$.

Ostendorf, R. J.: Dynamische Ökologieführerschaft: eine Wettbewerbsstrategie gewinnorientierter Unternehmen - theoretische Darstellung und praktische Überprüfung am Beispiel der Automobilindustrie, Sternenfels 2000.

Österreichisches Umweltministerium (Hrsg.): Lärm, in: http://www.bmu.gv. at/u verkehr laerm/ [06.11.00].

Ouchi, W. G.: A conceptual Framework for the Design of Organizational Control Mechanisms, in: Management Science, 9. Jg. (1979), Nr. 6, S. 833 -848 . 
Packard, K.; Reinhardt, F.: What Every Executive Needs to Know About Global Warming, in: Harvard Business Review, 78. Jg. (2000), Nr. 4, S. $129-135$.

Paul, Th.: Globales Management von Wertschöpfungsfunktionen, Wiesbaden 1998.

Pausenberger, E.: Globalisierung der Wirtschaft und die Machteinbußen des Nationalstaats, in: Engelhard, J.; Oechsler, W. A. (Hrsg.): Internationales Management: Auswirkungen globaler Veränderungen auf Wettbewerb, Unternehmensstrategie und Märkte, Festschrift zum 60. Geburtstag von Klaus Macharzina, Wiesbaden 1999, S. 75 - 92.

Pausenberger, E.: Plädoyer für einen „Internationale Betriebswirtschaftslehre“, in: Kirch, W.; Picot, A. (Hrsg.): Die Betriebswirtschaftslehre im Spannungsfeld zwischen Generalisierung und Spezialisierung, Wiesbaden 1989, S. $382-396$.

Pausenberger, E. (Hrsg.): Internationales Management, Stuttgart 1981.

Pausenberger, E.; Noelle, G. F.: Entsendung von Führungskräften in ausländische Niederlassungen, in: ZfbF, 29. Jg. (1977), Nr. 12, S. $346-$ 366.

Perlmutter, H. V.: The Tortuous Evolution of the Multinational Corporation, in: Columbia Journal of World Business, No. 4, 1969, S. 9 - 18.

Perlmutter, H. V.: Three Conceptions of a World Enterprise, in: Revue Economique et Sociale, May 1965, S. 9-18.

Petrick, K.: Das Konzept der Zusammenführung von Qualitätsmanagement und Umweltmanagement, in: Petrick, K.; Eggert, R. (Hrsg.): Umweltund Qualitätsmanagementsysteme: Eine gemeinsame Herausforderung, München, Wien 1995.

Petrick, K.; Eggert, R. (Hrsg.): Umwelt- und Qualitätsmanagementsysteme: Eine gemeinsame Herausforderung, München, Wien 1995.

Petschow, U. et al.: Nachhaltigkeit und Globalisierung - Herausforderungen und Handlungsansätze, Berlin u. a. 1998.

Pfaffenbichler, P.: Energie- und Schadstoffbilanz bei der Herstellung und Verteilung verschiedener Verkehrsmittel bis zur Auslieferung an den Kunden, in: Institut für Verkehrsplanung und -Technik der TU Wien (Hrsg.): Beiträge zu einer ökologisch und sozial verträglichen Verkehrsplanung, 1/98, Wien 1998.

Pfahl, S.: Internationaler Handel und Umweltschutz - Zielkonflikte und Ansatzpunkte des Interessenausgleichs, Berlin u. a. 2000. 
Pfriem, R.: Unternehmenspolitik in sozialökologischen Perspektiven, Marburg 1995.

Phil Watts: Globalisation and the Corporation: when preparation meets opportunity, Budapest 2000, http://www.shell.com/library/speech/ [26.08.2000].

Picot, A.: Ein neuer Ansatz zur Gestaltung der Leistungstiefe, in: Zeitschrift für betriebswirtschaftliche Forschung, 43. Jg. (1991), Nr. 4, S. 336 357.

Pietschmann, M.: Medienstrategien aus der Sicht einer Umweltschutzorganisation, in: Fichter, K.; Schneidewind, U. (Hrsg.): Umweltschutz im globalen Wettbewerb: Neue Spielregeln für das grenzenlose Unternehmen, Berlin et al. 2000, S. $163-170$.

Plump, D.: Die Koordination des Marketings in multinationalen Unternehmungen: Systemanalyse und Ansätze zur Systemgestaltung, Bamberg 1976.

Porter, M. E.: Nationale Wettbewerbsvorteile: Erfolgreich konkurrieren auf dem Weltmarkt, Sonderausgabe, Wien 1993.

Porter, M. E.: Towards a Dynamic Theory of Strategy, in: Strategic Management Journal, Vol. 12 (1991), S. 95 - 117.

Porter, M. E.: Der Wettbewerb auf globalen Märkten: Ein Rahmenkonzept, in: Porter, M. E. (Hrsg.): Globaler Wettbewerb: Strategien der neuen Internationalisierung, Wiesbaden 1989.

Porter, M. E. (Hrsg.): Globaler Wettbewerb: Strategien der neuen Internationalisierung, Wiesbaden 1989.

Porter, M. E.: Wettbewerbsvorteile, Frankfurt a. M. 1986.

Porter, M.; v. Linde, C.: Green and Competitive. Ending the Stalemate, Harvard Business Review, 73. Jg. (1995), Nr. 5. S. 97 - 118.

Prahalad, C. K.; Hamel, G.: The Core Competence of the Corporation, in: Bartlett, C.; Ghoshal, S. (Hrsg.): Transnational Management: Text, Cases, and Readings in Cross-Border Management, Third Edition, Boston u. a. 2000, S. $358-372$.

Proff, H.: Internationale Geschäftsfeldstrategien - Entwicklung einer umfassenden Klassifikation und Anwendung auf die Automobilindustrie, in: Proff, H.; Proff, H. V. (Hrsg.): Strategien für die Automobilindustrie: Ansatzpunkte im strategischen Management und in der Industriepolitik, Wiesbaden 1998, S. $103-124$. 
Proff, H.; Proff, H. V. (Hrsg.): Strategien für die Automobilindustrie: Ansatzpunkte im strategischen Management und in der Industriepolitik, Wiesbaden 1998.

Proff, H.; Proff, H. V.: Struktur und Entwicklung der weltweiten Automobilindustrie, in: Proff, H.; Proff, H. V. (Hrsg.): Strategien für die Automobilindustrie: Ansatzpunkte im strategischen Management und in der Industriepolitik, Wiesbaden 1998, S. 83 - 102.

Pugh, D. S. et al.: The Context of Organization Structures, in: Administrative Science Quarterly, 14. Jg. (1969), S. $91-114$.

Rall, W.: Der Netzwerkansatz als Alternative zum zentralen und hierarchisch gestützten Management der Mutter-Tochter-Beziehungen, in: Macharzina, K.; Oesterle, M.-J. (Hrsg.): Handbuch Internationales Management: Grundlagen - Instrumente - Perspektiven, Wiesbaden 1997, S. $663-680$.

Reichmann, Th. (Hrsg.): DV-gestütztes Unternehmens-Controlling, München 1993.

Reichmann, Th.: Trends und Entwicklungen im Euro-Controlling, in: Reichmann, Th. (Hrsg.): DV-gestütztes Unternehmens-Controlling, München 1993, S. $1-15$.

Remer, A.: Organisationslehre, 4. Aufl., Bayreuth 1996.

Reuter, G. C.: Nationale Regulierungsunterschiede als komparativer Wettbewerbsvorteil oder Wettbewerbsverzerrung? Eine Studie zur Ökodumpingdiskussion als Problem der Welthandelsordnung, Bamberg 1996.

Rugman, A. M.; Verbeke, A.: Corporate Strategies and Environmental Regulations, in: Strategic Management Journal, 19. Jg. (1998), S. $363-375$.

Rugmann, A. M.; Verbeke, A.: Corporate Strategy and International Environmental Policy, in: Journal of International Business Studies, 29. Jg. (1998), Nr. 4, S. $819-834$.

Sanchez, R.: Strategic Product Creation: Managing New Interactions of Technology, Markets and Organizations, in: European Management Journal, 14. Jg., Nr. 2, 1996, S. 121-138.

Sauernheimer, K.; Ködding, G.: Internationale Aspekte der Umweltökonomie, in: Junkernheinrich, M.; Klenner, P.; Wagner, G. R. (Hrsg.): Handbuch zur Umweltökonomie, Berlin 1995. 
Schaltegger, S.; Figge, F.: Ökologisierung von Finanzmärkten: Neu Steuerungssignale für Unternehmen?, in: Fichter, K.; Schneidewind, U. (Hrsg.): Umweltschutz im globalen Wettbewerb - Neue Spielregeln für das grenzenlose Unternehmen, Berlin u. a. 2000, S. 103-110.

Schaltegger, S.; Figge, F.: Umweltmanagement und Shareholder Value in den Kriterien des Unternehmenserfolgs, in: Koslowski, P. (Hrsg.): Shareholder Value und die Kriterien des Unternehmenserfolgs. Ethische Ökonomie. Beiträge zur Wirtschaftsethik und Wirtschaftskultur, Heidelberg 1999, S. $201-227$.

Schein, E. H.: Organizational Culture and Leadership - A Dynamic View, San Francisco et al. 1985.

Schenk, K. E. (Hrsg.): Systemanalyse in den Wirtschafts- und Sozialwissenschaften, Berlin 1971.

Schmalenbach-Gesellschaft/Deutsche Gesellschaft für Betriebswirtschaft e. V. (Hrsg.): Internationalisierung der Wirtschaft, Stuttgart 1993.

Schmid, S.: Multikulturalität in der internationalen Unternehmung: Konzepte Reflexionen - Implikationen, Wiesbaden 1996.

Schmitt, A.: Die psychologischen Rahmenbedingungen des Automobilmarketing, in: Hünerberg, R. et al. (Hrsg.): Internationales Automobilmarketing: Wettbewerbsvorteile durch marktorientierte Unternehmensführung, Wiesbaden 1995, S. 61 - 82.

Schneidewind, U.; Dyllick, Th.: Ökologisches Benchmarking, in: DBW, 57. Jg. (1997), Nr. 4, S. $569-572$.

Scholz, C.: Die richtige Kulturstrategie schafft Synergien, in: Personalwirtschaft, 20. Jg (1993), S. $31-34$.

Schönert, O.: Integrationspotentiale von Informations- und Kommunikationstechnologien für internationale Unternehmungen, in: Kutschker, $\mathrm{M}$. (Hrsg.): Integration in der internationalen Unternehmung, Wiesbaden 1998.

Schöttle, K. M. (Hrsg.): Jahrbuch des Marketing, Essen 1971.

Schreyögg, G.: Organisation: Grundlagen moderner Organisationsgestaltung, Wiesbaden 1996.

Schulz, E.; Schulz, W. (1994): Ökologiemanagement: So nutzen Sie den Umweltschutz im Betrieb, München 1994.

Schweitzer, M., Friedl, B.: Beitrag zu einer umfassenden Controlling-Konzeption, in: Spremann, K.; Zur, E. (Hrsg.): Controlling, Wiesbaden 1992, S. $141-167$. 
Seidel, E.: Instrumente umweltorientierter Unternehmensführung im internationalen Kontext, in: Kramer, M.; Reichel, M. (Hrsg.): Internationales Umweltmanagement und europäische Integration: Deutschland, Polen und Tschechien im Vergleich, Wiesbaden 1998, S. 53 - 70.

Serapio, M. G. jr.: Macro-Micro Analyses of Japanese Direct $R$ \& $D$ Investments in the U.S. Automotive and Electronics Industries, in: Management International Review, 1993, Nr. 3, S. $209-225$.

Shoham, A., Albaum, G.: The Effects of Transfer of Marketing Methods on Export Performance: An Empirical Examination, in: International Business Review, 3. Jg. (1994), Nr. 3, S. 219 - 241.

Shoham, A.: Global Marketing Standardization, in: Journal of Global Marketing, 9. Jg. (1995), Nr. 1 / 2, S. 91 - 119.

Simon, H. (Hrsg.): Wettbewerbsvorteile und Wettbewerbsfähigkeit, Stuttgart 1988.

Simon, H. A.; Guetzkow, H. et al.: Centralization vs. Decentralization in Organizing the Controller's Department, New York 1954.

Spremann, K.; Zur, E. (Hrsg.): Controlling, Wiesbaden 1992.

Steffenhagen, H.: Konflikt und Koordination in Distributionssystemen, Münster 1972.

Steger, U.: Ist die Deregulierung im Umweltschutz vermeidbar?, in: Akademie der Politischen Bildung (Hrsg.): Umweltschutz zwischen Globalisierung und Deregulierung - Die Grenzen des Rechts in der Risikogesellschaft, Bonn 1998, S. $35-44$.

Steger, U.; Winter, M.: Strategische Früherkennung zur Antizipation ökologisch motivierter Marktveränderungen, in: DBW, 59 Jg. (1996).

Steinmann, H.; Scherer, A. G.: Die multinationale Unternehmung als moralischer Aktor - Bemerkungen zu einigen normativen Grundlagenproblemen des interkulturellen Managements, in: Engelhard, J. (Hrsg.): Interkulturelles Management: Theoretische Fundierung und funktionsbereichsspezifische Konzepte, Wiesbaden 1997.

Stirn, A.: Wunschdenken oder Wunderauto?, in: Spiegel Online-Auto, 06.10.2000, http://www.spiegel.de/auto/showroom/0,1518,97076, 00.html [07.10. 2000].

Stitzel, M.: Ökologisch orientierte Unternehmensführung - Hemmung der Förderung der Wettbewerbsfähigkeit der Unternehmung, in: Dichtl, E.; Gerke, W.; Kieser A. (Hrsg.): Innovation und Wettbewerbsfähigkeit, Wiesbaden 1987, S. 377 - 397. 
Stocker, S.: Mitarbeiterorientierung als Basis eines umweltbewussten Unternehmens, in: Winter, G.: Das Umweltbewusste Unternehmen: Die Zukunft beginnt heute, 6. Aufl., München 1998.

Straubhaar, T.; Wyss M.: Ökologisch bedingte Standortarbitrage? Ausländische Direktinvestitionen in Nicht-OECD-Ländern, in: ZfU 1/94, S. $98-121$.

Strebel, H.: Zielsysteme und Zielforschung, in: Die Betriebswirtschaft, 41. Jg. (1981), Nr. 3, S. 457 - 475.

Strebel, H.: Umwelt und Betriebswirtschaftslehre, Berlin 1980.

Sustainable Asset Management (Hrsg.): Sustainability in der Automobilindustrie: Das Automobil im nächsten Jahrtausend, Zürich 1998.

Tannenbaum, R.; Schmidt, W.: How to Choose a Leadership Pattern, in: Litterer, J. A. (Hrsg.): Leadership and Organization, New York, u. a. S. $122-130$.

Teece, D. J.; Pisano, G.; Shuen, A.: Dynamic Capabilities and Strategic Management, in: Strategic Management Journal, 18. Jg (1997), Nr. 7, S. $509-533$.

Teufel, D., et al.: Externe Gesundheitskosten des Verkehrs in der Bundesrepublik Deutschland - im Auftrag von Greenpeace Deutschland, in: Umwelt- und Prognose-Institut e. V. (Hrsg.), UPI-Bericht Nr. 43, 2. Aufl., Heidelberg 1999.

Thimme, P. M.: Der Wettbewerb zwischen EG-Öko-Audit-Verordnung und DIN ISO 14.001, in: Doktoranden-Netzwerk Öko-Audit e. V. (Hrsg.): Umweltmanagementsysteme zwischen Anspruch und Wirklichkeit Eine interdisziplinäre Auseinandersetzung mit der EG-Öko-AuditVerordnung und der DIN ISO 14.001, Berlin u. a. 1998, S. 265 286.

Thompson, J. D.: Organizations in Action, New York u. a. 1967.

Tischler, K.: Ökologische Betriebswirtschaftslehre, München u. a. 1996.

Töpfer, A.: Planungs- und Kontrollsysteme industrieller Unternehmungen: Eine theoretische, technologische und empirische Analyse, Berlin 1976.

Troge, A.: Schritte zu einer nachhaltigen Weltumweltordnung, in: Fichter, K. Schneidewind, U. (Hrsg.): Umweltschutz im globalen Wettbewerb Neue Spielregeln für das grenzenlose Unternehmen, Berlin u. a. 2000, S. $15-22$.

Ulrich, H.: Die Unternehmung als produktives soziales System: Grundlagen der allgemeinen Unternehmungslehre, Bern 1968. 
United Nations (Hrsg.): A Compact for the New Century, http://www. unglobalcompact.org/GC/unweb.nsf/webprintview/thenine.htm [29. 11.2000].

United Nations (Hrsg.): Activities of the Transnational Corporations and Management Divisions and its Joint Units, New York 1993.

United Nations (Hrsg.): Environmental Management in Transnational Corporations: Report on the Benchmark Corporate Environmental Survey, New York 1993.

United Nations (Hrsg.): Rio Declaration on Environment and Development, Rio de Janeiro 1992.

United Nations (Hrsg.): Report of the United Nations Conference on the Human Environment, Stockholm 1972.

UPI (Hrsg.): Folgen einer globalen Motorisierung, UPI-Bericht Nr. 35, Heidelberg 1995.

VCD (Hrsg.): Zehn Jahre Auto-Umweltliste des VCD: Eine Erfolgsbilanz, Bonn 2000, http://www.vcd.org/themen/ auli10.html [31.08.2000].

VDA (Hrsg.): Auto 2000: Jahresbericht des Verbands der Automobilindustrie e. V., Frankfurt a. M. 2000.

Volkswagen (Hrsg.): Jahresbericht des produktionsbezogenen Umweltschutzes 1999/2000, Wolfsburg 2000.

Volkswagen (Hrsg.): Umweltbericht 1999/2000, Wolfsburg 1999.

Volkswagen (Hrsg.): Umweltbrief, Nr. 11/99, Wolfsburg 1999.

Volkswagen (Hrsg.): Sachkundige für Umweltschutz: Handbuch, Stand 6/99, Wolfsburg 1999.

Volkswagen (Hrsg.): Jahresbericht des fertigungsbezogenen Umweltschutzes 1998/1999, Wolfsburg 1999.

Volkswagen (Hrsg.): Geschäftsbericht 1993, Wolfsburg 1994.

Volkswagen (Hrsg.): Geschäftsbericht 1991, Wolfsburg 1992.

Volkswagen (Hrsg.): Geschäftsbericht 1988, Wolfsburg 1989.

Volkswagen, imug (Hrsg): Jahrbuch Umwelt und Verkehr 1999: Diskussionen und Ereignisse des Jahres 1999 im Themenfeld Umwelt und Verkehr, interne Studie, Wolfsburg 2000.

Wagner, G. R. (Hrsg.): Unternehmensführung, Ethik und Umwelt, Wiesbaden 1999. 
Wagner, G. R.: Betriebswirtschaftliche Umweltökonomie, Stuttgart 1997.

Walley, N.; Whitehead, B.: 'It's not easy being green', Harvard Business Review, 72. Jg. (1994), Nr. 3, S. 46-52.

Walter, I. (Hrsg.): Studies in International Environmental Economics, New York u. a. 1976

Waltermann, B.: Internationale Markenpolitik und Produktpositionierung: markenpolitische Entscheidungen im europăischen Automobilmarkt, Wien 1989.

Warhurst, A.: Corporate Citizenship and Corporate Social Investment: Drivers of Tri-Sector Partnerships, in: Journal of Corporate Citizenship, Nr. 1 (2001), S. $57-73$.

Weber, J., Schäffer, U.: Balanced Scorecard - Gedanken zur Einordnung des Konzeptes in das bisherige Controlling-Instrumentarium, WHUForschungspapier 1998.

Welge, M. K.; Berg, N.: Public Affairs-Management in Multinationalen Unternehmungen, in: Welge, M. K. (Hrsg.): Arbeitspapiere des Lehrstuhls für Unternehmensführung, Nr. 24, Dortmund 1998.

Welge, M. K. (Hrsg.); Böttcher, R.; Paul, Th.: Das Management globaler Geschäfte: Grundlagen, Analysen, Handlungsempfehlungen, München, Wien 1998.

Welge, M. K.: Die effiziente Gestaltung der Mutter-Tochter-Beziehungen in deutschen multinationalen Unternehmungen, in: Pausenberger, E. (Hrsg.): Internationales Management, Stuttgart 1981, S. 45 - 77.

Welge, M. K.: Management in deutschen multinationalen Unternehmungen: Ergebnisse einer empirischen Untersuchung, Stuttgart 1980.

Clarke R. A. et al.: The challenge of going green, in: Harvard Business Review, 72. Jg. (1994), Nr. 4, S. $37-50$.

Wermeyer, F.: Marketing und Produktion - Schnittstellenmanagement aus unternehmensstrategischer Sicht, Wiesbaden 1994.

Whisler, T. L.: The Impact of Computers on Organizations, Washington, London 1970.

Whitelock, J.; Pimblett, C.: The Standardisation Debate in International Marketing, in: Journal of Global Marketing, 10. Jg. (1997), Nr. 3, S. $45-65$.

Wied-Nebbing, S.: Wohlfahrtswirkungen des Freihandels bei negativen externen Effekten, in: WiSt, Nr. 11, 1997, S. 574 - 580.

Winkler, G.: Koordination in strategischen Netzwerken, Wiesbaden 1999. 
Winter, G. (Hrsg.): Das Umweltbewusste Unternehmen: Die Zukunft beginnt heute, 6. Aufl., München 1998.

Winter, J. (Hrsg.): Umweltmanagement, Stuttgart 1997.

Wirtschaft und Umwelt e. V. (Hrsg.): Welthandel und Umweltschutz - Wie handeln wir ökologisch?, Münster 1996.

Wittmann, W. et al. (Hrsg.): Handwörterbuch der Betriebswirtschaft, 5. Aufl., Stuttgart 1993.

Wöhler, K. (Hrsg.): Organisationsanalyse, Stuttgart 1978.

Wolf, J.: Internationales Personalmanagement: Kontext - Koordination - Erfolg, Wiesbaden 1994.

Wolter, F.: Umweltmanagement in Europa - eine empirische Untersuchung auf Grundlage des Europäischen Umweltmanagement-Barometers (EBEB), in: Meffert, H.; Backhaus, K.; Becker, J. (Hrsg.): Arbeitspapiere der Wissenschaftlichen Gesellschaft für Marketing und Unternehmensführung e. V., Nr. 130, Münster 1999.

Womack, J. P.: Die zweite Revolution in der Automobilindustrie: Konsequenzen aus der weltweiten Studie aus dem Massachusetts Institute of Technology, 6. Aufl., Frankfurt a. M., New York 1992.

World Business Council for Sustainable Development (Hrsg.): Stakeholder, http://www.wbcsd.org/sustain 1.htm-stakeholder [13.09.2000].

World Commission on Environment and Development (Hrsg.): Our common future, Oxford 1987.

World Ressource Institute (Hrsg.): Global Environmental Trends, 1997, http://www.wri.org/wri/ [29.11.00].

Wruk, H.-P.: Standardisierte Grundlagen für die Umsetzung von Umweltmanagementsystemen, in: Winter, G. (Hrsg.): Das umweltbewusste Unternehmen: Die Zukunft beginnt heute, 6. Aufl., München 1998, S. $833-844$.

Zeithaml, V. A.; Varadarajan, P., Zeithaml, C.: The Contingency Approach: It's Foundations and Relevance to Theory Building and Research in Marketing, in: European Journal of Marketing, Vol. 22 (1988), Nr. 8, S. $37-64$.

Zerfaß, A.: Machen Medien Märkte? - Zur Rolle der Medien im ökologischen Wettbewerb, in Fichter, K.; Schneidewind, U. (Hrsg.): Umweltschutz im globalen Wettbewerb: Neue Spielregeln für das grenzenlose Unternehmen, Berlin et al. 2000, S. 171-176. 


\section{SCHRIFTEN ZUM MARKETING}

Band 1 Friedrich Wehrle: Strategische Marketingplanung in Warenhäusern. Anwendung der Portfolio-Methode. 1981. 2. Auflage. 1984.

Band 2 Jürgen Althans: Die Übertragbarkeit von Werbekonzeptionen auf internationale Märkte. Analyse und Exploration auf der Grundlage einer Befragung bei europaweit tätigen Werbeagenturen. 1982.

Band 3 Günter Kimmeskamp: Die Rollenbeurteilung von Handelsvertretungen. Eine empirische Untersuchung zur Einschätzung des Dienstleistungsangebotes durch Industrie und Handel. 1982.

Band 4 Manfred Bruhn: Konsumentenzufriedenheit und Beschwerden. Erklärungsansätze und Ergebnisse einer empirischen Untersuchung in ausgewählten Konsumbereichen. 1982.

Band 5 Heribert Meffert (Hrsg.): Kundendienst-Management. Entwicklungsstand und Entscheidungsprobleme der Kundendienstpolitik. 1982.

Band 6 Ralf Becker: Die Beurteilung von Handelsvertretern und Reisenden durch Hersteller und Kunden. Eine empirische Untersuchung zum Vergleich der Funktionen und Leistungen. 1982.

Band 7 Gerd Schnetkamp: Einstellungen und Involvement als Bestimmungsfaktoren des sozialen Verhaltens. Eine empirische Analyse am Beispiel der Organspendebereitschaft in der Bundesrepublik Deutschland. 1982.

Band 8 Stephan Bentz: Kennzahlensysteme zur Erfolgskontrolle des Verkaufs und der MarketingLogistik. Entwicklung und Anwendung in der Konsumgüterindustrie. 1983.

Band 9 Jan Honsel: Das Kaufverhalten im Antiquitätenmarkt. Eine empirische Analyse der Kaufmotive, ihrer Bestimmungsfaktoren und Verhaltenswirkungen. 1984.

\section{SCHRIFTEN ZU MARKETING UND MANAGEMENT}

Band 10 Matthias Krups: Marketing innovativer Dienstleistungen am Beispiel elektronischer Wirtschaftsinformationsdienste. 1985.

Band 11 Bernd Faehsler: Emotionale Grundhaltungen als Einflußfaktoren des Käuferverhaltens. Eine empirische Analyse der Beziehungen zwischen emotionalen Grundhaltungen und ausgewählten Konsumstrukturen. 1986.

Band 12 Ernst-Otto Thiesing: Strategische Marketingplanung in filialisierten Universalbanken. Integrierte Filial- und Kundengruppenstrategien auf der Grundlage erfolgsbeeinflussender Schlüsselfaktoren. 1986.

Band 13 Rainer Landwehr: Standardisierung der internationalen Werbeplanung. Eine Untersuchung der Prozeßstandardisierung am Beispiel der Werbebudgetierung im Automobilmarkt. 1988.

Band 14 Paul-Josef Patt: Strategische Erfolgsfaktoren im Einzelhandel. Eine empirische Analyse am Beispiel des Bekleidungsfachhandels. 1988. 2. Auflage. 1990.

Band 15 Elisabeth Tolle: Der Einfluß ablenkender Tätigkeiten auf die Werbewirkung. Bestimmungsfaktoren der Art und Höhe von Ablenkungseffekten bei Rundfunkspots. 1988.

Band 16 Hanns Ostmeier: Ökologieorientierte Produktinnovationen. Eine empirische Analyse unter besonderer Berücksichtigung ihrer Erfolgseinschätzung. 1990.

Band 17 Bernd Büker: Qualitätsbeurteilung investiver Dienstleistungen. Operationalisierungsansätze an einem empirischen Beispiel zentraler EDV-Dienste. 1991.

Band 18 Kerstin Ch. Monhemius: Umweltbewußtes Kaufverhalten von Konsumenten. Ein Beitrag zur Operationalisierung, Erklärung und Typologie des Verhaltens in der Kaufsituation. 1993. 
Band 19 Uwe Schürmann: Erfolgsfaktoren der Werbung im Produktlebenszyklus. Ein Beitrag zur Werbewirkungsforschung. 1993.

Band 20 Ralf Birkelbach: Qualitätsmanagement in Dienstleistungscentern. Konzeption und typenspezifische Ausgestaltung unter besonderer Berücksichtigung von Verkehrsflughäfen. 1993.

Band 21 Simone Frömbling. Zielgruppenmarketing im Fremdenverkehr von Regionen. Ein Beitrag zur Marktsegmentierung auf der Grundlage von Werten, Motiven und Einstellungen. 1993.

Band 22 Marcus Poggenpohl: Verbundanalyse im Einzelhandel auf der Grundlage von Kundenkarteninformationen. Eine empirische Untersuchung von Verbundbeziehungen zwischen Abteilungen. 1994.

Band 23 Kai Bauche: Segmentierung von Kundendienstleistungen auf investiven Märkten. Dargestellt am Beispiel von Personal Computern. 1994.

Band 24 Ewald Werthmöller: Räumliche Identität als Aufgabenfeld des Städte- und Regionenmarketing. Ein Beitrag zur Fundierung des Placemarketing. 1995.

Band 25 Nicolaus Müller: Marketingstrategien in High-Tech-Märkten. Typologisierung, Ausgestaltungsformen und Einflußfaktoren auf der Grundlage strategischer Gruppen. 1995.

Band 26 Nicolaus Henke: Wettbewerbsvorteile durch Integration von Geschättsaktivitäten. Ein zeitablaufbezogener wettbewerbsstrategischer Analyseansatz unter besonderer Berücksichtigung des Einsatzes von Kommunikations- und Informationssystemen (KIS). 1995.

Band 27 Kai Laakmann: Value-Added Services als Profilierungsinstrument im Wettbewerb. Analyse, Generierung und Bewertung. 1995.

Band 28 Stephan Wöllenstein: Betriebstypenprofilierung in vertraglichen Vertriebssystemen. Eine Analyse von Einflußfaktoren und Erfolgswirkungen auf der Grundlage eines Vertragshändlersystems im Automobilhandel. 1996.

Band 29 Michael Szeliga: Push und Pull in der Markenpolitik. Ein Beitrag zur modellgestützten Marketingplanung am Beispiel des Reifenmarktes. 1996.

Band 30 Hans-Ulrich Schröder: Globales Produktmanagement. Eine empirische Analyse des Instrumenteeinsatzes in ausgewählten Branchen der Konsumgüterindustrie. 1996.

Band 31 Peter Lensker: Planung und Implementierung standardisierter vs. differenzierter Sortimentsstrategien in Filialbetrieben des Einzelhandels. 1996.

Band 32 Michael H. Ceyp: Ökologieorientierte Profilierung im vertikalen Marketing. Dargestellt am Beispiel der Elektrobranche. 1996.

Band 33 Mark Unger: Die Automobil-Kaufentscheidung. Ein theoretischer Erklärungsansatz und seine empirische Überprüfung. 1998.

Band 34 Ralf Ueding: Management von Messebeteiligungen. Identifikation und Erklärung messespezifischer Grundhaltungen auf der Basis einer empirischen Untersuchung. 1998.

Band 35 Andreas Siefke: Zufriedenheit mit Dienstleistungen. Ein phasenorientierter Ansatz zur Operationalisierung und Erklärung der Kundenzufriedenheit im Verkehrsbereich auf empirischer Basis. 1998.

Band 36 Irene Giesen-Netzer: Implementierung von Rücknahme- und Recyclingsystemen bei Gebrauchsgütern. 1998.

Band 37 Frithjof Netzer: Strategische Allianzen im Luftverkehr. Nachfragerorientierte Problemfelder inrer Gestaltung. 1999.

Band 38 Silvia Danne: Messebeteiligungen von Hochschulen. Ziele und Erfolgskontrolle. 2000.

Band 39 Martin Koers: Steuerung von Markenportfolios. Ein Beitrag zum Mehrmarkencontrolling am Beispiel der Automobilwirtschaft. 2001. 
Band 40 Frank Wolter: Koordination im internationalen Umweltmanagement. Dargestellt an Beispielen aus der Automobilindustrie. 2002. 


\section{Umweltrechtssicherheit für Unternehmen}

\section{Management produktbezogener umweltrechtlicher Infor- mationen für die Produktentwicklung}

Frankfurt/M., Berlin, Bern, Bruxelles, New York, Oxford, Wien, 2001. 220 S., 34 Abb., 8 Tab.

ISBN 3-631-38071-2 · br. DM 69.- /€ 35.30*

Die Gewährleistung von Umweltrechtssicherheit für Produkte stellt eine große und zunehmende Herausforderung für Unternehmen dar. Dennoch ist diese Problematik in der Managementliteratur bisher kaum behandelt worden. Dieses Buch schließt diese Lücke. Tobias Hahn hat ein umfassendes und praxistaugliches Konzept erarbeitet, wie Unternehmen Umweltrechtssicherheit managen können und dafür ein detailliertes Instrumentarium entwickelt. In Zusammenarbeit mit einem Unternehmen der Maschinenbaubranche entstand ein Rechtsinformationssystem, mit dem die Entscheidungsträger in der Produktentwicklung gezielt mit den relevanten Informationen zur Gewährleistung von Umweltrechtssicherheit versorgt werden können. Dieses Buch stellt das Konzept und seine theoretische Fundierung dar, zeigt Schritt für Schritt den Aufbau und den Ablauf des Rechtsinformationssystems auf, legt Zuständigkeiten fest und definiert alle dafür notwendigen Prozesse.

Aus dem Inhalt: Theoretische Grundlagen - Einsatz produktbezogener umweltrechtlicher Informationen in der Produktentwicklung · Ausgestaltung der Informationsinstrumente - Aufbau und Implementierung des Informationssystems · Schlußbetrachtung und Ausblick

Frankfurt/M - Berlin · Bern - Bruxelles - New York · Oxford · Wien

Auslieferung: Verlag Peter Lang AG

Jupiterstr. 15, CH-3000 Bern 15

Telefax (004131) 9402131

*inklusive der in Deutschland gültigen Mehrwertsteuer

Preisänderungen vorbehalten

Homepage http://wuw.peterlang.de 